\title{
Extending the Range of Low Energy Electron Diffraction (LEED) Surface Structure Determination: Co-adsorbed Molecules, Incommensurate Overlayers and Alloy Surface Order Studied by New Video and Electron Counting LEED Techniques
}

\author{
DISCLAIMER
}

This report was prepared as an account of work sponsored by an agency of the United States Government. Neither the United States Government nor any agency thereof, nor any of their employees, makes any warranty. express or implied, or assumes any lepal liability or responsibility for the accuracy, completeness, or usefulness of any information, apparatus, product, or process disclosed, or represents that its use would not infringe privately owned rights. Refer. erice herein to any specific commercial oroduct, process, or service by trade name, trademark. manufacturer, or otherwise does not necessarily constitute or imply its endorsement, recommendation, or favoring by the United States Government or any agency thereof. The views and opinions of authore expressed herein do not necessarily state or reflect those of the United States Government or any agency thereor.

\section{David Franklin Ogletree}

Ph.D. Thesis (1986)

Department of Physics and

Materials and Chemical Sciences Division

Lawrence Berkeley Laboratory

University of California, Berkeley, CA 94720

The United States Department of Energy has the right to use this thesis for any purpose whatsoever including

the right to reproduce all or any part thereol. 
Extending the Range of Low Energy Electron Diffraction (LEED) Surface Structure Determination: Co-adsorbed Molecules, Incommensurate Overlayers and Alloy Surface Order Studied by New Video and Electron Counting LEED Techniques

copyright 1986

David Franklin Ogletree 


\section{Extending the Range of Low Energy Electron Diffraction (LEED) Surface Structure Determination: Co-adsorbed Molecules, Incommensurate Overlayers and Alloy Surface Order Studied by New Video and Electron Counting LEED Techniques}

\section{David Franklin Ogletree}

\section{ABSTRACT}

LEED multiple scattering theory is briefly summarized, and aspects of electron scattering with particular significance to experimental measurements such as electron beam coherence, instrument response and phonon scattering are analyzed. Diffuse LEED experiments are discussed.

New techniques that enhance the power of LEED are described, including a real-time video image digitizer applied to LEED intensity measurements, along with computer programs to generate I-V curves. The first electron counting LEED detector using a "wedge and strip" position sensitive anode and digital electronics is described. This instrument uses picoampere incident beam currents, and its sensitivity is limited only by statistics and counting times.

Structural results on new classes of surface systems are presented. The structure of the $\mathrm{c}(4 \times 2)$ phase of carbon monoxide adsorbed on $\mathrm{Pt}(111)$ has been determined, showing that carbon monoxide molecules adsorb in both top and bridge sites, $1.85 \pm 0.10 \AA$ and $1.55 \pm 0.10 \AA$ above the metal surface, respectively. LEED patterns are analyzed to show that domain wall formation, not uni-axial 
compression, is the mechanism of phase transitions for carbon monoxide adsorbed on $\mathrm{Pt}(111)$.

The structure of an incommensurate graphite overlayer on $\operatorname{Pt}(111)$ is analyzed. The graphite layer is $3.70 \pm 0.05 \AA$ above the metal surface, with intercalated carbon atoms located $1.25 \pm 0.10 \AA$ above hollow sites supporting it. This is the second incommensurate overlayer to be analyzed by LEED.

The $(2 \sqrt{3} \times 4)$-rectangular phase of benzene and carbon monoxide coadsorbed on $\mathrm{Pt}(111)$ is analyzed. Benzene molecules adsorb in bridge sites parallel to and $2.10 \pm 0.10 \AA$ above the surface. The carbon ring is expanded, with an average $C$ $\mathrm{C}$ bond length of $1.72 \pm 0.15 \AA$. The carbon monoxide molecules also adsorb in bridge sites. This study, together with complementary studies on $\operatorname{Rh}(111)$, is the first structure determination for coadsorbed molecules or for aromatic molecules.

The structure of the $(\sqrt{3} \times \sqrt{3})$ reconstruction on the (111) face of the $\alpha$ $\mathrm{CuAl}$ alloy has been determined. Al atoms are substituted in $1 / 3$ of the top layer sites in the bulk copper lattice, without buckling or changes in the interlayer spacing. This is the first reconstructed alloy surface to be analyzed by LEED.

November 18, 1986 
"Concern for man himself and his fate must always form the chicf interest of all technical endeavors, concern for the great unsolved problems of the organization of labor and the distribution of goods - in order that the creations of our minds should be a blessing and not a curse to mankind. Never forget this in the midst of your diagrams and equations."

- A. Einstein 


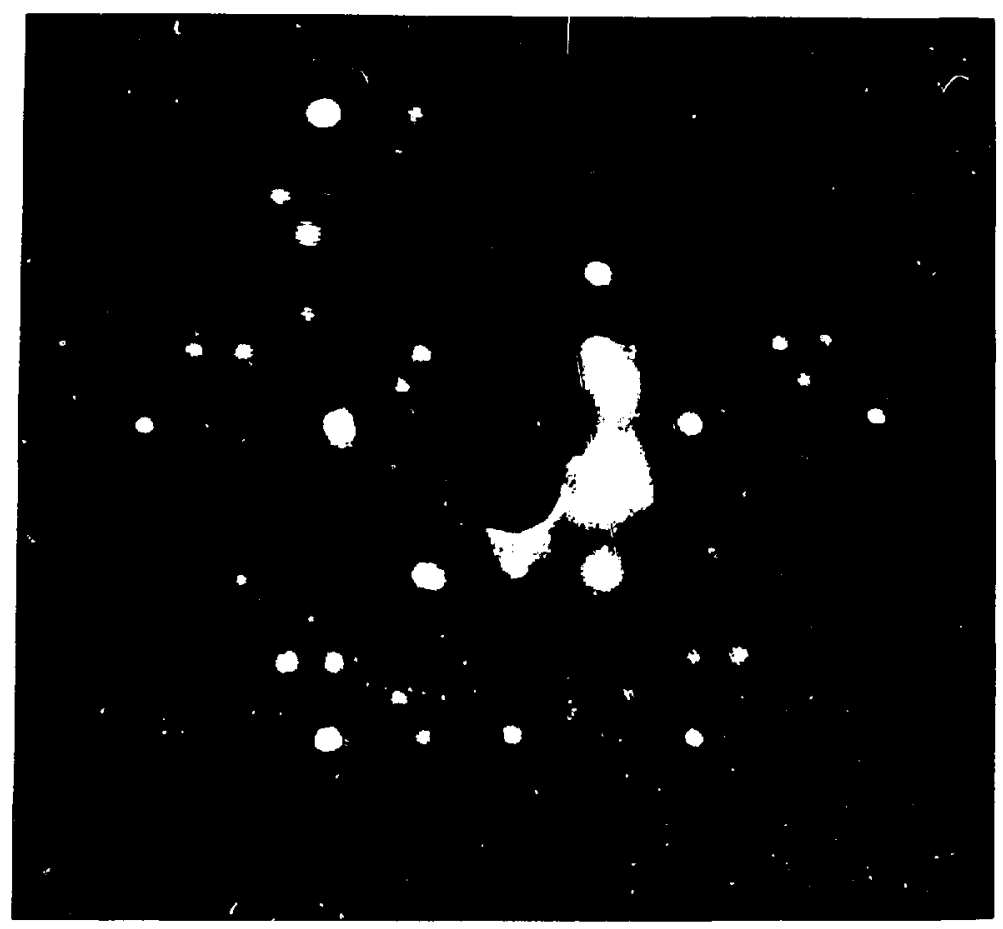

XBB 864-3004

\section{$(2 \sqrt{3} \times 4)$ rect}

Benzene and Carbon Monoxide Coadsorbed on $\operatorname{Pt(111)}$ 


\section{Acknowledgements}

I would like to thank Gabor Somorjai for his enthusiasm which first introduced me to surface seience, and for providing a creative and stimulating environment for my work in Berkeley. The constant exchange with the students and visitors from around the world who are drawn to Berkeley added a rich dimension to my experience as a graduate student. I would also like to express my appreciation to Michel Van Hove for his support and encouragement from my first day at LBL, for teaching me LEED theory and for stimulating discussions of surface science and of other ideas.

I am happy to acknowledge the help and friendship of many past and current members of the Somorjaj group. In particular I want to thank Eric Garfunkel and Roland Koestner for first intrcducing me to the surface characterization, vacuum techniques and LEED, and Lin Rong Fu and Greg Blackman for sharing the problems and successes of the LEED work.

Much of the scientific work described here was done in collaboration. Michel Van Hove was deeply involved in all of the structure determination work, and he performed all of the theoretical LEED structure calculations discussed in chapters 10, 11 and 13. Hu $\mathrm{Zi} \mathrm{Pu}$ also participated in the LCC.D calculations for the incommensurate graphite system described in chapter 12. Matthew Mate performed the HREELS measurements which helped to unravel the benzene coadsorption system, described in chapter 11. Ron Baird prepared and did the initial surface characterization for the the copper aluminum alloy single crystals used in 
the structure determination experiments described in chapter 13.

The development of the video and digital LEED systems would not have been possible without the active ard enthusiastic participation of Joe Katz of LBL's department of Instrument Science and Engineering. He is responsible for the design and construction of the digital LEED digitizer, amplifiers and power supplies, and his contribution of nights and weekends help uravel the mysteries of position-sensitive detection. Discussions with John Barton and Steve Robey were invaluable in getting the position sensitive detection to work. They were working in parallel to develop a parabolic mirror position-sensitive electron energy analyzer. Steve Robey worked out the right way (and several other ways) to produce wedge and strip anodes, and also figured out the significance of capacitive coupling for the wedge and strip anode.

One of the things that makes LBL a pleasant and productive place to work is the superlative technical support. In particular I would like to express my appreciation to Keith Franck, Weyland Wong and Dan Coulomb for mechanical construction and consultation, to Mike Press for maintenance and coddling of my electronics and to the real-time systems group for computer support and arcane iore. I would like to thank Sandra Stewart for shielding me from the pitfalls of purchasing and property accounting and the staff of the photolab for prompt and efficient responses to many requests.

This work was supported in part by the Director, Office of Energy Research, Office of Basic Energy Sciences, Materials Science Division, of the U.S. 
Department of Energy under Contract Number DE-AC03-76SF00098. Supercomputer time was also provided by the Office of Energy Research of the Department of Energy.

I would also like to acknowledge the patient assistance of 4.2 BSD UNIX in the preparation of this thesis. 


\section{Table of Contents}

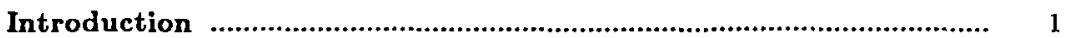

\section{Part I - The Theory and Practice of LEED}

Chapter 1: Surface Structure Determination …................................. 5

1.1 Surface Science ............................................................................ 5

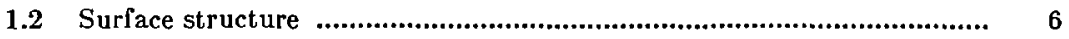

1.3 Structure determination .................................................................... 8

1.4 Complementary techniques ............................................................. 10

1.5 Structure sensitive techniques ............................................................. 11

1.5.1 Electron diffraction techniques ............................................. 11

1.5.2 Photoelectron Diffraction ........................................................ 13

1.5.3 Fine Structure Techniques ...................................................... 14

1.5.4 Surface topography techniques ................................................ 20

1.5.5 Ion scattering ...................................................................... 24

1.6 Non-structure sensitive techniques ..................................................... 25

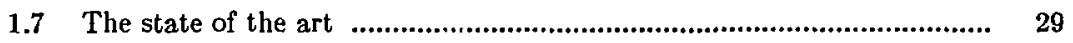

1.7.1 Clean metals ............................................................................. $\quad 30$

1.7.2 Reconstructed metal surfaces ................................................ 31

1.7.3 Metal surfaces and atomic adsorbates ...................................... 33

1.7.4 Semiconductors ........................................................................ 35

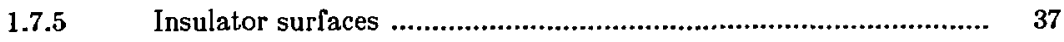

1.7.6 Molecular adsorbates ................................................................ 37

Figure Captions for Chapter 1 ....................................................... 46

Tables for Chapter 1 .................................................................... 50

Chapter 2: LEED Experimental Methods ........................................ 108

2.1 Introduction .................................................................................... 108

2.2 Types of LEED experiments ......................................................... 109

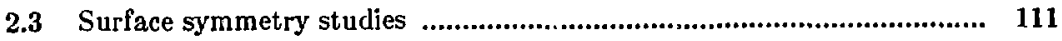


2.4 Surface order studies ................................................................... 113

2.4.1 LEED coherence and transfer width .................................... 114

2.4.2 Overlayer islands and coherence ............................................. 119

2.4.3 Extended surface defects ....................................................... 121

2.5 Surface structure studies ................................................................ 122

2.5.1 Diffuse LEED .......................................................................... 125

2.5.2 Instrument response and LEED int snsity ............................... 127

2.5.3 LEED intensity measurements ............................................ 129

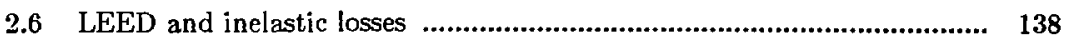

2.7 LEED and phonon scattering ..................................................... 140

2.8 LEED instrumentation .......................................................... 145

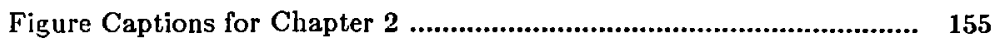

Chapter 3: LEED Scattering Theory ............................................... 160

3.1 Introduction .................................................................................... 160

3.2 Concepts of LEED scattering .......................................................... 161

3.3 Scattering theory ............................................................................. 164

3.4 The ion-core potential ................................................................. 167

3.5 The optical potential ..................................................................... 172

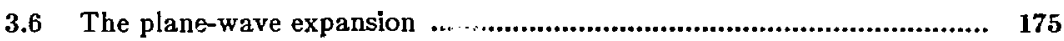

3.7 Layer scattering ................................................................................ 177

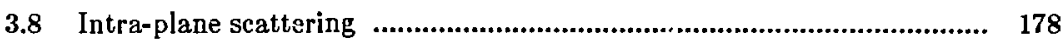

3.8.1 Multiple-scattering corrections ............................................. 179

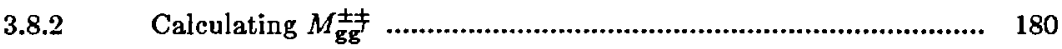

3.8.3 Composite layers ....................................................................... 181

3.8.4 Combined space method (CSM) ........................................... 182

$3.9 \quad$ Layer stacking ............................................................................... 184



3.9.2 Renormalized forward-scattering (RFS) .............................. 186

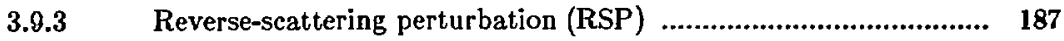

3.10 Diffuse LEED theory ................................................................... 188

3.11 Temperature effects .................................................................. 191

3.12 Symmetry considerations ........................................................... 194

Figure Captions for Chapter 3 ...................................................... 201

Chapter 4: Methods of LEED Structure Determination .................. 210 


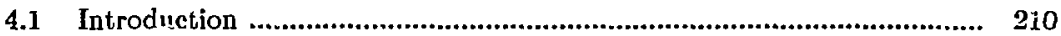

4.2 Reliability factors ....................................................................... 211

4.3 R-factor properties ..................................................................... 212

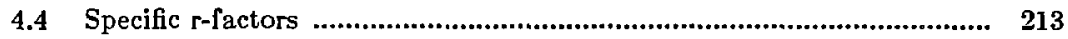

4.5 Data sub-division ........................................................................... 215

4.6 Model parameters ....................................................................... 217

4.7 Structural and non-structural parameters ...................................... 219

4.8 Structural searching ......................................................................... 221

4.9 Approximations in structural searches .............................................. 223

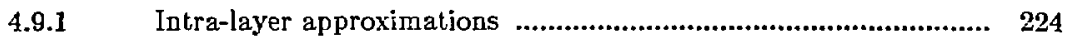

4.9.2 Inter-layer approximations ..................................................... 225

4.9.3 Beam set neglect and disorder (BSN) ..................................... 227

4.10 Conclusion ..................................................................................... 228

Figure Captions for Chapter 4 .................................................... 231

Part II - Experimental Developments in LEED

Chapter 5: Video Techniques and

LEED Intensity Measurement ................................................ 236

5.1 Introduction ............................................................................. 236

5.2 Video camera concepts .................................................................... 238

5.2.1 Optical components ............................................................... 239

5.2.2 Video transducer .................................................................. 239

$5.2 .3 \quad$ Video amplifier ............................................................... 242

5.2.4 Video scanning ................................................................... 243

5.3 Data Acquisition ............................................................................. 243

5.4 Video digitizer ................................................................................ 246

5.5 Video data processing .......................................................................... 248

5.6 Instrumentation ........................................................................... 249

5.7 Performance ................................................................................. 252

Figure Captions for Chapter 5 ................................................... 256

Chapter 6: Electron Counting Techniques and

LEED Intensity Measarement ....................................................... 262

6.1 LEED data acquisition ................................................................ 262 
6.2 Comparison of video and digital LEED ........................................... 263

6.2.1 Improvements in video techniques ........................................ 265

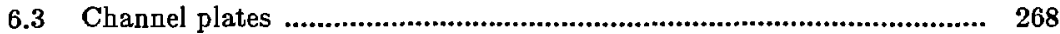

6.3.1 Counting rates for channel plates ............................................ 267

6.4 Position sensitive detectors ................................................................ 268

6.4.1 Resistive anode charge-division ............................................... 269

6.4.2 Wedge-and-strip anode charge-division ..................................... 270

6.5 Detector construction ...................................................................... 271

6.5.1 Data system ............................................................................... 273

6.5.2 Digitizer electronics ................................................................. 274

6.6 The wedge-and-strip anode .............................................................. 274

6.6.1 Anode resolution .................................................................. 275

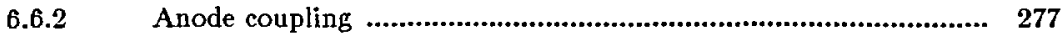

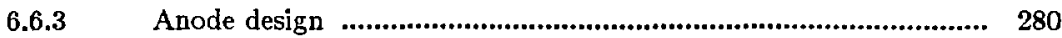

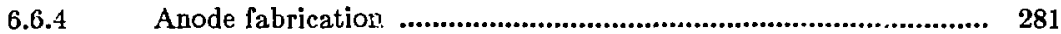

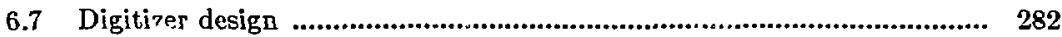

6.7.1 Pulse pile-up ............................................................................ 283

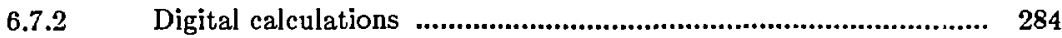

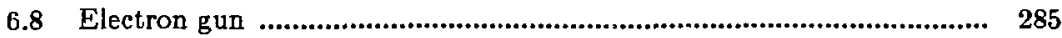

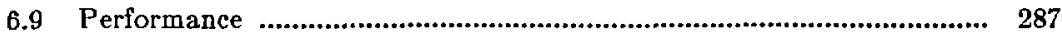

6.9.1 LEED statistics ................................................................ 288

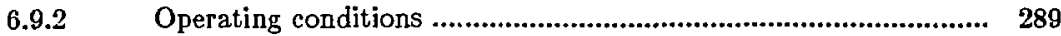



Figure Captions for Chaptier 6 ....................................................... 295

Chapter 7: I-V Curve Generation from

Digitized LEED Patterns ........................................................ 307

7.1 Introduction ........................................................................................ 307

$7.2 \quad$ LEED programs ........................................................................... 308

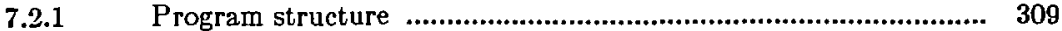

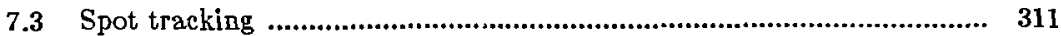

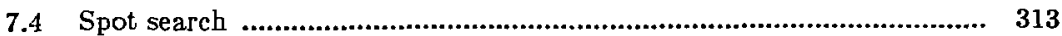

7.5 Spot evaluation ............................................................................. 314

7.5.1 Local background .................................................................... 315

7.5.2 Spot width ....................................................................... 316

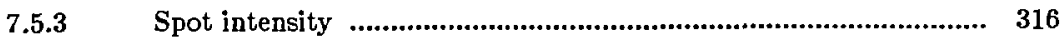




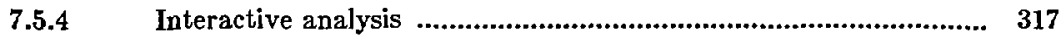

7.6 Performance ..................................................................................... 318

Figure Captions for Chapter 7 ............................................................. $\quad 320$

Tables for Chapter 7 .................................................................... 325

Chapter 8: An Improved LEED Manipulator ............................... 326

8.1 Introduction .................................................................................... 326

8.2 Design requirements ..................................................................... 327



8.4 Heating and cooling ....................................................................... 331

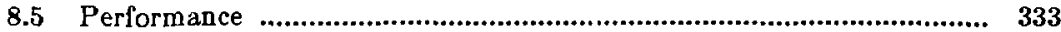

Figure Captions for Chapter 8 ........................................................... 336

Part III - Experimental Results for LEED Structure Determination

Chapter 9: Experimental Technique ................................................ 344

9.1 Introduction ........................................................................................ 344

9.2 Vacuum techniques ....................................................................... 344

9.3 Surface preparation ............................................................................ 347

9.3.1 Crystal preparation ............................................................ $\quad 347$

9.3.2 Sample cleaning in vacuum ....................................................... 348

9.3.3 Ion bombardment ............................................................... 348

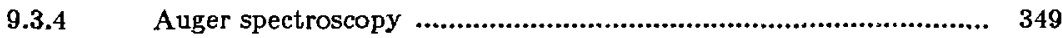

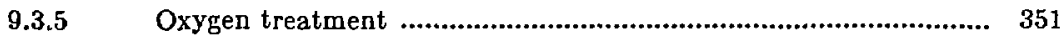

9.4 Crystal heating and cooling ........................................................ 352

9.4.1 Thermal desorption ........................................................................ $\quad 354$

9.5 Chemisorption techniques .............................................................. 355

9.6 LEED technique .......................................................................... $\quad 356$

9.6.1 The LEED optics .................................................................. 357

9.6.2 Voltage measurement ................................................................ 358

9.6.3 The LEED electron gun ............................................................. 358

9.6.4 Magnetic and electrostatic fields ............................................ 360

9.7 Crystal orientation ........................................................................... 362

9.7.1 Manipulator geometry ........................................................... 362

9.7.2 Crystal alignment .............................................................. 363 
9.7.3 Angle and spot labeling conventions ......................................... 367

9.8 LEED data reduction ........................................................................ 367

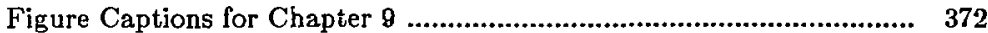

Chapter 10: Carbon Monoxide on Pt(111) ..................................... 377

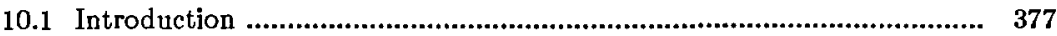

10.2 The carbon monoxide-Pt(111) system ............................................. 378

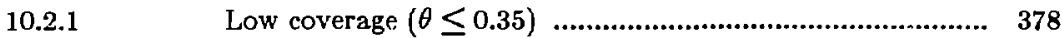

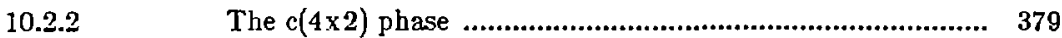

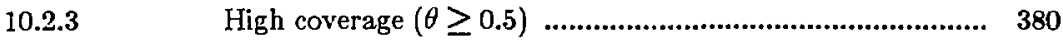

10.3 Uni-axial compression and domain-walls ......................................... 382

10.4 High coverage discussion .................................................................. $\quad 390$

10.5 Clean Pt(111) surface structure ....................................................... 391

10.5.1 New surface models ............................................................ 392



10.6 Carbon monoxjde structure determination ........................................ 394

10.7 Carbon monoxide surface models ....................................................... 398

10.8 Structural search ............................................................................. 399

10.9 Discussion of structural results ........................................................ 401

10.10 Conclusions ...................................................................................... 401

Figure Captions for Chapter 10 ................................................. 406

Chapter 11: Benzene Ordering on Pt(111) ......................................... 446



10.2 Benzene on platinum (111) ............................................................. 447

10.3 LEED observations .................................................................... 447

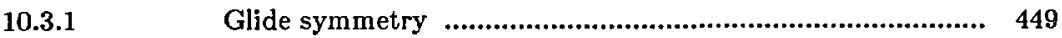

10.3.2 HREELS results .......................................................... 450

10.3.3 Thermal desorption results .......................................... 451

10.3.4 Photoemission results ........................................................ 453

10.4 Benzene chemisorption experiments ................................................ 454

10.4.1 Reproducibility ............................................................. 454

10.4.2 Benzene uptake ............................................................. 455

10.4.3 Benzene thermal desorption .............................................. 456

10.4.4 Ordering with large exposures ........................................... 458

10.5 Benzene - carbon monoxide coadsorption ......................................... 458 


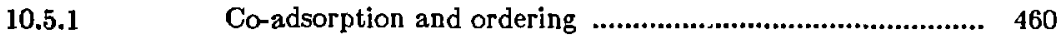

10.6 LEED I-V data ......................................................................... 461

10.6.1 Data acquisition ...................................................... 463

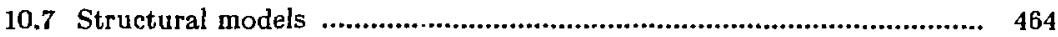

10.7.1 Model constraints ........................................................... 465

10.7.2 Internal degrees of freedom ........................................... 466

10.8 LEED Theory ............................................................................... 468

10.8.1 Overlayer calculations .................................................... 469

10.8.2 Glide symmetry theory ................................................... 470

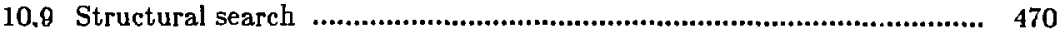

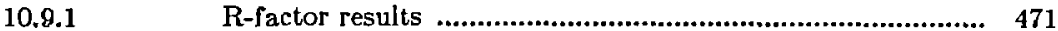



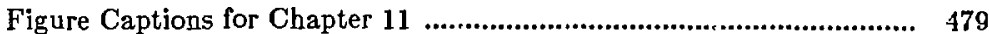

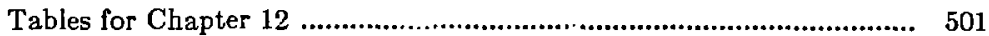

Chapter 12: Incommensurate Graphite on Pt(111) ...................... 508

12.1 Introduction .................................................................................. 508

12.2 Incommensurate overlayers ......................................................... 509

12.3 Incommensurate overlayer analysis ................................................. 510

12.4 Carbonaceous layers .......................................................................... 511

12.5 Graphite overlayers on platinum ............................................... 513

12.5.1 Preferred graphite orientations ......................................... 514

12.5.2 Overlayer growth ....................................................... 515

12.6 LEED beam types ........................................................................ 517

12.7 LEED data ..................................................................................... 520

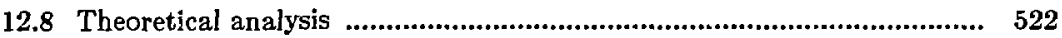

12.9 BSN approximation for incommensurate overlayers ......................... 524

12.10 Graphite-platinum calculations .................................................... 527

12.11 Quasi-kinematical analysis ....................................................... 530

12.12 Conclusion .................................................................................... 534

Figure Captions for Chapter 12 ...................................................... 541

Chapter 13: Ordering on the $\alpha$-CuAl(111) Alloy Surface .................. 563

12.1 Introduction .................................................................................. 563

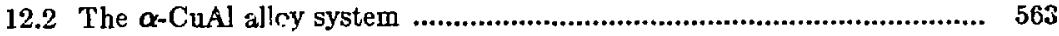

12.3 Surface characterization .................................................................. 564 
xii



12.5 LEED structure determination ....................................................... 568

12.5.1 LEED data ..................................................................... 569

12.5.2 Theoretical methods ..................................................... 570

12.5.3 Structure search ............................................................. 571

12.6 Discussion of results .................................................................... 572

12.7 Conclusion ........................................................................................ 574

Figure Captions for Chapter 13 ................................................... 578

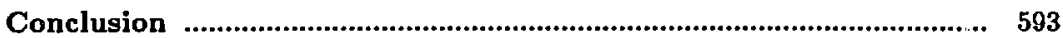

Appendix I - I-V data ........................................................................ 596

Appendix II - Computer programs ...................................................... $\quad 599$ 


\section{Introduction}

Most structure determination experiments to date have dealt with clean surfaces and various atomic adsorbates on low Miller index surfaces of metals and semiconductors. This work discusses the problem of surface structure determination, along with the theoretical and experimental tools needed to extend low energy eleciron diffraction (LEED) structure determination to new classes of surface systems, including molecular adsorbates, systems with large unit cells, incommensurate overlayers and disordered systems.

This work is organized in three parts which discuss the motivation, theory and state of the art of structure determination; new experimental techniques that have been developed for LEED, including video and electron counting data acquisition techniques and data reduction techniques for digitized LEED data; and new structural results for carbon monoxide molecules adsorbed on $\mathrm{Pt}(111)$, for coadsorbed carbon monoxide and benzene molecules on $\mathrm{Pt}(111)$, for incommensurate graphitic carbon adsorbed on $\operatorname{Pt}(111)$ and for the $(\sqrt{3} \times \sqrt{3})$ R30 reconstruction of the $\alpha-\operatorname{CuAl}(111)$ surface below the $(\sqrt{3} \times \sqrt{3}) \mathrm{R} 30^{\circ} \rightarrow(1 \times 1)$ phase transition temperature.

In Part I, chapter 1 discusses the problem of surface structure determination in the context of surface science, reviews the various experimental techniques used to study surface structure, and surveys the available results on surface structure. Chapter 2 discusses the experimental limitations of LEED and the correspondence between experiment and idealized LEED theory. Chapter 3 discusses LEED 
scattering theory and chapter 4 discusses the application of LEED theory to structure determination, including a discussion of the approximations used to study the complex systems discussed in part III.

Part II describes some new experimental techniques developed for LEED structural studies. Chapter 5 describes a video camera/real time video processor system used to digitize LEED patterns and chapter 6 describes a new electroncounting detector that greatly increases the sensitivity of LEED measurements. Chapter 7 describes computer software developed for the interactive analysis and generation of I-V curves from digitized LEED patterns collected with either video or electron-counting techniques. Chapter 8 concludes part II with the description of an off-axis LEED sample manipulator developed for the electron-counting LEED system.

Part III discusses the results of new structural studies. Chapter 9 covers general experimental techniques used in sample characterization and LEED measuremenis, chapter 10 presents the structural results for the $c(4 \times 2)$ structure on $\mathrm{Pt}(111)$ with two molecules per unit cell and analyzes the high-coverage phase diagram. Chapter 11 discusses the structure of benzene and carbon monoxide coadsorbed in the $(2 \sqrt{3} \times 4)$ rectangular structure on $\operatorname{Pt}(111)$. Chapter 12 presents the results of a structural investigation of incommensurate graphitic overlayers on $\mathrm{Pt}(111)$, with the new result that this layer is not in direct contact with the metal surface, but is instead supported by an intermediate layer of intercalateci carbon atoms. Chapter 13 concludes with the results of a LEE.D investigation of the structure of the $(\sqrt{3} \times \sqrt{3}) \mathrm{R} 30^{\circ}$ reconstruction on the (111) face of an $\alpha$-CuAl 
single crystal. 
Part I

The Theory and Practice of Low Energy Electron Diffraction 


\section{Chapter 1}

\section{Surface Structure Determination}

\subsection{Surface Science}

The understanding of surface properties can provide answers to many key questions in physics, chemistry and materials science. The study of surface and interface properties has expanded greatly over the last twenty years. Rapid progress in surface science started with the ability to prepare routinely well characterized surfaces in vacuum. The development of commercial ultra-high vacuum components ( $\leq 10^{-9}$ torr) made it possible to maintain a well-defined surface. Electrons were used to probe the surface chemical composition through Auger electron spectroscopy and the surface order through low energy electron diffraction (LEED). A wide range of tools are now available to study the solidvacuum interface and techniques are being developed to investigate solid-gas, solid-liquid and sclid-solid interfaces.

Surface science has now developed to the point that atomic-scale mechanisms are being explained for some important surface processes and many others are now being directly investigated. The range of problems under investigation covers many disciplines. These include, in physics, the electronic properties of surfaces and interfaces, the changes in bulk crystal structure at surfaces, including surface reconstructions and multilayer relaxations in lattice spacings, surface magnetism and electrical conduction, the properties of ordering and phase- 
transitions in quasi two-dimensional systems; in chemistry, the properties or the surface chemical bond, the mechanisms of heterogeneous catalysis, of oxidation, corrosion and of passivation and the kinetics of surface chemical reactions; in materials science, the mechanisms of lubrication and friction and the effects of surface coatings and interfaces on bulk mechanical properties.

\subsection{Surface structure}

Knowledge of the atomic structure of surfaces is crucial for understanding and describing the mechanisms of surface processes. The atomic geometry is a fundamental input for theories of surface e!ectronic properties. Experimental determination of surface structure is a necessary check for theoretical total-energy calculations of surface structure and chemisorption geometry. Surface chemical reactions cannot be explained without a basic understanding of surface chemical bonding, which in turn requires the knowledge of surface chemical bond-lengths and bond-angles.

Most quantitative information on surface structure and chemical bonding comes from studies of the solid-vacuum interface. In large part this is because the most powerful probes of surface structure rely on the propagation of electron, ion or atomic beams in vacuum. New developments such as the scanning tunneling microscope (STM), which can also investigate the solid-gas and solid-liquid interface and new non-linear optical techniques, which can potentially investigate all types of surfaces and interfaces, may greatly extend our understanding of surface structure in the future. At this time, however, the solid-vacuum interface is the 
focus of most of the active investigation of surface structure.

The first determinations of surface structure were made in the early 1970's with LEED multiple-scattering intensity calculations. Attempts at LEED structure determination were not successful until accurate methods for multiplescattering calculations were developed. Since that time a large number of increasingly complex structures have been analyzed through LEED multiple scattering calculations. Also, other surface structure sensitive techniques have been developed, such as ion scattering, atomic beam diffraction and extended X-ray emission fine-structure (EXAFS). Over 250 surface structures have now been determined using various techniques. ${ }^{1,2}$ These structures include clean and reconstructed metal surfaces, clean and reconstructed semiconductor surfaces, a few insulator surfaces and the structure of a number of atomic adsorbates on metal surfaces. Progress is also being made on more complex stepped metal surfaces, alloy surfaces, surfaces with molecular adsorbates and surfaces with disordered and incommensurate overlayers.

LEED is the most generally applicable technique for surface structure determination and a substantial majority of the known surface structures were investigated by LEED. Low energy electrotss penetrate several layers deep into the surface, so LEED is sensitive to structure throughout the near surface region, not just to the topography of the outermost surface layer like scanning tunneling microscopy or atomic beam diffraction. LEED is also sensitive to all chemical elements, although the electron scattering cross-section for hydrogen, in particular, is very small. Structures can be determined where atoms have different local 
geometries, which is dififult with fine-structure techniques. Surface structures have been successfully determined with LEED for clean and reconstructed metal and semiconductor surfaces, for some insulator surfaces and for metal and semiconductor surfaces with atomic and molecular adsorbates. The main limitation of conventional LEED structure determination has been the requirement of longrange order, needed for the formation of a diffraction pattern. However, there is no fundamental requirement for long range order in electron scattering calculations and it has been shown that LEED theory can be used to determine surface structure from the diffuse scattered electron intensities for some types of disordered systems, especially for disordered adsorbates on ordered crystal substrates. $^{3,4}$

\subsection{Structure determination}

The rajority of the known surface structures liave been solved by comparing experimental data with theoretical predictions based on models of the surface, since the data from many surface experiments cannot be directly interpreted in terms of surface structure. A theoretical description of the interaction of the electron, ion or photon probe with the surface is used to calculate experimental spectra for a given model geometry. Different model geometries are tried and structural parameters within a given model are varied, until a good fit is obtained between the Experimental data and the theoretically calculated spectra. This basic approach has been used to interpret data from low-energy electron diffraction (LEED) and other electron and angle-resolved photoemission 
experiments, from helium diffraction and from ion scattering.

Structural information has also been obtained by matching the observed band-structure of surface vibrational or electronic excitations with spectra calculated from structural models. ${ }^{5}$ This approach has been applied to the bandstructure of electronic excitations as measured by angle-resolved ultra-violet photo-emission (ARUPS) ${ }^{6,7}$ and to the band-structure of vibrational excitations as measured by high-resolution electron energy-loss spectroscopy (HREELS). $.8,9,10$

There has been an ongoing search for a technique that could determine surface structure directly from experimental results, without the need to fit experimental data to theoretical calculations for model systems. So far this search has not been successful, although certain techniques yield more direct solutions for some special cases. Structure sensitive techniques such as scanning tunneling microscopy (STM) $)^{11,12,13}$ and field ionization microscopy (FIM) ${ }^{14}$ do give results that can be interpreted as direct images of the surface, but these results do not provide complete information on atomic coordinates in the near-surface region. Extended fine-structure techniques provide direct information on bond-lengths for chemisorbed atoms, but this information may not be sufficient to determine fully the three dimensional surface structure.

Most surface structural studies combine results from several different surface science techniques applied to the same system. For an approach based on nodel calculations to be successful, it is necessary to construct reasonable structural models of surfaces and this requires a wide range of experimenial information. Techniques which cannot give direct information on the atomic coordinates of 
atoms in the near-surface region ofiten provide necessary information to develop appropriate surface structural models.

\subsection{Complementary techniques}

Certain combinations of experimental techniques have been valuable complements for different classes of surface systems. LEED and ion scattering studies have been used to study clean surface relaxation and reconstruction. Ion channeling effects are particularly sensitive to small changes in relative atomic positions. Although it is a new technique, scanning tunneling microscopy will clearly be valuable in developing models for surface reconstruction and possibly for chemisorption. Some of the first STM results confirmed the missing-row model for the surface reconstruction of gold $(110)^{11,12}$ and later results have made substantial contributions to the debate on the $(7 x 7)$ reconstruction on the silicon (111) surface. ${ }^{13}$ Auger electron spectroscopy complements LEED studies of atomic adsorbates. Knowledge of the surface chemical composition and approximate measures of the relative concentrations of different species, along with information on the surface unit cell from LEED is often enough to suggest reasonable structural models for simple surfaces. For studies of molecular chemisorption highresolution electron energy-loss spectroscopy (HREELS) complements LEED. Vibrational spectra can often determine if the the molecule has changed chemically during adsorption and it may be possible to determine the binding site from the type and number of vibrational modes that are excited. $15,16,17$ 
A wide array of techniques have been developed to study surfaces in vacuum and all too many are commonly referred to by acronyms. Table 1.1 lists acronyms and brief descriptions of most of the commonly used surface science techniques.

\subsection{Structure sensitive techniques}

The structure sensitivity of most techniques involving electrons comes from the interference and diffraction effects associated with the propagation of lowenergy electrons through solids. This class of techniques includes, in addition to LEED, all of the near-edge and extended fine-structure techniques, angle-resolve. photoemission techniques and some aspects of inelastic scattering techniques such as HREELS. Although the basic physical processes are the same as for LEED, some of these techniques can be interpreted with simpler theories than for LEED, because certain structural aspects of the surface are selectively emphasized. The structure-sensitivity of ion and atom seattering, non-linear optical probes and the surface topography techniques do not depend on electron propagation effects.

\subsubsection{Electron diffraction techniques}

The majority of known surface structures have been solved with electron diffraction techniques. Low energy electrons (below $\sim 400 \mathrm{eV}$ ) interact strongly with atoms through both elastic and inelastic processes. Inelastic scattering in solids limits electrons with energies below a few hundred $\mathrm{eV}$ to a mean free path of $\sim 2$ to $20 \AA$. Elastic interactions are strong enough that multiple scattering is 
important in this energy range, so scattered electrons are sensitive to the threedimensional geometry of the near-surface region. Electron scattering also depends on the chemical identity of the scattering atoms, so electron diffraction techniques are sensitive to both chemical composition and structure throughout the nearsurface region. Therefore low-energy electrons are an ideal probe of surface structural chemistry. LEED is discussed in detail in the following chapters.

The main difficulty with alectron diffraction methods is that the strong electron-atom scattering makes data analysis difficult -- it is not possible in most cases to "invert" electron diffraction data to obtain the original surface structure in the way X-ray diffraction data can be analyzed to determine bulk structure. At higher energies the surface sensitivity and the importance of multiple scattering is reduced. Diffraction techniques in this energy range have primarily been applied to the study of defects and deviations from long range order, as in the use of RHEED to monitor epitaxial growth on surfaces. ${ }^{18}$ Electron diffraction in the MEED energy range have not yet been much used for surface structure determination because the calculational approaches used in the LEED energy range become prohibitively complex at higher energies, while the simple kinematic scattering theory is not sufficient for structure determination. Theoretical approaches suited to this energy range are being developed $19,20,21,22,23$ and a few structures have been studied with this method, including clean and adsorbate covered aluminum. ${ }^{24}$ 


\subsubsection{Photoelectron Diffraction}

The interference and diffraction of photoemitted electrons can provide structural information, as in ARPEFS, ${ }^{25,26}$ ARUPS, ${ }^{27}$ ARXPS $^{28}$ and ARXPD. The main advantage of photoemission over LEED is that the initial state of the photoemitted electron is better defined and can be controlled to emphasized partirnlar structural features, especially in chemisorption systems. Because the initial state is well-defined, in some cases the data analysis can be simpler than full LEED multiple-scattering calculations.

In photoelectron diffraction experiments monoenergetic photons excite electrons from a particular atomic core-level. Angular momentum is conserved, so the emitted electron wave-function is a spherical wave centered on the source atom, with angular momentum components $l \pm 1$, where $l$ is the angular momentum of the core-level. If the incident photon beam is polarized, the orientation of the emitted electron wave-function can be controlled. These electrons then propagate through the surface and are detected and analyzed as in LEED experiments. A synchrotron X-ray source normally produces the intense beams of variable-energy polarized photons used for photoelectron diffraction.

In angle-resolved photoemission fine-structure (ARPEFS) electrons are detected at a given angle as a function of energy. The variation in intensity because of interference between different scattering paths for electrons over a 100 to $500 \mathrm{eV}$ energy range gives structural information. In this energy range the interference effects are dominated by single-scattering, so the scattering amplitude can be readily calculated for a model system. A Fourier transform of the 
interference pattern will show the distribution of different scattering path-lengths that contribute to the detected intensity. This can be a zuide to the proper surface structure, but this result may require confirmation by a calculation of the interference for a model geometry. ${ }^{29}$ ARPEFS has been used to solve several surface structures, including sulfur on nickel. ${ }^{30}$ Long-range order is not required for ARPEFS experiments. In spite of the name, ARPEFS is quite different from other "fine-structure" techniques. The structure-sensitivity of fine-structure techniques depends only on back-scattering, where APPEFS sensitivity depends on many scattering angles and requires angle-resolved detection. The variations in the detected intensity as a function of energy can be orders of magnitude larger than the fine-structure modulation in core-level excitation cross-sections.

Photoelectron diffraction is most useful for systems where the photoexcited atoms all have the same local geometry, as in a chemisorption problem. If there are source atoms in different local geometries, there will be a superposition of interference patterns and the resulting interference spectrum will be harder to interpret.

\subsubsection{Fine Structure Techniques}

There are a large number of "fine structure" techniques, all based on the same physical principle. In all of these techniques an electron is ejected from an atomic core-level by incident photons or other particles. The different names refer to different experimental arrangements for the excitation and detection of core-hole decay. 
As the excitation energy is varied the energy of the emitted electron varies. The emitted electron wave can be back-scattered by neighboring atoms, where it interferes with the source wave-function. This energy dependent interference changes the coupling of the incident excitation to the final state, producing a modulation or "fine-structure" in the excitation cross section.

If there are different local geometries for source atoms, the different fine structures will be superimposed in the experimental spectrum, which will be difficult to interpret if there are multiple source atom environments. Fine structure techniques are primarily used when a chemisorbed atom acts as the source atom. One important advantage of extended fine structure techniques is that they do not require long range order or single crystal substrates, so they can be directly applied to many systems of technological importance.

Near-edge techniques need a spectrum of $\sim 50 \mathrm{eV}$ and extended finestructure techniques need a spectrum of $150 \mathrm{eV}$ or more for decent resolution. If another strong core-level excitation-threshold falls in this region, it will be difficult or impossible to separate out the desired fine-structure, so these techniques are limited to certain systems. The wrong combination of atoms will prevent a sufficient range of the extended fine-structure modulations from being observed. 


\subsubsection{Near-edge Fine Structure Techniques}

In the "near-edge fine-structure" region, where emitted electrons have energies up to $\sim 50 \mathrm{eV}$, multiple scattering effects are predominant. An emitted electron wave can scatter several times and still have a significant amplitude at the source atom. Therefore the variation in the observed cross-section depends on the relative positions of all of the neighboring atoms, so the local three-dimensional geometry around the source atom can be determined. The theoretical analysis of the near-edge fine-structure is much more complex than for the extended finestructure region, where only single back-scattering is explicitly included. However, the near-edge region contains more structural information than the extended region.

This technique is referred to by two acronyms, XANES for X-ray adsorption near-edge structure and NEXAFS, for near-edge X-ray adsorption fine-structure. Pendry, et al. ${ }^{31}$ and others ${ }^{32}$ have developed theoretical methods to analyze XANES data that are generalized from LEED analysis methods. XANES has been applied to the structure of oxygen adsorbed on nickel (100), a benchmark system for structure techniques and the results were consistent with those of LEED and other techniques (see Table 1.2). ${ }^{33,34}$

A variation of NEXAFS has been used by Stöhr, et al..$^{35,36}$ to determine the structure of hydrocarbon molecules chemisorbed on metal surfaces. Structural information has been obtained without the need to do multiple scattering calculations. Instead, the molecular shape-resonances corresponding to the $\pi$ and $\sigma$ molecular orbitals can be recognized in the NEXAFS spectrum. The approximate 
orientation of the molecules can be determined by varying the polarization of the incident photon beam and using selection rules to identify the orientation of different molecular orbitals. Carbon-carbon bond lengths have also been determined using a quasi-empirical correlation between bond-length and the shift in the $\sigma$-orbital shape-resonance energy. This technique has been used to study the chemisorption of several hydrocarbon molecules on different transition metal surfaces. ${ }^{37}$

\subsubsection{Extended fine-structure techniques}

For emitted electrons above $\sim 50 \mathrm{eV}$, the "extended fine-structure" region, the modulation of the excitation cross section is dominated by single backscattering between near-neighbor atoms and the source atom. A Fourier transform of the extended fine-structure as a function of momentum transfer gives the distribution of radial distances between the source atom and neighboring atoms, providing the back-scattering phase-shifts are known as a function of energy. With empirical or theoretical correction for scattering phase shifts, which have been calibrated using results from bulk structures determined from X-ray diffraction, this gives chemical bond lengths with an accuracy of better than 0.05 A. 38

In fact, multiple scattering does contribute to extended fine-structure spectra. Strong forward-scattering along linear chains of neighboring atoms will modify the single back-scattering result. In effect, there will be an energydependent renormalization of the back-scattering phase-shift due to multiple 
forward scattering. Since experimental data are usually interpreted using experimentally-derived phase shifts from EXAFS studies of known bulk structures, the effects of multiple forward-scattering are implicitly included in many cases.

There are a number of extended fine-structure results available. These techniques, after LEED, have provided the largest amount of quantitative information on chemisorption structures and surface chemical bonding. The extended finestructure techniques give quantitative information on surface structure without the need for model calculations. In simple systems and when combined with qualitative data from other experiments, knowledge of the bond lengths may be sufficient to describe completely the surface geometrical structure. These techniques are most useful in multi-component or ch^misorption systems, where atoms of a particular chemical species have only one local geometry. By selectively exciting an appropriate core level, near neighbor bond lengths are determined. In systems with non-equivalent atomic sites, such as reconstructed surfaces, the extended fine-strurture will show a superposition of radial distribution functions for the different sites. Additional data will be needed to solve these structures. Data interpretation is generally simpler with photon excitation, since the polarization and orientation of final state electrons can be controlled by using single crystal samples and polarized photons from a synchrotron source. Fine-structure techniques can give useful information on the local chemisorption geometry without describing the complete surface structure. This is useful for investigations of chemisorption on semiconductors, where surfaces often exhibit complex 
reconstructions.

There are a number of different experimental methods to observe finestructure. ${ }^{39}$ In EXAFS X-ray photons excite core levels and the cross section is monitored by X-ray adsorption or by fluorescence from excited atoms. This is not intrinsically surface sensitive, although surface information can be obtained if the photoexcited atoms are concentrated on the surface, as is often true for chemisorption. When Auger (Auger-SEXAFS) or secondary electrons (PE-SEXAFS) emitted from the excited atoms are detected, the process is called surface EXAFS or SEXAFS. SEXAFS in the near edge region is called NEXAFS or XANES. SEXAFS experiments have aiso been done by detecting ions emitted from surfaces (PSD-SEXAFS).

There are analogous processes using electrons instead of photons to excite atomic core levels. Diffraction effects may perturb the cross-section fine-structure when electrons are used to excite core levels or monitor cross-section. This is not a problem for systems without long range order. For cases involving long range order it is desirable to integrate over detector angles and also electron beam incidence angles where this is practical, so that diffraction effects do not add additional structure to the extended fine-structure spectrum. In EAPFS incident electrons at $\sim 1 \mathrm{KeV}$ excite shallow core holes and the cross section is monitored by soft X-ray fluorescence (SXAPS-EAPFS), Auger emission (AEAPS-EAPFS), or the variation in elastic back-scattering (DAPS-EAPFS or SEELFS). With exc'tation by high energy electrons ( 60 to $300 \mathrm{KeV}$ ) and detection by fluorescence the process is called EXELFS, or ELNES in the near edge region. These last 
processes, like EXAFS, are not inherently surface sensitive.

Electron-excited fine-structure spectra are more difficult to interpret than photo-emitted spectra, since the emitted electron state is less well-defined. In photo-emission only one (for $s$-levels) or two partial waves contribute to the emitted electron wave-function. There is no such limit for electron excitation, also the advantage of polarization is lost. In a photon-excited fine-structure experiment most of the electrons collected outside the surface may be related to the initial core-level excitation cross-section, while for electron excitation many competing inelastic-loss mechanisms effect the primary beam, which reduces the experimental signal-to-noise ratio. The motivation, of course, for using electron excitation is that no synchrotron is required and high-flux electron beams can easily be produced in most laboratories. It remains to be seen if useful structural information will be provided by electron-excited fine-structure techniques.

\subsubsection{Surface topography techniques}

Field ionization microscopy, helium or atomic beam diffraction and scanning tunneling microscopy provide atomic scale information on surface topography. These techniques produce good qualitative images of surfaces. In most cases it is difficult to get atomic coordinates directly. However, knowledge of the surface topography can lead directly to structural models of a surface. 


\subsubsection{Field ion microscopy}

Field ion microscopy (FIM) is the oldest technique, developed in the 30 's. ${ }^{14} \mathrm{~A}$ sharp, single crystal metal tip with a radius of $\sim 1000 \AA$ is maintained at $\sim 10 \mathrm{kV}$ in a low pressure $\left(<10^{-4}\right.$ torr $)$ gas, usually helium. Gas molecules are polarized and attracted to the tip by the strong, inhomogeneous electrostatic fields. The field strength near the tip is on the order of several $V / \AA$. Valence electrons in the gas atom can tunnel into the tip as the neutrai atom approaches the surface, creating a positive ion which is repelled by the high field around the tip. The ions project an image of the high field regions of the tip onto a phosphor screen. If the tip is cooled, individual surface atoms can be imaged. Pairs of atoms separated by as little as $1.5 \AA$ have been resolved.

A field-io; microscopy image is a two-dimensional projection of the outermost surface layer. This image provides a qualitative 'mage of the surface, but very little information on distances normal to the surface. Although the direct ir . srmation on the surface chemical bond is limited, FIM has added a lot to the qualitative understanding of surface structure and in particular to the understanding of surface diffusion kineties. ${ }^{40}$ Because of the high field strengths required, application of FIM has been limited to refractory metals, although some chemisorption systems on these metals have been studied. ${ }^{40}$ 


\subsubsection{Atomic beam diffraction}

A thermal energy atomic beam (20-200 meV) has a wavelength on the order of inter-atomic distances. The atomic beam diffracts from a contour of the surface potential coiresponding to the beam energy. This contour is located $3-4 \AA$ outside the ion cores in the outermost layer of the surface. Atomic beam diffraction patterns are normally interpreted using model surface scattering calculations, where the atom-surface interaction is described by a Van der Waals potential. $^{41}$

Because of the low probe energies used, atomic diffraction does not damage even the most delicate physisorbed overlayers, and it is sensitive to hydrogen, which is an important component of many surface systems of current interest. Electron scattering techniques are relatively insensitive to hydrogen because of its small scattering cross section. The structure of the $(2 \times 1)$ and $(6 \times 2)$ phases of hydrogen chemisorbed on $\mathrm{Ni}(110)$ were described using helium diffraction. ${ }^{\mathbf{4 2 , 4 3}}$ Finally, atom diffraction is extremely sensitive to surface order and defects and it has been very useful in the study of disorder and kinetic processes on surfaces. Because neutral atoms scatter well outside the ion-cores details of the surface structure can be lost and no information on subsurface structure can be obtained.

\subsubsection{Scanning tunneling microscopy}

The scanning tunneling microscope (STM) is onc of the most recently developed surface sensitive techniques. ${ }^{11,12,13}$ In STM a metal probe is brought close enough to the surface under study for the electron wave înetions to 
overlap. A fixed potential difference between probe and surface is maintained and the probe approaches the surface until a given tunneling current is observed. The probe is mechanically scanned over the surface with a pieazoelectric drive and a feedback loop adjusts the vertical spacing to maintain the tunneling current. STM has achieved a horizontal resolution of $\sim 2 \AA$ and a vertical resolution of $\sim$ 0.1 \& under optimum conditions. By changing the probe-surface potential difference it is possible to map out different surface profiles of wave-function overlap. The STM is very flexible and can be applied to all kinds of electricallyconductive surfaces. Unlike electron based techniques it is not limited to the solid-vacuum interface, although the best resolution has been obtained in vacuum.

A variation on the STM, the atomic force microscope (AFM) is being developed to extend STM techniques to insulator surfaces. An extremely sensitive mechanical probe is scanned over the surface. The force-constant of the spring loaded probe is small enough that the probe will respond to single surface atoms. An STM tip is used to monitor the defiection of this mechanical probe, which provides a record of the surface topography. ${ }^{44}$

The STM can give a direct qualitative image of surface topography. An early STM experiment confirmed the LEED result that the "missing row" model described the $(2 \times 1)$ reconstruction of the gold (111) surface. ${ }^{11,12}$ There is no adequate theoretical description of the STM tunneling process at this time, so it is difficult to relate a constant tunneling current contour to the geometrical strecture of the surface if more than one type of atom is involved. The STM can alsn 
provide information on surface electronic structure. STM tips have been used to record a tunneling current "I-V" curve, i.e. a record of the tunneling current as a function of the tip-surface bias voltage. Since the tunneling current is related to the overlap of the probe and surface wave-functions, this measurement should be related to a local surface "density of states". 45,46 Changes in the probe-surface bias can produce dramatic changes in the appearance of the STM image, especially for surfaces with chemisorbed overlayers. The lateral resolution of STM images is not good enough to provide accurate quantitative information on chemical bond lengths and angles. As the experimental and theoretical tools develop, scanning tunneling microscopy should provide increasingly useful information on surface structure.

\subsubsection{Ion scattering}

At high energies $(\sim 100 \mathrm{KeV})$ the interaction of ions with surfaces can be described by classical Rutherford scattering. Ion scattering has been used to study surface structure by directing ion beams along bulk crystal plans of solid surfaces. The "channeling" and "blocking" of these beams is very sensitive to deviations from bulk structure. Ion scattering has been used in particular to study relaxation and reconsiruction at crystal surfaces. Measurements of surface relaxation have provided an accurate check of LEED structure determination results, as in the case of chalcogen adsorption on nickel surfaces. ${ }^{47,48}$ Ion channeling can also provide a direct test to discriminate between different model surfaces for surface reconstructions. Ion scattering has provided important information on 
some complex semiconductor surfaces where a complete LEED structure solution has not yet been obtained. Ion scattering can probe certain structural features, such as the degree of lateral motions, or surface layer spacings, without having to solve the entire surface structure.

If a surface structure involves a large departure from bulk crystal structure, it can be difficult to interpret ion scattering results. Because ion scattering is strongly modified by thermal vibrations of the surface atoms, experimental data often must be compared to Monte-Carlo simulations of model surfaces including the effects of thermal vibrations to obtain quantitative results. The available data base for structure-fitting is rather small compared to electron spectroscopies, so the sensitivity to structural parameters is limited. When the surface structure is close to the bulk structure, ion channeling data can be strongly sensitive to small variations in structural parameters.

\subsection{Non-structure sensitive techniques}

There are a number of surface probes that are sensitive to the local geometry of the surface or give important information on surface composition, but which do not give direct information on atomic coordinates in the surface region. These techniques provide vital information needed to construct reasonable models of surface structure, which are needed to interpret data from quantitative techniques such as LEED and SEXAFS. 


\subsubsection{Chemical compusition}

Chemical composition is the most basic information needed to describe a surface and a prerequisite for structure determination. Auger electron spectroscopy is the most generally used techniques for measuring surface compasition and it is sensitive to all elements except hydrogen and helium. X-ray photoemission spectroscopy (XPS) is also an important probe of chemical composition and XPS and Auger peak-shifts give information on chemical environment. Thermal desorption spectroscopy is particularly useful for chemisorption studies - it gives a general idea of the chemical identity of adsorbates and some information on the type of surface binding and number of binding sites. Secondary-ion mass spectrometry is another way to get information on surface chemical composition -- it is generally more complicated to interpret the spectra, but SIMS is sensitive to hydrogen and also gives some information on the surface molecular structure.

\subsubsection{Electronic spectroscopy}

Some techniques are sensitive to electronic structure at surfaces and can probe the electronic band structure and density of states near the surface. This electronic information is useful for understanding the electronic structure of surfaces and the bonding mechanisms responsible for chemical process operating at surfaces. Structural information can also be obtained by comparing experimentally observed electronic structure with theoretical calculations of electronic structure for model systems. 
Ultraviolet photoemission spectroscopy (UPS) ${ }^{27}$ probes the density of states and ion neutralization spectroscopy (INS) and surface Penning ionization (SPI) provide similar information with probes of low-energy ions and metastable atoms, respectively. Angle-resolved UPS can determine the valence band structure.

\subsubsection{Vibrational spectroscopy}

Another class of techniques monitors surface vibrations. High-resolution electron energy loss spectroscopy (HREELS) measures the inelastic scattering of low energy $(\sim 5 \mathrm{eV})$ electrons from surfaces to study the vibrational excitation of adsorbed atoms and molecules and the surface phonon spectrum. In principle LEED-like structural information may be obtained from HREELS measurements, although calculations are complicated since very low energy electrons have long mean-free paths. In practice HREELS spectra are normally collected at a fixed incident beam energy and angle, so interference and diffraction effects are constant and only the loss spectrum is analyzed. HREELS is particularly useful for chemisorption systems, allowing the identification of surface species. Application of normal mode analysis and selection rules can often determine the point symmetry of the adsorption sites. ${ }^{16}$ Infrared reflectance-adscrption spectroscopy (IRRAS) is also used to study surface systems, although it is not intrinsicly surface sensitive. IRRAS is less sensitive than HREELS but has much higher energy resolution. 49 


\subsubsection{Optical techniques}

Non-linear optical techniques, such as second harmonic generation (SHG), have recently been used as surface probes. Systems with inversion symmetry do not generate second-order non-linear optical signals, so the total SHG signal will come from the surface region for many systerns. Surface SHG signals can be dramatically modified by monolayer chemisorption. The components of the nonlinear polarizability tensor have been used to determine the orientation of chemisorbed molecules. 50

$\mathrm{X}$-ray diffraction techniques are also being developed that can analyze surface structure. At glancing angles of incidence total reflection will assure surface sensitivity. ${ }^{51,52}$ Optical techniques are not limited to solid-vacuum interfaces like charged particle techniques, so their further development can expand the range of surface structural studies.

\subsubsection{Electron-stimulated desorption}

Electron stimuiated desorption of chemisorbed species (ESDLAD) provides direct quantitative information on the orientation of adsorbed molecules on surfaces. Electrons incident on the surface can excite chemical bonds into nonbonding states, causing molecular decomposition. The electrostatic repulsion along the broken bond accelerates an ionic fragment of the molecule along the axis of the bond. The angular distribution of desorbed ions can be related to the orientation of the bonds in the unperturbed adsorbed molecules. This techniques can give direct information on the number and symmetry of sites for 
chemisorption and approximate information on bond angles. ${ }^{53,54}$

\subsection{The state of the art}

Over the last fifteen years a significant amount of information on the structure of surfaces in vacuum has been obtained. Some 240 structure determination experiments have been performed that gave direct information on atomic coordinates in the near-surface region (including studies of the same system with different techniques). The systems investigated include clean surfaces of metals, semiconductors and some insulators and also metals and semiconductors with adsorbed atoms and molecules.

About three quarters of these experiments used LEED multiple-scatterin: calculations for structure determination. The remainder are divided approximately evenly between ion scattering, fine-structure and other photoelectron diffraction techniques. The photoemission techniques have been applied to chemisorption systems and the ion scattering techniques have primarily been used to study relaxations and reconstructions in clean surfaces, although they have been applied to chemisorption problems.

These techniques are the only ones that have established a range of structural results. The remaining techniques discussed above are not generally applicable to surface structure determination, or have not yet been routinely applied. Some of these, such as scanning tunneling microscopy and non-inear optical techniques, can be expected to develop into powerful surface probes in the near future. 


\subsubsection{Clean metals}

The structures of many low Miller-index (close packed) metal surfaces have been determined by LEED and other techniques. Not all crystal planes are stable as free surfaces. Some surfaces, particularly those with a very open arrangement of surface atoms, will spontaneously facet into macroscopic regions of more stable crystal planes. The thermodynamic stability of high-index surfaces is temperature dependent and is also affected by chemisorption. ${ }^{55}$ The low-index faces, such as (111), (110) and (100) for bec and fcc crystals, are usually stable. In some cases clean metal surfaces reconstruct, changing the translational symmetry of the surface. More commonly there is no change in the translational symmetry and the only structural change is a possible relaxation in inter-layer spacing. Many metal surfaces show a contraction in the first interlayer spacing of $\sim 0.10$ to $0.20 \AA$. More open surfaces tend to relax somewhat more than close-packed surfaces (see Table 1.2).

With the increase in sophistication of LEED experimental and theoretical techniques over the last few years, it has become possible to determine changes in the the first several interlayer spacings. This requires a large experimental data base and an r-factor analysis of the optimum values for structural parameters. In most of the earlier studies only a few non-equivalent beams were used and theoretical and experimental I-V curves were compared by eye. In cases where multi-layer relaxations have been investigated contractions and expansions in layer spacings often alternate, with decreasing amplitude for deeper layers. Table

I.2 summarizes the results of structure determinations of clean, un-reconstructed 
metal surfaces. Where multiple inter-layer spacings have been measured these are listed on consecutive lines in the Table.

The existing surface structure determination techniques are sufficient to study low index metal surfaces and the range of data available on these surfaces is steadily growing. The high Miller index surfaces, the so-called stepped and kinked surfaces, are more difficult. The geometry of atoms at step and kink edges is of interest, because the small percentage of atoms at such sites are known to play a dominant role in certain surface chemical reactions. 55 Stepped surfaces present great difficulty for the standard layer approach to LEED structural calculations (see chapter 3 ). These surfaces have very large unit cells and very close layer spacings and both factors increase the number of plane-waves needed to represent the LEED wave-function in the crystal, leading to long calculation times. Some stepped surfaces with relatively small unit cells, such as the (311) surfaces of copper and aluminum have been analyzed using conventional techniques (see Table 1.2), however modifications to the traditional calculational schemes are needed to investigate effectively stepped surfaces.

\subsubsection{Reconstructed metal surfaces}

For some low index surfaces the terminated bulk structure is not stable, even with relaxed layer spacings and a more substantial rearrangement takes place at the surface. When this changes the translational geometry of the surface unit cell it is called a reconstruction. One example of this is the (2x1) reconstruction observed on the (110) face of several fec transition metals. This reconstruction is 
seen on iridium, gold, platinum and palladium, but not on nickel or copper. Another example is the (5x1) reconstruction observed on the (100) faces of platinum and iridium, with a related reconstruction seen on gold (100).

Metal reconstructions generally serve to increase the coordination of the toplayer metal atoms. This is seen in the fcc (100) reconstruction, where the relatively open surface layer, with four-fold coordination in the surface, reconstructs to form a denser, approximately hexagonal close-packed surface with 6-fold coordination in the surface. The fcc (110) missing-row reconstruction can be understood in a similar way. The removal of the "missing" [001] row reduces by half the number of exposed "edge" atoms, forming instead three atom wide (111)-like terraces.

The few stepped surfaces that have been analyzed show some similarities to surface reconstruction. Two stepped iron surfaces, (210) and (211), have been analyzed. In both cases the top-layer atoms make lateral as well as vertical motions away from the terminated bulk geometry. Since the symmetry for these stepped surfaces is generally lower than for low Miller index surfaces, some lateral motions are consistent with the terminated bulk symmetry. In other cases, such as the (311) surface for both copper and nickel, there is no evidence for lateral registry shifts, only for relaxations in inter-layer spacing.

There is no difficulty in principle in carrying out LEED calculations for a reconstructed surface. The difficulty is in the number of geometrical parameters in the model that are not constrained by symmetry. There is no easy solution to finding the best fit of model LEED calculations to experimental data in a multi- 
dimensional parameter-space (see chapter 4 ). The $(2 \times 1)$ fec (110) reconstruction was recently solved for gold by Moritz and Wolf..$^{56}$ Their solution involves a missing row in the first layer, paired (laterally displaced) rows in the second layer and a buckled third layer. Once a sufficiently complex model was used in LEED calculations, is was possible to distinguish clearly between the preferred "missingrow" model and the competing "saw-tooth" model. The example illustrates the difficulty of solving complicated structures by LEED and related techniques -- the method is clearly able to identify a good model geometry, but there are no guideposts toward an adequate model. In the case of the missing-row model, previous calculations without the second and third layer relaxations had given ambiguous results. At the same time, LEED calculations are complex enough that it is not practical to exhaust all options. Table 1.3 stmmarizes the existing structural results for reconstructed and stepped surfaces.

\subsubsection{Metal surfaces and atomic adsorbates}

The behavior of atomic adsorbates on metal surfaces is determined by the thermodynamic properties of the system. In many cases adsorbates are localized on the surface, forming various ordered structures depending on the surface temperature and adsorbate concentrations. In the case of physisorption the adsorbate-surface interaction energy is low, less than $\sim 0.1 \mathrm{eV}$ per atom, as in the case of inert gas adsorption. Chemisorption energies are higher, $\sim 1 \mathrm{eV}$ per atom. The structures of a number of atomic adsorbates on metals have been determined, including adsorbed alkali and transition metal atoms, chalcogen atoms 
and other light atoms.

Structure determination techniques today are sufficient to handle most systems with atomic adsorbates localized at the surface. In particular the system of chalcogen adscrption on nickel surfaces has become a "bench-mark" system, studied by many different structure-sensitive techniques. The results of these studies are summarizus in Table 1.4. In general chalcogen atoms tend to adsorb in the most highly coordinated sites on the surface and adsorbate atoms do not fill nearest-neighbor sites, but form overlayers with next-nearest neighbor sites occupied, such as (2x2) and $c(2 \times 2)$. In some cases oxygen is adsorbed in sub-surface sites as a precursor to the formation of a bulk oxide.

Table 1.5 lists surface structures resulting from the adsorption of other atomic species on metal surfaces. These systems follow the pattern of chalcogen adsorption. Transition metal atoms also adsorb in highly coordinated sites, as do several other species, including nitrogen, carbon and halogen atoms for the relatively few cases that have been investigated. Nearest neighbor sites may be occupied, depending on the atomic radii. Exceptions are found in cesium, which adsorbs on top-sites on $\mathrm{Cu}(111)^{57}$ and hydrogen, which adsorbs in neighboring bridge sites on $W(100) .58,59$

For these simple adsorbates, the substrate structure is mostly unchanged during chemisorption. Where the surface layer spacing has a significant contraction chemisorption may reduce or even reverse the change from the bulk layer spacing. Some surface reconstructions, such as the fcc (100) hexagonal reconstruction, are no longer stable in the case of chemisorption. In the case of carbon 
adsorption on $\mathrm{Fe}(100)$ a surface reconstruction is induced by clemisorption, with lateral shifts in top-layer atom positions. In other systems there may be smaller motions of substrate atoms which have not been investigated in most of the previous structure studies. For example, a recent analysis of ARPEFS data for sulfur adsorbed in a $c(2 \times 2)$ arrangement on nickel (100) showed a significant improvement in the fit between theory and experiment when buckling was included in the second nickel layer and for the (2x2) arrangement first-layer lateral motions were also important. 60

Other adsorption systems have more complicated thermodynamic properties, where instead of a simple overlayer more complex surface phases are formed involving multiple layers in the creation of new bulk phises, as in the case of iron oxidation, or carbon adsorption on rhodium at elevated temperatures. ${ }^{61}$ In still other cases there are no stable surface phases and adsorbates move directly into the bulk substrate, as in hydrogen adsorption on palladium. Most of the solved chemisorption surface structures are for cases where the adsorbate is localized on the surface, with little modification to the substrate structure.

\subsubsection{Semiconductors}

Semiconductor surfaces are characterized by stronger and more directional chemical bonds than metals. Broken bonds at the surface provide a thermo dynamic driving force for surface reconstructions and most semiconductors surfaces reconstruct. Even when the translational symmetry of the surface is unchanged, as for the (110) surfaces of several III-V semiconductors, there are 
substantial changes in the surface geometry. Theoretical studies and total-energy calculations suggest that semiconductor bond-lengths are preserved during reconstructions and bonds are rotated and bond angles changed as dangling bonds at the surface are taken up .by nearby atoms. Such bond-length preserving reconstructions can involve substantial atomic motions, $\sim 0.5$ to $1.0 \AA$, both lateraliy and vertically in the top several layers of the surface.

Chemisorption usually changes the surface layer, often changing the reconstruction or restoring the bulk periodicity to the surface. Some semiconductor surface structures have been solved, such as the $(2 \times 2)$ reconstruction of the (111) face of $\mathrm{GaAs}$ and $\mathrm{GaP}$. Other reconstructions such as the $(7 \times 7)$ reconstruction of the $\mathrm{Si}(111)$ surface, with 49 atoms per layer in the unit ceil and a reconstruction that goes several layers deep, are so complex with so many geometrical parameters that it is unclear what a structural "solution" would mean. Solved semiconductor sur"ace structures are described in Table 1.6.

Surface structurc has been investigated for only a few semiconductor chemisorption systems. These results are listed in Table 1.7. For several of these systems only the local adsorption geometry is known from fine-structure experiments, while the detailed effects of chemisorption on semiconductor surface geometry are not well understood. 


\subsubsection{Insulator surfaces}

Relatively few insulator surface structures have been determined. In part attention has been focused on semiconductors and metals because of their relative importance in the electronics and chemical industries. Further, electronic spectroscopies are experimentally complicated for non-conducting samples. The known insulator surface structures are tabulated in Table 1.8. There are a few trends evident in this list. The (100) surfaces of $\mathrm{CoO}, \mathrm{MgO}$ and $\mathrm{NiO}$ have been investigated and they all show close to terminated bulk structures, with little or no top-layer buckling or relaxations. For the A-B-A tayer compounds that have been investigated, including $\mathrm{Na}_{2} \mathrm{O}, \mathrm{MoS}_{2}$ and $\mathrm{NbSe}_{2}$ the surface terminates with a normally stacked A-B-A arrangement.

\subsubsection{Molecular adsorbates}

Structure determination for adsorbed molecules is generally more complicated than for atomic adsorbates. The chemical identity of a molecule may change on adsorption. The unit cells are often larger and the internal geometry of the molecule may be changed during chemisorption. These internal degrees of freedom must be determined along with the atomic structure of the substrate.

Molecular adsorbates in large unit cells have been studied with molecules adsorbed in in-equivalent sites within the unit cell (see chapter 10). Molecular co-adsorption systems have also been studied, where more than one type of molecule is adsorbed in a unit cell 16 to 20 times the size of the substrate unit cell (see chapter 11). 
Carbon monoxide is the most extensively studied molecular adsorbate. Table 1.9 lists the structural results of a number of investigations of carbon monoxide adsorption on metal surfaces. In general, carbon monoxide adsorbs intact on a number of metals, with the $\mathrm{C}-\mathrm{O}$ bond axis perpendicular to the surface and the carbon end bonded to the substrate. At low coverages top sites are usually occupied first, with bridge sites occupied as adsorbate-adsorbate interactions become significant. Adsorption in three-fold hollow sites on fcc (111) metal surfaces has been observed when carbon monoxide is coadsorbed with other molecules.

Sufficient structural information is now available to establish a correlation between carbon monoxide adsorption site, as determined by LEED and the C-O vibrational frequency, measured by HREELS for surface species. This information is shown in figure 1.1. The relationship between $\mathrm{C}-\mathrm{O}$ stretching frequency, $\mathrm{C}-\mathrm{O}$ bond length and the carbon-metal bond length is shown in figure 1.2. The studies on carbon monoxide help to establish another useful correlation -- that between surface species and urgano-metallic cluster compounds. A number of cluster compounds with adsorption sites analogous to surface can be synthesized. These clusters provide very high surface area samples, so their structure can be determined by X-ray diffraction and their vibrational properties measured by infrared spectroscopy. Figure 1.3 shows the structural correlation between metal carbonyl clusters and carbon monoxide adsorbed on surfaces. Cluster results cannot be carried directly over to surfaces, but they provide valuable structural analogies and also vibrational spectral signatures associated with chemical bonding geometries. 
Only a few molecular adsorbate structures are known for molecules other than carbon monoxide. These are summarized in Table 1.10. As discussed in chapters 10, 11 and 12 the tools to determine the structure of more complex molecular adsorption systems are being developed and this is an area of investigation that should see significant progress in the near future. 


\section{References}

1. G. A. Somorjai and M. A. Van Hove, "Adsorbed Monolayers on Solid Surfaces," in Structure and Bonding, vol. 38, Springer Verlag, Berlin, 1979.

2. J. B. Pendry and M. A. Van Hove, Surface Crystallography Information Service, to be published.

3. J. B. Pendry and D. K. Saldin, Sutface Science, vol. 145, p. 33, 1984.

4. D. K. Saldin, J. B. Pendry, M. A. Van Hove, and G. A. Somorjai, Physical Review B, vol. 31, p. 1216, 1985.

5. M. A. Van Hove, S. W. Wang, D. F. Ogletree, and G. A. Somorjai, Advances in Quantum Chemistry, in preparation.

6. J. E. Demuth and D. E. Eastman, Solid State Communications vol. 18, p. $1497,1976$.

7. C. Y. Yang, H. L. Yu, and D. A. Case, Chemical Physics Letters, vol. 81, p. 170,1651 .

8. T. S. Rahman, J. E. Black, and D. L. Mills, Physical Review Letters, vol. 46, p. $1469,1981$.

9. T. S. Rahman, D. L. Mills, J. E. Black, J. M. Szeftel, S. Lehwald, and H. Ibach, Physical Review B, vol. 30, p. 589, 1984.

10. M. Rocca, H. Ibach, S. Lehwald, M.-L. Xu, B. M. Hall, and S. Y. TOng, in Proceedings of ICSOS-1, The Structure of Surfaces, ed. M. A. Van Hove and S. Y. Tong, vol. 2, p. 156, Springer Series in Surface Science, Springer Verlag, Berlin, 1085. 
11. G. Binnig, H. Rohrer, C. Gerber, and E. Weibel, Applied Physics Letters, vol. 40, p. 178, 1982.

12. G. Binnig, H. Rohrer, C. Gerber, and E. Weibel, Applied Physics Letters, vol. 49, p. $57,1982$.

13. G. Binnig, H. Rohrer, C. Gerber, and E. Weibel, Applied Physics Letters, vol. 50, p. $1998,1983$.

14. E. W. Müller and T. T. Tsong, Field Ion Microscopy, American Elsevier Publishing Company, New York, 1969.

15. M. A. Van Hove, R. J. Koestner, J. C. Frost, and G. A. Somorjai, Surface Science, vol. 129, p. 482, 1983.

16. M. A. Van Hove, in Proceedings of ICSOS-1, The Structure of Surfaces, ed. M. A. Van Hove and S. Y. Tong, vol. 2, p. 100, Springer Series in Surface Science, Springer Verlag, Berlin, 1985.

17. B. E. Koel, J. E. Crowell, C. M. Mate, and G. A. Somorjai, Journal of Physical Chemistry, vol. 88, p. 1988, 1984.

18. P. Chen, J. Y. Kim, A. Madhukar, and N. M. Cho, Journal of Vacuum Science and Technology $B$, vol. 4, p. 890, 1986.

19. N. Masud and J. B. Pendry, Journal of Physics C, vol. 10, p. 1, 1976.

20. N. Masud, C. C. Kinniburg, and J. B. Pendry, Journal of Physics C, vol. 10, p. 1,1977 .

21. N. Masud, Journal of Physics C, vol. 13, p. 6359, 1980. 
22. P. A. Maksym and J. L. Beeby, Surface Science, vol. 140, p. 77, 1984.

23. J. J. Barton and M. A. Van Hove, to be published.

24. N. Masud, R. Baudoing, D. Aberdam, and C. Goubert, Surface Science, vol. 133, p. 580, 1983.

25. D. H. Rosenblatt, J. G. Tobin, M. G. Mason, R. F. Davis, S. D. Kevan, D. A. Shirley, C. H. Li, and S. Y. Tong, Physical Review B, vol. 23, p. 3828, 1981.

26. J. J. Barton, C. C. Bahr, Z. Hussain, S. W. Robey, J. G. Tobin, L. E. Klebanoff, and D. A. Shirley, Physical Review Letters, vol. 51, p. 272, 1983.

27. E. W. Plummer and W. Eberhardt, Advances in Chemical Physics, vol. 49, p. 533, Wiley, New York, 1982.

28. C. S. Fadley, in Progress in Surface Science, ed. S. G. Davidson, Pergammon, New York, 1983.

29. J. J. Barton, S. W. Robey, C. C. Bahr, and D. A. Shirley, in Proceedings of ICSOS-1, The Structure of Surfaces, ed. M. A. Van Hove and S. Y. Tong, vol. 2, p. 191, Springer Series in Surface Science, Springer Verlag, Berlin, 1985.

30. J. J. Barton, C. C. Bahr, Z. Hussain, S. W. Robey, L. E. Klebanoff, and D. A. Shirley, Journal of Vacuum Science and Technology A, vol. 2, p. 847, 1984.

31. P. J. Durham, J. B. Pendry, and C. H. Hodges, Solid State Communications, vol. 38, p. $159,1981$. 
32. G. Bunker and E. A. Stern, Physical Review Letters, vol. 52, p. 1990, 1984.

33. P. J. Durham, J. B. Pendry, and D. Norman, Journal of Vacuum Science and Techrology, vol. 20, p. 665, 1982.

34. D. Norman, J. Stöhr, R. Jaeger, P. J. Durham, and J. B. Pendry, Physical Review Lelters, vol. 51, p. 2052, 1983.

35. J. Stöhr, F. Sette, and A. L. Johnson, Physical Review Letters, vol. 53, p. 1684, 1984.

36. J. A. Horsley, J. Stöhr, and R. J. Koestner, Journal of Chemical Physics, vol. 83 , p. $3146,1985$.

37. J. Stöhr, in Proceedings of JCSOS-1, The Structure of Surface6, ed. M. A. Van Hove and S. Y. Tong, Springer Verlag, Berlin, 1985.

38. P. H. Citrin, in Proceedings of ICSOS-1, The Structure of Surfaces, ed. M. A. Van Hove and S. Y. Tong, vol. 2, p. 149, Springer Series in Surface Science, Springer Verlag, Berlin, 1985.

39. T. L. Einstein, Applications of Surface Science, vol. 11/12, p. 42, 1982.

40. G. Erlich, in Proceedings of ICSOS-1, The Structure of Surfaces, ed. M. A. Van Hove and S. Y. Tong, Springer Veriag, Berlin, 1985.

41. J. Lapujoulade, B. Salanon, and D. Gorse, in Proceedings of ICSOS-1, The Structure of Surfaces, ed. M. A. Van Hove and S. Y. Tong, vol, 2, p. 176, Springer Series in Surface Science, Springer Verlag, Berlin, 1985.

42. K. H. Reider and T. Engel, Physical Review Letters, vol. 45, p. 824, 1980. 
43. K. H. Reider, Surface Science, vol. 128, p. 325, 1983.

44. C. M. Mate, private communication.

45. J. Tersoff and D. R. Hamann, Physical Review Letters, vol. 50, p. 1998, 1983.

46. J. Tersoff, in Proceedings of ICSOS-1, The Structure of Surfaces, ed. M. A. Van Hove and S. Y. Tong, vol. 2, p. 54, Springer Series in Surface Science, Springer Verlag, Berlin, 1985.

47. J. F. van der Veen, R. M. Tromp, R. G. Smeenk, and F. W. Saris, Surface Science, vol. 82 , p. $468,1979$.

48. J. W. M. Frenken, J. F. van der Veen, and G. Allan, Physical Review Letters, vol. 51, p. 1876, 1983.

49. F. M. Hoffmann, Surface Science Reports, vol. 3, p. 107, 1983.

50. Y. R. Shen, in Proceedings of ICSOS-1, The Structure of Surfaces, ed. M. A. Van Hove and S. Y. Tong, vol. 2, p. 77, Springer Series in Surface Science, Springer Verlag, Berlin, 1985.

51. I. K. Robjinson, in Proceedings of ICSOS-1, The Structure of Surfaces, ed. M. A. Van Hove and S. Y. Tong, vol. 2, p. 60, Springer Series in Surface Science, Springer Verlag, Berlin, 1985.

52. K. S. Liang, P. H. Fuoss, G. J. Hughes, and P. Eisenberger, in Proceedings of ICSOS-1, The Structure of Surfaces, ed. M. A. Van Hove and S. Y. Tong, vol. 2, p. 60, Springer Series in Surface Science, Springer Verlag, Berlin, 1085. 
53. T. E. Madey, J. J. Czyzewski, and J. T. Yates, Jr., Surface Science, vol. 49, p. 465, 1975.

54. T. E. Madey, in Proceedings of ICSOS-1, The Structure of Surfaces, ed. M. A. Van Hove and S. Y. Tong, vol. 2, p. 264, Springer Series in Surface Science, Springer Verlag, Berlin, 1985.

55. G. A. Somorjai, Chemistry in Two Dimensions: Surfaces, Cornell University Press, Ithaca, New York, 1981.

56. W. Moritz and D. Wolf, Surface Science, vol. 163, p. L655, 1985.

57. S. A. Lindgren, L. Walldén, J. Rundgren, P. Westrin, and J. Neve, Physical Review B, vol. 28, p. 6707, 1983.

58. M. A. Passler, A. Ignatiev, B. W. Lee, D. Adams, and M. A. Van Hove, in Determination of Surface Structure by LEED, Plenum, New York, 1984.

59. M. A. Passler, B. W. Lee, and A. Ignatiev, Surface Science, vol. 150, p. 263, 1985.

60. J. J. Barton, private communication.

61. G. S. Blackman, private communication. 
Figure Captions for Chapter 1

1.1 The correlation between the carbon monoxide adsorption site as determined by LEED and the C-O stretching frequency as determined by HREELS or IRAS.

1.2 Carbon monoxide bond-lengths and $\mathrm{C}-\mathrm{O}$ stretching frequencies for adsorption on metal surfaces.

1.3 Structural correlations for carbon monoxide adsorbed on metal surfaces and metal-carbonyl clusters. 
Metal carbonyls: $C O$ stretch frequency versus $M-C$ and $C-O$ bond length

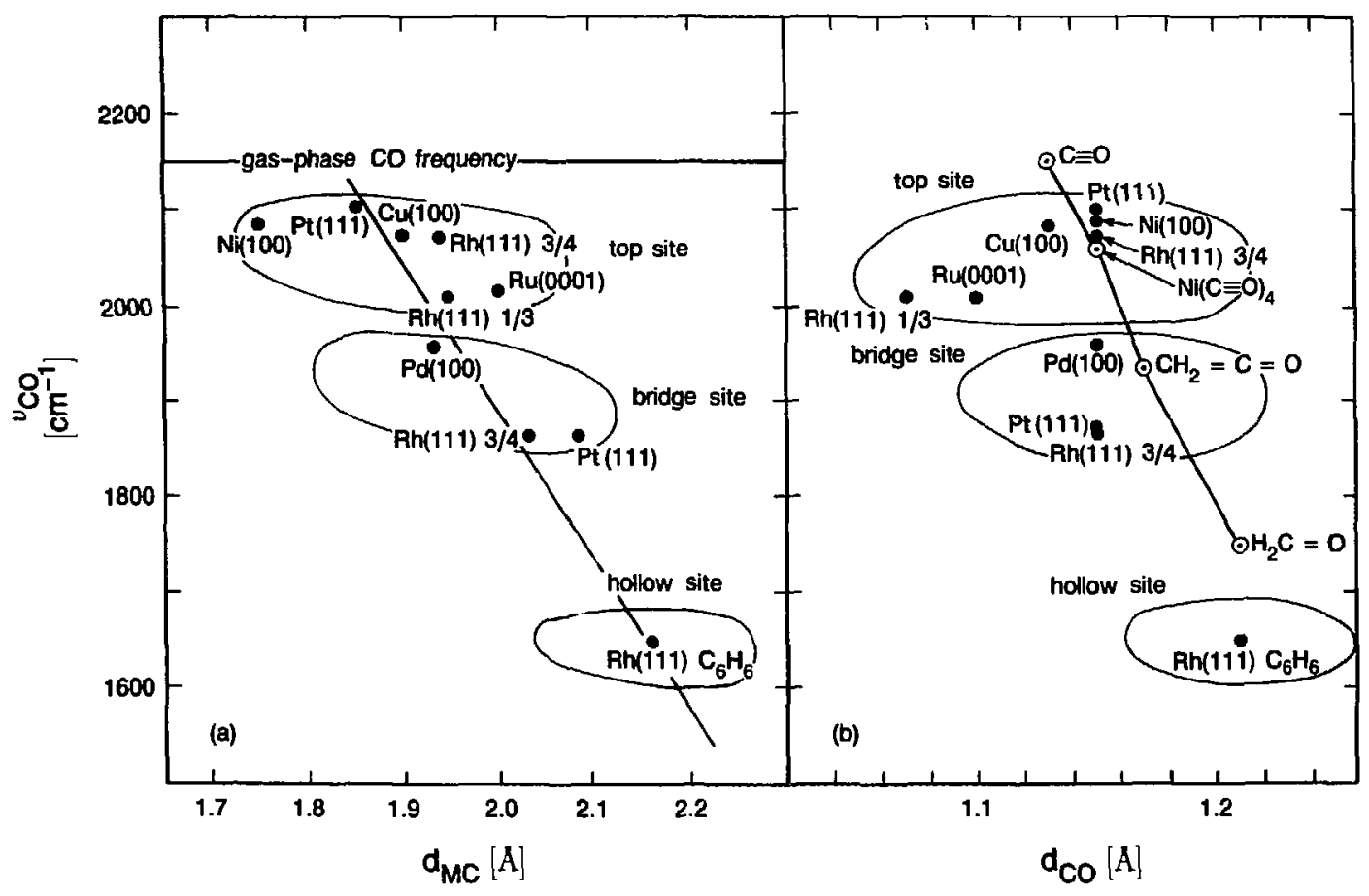

Figre 1.1

XBL 857-10612 
LEED CO adsorption site and $\mathrm{C} . \mathrm{O}$ stretch frequency

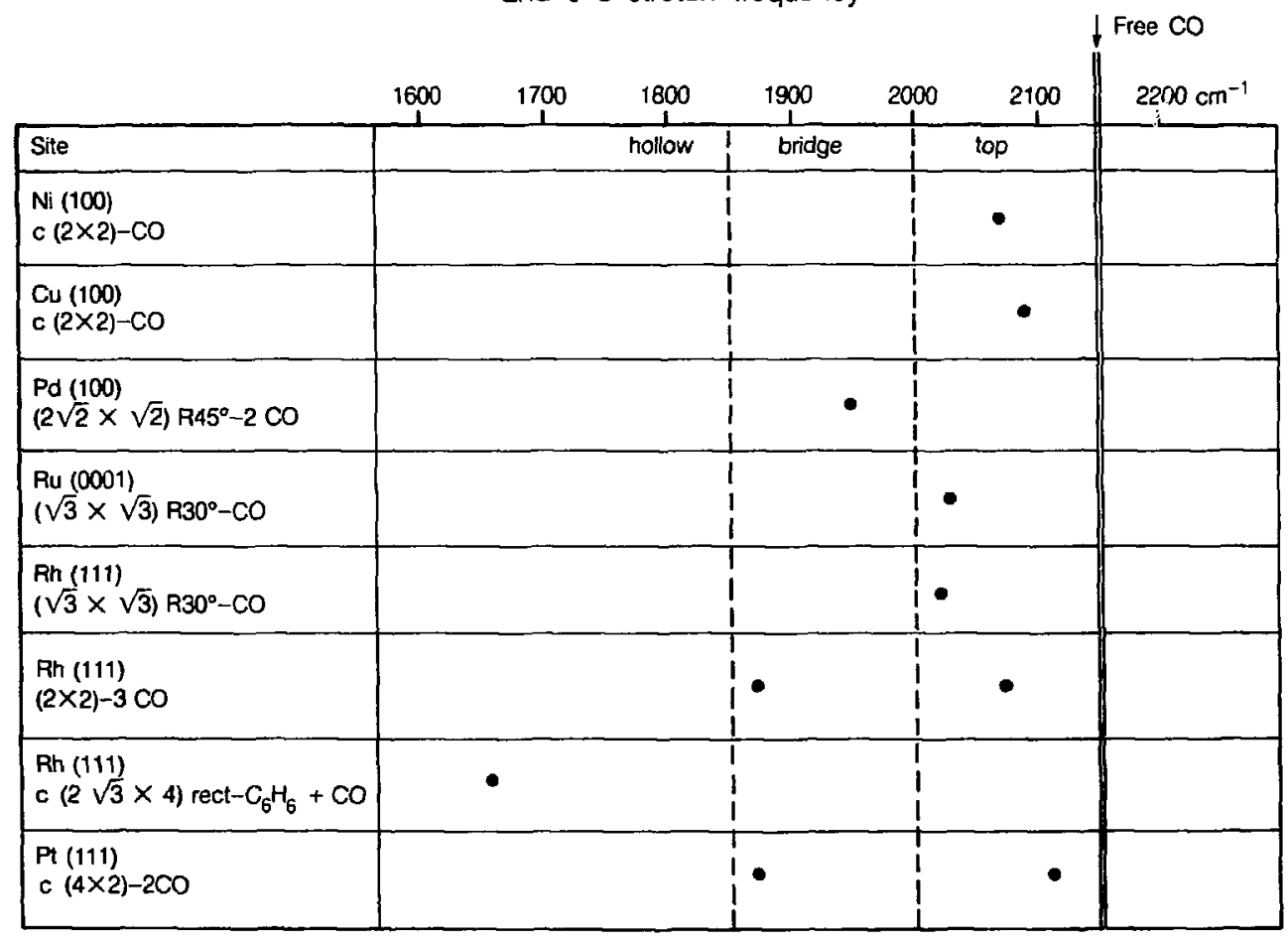


Metal Carbonyls: $\mathrm{M}-\mathrm{C}$ and $\mathrm{C}-\mathrm{O}$ bond length in clusters and at surfaces

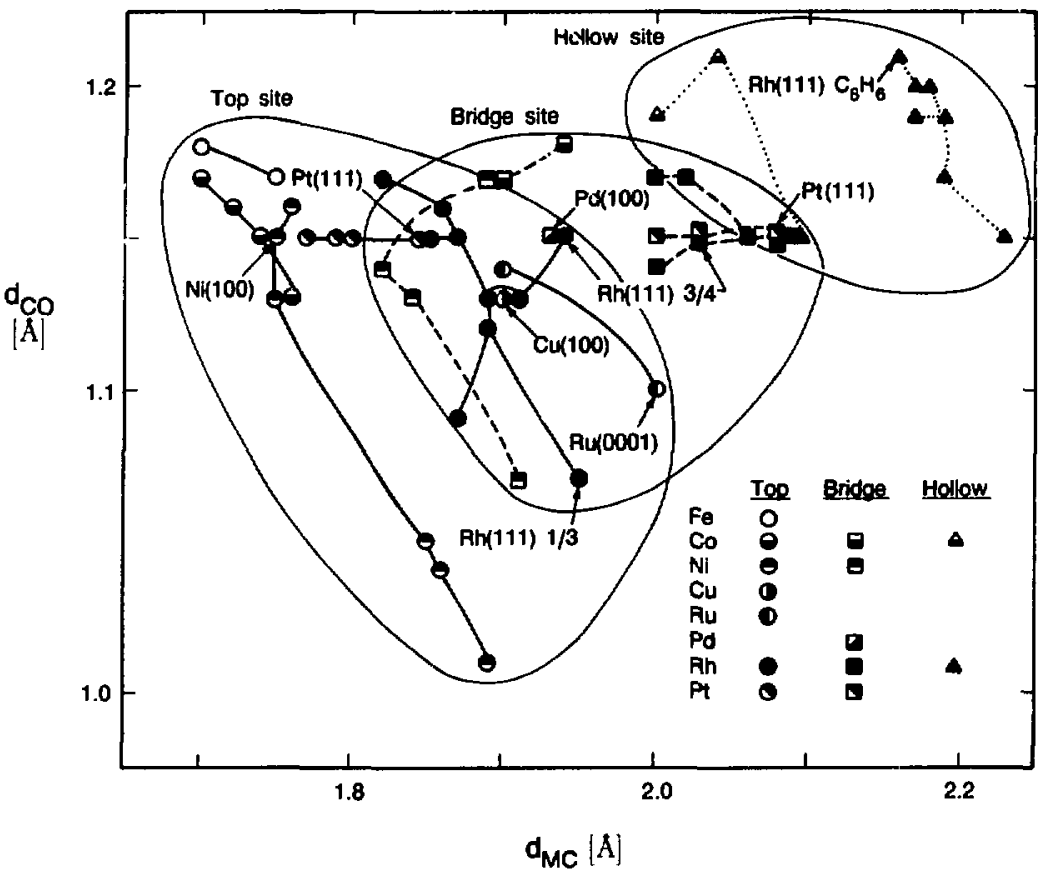

Figre 1.3 


\section{Tables for Chapter 1}

1.1 Surface science techniques

1.2 Clean, unreconstructed metal surface structures

1.3 Alloy and reconstructed metal surface structures

1.4 Chalcogen chemisorption on metals

1.5 Atomic chemisorption on metals

1.6 Clean semiconductor surface structures

3.7 Atomic adsorption on semiconductor surfaces

1.8 Insulator surface structures

1.9 Carbon monoxide chemisorption on metal surfaces

1.10 Molecular adsorption structures 


\section{Table 1.1 - Surface Science Techniques}

\begin{tabular}{|ll} 
Acronym Name & Description \\
\hline
\end{tabular}

AD Atom or Helium Diffraction
AEAPS Auger Electron Appearance Potential Spectroscopy

AES Auger Electron Spectroscopy

AFM

Atomic Force Microscopy
Moncenergetic beams of thermal energy neutral atoms are elasticly scattered off of ordered surfaces and detected as a function of scattering angle. This gives structural information on the outermost layer of the surface. Atom diffraction is extremely sensitive to surface ordering and defects.

The EAPFS cross-section is monitored by Auger tectron intensity. Also known as APAES.

Core-hole excitations are created, usually by $1-10 \mathrm{KeV}$ incident electrons, and Auger electrons of characteristic energies are emitted through a two-electron process as excited atoms decay to their ground state. AES gives information on the near-surface chemical composition.

Similar to STM. An extremely delicate mechanical probe is used to scan the topography of a surface, and a STMtype tunneling-current probe is used to measure the deflection of the mechanical surface probe. This is designed to provide STM-type images of insulating surfaces

APAES Appearance Poten- See AEAPS. tial Auger Electron Spectroscopy 


\section{Acronym}

Name

Description

APXPS Appearance Potential $X$-ray Photoemission Spectroscopy

ARAES

Angle-Resolved Auger Electron Spectroscopy

ARPEFS Angle-Resolved Photo-Emission Fine Structure

ARPES Angle-Resolved Photo-Emission Spectroscopy

ARUPS Angle-Resolved Ullraviolet Photoemission Spectros$\operatorname{cop} y$

ARXPD Angle-Resolved $X$ ray Photoemission Diffraction
The EAPFS excitation cross-section is monitored by fluorescence from core-hole decay (also known as SXAPS).

Auger electrons are detected as a function of angle to provide information on the spatial distribution or environment of the excited atoms (see AES).

Electrons are detected at given angles after being photoemitted by polarized synchrotron radiation. The interference in the detected photoemission intensity as a function of electron energy $\sim 100$ $500 \mathrm{eV}$ above the excitation threshold gives structural information.

A general term for structure sensitive photoemission techniques, including ARPEFS, ARXPS, ARUPS, and ARXPD.

Electrons photoemitted from the valence and conduction bands of a surface are detected as a function of angle. This gives information on the dispersion of these bands (which is related to surface structure), and also structural information from the diffraction of the emitted electrons.

Similar to ARXPS and ARPEFS. The angular variation in the photoemission intensity is measured at a fixed eneigy above the excitation threshold to provide structural information. 


\section{Acronym Name Description}

$\begin{array}{lll}\text { ARXPS Angle-Resolved } X- & \text { The diffraction of electrons photoemitted } \\ \text { rayPhotoemission } & \text { from core-levels gives structural informa- } \\ \text { Spectroscopy } & \text { tion on the surface. }\end{array}$

CEMS Conversion-Electron

A surface-sensitive version of Mössbauer Mössbauer Spectrosspectroscopy. Like Mössbauer Spectroscopy copy, this technique is limited to some isotopes of certain metals. After a nucleus is excited by $\gamma$-ray absorption, it can undergo inverse $\beta$-decay, creating a core-hole. The decay of core-holes by Auger processes within an electron mean free path of the surface produces a signal. Detecting emitted electrons as a function of energy gives some depthprofile information, because of the changing electron mean free path.

DAPS Dis-Appearance Potential Spectroscopy

The EAPFS cross-section is monitored by variations in the intensity of electrons elasticly back-scattered from the surface.

EAPFS Electron Appearance Potential Fine-Structure

ELNES Electron energyLoss Near-Edge Structure

A fine-structure technique (see EXAFS). Core-holes are excited by monoenergetic electrons at $\sim 1 \mathrm{KeV}$. The modulation in the excitation cross section may be monitored through adsorption, fluorescence, or Auger emission.

Similar to NEXAFS, except monoenergetic high-energy electrons $\sim 60-300$ $\mathrm{KeV}$ excite core-holes. 


\begin{tabular}{|c|c|c|}
\hline Acronym & Name & Description \\
\hline ELS & $\begin{array}{l}\text { Electron Energy } \\
\text { Loss Spectroscopy }\end{array}$ & $\begin{array}{l}\text { Monoenergetic electrons } \sim 5-50 \mathrm{eV} \text { are } \\
\text { scattered off a surface the energy losses } \\
\text { are measured. This gives information on } \\
\text { the electronic excitations of the surface } \\
\text { and adsorbed molecules (see HREELS). } \\
\text { Sometimes called EELS. }\end{array}$ \\
\hline ESCA & $\begin{array}{l}\text { Electron Spectros- } \\
\text { copy for Chemical } \\
\text { Analysis }\end{array}$ & Now generally called XPS. \\
\hline ESDLAD & $\begin{array}{l}\text { Electron Stimulated } \\
\text { Desorption Ion } \\
\text { Angular Distribution }\end{array}$ & $\begin{array}{l}\text { Electrons break chemical bonds in } \\
\text { adsorbed atoms or molecules, causing } \\
\text { ionized atoms or radicals to be ejected } \\
\text { from the surface along the axis of the } \\
\text { broken bond by Coulomb repulsion. } \\
\text { The aitgular distribution of these ions } \\
\text { gives information on the bonding } \\
\text { geometry of adsorbed molecules. }\end{array}$ \\
\hline EXAFS & $\begin{array}{l}\text { Extended } X \text {-ray } \\
\text { Adsorption Fine- } \\
\text { Structure }\end{array}$ & $\begin{array}{l}\text { Monoenergetic photons excite a core- } \\
\text { hole. The modulation of the adsurption } \\
\text { cross-section with energy } \sim 100-500 \mathrm{eV} \\
\text { above the excitation threshold yields } \\
\text { information on the radial distances to } \\
\text { neighboring atoms. The cross-section can } \\
\text { be monitored by fluorescence as core- } \\
\text { holes decay or by the attenuation of the } \\
\text { transmitted photon beam. EXAFS is } \\
\text { one of many "fine-structure" techniques. } \\
\text { This is not intrinsicly surface sensitive } \\
\text { (see SEXAFS). }\end{array}$ \\
\hline EXELFS & $\begin{array}{l}\text { Extended } X \text {-ray } \\
\text { Energy Loss Fine } \\
\text { Structure }\end{array}$ & $\begin{array}{l}\text { A fine-structure technique similar to } \\
\text { EXAFS, except that } 60-300 \mathrm{KeV} \text { elec- } \\
\text { trons rather than photons excite core- } \\
\text { holes. Like EXAFS, this techniques is } \\
\text { not explicitly surface sensitive. }\end{array}$ \\
\hline
\end{tabular}




\begin{tabular}{|c|c|c|}
\hline Acronym & Name & Description \\
\hline FIM & $\begin{array}{l}\text { Field-Ionization } \\
\text { Microscopy }\end{array}$ & $\begin{array}{l}\text { A strong electric field volts/angstrom } \\
\text { is created at the tip of a sharp, single } \\
\text { crystal wire. Gas atoms, usually He, are } \\
\text { polarized and attracted to the tip by the } \\
\text { strong electrostatic field, and then ion- } \\
\text { ized by electrons tunneling from the gas } \\
\text { atoms into the tip. These ions, } \\
\text { accelerated along radial trajectories by } \\
\text { Coulomb repulsion, map out the varia- } \\
\text { tions in the electric-field strength across } \\
\text { the surface with atomic resolution, show- } \\
\text { ing the surface topography. }\end{array}$ \\
\hline FTIR & $\begin{array}{l}\text { Fourier-Transform } \\
\text { Infra-Red spectros- } \\
\text { copy }\end{array}$ & $\begin{array}{l}\text { Broad-band IRAS experimerts are per- } \\
\text { formed, and the IR adsorption spectrum } \\
\text { is deconvoluted by using a doppler- } \\
\text { shifted source and Fourier analysis of } \\
\text { the data. This technique is not res- } \\
\text { tricted to surfaces. }\end{array}$ \\
\hline HEIS & $\begin{array}{l}\text { High-Energy Ion } \\
\text { Scattering }\end{array}$ & $\begin{array}{l}\text { High-energy ions, above } ~ 500 \mathrm{KeV} \text {, are } \\
\text { scattered off of a single crystal surface. } \\
\text { The "channeling" and "blocking" of scat- } \\
\text { tered ions within the crystal can be used } \\
\text { to triangulate deviations from the bulk } \\
\text { structure. HEIS has been used in partic- } \\
\text { ular to study surface reconstructions and } \\
\text { the thermal vibrations of surface atoms } \\
\text { (see also MEIS, ISS) }\end{array}$ \\
\hline HREELS & $\begin{array}{l}\text { High-Resolution } \\
\text { Electron Energy } \\
\text { Loss Spectroscopy }\end{array}$ & $\begin{array}{l}\text { A monoenergetic electron beam, usually } \\
\sim 2-10 \mathrm{eV} \text {, is scattered off a surface and } \\
\text { energy losses below } \sim 0.5 \mathrm{eV} \text { to bulk and } \\
\text { surface phonons and vibrational excita- } \\
\text { tions of adsorbates are measured as a } \\
\text { function of angle and energy (also called } \\
\text { EELS). }\end{array}$ \\
\hline
\end{tabular}




\begin{tabular}{|c|c|c|}
\hline Acronyn! & Name & Description \\
\hline INS & $\begin{array}{l}\text { Ion Neutralization } \\
\text { Spectroscopy }\end{array}$ & $\begin{array}{l}\text { Slow ionized atoms, typically } \mathrm{He}^{+} \text {, are } \\
\text { incident on a surface where they are } \\
\text { neutralized in a two-electron process } \\
\text { which can eject a surface electron, a pro- } \\
\text { cess similar to Auger emission from the } \\
\text { valence band. The ejected electrons are } \\
\text { detected as a function of energy, and the } \\
\text { surface density of states can be deter- } \\
\text { mined from the energy distribution. } \\
\text { The interpretation of the data is more } \\
\text { complicated than for SPI or UPS. }\end{array}$ \\
\hline IRAS & $\begin{array}{l}\text { Infrared Reflection } \\
\text { Adsorption Spec- } \\
\text { troscopy }\end{array}$ & $\begin{array}{l}\text { Monoenergetic IR photons are reflected } \\
\text { off a surface, and the attenuation of the } \\
\text { IR intensity is measured as a function of } \\
\text { frequency. This yields a spectrum of the } \\
\text { vibrational excitations of adsorbed } \\
\text { molecules. Recent improvements in the } \\
\text { sensitivity of this technique allow IRAS } \\
\text { measurements to be made on single cry- } \\
\text { stal surfaces. }\end{array}$ \\
\hline IRES & $\begin{array}{l}\text { Infra-Red Emission } \\
\text { Spectroscopy }\end{array}$ & $\begin{array}{l}\text { The vibrational modes of adsorbed } \\
\text { molecules on a surface are studied by } \\
\text { detecting the spontaneous emission of } \\
\text { infra-red radiation from thermally } \\
\text { excited vibrational modes as a function } \\
\text { of energy. }\end{array}$ \\
\hline
\end{tabular}




\section{Acronym Name Description}

ISS

Ion-Scattering Spectroscopy

Ions are inelasticly scattered from a surface, and the chemical composition of the surface is determined from the momentum transfer to surface atoms. The energy range is $\sim 1 \mathrm{KeV}$ to $10 \mathrm{MeV}$, and the lower energies are more surface sensitive. At higher energies this technique is also known as Rutherford Back-Scattering (RBS).

LEED Low Energy Electron Diffaction

LEIS Low-Energy Ion Scattering

LEPD Low Energy Positron Diffraction

MEED Medium Energy Electron Diffraction
Monoenergetic electrcns below $\sim 500 \mathrm{eV}$ are elasticly back-scattered from a surface and detected as a function of energy and angle. This gives information on the structure of the near surface region.

Low-energy ions, below $\sim 5 \mathrm{KeV}$, are scattered from a surface, and the ion "shadowing" gives information on surface structure. At these low energies the surface atom ion scattering cross-section is very large, resulting in large surface sensitivity. Accuracy is limited because the low energy ion scattering crosssections are not well known.

Similar to LEED with positrons as the incident particle. The interaction potential for positrons is somewhat different than for electrons, so the form of the structural information is modified.

Similar to LEED, except the energy range is higher, $\sim 300-1000 \mathrm{eV}$. LEED calculational methods break down in this energy range. New methods are being developed for glancing angle scattering, which emphasizes forward scattering. 


\section{$\begin{array}{lll}\text { Acronym Name Description } & \end{array}$}

MEIS Medium-Energy Ion Similar to HEIS, except that incident ion Scattering

Neutron Diffraction

NEXAFS Near-Edge $X$-ray Adsorption Fine Structure

NMR Nuclear Magnetic Resonance

NPD Normal Photoelectron Diffraction energies are $\sim 50-500 \mathrm{KeV}$.

Neutron diffraction is not an explicitly surface-sensitive technique, but neutron diffraction experiments on large surfacearea samples have provided important structural information on adsorbed molecules, and also on surface phase transitions.

A core-hole is excited as in fine-structure techniques (see EXAFS), except the fine-structure within $\sim 30 \mathrm{eV}$ of the excitation threshold is measured. Multiple scattering is much stronger at low electron energies, so this technique is sensitive to the local 3-dimensional geometry, not just the radial separation between the source atom and its neighbors. The excitation cross-section may be monitored by detecting the photoemitted electrons or the Auger electrons emitted during core-hole decay.

NMR is not an explicitly surfacesensitive technique, but NMR data on large surface-area samples has provided useful data on molecular adsorption geometries.

Similar to ARPEFS with a somewhat lower energy range. 


\begin{tabular}{|c|c|c|}
\hline Acronym & Name & Description \\
\hline RBS & $\begin{array}{l}\text { Rutherford Back- } \\
\text { Scattering }\end{array}$ & $\begin{array}{l}\text { Similar to ISS, except the main focus is } \\
\text { on depth-profiling and composition. The } \\
\text { momentum transfer in back-scattering } \\
\text { collisions between nuclei is used to iden- } \\
\text { tify the nuclear masses in the sample, } \\
\text { and the smaller, gradual momentum-loss } \\
\text { of the incident nucleus through } \\
\text { electron-nucleus interactions provides } \\
\text { depth-profile information. }\end{array}$ \\
\hline RHEED & $\begin{array}{l}\text { Reflection High } \\
\text { Energy Electron } \\
\text { Diffraction }\end{array}$ & $\begin{array}{l}\text { Monoenergetic electrons of } \sim 1-20 \mathrm{KeV} \\
\text { are elasticly scattered from a surface at } \\
\text { glancing incidence, and detected as a } \\
\text { function of angle and energy for small } \\
\text { forward-scattering angles. Back- } \\
\text { scattering is less important at high ener- } \\
\text { gies, and glancing incidence is used to } \\
\text { enhance surface sensitivity. }\end{array}$ \\
\hline SEELFS & $\begin{array}{l}\text { Surface Electron } \\
\text { Energy Loss Fine } \\
\text { Structure }\end{array}$ & $\begin{array}{l}\text { A fine structure technique similar to } \\
\text { EXELFS, except the incident electron } \\
\text { energies are } \sim 100-3000 \mathrm{eV} \text {. SEELFS is } \\
\text { surface sensitive because of the lower } \\
\text { excitation energy. }\end{array}$ \\
\hline SERS & $\begin{array}{l}\text { Surface Enhanced } \\
\text { Raman Spectroscopy }\end{array}$ & $\begin{array}{l}\text { Some surface geometries (rough surfaces) } \\
\text { concentrate the electric fields of incident } \\
\text { light sufficiently to enhance the Raman } \\
\text { scattering cross-section so that it is sur- } \\
\text { face sensitive. This gives information on } \\
\text { surface vibrational modes, and some } \\
\text { information on geometry via selection } \\
\text { rules. }\end{array}$ \\
\hline
\end{tabular}


Acronym

SEXAFS Surface Extended

$X$-ray Adsorption

Fine-Structure

SHG Second Harmonic Generation

SIMS Secondary Ion Mass Spectroscopy
Description

A surface-sensitive version of EXAFS, where the excitation cross-section finestructure is monitored by detecting the photoemitted electrons (PE-SEXAFS), Auger electrons emitted during core-hole decay (Auger-SEXAFS), or ions excited by photoelectrons and desorbed from the surface (PSD-SEXAFS).

A surface is illuminated with a highintensity laser, and photons are generated at the second-harmonic frequency through non-linear optical process. For many materials only the surface region has the appropriate symmetry to produce a SHG signal. The non-linear polarizability tensor depends on the nature and geometry of adsorbed atoms and molecules.

Ions and ionized clusters ejected from a surface during ion bombardment are detected with a mass spectrometer. Surface chemical composition and some information on bonding can be extracted from SMS ion fragment distributions.

Neutral atoms, usually $\mathrm{He}$, in excited states are incident on a surface at thermal energies. A surface electron may tunnel into the unoccupied electronic level, causing the incident atom to become ionized and eject an electron, which is then detected. This technique ineasures the density of states near the Fermi-level, and is Lighly surface sensitive. 


\section{Acronym \\ Name \\ Description}

SPLEED Spin-Polarized Low

Energy Electron

Diffraction

STM Scanning Tunneling Microscopy

SXAPS

Soft $X$-ray Appearance Potential Spectroscopy

TEM Transmission Electron Microscopy

TDS Thermal Desorption Spectroscopy
Similar to LEED, except the incident electron beam is spin-polarized. This is particularly useful for the study of surface magnetism and magnetic ordering.

The topography of a surface is measured by mechanically scanning a probe over a surface with Angstrom resolution. The distance from the probe to the surface is measured by the probe-surface tunneling current. Also known as Scanning Electron Tunneling Microscopy (SETM).

Another name for APXPS.

TEM can provide surface information for carefully prepared and oriented bulk samples. Real images have been formed of the edges of erystals where surface planes and surface diffusions have been observed. Diffraction patterns of reconstructed surfaces, superimposed on the bulk diffraction patlern, have also provided surface structural information.

An adsorbate-covered surface is heated, usually at a linear rate, and the desorbing atoms or molecules are detected with a mass spectrometer. This gives information on the nature of adsorbate species and some information on adsorption energies. 


\section{$\begin{array}{lll}\text { Acronym Name Description } & \end{array}$}

TPD Temperature Programmed Desorption

UPS Ultra-violet Photoemission Spectroscopy

WF Work Function measurements

XANES $X$-ray Adsorption Near-Edge Structure

XPS X-ray Photoemission Spectroscopy
Similar to TDS, except the surface may be heated at, a non-uniform rate to get more selective information on adsorption energies.

Electrons photoemitted from the valence and conduction bands are detected as a function of energy to measure the electronic density of states near the surface. This gives information on the bonding of adsorbates to the surface (see ARUPS).

Changes in the work-function during the adsorption of atoms and molecules provide information on charge-transfer and chemical bonding.

Another name for NEXAFS.

Electrons photoemitted from atomic core levels are detected as a function of energy. The shifts of core level energies gives information on the chenical environment of the atoms (see ARXPS, ARXPD). 


\begin{tabular}{|c|c|c|}
\hline Acronym & Name & Description \\
\hline XRD & X-Ray Diffraction & $\begin{array}{l}\text { X-ray diffraction has been carried out at } \\
\text { extreme glancing angles of incidence } \\
\text { where total reflection assures surface } \\
\text { sensitivity. This provides structural } \\
\text { information that can be interpreted by } \\
\text { well-known methods. An extremely high } \\
\text { x-ray flux is required to get useful data } \\
\text { from single crystal surfaces. Bulk x-ray } \\
\text { diffraction is used to determine the } \\
\text { structure of organo-metallic clusters, } \\
\text { which provice comparisons to molecules } \\
\text { adsorbed on surfaces. X-ray diffraction } \\
\text { has also given structural information on } \\
\text { large surface-area samples. }\end{array}$ \\
\hline
\end{tabular}




\section{Table 1.2 - Clean Metals (unreconstructed)}

(Where multiple layer spacir $r_{\ddot{3}}$ changes have been investigated these are listed in the table on successive lint:.)

\begin{tabular}{|c|c|c|c|c|}
\hline $\begin{array}{c}\text { Substrate } \\
\text { Face }\end{array}$ & $\begin{array}{c}\text { Bulk } \\
\text { Spacing }(\AA)\end{array}$ & $\begin{array}{c}\text { Surface } \\
\text { Spacing }(\AA)\end{array}$ & Expansion (\%) & Method \\
\hline $\mathrm{Ag}(110) \mathrm{fec}$ & 1.44 & $\begin{array}{l}1.33 \pm 0.04 \\
1.50 \pm 0.04\end{array}$ & $\begin{array}{r}-7.6 \\
4.2\end{array}$ & HEIS $^{1}$ \\
\hline $\mathrm{Al}(100) \mathrm{fcc}$ & 2.02 & $2.025 \pm 0.10$ & 0.0 & LEED $^{2}$ \\
\hline $\mathrm{Al}(100) \mathrm{fcc}$ & & 2.02 & 0.0 & LEED $^{3}$ \\
\hline $\mathrm{Al}(100) \mathrm{fcc}$ & & 2.052 & 1.0 & $\mathrm{MEED}^{4}$ \\
\hline $\mathrm{Al}(110) \mathrm{fcc}$ & 1.43 & 1.30 & -9.1 & LEED $^{3}$ \\
\hline $\mathrm{Al}(110) \mathrm{fcc}$ & & $\begin{array}{l}1.304 \pm 0.012 \\
1.499 \pm 0.15 \\
1.404 \pm 0.017 \\
1.429 \pm 0.018\end{array}$ & $\begin{array}{r}-8.8 \\
4.8 \\
-1.8 \\
0.0\end{array}$ & LEED $^{5}$ \\
\hline Al (110) fec & & $\begin{array}{l}1.310 \pm 0.014 \\
1.510 \pm 0.016 \\
1.463 \pm 0.019 \\
1.455 \pm 0.022\end{array}$ & $\begin{array}{r}-8.4 \\
5.6 \\
2.3 \\
1.7\end{array}$ & $\mathrm{LEED}^{6}$ \\
\hline $\mathrm{Al}(111) \mathrm{fcc}$ & 2.33 & $2.350 \pm 0.012$ & 0.9 & $\mathrm{LEED}^{7}$ \\
\hline $\mathrm{Al}(111) \mathrm{fcc}$ & & $2.41 \pm 0.05$ & 3.1 & LEED $^{8}$ \\
\hline $\mathrm{Al}(311) \mathrm{fcc}$ & 1.23 & $\begin{array}{l}1.68 \pm 0.01 \\
1.335 \pm 0.02\end{array}$ & $\begin{array}{r}-13.0 \\
8.8\end{array}$ & LEED $^{9}$ \\
\hline $\mathrm{Au}(100) \mathrm{fcc}$ & 2.04 & 2.04 & 0.0 & LEED $^{10}$ \\
\hline Co $(100)$ fcc & 1.77 & 1.70 & -4.0 & LEED $^{11}$ \\
\hline Co (111) fcc & 2.05 & $2.05 \pm c .05$ & 0.0 & LEED $^{12}$ \\
\hline Co $(0001)$ hep & 2.05 & $2.05 \pm 0.05$ & 0.0 & LEED $^{12}$ \\
\hline Co $(11 \overline{2} 0) h e p$ & 1.25 & $1.14 \pm 0.04$ & -8.8 & $\mathrm{LEED}^{13}$ \\
\hline $\mathrm{Cu}(100) \mathrm{fec}$ & 1.81 & $\begin{array}{l}1.785 \\
1.836 \\
1.832\end{array}$ & $\begin{array}{r}-1.1 \\
1.7 \\
1.5\end{array}$ & $\mathrm{LEED}^{14}$ \\
\hline $\mathrm{Cu}(110) \mathrm{fec}$ & 1.28 & $\begin{array}{l}1.159 \\
1.305\end{array}$ & $\begin{array}{r}-9.2 \\
2.3\end{array}$ & LEED $^{14}$ \\
\hline
\end{tabular}


$\begin{array}{cccc}\begin{array}{c}\text { Substrate } \\ \text { Face }\end{array} & \begin{array}{c}\text { Bulk } \\ \text { Spacing }(\AA)\end{array} & \begin{array}{c}\text { Surface } \\ \text { Spacing }(\hat{A})\end{array} & \text { Expansion (\%) }\end{array}$

\begin{tabular}{|c|c|c|c|c|}
\hline $\mathrm{Cu}(110)$ fec & & $\begin{array}{l}1.21 \pm 0.02 \\
1.32 \pm 0.02\end{array}$ & $\begin{array}{r}-5.3 \\
3.3\end{array}$ & MEIS $^{15}$ \\
\hline $\mathrm{Cu}(110) \mathrm{fcc}$ & & $\begin{array}{l}1.170 \pm 0.008 \\
1.307 \pm 0.010\end{array}$ & $\begin{array}{r}-8.5 \\
2.3\end{array}$ & $\operatorname{LEED}^{16}$ \\
\hline $\mathrm{Cu}$ (111) fcc & 2.09 & $2.076 \pm 0.02$ & -0.7 & LEED $^{17}$ \\
\hline $\mathrm{Cu}(311) \mathrm{fcc}$ & 1.09 & $1.035 \pm 0.02$ & -5.0 & $\operatorname{LEED}^{18}$ \\
\hline $\mathrm{Fe}(100) \mathrm{bcc}$ & 1.43 & $1.41 \neq 0.04$ & -1.6 & LEED $^{19}$ \\
\hline $\mathrm{Fe}(110) \mathrm{bcc}$ & 2.02 & $2.04 \pm 0.04$ & 0.5 & LEED $^{20}$ \\
\hline $\mathrm{Fe}(111) \mathrm{bcc}$ & 0.83 & $0.70 \pm 0.03$ & -15.4 & LEED $^{21}$ \\
\hline $\mathrm{Fe}(111) \mathrm{bec}$ & & $\begin{array}{l}0.69 \pm 0.025 \\
0.75 \pm 0.025 \\
0.86 \pm 0.03 \\
0.81 \pm 0.025\end{array}$ & $\begin{array}{r}-16.6 \\
-9.3 \\
4.0 \\
-2.1\end{array}$ & LEED $^{22}$ \\
\hline $\mathrm{Fe}(210)^{*} \mathrm{bcc}$ & 0.64 & $\begin{array}{l}0.50 \pm 0.03 \\
0.57 \pm 0.03 \\
0.61 \pm 0.03 \\
0.64 \pm 0.03\end{array}$ & $\begin{array}{r}-21.9 \\
-10.9 \\
-4.7 \\
0.0\end{array}$ & LEED $^{23}$ \\
\hline $\mathrm{Fe}(211)^{*} \mathrm{bcc}$ & 1.17 & $\begin{array}{l}1.05 \pm 0.03 \\
1.23 \pm 0.03 \\
1.15 \pm 0.04\end{array}$ & $\begin{array}{r}-10.3 \\
5.1 \\
-1.7\end{array}$ & $\mathrm{LEED}^{24}$ \\
\hline Ir $(100)$ fec & 1.92 & $1.85 \pm 0.01$ & -3.6 & LEED $^{25}$ \\
\hline $\operatorname{Ir}(110)$ Icc & 1.36 & $1.26 \pm 0.10$ & -7.4 & LEED $^{26}$ \\
\hline $\operatorname{Ir}(111)$ fec & 2.22 & $2.16 \pm 0.10$ & -2.6 & LEED $^{27}$ \\
\hline $\mathrm{Na}(110) \mathrm{bcc}$ & 3.03 & 3.03 & 0.0 & $\mathrm{LEED}^{28}$ \\
\hline $\mathrm{Na}(110) \mathrm{bcc}$ & & $3.0 \pm 0.01$ & -1.0 & LEED $^{29}$ \\
\hline $\mathrm{Na}(0001) \mathrm{hcp}$ & 2.87 & 2.87 & 0.0 & $\operatorname{LEED}^{30}$ \\
\hline $\mathrm{Ni}(100)$ fcc & 2.22 & $1.78 \pm 0.02$ & 1.1 & LEED $^{31}$ \\
\hline $\mathrm{Ni}(100)$ fec & & $1.604 \pm 0.008$ & -8.9 & $\mathrm{MEIS}^{32}$ \\
\hline $\mathrm{Ni}(110)$ fec & 1.25 & $1.195 \pm 0.01$ & -4.0 & $\mathrm{MEIS}^{33}$ \\
\hline $\mathrm{Ni}(110)$ fcc & & $\begin{array}{l}1.18 \pm 0.02 \\
1.27 \pm 0.02\end{array}$ & $\begin{array}{r}-4.8 \\
2.4\end{array}$ & HEIS $^{34}$ \\
\hline
\end{tabular}


The superiattice spots in the $c(4 \times 2)$ LEED pattern become very dim for $\mathrm{T}>$ $270 \mathrm{~K}$ and they cannot be seen for $\mathrm{T}>300 \mathrm{~K}$. This appears to be due to a reversible $\mathrm{c}(4 \times 2) \underset{300 \mathrm{~K}}{\rightarrow}$ lattice gas order-disorder transition, since no appreciable amount of carbon monoxide desorption occurs below $\sim 350 \mathrm{~K}$ for $\theta=1 / 2$ and the $c(4 \times 2)$ LEED pattern reappears on cooling below $\sim 270 \mathrm{~K}$. LEED I-V curves were recorded for the $c(4 \times 2)$ phase and the structure was determined through LEED intensity calculations as described below.

\subsubsection{High coverage $(\theta \geq 0.5)$}

Three different LEED patterns have been observed for $\theta \geq 0.5$, along with streaked LEED patterns at intermediate coverages. In 1977 Ert] et al. ${ }^{1}$ reported ordered LEED patterns at $\theta=3 / 5$ (see figure 10.4) and at $\theta=2 / 3$ (see figure 10.5). These high coverage structures have unit cells that are best described with the rectangular unit cell notation introduced by Bibérian and Van Hove.11,12 in this notation the overlayer is described by a rectangular unit cell on a substrate witb three-fold rotational symmetry. The first number is the width of the unit cell in the [11 $\overline{2}]$ dirention (perpendicular to rows of atoms) and the second number is the length of the unit cell in the $[1 \overline{1} 0]$ direction (along a row). A "c" denotes a centered unit cell, as in the Wood notation. In this notation the $\mathrm{c}(4 \times 2)$ unit cell at $\theta=1 / 2$ is called $(\sqrt{3} \times 2)$-rect, the $\theta=3 / 5$ phase is $c(\sqrt{3} \times 5)$ rect and the $\theta=$ $2 / 3$ phase is $(\sqrt{3} \times 3)$-rect.

Ertl at al. determined the equilibrium surface coverage $\theta$ as a function of temperature and carbon monoxide background pressure for $\theta \geq 0.5$. They found 


\begin{tabular}{|lcccc|}
\hline $\begin{array}{c}\text { Substrate } \\
\text { Face }\end{array}$ & $\begin{array}{c}\text { Bulk } \\
\text { Spacing }(\AA)\end{array}$ & $\begin{array}{c}\text { Surface } \\
\text { Spacing( }(\AA)\end{array}$ & Expansion (\%) & Method \\
\hline \hline Ru (0001) hcp & 2.14 & $2.10 \pm 0.02$ & -1.9 & LEED $^{54}$ \\
Sc (0001) hcp & 2.64 & $2.59 \pm 0.02$ & -1.9 & LEED $^{55}$ \\
Ta (100) bcc & 1.65 & $1.47 \pm 0.03$ & -10.9 & LEED $^{56}$ \\
Te (1010) hcp & & $1.67 \pm 0.03$ & 1.2 & \\
Ti (0001) hcp & 2.34 & $2.29 \pm 0.05$ & & LEED $^{57}$ \\
V (100) bcc & 1.51 & $1.41 \pm 0.01$ & -2.1 & LEED $^{58}$ \\
V (110) bcc & 2.14 & $2.13 \pm 0.10$ & -6.6 & LEED $^{59}$ \\
W (100) fcc & 1.58 & $1.46 \pm 0.03$ & -0.5 & LEED $^{60,61}$ \\
W (110) fcc & 2.23 & $2.23 \pm 0.10$ & -7.6 & LEED $^{62}$ \\
Zr (0001) hcp & 2.57 & $2.54 \pm 0.05$ & 0.0 & LEED $^{63}$ \\
\hline
\end{tabular}

* There are relaxations in the layer registries for the stepped iron (211) and (210) surfaces in addition to layer spacing relaxations.

\section{References}

1. Y. Kuk and L. C. Feldman, Physical Review B, vol. 30, p. 5811, 1984.

2. M. A. Van Hove, S. Y. Tong, and N. Stoner, Surface Science, vol. 54, p. 259, 1976.

3. Groupe d'Etude des Surfaces, Surface Science, vol. 62, p. 567, 1977.

4. N. Masud, R. Baudoing, D. Aberdam, and C. Goubert, Surface Science, vol. 133 , p. $580,1983$.

5. J. N. Andersen, H. B. Nielsen, L. Petersen, and D. L. Adams, Journal of Physics $C$, vol. 17, p. $173,1984$. 
6. J. R. Noonan and H. L. Davis, Physical Review B, vol. 29, p. 4349, 1984.

7. H. B. Nielsen and D. L. Adams, Journal of Physics C, vol. 15, p. 615, 1982.

8. V. Martinez, F. Soria, M. C. Munoz, and J. L. Sacedon, Surface Science, vol. 128, p. 424, 1983.

9. J. R. Noonan, H. L. Davis, and W. Erley, Surface Science, vol. 152/153, p. $142,1985$.

10. E. Lang, W. Grimm, and K. Heinz, Surface Science, vol. 177, p. 169, 1982.

11. M. Maglietta, E. Zanazzi, F. Jona, D. W. Jepsen, and P. M. Marcus, Applied Physics, vol. 15, p. 409, 1978.

12. B. W. Lee, R. Alsenz, A. Ignatiev, and M. A. Van Hove, Physical Review B, vol. 17, p. 1510, 1978.

13. M. Weltz, W. Moritz, and D. Wolf, Surface Science, vol. 125, p. 473, 1983.

14. H. L. Davis and J. R. Noonan, Surface Science, vol. 126, p. 245, 1983.

15 I. Stensgaard, R. Feidenhans'l, and J. E. Sorensen, Surface Science, vol. 128, p. $281,1983$.

16. D. L. Adams, H. B. Nielsen, and J. N. Andersen, Surface Science, vol. 128, p. $284,1983$.

17. S. A. Lindgren, L. Walldén, J. Rundgren, and P. Westrin, Physical Review $B$, vol. 29 , p. 576, 1984 .

18. R. W. Streater, W. T. Moore, P. R. Watson, D. C. Frost, and K. A. R. Mitchell, Surface Science, vol. 72, p. 744, 1978. 
19. K. O. Legg, F. Jona, D. W. Jepsen, and P. M. Marcus, Journal of Physics C, vol. 10, p. $937,1977$.

20. H. D. Shih, F. Jona, and P. M. Marcus, Journal of Physics C, vol. 13, p. $3801,1980$.

21. F. E. Shih, F. Jona, D. W. Jepsen, and P. M. Marcus, Physical Review Letters, vol. 46, p. 731, 1981.

22. J. Sokolov, F. Jona, and P. M. Marcus, Physical Review B, vol. 33, p. 1397, 1986.

23. J. Sokolov, F. Jona, and P. M. Marcus, Physical Review B, vol. 31, p. 1929, 1985.

24. J. Sokolov, H. D. Shih, U. Bardi, and F. Jona, Journal of Physics C, vol. 17, p. 371,1984 .

25. K. Heinz and G. Besold, Surface Science, vol. 125, p. 515, 1983.

26. C. M. Chan, S. M. Cunningham, K. L. Luke, W. H. Weinberg, and S. P. Withrow, Surface Science, vol. 78, p. 15, 1978.

27. C. M. Chan, S. L. Cunningham, M. A. Van Hove, W. H. Weinberg, and S. P. Withrow, Surface Science, vol. 66, p. 394, 1977.

28. P. M. Echenique, Journal of Physics C, vol. 9, p. 3193, 1976.

29. S. Andersson, J. B. Pendry, and P. M. Echenique, Surface Science, vol. 65, p. 539,1977 .

30. S. A. Lindgren, J. Paul, L. Walldén, and P. Westrin, Journal of Physics $C$, vol. 15, p. 6285, 1882. 
31. J. E. Demuth, P. M. Marcus, and D. W. Jepsen, Physical Review B, vol. 11, p. $1460,1975$.

32. J. W. M. Frenken, J. F. van der Veen, and G. Allan, Physical Review Letters, vol. 51, p. 1876, 1983.

33. J. F. van der Veen, R. M. Tromp, R. G. Smeenk, and F. W. ris, Surface Science, vol. 82, p. 468, 1979.

34. R. Feidenhans'l, J. E. Sorensen, and I. Stensgaard, Surface Science, vol. 134, p. 329,1983 .

35. Y. Gauthier, R. Baudoing, Y. Joly, and C. Gaubert, Journal of Physics C, vol. 17, p. 4547,1984 .

36. E. Törnquist, E. D. Adams, M. Copel, T. Gustafsson, and W. R. Graham, Journal of Vacuum Science and Technology A, vol. 2, p. 939, 1984.

37. M. L. Xu and S. Y. Tong, Physical Review B, vol. 31, p. 6332, 1985.

38. D. L. Adams, L. E. Peterson, and C. S. Sorenson, Journal of Physics C, vol. 18, p. $1753,1985$.

39. T. Narusawa, W. M. Gibson, and E. Törnquist, Physical Review Letters, vol. 47, p. $417,1981$.

40. D. L. Adams, W. T. Moore, and K. A. R. Mitchell, Surface Science, vol. 149, p. $407,1885$.

41. L. J. Clarke, Surface Science, vol. 91, p. 131, 1880.

42. L. Morales, D. O. Garza, and L. J. Clarke, Journal of Physics C, vol. 14, p. $5391,1881$. 
43. R. J. Behm, K. Christmann, G. Ertl, and M. A. Van Hove, Journal of Chemical Physics, vol. 73, p. 2984, 1980.

44. C. J. Barnes, M. Q. Ding, M. Lindroos, R. D. Diehl, and D. A. King, Surface Science, vol. 162, p. 59, 1985.

45. Y. Kuk, L. C. Feldman, and P. J. Silverman, Physical Review Letters, vol. 50, p. $511,1983$.

46. R. Feder, Surface Science, vol. 68, p. 229, 1977.

47. J. A. Davies, T. E. Jackman, D. P. Jackson, and P. R. Norton, Surface Science, vol. 109, p. 20, 1981.

48. D. L. Adams, H. B. Nielsen, and M. A. Van Hove, Physical Review B, vol. 20, p. $4788,1979$.

49. R. Feder, H. Pleyer, P. Bauer, and N. Müller, Surface Science, vol. 109, p. $419,1981$.

50. K. Hayek, H. Glassl, A. Gutmann, H. Leonhard, P. Prutton, S. P. Tear, and M. R. Welton-Cook, Surface Science, vol. 152, p. 419, 1985.

51. H. L. Davis and D. M. Zehner, Journal of Vacuum Science and Technology, vol. 17, p. $190,1980$.

52. S. Hengrasmee, K. A. R. Mitchell, P. R. Watson, and S. J. White, Canadian Journal of Physics, vol. 58, p. 200, 1980.

53. M. A. Van Hove and R. J. Koestner, in Surface Structure by LEED, ed. P. M. Marcus and F. Jona, Plenum, New York, 1984. 
54. G. Michalk, W. Moritz, H. Pfnur, and D. Menzel, Surface Science, vol. 129, p. $92,1983$.

55. S. Tougaard, A. Ignatiev, and D. L. Adams, Surface Science, vol. 115, p. 270, 1982.

56. A. Titov and W. Moritz, Surface Science, vol. 123, p. L709, 1982.

57. R. J. Meyer, W.R. Salaneck, C. B. Duke, A. Paton, C. H. Griffiths, L. Kovnat, and L. E. Meyer, Physical Review B, vol. 21, p. 4542, 1980.

58. H. D. Shih, F. Jona, D. W. Jepsen, and P. M. Marcus, Journal of Physics $C$, vol. 9, p. 1405, 1976.

59. V. Jensen, J.N. Andersen, H. B. Nielsen, and D. L. Adams, Surface Science, vol. 116, p. 66, 1982 .

60. D. L. Adams and H. B. Nielsen, Surface Science, vol. 107, p. 305, 1981.

61. D. L. Adams and H. B. Nielsen, Surface Science, vol. 116, p. 598, 1982.

62. F. S. Marsh, M. K. Debe, and D. A. King, Journal of Physics C, vol. 13, p. $2799,1980$.

63. M. A. Van Hove and S. Y. Tong, Surface Science, vol. 54, p. 91, 1976.

64. W. T. Moore, P. R. Watson, D. C. Frost. and K. A. R. Mitchell, Journal of Physics C, vol. 12, p. L887, 1979. 


\section{Table 1.3 - Alloys and Reconstructed Metals}

\begin{tabular}{|c|c|c|c|}
\hline Substrate & Unit Cell & Structure & Method \\
\hline$A u(1)$ & $(2 \times 1)$ & $\begin{array}{l}\text { Missing-row reconstruction similar to } \\
\text { gold (110). One top layer row is missing, } \\
\text { the second layer lateral displacements } \\
\text { are } \sim 0.07 \AA \text {, and the second row is } \\
\text { buckled by }-0.24 \AA \text {. The first layer spac- } \\
\text { ing is }-20.1 \% \text {, and the second and third } \\
\text { is }-6.3 \% \text { relative to the } 1.44 \AA \text { bulk layer } \\
\text { spacing. }\end{array}$ & LEED $^{1}$ \\
\hline$\alpha-\mathrm{CuAl}(111)$ & $\sqrt{ } 3 \times \sqrt{ } 3) R 30^{\circ}$ & $\begin{array}{l}\text { Al substituted in } 1 / 3 \text { of top layer Cu } \\
\text { sites, second layer pure } \mathrm{Cu} \text {, no buckling } \\
\text { in top layer, layer spacing is } 2.05 \pm 0.05 \\
\AA \text {, the same as bulk copper. Alloy com- } \\
\text { position } 16 \% \mathrm{Al} \text { atoms in } \mathrm{Cu} \text {. }\end{array}$ & LEED $^{2}$ \\
\hline $\operatorname{Ir}(100)$ & $(5 \times 1)$ & $\begin{array}{l}\text { The top layer of the surface reconstructs } \\
\text { to form a compact hexagonal surface, } \\
\text { with } 6 / 5 \text { the density of unreconstructed } \\
\text { surface. The layer spacing expands by } \\
14.6 \pm 5.2 \% \text { from the bulk value of } 1.82 \\
\AA \text {. Some top-layer atoms are buckled } \\
\text { outward by up to an additional } 0.2 \pm 0.02 \\
\AA \text { so the hexagonal layer can fit the } \\
\text { square layer below. This is } 1 / 2 \text { to } 2 / 3 \text { of } \\
\text { the buckling required to have top layer } \\
\text { atoms in hard-sphere contact with all } \\
\text { substrate atoms. }\end{array}$ & $\operatorname{LEED}^{3}$ \\
\hline $\operatorname{Ir}(100)$ & $(5 \times 1)$ & $\begin{array}{l}\text { The top layer of the surface reconstructs } \\
\text { to form a compact hexagonal surface, } \\
\text { with } 6 / 5 \text { the density of unreconstructed } \\
\text { surface. The layer spacing expands by } \\
7.3 \pm 2.6 \% \text { from the bulk value of } 1.92 \AA \text {. } \\
\text { Some top-layer atoms are buckled out- } \\
\text { ward by up to an additional } 0.48 \pm 0.02 \AA \\
\text { so the hexagonal layer can fit the square } \\
\text { layer below. This puts the top layer } \\
\text { atoms in hard-sphere contact with all } \\
\text { substrate atoms. }\end{array}$ & LEED $^{4}$ \\
\hline
\end{tabular}




\begin{tabular}{|c|c|c|c|}
\hline Substrate & Unit Cell & Structure & Method \\
\hline Ir (110) & $(2 \times 1)$ & $\begin{array}{l}\text { Missing-row reconstruction similar to } \\
\text { gold (110). One top layer row is missing, } \\
\text { the second layer lateral displacements } \\
\text { are } \sim 0.04 \AA \text {, and the second row is } \\
\text { buckled by }-0.23 \AA \text {. The first layer spac- } \\
\text { ing is }-12.4 \% \text {, and the second and third } \\
-5.8 \% \text { relative to the } 1.36 \AA \text { bulk layer } \\
\text { spacing. }\end{array}$ & LEED $^{5}$ \\
\hline $\mathrm{NiAl}(110)$ & $(1 \times 1)$ & $\begin{array}{l}\text { Top layer spacing } 1.92 \AA \text {, contracted } 6 \% \\
\text { from bulk value of } 2.04 \AA . \mathrm{Al} \text { atoms } \\
\text { buckled out by } 0.22 \AA .\end{array}$ & LEED $^{6}$ \\
\hline $\mathrm{Ni}_{3} \mathrm{Al}(100)$ & $(1 \times 1)$ & $\begin{array}{l}\text { Top layer is } 50-50 \text { nickel and aluminum, } \\
\text { second layer nickel, etc. Top layer spac- } \\
\text { ing is } 1.73 \pm 0.03 \AA \text { with } A \text { l atoms buck- } \\
\text { led outward by } 0.02 \pm 0.03 \AA \text {, second } \\
\text { layer spacing is the bulk value of } 1.78 \AA \\
\text { within } \pm 0.03 \AA \text {. }\end{array}$ & $\mathrm{LEED}^{7}$ \\
\hline $\operatorname{Pd}(110)$ & $(2 \times 1)$ & $\begin{array}{l}\text { Missing-row model with third layer } \\
\text { assumed bulk-like. Second layer found } \\
\text { to by bulk-like, fist layer spacing }-\% .1 \% \\
\text { relative to } 1.37 \AA \text { bulk spacing. Saw- } \\
\text { tooth model almost as good. }\end{array}$ & $\mathrm{LEED}^{8}$ \\
\hline $\operatorname{Pt}(110)$ & $(2 \times 1)$ & $\begin{array}{l}\text { Missing-row model is better than } \\
\text { buckled-row, paired-row or saw-tooth } \\
\text { models. Terminated-bulk positions } \\
\text { assumed for remaining atoms. }\end{array}$ & LEIS $^{9}$ \\
\hline$W(100)$ & $c(2 \times 2)$ & $\begin{array}{l}\text { Below room temperature the "zig-zag" } \\
\text { reconstruction occurs on the } W(100) \text { sur- } \\
\text { face. Alternate atoms move along the } \\
(011) \text { and }(0 \overline{1}) \text { directions by } 0.22 \pm 0.07 \\
\AA \text {, while the top layer spacing contracts } \\
\text { by } 0.05 \pm 0.05 \AA \text { from the bulk value of } \\
1.58 \AA \text {. There are two domains, since } \\
\text { atoms may also move along the }(0 \overline{1} 1) \\
\text { and }(01 \overline{1}) \text { directions. }\end{array}$ & LEED $^{10}$ \\
\hline
\end{tabular}




\section{References}

1. W. Moritz and D. Wolf, Surface Science, vol. 163, p. L655, 1985.

2. R. J. Baird, D. F. Ogletree, M. A. Van Hove, and G. A. Somorjai, Surface Science, vol. 165, p. 345, 1986.

3. M. A. Van Hove, R. J. Koestner, P. C. Stair, J. P. Bibérian, L. L. Kesmodel, I. Bartos, and G. A. Somorjai, Surface Science, vol. 103, p. 218, 1981.

4. E. Lang, K. Müller, K. Heinz, M. A. Van Hove, R. J. Koestner, and G. A. Somorjai, Surface Science, vol. 127, p. 347, 1983.

5. C.-M. Chan and M. A. Van Hove, Surface Science, to be published.

6. H. L. Davis and J. R. Noonan, Physical Review Letters, vol. 54, p. 566, 1985.

7. D. Sondericker, F. Jona, and P. M. Marcus, Physical Review B, vol. 33, p. $900,1986$.

8. C. J. Barnes, M. Q. Ding, M. Lindroos, R. D. Diehl, and D. A. King, Surface Science, vol. 162, p. 59, 1985.

0. H. Niehus, Surface Science, vol. 145, p. 407, 1984.

10. R. A. Barker, P. J. Estrup, F. Jona, and P. M. Marcus, Solid State Communications, vol. 25, p. 375, 1978. 


\section{Table 1.4 - Chalcogen Chemisorption on Metals}

(If the metal layer spacing has been investigated this is listed under the adsorbate information.)

\begin{tabular}{|c|c|c|c|c|}
\hline$F_{\mathrm{g}}$ & $\begin{array}{c}\text { Overlayer } \\
\text { Unit Cell }\end{array}$ & $\begin{array}{l}\text { Adsorption } \\
\text { Site }\end{array}$ & $\begin{array}{l}\text { Adsorbate } \\
\text { Spacing }(\AA)\end{array}$ & $\begin{array}{c}\text { Bond } \\
\text { Length }(\AA)\end{array}$ \\
\hline
\end{tabular}

\section{Oxygen}

\begin{tabular}{|c|c|c|c|c|c|}
\hline $\mathrm{Ag}(110)$ & $(2 \times 1)$ & long bridge & 0.20 & 2.05 & SEXAFS 1 \\
\hline $\mathrm{Al}(111)$ & $(1 \times 1)$ & 3-fold fec & $0.70 \pm 0.12$ & $1.79 \pm 0.05$ & LEED $^{2}$ \\
\hline $\mathrm{Al}(111)$ & $(1 \times 1)$ & 3-fold fec & $0.60 \pm 0.10$ & $1.75 \pm 0.03$ & NEXAFS 3 \\
\hline $\mathrm{Al}(111)$ & $(1 \times 1)$ & $\begin{array}{l}\text { sub-surface } \\
\text { tetrahedral }\end{array}$ & $0.60 \pm 0.10$ & $1.75 \pm 0.03$ & NEXAFS $^{3}$ \\
\hline $\mathrm{A} \backslash(111)$ & $(1 \times 1)$ & 3-fold fec & 0.70 & 1.79 & LEED4 \\
\hline $\mathrm{Al}(111)$ & undetermined & 3-fold fec & $0.98 \pm 0.10$ & $1.92 \pm 0.05$ & EXAFS 6 \\
\hline Co $(100)$ & $c(2 \times 2)$ & 4-fold & 0.80 & 1.94 & LEED $^{\circ}$ \\
\hline $\mathrm{Cu}(100)$ & $c(2 \times 2)$ & 4 -fold & 1.40 & 2.28 & LEED? \\
\hline $\mathrm{Cu}(100)$ & $c(2 \times 2)$ & 4-fold & $0.70 \pm 0.01$ & $1.94 \pm 0.01$ & SEXAFS \\
\hline $\mathrm{Cu}(100)$ & $c(2 \times 2)$ & 4-fold & 0.80 & 1.97 & $\mathrm{NPD}^{\circ}$ \\
\hline $\mathrm{Cu}(410)$ & $(1 \times 1)$ & $\begin{array}{l}\text { quasi 4-fold } \\
\text { at step edge }\end{array}$ & $0.4 \pm 0.2$ & $1.85 \pm 0.04$ & ARXPD 10 \\
\hline $\mathrm{Cu}(410)$ & $(1 \times 1)$ & $\begin{array}{l}\text { quasi } 4 \text {-fold } \\
\text { at step edge } \\
\text { and } 4 \text {-iold }\end{array}$ & $0.4 \pm 0.2$ & $1.85 \pm 0.04$ & ARXPD 10 \\
\hline
\end{tabular}




\section{Substrate Overlayer Adsorption Adsorbate Bond Method Face Unit Cell Site \\ Spacing $(\AA)$ Length $(\AA)$}

\begin{tabular}{|c|c|c|c|c|c|}
\hline $\mathrm{Fe}(100)$ & $(1 \times 1)$ & $\begin{array}{l}\text { 4-fold } \\
\text { top layer }\end{array}$ & $\begin{array}{l}0.48 \pm 0.10 \\
1.54 \pm 0.10\end{array}$ & $\begin{array}{l}2.08 \pm 0.02 \\
+7.7 \%\end{array}$ & LEED" \\
\hline $\operatorname{Ir}(110)$ & $c(2 \times 2)$ & $\begin{array}{l}\text { short bridge } \\
\text { top layer' }\end{array}$ & $\begin{array}{l}1.37 \pm 0.05 \\
1.33 \pm 0.07\end{array}$ & $\begin{array}{l}1.93 \pm 0.04 \\
-2.2 \%\end{array}$ & LEED 12 \\
\hline $\operatorname{Ir}(111)$ & $(2 \times 2)$ or $(2 \times 1)$ & 3 -fold fec & $1.30 \pm 0.05$ & $2.04 \pm 0.03$ & LEED $^{13}$ \\
\hline $\mathrm{Ni}(100)$ & $(2 \times 2)$ & 4-fold & $0.86 \pm 0.07$ & $1.96 \pm 0.03$ & EXAFS 14 \\
\hline $\mathrm{Ni}(\mathbf{1 0 0})$ & $c(2 \times 2)$ & 4-fold & $0.86 \pm 0.07$ & $1.96 \pm 0.03$ & EXAFS 14 \\
\hline $\mathrm{Ni}(100)$ & $(2 \times 2)$ & 4-fold & $0.90 \pm 0.10$ & $1.98 \pm 0.05$ & LEFD'16 \\
\hline $\mathrm{Ni}(100)$ & $\mathrm{c}(2 \times 2)$ & 4-fold & $0.92 \pm 0.05$ & $1.99 \pm 0.02$ & LEED 10 \\
\hline $\mathrm{Ni}(100)$ & $c(2 \times 2)$ & 4-fold & $0.85 \pm 0.05$ & $1.96 \pm 0.02$ & EXAFS 17 \\
\hline $\mathrm{Ni}(100)$ & $c(2 \times 2)$ & 4-fold & $0.90 \pm 0.04$ & $1.98 \pm 0.02$ & $\mathrm{NPD}^{18}$ \\
\hline $\mathrm{Ni}(100)$ & $c(2 \times 2)$ & 4-fold & $0.85 \pm 0.04$ & $1.96 \pm 0.02$ & $\mathrm{NPD}^{19}$ \\
\hline $\mathrm{Ni}(100)$ & $c(2 \times 2)$ & 4-fold & $0.86 \pm 0.10$ & $1.96 \pm 0.04$ & HEIS'O \\
\hline $\mathrm{Ni}(100)$ & $c(2 \times 2)$ & 4-fold & $0.90 \pm 0.10$ & $1.98 \pm 0.05$ & XANES 21 \\
\hline $\mathrm{Ni}(100)$ & $c(2 \times 2)$ & 4-fold & 0.90 & 1.98 & HREELS2 \\
\hline $\mathrm{Ni}(111)$ & $(\sqrt{ } 3 \times \sqrt{ } 3) R^{2} 30^{\circ}$ & 3-fold hep & 1.20 & 1.87 & HEIS? \\
\hline $\mathrm{Ta}(100)$ & $(3 \times 1)$ & $\begin{array}{l}\text { sub-surface } \\
\text { tetrahedral }\end{array}$ & -0.43 & 1.95 & LEED 24 \\
\hline$W(100)$ & disordered & 4-fold & $0.55 \pm 0.10$ & $2.30 \pm 0.02$ & LEED ${ }^{20}$ \\
\hline$W(110)$ & $(2 \times 1)$ & 3-fold & $1.25 \pm 0.01$ & $2.09 \pm 0.01$ & LEED ${ }^{20}$ \\
\hline
\end{tabular}




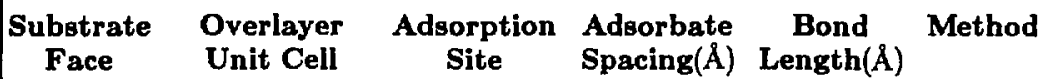

\begin{tabular}{|c|c|c|c|c|c|}
\hline $\mathrm{Zr}(0001)$ & $(2 \times 2)$ & $\begin{array}{l}\text { sub-surface } \\
\text { octahedral }\end{array}$ & 1.37 & 2.31 & LEED ${ }^{27}$ \\
\hline \multicolumn{6}{|c|}{ Sulfur } \\
\hline $\mathrm{Co}(100)$ & $c(2 \times 2)$ & 4-fold & 1.30 & 2.20 & LEED ${ }^{28}$ \\
\hline $\mathrm{Cu}(100)$ & $(2 \times 2)$ & 4-fold & 1.39 & 2.28 & ARXPS 20 \\
\hline $\mathrm{Fe}(100)$ & $c(2 \times 2)$ & 4-fold & $1.09 \pm 0.05$ & $2.30 \pm 0.02$ & LEED ${ }^{30}$ \\
\hline $\mathrm{Fe}(110)$ & $(2 \times 2)$ & $\begin{array}{l}\text { 4-fold recon- } \\
\text { structed }\end{array}$ & 1.43 & 2.02 & LEED $^{31}$ \\
\hline $\mathrm{I} r(111)$ & $\left(V_{3} \times V_{3}\right) \mathrm{R}_{30}{ }^{\circ}$ & 3-fold fec & $1.65 \pm 0.07$ & $2.28 \pm 0.05$ & LEED $^{22}$ \\
\hline $\mathrm{Ni}(100)$ & $(2 \times 2)$ & 4 -fold & $1.30 \pm 0.10$ & $2.19 \pm 0.06$ & LEED ${ }^{16}$ \\
\hline $\mathrm{Ni}(100)$ & $c(2 \times 2)$ & 4-fold. & $1.28 \pm 0.05$ & $2.18 \pm 0.03$ & LEED ${ }^{16}$ \\
\hline $\mathrm{Ni}(100)$ & $c(2 \times 2)$ & 4-fold & $1.30 \pm 0.05$ & $2.19 \pm 0.03$ & LEED*s \\
\hline $\mathrm{Ni}(100)$ & $c(2 \times 2)$ & 4-fold & $1.30 \pm 0.04$ & $2.19 \pm 0.02$ & NPD 18 \\
\hline $\mathrm{Ni}(100)$ & $c(2 \times 2)$ & 4-fold & $1.39 \pm 0.04$ & $2.24 \pm 0.02$ & SEXAFS \\
\hline $\mathrm{Ni}(100)$ & $c(2 \times 2)$ & 4-fold & $1.37 \pm 0.05$ & $2.23 \pm 0.03$ & ARrETS \\
\hline $\mathrm{Ni}(100)$ & $c(2 \times 2)$ & 4-fold & $1.35 \pm 0.10$ & $2.22 \pm 0.06$ & ARXPSsa \\
\hline $\mathrm{Ni}(100)$ & $c(2 \times 2)$ & 4 -fold & 1.35 & 2.22 & $\operatorname{ARX} r S^{20}$ \\
\hline $\mathrm{Ni}(100)$ & disordered & 4-fold & $1.36 \pm 0.03$ & $2.22 \pm 0.02$ & SEXAFS' \\
\hline $\mathrm{Ni}(110)$ & $c(2 \times 2)$ & 4-fold & 0.9 & 2.34 & ARXPS \\
\hline
\end{tabular}




\section{Substrate Overlayer Adsorption Adsorbate Bond Method Face Unit Cell Site Spacing $(\AA)$ Length $(\AA)$}

\begin{tabular}{|c|c|c|c|c|c|}
\hline $\mathrm{Ni}(110)$ & $c(2 \times 2)$ & $\begin{array}{c}\text { 4-fold } \\
\text { top layer } \\
\text { next layer }\end{array}$ & $\begin{array}{l}0.84 \pm 0.03 \\
1.372 \pm 0.02 \\
1.201 \pm 0.02\end{array}$ & $\begin{array}{l}2.31 \pm 0.01 \\
+9.6 \% \\
-4.0 \%\end{array}$ & LEED ${ }^{\text {So }}$ \\
\hline $\mathrm{Ni}(110)$ & $c(2 \times 2)$ & $\begin{array}{l}\text { 4-fold } \\
\text { top layer }\end{array}$ & $\begin{array}{l}0.87 \pm 0.03 \\
1.31 \pm 0.04\end{array}$ & $\begin{array}{l}2.32 \pm 0.01 \\
+4.8 \%\end{array}$ & MEIS* \\
\hline $\operatorname{Pd}(100)$ & $c(2 \times 2)$ & 4-fold & $1.30 \pm 0.05$ & $2.33 \pm 0.03$ & LEED 4 \\
\hline $\operatorname{Pd}(111)$ & $\left(V_{3} \times V_{3}\right) \mathrm{R}_{30}$ & 3-fold fcc & $1.53 \div 0.05$ & $2.20 \pm 0.03$ & LEED" \\
\hline Pt (111) & $\left(V_{3} \times V_{3}\right) \mathrm{R} 30^{\circ}$ & 3-fold fcc & $1.62 \pm 0.05$ & $2.28 \pm 0.04$ & LEED 43 \\
\hline $\mathrm{Rh}(100)$ & $(2 \times 2)$ & 4-fold & 1.29 & 2.30 & LEED ${ }^{41}$ \\
\hline$R h(110)$ & $c(2 \times 2)$ & 4-fold & 0.77 & 2.45 & LEED*S \\
\hline $\mathrm{Rh}(111)$ & $\left(V_{3} \times V_{3}\right) R^{2} 30^{\circ}$ & 3-fold fec & 1.53 & 2.18 & LEED 40 \\
\hline \multicolumn{6}{|c|}{ Selenium } \\
\hline $\mathrm{Ag}(100)$ & $c(2 \times 2)$ & 4-fold & $1.91 \pm 0.04$ & $2.80 \pm 0.03$ & LEED" \\
\hline $\mathrm{Ni}(100)$ & $(2 \times 2)$ & 4-fold & $1.55 \pm 0.10$ & $2.35 \pm 0.07$ & LEED 15 \\
\hline $\mathrm{Ni}(100)$ & $c(2 \times 2)$ & 4-fold & $1.45 \pm 0.10$ & $2.28 \pm 0.06$ & NPD 48 \\
\hline $\mathrm{Ni}(100)$ & $c(2 \times 2)$ & 4-fold & 1.55 & 2.35 & NPD \\
\hline $\mathrm{Ni}(110)$ & $c(2 \times 2)$ & 4-fold & $1.10 \pm 0.04$ & $2.42 \pm 0.02$ & NPD ${ }^{60}$ \\
\hline $\mathrm{Ni}(111)$ & $(2 \times 2)$ & 3-fold fcc & $1.80 \pm 0.04$ & $2.30 \pm 0.03$ & NPD ${ }^{60}$ \\
\hline
\end{tabular}




\begin{tabular}{|c|c|c|c|c|c|}
\hline $\begin{array}{l}\text { Substrate } \\
\text { Face }\end{array}$ & $\begin{array}{l}\text { Overlayer } \\
\text { Unit Cell }\end{array}$ & $\begin{array}{c}\text { Adsorptiona } \\
\text { Site }\end{array}$ & $\begin{array}{l}\text { Adsorbate } \\
\text { Spacing }(\AA)\end{array}$ & $\begin{array}{c}\text { Bond } \\
\text { Length }(\AA)\end{array}$ & Method \\
\hline \multicolumn{6}{|c|}{ Tellurium } \\
\hline $\mathrm{Cu}(100)$ & $(2 \times 2)$ & 4 -fold & $1.70 \pm 0.15$ & $2.48 \pm 0.10$ & LEED's \\
\hline $\mathrm{Cu}(100)$ & $(2 \times 2)$ & 4 -fold & $1.90 \pm 0.04$ & $2.62 \pm 0.03$ & SEXAFS ${ }^{52}$ \\
\hline $\mathrm{Ni}(100)$ & $(2 \times 2)$ & 4 -fold & $1.80 \pm 0.10$ & $2.52 \pm 0.07$ & LEED 15 \\
\hline $\mathrm{Ni}(100)$ & $c(2 \times 2)$ & 4 -fold & $1.90 \pm 0.10$ & $2.59 \pm 0.07$ & LEED 53 \\
\hline
\end{tabular}

\section{References}

1. A. Puschmann and J. Haase, Surface Science, vol. i44, p. 559, 1984.

2. V. Martinez, F. Soria, M. C. Munoz, and J. L. Sacedon, Surface Science, vol. 128 , p. $424,1983$.

3. D. Norman, S. Brennan, R. Jaeger, and J. Stöhr, Surface Science, vol. 105, p. L297, 1981.

4. J. Neve, J. Rundgren, and P. Westrin, Journal of Physics C, vol. 15, p. 4391, 1982.

5. R. Z. Bachrach, G. V. Hansson, and R. S. Bauer, Surface Science, vol. 109, p. L560, 1981.

6. M. Maglietta, E. Zanazzi, U. Bardi, and F. Jona, Surface Science, vol. 77, p. 101, 1978. 
7. J. Onuferko and D. P. Woodruff, Surface Science, vol. 95, p. 555, 1980.

8. U. Döbler, K. Baberschke, J. Stöhr, and D. A. Outka, Physical Review B, vol. 31, p. 2532, 1985.

9. J. G. Tobin, L. E. KJebanoff, D. H. Rosenblatt, R. F. Davis, E. Umbach, A. G. Baca, D. A. Shirley, y. Huang, W. M. Kang, and S. Y. Tong, Physical Review B, vol. 26, p. 7076, 1982.

10. K. A. Thompson and C. S. Fadley, Surface Science, vol. 146, p. 281, 1984.

11. K. O. Legg, F. Jona, D. W. Jepsen, and P. M. Marcus, Physicai Review B, vol. 16, p. 5271,1977 .

12. C. M. Chan, K. L. Luke, M. A. Van Hove, W. H. Weinberg, and S. P. Withrow, Surface Science, vol. 78, p. 386, 1978.

13. C.-M. Chan and W. H. Weinberg, Journal of Chemical Physics, vol. 71, p. $2788,1979$.

14. J. Stöhr, R. Jaeger, and T. Kende! wicz, Physical Review Letters, vol. 49, p. $142,1982$.

15. M. A. Van Hove and S. Y. Tong, Journal of Vacuum Science and Technology, vol. 12, p. $230,1975$.

16. P. C. Marcus, J. E. Demuth, and D. W. Jepsen, Surface Science, vol. 53, p. $501,1975$.

17. M. de Crescenzi, F. Antonangeli, C. Bellini, and R. Rosei, Physical Review Letters, vol. 50, p. 1949, 1983. 
18. D. H. Rosenblatt, J. G. Tobin, M. G. Mason, R. F. Davis, S. D. Kevan, D. A. Shirley, C. H. Li, and S. Y. Tong, Physical Review B, vol. 23, p. 3828, 1981.

19. S. Y. Tong, W. M. Kang. D. H. Rosenblatt, J. G. Tobin, and D. A. Shirley, Physical Review B, vol. 27, p. 4632, 1983.

20. J. W. M. Frenken, J. F. van der Veen, and G. Allan, Physical Review Letters, vol. 51, p. 1876, 1983.

21. D. Norman, J. Stöhr, R. Jaeger, P. J. Durham, and J. B. Pendry, Physical Reviev Letters, vol. 51, p. 2052, 1983.

22. T. S. Rahman, D. L. Mills, J. E. Black, J. M. Szeftel, S. Lehwald, and H. Ibach, Physical Review B, vol. 30, p. 589, 1984.

23. T. Narusawa and W. M. Gibson, Surface Science, vol. 114, p. 331, 1981.

24. A. V. Titov and H. Jagodzinski, Surface Science, vol. 152/153, p. 409, 1985.

25. K. Heinz, D. K. Saldin, and J. B. Pendry, Physical Revicw Letters, vol. 55, p. $2312,1985$.

26. M. A. Van Hove and S. Y. Tong, Physical Review Letters, vol. 35, p. 1092, 1975.

27. K. C. Hui, R. H. Milne, K. A. R. Mitchell, W. T. Moore, and M. Y. Zhou, Solid State Communications, vol. 56, p. 83, 1985.

28. M. Miaglietta, Solid State Communications, vol. 43, p. 395, 1982.

29. E. J. Bullock, C. S. Fadley, and P. J. Orders, Physical Review B, vol. 28, p. 4867, 1983. 
30. K. O. Legg, F. Jona, D. W. Jepsen, and P. M. Marcus, Surface Science, vol. 66, p. 25, 1977.

31. F. E. Shih, F. Jona, D. W. Jepsen, and P. M. Marcus, Physical Review Letters, vol. 46, p. 731, 1981.

32. C.-M. Chan and W. H. Weinberg, Journal of Chemical Physics, vol. 71, p. 3988,1979 .

33. Y. Gauthier, D. Aberdam, and R. Baudoing, Surface Science, vol. 78, p. 339, 1978.

34. J. Stöhr, R. Jaeger, and S. Brennan, Surface Science, voi. 117, p. 503, 1982.

35. J. J. Barton, C. C. Bahr, Z. Hussain, S. W. Robey, L. E. Klebanoff, and D. A. Shirley, Journal of Vacuum Science and Technology A, vol. 2, p. 847, 1984 .

36. P. J. Orders, B. Sinkovic, C. S. Fadley, R. Trehan, Z. Hussain, and J. Lecante, Physical Review B, vol. 30, p. 1838, 1984.

37. J. Stöhr, E. B. Kollin, D. A. Fischer, J. B. Hastings, F. Zaera, and F. Sette, Physical Review Letters, vol. 55, p. 1468, 1985.

38. R. Baudoing, E. Blanc, C. Gaubert, Y. Gautheir, and N. Gnuchev, Surface Science, vol. 128, p. $22,1983$.

39. R. Baudoing, Y. Gauthier, and Y. Joly, Journal of Physics C, vol. 18, p. $4061,1985$.

40. J. F. van der Veen, R. M. Tromp, R. G. Smeenk, and F. W. Saris, Surface Science, vol. 82, p. 468, 1979. 
41. W. Berndt, R. Hora, and M. Scheffler, Surface Science, vol. 117, p. 188, 1982.

42. F. Maca, M. Scheffler, and W. Berndt, Surface Science, vol. 160, p. 467, 1985.

43. K. Hayek, H. Glassl, A. Gutmann, H. Leonhard, P. Prutton, S. P. Tear, and M. R. Welton-Cook, Surface Science, vol. 152, p. 419, 1985.

44. S. Hengrasmee, P. R. Watson, D. C. Frost, and K. A. R. Mitchell, Surface Science, vol. 87, p. L249, 1979.

45. S. Hengrasmee, P. R. Watson, D. C. Frost, and K. A. R. Mitchell, Surface Science, vol. 92, p. 71, 1080.

46. P. C. Wong, M. Y. Zhou, K. C. Hui, and K. A. R. Mitchsll, Surface Science, vol. 163 , p. 172,1985 .

47. A. Ignatiev, F. Jona, D. W. Jepsen, and P. M. Marcus, Surface Science, vol. 40, p. $439,1973$.

48. J. E. Demuth, D. W. Jepsen, and P. M. Marcus, Physical Review Letters, vol. 31 , p. $540,1973$.

49. D. H. Rosenblatt, S. D. Kevan, J. G. Tobin, R. F. Davis, M. G. Mason, D. A. Shirley, J. C. Tang, and S. Y. Tong. Physical Review B, vol. 26, p. 3181, 1982.

50. D. H. Rosenblatt, S. D. Kevan, J. G. Tobin, R. F. Davis, M. G. Mason, D. R. Denley, D. A. Shirley, Y. Huang, and S. Y. Tong, Physical Review B, vol. 26, p. 1812, 1982. 
51. A. Salwén and J. Rundgren, Surface Science, vol. 53, p. 523, 1975.

52. F. Comin, P. H. Citrin, P. Eisenberger, and J. E. Rowe, Physical Review B, vol. 26, p. 7060, 1982.

53. J. E. Demuth, D. W. Jepsen, and P. M. Marcus, Journal of Physics C, vol. 6, p. L307, 1973. 


\section{Table 1.5 - Other Atomic Adsorbates on Metal Surfaces}

(If the metal layer spacing has been investigated this is listed under the adsorbate information.)

\begin{tabular}{|c|c|c|c|c|}
\hline $\begin{array}{l}\text { Adsorption } \\
\text { System }\end{array}$ & $\begin{array}{l}\text { Overlayer } \\
\text { Unit Cell }\end{array}$ & $\begin{array}{c}\text { Adsorbate } \\
\text { Site }\end{array}$ & $\begin{array}{c}\text { Layer } \\
\text { Spacing }(\AA)\end{array}$ & Method \\
\hline $\mathrm{Ag}(100)-\mathrm{Cl}$ & $c(2 \times 2)$ & 4-fold & 1.96 & $\begin{array}{l}\text { Helium } \\
\text { Diffraction }\end{array}$ \\
\hline$A g(111)-I$ & $\begin{array}{c}(\sqrt{ } 3 \times \sqrt{ } 3) \mathrm{R} 30^{\circ} \\
\text { top layer }\end{array}$ & $\begin{array}{c}\text { 3-fold fcc and hep } \\
0.0 \%\end{array}$ & $\begin{array}{l}2.29 \pm 0.06 \\
2.36 \pm 0.06\end{array}$ & LEED $^{2}$ \\
\hline $\operatorname{Ag}(111)-\mathrm{Xe}$ & incommensurate & $\begin{array}{l}\text { hexagonal Xe lat- } \\
\text { tice, random } \\
\text { orientation, Xe- } \\
\text { Xe spacing } \\
1.51 \AA\end{array}$ & $3.55 \pm 0.10$ & LEED $^{3}$ \\
\hline $\mathrm{Al}(100)-\mathrm{Na}$ & $c(2 \times 2)$ & 4 -fold & $2.08 \pm 0.12$ & LEED $^{4}$ \\
\hline $\mathrm{Nl}(100)-\mathrm{Na}$ & $c(2 \times 2)$ & 4-fold & $2.05 \pm 0.10$ & LEED $^{5}$ \\
\hline $\mathrm{Cu}(100)-\mathrm{Cl}$ & $\begin{array}{c}c(2 \times 2) \\
\text { top layer }\end{array}$ & $\begin{array}{l}4 \text {-fold } \\
+3 \%\end{array}$ & $\begin{array}{l}1.60 \pm 0.03 \\
1.85 \pm 0.03\end{array}$ & LEED $^{6}$ \\
\hline $\mathrm{Cu}(111)-\mathrm{Cs}$ & $(2 \times 2)$ & top & $3.01 \pm 0.05$ & LEED $^{7}$ \\
\hline $\mathrm{Cu}(100)-\mathrm{I}$ & $(2 \times 2)$ & 4-fold & $1.98 \pm 0.02$ & SEXAFS $^{8}$ \\
\hline $\mathrm{Cu}(111)-\mathrm{I}$ & $(\sqrt{ } 3 \times \sqrt{ } 3) R 30^{\circ}$ & 3-fold hcp & $2.21 \pm 0.02$ & SEXAFS $^{8}$ \\
\hline $\mathrm{Cu}(111)-\mathrm{Ni}$ & $(1 \times 1)$ & 3 -fold fec & $2.04 \pm 0.02$ & LEED $^{9}$ \\
\hline $\mathrm{Cu}(100)-\mathrm{Pb}$ & $c(2 \times 2)$ & 4-fold & $2.05 \pm 0.05$ & $\operatorname{LEED}^{10}$ \\
\hline
\end{tabular}




\begin{tabular}{|c|c|c|c|c|}
\hline $\begin{array}{l}\text { Adsorption } \\
\text { System }\end{array}$ & $\begin{array}{l}\text { Overlayer } \\
\text { Unit Cell }\end{array}$ & $\begin{array}{l}\text { Adsorbate } \\
\text { Site }\end{array}$ & $\begin{array}{c}\text { Layer } \\
\text { Spacing }(\AA)\end{array}$ & Method \\
\hline $\mathrm{Cu}(100)-3 \mathrm{~Pb}$ & $\mathrm{c}\left(5 V_{2} \times V_{2}\right) \mathrm{R} 5^{\circ}$ & $\begin{array}{l}\text { 4-fold with anti- } \\
\text { phase domain } \\
\text { walls and off- } \\
\text { center atoms }\end{array}$ & $2.4 \pm 0.05$ & LEED $^{10}$ \\
\hline $\mathrm{Fe}(110)-\mathrm{H}$ & $(2 \times 1)$ & quasi 3-fold & $0.90 \pm 0.10$ & $\operatorname{LEED}^{11}$ \\
\hline $\mathrm{Fe}(110)-2 \mathrm{H}$ & $(3 \times 1)$ & quasi 3-fold & $1.00 \pm 0.10$ & LEED $^{11}$ \\
\hline $\mathrm{Fe}(100)-\mathrm{N}$ & $\begin{array}{c}c(2 \times 2) \\
\text { top layer }\end{array}$ & $\begin{array}{l}\text { 4-fold } \\
+8 \%\end{array}$ & $\begin{array}{l}0.25 \pm 0.05 \\
1.54 \pm 0.05\end{array}$ & LEED $^{12}$ \\
\hline Mo $(100)-N$ & $c(2 \times 2)$ & 4-fold & 1.02 & LEED $^{13}$ \\
\hline $\mathrm{Mo}(100)-\mathrm{Si}$ & $(1 \times 1)$ & 4-fold & $1.16 \pm 0.10$ & LEED $^{14}$ \\
\hline $\mathrm{Ni}(111)-2 \mathrm{H}$ & $(2 \times 2)$ & 3-fold hep and fec & $1.15 \pm 0.10$ & $\operatorname{LEED}^{15}$ \\
\hline $\mathrm{Ni}(100)-2 \mathrm{C}$ & $\begin{array}{c}(2 \times 2) \\
\text { top layer } \\
\text { reconstructed }\end{array}$ & $\begin{array}{c}\text { 4-fold } \\
+22 \% \\
\text { lateral motions }\end{array}$ & $\begin{array}{l}0.10 \pm 0.10 \\
1.96 \pm 0.05 \\
25 \pm 0.35 \AA\end{array}$ & $\operatorname{LEED}^{16}$ \\
\hline $\mathrm{Ni}(100)-\mathrm{Cu}$ & $(1 \times 1)$ & 4-fold & $1.80 \pm 0.03$ & LEED $^{17}$ \\
\hline $\mathrm{Ni}(100)-\mathrm{Na}$ & $c(2 \times 2)$ & 4-fold & $2.23 \pm 0.10$ & $\operatorname{LEED}^{18}$ \\
\hline$P d(111)-A u$ & $(1 \times 1)$ & 3-fold fec & $2.25 \pm 0.19$ & HEIS $^{19}$ \\
\hline $\mathrm{Ti}(0001)-\mathrm{Cd}$ & $(1 \times 1)$ & 3-fold fec & 2.57 & LEED $^{20}$ \\
\hline $\mathrm{T}:(0001)-\mathrm{N}$ & $(1 \times 1)$ & $\begin{array}{l}\text { sub-surface } \\
\text { tetrahedral }\end{array}$ & $-1.22 \pm 0.05$ & LEED $^{2 I}$ \\
\hline$W(100)-2 H$ & $\begin{array}{c}(1 \times 1) \\
\text { top layer }\end{array}$ & $\begin{array}{l}\text { 2-fold } \\
-2.7 \%\end{array}$ & $\begin{array}{l}1.35 \pm 0.10 \\
1.54 \pm 0.04\end{array}$ & $\operatorname{LEED}^{22}$ \\
\hline$W(100)-2 H$ & $\begin{array}{c}(1 \times 1) \\
\text { top layer }\end{array}$ & $\begin{array}{l}2 \text {-fold } \\
-1.3 \%\end{array}$ & $\begin{array}{l}1.17 \pm 0.04 \\
1.56 \pm 0.02\end{array}$ & $\mathrm{LEED}^{23}$ \\
\hline
\end{tabular}




\begin{tabular}{|ccccc|}
\hline $\begin{array}{c}\text { Adsorption } \\
\text { System }\end{array}$ & $\begin{array}{c}\text { Overlayer } \\
\text { Unit Cell }\end{array}$ & $\begin{array}{c}\text { Adsorbate } \\
\text { Site }\end{array}$ & $\begin{array}{c}\text { Layer } \\
\text { Spacing }(\AA)\end{array}$ & Method \\
\hline $\mathrm{W}(100)-\mathrm{N}$ & $\mathrm{c}(2 \times 2)$ & $\begin{array}{l}4-\text { fold } \\
+1.3 \%\end{array}$ & $\begin{array}{c}0.49 \pm 0.06 \\
1.60 \pm 0.06\end{array}$ & LEED \\
& top layer & & \\
\hline
\end{tabular}

\section{References}

1. M. J. Cardillo, G. E. Becker, D. R. Hamann, and J. A. Serri, Physical Review $B$, vol. 28, p. 494, 1983.

2. M. Maglietta, E. Zanazzi, U. Bardi, D. Sondericker, and F. Jona, Surface Science, vol. 123, p. 141, 1982.

3. N. Stoner, M. A. Van Hove, S. Y. Tong, and M. B. Webb, Physical Review Letters, vol. 40, p. 243, 1978.

4. M. A. Van Hove, S. Y. Tong, and N. Stoner, Surface Science, vol. 54, p. 259, 1976.

5. B. A. Hutchins, T. N. Rhodin, and J. E. Demuth, Surface Science, vol. 54, p. $419,1976$.

6. F. Jona, D. Westphal, A. Goldman, and P. M. Marcus, Journal of Physics C, vol. 16, p. 3001, 1983.

7. S. A. Lindgren, L. Walldén, J. Rundgren, P. Westrin, and J. Neve, Physical Review $B$, vol. 28, p. 6707, 1983. 
8. P. H. Citrin, P. Eisenberger, and R. C. Hewitt, Physical Revier Letters, vol. 45, p. 1948, 1980.

9. S. P. Tear and K. Roell, Journal of Physics C, vol. 15, p. 5521, 1982.

10. W. Hoesler and W. Moritz, Surface Science, vol. 117, p. 196, 1982.

11. W. Moritz, R. Imbihl, R. J. Behm, G. Ertl, and T. Matsushima, fournal of Chemical Physics, vol. 83, p. 1959, 1985.

12. R. Imbihl, R. J. Behm, G. Ertl, and W. Moritz, Surface Science, vol. 123, p. $129,1982$.

13. A. Ignatiev, F. Jona, D. W. Jepsen, and P. M. Marcus, Surface Science, vol. 49 , p. $189,1975$.

14. A. Ignatiev, F. Jona, D. W. Jepsen, and P. M. Marcus, Physical Review B, vol. 11, p. 4780, 1975.

15. K. Christmann, R. J. Behm, G. Ertl, M. A. Van Hove, and W. H. Weinberg, Journal of Chemical Physics, vol. 70, p. 4168, 1979.

16. J. H. Onuferko, D. P. Woodruff, and B. W. Holland, Surface Science, vol. 87, p. 357, 1979 .

17. M. Abu-Joudeh, P. P. Vaishnava, and P. A. Montano, Journal of Physics $C$, vol. 17 , p. 6899,1984 .

18. J. E. Demuth, D. W. Jepsen, and P. M. Marcus, Journal of Physics C, vol. 8, p. L25, 1975.

19. Y. Kuk, L. C. Feldman, and P. J. Silverman, Physical Review Letters, vol. 50, p. 511, 1983. 
20. H. D. Shih, F. Jona, D. W. Jepsen, and P. M. Marcus, Physical Keview B, vol. 15, p. $5550,1977$.

21. H. D. Shih, F. Jona, D. W. Jepsen, and P. M. Marcus, Surface Science, vol. 60, p. $445,1976$.

22. M. A. Passler, A. Ignatiev, B. W. Lee, D. Adams, and M. A. Van Hove, in Determination of Surface Structure by LEED, Plcœum, New Ycrk, 1984.

23. M. A. Passler, B. W. Lee, and A. Ignatiev, Surface Science, vol. 150, p. 263, 1985.

24. K. Griffiths, D. A. King, G. C. Aers, and J. B. Pendry, Journal of Physics C, vol. 15, p. 4921, 1982. 
Table 1.6 - Semiconductor Surface Structures

Substrate Unit Cell

Structure

Method

CdTe (110)

(1x1)

Te is buckled out from the top layer by

LEED $^{1}$

$0.82 \pm 0.05$. T. Top layer Cd atoms con-

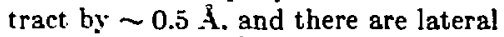
motions of $\sim 0.4 \AA$ to conserve bond lengths.

GaAs (110)

As is buckled out from the top layer by

LEED $^{2}$ $0.70 \AA$. Top layer $\mathrm{Ga}$ atoms contract by $\sim 0.5 \AA$, and there are lateral motions of $\sim 0.4 \AA$ to conserve bond lengths. The lateral motions may be reduced by $3 / 4$ to give a better agreement with MEIS results without significantly worsening the LEED fit.

GaAs (110)

As is buckled out from the top layer by

LEED $^{3}$ $0.69 \AA$. Top layer $\mathrm{Ga}$ atoms contract by $\sim 0.5 \AA$, and there are lateral motions of $\sim 0.3 \AA$ to conserve bond lengths.

GaAs (110)

As is buckled out from the top layer by $0.40 \pm 0.30 \AA$. Top layer $\mathrm{Ga}$ atoms contract by $\sim 0.20 \pm 0.30 \AA$, with no lateral motjons.

GaAs (111)

(2x2) One quarter of the top layer $\mathrm{Ga}$ atoms are missing, and the remaining atoms are almost co-planar with the first As layer, within $0.20 \AA$. Ga bonding is $s p^{2}$ rehybridized, instead of the normal $s p^{3}$ configuration. There are first bi-layer lateral motions of $\sim 0.2 \AA$, and some buckling in the third layer to maintain optimum bond-lengths and angles.

HEIS $^{4}$

LEED $^{5}$ 
$\mathrm{GaP}(111)$

$\mathrm{GaSb}(110)$

$\mathrm{GaSb}(110)$

(1x1)

(1x1)

Ge (100)

InAs (110)

$(1 \times 1)$

$(2 \times 1)$

$(2 \times 2)$

One quarter of the top layer Ga atoms

LEED $^{5}$ are missing, and the remaining atoms are almost co-planar with the first $P$ layer. $\mathrm{Ga}$ bonding is $s p^{2}$ rehybridized, instead of the normal $s p^{3}$ configuration. There are lateral motions up to $\sim 2 \AA$, and some buckling in the third layer to maintain optimum bond-lengths and angles.

$\operatorname{InP}(110)$
$\mathrm{Sb}$ is buckled out from the top layer by $0.77 \pm 0.05 \AA$. Top layer $\mathrm{Ga}$ atoms contract by $\sim 0.5 \AA$, and there are lateral motions of $\sim 0.4 \AA$ to conserve bond lengths.

$\mathrm{Sb}$ is buckled out from the top layer by $0.77 \pm 0.05 \AA$. Top layer $\mathrm{Ga}$ atoms contract by $\sim 0.5 \AA$, and there are lateral motions of $\sim 0.4 \AA$ to conserv bond lengths. Consistent with LEED results for layer displacements and lateral motions.

The top layer buckles, with one Ge atom moving out by $0.62 \pm 0.04 \AA$ and the other moving in by $0.66 \pm 0.04 \AA$. There are lateral displacements of $\sim 0.9 \AA$ in the first layes and $\sim 0.1 \AA$ in the second layer.

The top layer has As buckled outward

LEED 10 by $\sim 0.8 \AA$ with lateral motions of $\sim 0.6$ $\AA$ in the first three layers to conserve bond lengths.

LEED $^{6,7}$

MEIS $^{8}$

$\mathrm{XRD}^{9}$

Preliminary results show layer buckling with the $\mathrm{P}$ atom buckled out, and lateral and vertical shifts in the top layer $\sim 0.4$ $\AA$. Second and deeper layer shifts were not investigated. 


\begin{tabular}{|c|c|c|c|}
\hline Substrate & Unit Cell & Structure & Method \\
\hline $\operatorname{InP}(110)$ & $(1 \times 1)$ & $\begin{array}{l}\text { Top layer buckling with the } P \text { atom } \\
\text { buckled out by } 0.69 \pm 0.10 \AA \text {, and lateral } \\
\text { and vertical shifts in the top layer } \sim 0.4 \\
\AA \text {. The second layer spacing is con- } \\
\text { tracted by } 0.41 \pm 0.10 \AA \text { with a slight } \\
\text { buckling of } 0.07 \pm 0.10 \AA \text {. Only first layer } \\
\text { lateral displacements were investigated. }\end{array}$ & LEED $^{12}$ \\
\hline $\mathrm{Si}(100)$ & $(2 \times 1)$ & $\begin{array}{l}\text { Buckled dimer model is best fit to data. } \\
\text { Surface atoms dimerize to take up dan- } \\
\text { gling bonds, surface buckles for best } \\
\text { bond angles. There are lateral and verti- } \\
\text { cal ion-core motions for at least } 4 \text { layers } \\
\text { into the bulk crystal. Auihors conclude } \\
\text { that ( } 2 \times 1),(2 \times 2) \text { and c(4x2) buckled } \\
\text { dimer domains may exist on the surface. }\end{array}$ & MEis $^{13}$ \\
\hline Si $(100)$ & $(2 \times 1)$ & $\begin{array}{l}\text { LEED analysis considering vertical and } \\
\text { lateral displacements in the top three } \\
\text { atomic layers supports a buckled dimer } \\
\text { model. }\end{array}$ & LEED $^{14}$ \\
\hline $\mathrm{Si}(111)$ & $\begin{array}{l}\text { (1xi) laser } \\
\text { annealed }\end{array}$ & $\begin{array}{l}\text { The first two layers are almost co- } \\
\text { planar, instead of the normal } 0.78 \AA \\
\text { separation. The first layer spacing is } \\
0.08 \pm 0.02 \text {, a contraction of } 90 \% \text {, and the } \\
\text { second layer spacing is } 2.95 \pm 0.20 \AA \text {, a } \\
25.5 \% \text { expansion from } 2.35 \AA \text {. }\end{array}$ & LEED $^{15}$ \\
\hline Si (111) & $(2 \times 1)$ & $\begin{array}{l}\text { The top layer is buckled by } 0.30 \pm 0.05 \AA \text {, } \\
\text { the second layer spacing is } 0.70 \pm 0.05, \text { a } \\
\text { change of }+2.9 \% \text {, and the third layer } \\
\text { spacing is contracted by } 3.4 \% \text { to } \\
2.27 \pm 0.02 \text { from } 2.35 \AA \text {. There are } \\
\text { second-layer lateral shifts of } \sim 0.12 \AA \text {. }\end{array}$ & LEED $^{16}$ \\
\hline Si (111) & $(2 \times 1)$ & $\begin{array}{l}\text { Analysis of LEED data supports a " } \pi \text { - } \\
\text { bonded chain" model for the }(2 \times 1) \text { recon- } \\
\text { struction. Trial geometries based on } \\
\text { strain-minimization calculations for the } \\
\text { model, involving vertical motions four } \\
\text { layers deep with lateral motions along } \\
\text { the long side of the unit cell. }\end{array}$ & LEED $^{17}$ \\
\hline
\end{tabular}




\begin{tabular}{|c|c|c|c|}
\hline Substrate & Unit Cell & Structure & Method \\
\hline $\mathrm{Si}(111)$ & $(2 \times 1)$ & $\begin{array}{l}\text { Best fit to " } \pi \text {-bonded chain" model. This } \\
\text { model involves buckling in layers } 2 \text { to } 6 \\
\text { of up to } 0.27 \AA \text { and small lateral shifts } \\
\text { in the first six layers. }\end{array}$ & MEIS $^{18}$ \\
\hline $\mathrm{ZnSe}(110)$ & $(1 \times 1)$ & $\begin{array}{l}\text { Two different mudels were consistent } \\
\text { with LEED data. First; Se is buckled } \\
\text { outward by } \sim 0.70 \AA \text { and the second } \\
\text { layer spacing is contracted by } \sim 0.60 \AA \text {, } \\
\text { with lateral notions of } \sim 0.7 \AA \text {. Second; } \\
\text { Se buckled out by } 0.10 \AA \text { and the second } \\
\text { layer spacing contracted by } 0.09 \AA \text {, with } \\
\text { lateral motions of } \sim 0.07 \AA .\end{array}$ & LEED $^{19}$ \\
\hline $\operatorname{ZnTe}(110)$ & $(1 \times 1)$ & $\begin{array}{l}\text { Te is buckled out from the top layer by } \\
0.71 \pm 0.05 \AA \text {. Top layer } \mathrm{Zn} \text { atoms con- } \\
\text { tract by } \sim 0.5 \AA \text {, and there are lateral } \\
\text { motions of } \sim 0.4 \AA \text { to conserve bond } \\
\text { lengths. }\end{array}$ & $\mathrm{LEED}^{7}$ \\
\hline
\end{tabular}

\section{References}

1. C. B. Duke, A. Paton, W. K. Ford, A. Kahn, and G. Scott, Journal of Vacuum Science and Technology, vol. 20, p. 778, 1982.

2. C. B. Duke and A. Paton, Journal of Vacuum Science and Technology B, vol. 2, p. 327, 1984.

3. S. Y. Tong, W. M. Mei, and G.Xu, Journal of Vacuum Science and Technology $B$, vol. 2, p. 393, 1984 .

4. H. J. Grossman and W.M. Gibson, Journal of Vacuum Science and Technology $B$, vol. 2, p. 343, 1984. 
5. G. Xu, W. Y. Hu, M. W. Puga, S. Y. Tong, J. L. Yeh, S. R. Wang, and B. W. Lee, Physical Review B, vol. 32, p. 8473, 1985.

6. C. B. Duke, A. Paton, and A. Kahn, Physical Review B, vol. 27, p. 3436, 1983.

7. C. B. Duke, A. Paton, and A. Kahn, Journal of Vacuum Science and Technology $A$, vol. 1, p. 672, 1983.

8. L. Smit, R. M. Tromp, and J. F. van der Veen, Physical Review B, vol. 29, p. 4814,1984 .

8. P. Eisenberger and W. C. Marra, Physical Review Letters, vol. 46, p. 1081, 1981.

10. C. B. Duke, A. Paton, A. Kahn, and C. B. Bonapace, Physical Review B, vol. 27 , p. $6189,1983$.

11. S. P. Tear, M. R. Welton-Cook, M. Prutton, and J. A.. Walker, Surface Science, vol. 99, p. 598, 1980.

12. R. J. Meyer, C. B. Duke, A. Paton, J. C. Tsang, J. L. Yeh, A. Kahn, and P. Mark, Physical Review B, vol. 22, p. 6171, 1980.

13. R. M. Tromp, R. G. Smeenk, F. W. Saris, and D. J. Chadi, Surface Science, vol. 133, p. 137,1983 .

14. B. W. Holland, C. D. Duke, and A. Paton, Surface Science, vol. 140, p. L269, 1984.

15. G. J. R. Jones and B. W. Holland, Solid State Communications, vol. 53, p. $45,1985$. 
16. R. Feder, W. Mönch, and P. P. Auer, fournal of Physics C, vol. 12, p. L179, 1979.

17. F. J. Himpsel, P. M. Marcus, R. Tromp, I. P. Batra, M. R. Cook, F. Jona, and H. Liu, Physical Review B, vol. 30, p. 2257, 1984.

18. L. Smit, R. M. Tromp, and J. F. van der Veen, Surface Science, vol. 163, p. $315,1985$.

19. C. B. Duke, A. Paton, A. Kahn, and D. W. Tu, Journal of Vacuum Science and Technology $B$, vol. 2, p. 366, 1984. 


\section{Table 1.7 - Atomic Adsorption on Semiconductor Surfaces}

(If the substrate layer spacing has been investigated this is listed under the adsorbate information.)

\begin{tabular}{|c|c|c|c|c|}
\hline $\begin{array}{l}\text { Adsorption } \\
\text { System }\end{array}$ & $\begin{array}{l}\text { Overlayer } \\
\text { Unit Cell }\end{array}$ & $\begin{array}{c}\text { Adsorbate } \\
\text { Site }\end{array}$ & $\begin{array}{c}\text { Layer } \\
\text { Spacing }(\AA)\end{array}$ & Method \\
\hline GaAs (110)-Sb & $(3 \times 1)$ & 2-fold & note 1 & LEED $^{1}$ \\
\hline $\mathrm{Ge}(111)-\mathrm{Cl}$ & $(1 \times 1)$ & top & $2.07 \pm 0.03$ & SEXAFS $^{2}$ \\
\hline $\mathrm{Si}(111)-\mathrm{Br}$ & undetermined & top & $2.18 \pm 0.06$ & $\begin{array}{l}\text { X-ray stand- } \\
\text { ing wave } \\
\text { resonance } \\
\text { r,4 }\end{array}$ \\
\hline $\mathrm{Si}(111)-\mathrm{Cl}$ & $(1 \times 1)$ & top & $1.98 \pm 0.04$ & SEXAFS $^{2}$ \\
\hline $\mathrm{Si}(111)-\mathrm{Cl}$ & $(7 \times 7)$ & top & $2.03 \pm 0.03$ & SEXAFS $^{2}$ \\
\hline $\mathrm{Si}(111)-\mathrm{I}$ & $\begin{array}{c}(7 \times 7) \\
\text { top layer }\end{array}$ & $\begin{array}{l}\text { top } \\
+15 \%\end{array}$ & $\begin{array}{l}2.44 \pm 0.03 \\
0.90 \pm 0.05\end{array}$ & SEXAFS $^{5}$ \\
\hline $\mathrm{Si}(111)-\mathrm{NiSi}_{2}$ & $(1 \times 1)$ & tetrahedral & note 2 & $\operatorname{LEED}^{6}$ \\
\hline $\mathrm{Si}(111)-\mathrm{NiSi}_{2}$ & $(1 \times 1)$ & tetrahedral & note 3 & MEIS $^{7}$ \\
\hline $\mathrm{Si}(111)-\mathrm{Te}$ & $(7 \times 7)$ & 2 -fold & 1.51 & SEXAFS $^{5}$ \\
\hline
\end{tabular}

Note 1 Sb atoms fill As and Ga type sites in a slightly buckled $(0.10 \AA)$ first layer spaced $2.3 \AA$ above the GaAs surface, with lateral distortions to form a $s p^{3}$ bonded chain.

Note $2 \quad \mathrm{NiSi}_{2}$ grown on $\mathrm{Si}(111)$ substrate. Forms fluorite structure layer compound Si-Ni-Si with nickel in tetrahedral sites. Silicon layer 
terminates crystal, with a first-layer contraction of $\sim 25 \%$.

Note 3 Ion seattering investigation of $\mathrm{NiSi}_{2}$ - bulk Si interface. Determine $\mathrm{Si}-\mathrm{Ni}$-Si layer is $3.06 \pm 0.08 \AA$ above the next non-collinear $\mathrm{Si}$ atom (the bulk value is $0.77+2.35=3.12$ ). Of the two possible terminations this most closely matches the bulk silicon structure.

\section{References}

1. C. B. Duke, A. Paton, W. K. Ford, A. Kahn, and J. Carelli, Physical Review $B$, vol. 26, p. 803,1982 .

2. P. H. Citrin, J. E. Rowe, and P. Eisenberger, Physical Review B, vol. 28, p. $2299,1983$.

3. J. A. Golovchenko, J. R. Patel, D. R. Kaplan, P. L. Cowan, and M. J. Bedzyk, Physical Review Letters, vol. 49, p. 1560, 1982.

4. G. Materlik, A. Frohm, and M. J. Bedzyk, Physical Review Letters, vol. 52, p. $44 !, 1984$.

5. P. H. Citrin, P. Eisenberger, and J. E. Rowe, Physical Review Lellers, vol. 48 , p. $802,1982$.

6. W. S. Yang, F. Jona, and P. M. Marcus, Physical Review B, vol. 28, p. 7377, 1983.

7. E. J. van Loenen, J. W. M. Frenken, J. F. van der Veen, and S. Valeri, Physical Review Letters, vol. 54, p. 827, 1985. 


\section{Table 1.8 - Insulator and Other Compound Surface Stru:- tures}

\begin{tabular}{|c|c|c|c|}
\hline Substrate & Unit Cell & Structure & Method \\
\hline$C(111)$ & $(1 \times 1)$ & $\begin{array}{l}\text { Terminated bulk diamond, no relaxation } \\
\text { in layer spacing. }\end{array}$ & LEED $^{1}$ \\
\hline $\mathrm{C}(111)-\mathrm{H}$ & $(1 \times 1)$ & $\begin{array}{l}\text { Hydrogen in ( } 1 \times 1) \text { arrangement. Deter- } \\
\text { mined to by in top site, assumed } 1.09 \AA \\
\mathrm{H}-\mathrm{C} \text { bond length. }\end{array}$ & $\begin{array}{l}\text { Helium } \\
\text { diffraction }\end{array}$ \\
\hline$C(0001)$ & $(1 \times 1)$ & $\begin{array}{l}\text { Normal graphite layer stacking, first } \\
\text { layer contracted } 1.4 \% \text { to } 3.30 \AA \text { from } \\
\text { bulk spacing of } 3.35 \AA \text {. }\end{array}$ & LEED $^{3}$ \\
\hline $\mathrm{C}(0001)-K$ & intercalated & $\begin{array}{l}\text { When } K \text { is adsorbed on } C(0001) \text { it is } \\
\text { intercalated between layers, changing } \\
\text { the stacking sequence from ABAB... to } \\
\text { AAAA... and increasing the layer spac- } \\
\text { ing to } 5.35 \AA \text { from } 3.35 \AA \text {. }\end{array}$ & $\mathrm{LEED}^{4}$ \\
\hline $\mathrm{CaO}(111)$ & $(1 \times 1)$ & $\begin{array}{l}\text { Top layer contracts by } 1.2 \% \text { to } 2.38 \\
\text { from } 2.41 \AA \text {. No top-layer buckling. }\end{array}$ & LEED $^{5}$ \\
\hline $\mathrm{CoO}(111)$ & $(1 \times 1)$ & $\begin{array}{l}\text { Oxygen termination with fcc stacking, } \\
\text { top layer contraction of } 17 \% \text { to } 1.06 \AA \\
\text { from bulk } 1.27 \AA \text {. }\end{array}$ & LEED $^{6}$ \\
\hline $\mathrm{CoO}(100)$ & $(1 \times 1)$ & $\begin{array}{l}\text { Terminated bulk structure, top layer } \\
\text { spacing is } 2.85 \pm 0.08 \AA \text {. }\end{array}$ & LEED $^{7}$ \\
\hline $\mathrm{MgO}(100)$ & $(1 \times 1)$ & $\begin{array}{l}\text { Top-layer oxygen buckled out by } \\
0.04 \pm 0.05, \text { and top layer contracted by } \\
0.02 \pm 0.07 \AA . \text { Bulk layer spacing } 2.10 \AA \text {. }\end{array}$ & $\mathrm{LEED}^{8}$ \\
\hline $\mathrm{MgO}(100)$ & $(1 \times 1)$ & $\begin{array}{l}\text { Terminated bulk structure, no change } \\
\text { from bulk layer spacing of } 2.10 \AA \text {. }\end{array}$ & $\mathrm{LEED}^{9}$ \\
\hline $\mathrm{MoS}_{2}(0001)$ & $(1 \times 1)$ & $\begin{array}{l}\text { Normal stacking, S-Mo-S termination, } \\
\text { top layer contraction by } 5 \% \text { to } 1.51 \AA \\
\text { from bulk } 1.59 \AA \text {. No second layer con- } \\
\text { traction. }\end{array}$ & LEED $^{10}$ \\
\hline $\mathrm{Na}_{2} \mathrm{O}(111)$ & $(1 \times 1)$ & $\begin{array}{l}\text { Oxidation of epitaxial } \mathrm{Na}(110) \text { on } \\
\mathrm{Ni}(100) \text { substrate. Determine fluorite } \\
\text { lattice with } \mathrm{Na}-\mathrm{O}-\mathrm{Na} \text { termination. }\end{array}$ & $\mathrm{LEED}^{11}$ \\
\hline
\end{tabular}




\begin{tabular}{|c|c|c|c|}
\hline Substrate & Unit Cell & Structure & Method \\
\hline $\mathrm{NbSe}_{2}(0001)$ & $(1 \times 1)$ & $\begin{array}{l}\text { Normal layer stacking with Se-Nb-Se } \\
\text { termination, no evidence for relaxation } \\
\text { of first two layer spacings. }\end{array}$ & LEED $^{10}$ \\
\hline $\mathrm{NiO}(100)$ & $(1 \times 1)$ & $\begin{array}{l}\text { Top layer unbuckled, contracted } 2 \% \text { to } \\
2.04 \AA \text { from bulk value of } 2.08 \AA \text {. }\end{array}$ & LEED $^{12}$ \\
\hline $\mathrm{ZnO}(0001)$ & $(1 \times 1)$ & $\begin{array}{l}\text { Zn termination, top layer spacing } \\
0.60 \pm 0.10 \AA \text {, a } 25 \% \text { contraction from } \\
\text { the bulk value of } 0.80 \AA \text {. }\end{array}$ & LEED $^{13}$ \\
\hline $\mathrm{ZnO}(10 \overline{10})$ & $(1 \times 1)$ & $\begin{array}{l}\text { Oxygen buckled outward by } \sim 0.8 \AA \\
\text { and } \mathrm{Zn} \text { contracted by } 1.2 \AA \text { in the first } \\
\text { layer. Bulk layer spacing } 1.88 \AA \text {. }\end{array}$ & LEED ${ }^{14}$ \\
\hline $\mathrm{ZnO}(11 \overline{2} 0)$ & $(1 \times 1)$ & $\begin{array}{l}\text { Terminated bulk structure, no evidence } \\
\text { for reconstruction or relaxation in layer } \\
\text { spacings. }\end{array}$ & LEED $^{14}$ \\
\hline
\end{tabular}

\section{References}

1. W. S. Yang, J. Sokolor, F. Jona, and P. M. Marcus, Solid State Communications, vol. 41, p. 191, 1982.

2. G. Vidali, M. W. Cole, W. H. Weinberg, and W. A. Steele, Physical Review Letters, vol. 51, p. 118, 1983.

3. N. J. Wu and A. Ignatiev, Physical Review B, vol. 25, p. 2983, 1982.

4. N. J. Wu and A. Ignatiev, Physical Review B, vol. 28, p. 7288, 1883.

5. M. Prutton, J. A. Ramsey, J. A. Walker, and M. R. Welton-Cook, Journal of Physics C, vol. 12, p. 5271, 1979.

6. A. Ignatiev, B. W. Lee, and M. A. Van Hove, in Proceedings of the $7^{\text {th }}$ International Vacuum Concress and $3^{r d}$ International Conference on Solid 
Surfaces, Vienna, 1977.

7. R. C. Felton, M. Prutton, S. P.Tear, and M. R. Welton-Cook, Surface Science, vol. 88, p. 474, 1979.

8. M. R. Welton-Cook and W. Berndt, Joutrnal of Physics C, vol. 15, p. 569, 1982.

9. T. Urano, T. Kanaji, and M. Kaburagi, Surface Science, vol. 134, p. 109, 1983.

10. B. J. Mrstik, R. Kaplan, T. L. Reinecke, M. A. Van Hove, and S. Y. Tong, Physical Review B, vol. 15, p. 897, 1977.

11. S. Andersson, J. B. Pendry, and P. M. Echenique, Surface Science, vol. 65, p. $539,1977$.

12. M. R. Welton-Cook and M. Prutton, Journal of Physics C, vol. 13, p. 3993, 1980.

13. A. R. Lubinsky, C. B. Duke, S. C. Chang, B. W. Lee, and P. Mark, Journal of Vacuum Science and Technology, vol. 13, p. 189, 1976.

14. C. B. Duke, A. R. Lubinsky, B. W. Lee, and P. Mark, Journal of Vacuum Science and Technology, vol. 13, p. 761, 1976. 


\section{Table 1.9 - Carbon Monoxide Chemisorption on Metals}

(In all the listed structures $\mathrm{CO}$ is believed to adsorb perpendicular to the surface with the carbon end down. For CO structures with multiple non-equivalent adsorption sites these are listed on consecutive lines. If the metal layer spacing has been investigated this is listed under the adsorbate information.)

\begin{tabular}{|c|c|c|c|c|c|}
\hline $\begin{array}{c}\text { Substrate } \\
\text { Face }\end{array}$ & $\begin{array}{l}\text { Overlayer } \\
\text { Unit Cell }\end{array}$ & $\begin{array}{l}\text { Adsorption } \\
\text { Site }\end{array}$ & $\begin{array}{c}\text { C-Metal } \\
\perp \text { Spacing }(\AA)\end{array}$ & $\begin{array}{l}\text { C-O Bond } \\
\text { Length }(\AA)\end{array}$ & Method \\
\hline $\mathrm{Cu}(100)$ & $c(2 \times 2)$ & top & $1.90 \pm 0.10$ & $1.13 \pm 0.10$ & LEED $^{\text {I }}$ \\
\hline $\mathrm{Ni}(100)$ & $c(2 \times 2)$ & top & 1.72 & 1.15 & LEED $^{2}$ \\
\hline $\mathrm{Ni}(100)$ & $c(2 \times 2)$ & top & $1.80 \pm 0.10$ & $1.15 \pm 0.05$ & ARXPS $^{3}$ \\
\hline $\mathrm{Ni}(100)$ & $c(2 \times 2)$ & top & $1.80 \pm 0.10$ & $1.15 \pm 0.05$ & LEED $^{4}$ \\
\hline $\mathrm{Ni}(100)$ & $c(2 \times 2)$ & top & $1.70 \pm 0.10$ & $1.13 \pm 0.10$ & LEED $^{5}$ \\
\hline $\mathrm{Ni}(100)$ & $c(2 \times 2)$ & top & $1.71 \pm 0.10$ & $1.15 \pm 0.10$ & $\mathrm{LEED}^{6}$ \\
\hline $\mathrm{Ni}(100)$ & $c(2 \times 2)$ & top & $1.80 \pm 0.04$ & 1.13 & $\mathrm{NPD}^{7}$ \\
\hline $\mathrm{Ni}(111)$ & $(\sqrt[V]{ } 3 \times \sqrt{ } 3) \mathrm{R} 30^{\circ}$ & bridge & $1.27 \pm 0.05$ & $1.13 \pm 0.05$ & $\mathrm{NPD}^{7}$ \\
\hline $\mathrm{Pd}(100)$ & $(2 \sqrt{ } 2 \times \sqrt{ } 2) \mathrm{R} 45^{\circ}$ & $\begin{array}{l}\text { bridge } \\
\text { top layer }\end{array}$ & $\begin{array}{l}1.36 \pm 0.10 \\
1.945 \pm 0.10\end{array}$ & $\begin{array}{l}1.15 \pm 0.10 \\
+0.4 \%\end{array}$ & LEED $^{8}$ \\
\hline Pt (111) & $c(4 \times 2)$ & $\begin{array}{c}\text { top } \\
\text { bridge } \\
\text { top layer }\end{array}$ & $\begin{array}{l}1.85 \pm 0.05 \\
1.55 \pm 0.05 \\
2.26 \pm 0.025\end{array}$ & $\begin{array}{l}1.15 \pm 0.10 \\
1.15 \pm 0.10 \\
0.0 \%\end{array}$ & LEED $^{9}$ \\
\hline $\operatorname{Pt}(111)$ & $(2 \sqrt[V]{ } 3 \times 4)$ rect ${ }^{*}$ & bridge & 1.45 & 1.15 & LEED $^{10}$ \\
\hline$R h(111)$ & $(3 \times 3)^{*}$ & 3-fold hep & 1.30 & 1.17 & LEED $^{11}$ \\
\hline
\end{tabular}




\begin{tabular}{|c|c|c|c|c|c|}
\hline $\begin{array}{l}\text { Substrate } \\
\text { Face }\end{array}$ & $\begin{array}{l}\text { Overlayer } \\
\text { Unit Cell }\end{array}$ & $\begin{array}{c}\text { Adsorption } \\
\text { Site }\end{array}$ & $\begin{array}{c}\text { C-Metal } \\
\perp \text { Spacing }(\AA)\end{array}$ & $\begin{array}{l}\text { C-O Bond } \\
\text { Length }(\AA)\end{array}$ & Method \\
\hline Rh (111) & $c(2 \vee 3 \times 4)$ rect & 3-fold fcc & $1.50 \pm 0.05$ & $1.21 \pm 0.10$ & LEED $^{12}$ \\
\hline $\mathrm{Rh}(111)$ & $(2 \times 2)$ & $\begin{array}{c}\text { quasi-top } \\
\text { quasi-top } \\
\text { bridge }\end{array}$ & $\begin{array}{l}1.87 \pm 0.10 \\
1.87 \pm 0.10 \\
1.52 \pm 0.10\end{array}$ & $\begin{array}{l}1.15 \pm 0.10 \\
1.15 \pm 0.10 \\
1.15 \pm 0.10\end{array}$ & LEED $^{13}$ \\
\hline Rh (111) & $(\sqrt{ } / 3 \times \sqrt{ } 3) \mathrm{R} 30^{\circ}$ & $\begin{array}{c}\text { top } \\
\text { top layer }\end{array}$ & $\begin{array}{l}1.95 \pm 0.10 \\
2.19 \pm 0.10\end{array}$ & $\begin{array}{l}1.07 \pm 0.10 \\
0.0 \%\end{array}$ & LEED $^{14}$ \\
\hline $\mathrm{Ru}(0001)$ & $(\sqrt{ } 3 \times \sqrt{ } 3) R^{2} 30^{\circ}$ & top & $2.00 \pm 0.10$ & $1.09 \pm 0.10$ & LEED $^{15}$ \\
\hline
\end{tabular}

* These structures involve benzene co-adsorbed with carbon monoxide. See table 1.10 and chapter 12 for details.

\section{References}

1. S. Andersson and J. B. Pendry, Journal of Physics C, vol. 13, p. 2547, 1980.

2. M. Passler, A. Ignatiev, F. Jona, D. W. Jepsen, and P. M. Marcus, Physical Review Letters, vol. 43, p. 360, 1979.

3. L. G. Petersson, S. Kono, N. F. T. Hall, C. S. Fadley, and J. B. Pendry, Physical Review Letters, vol. 42, p. 1545, 1979.

4. K. Heinz, E. Lang, and K. Müller, Surface Science, vol. 87, p. 595, 1979.

5. S. Y. Tong, A. Maldonado, C. H. Li, and M. A. Van Hove, Surface Science, vol. 94, p. 73, 1980. 
6. S. Andersson and J. B. Pendry, Journai of Physics C, vol. 13, p. 3547, 1980.

7. S. D. Kevan, R. F. Davis, D. H. Rosenblatt, J. G. Tobin, M. G. Masoin, D. A. Shirley, C. H. Li, and S. Y. Tong, Physical Review Letters, vol. 46, p. 1629, 1981.

8. R. J. Behm, K. Christmann, G. Erti, and M. A. Van Hove, Journal of Chemical Physics, vol. 73, p. 2984, 1980.

9. L. F. Ogletree, M. A. Van Hove, and G. A. Somorjai, Surface Science, vol. 173, p. $351,1986$.

10. D. F. Ogletree, M. A. Van Hove, and G. A. Somorjai, Surface Science, in press.

11. M. A. Van Hove, R. F. Lin, G. A. Blackman, and G. A. Somorjai, Acta Crystallographica, to be published.

12. M. A. Van Hove, R. F. Lin, and G. A. Somorjaj, Journal of the American Cinemical Society, vol. 108, p. 2532, 1986.

13. M. A. Van Hove, R. J. Koestner, J. C. Frost, and G. A. Somorjai, Surface Science, vol. 129, p. $482,1983$.

14. R. J. Koestner, M. A. Van Hove, and G. A. Somorjai, Surface Science, vol. 107, p. 439, 1981.

15. G. Michalk, W. Moritz, H. Pfnur, and D. Menzel, Surface Science, vol. 129, p. $92,1983$. 
Table 1.10 - Other Molecular Adsorption Structures

\begin{tabular}{|c|c|c|}
\hline System & Structure & Method \\
\hline $\begin{array}{l}\mathrm{Cu}(100) \mathrm{HCO}_{2} \\
\text { disordered }\end{array}$ & $\begin{array}{l}\text { The formate radical is in a plane } \perp \text { to } \\
\text { the surface with the two oxygens closest } \\
\text { to the surface, and a formate O-C-O } \\
\text { bond angle of } 125^{\circ} \text { is assumed. The O } \\
\text { atoms are slightly off-center above two } \\
\text { adjacent } 4 \text {-fold hollow sites. The } O \\
\text { atoms are } 1.54 \AA \text { above the Cu surface } \\
\text { and separated by } 2.17 \AA \text {. The C atom is } \\
2.11 \AA \text { above the surface. }\end{array}$ & NEXAFS ${ }^{1}$ \\
\hline $\begin{array}{l}\mathrm{Cu}(110) \mathrm{HCO}_{2} \\
\text { disordered }\end{array}$ & $\begin{array}{l}\text { The formate radical is in a plane } \perp \text { to } \\
\text { the surface along the [001] direction. } \\
\text { The oxygen atoms are closest to the sus- } \\
\text { face, slightly off-center from two adja- } \\
\text { cent bridge sites, } 1.51 \AA \text { above the sur- } \\
\text { face and } 2.29 \AA \text { apart. The } O \text { atom is } \\
2.04 \AA \text { above the Cu atom. }\end{array}$ & $\mathrm{NEXAFS}^{2}$ \\
\hline $\begin{array}{l}\mathrm{Fe}(100) \mathrm{C}+\mathrm{O} \\
\mathrm{c}(2 \times 2)\end{array}$ & $\begin{array}{l}\mathrm{CO} \text { is decomposed on the } \mathrm{Fe}(100) \text { sur- } \\
\text { face. } \mathrm{C} \text { and } \mathrm{O} \text { occupy random } 4 \text {-fold } \\
\text { hollow sites } 0.48 \AA \text { above the surface. }\end{array}$ & LEED $^{3}$ \\
\hline $\begin{array}{l}\mathrm{Ni}(100) \mathrm{N}+\mathrm{O} \\
\mathrm{c}(2 \times 2)\end{array}$ & $\begin{array}{l}\text { NO dissociates on } \mathrm{Ni}(100) . \mathrm{N} \text { and } \mathrm{O} \\
\text { atoms randomly occupy } 4 \text {-fold hollow } \\
\text { sites } 0.93 \pm 0.10 \AA \text { above the surface. }\end{array}$ & LEED ${ }^{4}$ \\
\hline $\mathrm{Ni}(111) \mathrm{C}_{2} \mathrm{H}_{2}(2 \times 2)$ & $\begin{array}{l}\text { The acetylene molecules are adsorbed } \\
\text { with the C-C bond parallel to the sur- } \\
\text { face and the center of the C-C bond is } \\
\text { over a bridge site. The C-C bond is per- } \\
\text { pendicular to the Ni-Ni bridge. The C- } \\
\mathrm{C} \text { bond length is } 1.50 \AA \text { and the carbon } \\
\text { atoms are } 2.1 \pm 0.10 \AA \text { above the surface. }\end{array}$ & LEED $^{5}$ \\
\hline $\operatorname{Pt}(111) \mathrm{C}_{2} \mathrm{H}_{3}(2 \times 2)$ & $\begin{array}{l}\text { Ethylidyne }\left(\mathrm{CCH}_{3}\right) \text { bonded } \perp \text { to surface } \\
\text { in 3-fold fec sites, } \mathrm{C}-\mathrm{C} \text { bond } 1.50 \pm 0.05 \AA \\
\text { and C-surface } \perp \text { distance } 1.20 \pm 0.05 \AA \text {. }\end{array}$ & LEED $^{6}$ \\
\hline
\end{tabular}




\begin{tabular}{|c|c|c|}
\hline System & Structure & Method \\
\hline $\begin{array}{l}\mathrm{Pt}(111) \\
2 \mathrm{C}_{6} \mathrm{H}_{6}+4 \mathrm{CO} \\
(2 \sqrt{3 \times 4}) \text { rect }\end{array}$ & $\begin{array}{l}\text { Benzene ring parallel to surface over } \\
\text { bridge sites, } 2.10 \AA \text { above surface, with } \\
\text { two benzenes and four CO's per unit } \\
\text { cell. CO also over bridge sites, } 1.45 \AA \\
\text { above the metal surface. Benzene ring is } \\
\text { expanded with small in-plane distortions } \\
\text { consistent with local symmetry. }\end{array}$ & $\mathrm{LEED}^{?}$ \\
\hline $\mathrm{Rh}(111) \mathrm{C}_{2} \mathrm{H}_{3}(2 \times 2)$ & $\begin{array}{l}\text { Ethylidyne }\left(\mathrm{CCH}_{3}\right) \text { is adsorbed with the } \\
\mathrm{C}-\mathrm{C} \text { axis } \perp \text { to the surface with a } \\
1.45 \pm 0.10 \AA \text { bond length. The terminal } \\
\text { carbon atom is } 1.31 \pm 0.10 \AA \text { above a } 3- \\
\text { fold hcp hollow site. }\end{array}$ & LEED $^{8}$ \\
\hline $\begin{array}{l}\mathrm{Rh}(111) \\
\mathrm{C}_{6} \mathrm{H}_{6}+2 \mathrm{CO}(3 \times 3)\end{array}$ & $\begin{array}{l}\text { One benzene and two CO's per unit cell. } \\
\text { CO is } \perp \text { to the surface, adsorbed } 1.30 \AA \\
\text { above } 3 \text {-fold hcp sites. Benzene is paral- } \\
\text { lel to the surface, centered } 2.20 \AA \text { over a } \\
3 \text {-fold hcp site. Slight in-plane Kekulé } \\
\text { distortion of the benzene molecule. }\end{array}$ & LEED $^{9}$ \\
\hline $\begin{array}{l}\mathrm{Rh}(111) \mathrm{C}_{6} \mathrm{H}_{6}+\mathrm{CO} \\
\mathrm{c}(2 \sqrt{3 \times 4}) \text { rect }\end{array}$ & $\begin{array}{l}\text { Benzene is coadsorbed with } C O \text {, each } \\
\text { with one molecule per unit cell, both } \\
\text { centered over } 3 \text {-fold hcp sites, benzene is } \\
\text { parallel to and } 2.25 \pm 0.05 \AA \text { above the } \\
\text { surface. CO is } 1 \text { to the surface and the } \\
\text { metal-carbon spacing is } 1.50 \pm 0.05 \AA \text {. } \\
\text { The benzene molecule has an in-plane } \\
\text { Kekule distortion, with alternating long } \\
\text { and short bonds. }\end{array}$ & $\mathrm{LEED}^{10}$ \\
\hline
\end{tabular}

\section{References}

1. D. A. Outka, R. J. Madix, and J. Stöhr, Surface Science, vol. 164, p. 235, 1985.

2. A. Puschman, J. Haase, M. D. Crapper, C. E. Riley, and D. P. Woodruff, Physical Review Lettere, vol. 54, p. 2250, 1985. 
3. K. O. Legg, F. Jona, D. W. Jepsen, and P. M. Marcus, Physical Review B, vol. 16, p. 5271, 1977.

4. M. A. Passler, T. H. Lin, and A. Ignatiev, Journal of Vacuum Science and Technology, vol. 18, p. 481, 1981.

5. G. Casalone, M. G. Cattania, F. Merati, and M. Simonetta, Surface Science, vol. 120, p. 171, 1982.

6. L. L. Kesmodel, I. H. Dubois, and G. A. Somorjai, Journal of Chemical Physics, vol. 70 , p. $2180,1979$.

7. D. F. Ogletree, M. A. Van Hove, and G. A. Somorjai, Surface Science, in press.

8. R. J. Koestner, M. A. Van Hove, and G. A. Somorjai, Strface Science, vol. 121, p. 321, 1982 .

9. M. A. Van Hove, R. F. Lin, G. A. Blackman, and G. A. Somorjai, Acta Crystallographica, to be published.

10. M. A. Van Hove, R. F. Lin, and G. A. Somorjai, Journal of the American Chemical Society, vol. 108, p. 2532, 1986. 


\section{Chapter 2}

\section{LEED Experimental Methods}

\subsection{Introduction}

In the low energy electron diffraction (LEED) experiment a beam of electrons of well defined energy and momentum is incident on a surface and the backscattered electrons are detected as a function of energy and angular momentum. Normally attention is restricted to electrons that are elastically scattered from the surface and so LEED is sometimes referred to as ELEED, or elastic low-energy electron diffraction. The principle application of LEED is the study surface ordering and structure, so the target surface is usually a well-defined single crystal plane.

The experimental parameters that enter into a LEED experiment are the energy $E_{o}$ and the angle of incidence $\Omega_{0}$ of the incident electrons and the angle $\Omega$ and energy $E$ at which back-scattered electrons are detected. There is always some degree of error in these parameters, which limits the information that can be extracted from a LEED experiment. The sensitivity, dynamic range and signal-to-noise ratio of the measurement of diffracted electron currents also limits the information available in a LEED experiment.

The rest of this chapter will outline the different types of information that can be obtained through LEED experiments, along with a discussion of the physical processes and experimental factors which limit such investigations. 
Measurement techniques and the effects of inelastic processes on LEED experiments are discussed in this context. A critical survey is made of existing LEED instrumentation and motivation is given for the experimental developments described in part II.

\subsection{Types of LEED experiments}

A LEED experiment can give three basic types of information about a surface - information on the long range order and symmetry, on the deviations from long-range order and on the local geometry or structure. This can be illustrated by factoring the intensity of elastic electron scattering from a periodic surface into the product of a geometrical form-factor and a structure-factor as is done for X-ray diffraction.

$$
I\left(\mathbf{k}, \mathbf{k}_{\mathbf{o}}\right)=F\left(\mathbf{k}, \mathbf{k}_{\mathbf{o}}\right) S(\Delta \mathbf{s})
$$

Here $\mathbf{k}_{\mathbf{o}}$ is the momentum of the incident electron and $\mathbf{k}$ is the momentum of the scattered electron. The form-factor $F$ describes the electron scattering by a surface unit cell, including multiple scattering and the structure-factor $S$ depends on the periodic arrangement of the surface. $S$ is a function only of parallel momentum transfer

$$
\Delta \mathbf{s} \equiv\left(\mathbf{k}-\mathbf{k}_{\mathbf{o}}\right) \cdot(1-\hat{\mathbf{n}})=\Delta \mathbf{k}_{\|}
$$

where $\hat{\mathbf{n}}$ is the unit vector normal to the surface.

This factorization is exact for a perfectly ordered system or for any system described by kinematic (single) scattering. For a system with some deviation 
from perfect long-range order it is only approximate, since certain multiple scattering contributions are neglected. 1

When multiple scattering is important the scattering properties of a particular unit cell are affected by the positions of all neighboring atoms within a few times the electron mean-free path. The scattering of a unit cell located near a defect, domain boundary or impurity atom is different from the same unit cell in a large ordered region. The factorization of Eq. 2.1 is still useful as a guide to understanding the effects of disorder, thermal scattering and imperfect instruments on LEED experiments. In most cases the LEED patterns (as opposed to intensities) are dominated by the kinematic effects described by $S(\Delta \mathbf{s})$.

Information on long-range order and deviations from long range order are contained in the structure factor $S$. A perfectly periodic surface can be described by a set of reciprocal-space vectors $\{\mathbf{g}\}$ where $\mathbf{g} \cdot \hat{\mathbf{n}}=0$. The structure factor for this perfect surface is then

$$
S(\Delta \mathbf{s})=\sum_{i} g_{i} \delta\left(\Delta \mathbf{s}-\mathbf{g}_{i}\right)
$$

The electrons diffracted from this surface can be described as a number of backscattered diffraction "beams" with momentum $\mathbf{k}_{i}=\mathbf{k}_{\mathrm{o}}+\mathbf{g}_{\mathbf{j}}$. These beams are often labeled in terms of surface reciprocal-space unit cell vectors $\mathbf{a}$ and $\mathbf{b}$ which span the set of reciprocal-space vectors $\{\mathrm{g}\}$ so that

$$
\mathbf{k}_{i j}^{ \pm}=\mathbf{k}_{\mathrm{oll}} \pm \mathbf{k}_{\mathrm{oL}}+i \mathrm{a}+j \mathrm{~b}
$$

Because of energy conservation only the finite subset of these beams with $\left|\mathbf{k}_{\mathbf{i j}}\right| \leq\left|\mathbf{k}_{\mathbf{o}}\right|$ are observable. 


\subsection{Surface symmetry studies}

A major application of LEED is the determination of the surface unit cell vectors $(\mathbf{a}, \mathbf{b})$ through observation of the set of back-diffracted beams $\{\mathbf{k}\}$. This technique is especially useful in detecting surface reconstructions, where the surface symmetry is different from that of the terminated bulk crystal structure and also in studying overlayers of adsorbed atoms and molecules, which often form ordered super-lattices with reduced translational or rotational symmetry relative to the clean surface.

There are two important special cases involved in determination of the surface symmetry -- magnetic ordering and glide-symmetries. Surface magnetic ordering has been detected using spin-polarized LEED, or SPLEED and in some cases magnetic ordering effects have been detected with conventional LEED, for example Palmberg et al. ${ }^{2}$ saw $1 / 2$ order LEED beams from anti-ferromagnetic NiO due to spin-ordering. Glide-plane symmetries have been observed for several chemisorption systems, especially for large chemisorbed molecules. ${ }^{3}$ A glide-plane symmetry is a surface symmetry operation composed of a translation parallel to the glide plane by one half of a reciprocal space vector $1 / \mathrm{gg}^{\prime}$, where $1 / 2 \mathrm{~g}^{\prime} \neq\{\mathrm{g}\}$, combined with a reflection across the glide plane. The incident electron wave $\mathbf{k}_{\mathbf{o}}$ must be considered a part of the system when the LEED symmetry is determined, so a glide-symmetry will only be seen in LEED when the incident electron beam is in the glide-plane, i.e. when $\mathbf{k}_{\mathrm{o}}$ il is parallel to $\mathbf{g}^{\prime}$ (see chapter $\mathbf{3}$ on symmetry).

There is an additional practical complication in the determination of surface symmetry. When a surface reconstruction or adsorbed overlayer reduces the 
surface symmetry, multiple domains of different orientations may be present on the surface (see chapter 10). Normally these domains are much smaller than the spot-size of the LEED electron gun $(\sim 0.5 \mathrm{~mm})$ z.nd so multiple domains are superimposed in the observed LEED patterns, $4,5,6$ which can complicate the analysis of surface symmetry. Sometimes different domains will be observed with different apparent intensities, which simplifies interpretation. ${ }^{4}$ In some cases domains have been formed preferentially, by using stepped crystal substrates, as in the case of oxygen adsorbed in a (2x1) overlayer on a stepped $\operatorname{Re}(0001)$ crystal surface, ${ }^{7}$ or by sputtering the substrate along a preferred direction. LEED may not bc able to distinguish between a high-symmetry arrangement or a superposition of lower symmetry domains if preferential domain occupation is not observed. If the presence of the adsorbate does not affect the substrate structure,

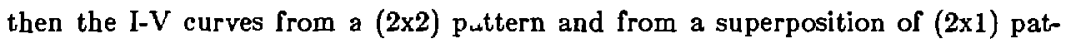
terns on a (111) or (100) surface can be very similar. The calculated LEED I-V curves for these two cases will differ primarily because of multiple scattering between adsorbates, which can be rather weak at large separations. The I-V curves common to both $c(2 \times 2)$ and $(2 \times 2)$ chalcogen overlayers on $\mathrm{Ni}(100)$ have been observed to be almost identical. Likewise tìe I-V curve for the $(1 / 2,1 / 2)$ superlattice beam of copper (100) changes very little except in overall intensity as oxygen coverage increases. ${ }^{8}$

Modern commercial LEED systems are generally adequate for determining surface symmetry. The only limitations are for cases where adjacent spots cannot be separated due to insufficient instrumental angular resolution, or where some 
superlattice spots are so weak in relative intensity that they cannot be distinguished from the diffuse background scattering (from phonon scattering, or incoherent scattering from defects in the surface lattice).

\subsection{Surface order studies}

A second major application of LEED is the study of surface defects and ordering. The basic properties of ordering and phase-transitions in quasi-two dimensional systems provide an important test case for theories of universality classes and critical exponents in phase transitions." (Surfaces are referred to as "quasitwo dimensional" since the properties of the bulk lattice iz liuence surface ordering. There are surface ordering phenomena that cannot be explained theoretically as a true two-dimensional system.) These theories, coming out of renormalization-group methods applied to the study of three dimensional phase transitions, predict that many kinds of surface phase transitions will have very general properties that depend on the surface symmetry, but not on the details of chemisorption or adatom-adatom interactions. There have been a number of experimental studies to confirm the properties of these "universality classes". Diffraction techniques such as LEED are natural tools to study the changes in long-range order associated with surface phase-transitions. The surface phase transition for oxygen chemisorbed on nickel (111) is a good example of such studies. 10,11

Defects and imperfections in a surface system with long-range order are another important aren of investigation. This category includes point defects, 
such as vacancies and random ad-atoms or impurity atoms and extended defects, such as surface steps and kinks. Deviations from long-range order are also associated with the ordering of adsorbates. In many cases adsorbates form ordered islands on the substrate. The distribution and growth of such islands can be studies by LEED and when islands grow together domain-walls may be formed. When ordered adsorbate islands have lower translational or rotational symmetry than the substrate there is the additional possibility of interference between outof-phase domains.

Studies of surface defects and overlayer islands can provide important information on surface thermodynamics and the kinetics of adsorption, surface diffusion and island growth. Also, surface defects such as vacancies, steps and kinks play a vital role in a number of catalytic reactions on surfaces. ${ }^{12}$

In this section only the statistical distribution of defects will be discussed. All information on the statistical distribution of various types of point defects is contained in the structure-factor $S(\Delta s)$. The actual structure of these defects, such as the position of atoms at step edges, cannot be determined from investigation of the structure-factor -- this information is contained in the geometrical form factor $F$.

\subsubsection{LEED coherence and transfer width}

In order to understand studies of the surface structure-factor $S$ it is necessary to clarify the concept of "coherence length", i.e. the range over which LEED is sensitive to surface order. There has been a great deal of confusion generated 
about the concepts of "coherence length" and "transfer width". The source of this confusion lies in the failure to distinguish clearly between the basic physics of the LEED process and the limitations of LEED instruments, as was explained by Comsa. ${ }^{13}$

LEED can be understood in terms of the diffraction of a single electron from a surface. In fact the electron counting detector described in chapter 6 comes very close to the idealized wave/particle experiment of introductory quantum mechaniss. Discrete electrons are counted one at a time, and the resulting probability distribution at the detector corresponds to the magnitude of the diffracted wavefunction. In a LEED experiment the detected signal is the superposition of many different electrons each diffracted from the surface. Because of the finite size and angular divergence of the electron source, there is a statistical distribution of incident electron momenta $\mathbf{k}_{\mathbf{o}}$ and when the diffraction patterns of electrons with different $\mathbf{k}_{\mathbf{o}}$ are averaged together, some information is lost. The electron detector also has a finite angular resolution and this further limits the information that can be obtained from a LEED experiment.

The occupation density of electron states in phase space is quite low, even in a very good beam, so interactions between electrons in the beam can be neglected. This density is give by

$$
\rho=\frac{n}{\Delta \mathbf{k} \Delta \mathbf{r}}
$$

where $n$ is the number of electrons, $\Delta \mathbf{k}$ the volume in momentum space and $\Delta \mathbf{r}$ the volume of the beam. The volume in momentum-space can easily be 
estimated:

$$
\Delta \mathbf{k}=\left(\frac{\partial k}{\partial E} \Delta E\right)\left(k^{2} d \Omega\right)=\sqrt{2}\left(\frac{m c}{h}\right)^{3} \frac{\sqrt{E} \Delta E}{\left(m c^{2}\right)^{3 / 2}} \frac{\gamma^{2}}{4}
$$

where $\gamma$ is the half-angle of divergence of the electron beam. The number of electrons in the beam volume is just

$$
\frac{n}{\Delta \mathbf{r}}=\frac{I}{e} \frac{D}{v} \frac{1}{\pi d^{2} D}
$$

where $I$ is beam current, $d$ is the beam radius and $v$ the electron velocity. Therefore

$$
\rho=\frac{2 I}{\pi e c \alpha^{2} d^{2}}\left(\frac{m^{2} c^{4}}{E \Delta E}\right)\left(\frac{h}{m c}\right)^{3}
$$

For reasonable values of the beam parameters $I=1 \mu \mathrm{A}, \alpha=0.01 \mathrm{rad}, d=0.25$ $\mathrm{mm}, E=100 \mathrm{~V}$ and $\Delta E=1 \mathrm{~V}$, this expression gives $\rho=3.1 \cdot 10^{-13}$, so it is indeed valid to consider LEED in terms of the diffraction of a single electron.

The effect of a LEED instrument on a diffraction pattern can be understood in terms of an instrument response function, as defined by Park et al. ${ }^{14}$ The diffraction pattern of a perfect infinite crystal should be a sum of $\delta$ functions, as defined in Eq. 2.3. The response of a real instrument to such a perfect diffraction pattern is given by an instrument response function $t(\mathbf{k})$. Now if a real surface would produce a diffraction pattern $i(\mathbf{k})$ in an ideal instrument, the diffraction pattern observed by the real instrument is given by $j(\mathbf{k})$, where

$$
j(\mathbf{k})=\int i\left(\mathbf{k}^{\prime}\right) t\left(\mathbf{k}-\mathbf{k}^{\prime}\right) d \mathbf{k}^{\prime} \equiv i(\mathbf{k})^{*} t(\mathbf{k})
$$


The instrument response function is normally assumed to be a Gaussian function. The main factors contribution to the instrument response function are the apparent angular size of the electron source, as seen through the electror gun lenses from the sample, the width of the incident beam, the angular resolution of the detector and the energy spread of the incident beam. These factors can be treated as independent contributions for a typical LEEL instrument and the resulting instrument response function is a Gaussian whose width $w$ is a quadratic sum of the individual contributions. The contributions for the different factors can be calculated from the LEED geometry, so that the angular width of the instrum .nt response function is ${ }^{13,15}$

$$
\begin{gathered}
\left(\Delta \theta_{\text {in } \delta t}\right)^{2}=\left(\frac{\left|\sin \theta_{i}-\sin \theta_{f}\right|}{\cos \theta_{f}} \frac{\Delta E}{E}\right)^{2}+\left(2 \gamma \frac{\cos \theta_{f}}{\cos \theta_{i}}\right)^{2} \\
+\left(\frac{\cos ^{2} \theta_{i}}{\cos ^{2} \theta_{f}} \frac{d}{D}\right)^{2}+\left(\Delta \theta_{d}\right)^{2}
\end{gathered}
$$

where $E$ is the incident beam energy, $\theta_{0}$ the incidence angle (where $\theta_{i}=0$ is normal incidence), $\theta_{f}$ the detector angle, $\gamma$ is the effective angular half-width of the electron source, $d$ the incident beam width, $D$ the distance between the source and the surface and $\Delta \theta_{d}$ is the angular resolution of the detector. This derivation assumes that $\mathbf{k}_{\boldsymbol{i} \|}$ and $\mathbf{k}_{\boldsymbol{f} \|}$ are parallel.

The source angular size $\gamma$ and the detector angular resolution are usually the dominant factors in this expression and the detector resolution can be improved simply by moving the detector away from the surface. Most attempts to increase 
the angular resolution of LEED instruments have concentrated on improving the electron gun, as is discussed below.

The instrument response-function $t(\theta)$ is a Gaussian with an angular width defined in terms of instrumental parameters by Eq. 2.10. If a perfect surface is observed by a real instrument, the diffaction beams will have a width $\Delta \theta$ given by $t$. The "transfer width" is defined to be the width of a diffraction grating that, in a perfect instrument, would give observed diffraction beams of the same width $\Delta \theta$. Formally, the transfer width is defined by

$$
w=\frac{\lambda}{\Delta \theta_{i n s t} \cos \theta_{f}}
$$

where $\lambda$ is the electron wavelength. A typical commercial display LEED system has a transfer width of $\sim 100 \AA$.

This definition of transfer width has often been mis-used to say that "LEED is only sensitive to structure up to a distance $w$ ", or "LEED clectrons are only 'coherent' on a scale $w$ ". This is not correct. A LEED electron is in principal sensitive to interference and diffraction effects over the width of the electron beam, $\sim$ $0.5 \mathrm{~mm}$. Much of this information is obscured by the response of a real instrument. An instrument will show obvious spot-broadening for domains less than the transfer width, however, this same instrument is still sensitive to the effects of domain size for domains much larger than the transfer wudth. This information can be obtained by deconvolution from the observed signal and thus is limited by the accuracy with which LEED beams can be measured. This point has been emphasized by Lagally, et al. ${ }^{15,16}$ The true angular width of a diffraction beam, 
assuming Gaussian shapes for the beam and the instrument response, is

$$
\Delta \theta_{\text {surface }}=\left[\left(\Delta \theta_{\text {observed }}\right)^{2}-\left(\Delta \theta_{\text {instrument }}\right)^{2}\right]^{1 / 2}
$$

If the transfer width and the experimental beam width can be measured with a fractional error $\epsilon$, then the minimum angle $\Delta \theta_{\text {surfoce }}$ that can be resolved is $2 \sqrt{\epsilon} \Delta \theta_{\text {inetrament }}$. So if angular widths can be measured to $1 \%$ of the instrumental resolution $\Delta \theta_{\text {instrument }}$, which is reasonable when the instrument response is dominated by the properties of the electron source, not the detector, then the LEED instrument is sensitive to island size up to five times the "transfer width". For this reason Lu and Lagally ${ }^{16}$ have suggested that the "minimum angle of resolution", which includes both the effect of the instrumental broadening and the precision with which measurements can be made, is a better way to characterize the performance of a LEED instrument.

\subsubsection{Overlayer islands and coherence}

It is often stated that there is no interference between islands separated by more than the transfer width or coherence length and so scattered intensities are summed. This is not correct as the above discussion shows. In fact electrons scatter "coherently" from all the islands illuminated by the electron beam, although instrumental broadening usually masks the effects. Improvements in LEED instrumentation may make it possible to distinguish between these different descriptions. 
Consider the case of an adsorbate on a one-dimensional substrate that fornis ordered islands with twice the substrate periodicity (see figure 2.1). Let $l$ be the typical island size and $L$ be the dimension of the surface (either the crystallite size or the beam dimension). The overlayer scattering may be described as

$$
\psi=\sum \phi_{i} e^{i \mathbf{E} \cdot \mathbf{R}_{i}}
$$

where the $\phi$ 's describe the scattering of individual islands and the $\mathbf{R}$ 's give the island positions. Now the scattered intensity is given by

$$
I=\psi^{*} \psi=\sum_{i} \phi_{i}^{*} \phi_{i}+\sum_{i \neq j} \phi_{i}^{*} \phi_{j} e^{i \theta^{*}\left(\mathbf{R},-\mathbf{R}_{i}\right)}
$$

The first term is the "incoherent" sum of the contributions of the individual islands and the second term contains the effects of interference between islands. The first term has a substantial amplitude for a range $\Delta s \approx \frac{\pi}{l}$ around the reciprocal lattice vector $\mathbf{g}_{h}$, while phases in the interference term add randomly and the sum averages to zero. However, for $\mathbf{s}$ sufficientiy close to $\mathbf{g}_{h}$ the interference terms will start to add in phase, i.e. over a region $\Delta \approx \frac{\pi}{L}$. If $h$ is even, then the interference terms add in phase and the diffracted intensity is much greater. If $h$ is odd, then the phases of the domain cross-terms will vary by $\pm \pi$ and the overall scattered intensity will average to zero. This is because of anti-phase domains - since the overlayer islands have twice the periodicity of this substrate, each overlayer islands may align with the "even numbered" or the "odd numbered" substrate atoms, leading to constructive or destructive interference very close to $\mathbf{g}_{h}$. This long range interference produces a "spike" or a "hole" in the 
overlayer diffraction beam profile, as shown in figure 2.1 after Lagally et al. ${ }^{16}$

This effect is not resolved in a typical LEED instrument where the transfer width is $\sim 100$ to $1000 \AA$ and the beam diameter is $\sim 10^{6} \AA$. However, LEED electrons guns with spot sizes as small as $\sim 10^{4} \AA$ and smaller beam divergence angles are being developed, so it may soon be possible to demonstrate experimentally the effects of long-range interference between islands (see chapter 10 for further discussion of island and domain-wall effects in LEED).

\subsubsection{Extended surface defects}

Henzler ${ }^{17}$ and Lagally ${ }^{18}$ have shown that various kinds of extended surface defects can be studied quantitatively by observing the behavior of the structurefactor. Monatomic steps on the surface were the first defect to be studied. When steps are present on the surface, wavelength-dependent oscillations can be observed in the widths of the diffraction beams. The period of these oscillations is a function of step height and their amplitude is a function of step density. ${ }^{17}$ This analysis was used to demonstrate the existence of monatomic surface steps and this approach has been used to study annealing on various surfaces. ${ }^{19}$ Other types of extended defects have different effects on $S(\Delta \mathbf{g}) .{ }^{18}$ For example, surface strain, as in an epitaxially grown thin-film, will cause increasing spot broadening with increasing beam order, or $\Delta s$, when the beam widths of different orders are measured at the same value of $\mathbf{k}_{\mathrm{ol}}$. Strain broadening is zero for specular reflection.

Another type of extended surface defect is a mosaic structure, where different surface domains are tilted out of the average surface plane. This will cause beam 
broadening that increases linearly with the component of momentum transfer normal to the surface $\Delta \mathbf{k}_{1}$ and the rate of increase is proportional to the average out-of-plane tilt. The limit of the beam broadening as momentum transfer normal to the surface goes to zero is characteristic of the finite size of in-plane domains, as in the case of island formation. Average island size can be determined from this value. ${ }^{18}$

To summarize, measurement of LEED beam size as a function of momentum-transfer, after correction for instrumental broadening, can show the average domain size, the degree of domain angular out-of-plane misalignment, the step height and step density and the amount of surface strain.

\subsection{Surface structure studies}

Surface structure determination is the third major application of LEED. All information on the local structure and geometry, including bond-lengths and bond-angles for chemisorbed atoms and molecules, is contained in the form-factor $F\left(\mathbf{k}, \mathbf{k}_{\mathrm{o}}\right)$. In LEED studies of surfaces with long-range order, the structure-factor $S$ describes a set of diffraction beams. Within the diffraction pattern the beam intensities are modulated by the geometrical form-factor. LEED structural data is collected by measuring the intensity of a particular diffraction beam $\left(\Delta s=\mathbf{g}_{h, k}\right)$ as a function of $\mathbf{k}_{\mathrm{o}}$. For a perfect surface $S$ is given by Eq. 2.3, so the LEED beam intensity is

$$
I_{h, k}\left(\mathbf{k}_{\mathbf{o}}\right)=g_{h, k} F\left(\mathbf{k}_{\mathbf{o}}+\mathbf{g}_{h, k}, \mathbf{k}_{\mathbf{o}}\right)
$$


When a real surface is studied with a real instrument, there is a range of incident electron momenta $\Delta \mathbf{k}_{\mathbf{o}}$ around the nominal value $\mathbf{k}_{\mathbf{o}}$ and the detector measures the diffracted electron current over a solid angle $\Delta \Omega$ around the nominal diffracted beam direction $\mathbf{k}_{h, k}$. So the effective measured intensity is

$$
I_{h, k}\left(\mathbf{k}_{\mathrm{o}}\right)=\int_{\Delta \mathbf{k}_{\mathrm{o}}} d \mathbf{k}_{\mathbf{o}} \int_{\Delta \Omega} d \mathbf{k} F\left(\mathbf{k}, \mathbf{k}_{\mathrm{o}}\right) S\left(\mathbf{k}, \mathbf{k}_{\mathrm{o}}\right)
$$

In most cases the angular variation of the structure-factor is much faster than that of the geometrical form factor and the form-factor may be taken to be approximately constant across the finite width of a diffraction beam. Electron source broadening then just smears out the intensity of the sharp diffraction beam that wouid be observed for a incident plane wave into a wider Gaussian shaped beam.

$$
\Gamma_{h, k}^{\prime}\left(\mathbf{k}_{\mathrm{o}}\right) \approx F\left(\mathbf{k}_{h, k}, \mathbf{k}_{\mathrm{o}}\right) \int_{\Delta \mathbf{k}_{\mathrm{o}}} d \mathbf{k}_{\mathrm{o}} \int_{\Delta \Omega} d \mathbf{k} S\left(\left(\mathbf{k}-\mathbf{k}_{\mathrm{o}}\right) \cdot \hat{\mathbf{n}}\right)
$$

Note that the remaining integral in Eq. 2.17 depends on energy only if the limits of integration change with energy. If different diffraction beams do not overlap then the detector acceptance angle $\Delta \Omega$ can be chosen to include the entire diffracted beam. The integral over $S$ will then be close to the $g_{h, k}$ of Eq. 2.3 and Eq. 2.17 reduces to Eq. 2.15. The measured intensity will be smaller since phonon scattering and incoherent scattering from surface defects takes some intensity out of each diffraction beam and some background intensity from diffuse defect scattering will be included in $\Delta \Omega$. 
The integrated intensity in a diffaction beam is then the quantity to be compared with the results of theoretical scattering calculations. Some LEED detectors include only part of the beam in $\Delta \Omega$. This will distort the intensity measurements since the beam width changes with energy and scattering angles, so the collected fraction of the integrated beam intensity will not be constant.

There are some surface systems where the beam intensities can change significantly over an angle as small as $1 / 2{ }^{\circ}$. This is on the order of the width of the beam from a typical LEED electron gun, so some caution should be used to keep the LEED gun focused. Even if there are small variations in intensity across the width of an incident LEED beam these will average out to first order.

The most common method of collecting JEEED intensity data is to measure the intensity of an individual beam while varying the energy (voltage) of the incident beam. This produces an intensity-voltage (I-V) curve. On occasion the intensity of diffraction beams has been measured as a function of the incidence angle, either the polar angle (I- $\theta$ curves) or the azimuthal angle (I- $\phi$ curves). I-V data collection has some significant advantages. First, theoretical LEED calculations are much more efficient when $\mathbf{k}_{\mathbf{o}}$ is along a high symmetry direction. Calculations at normal incidence can be more than an order of magnitude faster, depending on the symmetry of the surface and a factor of four to eight faster when $k_{o}$ is in a mirror-plane. Complete I-V curves can be collected at hignsymmetry directions, while I- $\theta$ curves can have at most mirror symmetry and I- $\phi$ curves have no symmetry advantage. Secondly, angular alignment is difficult. Errors in alignment of $\leq 12^{\circ}$ can have a significant effect on the intensity. At high 
symmetry incidence angles comparison of the intensities of symmetrically degenerate beams provides an internal check on LEED data. It is difficult to change mechanically the incidence angle while maintaining accurate alignment during the collection of I- $\theta$ or I- $\phi$ curves, while it is fairly simple to scan the incident electron beam voltage for a fixed scattering geometry.

\subsubsection{Diffuse Low Energy Electron Diffraction}

The experimental situation is different for LEED structural studies of systems without long-range order. Conventional LEED experiments are done on systems with long range order but this is not a fundamental restriction. The energydependent variation in diffracted intensity at a given point in the Brillouin zone is primarily determined by the local scattering geomeiry at the surface. Long range order gives rise to diffraction beams which reflect this intensity dependence. If angle resolved intensity data are collected from a system with definite local geometry but without long range order, the local geometry can still be determined by LEED calculations. ${ }^{20,1}$ This "diffuse" LEED experiment will be difficult to interpret unless there is one predominant local scattering geometry. Unlike the conventional LEED experiment, the diffraction process does not "filter-out" the contributions of defects and impurities from the contribution of the equilibrium structure in the diffuse LEED experiment. If the scattering from different kinds of sites is superimposed in the experimental data it will be difficult to construct a reasonable model of the surface. In this sense diffuse LEED is similar to finestructure and photoemission techniques. 
There are a number of "lattice-gas" chemisorption systems, where atoms or molecules are adsorbed in well-defined sites on the surface, as determined by vibrational spectroscopy, but where there is no long range order. The adsorption sites and bond lengths for such systems can be determined by diffuse LEED calculations, as has been done for oxygen on the tungsten (100) surface. ${ }^{21}$

In a diffuse LEED experiment the geometrical form-factor is a continuous function of $\mathbf{k}$, since the structure-factor does not pick out particular diffracted beams. (Substrate diffraction beams may still be present, but they will contain information on the periodic substrate structure, with almost no contribution from the disordered part of the surface.) The structure-factor $S$ still contains information on the substrate order plus information on the distribution of defects. The form-factor $F$ contains the effects of multiple scattering within the substrate, as well as structural information on the defect and on scattering between the defect and the substrate. It is still desirable, however, to separate the effects of the form-factor from the structure-factor. This is done by taking a logarithmic derivative of the scattered intensity at constant momentum transfer. ${ }^{20}$ First take the derivative of Eq. 2.1

$$
\frac{d I\left(\mathbf{k}, \mathbf{k}_{o}\right)}{d \mathbf{k}}=\left.\frac{\partial F\left(\mathbf{k}, \mathbf{k}_{\mathbf{o}}\right)}{\partial \mathbf{k}}\right|_{\mathbf{k}_{\mathrm{o}}} S\left(\mathbf{k}, \mathbf{k}_{\mathrm{o}}\right)+\left.F\left(\mathbf{k}, \mathbf{k}_{\mathrm{o}}\right) \frac{\partial S(\Delta \mathbf{s})}{\partial \mathbf{k}}\right|_{\mathbf{k}_{\mathrm{o}}}
$$

and now form the logarithmic derivative at constant $\Delta s$

$$
\frac{\mathbf{l}}{I} \frac{\partial I}{\partial \mathbf{k}_{\perp}}=\frac{1}{F} \frac{\partial F}{\partial \mathbf{k}_{\perp}}
$$

In experimental terms, this means that the diffracted intensity is measured as a 
function of angle $\Omega$ for a given incidence angle $\Omega_{o}$ at two adjaccnt energies $E$ and $E+\Delta E$ and then

$$
\left.\frac{I(E+\Delta E)-I(E)}{I(E)}\right|_{\Delta s, \Omega}
$$

is compared to the results of theoretical calculations.

\subsubsection{Instrument response and LEED intensity}

In genera! LEED intensity measurements do not require a particularly high angular resolution - the resolution of commercial LEED instruments is usually adequate since the geometrical form-factor may be considered constant over the angular area of the LEED beam. However, there are advantages to having an instrument with good angular resolution. For structures with large unit cells diffraction beams may be separated by only a few percent of the substrate unit cell size. ${ }^{3}$ In this case the instrument response is important, for the I-V curves cannot be accurately measured once the LEED spots begin to overlap.

Background correction is another factor that benefits from improved instrument response. In LEED measurements the diffraction pattern from the ordered part of the surface is superimposed with incoherent scattering from disordered defects and from electron-phonon scattering. Normally the diffraction contribution dominates the background scattering, since the diffracted intensity is $\sim n^{2}$, where $n$ is the number of atoms in an ordered domain and the defect contribution is linearly proportional to the defect density. However, near I-V curve minima or at high temperatures the background intensity may be larger than the diffraction 
contribution. This problem is worse for poorly ordered surfaces, such as for some molecular chemisorption systems, where the background scattering due to imperfect order is relatively large. ${ }^{3}$ An instrument with a better instrument response will produce sharper beams, so the integration area can be decreased and the relative background contribution reduced.

Although the background contributions distort I-V curves somewhat, this usually does not prevent successful structure determination. Peaks in I-V curves are typically $\sim 10 \mathrm{eV}$ wide and background contributions vary much more slowly with energy. The fitting of theoretical to experimental data (see chapter 4) depends primarily on peak position and peak shape and is much less sensitive to the absolute intensity, or to variations in relative intensity from one end of an I-V curve to the other. A slowly varying background contribution superimposed on an I-V curve is not likely to cause much change in the LEED structure determination results, although the sensitivity may be decreased as the relative intensity modulation in the I-V curve is reduced. In some experimental arrangements local background corrections to I-V curves are possible. This can significantly increase the resolution of I-V curve structure. Background correction is discussed further in chapter 8 on LEED data reduction.

For diffuse LEED studies of disordered systems there is no experimental method of distinguishing the "background" from the "signal". Electron-phonon scattering can be minimized if measurements are carried out at the lowest possible temperature, however the contributions from unwanted defects and from the disordered feature under study cannot be separated experimentally. Surface 
preparation is therefore much more important for diffuse LEED experiments. In LEED experiments on ordered surfaces the structure-factor effectively "filters out" contributions from disordered parts of the surface and useful I-V curves can be collected even in the presence of a significant amount of disorder. In a diffuse LEED experiment the surface preparation must insure that the scattering contribution of the feature under investigation is dominant. The use of the logarithmic derivative in data analysis will remove a significant part of the phonon background. The angular variation of diffuse LEED intensities is small compared to the angular resolution of a typical LEED instrument, so instrument response is not a limitation for diffuse intensity measurements.

\subsubsection{LEED intensity measurements}

About $0.1 \%$ to $1 \%$ of electrons in the LEED energy range are elastically back-scattered. Below the plasmon excitation threshold ( 10 to $20 \mathrm{eV}$ for metals) the elastic reflectivity increases significantly and can exceed $10 \%$. For an ordered surface the total diffracted intensity is divided among the diffracted beams, so the average intensity of a given beam tends to decrease with increasing energy. The intensity of a beam from a surface with a large unit cell will also have a lower average intensity. For example, when the metastable Si(111)-(1x1) surface reconstructs to the stable $(7 \times 7)$ surface symmetry, the total scattering strength of the surface will not be changed significantly, but this intensity will now be divided among 40 individual beams. 
Cohen and $\mathrm{Webb}^{22}$ have made very careful I-V curve measurements with a high-precision LEED instrument on the $\mathrm{Ag}(111)$ surface with adsorbed xenon overlayers. They have shown experimentally reproducible I-V curve structure over more than three orders of magnitude. The specular beam intensity varied between $3 \%$ and $0.001 \%$ of the incident beam intensity and the xenon overlayer beams were between $\sim 10^{-3}$ and $10^{-6}$ of the incident intensity.

A LEL. instrument that will be used to measure integrated intensities should be able to measure diffracted currents $\leq 10^{-6}$ of incident beam currents. To make accurate beam profile measurements for surface order studies the instrument should be able to measure $\sim 1 \%$ of the diffracted beam intensity; however, these measurements would not normally be made at intensity minima, so this requirement is not necessarily more stringent than for I-V curve measurements. Measurements of diffuse intensities are more difficult. This can be estimated for a typical surface: a reasonable diffraction beam width is $\sim 5 \%$ of the width of the Brillouin zone, or $\sim 0.25 \%$ of the area, so diffuse LEED intensities will be $\sim 0.1$ to $1 \%$ of normal LEED beam intensities, or $\sim 10^{-5}$ to $10^{-9}$ of the incident beam intensity.

A number of different methods have been used to measure LEED intensities. In many cases only relative measurements of diffracted intensities are made, instead of absolute measurements of LEED beam reflectivities. The main types of LEED intensity measurements are direct current measurements, either using a Faraday cup and conventional current measurements, or some kind of direct electron counting and photometric measurements, where the diffracted LEED beams 
are accelerated into a phosphor screen and the light intensity is mes "red. Most systems equipped with Faraday cups can make absolute intensity measurements, while photometric measurements are usually relative.

Intensity measurements may be characterized by speed, dynamic range, linearity and sensitivity. Here sensitivity is the minimum current that can be measured, which is closely related to noise in the system. Dynamic range is the ratio of the minimum detectable signal, or sensitivity, to the maximum signal that can be measured. Linearity is the ratio of the detector output to the true electron current over the dynamic range of the detector.

LEED measurements are made with single-channel detectors, where intensity is measured over a smill angle $\Delta \Omega$, and with large area detectors, where much of the diffraction pattern is observed at one time. Most studies of surface ordering are done with single-channel detectors. Structure studies can be done this way, but data collection is then quite slow, so large area detectors are often used.

\subsubsection{Current measurement techniques}

A Faraday cup was first used to measure LEED intensities ${ }^{23}$ and is still the most direct method for single-channel measurements. ${ }^{22}$ Sensitivity is the main limitation of a Faraday cup. Currents down to $10^{-i 2}$ A may be measured directly and the sensitivity may be extended to $10^{-14} \mathrm{~A}$ with careful construction and shielding of the Faraday cup and long measurement time-constants. The sensitivity is limited by amplifier Isise and leakage currents. The speed is also reasonably high except for very low currents - spot tracking is slower than the 
picoammeter time-constant for most applications.

The sensitivity of a Faraday cup can easily be increased by adding a channeltron electron multiplier, which has sufficient gain for pulse counting. Single electrons can be counted with such a detector with a quantum efficiency of $>$ $50 \%$. Detector noise is very low in such systems, $\leq 1 \mathrm{~Hz}$. Dynamic range is the main limitation with a pulse counting system since the maximum count rate is $~$ $10^{6}$. This is sufficient to measure I-V curves, but not enough to measure incident beam currents. For pulse-counting statistical noise becomes significant - the signal to noise ratio due to fluctuations in the detected electron current is $\sqrt{N}$ where $N$ is the total number of counts and this limits the spred of a pulse counting detector for weak signals. The basic sensitivity of ar electron counting Faraday cup is $\sim 10^{4}$ greater than with direct current measurement, so much lower incident beam currents are required, $\sim 10^{-10} \mathrm{~A}$ for pulse-counting compared to $\sim$ $10^{-6}$ A for current measurements. This means either the incident beam current density may be it Juced, reducing beam damage, or the incident beam diameter may be reduced by $\sim 10^{2}$ to probe the structure of smaller regions or crystallites.

\subsubsection{Photometric measurements}

Phosphor screens were introduced so that a visible image of the entire diffraction pattern could be observed from behind the crystal. This type of "display LEED" system is in wide use and it is especially useful for characterizing surface order:mij in conjunction with studies involving other techniques. Because of the wide use of commercial LEED systems, photometric techniques have been 
developed to measure LEED intensities. In this approach all of the detector electronics and beam tracking equipment is located outside the vacuum system, which is a significant advantage in terms of maintenance and reliability.

Phosphor screens of the type used for LEED have a sufficiently linear response to incident electrons for the current densities used in LEED and sufficient spatial resolution that they do not contribute significantly to the instrument response function. ${ }^{19}$ Absolute measuremeuts of LEED beam currents are difficult to make with photometric measurements. A calibration depends on the spectral match between the phosphor and the detector, the efficiency of the phosphor layer which depends on its structure and the sensitivity of the photometer. Usually relative measurements are made along an I-V curve or across the profile of a LEED beam.

The light emitted by a phosphor in typicai phosphor screen applications can be described by the empirical relationship

$$
B=\frac{\epsilon_{L} \epsilon_{w}}{\pi} J V
$$

where $\mathrm{J}$ is current density, $\mathrm{V}$ is accelerating voltage and $\mathrm{B}$ is screen brightness (energy/area-steradian). For a type P-11 phosphor used in many commercial display LEED systems, $\epsilon_{w}=0.10$ radiated watt $/$ watt excitation and $\epsilon_{L}=140$ lumens/watt. ${ }^{24}$ For a detector illuminated through a lens system the surface illuminance $I_{L}$ is

$$
I_{L}=\frac{\pi B T}{4 f^{2}(m+1)^{2}+m^{2}}
$$


where $m$ is the image magnification, $f$ is the lens $\mathrm{f} / \mathrm{stop}$ and $T$ is the optical transmission of the system. ${ }^{24}$

The brightness of the phcsphor screen is changed by the retarding-field grids and the angle of view. At normal incidence the transmission of a single grid is typically $\sim 80 \%$. For a square-mesh grid of finite thickness the transmission at glancing incidence will be $t(\theta)=V_{t_{o}}\left(\cos \theta-1+V_{i_{0}}\right)$. Also, the brightness of a diffuse light source viewed at an angle is reduced by a factor of $\cos (\theta)$, sc: the effective spot brightness will be reduced by $t^{4}(\theta) \cos \theta$. This fartor can be included in the factor $T$ in Eq. 2.22. A set of four $80 \%$ grids will reduce the effective spot brightness by $59 \%$ at normal incidence, $64 \%$ at $30^{\circ}$ and $98 \%$ at $60^{\circ}$. Electrons always strike the grids at normal incidence, so the electron transmission term is constant.

\subsubsection{Spot photometry}

Three basic techniques have been used to measure spot brightness: spot photometry, film photography and video photography. A spot photometer is basically a telescope coupled to a photomultiplier tube. Variations include a photomultiplier coupled to the back of a transparent phosphor screen with an optical fiber and mirror systems which image a particular LEED beam onto a photomultiplier tube. ${ }^{25,26}$ Mirror systems are better than telescopes for automated spot tracking since the lower mass allows more rapid motion. The photomultiplier system has a detector performance comparable to a Faraday cup. The sensitivity is somewhat less than that of a current measuring Faraday cup, while the speed, 
linearity and dynamic range are good. Photomultiplier tubes have a dark current that must be subtracted from the measured intensity. Electronic noise in the photomultiplier tube and amplifier circuit limits the sensitivity.

\subsubsection{Film photography}

Photographic methods are fast. The entire diffraction pattern may be recorded in $\leq 1 \mathrm{~s}$, as quickly as a photomultiplier or spot photometer can measure a single beam. In addition, there is no need for spot tracking during an experiment and a permanent record may be made of the experiment.

Film photography was first used for LEED intensity measurements. ${ }^{27,28}$ After the film was processed a "scanning optical densitometer" was used to measure the optical transmittance of the negative images as a function of position. More intense a LEED spots produce darker (lower transmittance) negative images. Film has limitations on dynamic range, sensitivity and linearity. The dynamic range of film is $\sim 10^{3}$ and its response to light is highly non-linear. Over the center of the dynamic range the response is logarithmic and it saturates at the upper end of the range. Experimentally, a calibration table was used to correct for film response. ${ }^{27}$ Film response depends on many environmental factors, including temperature, humidity, film age, processing conditions, etc. In practice $\mathrm{I}-\mathrm{V}$ curves recorded on film have an effective dynamic range $\leq 10^{2}$ and relative peak heights may be distorted due to calibration errors. However, as discussed in section 2.5.1, such distortions do not prevent successful structure determination. 


\subsubsection{Video photography}

Video photography shares the advantages of fasi and permanent recording of entire diffraction patterns and overcomes some of the drawbacks of film. A vidicon camera pickup tube has a linear response to light and its electrical output can be analyzed immediately without further processing. Video data acquisition is also fast, with 30 full-resolution images recorded per second under U.S. video standards and the spatial resolution of typical video components is quite good. The entire phosphor screen of a display LEED system may be imaged without degrading the angular resolution of the instrument. ${ }^{19}$ Dynamic range and sensitivity are the main limitations of a video camera detection system. The main sources of noise are in noise in the photoconductive laysr inside the vidicon transducer tube and in the video camera amplifier. Noise is a problem since video requires a high bandwidth, above $20 \mathrm{MHz}$ for full spatial resolution. A highsensilivity video camera designed for scientific applications may have a rms output noise level of $\sim 5 \mathrm{mV}^{29}$ and the total light-to-dark range in a standard (RS170) video signal is $650 \mathrm{mV}$, so the dynamic range is limited to $\sim 100.24$ This is smaller than the range required to record LEED I-V dats. However, the same diffraction patterns may be recorded at different video sensitivity (i.e. lens opening) and the data combined to give a larger effective dynamic range. This is more practical than with film photography because of the linear response of a video camera and the ease of data reduction.

The limit on sensitivity is a more basic problen. A high-sensitivity vidicon tube can produce an output current of $\sim 100 \mathrm{nA}$ with a surface illumination of $\sim$ 
1 lumen $/ \mathrm{m}^{2}$. This output current corresponds approximately to the maximum linear output for a high-sensitivity video camera. It is possible to estimate the incident current in a diffraction beam required to produce this illumination level. Using Eqs. 2.21 and 2.22 above along with typical parameters for a display LEED system, a diffraction beam current density of $\sim 2 \cdot 10^{-10} \mathrm{~A} / \mathrm{mm}^{2}$ is required to produce a vidicon tube faceplate illumination of $\sim 1$ Jumen $/ \mathrm{m}^{2}$ for a camera with an $f / 0.85$ lens imaging the entire LEED pattern. The minimum de : table current density for a video camera without image amplification is then $\sim 10^{-12} \mathrm{~A} / \mathrm{mm}^{2}$. In practice a video camera works well for good quality LEED patterns, and it is zble to resolve I-V curves. However, for weak LEED patterns where there are many spots and the overall intensity is low, I-V curve minima get lost in the system noise. This limitation has been overcome by using channel-plates inside the vacuum system as a LEED amplifier, ${ }^{30}$ cr by using a channel-plate based inageintensifier lens on the video camera. ${ }^{31}$ This solves the sensitivity problem, at the cost of increased noise and decreased spatial resolution. Video LEED detection is discussed further in chapter 5.

LEED currents can also be measured with position-sensitive electron counting. This approach uses a channel-plate amplifier in a pulse-countinf, mode so that individual diffracted electrons can be detected as a function of position. As in single-channel electron counting, the intrinsic detector noise is very low and only statistical noise is significant. As with any digital counting technique, there is no non-linearity and the dynamic range and sensitivity is limited only by the counting time required to reach a given level of statistical accuracy. Position 
sensitive detection is discussed in chapter 6 .

\subsection{LEED and inelastic losses}

Low anergy electrons are surface-sensitive because inelastic scattering events limit the depth of penetration of electrons into the surface. In most LEED experiments inelastic losses are treated empirically by adding an attenuation factor to the LEED electron wave-function in the solid and only elastically seattered electrons are detected. Some surface-sensitive techniques depend on the inelastic losses, combined with LEED-like electron propagation before or after the energy loss, including fine-structure techniques like EXAFS (extended X-ray adsorption fine-structure).

LEED electrons can transfer energy to the electrons of the solid in several ways (phonon scatterir. is discussed in detail in the next section). Figure 2.2 shows a typical energy spectrum for electrons back-scattered from a surface. In metals conduction electrons may be scattered by LEED electrons. This process is weak just above the Fermi energy, since the number of final states where both electrons are above the Fermi energy is limited. At higher energies electrons may be scattered between bands or to unbound states. The bulk of these emitted "secondary" electrons have energies $\leq 50 \mathrm{eV}$. Electrons can also be excited from ion core levels, with the subsequent decay of these core-holes giving rise to Auger electrons and X-ray fluorescence. The dominant loss mechanism over the LEED energy range is the collective excitation of the valence electrons in the solid (plasmon modes). Below the plasmon excitation threshold, $\sim 20 \mathrm{eV}$, the main 
loss mechanism is electron-electron scattering and the elastic mean free path is $>$ $10 \AA$. Above the plasmon threshold the mean free path is reduced to $\sim 5$ to $7 \AA$ for most materials in the $\sim 20$ to $200 \mathrm{eV}$ range, rising slowly with energy to $\sim 15$ $\AA$ at $1000 \mathrm{eV}$. There have been some studies of low energy loss mechanisms ${ }^{32}$ but these processes have not bern investigated in detail. A part of the incident electron beam is also reflected at the potential step at the surface of the crystal. This effect is usually neglected in LEED studies, except for some specialized investigations of the nature of the surface electronic structure. ${ }^{33}$ Most of these processes involve the loss of several $\mathrm{eV}$ or more, so they can be easily separated from the elastically scattered electrons by most LEED detectors. LEED electron wavefunctions are represented in the crystal by $\psi=\psi_{\text {alstis }} e^{-r / \lambda}$ where $\lambda$ is the electron mean free path. This can be interpreted as a small imaginary component of the average potential $V_{o}$ in the solid. Since $\left(E-V_{o}\right)=\frac{h^{2} k^{2}}{2 m}$ then

$$
\left(E-V_{o r}\right)-i V_{o i}=\frac{h^{2}}{2 m}\left(k_{r}^{2}+2 i k_{r} k_{i}-k_{i}^{2}\right)
$$

therefore $k_{i}=\frac{m}{h^{2} k} V_{o i}$ and $\lambda=1 / k_{i}$. Because of this inelastic damping term peaks in LEED I-V curves will have a characteristic width $\geq 2 V_{a i}$. The observed LEED I-V peak widths of $\sim 10$ to $15 \mathrm{eV}$ are consistent with $\lambda \sim 5$ to $7 \AA$. Much sharper I-V features may be seen below the plasmon threshold. ${ }^{33}$ 


\subsection{LEED and Phonon scattering}

The discussion of LEED up to this point has assumed that electrons are scattered from a perfectly ordered crystal. In a real crystal atoms are displaced from their equilibrium positions by thermal vibrations and zero-point motion, which modifies the diffraction and interference effects. Phonon scattering has the largest effects for large momentum transfer, since interference effects are proportional to $\Delta \mathbf{k} \cdot \mathbf{r}$. In theoretical LEED calculations phonon scattering is usually treated as another loss mechanism that simply attenuates scattered waves by a DebyeWaller factor $e^{-M}$ where $M=\left\langle(\Delta \mathbf{k} \cdot \Delta \mathbf{r})^{2}\right\rangle$ and $\Delta \mathbf{r}$ is the displacement from equilibrium positions. Since $M$ increases with $\Delta \mathbf{k}$, thermal attenuation of LEED intensities is most apparent at higher energies. This is one reason why RHEED, with small scattering angles, can be done at much higher energies than LEED, which involves back-scattering. Experimentally, phonon scattering is significant since most LEED instruments do not have sufficient energy resolution to separate elastically scattered electrons from the "quasi-elastically" scattered electrons that have interacted with phonons. Phonon energies are typically $\leq 50 \mathrm{meV}$ and most LEED electron guns produce a beam with an energy spread of $\sim 0.2$ to $2 \mathrm{eV}$, although specialized instruments have been constructed to study electron-phonon interactions which have much higher energy resolution. ${ }^{34}$ Phonon scattering can have a significant effect on experimental LEED intensity measurements, in particular for diffuse scattering experiments, since phonon scattering redistributes intensity within the surface Brillouin zone. 


\subsubsection{Electron-phonon scattering distribution}

Most of the analysis of electron-phonon scattering has been restricted to the kinematic (single-scattering) case, by analogy with X-ray diffraction. Although the effects of electron multiple scattering are neglected, the basic features of the kinematic analysis are consistent with experimental observations of phonon scattering. This is plausible since forward scattering is dominant and phonon scattering has relatively little effect on forward scattering. If most multiple scattering paths include only one large-angle scattering event, then a kinematic description of multiple scattering should give reasonable results. This type of "Debye-Waller" treatment of scattering neglects the effects of correlated motions of neighboring atoms on multiple scattering.

One basic point about phonon scattering is that it only redistributes the quasi-elastically scattered intensity, but the total scattered intensity $\int I\left(\mathbf{k}_{\mathbf{o}}, \mathbf{k}\right) d \mathbf{k}$ is constant. This has been verified experimentally by $W e b b,{ }^{32}$ who measured the integrated scattered intensity as a function of crystal temperature for different values of $\mathbf{k}_{\mathrm{o}}$. This intensity was constant even when the diffraction beams were completely lost in the thermal scattering background.

The effects of thermal vibrations on kinematic electron scattering are described by

$$
I(\mathbf{k})=|f(\theta, E)|^{2} \sum_{i, j} e^{i k \cdot\left(r_{1}+u_{1}-r_{1}-u_{j}\right)}
$$

where the $\mathbf{u}_{i}$ 's are the displacements from equilibrium positions and $k \equiv \mathbf{k}_{f}$ $-\mathbf{k}_{\mathbf{i}}$. The atomic vibrations are slow compared to electron scattering, so the $\mathbf{u}$ 's 
can be considered constant during scattering. LEED measurements are an average of many electron scattering events, so the observed LEED intensity is described by a time average

$$
\begin{aligned}
& I(\mathbf{k})=|f(\theta, E)|^{2} \sum_{i, j} e^{i \mathbf{k} \cdot\left(\mathbf{r}_{1}-\mathbf{r}_{\jmath}\right)} e^{\left.-\mathbf{k}_{2}<\left[\mathbf{k} \cdot\left(\mathbf{u}_{1}-u_{j}\right)\right]^{2}\right\rangle} \\
& =|f(\theta, E)|^{2} e^{-\left\langle(k \cdot u)^{2}\right\rangle} \sum_{i, j} e^{i k \cdot\left(r_{1}-r, j\right.} e^{\left\langle k \cdot u_{l}\right\rangle\left\langle k \cdot u_{p}\right\rangle}
\end{aligned}
$$

The term $e^{-\left\langle(k \cdot u)^{2}\right\rangle} \equiv e^{-2 M}$ is the Debye-Waller factor. The mean square displacement $\left\langle(\mathbf{u})^{2}\right\rangle$ can be calculated if the phonon dispersion is known. If the Debye model is assumed, then

$$
\begin{gathered}
M=\frac{12 h^{2} k^{2}}{\pi^{2} m k_{B} \theta_{D}} \sin ^{2} \frac{\theta}{2}\left\{\begin{array}{l}
0.25+1.642 \frac{T^{2}}{\theta_{D}^{2}}+\cdots, T \ll \theta_{D} \\
\frac{T}{\theta_{D}}, \quad T \rightarrow \infty
\end{array}\right. \\
=76 K / V \frac{\left(V-V_{o}\right) T}{A \theta_{D}^{2}} \sin ^{2} \frac{\theta}{2}
\end{gathered}
$$

where $V$ is beam voltage, $V_{0}$ is the inner potential, $\theta_{D}$ is the Debye temperature, $\theta$ is the scattering angle and $A$ is the mass of the scattering atoms in amu. For surfaces this expression gives a reasonable fit to experiment, although the value of the parameter $\theta_{D}$ is larger than for the bulk crystal and the value tends to increase when measured at lower energies. This is usually explained by saying that the vibrational amplitude is larger near the surface of the crystal.

The term $e^{\left\langle k \cdot u_{1}\right\rangle\left\langle k \cdot u_{1}\right\rangle}$ in Eq. 2.25 may be rewritten as

$$
1+\left\langle\left(\mathbf{k} \cdot \mathbf{u}_{i}\right)\left(\mathbf{k} \cdot \mathbf{u}_{j}\right)\right\rangle+\left[e^{\left.<k \cdot u_{s}\right\rangle\left\langle k \cdot \mathbf{u}_{j}\right\rangle}-1-\left\langle\left(\mathbf{k} \cdot \mathbf{u}_{i}\right)\left(\mathbf{k} \cdot \mathbf{u}_{j}\right)>\right]\right.
$$


Now the first term can be identified as zero-phonon scattering, the second as onephonon scattering and the final term as multi-phonon scattering. Evaluation of these terms would again require knowledge of the phonon dispersion of the system under study.

Using Eq. 2.27 in Eq. 2.25 the scattering may be defined as

$$
I(\mathbf{k})=I_{0}\left(J^{(0)}(\mathbf{k}) e^{-2 M}+J^{(1)}(\mathbf{k}) 2 M e^{-2 M}+J^{(n)}(\mathbf{k})\left[1-(1+2 M) e^{-2 M}\right]\right)
$$

where $I_{0}$ is the integrated intensity of the diffraction beam for a rigid, perfect crystal and the $r$ 's are functions that describe the distribution of zero-, one- and multi-phonon scattered electrons across the Brillouin zone. The integral of $f^{(i)}$ over the Brillouin zone is one and the temperature dependence is contained in the terms involving $M$.

The relative intensity of these different terms is shown in figure 2.3. Under normal LEED conditions there is a significant amount of phonon scattering; for example $M=0.6$ for back-scattering from platinum at $150 \mathrm{eV}$, over $2 / 3$ of the intensity of the beam has been scattered out by phonons.

Because of the different temperature dependence the zero-, one- and multiphonon terms can be experimentally separated. The work of Dennis and $\mathrm{Webb}^{35}$ shows that multi-phonon scattering is isotropic, so $J^{(n)}(q)$ is constant for phonon momentum $\mathbf{q}$ in the Brillouin zone. This creates a uniform, temperaturedependent quasi-elastic background intensity in the LEED pattern.

Tha contribution of the background scattering to an integrated LEED intensity measurement can be estimated. Assuming that the intensity detector 
subtends a constant solid angle, the fraction of the Brillouin zone included in the detector is $\sim \frac{V}{10^{4} \text { volt }}$ for a detector half width of $\sim 1^{\circ}$. When this number is multiplied by the ratio of $J^{(n)}$ to $I_{o}$ it gives the background intensity as a function of $\mathrm{T}$ and $\mathrm{V}$. For example, on platinum at $100 \mathrm{eV}$ the background is $\sim 0.4 \%$; however, at $300 \mathrm{eV}$ the background contribution is $\sim 23 \%$ at room temperature.

The single phonon function $f^{(1)}$ has been observed to have the form $|q|^{-x}$ where $x$ is $\sim 1$ and depends on the material and on the beam being studied. ${ }^{32}$ This distribution flattens out near the zone boundary and also for small q.

\subsubsection{Large unit cells and phonon scattering}

As a first approximation the electron-phonon scattering can be assumed to be unchanged when an overlayer is adsorbed or when a surface reconstructs. If the surface symmetry is reduced during such a process there will be additional beams appearing in the substrate Brillouin zone. The uniform multiple-scattering background will now have a contribution from all diffraction beams. This uniform background will be approximately

$$
\pi k_{\circ}^{2} A\left[1-(1+2 M) e^{-2 M}\right] \sum_{\mathbf{k}^{\prime} \leq k_{o}} I_{k^{\prime}}\left(\mathbf{k}_{\mathrm{o}}\right)
$$

for a unit cell of area $A$, where the first term is the multi-phonon intensity and the second term is the sum of all back-scattered LEED beam intensities. Since this background includes contributions from all beams, the relative background scattering will be even larger for a weak beam. 
The more structured one-phonon scattering is more significant for a lerge unit cell. The separation in momentum space $\Delta \mathbf{s}$ between adjacent beams may $b \in$ substantially smaller than for a clean surface. Consider the case of benzene adsorbed on the $\mathrm{Pt}(111)$ surface, as discussed in chapter 11 . For the $(2 \mathrm{~V} / 3 \times 4)$ rect superlattice, when rotated domains are included, adjacent beams mry be separated by only $1 / 8$ of the clean $\operatorname{Pt}(111)\left|g_{01}\right|$; and for the $\left(2 V_{3} \times 5\right)$ rect superlattice the separation is only $1 /\left.20\right|_{g_{01}} \mid$. If a weak (superlattice) beam is near a strong (substrate) beam, the one-phonon tail of the strong beam can obscure the intensity of the weak beam. The fractional intensity of this tail is $\sim \frac{M q_{0}^{2}}{\pi q}$ where $q_{o}$ is the width (including instrumental resolution) of the zero-phonon peak and $q$ is a point in the Brillouin zone and the $q$ 's are given as a fractions of $\left|g_{01}\right|$. If $q_{o}$ $=0.03$ then the tail will contribute $\sim 1 \%$ for $q=1 / 8$ and $\sim 2.5 \%$ for $q=1 / 20$ at $100 \mathrm{~V}$ and room temperature. This contribution can be a significant problem for adjacent beams of differing intensities and shows the advantages of data collection at low temperatures.

\subsection{LEED instrumentation}

The basic display LEED optics, equipped with a retarding-field energy analyzer and a post-accelerated phosphor screen display, is found all around the world and the basic design has changed very little in the past 30 years. The only recent change is the addition of a transparent phosphor screen, so that the LEED pattern may be seen without interference from the crystal-holder or other 
bistruments ${ }^{36}$ and in fact Scheibner and Germer used this method twenty years before. ${ }^{37}$ The main new developments have been in improved electron guns, to increase the transfer width of a system so that surface order may be investigated and in improved detectors, so that LEED intensities may be measured with greater speed, precision, sensitivity or reduced electron beam-damage to the surface. There have also been some specialized instruments developed, for example those using polarized electrons.

\subsubsection{High angular resolution instruments}

The motivation for increasing the angular resolution of a LEED system is to have a more sensitive probe of surface order. Dennis and $\mathrm{Webb}^{35}$ developed a system using magnetic deflection to study beams one at a time. This system is able to work at normal incidence and is much less sensitive to residual magnetic fields than a display LEED system. Since the crystal position is fixed, this design also works well for cryogenic applications.22 Henzler has used a somewhat different approach, ${ }^{38}$ adding electrostatic deflection plates to a channeltron equippe 1 Faraday cup. This allows a diffracted beam to be scanned across a very small aperture, quickly producing a high resolution, two dimensional profile of the diffracted electron beam.

The electron gun is the main limiting factor in LEED angular resolution. A commercial display LEED gun has a spot size of 250 to $1000 \mu \mathrm{m}$, a beam current of $\sim 1 \mu \mathrm{A}$ and a bean divergence of .25 to $1^{\circ}$. Using a stable power supply and a beam limiting aperture its possible to get down to a $200 \mu \mathrm{m}$ beam width at 0.1 
$\mu \mathrm{A}$ with $0.2^{\circ}$ divergence. Its difficult to improve this without excessive reductions in beam current.

Field-emission sources have been used for improved LEED guns because of the much higher brightness of a field-emission source. Energy resolution is also improved to $\sim 0.2 \mathrm{eV}$. Field-emission sources typically work at $\geq 1 \mathrm{KeV}$ and the need to decelerate the electron beam can lead to problems in focusing because of lens aberrations. Lagally has resolved this problems by using three field-emission tips as anodes in a gun with a field-emission cathode: ${ }^{39}$ This design can produce a $1 \mathrm{nA}$ current at $150 \mathrm{eV}$ with a $5 \mu \mathrm{m}$ spot size. Williams ${ }^{40}$ has built a computerdesigned field-emission gun with a more conventional anode geometry. This gun is optimized for a low divergence angle and can produce a low-voltage, $1 \mathrm{nA}$ beam with $500 \mu \mathrm{A}$ spot-size and a beam divergence of $\leq 0.1^{\circ}$. When such low-current guns are used some kind of image intensification or electron multiplication is needed for the detector.

\subsubsection{Large-area LEED detectorg}

For structure determination and surface crystallography the intensities of a number of different LEED beams must be measured as functions of energy and angle. Surface structure determination is most reliable when theoretical scattering calculations can be fit to a large experiulental data base. The instruments discussed in the previous section are designed for point measurements of LEED intensities and provide the highest resolution so far obtained for LPED instruments. However, several thousand or more LEED intensity measurements at 
different diffraction angles are required for a typical structure determination experiment. This provides the motivation for developing large-area or positionsensitive LEED detectors. Two basic approaches have been used -- the phosphor-screen display LEED optics and photometric measurements and position-sensitive electron detectors built around channel plate electronmultipliers.

The techniques of photometric measurements have been discussed above. The most practical method of making large-area photometric measurements is with a video camera. In particular Müller et al. ${ }^{41,42}$ and Lagally et al. ${ }^{19}$ have developed such systems. These systems and some advances in data acquisition techniques are discussed in detail in chapters $\mathbf{5}$ and $\mathbf{8}$.

Position sensitive electron detectors are also being applied to LEED intensity measurements. These detectors provide the maximum sensitivity and dynamic range achievable for large area. LEED detectors. The main disadvantage is in the limited speed compared to camera based systems. This restriction comes from the limitation on total counting rates imposed by the channel plates and the need for adequate statistics for a decent signal/noise ratio.

These detectors are based on instruments developed for astronomical applications $^{43}$ and were first applied to LETD by Stair. ${ }^{44}$ Position-sensitive LEED systems are discussed in detail in chapter 6. 


\subsubsection{Special purpose LEED instruments}

Most LEED investigations of surface structure and surface ordering use the basic techniques described above. Several special purpose instruments have been developed to make particular kinds of measurements. Price, ${ }^{33}$ for example, has improved the energy resolution of a display LEED system operated at low beam voltages in order to study surface resonances and surface state structure in detail. Wendelken has also developer a LEED detector with high energy-resolution to study the details of the electron-phonon int craction. ${ }^{34}$

Spin-polarized LEED experiments can provide additional information on surface structure. These experiments have been performed using a traditional electron source and spin-sensitive detection with either a Mott detector ${ }^{45}$ or a double-scattering LEED detector. ${ }^{46}$ It is also possible to perform spin-sensitive experiments using a partially polarized electron source and a conventional LEED detectors. ${ }^{47}$ Experimentally it is probably simpler to produce spin-polarized electrons than to eperate a spin-sensitive detector. Positron beams have also been used for surface diffaction experiments. Since positrons have a somewhat different interaction with the solid surface than electron, in principle this technique can $T$ de useful information. Positron diffraction, or LEPD, has been observed from the $\mathrm{Cu}(111)$ surface. ${ }^{48}$ 


\section{References}

1. D. K. Saldin, J. B. Pendry, M. A. Van Hove, and G. A. Somorjai, Physical Review B, vol. 31, p. 1216, 1985.

2. P. W. Palmberg, R. De Wames, and L. A. Vredevoe, Physical Review Letters, vol. 21, p. 682, 1968.

3. D. F. Ogletree, M. A. Var Hove, and G. A. Somorjai, Surface Science, in press.

4. D. F. Ogletree, M. A. Van Hove, and G. A. Somorjai, Surface Science, vol. 173, p. 351, 1986.

5. G. Ertl and J. Küppers, Low Energy Electrons and Surface Chemistry, p. 53, Verlag Chemie, Weinheim, 1974.

6. L. J. Clarke, Surface Crystollagraphy; An Introduction to Low Energy Electron Diffraction, Wiley-Interscience, Chichester, 1985.

7. R. Pantel, M. Bujor, and J. Bardolle, Surface Science, vol. 83, p. 228, 1979.

8. W. S. Yang, F. Jona, and P. M. Marcus, Physical Review B, vol. 27, p. 1394, 1983.

9. M. Schick, Progress in Surface Science, vol. 11, p. 245, Pergammon Press, 1881.

10. R. L. Park, T. L. Einstein, A. R. Kortan, and L. D. Roelofs, in Ordering in Two Dimensions, ed. S. Sinha, p. 17, Elsevier, New York, 1980. 
11. L. D. Roelofs, A. R. Kortan, T. L. Einstein, and R. L. Park, Physical Review Letters, vol. 46, p. 1495, 1981.

12. G. A. Somorjai, Chemistry in Two Dimensions: Surfaces, Cornell University Press, Ithaca, New York, 1981.

13. G. Comsa, Surface Science, vol. 81, p. 57, 1979.

14. R. L. Park, J. E. Houston, and D. G. Schrainer, Review of Scientific Instruments, vol. 42 , p. 60, 1971.

15. G.-C. Wang and M. G. Lagally, Surface Science, vol. 81, p. 69, 1978.

16. T.-M. Lu and M. G. Lagally, Surface Science, vol. 98, p. 695, 1980.

17. M. Henzler, in Electron Spectroscopy for Surface Analysis, ed. H. Ibach, Springer, Berlin, 1977.

18. D. G. Welkie, M. G. Lagally, and R. L. Palmer, Journal of Vacuum Science and Technology, vol. 17, p. 453, 1980.

19. D. G. Welkie and M. G. Lagally, Applications of Surface Science, vol. 3, p. $272,1979$.

20. J. B. Pendry and D. K. Saldin, Surface Science, vol. 145, p. 33, 1984.

21. K. Heinz, D. K. Saldin, and J. B. Pendry, Physical Review Letters, vol. 55, p. $2312,1985$.

22. P. I. Cohen, J. Unguris, and M. B. Webb, Surface Science, vol. 58, p. 429, 1976. 
23. C. J. Davisson and L. H. Germer, Physical Review, vol. 30, p. 705, 1927.

24. "Light-sensing and Light-emitting tubes," in Reference Data for Radio Engineers, pp. 16:30-45.

25. L. de Bersuder, Review of Scientific Instruments, vol. 45, p. 1569, 1972.

26. E. Zanazzi, F. Jona, D. W. Jepsen, and P. M. Marcus, Journal of Physics C, vol. 10, p. $375,1977$.

27. P. C. Stair, T. J. Kaminska, L. L. Kesmodel, and G. A. Somorjai, Physical Review B, vol. 11, p. 623, 1975.

28. M. A. Van Hove, R. J. Koestner, J. C. Frost, and G. A. Somorjai, Surface Science, vol. 129, p. 482, 1983.

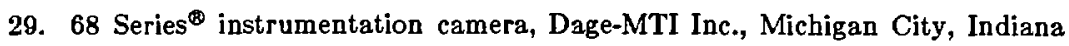
46360.

30. M. D. Chinn and S. C. Fain, Jr., Physical Review Letters, vol. 39, p. 146, 1977.

31. S. P. Weeks, J. E. Rowe, S. B. Christman, and E. E. Chaban, Review of Scientific Instruments, vol. 50, p. 1249, 1979.

32. M. B. Webb and M. G. Lagally, Solid State Physics, vol. 28, p. 301, 1973.

33. G. L. Price, Review of Scientific Instruments, vol. 51, p. 605, 1980.

34. J. F. Wendelken and F. M. Propst, Review of Scientific Instruments, vol. 47, p. $1069,1976$. 
35. R. L. Dennis and M. B. Webb, Journal of Vacuum Science and Technology, vol. 10, p. 192, 1973.

36. J. F. Wendelken, S. P. Withrow, and P. S. Herrell, Review of Scientific Instruments, vol. 51, p. 255, 1980.

37. E. J. Scheibner, L. H. Germer, and C. D. Hartman, Review of Scientific Instruments, vol. 31, p. 112, 1960.

38. K. D. Gronwald and M. Henzler, Surface Science, vol. 117, p. 180, 1982.

39. J. A. Martin and M. G. Lagally, Journal of Vacurm Science and Technology $A$, vol. 1, p. $1210,1983$.

40. E. D. Williams, R. Q. Hwang, and R. L. Park, unpublished, Department of Physies, University of Maryland, College Park., MD 20742.

41. P. Heilmann, E. Lang, K. Heinz, and K. Müller, Applied Physics, vol. 9, p. $247,1876$.

42. E. Lang, P. Heilmann, G. Hanke, K. Heinz, and K. Müller, Applied Physics, vol. 19, p. $287,1979$.

43. M. Lampton and F. Paresce, Review of Scientific Instruments, vol. 45, p. 1098, 1974.

44. P. C. Stair, Review of Scientific Instruments, vol. 51, p. 132, 1980.

45. J. Kirschner and R. Feder, Physical Review Letters, vol. 42, p. 1008, 1878.

46. R. Feder and J. Kirschner, Surface Science, vol. 103, p. 75, 1981. 
47. D. T. Pierce, R. J. Cellotta, G.-C. Wang, W. N. Unertl, A. Galejs, C. E. Kuyatt, and S. R. Meilczarek, Review of Scientific Instruments, vol. 51, p. $478,1980$.

48. L. J. Rosenberg, A. H. Weiss, and K. F. Canter, Physical Review Letters, vol. 44, p. 1139, 1980. 


\section{Figure Captions for Chapter 2}

2.1 a) Anti-phase domains on a one-dimensional surface.

b) LEED beam profilus before and after convolution with the instrumental response function, for substrate beams on the clean and adsorbate island covered surface and for even (in phase) and odd (out of phase) order superlattice beams.

2.2 Electron back-scattering spectrum at typical LEED energies.

2.3 Integrated intensity of zero, one and multi-phonon contributions to the quasi-elastic back-scattering as a function of the Debye-Waller factor $M$, given by Eq. 2.26. 


\section{Long Range Coherence Effects}



clean substrate beam

substrate beam with adsorbate

super lattice beam
Plane Wave



Instrument Response
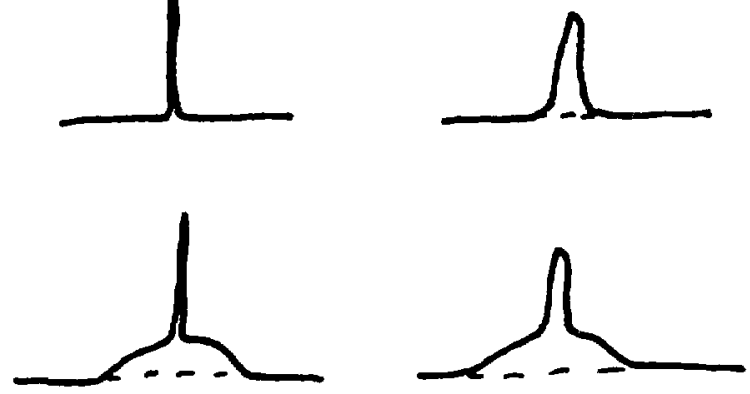


\section{Electron Energy Spectrum}

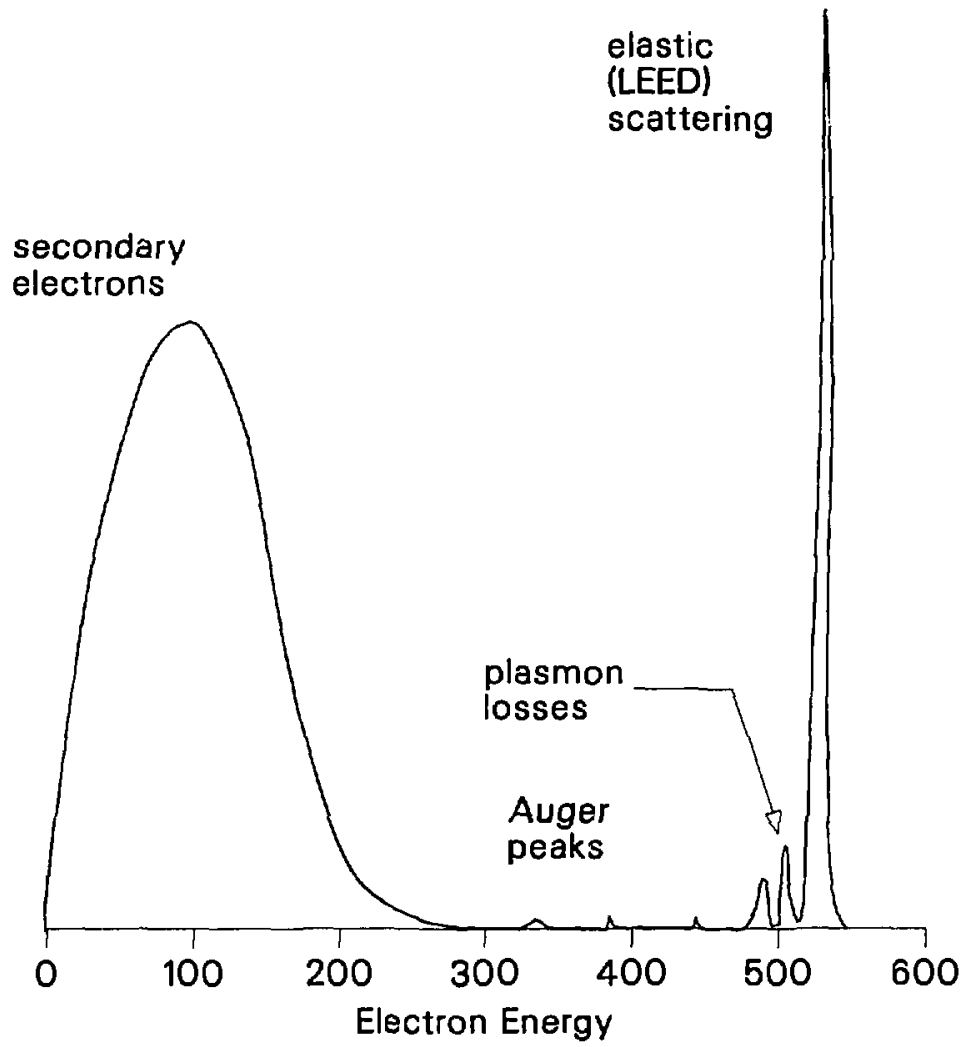

XBL $8612-4846$

FIgure 2.2 


\section{Phonon Scattering Attenuation}

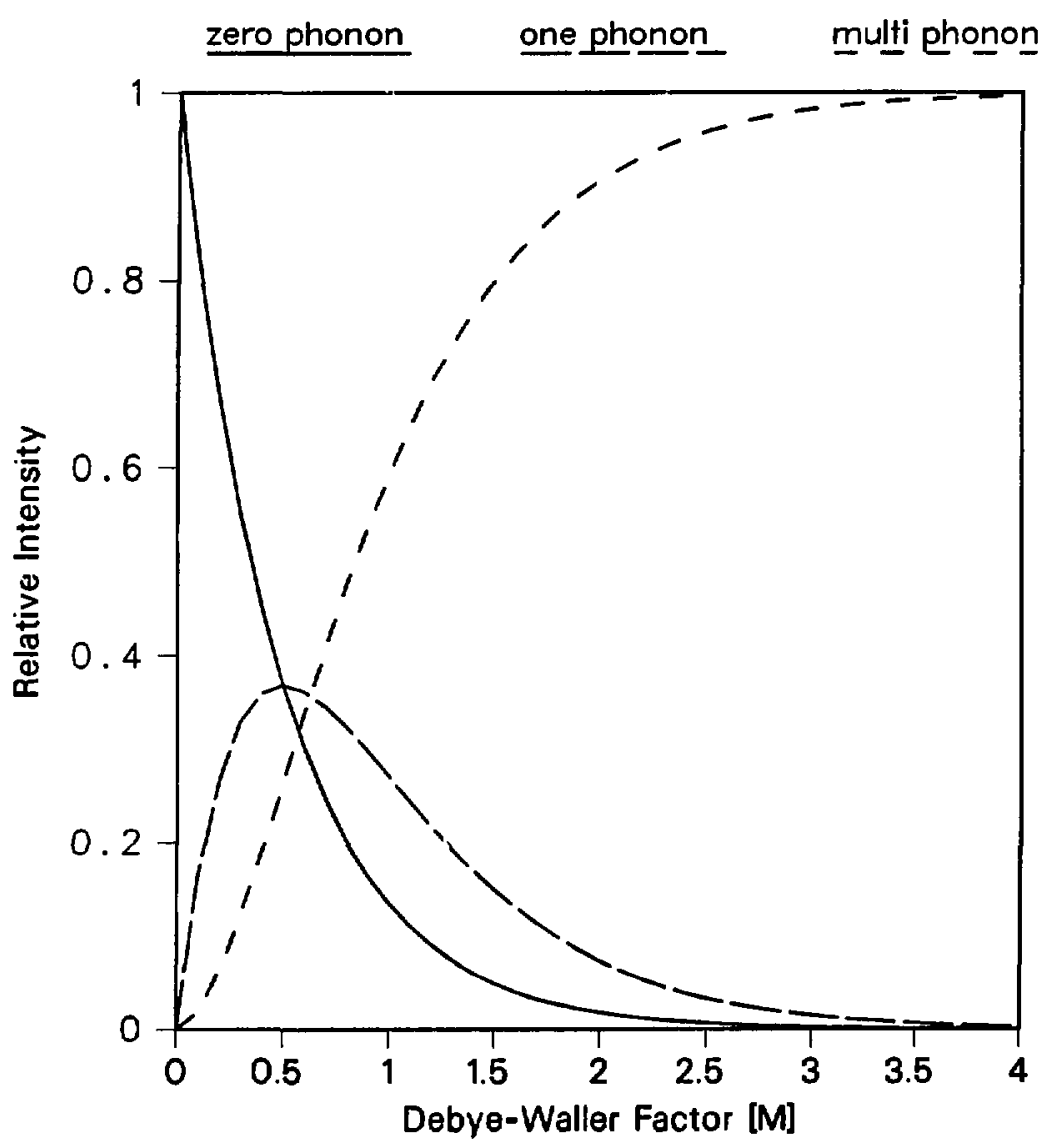

XBL 8611-4252

Fore 2.3 


\section{Chapter 3}

\section{LEED Scattering Theory}

\subsection{Introduction}

LEED multiple scattering calculations are nornally performed for idealized systems, with a plane wave of well-defined momentum $\mathbf{k}_{\mathbf{o}}$ incident on an infinite, perfect crystal lattice. Phonon scattering and other inelastic losses are included in the theory only through quasi-empirical damping terms. The effects of imperfect order and limited instrumental resolution do not enter into multiple-scattering calculations for structure determination. If required, these effects are considered when comparing the results of theoretical calculations to measured LEED intensities.

The next section will discuss the basic approximations commonly used in LEED calculations along with a conceptual outline of multiple scattering calculations. This will be followed with a more in-depth discussion of scattering theory and a justification of the approximations used. This chapter will conclude with a discussion of temperature effects and symmetry in LEED calculations. The next chapter will discuss structure determination using calculated LEED intensity data and the application of LEED scattering theory and structure determination methods to new classes of surface structures, including surfaces with large unit cells, molecular overlayers and incommensurate structures. 


\subsection{Concepts of LEED scattering}

The fundamental assumption made in LEED theory is the choice of the "muffin-tin" potential to describe the scattering of LEED electrons by solid surfaces. In this approximation each ion-core is replaced with a spherically symmetrical potential of finite radius and the potential is taken to be constant outside of the muffin-tin scattering spheres. The assumption of elastic scattering from a spherical jon-core potential vastly simplifies the description of the electron-ion interaction.

The scattering from a spherical potential can be characterized by phaseshifts when the electron wave-function is represented in terms of spherical waves. Since angular momentum is conserved in a spherical potential, partial waves of different angular momentum quantum numbers are not coupled during scattering. The total scattered flux is conserved since only elastic ion-core scattering is allowed, therefore only the overall phase of a partial wave may change. All of the effects of ion-core scattering are included in one energy-dependent phase-shift for each value of angular momentum. Furthermore, the number of angular momentum components that are required to describe a potential of finite radius is limited. The maximum angular momentum of a classical particle interacting with a potential of finite radius, the muffin-tin radius $r_{m}$, is $h \kappa r_{m}$, so partial waves with $l>\kappa r_{m}$ will probably not contribute much to the ion-core scattering. Calculated ion-core scattering phase-shifts are consistent with this estimate, and it is possible to show that phase-shifts must vanish for $l \gg l_{\max }{ }^{1}$ 
LEED beam intensities are usually calculated in terms of scattering by individual planes of atoms parallel to the surface. This approach takes advantage of the limited depth of electron penetration irto the surface, sirce only those planes within 20 to $30 \AA$ of the surface contribute to LEED scattering. Each plane in the solid has translational symmetry as high or higher than the surface, so the LEED wavefunction between scattering planes can be expressed as the sum of a finite number of plane-waves $\left\{\mathbf{k}_{\mathrm{g}}^{ \pm}\right\}$related to the incident beam $\mathbf{k}_{\mathrm{o}}$ by reciprocallattice vectors. The number of plane-waves required depends on the electron energy and the inter-plane spacing.

The scattering of an individval plane of atoms is represented by a diffraction matrix $M$ which couples an incident beam $\mathbf{k}_{\mathbf{B}}^{ \pm}$into other diffracted beams $\mathbf{k}_{\mathbf{g}}$. Various calculational schemes have been developed to combine the diffraction matrices of different layers, including multiple scattering effects, to obtain the overall reflection coefficients for a surface $\boldsymbol{R}_{\mathbf{g}}\left(\mathbf{k}_{\mathrm{o}}\right)$, the relative amplitude of a back-diffracted beam $\mathbf{k}_{\mathrm{g}}$ as a function of the incident beam momentum $\mathbf{k}_{\mathbf{o}}$. Structures are determined by varying the geometrical parameters of a structural model until the calculated $R_{\mathrm{g}}$ 's match the experimentally measured $I_{\mathrm{g}}\left(\mathbf{k}_{\mathrm{o}}\right)$ 's.

The diffraction matrix $M$ for a given atomic plane depends on the structure of the plane and on multiple-scattering within the plane. Intra-plane scattering is usually calculated in the spherical-wave representation. The wave incident on the $i^{\text {th }}$ atom in a plane is a combination of the wave incident on the plane and the amplitude at the $i^{\text {th }}$ atom of waves scattered from all other atoms in the plane. The second term can be represented by a intra-plane scattering matrix $X$ of the 
form $F \delta$, where $F$ is a sum over all other atoms in the plane, which depends on the geometry of the plane and the radial dependence of the spherical waves scattered from the other atoms. The factor $\delta$ describes the scattering properties of the ion-cores that make up the plane. The effects of multiple scattering within the plane can be summed and all orders of multiple scattering are included when $X$ is replaced by $(1-X)^{-1}$ times the amplitude of the wave incident on the plane.

A practical LEED calculation within this scheme consists of determining the intra-layer scattering matrices $X$ from lattice sums $F$ for each type of layer, inverting $X$ to account for multiple scattering, then expressing the result in a plane-wave diffraction matrix $M$. The scattering of the different layers is combined to yield the overall reflection coefficients $R_{8}$ for the surface. This basic calculational scheme $\mathrm{e}^{2,3}$ is quite efficient for many surfaces and it has been used for almost all LEED structure determinations to date.

There are alternate schemes for LEED calculations. The calculation of diffuse LEED scattering from partly disordered systems involves a modification of this layer scheme. ${ }^{4}$ Cluster-type approaches to LEED scattering are also being developed, where multiple-scattering paths through a cluster are summed for individual atoms. ${ }^{5}$ This approach is much more complicated for simple structures, but the complexity of the calculation is proportional to the number of atoms $n$ in the unit cell. In the traditional layer scheme, assembling $X$ scales as $n^{2}$ and calculating $X^{-1}$ scales as $n^{3}$. Different schemes are also being explored for calculations at higher energies, ${ }^{6}$ since the time required for traditional layer calculations 
is approximately proportional to $E^{3}$. These higher-energy methods take advantage of the decreasing importance of back-scattering relative to forward-scattering with increasing energy, building up scattering calculations in terms of scattering paths or chains of atoms instead of layers parallel to the surface.

\subsection{Scattering theory}

In a region of constant potential any wave-function $\psi$ may be represented in terms of plane-waves

$$
\psi=\sum_{\mathbf{k}} c_{\mathbf{k}} e^{i \mathbf{k} \cdot \mathbf{r}}
$$

or in terms of spherical-waves centered on a particular origin

$$
\psi=\sum_{\kappa} \sum_{l} \sum_{m=-l}^{m_{l}-l} \alpha_{\kappa l m} j_{l}(\kappa r) Y_{l m}(\boldsymbol{r})
$$

where the $j$ 's are Bessel functions, the $Y$ 's are the spherical harmonics and $\kappa=|\mathbf{k}|$ ( $\kappa$ is a scaler, but it can be a complex number). The complex constants $c_{\mathbf{k}}$ and $\alpha_{\kappa l m}$ define the waves in terms of the basis functions $|\mathbf{k}\rangle$ and $|\kappa l m\rangle$ A wave-function represented by spherical waves centered around one origin may also be represented in terms of spherical-waves centered around a different origin. A wave-function $\psi$ in one representation is transformed into a "aw representation by projection onto the new basis set

$$
\psi=\sum_{i} c_{i} \phi_{i}=\sum_{j} c_{j}^{\prime} \phi_{j}^{\prime} \text { where } c_{j}^{\prime}=\sum_{i} c_{i} \int \phi_{j}^{\prime \prime} \phi_{i} d \mathbf{r}
$$

LEED scattering calculations require repeated transformations between plane- 
wave and spherical-wave representations and between spherical-waves defined around different origins.

Elastic scattering of electrons of a given energy from a spherical potential of finite range (actually, such that $\lim _{r \rightarrow \infty} V(r)<r^{-1}$ ) can be represented as 7

$$
\psi=\sum_{l m} \alpha_{l m}\left[h_{l}^{(1)}(r \kappa) e^{2 i \delta_{l}}+h_{l}^{(2)}(r \kappa)\right] Y_{l m}(\mathbf{r})
$$

outside of the range of the potential, where the $h$ 's are Hankel functions of the first and second kinds. These are interpreted as incoming and outgoing waves since the flux carried by a wave-function $\psi$ is

$$
\begin{gathered}
-\frac{i \hbar}{2 m}\left[\psi^{*} \nabla \psi-\nabla \psi \psi^{*}\right] \\
\text { and } \lim _{r \rightarrow \infty} h_{l}^{( \pm)}(\kappa r)=\frac{1}{\kappa r} e^{ \pm i|\kappa r-(l+1) \pi|}
\end{gathered}
$$

where the upper and lower signs apply to $h^{(1)}$ and $h^{(2)}$, respectively. The flux carried toward the ion-core by the waves in Eq. 3.6, integrated over all angles, is just $\pm \hbar \kappa / m$. Elastic scattering requires that the amplitude $\alpha_{l m}$ of each angular momentum component remain unchanged during scattering, so the total effect of ion-core scattering is contained in the energy-dependent phase-shifts $\delta_{l}$.

This simple description of scattering is the result of assuming spherical symmetry. If this assumption were relaxed, different angular momentum components would be coupled and instead of $l_{\max }$ phase shifts, a matrix of $\left(l_{\max }+1\right)^{4}$ complex scattering amplitudes would be required to describe the ion-core scattering, subject to the restriction of overall flux conservation. 
The phase-shifts $\delta_{l}$ are determined by solving the Schrödinger equation for $r \leq r_{m}$ of the ion-core potential $V(r)$ and matching the resulting wave-functions to solutions of the form of Eq. 3.4 at $r=r_{m}$. For a static potential $V(r)$ (a potential that is not affected by the scattered wave $\psi$ ) the phase-shift $\delta_{l}$ is given by 1

$$
\sin \delta_{l}=-\frac{2 m}{h^{2}} \int_{0}^{\infty} j_{l}(\kappa r) V(r) \phi_{l}(r) d r
$$

where $j_{l}$ is a Bessel function and $\phi_{l}$ is the radial part of the wave-function of the $l^{\text {th }}$ eigenvalue of $V(r)$.

The total scattered wave from a plane-wave $e^{i \mathbf{k} \cdot \mathbf{r}}$ incident on a spherical potential described by phase-shifts $\delta_{l}$ is $t(\theta) \frac{e^{i \kappa r}}{r}$, with

$$
t(\theta)=\frac{1}{\kappa} \sum_{l}(2 l+1) e^{i \delta_{l}} \sin \delta_{l} P_{l}(\cos \theta)
$$

where the $P$ 's are Legendre polynomials. Integrating $t(\theta)$ over all scattering angles gives the total scattering cross-section

$$
\sigma(\kappa)=\frac{4 \pi}{\kappa^{2}} \sum_{l}(2 l+1) \sin ^{2} \delta_{l}(\kappa)
$$

The above discussion has been in terms of an infinite plane-wave $e^{i k \cdot r}$ incident on a potential. For a real LEED experiment the incident beam has a finite width and energy spread, corresponding to a certain spread in momenicum $\Delta \mathbf{k}$ around $\mathbf{k}_{\mathbf{o}}$, as discussed in section 2.4 , so it must be represented by a wave packet. As long as the wave-packet dimensions $|\Delta \mathbf{k}|^{-1}$ are large compared to 
the electron wavelength $\lambda$ and the radius of the potential $r_{m}$ and $\frac{\partial \delta}{\partial \kappa}|\Delta \mathbf{k}|<<\delta(\kappa)$, the finite extent of the incident wave-packet does not change the results of the scattering calculation. This is the normal case for LEED.

\subsection{The ion-core potential}

LEED calculations are normally carried out at energies well above the Fermi energy. This changes the LEED scattering problem signifi antly from the case of band-structure calculations. At the Fermi energy the ion cores are efficiently screened by the conduction electrons. This is the basis for the success of pseudopotential methods. When conduction-electron screening is included the ion-core potential may be replaced with a weak pseudo-potential without changing the conduction-electron band-structure. For the nearly free electron metals like aluminum ion-core scattering is almost negligible. ${ }^{8}$ At LEED energies the situation is quite different. Now the interaction of the electron with the solid is dominated by ion-core scattering; the only source of back-scattering and the effect of the conduction band dispersion may be neglected. In principle the ion-core potential should have the symmetry of the bulk crystal lattice. Since the coreelectron wave-functions are difficult to polarize the assumption of spherical symmetry gives reasonable results. This also implies that the same ion-core potential can be used to describe both surface and bulk atoms and atoms in different chemical environments (alloys, sub-surface adsorpticn sites, etc). 


\subsubsection{Ion-core charge distribution}

The ion-core charge-distribution must be modified from that of the free atom to satisfy the requirements of spherical symmetry and charge-conservation. Spherical symmetry requires that the ion-core have no net orbital angular momentum $j$. If the ion-core does not have a filled outer shell, the electrons are redistributed among the valence orbitals so that each state $m$ for a given orbital angular momentum $l$ has the same average occupation. Sirce the inverstitial potential in the muffin-tin model is constant, there can be no charge outside the muffin tin radius. Various methods have been used to achieve charge-neutrality, including distributing the interstitial charge uniformly through the muffin-tin sphere, distributing the charge in the form of the conduction-electron wavefunction inside the sphere, or adding a discontinuous step in the potential at the muffin-tin radius. ${ }^{2,9}$ Phase-shift calculations with different distributions of excess charge give very similar results. ${ }^{2}$ With these modifications to the ion-core charge distribution the ion-core wave-functions can be calculated.

\subsubsection{Ion-core wave-functions}

Ion-core scattering is still a complex problem even with the assumption of spherical symmetry. In principle this problem requires the solution of a multielectron wave-funetion $\Psi\left(\mathbf{r}_{0}, \mathbf{r}_{1}, \cdots, \mathbf{r}_{n}\right)$ for the incident electron and $n$ core electrons, with the Hamiltonian ${ }^{2}$

$$
H=\sum_{i=0}^{n}\left[-\frac{h^{2}}{2 m} \nabla_{i}^{2}-\frac{e Z}{\left|\mathbf{r}_{i}\right|}+v_{8}\left(\mathbf{r}_{i}\right)\right]+\sum_{i=0}^{n} \sum_{j=i+1}^{n} \frac{1}{\left|\mathbf{r}_{i}-\mathbf{r}_{j}\right|}
$$


where $v_{s}$ is the conduction electron screening potential and $Z$ is the nuclear charge.

This is an extremely difficult problem and some approximation scheme is required for a solution. One major advantage of the muffin-tin approximation is that the ion-core scattering enters only through the phase-shifts, so the most sophisticated available calculations of the ion-core wave-functions may be used as an input for the remainder of a LEED multiple-scattering calculation.

One common scheme is to express the multi-elecíron wave-function $\Psi$ as a properly anti-symmetrized produci of one-electron wave-functions $\psi(\mathbf{r})$. This leads to the Hartree-Fock poteritial, with the Schrödinger equation for the radial part of the incident electron wave-function

$$
\begin{aligned}
E \psi_{l}(r) & =-\frac{h^{2}}{2 m} \frac{1}{r^{2}} \frac{d}{d r}\left[r^{2} \frac{d}{d r} \psi_{l}(r)\right]+\frac{l(l+1)}{2 r^{2}} \psi_{l}(r) \\
& +V_{H}(r) \psi_{l}(r)+\int V_{e z}^{(l)}\left(r, r^{\prime}\right) \psi_{l}\left(r^{\prime}\right) r^{\prime 2} d r^{\prime}
\end{aligned}
$$

where $V_{H}$ is the potential due to the remaining electrons and the nucleus

$$
V_{H}(r)=v_{s}(r)-\frac{e Z}{r}+\sum_{j}^{n} \int \frac{\left|\phi_{j}\left(r^{\prime}\right)\right|^{2}}{\left|r-r^{\prime}\right|} d x^{\prime}
$$

and $V_{e z}$ is the exchange potential

$$
\begin{gathered}
V_{e x}^{(l)}=-\sum_{l^{\prime \prime}} \phi_{l^{\prime}}(r) \phi_{l^{\prime}}^{\prime \prime}\left(r^{\prime}\right) \frac{r_{<}^{l^{\prime \prime}}}{r_{>}^{\prime \prime}+1} C\left(l^{\prime}, l^{\prime \prime}, l\right) \quad \text { with constants } \\
C\left(l^{\prime}, l^{\prime \prime}, l\right)=\frac{2 l+1}{2} \int_{-1}^{+1} P_{l l^{\prime}}(x) P_{l \prime l}(x) P_{l}(x) d x
\end{gathered}
$$


The $\phi_{l}$ 's are the radial wave-functions of the $n$ ion-core electrons.

The Hartree-Fock equation is solved self-consistently to give the best oneelectron solution for Eq. 3.12. This equation can be solved numerically for $\psi$ and the $\phi$ 's and phase-shifts can be determined from the solution. This method has been applied to LEED calculations with reasonable success. ${ }^{2}$ The Hartree-Fock approximation does not include correlations between the incident and the ion-core electrons since it is a one-electron solution. In principle correlation effects can be included, for example with the configuration-interaction (CI) method used for quantum chemistry calculations. In practice one-electron calculations without corrections for electron correlation give reasonable results in LEED scattering calculations. This is plausible because of the limited polarizability of the n-core wave-functions cited above.

Simpler potentials such as the Slater and $X \alpha$ schemes, where the complex non-local exchange potential is replaced by a local-density approximation

$$
V_{e x}(r)=-3 \alpha\left(\frac{3 \rho(\mathbf{r})}{8 \pi}\right)^{\frac{1}{3}}
$$

where $\rho(r)$ is the local charge-density, proportional to $\Psi^{*}(r) \Psi(r)$. When $\alpha=1$ this is known as the Slater potential and when $\alpha$ is used as a variational parameter to minimize the total energy this is called the $\boldsymbol{X} \alpha$ method. Phase-shifts calculated under these different approximations lead to very similar calculated I-V curves. 9 
Unlike $x$-ray scattering, LEED scattering cross-sections show no simple $Z$ dependence. This is explained by arguing that electrons at LEED energies do not effectively see the full ion-core potential. A simple semi-classical argument says that for deep parts of the potential where $E<<|V|$ the electron moves across that part very rapidly and does not have time to feel the full effects. For $E \sim|V|$ the change in the electron "velocity" is much less and the full effect of the deep potential is felt. This is consistent with the observation that the scattering cross-section is approximately proportional to $Z$ for transmission electron microscopy, where the electron beam voltage is $>100 \mathrm{KeV} .{ }^{2}$

\subsubsection{Relativistic effects}

Relativistic effects can be important in electron-atom scattering for heavy atoms, where the ion-core potential can reach values approaching the electron rest-mass. Electron polarization from electron-ion scattering was predicted by Mott ${ }^{10}$ and polarization effects have been observed in LEED diffraction from heavy elements. ${ }^{11,12,13}$ Relativistic effects are included in the basic LEED scheme by using the Dirac equation in place of the Schrödinger equation to calculate spin-dependent phase shifts $\delta_{l}^{\ddagger}$ and spin-flip scattering probabilities, doubling the number of spin-quantized plane waves or spherical waves needed to represent the electron wave-function ontside the ion-cores. Doubling the dimensions of the $X$ and $M$ matrices in the I-V curve calculations will increase the overall calculation time by a factor of $\sim 8$. For most applications not involving a spin-polarized electron beam or spin-polarized detection it is sufficient to average the phase 
shifts, replacing $\delta_{l}$ with $\delta_{l}=1 / 2\left(\delta_{l}^{+}+\delta_{l}^{-}\right) .{ }^{14}$

\subsubsection{The platinum potential}

Phase-shifts calculated by Wang $^{15}$ for platinum are shown in figure 3.1. This plot shows $\delta_{l}(\kappa)$ for $l=0$ to 7 . These phase-shifts were determined from a relativistic Hartree-Fock calculation with confguration interaction (CI) corrections for electron correlation. The total LEED scattering cross-section is plotted as a function of energy at $\mathrm{T}=0 \mathrm{~K}$ and at room temperature in figure 3.2 , along with the "forward-scattering" cross section, the integrated cross-section for $\theta \geq$ $60^{\circ}$, calculated using Eqs. 3.8 and 3.9. The sharp spike in $\sigma(\kappa) \cdot$ round $15 \mathrm{eV}$ is associated with the rapid change in $\delta_{2}$ and the maximum around $60 \mathrm{eV}$ with the combined effects of $\delta_{l}$ for $l=0,1,2$ and 3 . Above $\sim 20 \mathrm{eV}$ back-scattering is significantly weaker than forward scattering.

The platinum scattering amplitude $|t(\theta)|$ is plotted in figure 3.3 for several energies. The relative scattered intensity at large angles is greater than that suggested by figure 3.3 as the scattering probability is multinlied by $\frac{d \Omega}{d \theta}=2 \pi \sin \theta$.

\subsection{The optical potential}

Electrons in the valence bands of a solid interact strongly with LEED electrons. Unlike the deep core-level electron wave-functions, these electrons are easily polarized by LEED electrons and inelastic interactions with these electrons are the main source of inelastic losses at LEED energies. It is not necessary to 
solve the multi-electron problem completely since the only information needed is the wave-function of the elastically scattered electrons and not the details of the inelastic excitations. This part of the problem requires the solution of a wavefunction ${ }^{2}$

$$
E \psi(\mathbf{r})=\left[-\frac{h^{2}}{2 m} \nabla^{2}+V_{c}(\mathbf{r})\right] \psi(\mathbf{r})+V_{o}(\mathbf{r}) \psi(\mathbf{r})
$$

where $\psi$ is the incident electron wave-function, $V_{c}$ is the potential of the ion-cores and $V_{o}$ is a non-local potential, which depends on the coordinates of the incident and valence-band electrons. Flux is not conserved in this expression, due to inelastic excitations of the valence electrons. This problem is still to hard to solve and one-electron approximations cannot be expected to work well because of the strong interactions.

Some calculations have been made for free-electron solids and these show that the variation of $V_{0}$ with energy is relatively slow above the plasmonexcitation threshold. ${ }^{2}$ The real part of the optical potential should be approximately the sum of the Fermi energy and the work function. valcuiated values for a free electron metal with the conduction-electron density of aluminum gave $V_{o}=$ - $13.6 \mathrm{eV}-i 5.5 \mathrm{eV}$. A calculation of the contribution of the aluminum core-levels to $V_{a i}$ gave an upper limit of $-0.015 \mathrm{eV} .^{2}$

The situation is more complicated for transition metals and semiconductors where there is a lot more structure to the distribution of charge density in the unit cell. Pendry ${ }^{2}$ reports estimates that the variation of the optical potential within the copper unit cell are less than $\sim 10 \%$. The variation of the optical 
potential should be even greater for adsorbates that are not closely bound to the substrate and for molecular adsorbates in particular.

Plasmon excitations are the dominant loss mechanisms for LEED electrons. Below the plasmon excitation threshold the optical potential will be more sensitive to the details of the band structure in the solid. Incident electrons with energies in the band-gaps of insulators or semiconductors could have a very long mean-free paths; however, LEED I-V measurements and calculations for structure determination are not usually done for this energy range.

In practice a uniform value of $V_{o}$ is used in making LEED calculations. Changing the real part $V_{o \tau}$ to first order just causes a rigid shift of the calculated $\mathrm{I}-\mathrm{V}$ curves at normal incidence where refraction does not change the incident angle, so it is easily optimized. Likewise $V_{o i}$, the damping term, is chosen to optimize the fit of peak widths. Usually $V_{o i}$ is kept constant or varied as $E^{1 / 3}$ above the plasmon excitation-threshold. LEED calculations using this approximation are successful and agree well with other structural techniques, so this approach seems adequate over the LEED energy range.

An inhomogeneous optical potential will have relatively greater effects for lower energy electrons. This is a significant problem for HREELS and XANES calculations where energies are lower than for LEED, ${ }^{16}$ especially for molecular adsorbates, where the inter-atomic potential is much less uniform than for a solid. 


\subsubsection{The surface potential barrier}

At the surface the optical potential $V_{0}$ decays to zero outside the surface. This surface potential step will reflect a part of both incoming and outgoing waves. In principle the surface potential step will diffract incident waves since it has some structure along the surlace. In practice this effect is not significant in the LEED energy range and neglecting the surface potential step (except for refraction) is a reasonable approximation. The exact form of this potential step is difficult to calculate, but it is relatively smooth since the effects on calculated I-V curves are not dramatic. I-V curves calculated assuming a sharp step give a much worse fit to experimental data than calculations which neglect the surface reflection and diffraction completely. The details of the surface potential are most important in calculating surface states and surface resonance effects just as beams emerge from surface. At LEED energies, when diffracted beams are not emerging at glancing angles from the surface, the details of the surface potential can usually be neglected.

\subsection{The plane-wave expansion}

The incident beam momentum $\mathbf{k}_{\mathbf{0}}$ can be divided into components parallel and perpendicular to the surface:

$$
k_{o \perp} \equiv\left(\mathbf{k}_{\mathbf{o}} \cdot \hat{\mathbf{n}}\right), \quad \mathbf{k}_{\mathrm{o}} \| \mathbf{k}_{\mathrm{o}}-k_{o \perp} \hat{\mathbf{n}}
$$

where $\hat{\mathbf{n}}$ is the surface normal. 
The translational symmetry of the surface defines a set of reciprocal lattice vectors $\{\mathbf{g}\}$. When a plane-wave scatters from the surface the momentum transfer parallel to the surface $\Delta \mathbf{s}$ must be equal to a reciprocal lattice vector $\mathbf{g}$ for a perfectly ordered surface. Between atomic layers the electron wave-function can be represented as a sum of plane-waves of momentum $\mathbf{k}_{\mathrm{g}}^{ \pm}$where $\mathbf{k}_{\mathbf{g} \|}=\mathbf{k}_{\mathbf{o} \|}+\mathbf{g}$ and

$$
k_{01}= \pm\left(\frac{2 m}{h^{2}}\left(E-V_{o}\right)-\left|\mathbf{k}_{\mathrm{g} \|\left.\right|^{2}}\right|^{2}\right)^{\frac{1}{2}}
$$

where a "+" wave is traveling into the solid and a "-" wave is traveling back toward the surface.

Only those beams $\mathbf{k}_{\mathbf{g}}{ }^{-}$with $\left|\mathbf{k}_{\mathbf{g}}\right||<| \mathbf{k}_{\mathrm{o}} \mid$ can be observed in the LEED experiment. The other beams are known as evanescent beams because they have an imaginary $\mathbf{k}_{1}$ and decay exponentially outside the surface. Inside the surface all beams have an imaginary component of $\mathbf{k}_{\perp}$ because of the imaginary part of the optical potential $V_{o i}$. The amplitude of a beam will decay between layers by $e^{-k_{1} d_{\text {lager }}}$ where $k_{i}$ is the imaginary part of the wave vector and $d_{\text {layer }}$ is the interlayer spacing. In a LEED calculation beams which are attenuated by a factor $t$ or more can be left out of the plane-wave expansion without causing significant errors in the calculated LEED intensities. A value of $t=0.002$ has been found to give good results and if $t$ is increased to 0.008 LEED peak positions will still be fairly accurate, although there may be errors in relative peak intensities. ${ }^{3}$ The density of beams in reciprocal space is $\frac{A}{4 \pi^{2}}$ where $A$ is the area of the surface 
unit cell, so the number of beams required in a plane-wave expansion of $\psi$ is approximately $\frac{A}{4 \pi^{2}}\left(\pi^{2} \mathbf{k}_{1 \max }^{2}\right)$. Using Eq. 3.16, the total number of beams in the expansion, including evanescent beams, is given by

$$
N \approx \frac{A}{4 \pi}\left|\frac{2 m}{h^{2}}\left(E-V_{o r}\right)+\left[\frac{\ln t}{d_{\min }}\right]^{2}\right|
$$

The plane-wave expansion becomes increasingly unwieldy with increasing unit-cell size and decreasing interlayer spacings. For example, at $200 \mathrm{eV}$ with $t=0.002$ the number of evanescent beams is $\sim 1 / 4$ of the number of real beams if $d_{\text {layer }}=$ $1.08 \AA$. This doubles the time needed to invert the layer scattering matrix, a step involved in layer stacking.

\subsection{Layer scattering}

The scattering properties of an atomic layer can be described by scattering matrices $M_{\mathbf{g g}^{\prime}}^{ \pm t}$ in the plane-wave representation. The subscripts are read from right to left, so $M_{\mathrm{Eg}^{\prime}}^{ \pm \pm}$gives the (complex) ratio of the amplitude of a scatterec wave of momentum $\mathbf{k}_{\mathbf{g}}^{ \pm}$to the amplitude of the incident wave of momentum $\mathbf{k}_{\mathbf{g}^{\prime}}$. Such a matrix can describe the scattering of a single atomic layer, or of several layers combined. If the scattering layer has inversion symmetry, as a co-planer layer always does, then $M^{++}=M^{--}$and $M^{+-}=M^{-+}$. The dimension of $M$ is given by Eq. 3.17. For the (1x1) lattice on platinum (111) this number ranges from $\sim 7$ at $50 \mathrm{eV}$ to $\sim 30$ at $200 \mathrm{eV}$. 


\subsection{Intra-plane scattering}

Scattering within an atomic plane is normally calculated in the sphericalwave representation. First consider a simple layer of a single kind of atom on a Bravais lattice. For an atom at the origin the incident spherical-wave amplitude is, neglecting intra-plane scattering,

$$
\alpha_{l m}^{(0)}=\sum_{\mathbf{g}} c_{\mathbf{g}}^{+} 4 \pi i(-1)^{m} Y_{l-m}\left(\mathbf{k}_{\mathbf{g}}^{+}\right)
$$

after transforming the representation of the incident plane-wave $\psi=\Sigma c_{\mathbf{g}}^{+} e^{i \mathbf{k}_{\mathbf{z}}^{+\cdot r}}$ using Eq. 3.3. The scattered wave, in terms of phase-shifts $\delta_{l}$ as in Eq. 3.4, is

$$
\alpha l_{m}^{(o)}=\sum_{\mathbf{g}} c_{\mathbf{g}}^{+} 4 \pi i(-1)^{m} Y_{l-m}\left(\mathbf{k}_{\mathrm{B}}^{+}\right) h_{l}^{(1)}(r \kappa) Y_{l m}(\mathbf{r}) e^{i \delta_{l}} \sin \delta_{l}
$$

This expression is summed over all atoms in the layer and transformed back into the plane wave representation. After transforming the Hankel functions ${ }^{2}$ and dividing by the incident plane-wave amplitudes $c_{\mathrm{g}}^{ \pm}$, this gives the layer scattering matrix calculated in the kinematic, or single-scattering model

$$
M_{\mathrm{gg}^{\dagger}}^{ \pm \dagger}=\frac{8 \pi^{2} i}{A\left|\mathbf{k}_{\mathrm{o}}\right| k_{\mathrm{g}^{\prime} \perp}^{+}} \sum_{L}\left[i^{l}(-1)^{m} Y_{l-m}\left(\mathbf{k}_{\mathrm{g}}{ }^{ \pm}\right)\right]\left[i^{l}(-1)^{m} Y_{l m}\left(\mathbf{k}_{\mathbf{g}^{\prime}}^{ \pm}\right)\right] e^{i \delta_{l}} \sin \delta_{l}
$$

This kinematic expression completely neglects intra-plane multiplescattering. This assumption is almost never valid in LEED. Prehaps the only case where the approximation works is for solid xenon, where the unit cell is extremely large and inelastic damping effectively prevents significant multiple-scattering. 


\subsubsection{Multiple-scattering corrections}

In addition to the spherical-wave amplitude $\alpha l_{m}^{0)}$ directly incident on the atom at the origin, there is an additional contribution $\alpha l_{m}^{(s)}$ scattered from all the other atoms in the plane. The total amplitude incident on the atom is

$$
\alpha_{l m}=\alpha_{l m}^{(o)}+\alpha_{l m}^{(s)}
$$

The wave incident on an atom at lattice-site $\mathbf{R}_{i}$ is just $\alpha_{l m} e^{i \mathrm{R}, \mathrm{g}}$ since $e^{i \mathbf{R}_{\mathrm{r}} \cdot \mathrm{B}}=1$ by symmetry. The scattered wave at the origin is then

$$
\sum_{\mathbf{R}_{1} \neq 0} \sum_{l, m} \alpha_{l m} e^{i \mathbf{k}_{o} \| \mathbf{R}_{1}} h_{l}^{(1)}\left(\kappa R_{i}\right) Y_{l m}{ }^{\prime}\left(\mathbf{R}_{i}\right) e^{i \delta_{l}} \sin \delta_{l}
$$

The prime on the spherical harmonic denotes that $Y_{l m}^{\prime}$ is not expanded around the origin. After projecting the $Y_{l m}$ 's at $\mathbf{R}_{i}$ onto the $Y_{l m}$ 's at $\mathbf{R}=0$, the result is $^{2}$

$$
\alpha\}_{m}^{(s)}=\sum_{L^{\prime \prime}} X_{L^{\prime \prime}} \alpha_{L^{\prime \prime}}=\sum_{L^{\prime} L^{\prime \prime}} C\left(L, L^{\prime}, L^{\prime \prime}\right) F_{L^{\prime}} e^{i \delta r} \sin \delta_{l^{\prime \prime}} \alpha_{L^{\prime \prime}}
$$

The C's are numerical Clebsch-Gordan coefficients, defined

$$
C \equiv A \pi(-1)^{\frac{1-l^{\prime}-l^{\prime \prime}}{2}+m^{\prime}+M^{\prime \prime}} Y_{l^{\prime}-m}\left(\frac{\pi}{2}, 0\right) \int Y_{L}(\Omega) Y_{L^{\prime}}(\Omega) Y_{L^{\prime \prime}}(\Omega) d \Omega
$$

and $F_{L}$ is a lattice sum

$$
F_{L}=\sum_{\mathbf{R}_{i} \neq 0} e^{i \mathbf{k}_{o} \| \cdot R_{1}} h_{l}^{(1)}\left(\kappa R_{i}\right) e^{i m \phi\left(\mathbf{R}_{i}\right)}
$$

where $\phi(\mathbf{R})$ is the azimuthal angle of $\mathbf{R}_{i}$ in the plane and $L$ represents the angular momentum quantum numbers $(l, m)$. The lattice sum $F_{L}$ correrges because $\kappa$ has an imaginary component $\kappa_{i}=\frac{m V_{o i}}{h \kappa r}$ to account for damping due to inelastic 
losses.

When the results of Eq. 3.23 are substituted into Eq. 3.21,

$$
\alpha_{l m}=\alpha l_{m}^{0)}+\sum_{l^{\prime} m^{\prime}} X_{l m, l^{\prime} m^{\prime}} \alpha_{l^{\prime} m^{\prime}}
$$

This equation can be solved by matrix inversion and so the effects of multiple scattering can be included by substituting $\alpha_{l m}$ for $\alpha_{m}^{(0)}$, with

$$
\alpha_{J m}=\sum_{l^{\prime} m^{\prime}}(1-X)_{l_{m}, I^{\prime} m^{\prime}}^{-1} \alpha(0)
$$

and the dynamical (multiple-scattering) result for the scattering matrix is, after substituting Eq. 3.27 in 3.20

$$
\begin{aligned}
M_{\mathbf{g} \mathbf{g}}^{ \pm \pm}= & \frac{8 \pi^{2} i}{A\left|\mathbf{k}_{\mathbf{o}}\right| k_{\mathbf{g}^{\prime} \perp}^{+}} \sum_{L, L^{\prime}}\left[i^{l}(-1)^{m} Y_{l-m}\left(\mathbf{k}_{\mathbf{g}}^{ \pm}\right)\right] \\
& \times(1-X)_{L L^{\prime}}^{-1}\left[i^{l^{i}}(-1)^{m^{\prime}} Y_{l^{\prime} m}\left(\mathbf{k}_{\mathbf{g}^{\prime}}^{ \pm}\right)\right] e^{i \delta_{l} \sin \delta_{l}}
\end{aligned}
$$

\subsubsection{Calculating $M_{\mathrm{Bg}}^{ \pm \neq}$}

The lattice sum $F$ of Eq. 3.26 is evaluated by direct summation and depends on the layer geometry, given by $\left\{\mathbf{R}_{i}\right\}$ and the incident beam $\mathbf{k}_{\mathrm{o}} \cdot F$ also depends on both the real and imaginary parts of the optjcal potential $V_{a}$. The time required to assemble $F$ scales as $\frac{L \lambda^{2}}{A}$ where $L$ is the number of angular momentum components $L=\left(l_{\max }+1\right)^{2}, A$ is the area of the unit cell and $\lambda$ is the electron mean-free path. This is often the most time consuming step in a LEED calculation. Calculating the inverse $(1-X)^{-1}$ once $X$ has been assembled scales as 
$L^{3}$. In principle the multiple-scattering correction can be approximated as a power series in orders of multiple scattering $(1-X)^{-1}=1+X+X^{2}+\cdots$; however, this series will often fail to converge for common LEED problems, such as scattering from metal surfaces. ${ }^{2}$ Once a wave is scattered into a layer, strong multiple-scattering within the plane prevents convansense of the perturbation series. In general attempts to treat LEED scattering as a perturbation have not succeeded. There is not much to gain in calculational efficiency in any case since it often takes longer to assemble $X$ than to calculate $(1-X)^{-1}$.

\subsubsection{Composite layers}

Intra-plane scattering for a Bravais lattice was discussed in section 3.8.2 above. This basic approach can easily be extended to layers where the unit cell contains several atoms of different types. Atoms in a composite layer need not be co-planar. For a unit cell containing $N$ atoms the intra-layer scattering problem can be solved by assembling a matrix of dimension NL and inverting it. The time to assemble the matrix scales as $N^{2} L \frac{\lambda^{2}}{A}$ and the time to invert the matrix scales as $(N L)^{3}$. In principle the entire LEED problem could be solved by considering the first ten or so atomic layers as one unit cell of a single thick composite layer, to be solved with one enormous matrix inversion. This would be an extremely inefficient calculational method, however and non-coplanar layers are treated by matrix inversion only when there are problems with plane-wave methods, due to small layer separations and the large number of terms in the expansion. 


\subsubsection{Combined space method}

The combined space method (CSM) has been developed to solve the problem of intra-layer scattering for composite layers of $i=1,2, \cdots n$ sublayers, each a simple Bravais lattice of a single kind of atom. Scattering will be described by a diagonal phase-shift matrix $t^{(i)}$ for each sublayer, with Green function propagators $G^{i j}$ which link spherical-wave expansions around atoms in different sublayers, scattering matrices $\pi^{(i)}$ which include all scattering paths within the $i^{\text {th }}$ sublayer and matrices $T^{(i)}$ which include all scattering paths within the composite layer that end on the atoms of the $i^{\text {th }}$ sublayer. The terms $\tau$ and $G^{i i}$ are similar to the $F_{L}$ and $X$ functions used in single layer scattering, while the $T$ and $G^{i j}$ terms are generalizations to the composite layer case. ${ }^{3}$

The composite layer scattering and propagation matrices $t$ and $G$ are defined below. The vector $\mathbf{r}_{i}$ gives the position of a reference atom in the $i^{\text {th }}$ sublayer and the set of vectors $\left\{\mathbf{R}^{(i)}\right\}$ define the positions of the other atoms relative to the reference atom at $\mathbf{r}_{i}$. A plane-wave propagator $K_{\mathrm{g}}^{i \pm}$ brings a plane-wave into the $i^{\text {th }}$ sublayer reference atom, to link the composite-layer sphe: ical-wave representation with the inter-layer plane-wave representation. In the rest of this section repeated spherical-wave indices $L$ will imply matrix multjplication.

$$
\begin{gathered}
t\left(j L^{\prime} \equiv \delta_{L L^{\prime}} \frac{1}{2 \kappa} e^{i \delta_{l}} \sin \delta_{l}\right. \\
R_{\mathrm{g}}^{i \pm} \equiv e^{ \pm i \mathrm{k}_{\mathrm{z}}^{ \pm} \cdot \mathbf{r}_{\mathbf{l}}}
\end{gathered}
$$




$$
\begin{gathered}
G_{L L^{\prime}}^{i j} \equiv-8 \pi i \kappa \sum_{\mathbf{R}^{(i)} \neq 0} i^{l_{1}} a\left(L, L^{\prime}, L_{1}\right) h_{1}^{(1)}\left(\kappa\left|\mathbf{R}^{(i)}+\mathbf{r}_{i}-\mathbf{r}_{j}\right|\right) \\
\times Y_{L_{1}}\left(\mathbf{R}^{(i)}+\mathbf{r}_{i}-\mathbf{r}_{j}\right) e^{-i \mathbf{k}_{0} \cdot \mathbf{R}^{(i)}}
\end{gathered}
$$

$$
\text { with } \quad a\left(L, L^{\prime}, L_{1}\right) \equiv \int Y_{L}^{*}(\Omega) Y_{L^{\prime}}(\Omega) Y_{L_{1}}^{*}(\Omega) d \Omega
$$

The intra-sublayer scattering matrix $\tau$ for a layer $i$ is equivalent to the simple layer problem solved in section 3.8 .2 , so by analogy to Eqs. 3.23 to 3.25 the $\tau^{(i)}$ matricies are

$$
\tau\left(i L^{\prime}=\left[1-t L_{L_{1}}^{(i)} G_{L_{1} L_{2}}^{i i}\right]^{-1} t_{L_{2} L^{\prime}}^{(i)}\right.
$$

The new part of the problem is to calculate the matrices $T^{(i)}$ which include multiple scattering between sublayers. For single inter-sublayer scattering the contribution to $T^{(i)}$ should have the form

$$
T^{(i)}(1)=\tau^{(i)}+\sum_{j \neq i} \gamma^{(i)} G^{i j} \tau^{(j)}
$$

where matrix multiplication is from righi to left. A perturbation expansion in orders of scattering is not adequate, but this series can be put in the form of a matrix multiplication power-series and summed by matrix inversion. ${ }^{3}$

$$
\left(\begin{array}{c}
T^{(1)} \\
T^{(2)} \\
\cdot \\
T^{(n)}
\end{array}\right)=\mathrm{R}^{-1}\left(\begin{array}{cccc}
I & -T^{(1)} G^{12} & \cdots & -T^{(1)} G^{1 n} \\
-T^{(2)} G^{21} & I & \cdots & -T^{(2)} G^{2 n} \\
\vdots & \vdots & \cdot & \vdots \\
-T^{(n)} G^{n 1} & -T^{(n)} G^{n 2} & \cdots & I
\end{array}\right)^{-1} \mathrm{R}\left(\begin{array}{c}
\tau^{(1)} \\
\tau^{(2)} \\
\vdots \\
T^{(n)}
\end{array}\right)
$$

where $R$ is a diagonal matrix of plane-wave propagators whose elements are $\delta_{(i)(j)} \delta_{L L^{\prime}} R_{\mathrm{g}}^{i \pm}$. After the $T$ 's are determined through matrix multiplication and inversion the composite layer scattering matrix can be calculated by substituting 
the spherical-wave multiple-scattering expression in place of $(1-X)^{-1}$ in Eq. 3.28. With a few small differences in the way phase-factors are accounted for and noting that $T$ contains a factor of $\kappa^{-1}$, one obtains

$$
\begin{aligned}
& M_{\mathbf{g g}^{\prime}}^{ \pm \pm}=-\frac{16 \pi^{2} i}{A k_{\mathbf{g}_{1}^{\prime}}^{+}} \sum_{L, L^{\prime}}\left[i^{i}(-1)^{m} Y_{l-m}\left(\mathbf{k}_{\mathbf{g}}{ }^{ \pm}\right)\right] \sum_{i=1}^{n}\left(e^{i\left(\mathbf{k}_{\mathbf{b}}^{ \pm}-\mathbf{k}_{\mathbf{8}}^{+}\right) \cdot \mathrm{r}^{\prime}} \tau T_{L L^{\prime}}^{(i)}\right) \\
& \times\left[i^{l^{\prime}(-1)^{m^{\prime}}} Y_{l^{\prime} m}\left(\mathbf{k}_{\mathbf{g}^{\prime}}{ }^{ \pm}\right)\right] e^{i \delta_{l}} \sin \delta_{l}
\end{aligned}
$$

An approximation to the large matrix inversion of the CSM is discussed below.

\subsection{Layer stacking}

Once the layer diffraction matrices have been calculated the effects of the different layers must be combined, including the effects of multiple scattering between layers. Several schemes have been used to solve this problem. The most complicated is the Bloch-wave scheme. The idea is to find the normal modes of the layer scattering matrix for the substrate layers. The effect of the layer scattering matrix is the same as multiplying the normal mode by a constant factor. This means solving the eigenvalue problem for the scattering matrix $M$. A second approach is the layer doubling method. Multiple scattering between layers results in a scattering power series, which can be solved by matrix inversion. Layers are stacked in this way until the scattering matrix is determined for a slab too thick for the electrons to penetrate. Matrix inversion is significantly faster than solving the eigenvalue problem. A third method involves a perturbation expansion in orders of back-scattering, with all orders of forward-scattering 
included. This requires only matrix multiplication and is by far the fastest method, however it can have convergence problems when interlayer scattering is too strong.

\subsubsection{Layer doubling}

To simplify the discussion define transmission and reflection matrices $t^{+}=M_{\mathbf{g g}^{\dagger}}^{++}$and $r^{+}=M_{\mathbf{g g}^{\prime}}^{+-}$. The subscripts 1 and 2 will denote the layers and the superscript indicates if the incident plane-waves came from above $(+)$ or below (-) the layer. In general $r_{1}^{+} \neq r_{1}^{-} \neq r_{2}^{+}$, however for identical, symmetrical layers only two matrices $r$ and $t$ are needed, instead of eight. The combined reflection and transmission matrices are $R^{ \pm}$and $T^{ \pm}$and $F^{ \pm}$is a plane-wave propagator between the layers, a diagonal matrix of the form $P^{ \pm}=\delta_{\mathrm{Bg}^{\prime}} e^{i \mathrm{k}_{\mathrm{E}}^{ \pm \cdot} \cdot \mathbf{r}}$.

The calculation of the combined reflection and transmission matrices by layer doubling is shown graphically in figure 3.4(a). Multiple scattering leads to a power series of the form $1+x+x^{2}+\cdots=(1-x)^{-1}$, so the layer doubling solution may be written by inspection from figure 3.4(a):

$$
\begin{gathered}
T^{+}=\iota_{1}^{+} P^{+}\left(1-r_{2}^{+} P^{-} r_{1}^{-} P^{+}\right)^{-1} \iota_{2}^{+} \\
R^{+}=r_{1}^{+}+t_{1}^{+} P^{+} r_{2}^{+} P^{-}\left(1-r_{1}^{-} P^{+} r_{2}^{+} P^{-}\right)^{-1} t_{1}^{-}
\end{gathered}
$$

where the matri: multiplication goes from right to left. The matrices $T^{-}$and $R^{-}$ are obtained from Eq. 3.36 by permuting subseripts and superscripts. 
This is called the layer doubling method and in the bulk $2,4,8, \ldots$ identical layers may be combined. The time to combine $M$ layers using $N$ plane-waves scales as $N^{3} \ln M$. Usually a slab of 8 or 16 layers is sufficient to describe the substrate scattering.

\subsubsection{Renormalized forward-scattering}

It was quickly realized that treating scattering as a perturbation will not work for LEED calculations. Back-scattering, however, is relatively weak, as shown in figure 3.2 and can be treated as a perturbation. This is the basis of the renormalized forward-scattering method (RFS). The amplitudes of the forward and backward traveling waves are iterated, with the amplitude of a forwardtraveling component after the $i^{\text {th }}$ layer consisting of the transmitted wave through the $i^{\text {th }}$ layer in the current iteration, plus the reflected wave from the $i+1^{\text {th }}$ layer in the previous iteration. This calculation is repeated for backwardtraveling waves and the process is iterated until the amplitudes are self-consistent. Layer scattering amplitudes are calculated up to $i_{\max }$, where the wave amplitude is negligible. Typically 12 to 15 layers and three or four passes are needed for convergence. ${ }^{3}$ The RFS method may fail to converge if layers are too close (less than $\sim 0.9 \AA$ ), damping is too weak (below the plasmon threshold), or scattering is too strong (such as resonant scattering between the surface potential step and and the crystal at low energies). The RFS calculation is very fast relative to the calculation of the layer scattering matrices $M_{\mathrm{gg}}^{ \pm \ddagger}$, since only matrix multiplication is involved. When RFS fails another method must be used, either the layer 
doubling method, or for very small layer spacings (less than $\sim 0.5 \AA$ ) the CSM method for composite layers.

The expressions for RFS scattering may be determined using the conventions shown in figure 3.4(b). The vectors $a_{i}^{+}(n)$ and $a_{i}^{-}(n)$ represent the amplitudes or the forward and backward traveling plane-waves $e^{i \mathbf{k}_{\mathrm{f}}^{ \pm} \cdot \mathbf{r}}$ between the $i^{\text {th }}$ ard $i+1^{\text {th }}$ layers after the $n^{\text {th }}$ pass. First $a_{i}^{+}(n)$ is calculated going into the suriace $i=1,2, \cdots, i_{\max } ;$ then $a_{i}^{-}(n)$ is calculated coming out, for $i=i_{\max }, i_{\max }-1, \ldots, \mathbf{i}, 0$. The vector $a_{0}^{-}$gives the amplitudes of the diffracted beams. The transmission and diffraction matrices for the $i^{\text {th }}$ layer are $t_{i}^{ \pm}$and $r_{i}^{ \pm}$ and the propagators $P_{i}^{ \pm}$are diagonal matrices as in Eq. 3.36. The RFS amplitudes are

$$
\begin{gathered}
a_{i}^{+}(n+1)=t_{i}^{+} P_{i-1}^{+} a_{i-1}^{+}(n+1)+r_{i}^{-} P_{i}^{-} a_{i}^{-}\left(n_{t}\right) \text { going in } \\
a_{i}^{-}(n+1)=t_{i+1}^{-} P_{i+1}^{-} a_{i+1}^{-}(n+1)+r_{i}^{+} P_{i}^{+} a_{i}^{+}(n) \text { going out }
\end{gathered}
$$

Matrix multiplication from right to left is implied and the initial conditions are $a_{i}^{ \pm}(0)=0$ with the boundary conditions that $a_{0}^{+}(n)=0$ for $g \neq 0, a_{0}^{+}(n)=1$ for $\mathbf{g}=0$ and $a_{i_{\max }}(n)=0$.

\subsubsection{Reverse-scattering perturbation}

In the reverse-scattering perturbation (RSP) scheme the concept of the RFS method is applied to composite layers instead of the CSM matrix inversion method. For this to work the sublayers must be ordered in terms of distance from the surface. Co-planar sub-layers must be treated by CSM matrix inversion. 
When the sublayers $i=1, \cdots, n$ are stacked in order, the $n^{\text {th }}$ approximation to $T_{L L}^{[i}$, the matrix representing all scattering paths terminating in the $i^{\text {th }}$ sublayer (see section 3.8.4 above), includes contributions from the sublayers $j<i$ in the $n^{\text {th }}$ iteration, propagated by $G_{L L^{\prime}}^{i j}$, plus back-scattered contributions from the sublayers $k>i$ in the $n-1^{\text {th }}$ iteration, propagated by $G_{L L^{\prime}}^{i k}$. The $T(i)$ 's are iterated in forward and backward passes until convergence. As in RFS, if layers are close, damping small, or scattering strong the RSP method will not converge and CSM matrix inversion is required. As with RFS, groups of strong-scattering layers may be combined with matrix inversion and the other layers with RSP, to generate the overall composite layer scattering matrix most efficiently.

\subsection{Diffuse LEED theory}

LEED beams are the result of long-range order on the surface. When parts of the surface are disordered - because of point defects like vacancies, or onedimensional defects such as steps, kinks and domain-wall boundaries, or adsorbed atoms or molecules without long-range order, there will be elastically scattered electron intensity outside of the LEED beams (in addition to phonon scattering). This is the same type of scattering measured with photoelectror techniques like XANES, ARPEFS and SEXAFS.

Diffuse LEED scattering has the same physical mechanism as normal LEED and it can be calculated by a generalization of the methods used for ordered LEED calculations. These calculations are more complex, since the diffraction structure factor $S(\Delta \mathbf{s})$ does not pick out certain angles and instead all angles are 
important, but calculational tools have been developed to handle these systems. ${ }^{17,4}$

\subsubsection{Diffuse LEED calculations}

Diffuse LEED scattering can be described as a three step process (see figure 3.5). This discussion will focus on the case of an adsorbed molecule, which may have internal structure, adsorbed on a well ordered substrate. Adsorbed molecules are assumed to be separated, so there is no significant adsorbateadsorbate scattering. This analysis could easily be applied to an adsorbed atom or to a point defect like a vacancy. The treatment of an extended feature like a step edge would require developing new calculational schemes.

In the first step the electron wave-function incident on the molecule is calculated. This includes the initial plane-wave $\mathbf{k}_{\mathrm{o}}$ from the electron gun and the plane-waves back-diffracted from the substrate $\left\{k_{\mathrm{g}}^{-}\right\}$. This part of the calculation is identical to a normal LEED calculation for a clean surface, except that backdiffracted evanescent waves may have non-negligible amplitude at the adsorbed molecule.

The second step describes multiple-scattering between the adsorbed molecule and substrate and includes all multiple-scattering paths that start and end within the adsorbed molecule. This step is equivalent to the XANES problem with the electron incident from the outside instead of being photoemitted from the adsorbed molecule and the methods developed for XANES calculations can be applied. ${ }^{18,19}$ Scattering paths can be calculated in the spherical wave 
representation as in the CSM method for thick layers, or they can be calculated with a modified RSP scheme using concentric shells of atoms instead of parallel layers. ${ }^{20}$ This step can also be approximately calculated using a variation of the beam-set neglect (BSN) method discussed in section 4.9 .

The final step in the diffuse LEED calculation involves the propagation of the scattered electrous from the adsorbed molecule to the detector, both directly and after scattering from the substrate. This is the inverse LEED problem. To calculate the diffuse intensity at a point $\mathbf{k}_{\boldsymbol{s}}$ in the first Brillouin zone, the final outgoing spherical wave from the adsorbed atom can be decomposed into a plane wave $\mathbf{k}_{s}^{-}$traveling directly to the detector and a set of plane waves $\left\{\mathbf{k}_{s}^{+}+\mathbf{g}\right\}$. The set of plane-waves going toward the surface are coupled and back-diffracted by the substrate diffraction matrix $M_{\mathrm{gg}^{\prime}}^{+-}\left(\mathbf{k}_{s}^{-}\right)$. This calculation gives the diffracted intensity at $\mathbf{k}_{s}^{-}$in the first Brillouin zone and also at the points $\mathbf{k}_{\mathrm{s}}^{-}+\mathbf{g}$.

The calculation of the diffuse LEED back-scattered intensity distribution involves solving the XANES problem and then calculating the substrate reflection matrix at enough different points in the Brillouin zone to map out the diffuse intensity variation. This involves more effort than a normal LEED calculation at a single energy, but there is much more information in a diffuse pattern so a single energy may be sufficient for structure determination. 


\subsection{Temperature effects}

LEED I-V curves are strongly affected by temperature. This can be seen from figure 3.2, which shows that the back-scattering cross section is much more strongly attenuated than forward-scattering. The Debye-Waller intensity attenuation factor for back-scattering is plotted in figure 3.6 for platinum. As temperature increases the overall intensity of LEED diffraction peaks drop off exponentially, with the strongest effects at large momentum transfer $\Delta \mathbf{k}$. The simple Debye-Waller attenuation treatment used in x-ray scatter̈ing and in the kinematic model is not sufficient for LEED. For example, individual LEED peaks do show a $I=I_{o} e^{-\alpha T}$ temperature dependence, but $\alpha$ is different for different peaks in the same I-V curve. The intensity ratio of adjacent peaks in the same I$\mathrm{V}$ curve can change significantly with temperature.

Thermal effects are caused by the displacements $\Delta \mathbf{R}_{i}$ of ion-cores from their equilibrium positions $\mathbf{R}_{\boldsymbol{i}}$ by lattice vibrations (phonons). Vibrations of ion-cores are about $10^{4}$ times slower than electron velocities, so ion-cores may be considered to be stationary during a single LEED scattering event. This is known as the Born approximation. In this approximation there is no energy transfer between electron and phonon modes. Since phonon energies $(<0.1 \mathrm{eV})$ are insignificant compared to LEED energies this is a good approximation. In a LEED experiment many electrons are collected, so terms like $\Delta \mathbf{k} \cdot \Delta \mathbf{R}$ are replaced by their thermal averages.

If a LEED multiple scattering expression, including displacements $\Delta R_{i}$, is expanded in plane-waves, ${ }^{2}$ ion-core scattering events are represented by a 
modified scattering amplitude $t(\Delta \mathbf{k}) e^{i \Delta \mathbf{k} \cdot \Delta \mathbf{R}}$ A typical multiple-scattering path will involve several terms of this kind. The thermal average $e^{\left.i<\Delta k \cdot \Delta \mathbf{R}_{1}\right\rangle_{T}}$ is evaluated in terms of the phonon spectrum of the solid. Using the Debye model for the phonon distribution gives $t_{T}(\Delta k)=e^{-M} t(\Delta \mathbf{k})$, with the Debye-Waller factor $e^{-M}$ which was discussed in section 2.7 .

If $\left\langle(\Delta \mathbf{R})^{2}\right\rangle_{T}$ is assumed to be isotropic, then $t_{T}(\theta)=t(\theta) e^{-\alpha \cos \frac{\theta}{2}}$ can be given the same functional form as the temperature independent $t$-matrix of Eq. 3.8, except with temperature-dependent phase-shifts $\delta_{l}(T)$. These phase-shifts are complex numbers, to account for the "attenuation" of the wave-function during ion-core scattering. As discussed in section 2.7, these "attenuated" electrons are just re-distributed as a slowly varying quasi-elastically scattered background between the LEED beams.

\subsubsection{Surface vibrations}

The amplitude of thermal vibrations may be greater for ion-cores near the surface than in the bulk material, particularly for vibrations normal to the surface. This can give a larger "effective" Debye temperature for LEED than for $\mathrm{x}$ ray diffraction. The mean square displacement $\left\langle\left(\Delta \mathbf{R}_{\perp}\right)^{2}\right\rangle$ at the surface has been estimated to be approximately twice the bulk value, with a smaller surface enhancement in $\left\langle\left(\Delta \mathbf{R}_{\|}\right)^{2}\right\rangle$. Different values of $\delta_{l}(T)$ can be used for the surface layer and for bulk layers in LEED calculations to approximate more closely the effects of phonon scattering. The surface Debye temperature $\theta_{D}$ can be treated as 
a parameter to be optimized during the LEED calculation. More often a value $\theta_{\text {Dsurface }}=\frac{1}{\sqrt{2}} \theta_{D b u l k}$ is used to approximate surface vibrations.

\subsubsection{Thermal scattering assumptions}

There are two major assumptions made in treating lattice vibrations with temperature-dependent phase-shifts: that lattice vibrations are isotropic and that ion-core motions are uncorrelated. Both of these assumptions involve substantial simplifications to the actual physical situation. Lattice vibrations certainly reflect the structure and symmetry of the crystal lattice - they are not isotropic. Introducing non-isotropic ion-core scattering would greatly complicate the mathematical description of the LEED process. The representation of ion-core scattering would change from a diagonal matrix with $l_{\max }$ different elements to a nondiagonal matrix with $\left(l_{\max }+1\right)^{4}$ elements and the appropriate form of this matrix would be difficult to calculate. No calculations for non-isotropic scattering have been performed that improved on the results of isotropic scattering calculations.

Neighboring ion-core motions are correlated in solids. The correlation in the motions of the $i^{\text {th }}$ and $j^{\text {th }}$ atoms can be defined

$$
C_{i j} \equiv \frac{<\Delta \mathbf{R}_{i} \cdot \Delta \mathbf{R}_{j}>_{T}}{\left[<\left(\Delta \mathbf{R}_{i}\right)^{2}>_{T}<\left(\Delta \mathbf{R}_{j}\right)^{2}>_{T}\right]^{1 / 2}}
$$

In the high temuerature limit of the Debye model for lattice vibrations, $C_{i j}$ is temperature independent, with the limiting values of 0.46 and 0.20 for atoms separated by one and two lattice constants, respectively. ${ }^{2}$ 
A multiple scattering path that involves near-neighbor atoms will be modified by correlated ion-core motions. An empirical estimate of an effective surface Debye temperature for uncorrelated motion will over-estimate the attenuation of scattering-paths involving near-neighbors and under-estimate the attenuation of widely separated atoms. In practice the assumption of uncorrelated motion gives reasanable results. Since scattering path-lengths are several times the lattice constants, prehaps 10 or $20 \AA$, only a small fraction of scattering-paths will involve highly correlated atoms, so this result is plausible.

One crude approximation to corrections for anisotropic motion is to calculate the layer scattering matrix $M$ using isotropic (or no) ion-core displacements and then to include an additional Debye-Waller type factor with $\left\langle\left(\Delta \mathbf{R}_{1}\right)^{2}\right\rangle$ $\neq<\left(\Delta \mathbf{R}_{\|}\right)^{2}>$ when combining layers. This is equivalent to a $100 \%$ correlation of ion-core motions within a layer and uncorrelated motion between layers. This type of anisotropy is easy to add in the plane-wave representation, but its not clear that it produces better results in I-V calculations.

\subsection{Symmetry considerations}

There are a number of aspects of symmetry which can reduce the complexity of LEED calculations. Some of these are general, depending only on the structure of the surface and others apply only for certain values of $\mathbf{k}_{\mathbf{o}}$. 


\subsubsection{Translational symmetry}

The translational symmetry of the surface determines the plane-wave representation $\left\{\mathbf{k}_{\mathrm{g}}^{ \pm}\right\}$of the LEED wave-function. In the case of surface reconstructions and chemisorption the surface layer often has lower translational symmetry than the terminated bulk structure. (Terminated bulk structure refers to a solid where the ion-cores are fixed at their equilibrium positions while the crystal is cut along a given crystal plane. A real surface may relax, reconstruct, or facet, depending on the surface temperature and chemical composition, to minimize the overall free-energy of the system. ${ }^{21}$ ) If the surface unit cell area $A$ is a multiple $n$ of the terminated bulk unit cell $A_{a}$, the only the lowest symmetry layers, usually the top-most layers, will couple all the plane-waves in $\left\{\mathbf{k}_{\mathrm{g}}^{ \pm}\right\}$. If the layer scattering matrix $M_{\mathbf{g g}^{\prime}}^{ \pm \neq}$has dimension $N$, the scattering matrix for the highsymmetry layers can be block-diagonalized into $n$ smaller blocks. The time required to assemble these matrices will be reduced by a factor $\frac{1}{n}$ and the time for matrix multiplication or inversion will be reduced by $\frac{1}{n^{2}}$. This is the basis for the beam-set neglect (BSN) approximation scheme discussed in the next chapter. The plane-wave representation $\left\{\mathbf{k}_{\mathrm{g}}^{ \pm}\right\}$is divided into $n$ independent beam-sets $\left\{k_{\mathbf{g}}^{ \pm}\right\}_{i}$ which are not coupled by scattering in the high-symmetry substrate.

Translational symmetry does not affect calculations in the angular momentum representation very much. Certain symmetries can be used to speed up the lattice sums in the spherical-wave propagators $X_{L L^{\prime}}$ and $G_{L L^{\prime}}^{i j}$, but there is no 
straight-forward reduction in calculational effort.

\subsubsection{Layer inversion symmetry}

If a layer or group of layers has a mirror plane parallel to the surface, then the scattering matrices must be symmetrical and $M^{+-}=M^{-+}=r, M^{++}=$ $M^{--}=t$. This is always true for a single plane. In the angular momentum representation spherical harmonics expanded around different origins in the same plane will couple only if $l+m$ and $l^{\prime}+m^{\prime}$ are both even or both odd. This means that $X_{L L^{\prime}}$ and $G_{L L^{\prime}}^{i i}$ can be block-diagonalized, with one block of $\left(l_{\max }+1\right)\left(l_{\max }+2\right) / 2$ terms and one block of $l_{\max }\left(l_{\max }+1\right) / 2$ terms. $G_{L L^{\prime}}^{i j}$ can also be block-diagonalized if sublayers $i$ and $j$ are co-planar.

\subsubsection{Wave-function symmetry}

Unlike translational symmetries, the point group symmetry elements (rotations, mirror planes perpendicular to the surface) depend on the incident beam mcmentum $\mathbf{k}_{\mathbf{o}}$. At normal incidence the LEED wave-function $\psi(\mathbf{r})$ has the full symmetry of the surface, so LEED beams related by symmetry operations have the same I-V curves. For off-normal incidence the wave-function can have at most mirror symmetry when $\mathbf{k}_{\mathrm{o}}$ lies in the mirror plane. 


\subsubsection{Plane-waves}

Linear combinations of LEED beams related by symmetry operations can be formed that transform as irreducible representations of the point group. ${ }^{22}$ Only the fully symmetrized combination: that transform as the representation $a$ are excited and other representations may be dropped from $M_{L L}^{t}$. In other words any group of beams related by symmetry operations needs only one entry in $M_{L L^{7}}^{ \pm}$. A 4-fold rotation axis, for example, reduces the dimensions of $M$ from $N$ to $\frac{N+3}{4}$ (the specular beam is unchanged by rotation at normal incidence). This reduction in the size of the plane-wave representation $\left\{\mathbf{k}_{\mathrm{g}}^{ \pm}\right\}$will be different for layers of lower symmetry. It may also be lower for combinations of layers, for example a pair of layers from the (111) surface of an fcc crystal has a 6-fold rotation axis, but three or more layers have only a 3-fold axis because of the layer stacking registry. To take full advantage of the point group symmecry the layer origin for the wave-function calculations must be on the rotation axis or in the mirror plane. This requires appropriate definitions of the plane-wave propagators between layers. ${ }^{3}$

\subsubsection{Spherical-waves}

The wave-function symmetry is preserved in the spherical-wave representation. At normal incidence an $n$-fold rotation axis implies that $X_{L L^{\prime}}$ and $G_{L L^{\prime}}^{i j}$ have factors of $Y_{l m}$ only for $|m|=0, n, 2 n, \cdots$, since only these $Y_{l m}$ 's transform properly under rotation. This greatly reduces the size of the 
propagation matricies and increases the speed of all calculations in the sphericalwave representation. Further, the lattice sums in $F_{L}$ and $G_{L L^{\prime}}^{i j}$ need only be calculated over a fraction $\frac{1}{n}$ of the plane. For mirror symmetries coordinate representations of the $Y_{l m}$ 's can be chosen so that the azimuth $\phi$ equals zero in the mirror plane. Then only symmetrized contributions of $\pm m$ appear in the spherical wave expansion and instead of $(2 l+1)$ terms for each $l$ value, only $(l+1)$ are needed. Likewise, the lattice sums are done over the half-plane only. 


\section{References}

1. A. Messiah, Quantum Mechanics, pp. 369-410, John Wiley and Sons, New York, 1958.

2. J. B. Pendry, Low Energy Electron Diffraction, Academic Press, London, 1974.

3. M. A. Van Hove and S. Y. Tong, Surface Crystallography by LEED, Springer, Berlin, 1979.

4. D. K. Saldin, J. B. Pendry, M. A. Van Hove, and G. A. Somorjai, Physical Review B, vol. 31, p. 1216, 1985.

5. F. Jona, J. A. Strozier, and P. M. Marcus, in Proceedings of ICSOS-1, The Slructure of Surfaces, ed. M. A. Van Hove and S. Y. Tong, vol. 2, p. 92, Springer Series in Surface Science, Springer Verlag, Berlin, 1985.

6. J. J. Barton and M. A. Van Hove, to be published.

7. L. J. Schiff, Quantum Mechanics, McGraw Hill, New York, 1968.

8. W. A. Harrison, Solid State Theory, Dover, New York, 1980.

9. L. J. Clarke, Surface Crystallography; An Introduction to Low Energy Electron Diffraction, Wiley-Interscience, Chichester, 1985.

10. N. F. Mott, Proceedings of the Royal Society A, vol. 135, p. 429, 1932.

11. J. Kisschner and R. Feder, Physical Review Letters, vol. 42, p. 1008, 1979.

12. R. Feder and J. Kirschner, Surface Science, vol. 103, p. 75, 1981. 
13. D. T. Pierce, R. J. Cellotta, G.-C. Wang, W. N. Unertl, A. Galejs, C. E. Kuyatt, and S. R. Meilczarek, Review of Scientific Instruments, vol. 51, p. $478,1980$.

14. R. Feder, in Surface Structures by LEED, ed. P. M. Marcus and F. Jona, Plenum Press, New York, 1981.

15. S.-W. Wang, unpublished.

16. M. A. Van Hove, S. W. Wang, D. F. Ogletree, and G. A. Somorjai, Advances in Quantum Chemistry, in preparation.

17. J. B. Pendry, in Proceedings of ICSOS-1, The Structure of Surfaces, ed. M. A. Van Hove and S. Y. Tong, vol. 2, p. 124, Springer Series in Surface Science, Springer Verlag, Berlin, 1985.

18. P. J. Durham, J. B. Pendry, and C. H. Hodges, Solid State Communications, vol. 38, p. 159, 1981.

19. G. Bunker and E. A. Stern, Physical Review Leiters, vcl. 52, p. 1990, 1584.

20. D. K. Saldin, D. D. Vvedensky, and J. B. Pendry, in Proceedings of ICSOS1, The Structure of Surfaces, ed. M. A. Van Hove and S. Y. Tong, vol. 2, p. 131, Springer Series in Surface Science, Springer Verlag, Berlin, 1985.

21. G. A. Somorjai, Chemistry in Two Dimensions: Surfaces, Cornell University Press, Ithaca, New York, 1981.

22. L. M. Falicov, Group Theory and Its Physical Applications, University of Chicago Press, Chicago and London, 1966. 


\section{Figure Captions for Chapter 3}

3.1 Platinum scatteing phase-shifts calculated by S-W. Wang with a relativistic Hartree-Fock configuration interaction potential, for $l=0$ to 7 . The horizontal dotted lines cr.respond to phase-shifts of $\pm \pi$. Voltage is measured relative to the muffin-tin zero.

3.2 Cross-sections calcuiated using the phase-shifts of figure 3.1. The backscattering cross-section includes all angles $\geq 60^{\circ}$, or $75 \%$ of the tot:i solid angle. Note the much weaker temperature dependence of the total scattering cross-section, which is dominated by forward scattering.

3.3 Teattering amplitude at four different electron energies calculated from the phase-shifts of figure 3.1. The partial cross-section is the square of the scattering amplitude.

3.4 Layers are stacked to give the surface reflection matrix, including the effects of multiple scattering between layers.

(a) A graphical representation of the inter-layer multiple-scattering power series that is summed by matrix inversion (Eq. 3.36) in the layer-doubling scheme.

(b) The conventions used in setting up the RFS expressions of Eq. 3.37.

3.5 The three-step description of diffuse LEED scattering.

(a) The beam from the electron gun and beams diffracted from the substrate arr incident on the adsorbed molecule.

(b) Electrons scatter within the molecule and between the molecule and the 
substrate.

(c) Electrons leave the adsorbed molecule and are detected both directly and after scattering from the surface.

3.6 The Debye-Waller factor $e^{-2 M}$ plotted as a function of beam voltage for a scattering angle of $180^{\circ}\left(M \sim V \sin \frac{\theta}{2}\right)$. This gives the attenuation of scattered intensities, using a value of $229 \mathrm{~K}$ for the bulk Debye temperature. The attenuation at $\mathrm{T}=0 \mathrm{~K}$ is due to zero-point motion and the attenuation does not go to zero at zero volts because the inner potential was set at $-18 \mathrm{~V}$. 
203

\section{Platinum Phase-shifts}

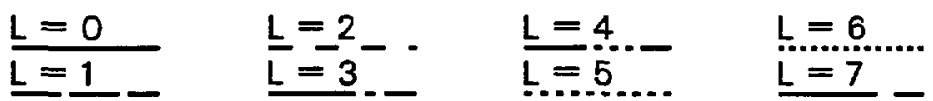

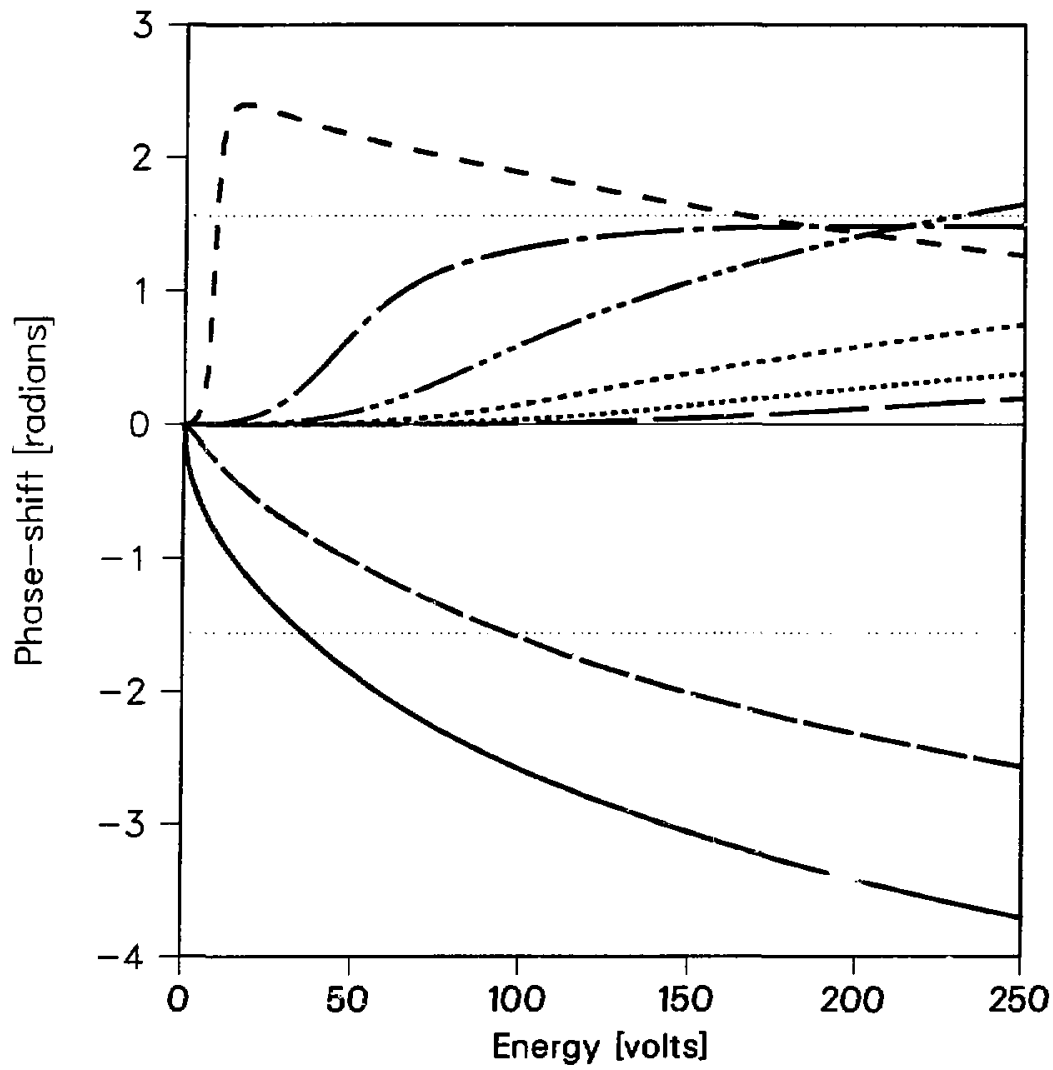

XBL 8611-4245

Figre 31 


\section{Platinum Scattering Cross-section}

Total at $0 \mathrm{~K}$

Total af $300 \mathrm{~K}$

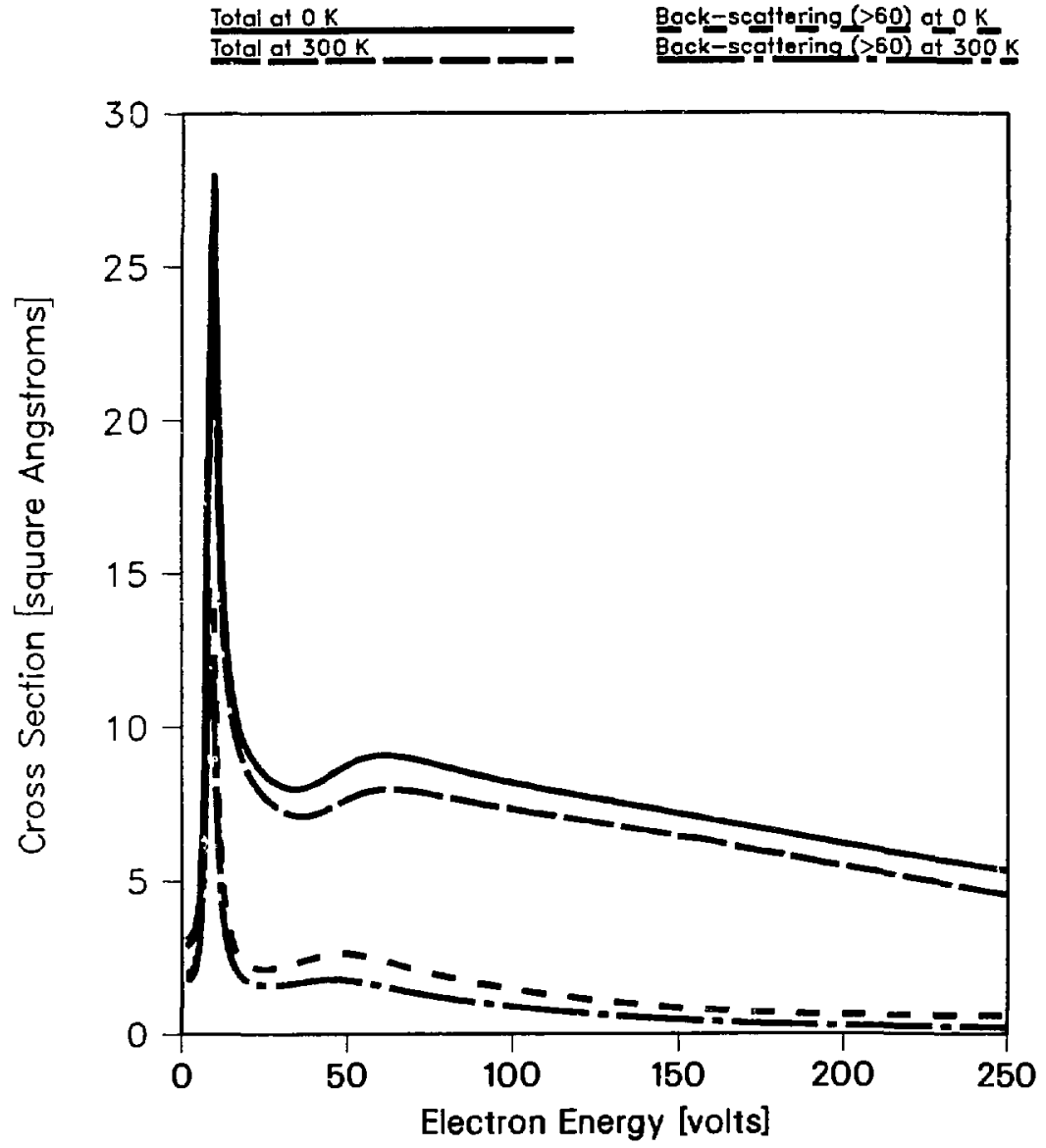

Back-scattering $(>60)$ at $0 \mathrm{~K}$ Back-segtering $(>60)$ at $300 \mathrm{~K}$

XBL $8611-4246$

Figne 32 
205

Platinum Scattering Amplitude



X8L 8611-4244

Figre 3.3 


\section{Layer Doubling Series Sum}

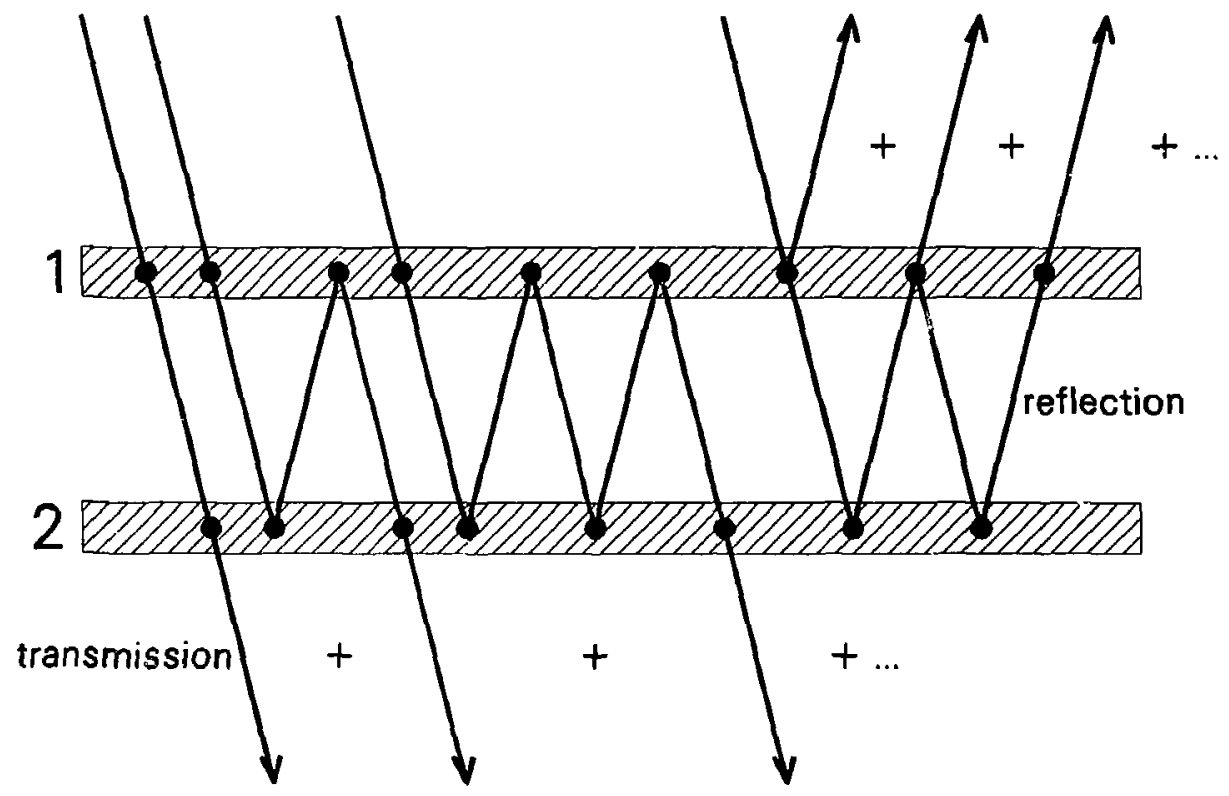




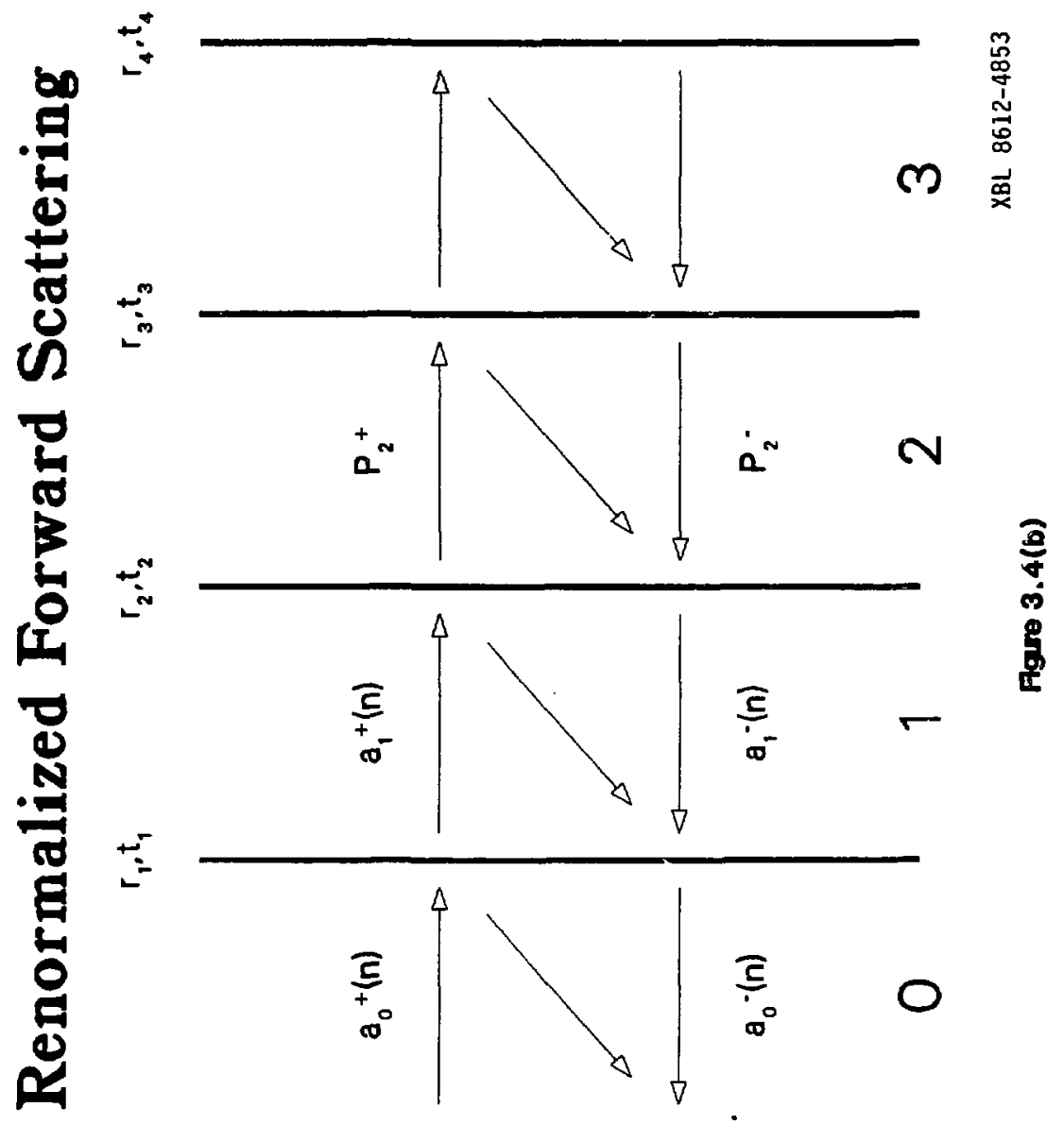




\section{Diffuse LEED Scattering}

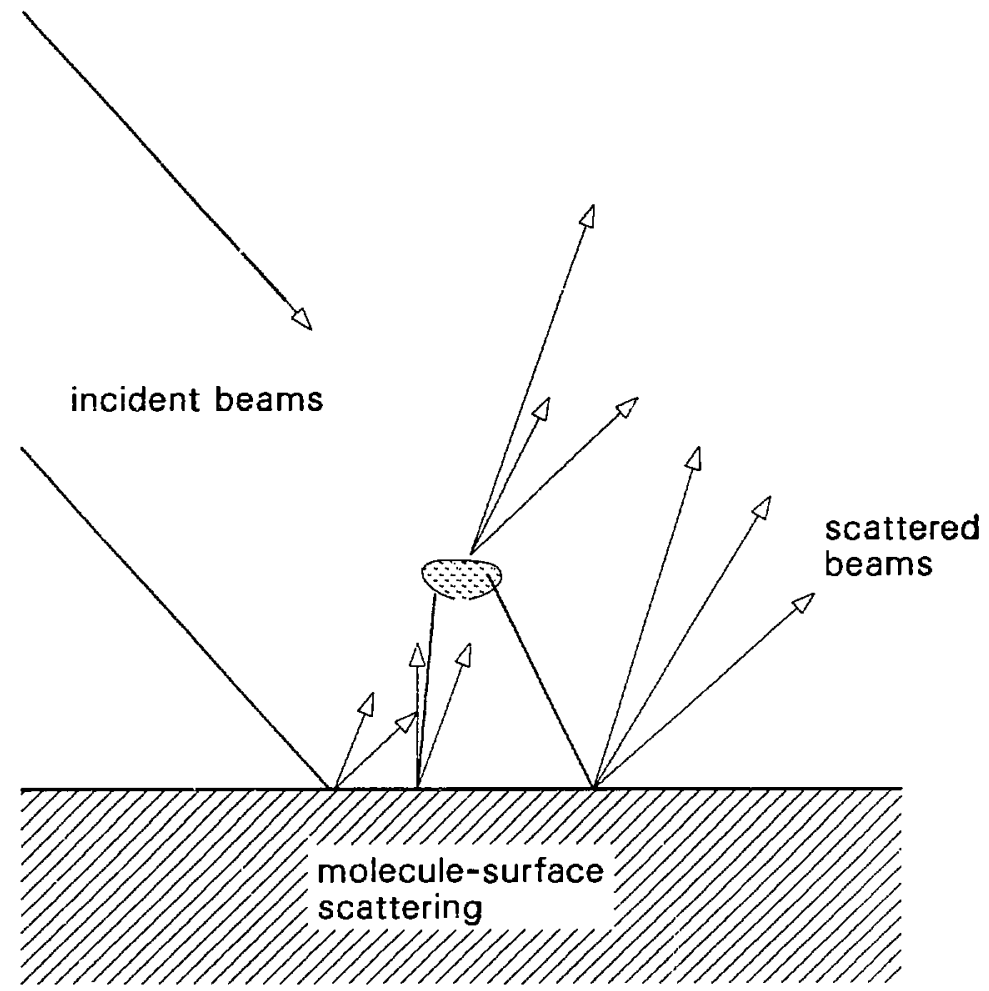

XBL 8612-4849

Figre 3.5 


\section{Debye-Waller Factor for Platinum}

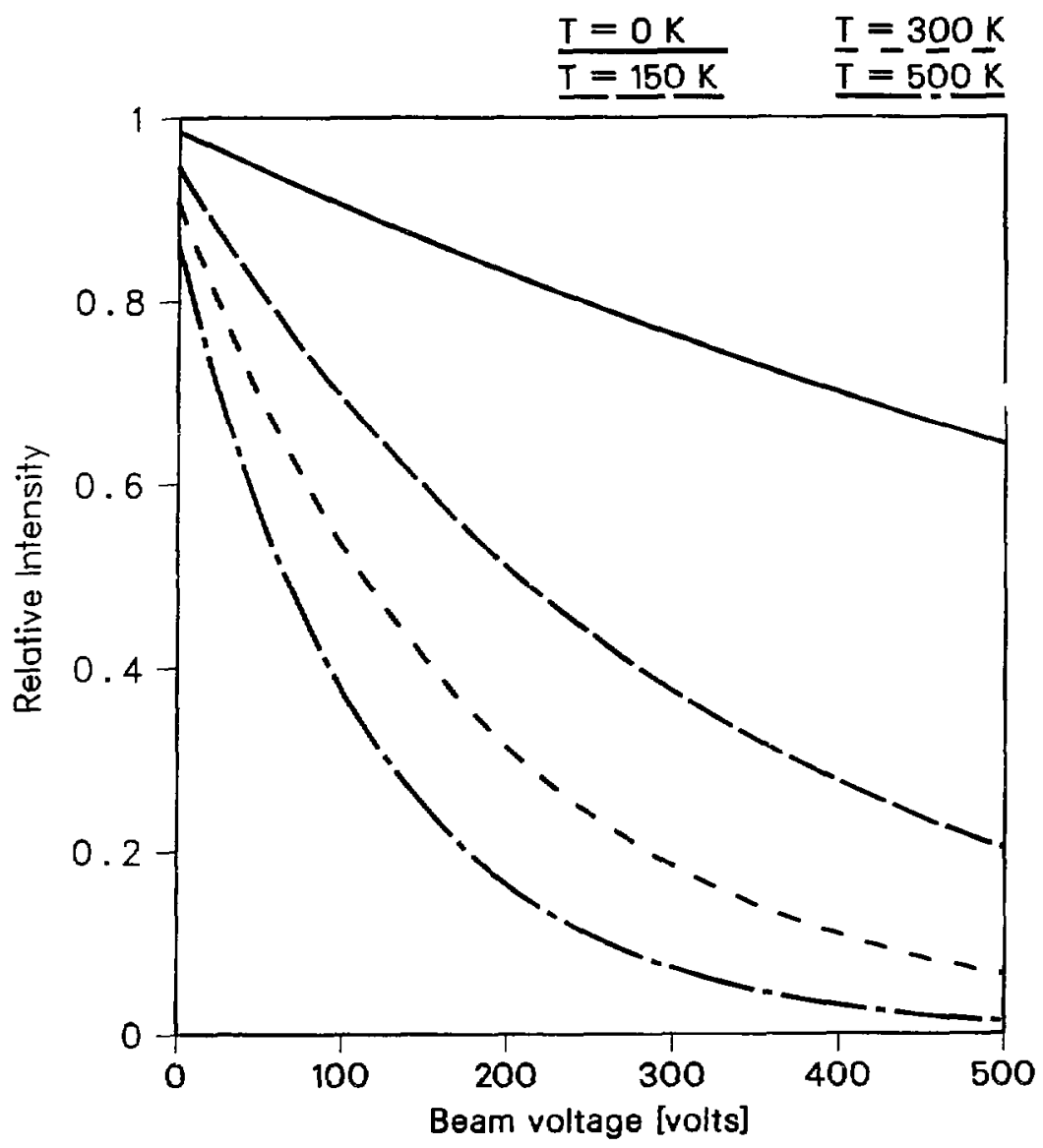

XBL 8611-4243

Figre 3.6 


\section{Chapter 4 \\ Methods of LEED Structure Determination}

\subsection{Introduction}

The previous chapter discussed the calculation of LEED intensities for a given surface geometry. This is only one part of the problem of structure determination. Calculated intensities must be compared to experimental data to choose the best model of surface geometry and to optimize this model.

The early LEED structural studies car'ied out in the mid 70's examined relatively simple surface structures, such as low Miller-index metal surfaces, either clean or with atomic adsorbates. For these systems usually only one geometrical parameter was involvad in the structural calculations, either the adsorbatesubstrate spacing or the first inter-layer spacing. Structures were solved by visually comparing the I-V curves for a few LEED beams at three or four different values of this geometrical parameter. Today much more complicated structures are being investigated by LEED: surface reconstructions, systems with large unit cells, molecular adsorption systems and systems with adsorbates in inequivalent sites. Structure determination for such systems may involve the comparison of many I-V curves calculated for dozens or hundreds of different trial geometries in order to pick the optimum model geometry. R-factor, or reliability factor methods have been developed to aid in structure determination for complex systems. 


\subsection{Reliability factors}

A reliability factor is used as a measure of the extent to which LEED I-V curves calculated for model geometries agree with experimertal LEED data. A small $r$-factor value indicates good agreement and a larger value implies a relatively worse fit. Structure determination involves a search through various model geometries to minimize the value of the r-factor.

Mathematirally, an r-factor is a functional of the experimental LEED intensity $I$ and a theoretical intensity $J$ calculated for a given model geometry, of the form

$$
r=r\left[I\left(\mathbf{k}_{\mathbf{o}}, \Omega\right), J\left(\mathbf{k}_{\mathbf{o}}, \Omega\right)\right]
$$

Since $I$ and $J$ represent ratios of the diffracted LEED intensity to the incident beam intensity, they have the properti $\epsilon I\left(\mathbf{k}_{\mathbf{o}}, \Omega\right) \geq 0$ and $\int I\left(\mathbf{k}_{\mathbf{o}}, \Omega\right) d \Omega \leq 1$. The functional $r$ is a metric, a measure of the "distance" between the functions $I$ and $J$. To be useful, $r$ must have certain properties. These include ${ }^{1}$

$$
\begin{gathered}
r[I, I]=0, \quad r[I, J] \geq 0, \quad \text { and } \\
r[I, J]+r[J, K] \geq r[I, K]
\end{gathered}
$$

Further, + must be "smooth" in some sense, so that

$$
\lim _{\alpha \rightarrow 0} r\left[I, J+\alpha J^{\prime}\right]=r[I, J]
$$

where $\alpha$ is a real parameter and $I, J$ and $J^{\prime}$ are LEED-like functions. For difiraction from an ordered surface, the diffracted intensity $I\left(\mathbf{k}_{o}, \Omega\right)$ is replaced by a set of integrated beam intensities $\left\{I_{\mathbf{g}}\left(\mathbf{k}_{\mathbf{o}}\right)\right\}$, so 


$$
r=r\left[\left\{I_{\mathbf{g}}\left(\mathbf{k}_{\mathbf{o}}\right)\right\},\left\{J_{\mathbf{g}}\left(\mathbf{k}_{\mathbf{o}}\right)\right\}\right]
$$

\subsection{R-factor properties}

For r-factor based surface structure determination to work, the r-factors must reach a clear global minimum at the optimum geometry. Further, small changes of the model parameters around the optimum value should produce only small increases in the r-factors. Given experimental data for $I$ the r-factor functional $r[I, J]$ can be considered as a function of the structural and non-structural parameters in the LEED model calculation. This function can be represented as an $n$-dimensional surface and the optimum geometry will be the global minimum of this surface. Sufficiently close to the minimum the $\mathbf{r}$-factor surface should resemble an $n+1$-dimensional ellipsoid. If the structural model does not represent the basic features of the surface, there should not be a clear global minimum.

To be of practical use the shape of the r-factor surface should be similar when calculated with any sufficiently large sub-set of the available range of data. Most LEED experiments measure only three to ten symmetry-independent I.V curves at one to three different angles of incidence. There has been no mathematical proof that r-factors actually have the useful properties described above. Instead r-factors have been devised and used empirically. When used with caution and a sufficient range of data, $r$-factor structure determination has been quite successful. 


\subsection{Specific r-factors}

There are several different $r$-factors in use and in fact from the discussion above, almost any smooth, well behaved metric functional $r[I, J]$ should work for LEED structure determination. Clarke compares the results of LEED structure determination using different $r$-factors and shows that similar optimum geometries can be obtained. ${ }^{2}$ The first $r$-factor used was the $x$-ray diffraction structure factor $r_{2}$, defined for a single I-V curve as

$$
r_{2}=\frac{\int(I-c J)^{2} d E}{\int I^{2} d E} \text { where } c \equiv \frac{\int I d E}{\int J d E}
$$

This puts a lot of emphasis on peak intensities, which is appropriate for $\mathrm{x}$-ray analysis, since peak positions are determined by the unit cell and most of the geometrical information is contained in peak intensities. In LEED peak positions have much more informational content because of multiple scattering and the lack of a three-dimensional Bragg condition. Further, LEED intensities are much more sensitive than peak positions to small changes in the non-structural parameters, as demonstrated by Pendry ${ }^{3}$ for changes in the Debye temperature (vibrational amplitude) and for small changes in the ion-core potential used to calculate the scattering phase-shifts. In addition, there are often significant differences between calculated and experimental intensities since plane-wave calculations for perfect surfaces are compared to real experiments.

Zanazzi and Jona constructed an r-factor based on the derivatives of the LEED intensities that would put more emphasis on the peak positions, defined ${ }^{2}$ 


$$
\tau_{Z J}=\frac{1}{0.027 \int|I| d E} \int \frac{\left|I^{\prime \prime}-c J^{\prime \prime}\right|}{\left|I^{\prime}\right|+\left|I^{\prime}\right|_{\max }}\left|I^{\prime}-c J^{\prime}\right| d E
$$

where $I^{\prime}$ and $I^{\prime \prime}$ denote $\frac{\partial I}{\partial E}$ and $\frac{\partial^{2} I}{\partial E^{2}}$, respectively and $c$ is defined as in Eq. 4.5. The numerical factor 0.027 is empirically chosen so I-V curves from unrelated surfaces will give values of $r_{Z J} \sim 1$.

Pendry has also defined an r-factor to emphasize peak positions by considering an I-V curve as a superposition of Lorentzian peaks of the form

$$
I_{j}=\frac{a_{j}}{\left(E-E_{j}\right)^{2}+V_{o i}^{2}}
$$

A r-factor based on the logarithmic derivative $L_{I} \equiv I^{t} / I$ will depend only on peak positions $E_{i}$. Pendry's r-factor is defined as ${ }^{2}$

$$
r_{P e n d r y}=\frac{\int\left(Y_{I}-Y_{J}\right)^{2} d L}{\int\left(Y_{I}^{2}+Y_{J}^{2}\right) d E} \quad \text { where } \quad Y_{I} \equiv \frac{L_{I}}{1+V_{o i}^{2} L_{I}^{2}}
$$

Van Hove and Koestner ${ }^{4}$ have proposed using an average of five r-factors, including $r_{2}, r_{Z J}$ and $r_{P e n d r y}$ plus two additional r-factors:

$$
\begin{gathered}
r_{1}=\frac{\int|I-c J| d E}{\int|I| d E} \text { and } \\
r_{\text {slope }}=\int \delta\left(\frac{I^{\prime}}{\left|I^{\prime}\right|}-\frac{J^{\prime}}{J^{\prime} \mid}\right) d E \text { so } \\
r_{5}=\frac{1}{5}\left[3 / 3 r_{1}+1 / 2 r_{2}+r_{\text {slope }}+1 / 2 r_{Z J}+1 / 2 r_{\text {Pendry }}\right]
\end{gathered}
$$

In this average the r-factors are scaled so that they will have approximately the same size for good agreement between theoretical and experimental I-V curves. 
Since $r$-factor computation is very fast compared to LEED calculations, Van Hove and Koestner ${ }^{4}$ do not try to choose the "best" r-factor. Instead, in addition to the five r-factor average, the individual $\mathrm{r}$-factors are also calculated for each $\mathrm{I}-\mathrm{V}$ curve and for the weighted average of all $\mathrm{I}-\mathrm{V}$ curves.

Any modification or processing of the I-V curves should be done before calculating the r-factors. For $r_{1}, r_{2}$ and $r_{Z J}$ the I-V curves are sometimes multipled by an exponential weighting function, so that Debye-Waller attenuation does not result in disproportionate emphasis for the low-energy part of the I-V curve. This is not a problem for $\tau_{\text {Pendry }}$ and $\tau_{\text {slope }}$ as they are almost independent of peak intensities. I-V curves are usually normalized for incident heam current and symmetry-related I-V curves are oíten averaged together. If instrument response is important, tive theoretical I-V curve $J$ can be convoluted with the instrument response function $T$ before $r$-factor calculation (see chapter 2). Usually it is sufficient to compare the experimental integrated peak intensities to the theoretical intensities. I-V curves are sometimes smoothed before calculating $r_{Z J}$ or $\tau_{P_{e n d r y}}$, since these involve factors of $\frac{\Delta I}{\Delta V}$ which can be disproportionately affected by experimental noise.

\subsection{Data sub-division}

Multiple r-factor minima are often encountered in LEED calculations. Of course the correct $r$-factor minima should have the lowest absolute value of the $r$ factor, but this is not a completely reliable guide when approximations are used 
in surface models or in LEED calculations. Surface medels are often oversimplified, for example in assuming a terminated bulk substrate as part of the model in chemisorption problems. Approximate descriotions of multiple scattering may also be used to make faster calculations possible to explore many surface geometries. These approximatious will have an undetermined effect on the absolute $\mathrm{r}$-factor values.

The data subdivision method ${ }^{5}$ is a way of distinguishing the correct minima. The basic idea is that $\mathbf{r}$-factors calculated with sufficiently large sub-seis of LEED data should give the same results. When r-factors are calculated with different sub-sets of data, the minima due to accidental correlations in I-V curves should change positions, while the true minima should not change. If all the individual I-V curves for the different r-factors and the average over all I-V curves, weighted by energy range of each curve, agree, then the surface model being used is probably a reasonable representation of the actual surface structure. When the model is not correct, although there will be some kind of overall minimum in the rfactor, the individual I-V curves will have minimum values over a range of parameter values.

The analysis of chemisorbed carbon monoxide on $\mathrm{Rh}(111)^{4}$ is an example of the data subdivision method. Here most of the individual $\mathrm{I}-\mathrm{V}$ curves $r_{5}$ minima agreed for top-site adsorption, while they were widely scattered for bridge-site adsorption (see figure 4.1). In this case $r_{Z J}$ did not distinguish clearly between the top and bridge sites $\left(r_{Z J}=0.40\right.$ vs 0.42$)$ although there was a clear difference for $r_{5}\left(r_{5}=0.23\right.$ vs 0.30$)$. Another example is the analysis of the incommensurate 
graphite layer on $\mathrm{Pt}(111)$. This analysis (see chapter 12) was based on the specular beam at different angles. Several minima appeared as the graphite-platinum distance was varied with little difference in the absolute value of $r_{5}$. When the rfactors were calculated for different ranges of data the false minima changed positions, while the position of the correct minima was almost independent of the range of experimental data used to calculate the r-factors. ${ }^{5}$

\subsection{Model parameters}

The theoretical diffracted intensity $J$ is calculated from a surface model described by a number of structural and non-structural parameters. The nonstructural parameters include the phase-shifts $\delta_{l}$, the components of the inner potential $V_{a i}$ and $V_{o r}$ and the effective surface Debye temperature $\theta_{D}$. The phase-shifts in turn depend on the assumptions used to calculate the ion-core potential and wave-functions and the muffin-tin radius. The structural parameters describe the geometry of the surface. In principle these could be the coordinates of each atom in the two-dimensional unit cell within a few mean-free paths of the surface, for prehaps $15 n$ coordinates, where $n$ is the number of atoms in the two dimensional unit cell. To solve for this number of coordinates through LEED structure determination procedures is quite impractical. Instead parameterized surface models are constructed. In a simple case of atomic adsorption, the model might consist of an atom adsorbed in a high-symmetry adsorption site, such as a bridge, hollow or top site for a fec (100) surface, with the substrate-adsorbate distance the only structural parameter. 
There is no simple rule to determine how complex a model is required to represent accurately a surface structure. In general, if a model is "reasonably" close then LEED structure determination procedures should produce the best fit passible within the range of the model parameters. On the other hand, if a model is completely inappropriate, then hopefully a LEED structure determination will not produce any satisfactory fit.

Consider the case of a clean metal surface. In the first LEED studies the only structural parameter used was the first inter-layer spacing. Structure determinations for many metals indicated a contraction in the fir $s$ inter-laver spacing. Later, more sophisticated studies varied several inter-layer spacings. These studies supported the earlier results of contractions in the first inter-layer spacing, with additional, smaller changes in deeper interlayer spacings. The optimized value for first inter-layer contraction was sometimes different. (See the tables at the end of chapter 1 .)

Another example is the case of atomic chemisorption. Again early studies varied only the adsorbate-substrate spacing. Later studies also varied the substrate inter-layer spacing(s). Although these later studics sometimes gave different adsorbate-substrate spacings, there is no case where the introduction of a second inter-layer spacing changed the LEED determised adsorption site. In these cases the initial models reproduced the dominant features of the surface structure under investigation and later more complex models only refined the initial results. 
A contrasting case is the $\mathrm{Au}(110)(2 \times 1)$ reconstruction. Several surface models were proposed for this surface, including the "missing-row" model, the "saw-tooth" model and the dimerized row model. The early LEED studies tended to support the missing-row model, but the results were somewhat ambiguous and different attempts to determine the change in the first inter-layer spacing gave different results and even different directions. A recent study by Moritz and Wolf ${ }^{6}$ used a more sophisticated model that allowed for changes in the first three inter-layer spacings, horizontal motions in the second layer and vertical bucking in the third layer, for a total of five structural parameters. The inclusion of third-layer buckling gave a significant improvement in the fit of the theory to experiment and gave clear evidence for a first-layer contraction of $\sim 20 \%$.

This discussion shows the importance, especially for complex structures, of obtaining all available information on the surface, using a range of techniques, in order to make reasonable judgements of what features are necessary to include in a surface model.

\subsection{Structural and non-structural parameters}

The results of LEED structure determination depend on both structural and non-structural parameters. Several investigations have shown that structural results are relatively insensitive to variations of the non-structural parameters over a reasonable range, a fact that considerably simplifies structure determination. Adams, Nielsen and Van $\mathrm{Hove}^{7}$ made a careful LEED structure analysis of the surface of clean $\mathrm{Pt}(111)$. The first inter-layer spacing $d_{1}$ was varied, along 
with $V_{o i}, V_{o r}$ and $\theta_{D}$. The optimum value of $r_{2}$ was 0.16 . These parameters were varied one at a time while the rest were held at near-optimum values. The fit between theory and experiment as measured by $r_{2}$ was about ten times as sensitive to a fractional change in $d_{\perp}$ as to the same change in any of the nonstructural parameters. Furthermore, changes in the non-structural parameters produced relatively small changes in $d_{\perp}$.

The non-structural parameter with the strongest influence on the structural parameters is $V_{o r}$. At normal incidence the main effect of a change in $V_{o r}$ is to shift the energy of the incident beam. There is also a change in the surface potential barrier height, which will change the reflection coefficient of the surface barrier, but this factor is usually small and is neglected in many LEED calculations. $V_{o r}$ is easily optimized by shifting the origin of the energy scale to produce the smallest r-factor, without any need to do additional scattering calculations. The imaginary component $V_{o i}$ ulso has a direct effect on the I-V curves through the peak widths, so $V_{o i}$ is easily optimized, although new calculations are required when $V_{o i}$ is changed.

Noonan and Davis ${ }^{8}$ have intensively investigated the effects of non-structural parameters on the determination of inter-layer spacing relaxations at the $\mathrm{Al}(110)$ surface. By self-consistently optimizing the first three inter-layer spacings along with the non-structural parameters they achieved an $r$-factor value $r_{Z J}=0.034$, probalily the best fit achieved to data. They varied the non-structural values over a wide range, used $r_{Z J}$ and $r_{2}$ independently for structure optimization and used different sets of phase-shifts calculated from different atomic potentials. 
Their study found the optimum values for layer spacing changes of $\Delta d_{12}=-8.5 \pm 1.0 \%$ and $\Delta d_{23}=+5.5 \pm 1.1 \%$. Although the minimum r-factor changed by more ìian a factor of 2 as phase-shifts and non-structural parameters were varied, the "best" values for $\Delta d_{12}$ and $\Delta d_{23}$ changed by less than $1 \%$. These results indicate that structure determination will give similar results with most "reasonable" values for non-structural parameters.

\subsection{Structural searching}

Structure determination is usually carried out by calculating theoretical I-V curves over a range of values for each structural parameter. The range is chosed to span "reasonable" values for the parameter. Typically four or five values will be used for each parameter, separated by 0.05 to $0.10 \AA$. For three independent geometrical parameters this will involve $\sim 100$ sets of I-V curves, with intensities calculated $\sim 5 \mathrm{eV}$ apart.

There are various ways to economize in these calculations by re-using partial results. In particular once the layer diffraction matrices $M_{\mathbf{g g}^{\prime}}^{ \pm \neq}$have been calculated in the plane wave representation it is easy to vary the inter-layer spacing, especially for layer spacings greater than $\sim 1.1 \AA$, where the very efficient RFS scheme can be used to stack the layers. Changing layer registries is more complicated. The intra-plane scattering, calculated using spherical-wave p1 opagators $\boldsymbol{X}$ or $G^{i j}$ and matrix inversion or the RSP scheme need not be changed, but the relatively slow step of projecting plane-waves onto spherical-waves must be repeated for each registry. When the internal geometry of a layer is changed then 
the layer scattering matrix $M_{\mathbf{g g}}^{ \pm \ddagger}$ must be completely recalculated. In any problem there will be a point beyond which the substrate has the bulk geometry. The total scattering properties of the rest of the substrate can be expressed by a reflection matrix $R_{\mathbf{g g}^{\prime}}$ which need be calculated only once for each value of incident momentum, provided the non-structural parameters remain constant (except for $V_{o r}$ at normal incidence).

Once the r-factors have been calculated for a range of structural parameters, a projection of the r-factor surface for a pair of parameters can by illustrated with a contour plot (see figure 4.2). A well-defined ellipsoid centered on the rfactor minimum is an indication of a good fit for the model geometry. The curvature of the ellipsoid at the minimum is a measure of the sensitivity of the structure determination calculation to the particular geometrical parameters. $\bar{F}$ urthermore, the contour plot can be used to interpolate optimum parameter values, which will usually fall between the calculated values.

In principle a structural model could be optimized by a steepest-descent fitting procedure. This has not been done in practice and there are several drawbacks. First, the partial results cannot be re-used efficiently, unless partial results at all energies are stored, a formidable task. Secondly, if the initial condition is not "sufficiently close" to the true minimum a fitting procedure will not find it. Third, such procedures can be quite unstable, even for relatively simple problems like the Gaussian deconvolution of a one-dimensional spectrum. Finally, knowledge of the r-factor surface over a range of parameter space allows useful internal checks of the structure determination process. 


\subsection{Approximations in structural searches}

LEED scattering theory was first applied to metal surfaces. Experience has shown that multiple scattering must be treated "exactly" to yield reliable results for these systems. The calculational schemes discussed in chapter 3 such as RFS, CSM and RSP are all "exact" when iterated to convergence in the context the basic LEED model: the muffin tin potential with isotropic, uncorrelated thermal motions.

The situation is different for molecular overlayers. Structure determination problems are complex for molecular systems. There are generally more possible adsorption sites than for atoms and there are also internal geometrical parameters to be determined. For example, for carbon monoxide adsorbed on $\mathrm{Rh}(111)$ in the (2x2) structure, the model geometry included five structural parameters just for the overlayer. ${ }^{9}$ A large number of calculations are needed to solve such a structure; however, the possibility of making useful approximations is better than in the metal substrate. Overlayers may scatter more weakly, being composed of light atoms with a lower density than metal layers. This is especially true for hydrocarbons. Hydrogen seatters weakly in LEED and can often be neglected completely when the overlaver includes heavier atoms. The hydrogen atoms form a "cushion" separating the carbon atom skeletons of neighboring molecules, so inter-molecular scattering is much reduced.

Van Hove and Somorjai ${ }^{10}$ have proposed the use of approximate calculations of scattering within the overlayer and between the overlayer and the substrate, combined with full multiple scattering within the substrate, to search rapidly 
through many possible adsorption geometries. Once the approximate structure has been determined, increasingly accurate calculations can be used to refine the result. This approach has been successfully applied to several molecular adsorption systems, including carbon monoxide ${ }^{9}$ and benzene. ${ }^{11,12,13,14}$

\subsubsection{Intra-layer approximations}

Multiple scattering within an overlayer can be treated in varying degrees of approximation. The validity of the various approximations depends on the details of the system under investigation and approximations must be used with caution to achieve reliable results. The simplest approach is to ignore intra-layer scattering (kinematic or quasi-dynamical approximation). In most cases this is not valid. Exceptions include solid xenon with a very large unit cell and possibly low-density overlayers, like the case of $(2 \times 2)$ chalcogen adsorption on $\mathrm{Ni}(111)$, where investigation has shown that the shape of the superlattice I-V curves is almost independent of coverage. ${ }^{15}$

For molecular adsorbates intra-molecular scattering is almost always important -- atoms may be separated by only 1.0 to $1.5 \AA$, even closer than in most solids. The kinematic subplane addition (KSA) approximation takes advantage of this. A subplane is defined as a single atom on a Bravais lattice. In the case of benzene adsorption discussed in chapter 11, for example, there are two benzene molecules per unit cell adsorbed parallel to the surface. Each of the twelve carbon atoms is on a different sublattice. The KSA approximation neglects intrasubplane scattering, including only scattering within the unit-cell. The KSA 
scheme is implemented by setting the intra-subplane propagators $G_{L L^{\prime}}^{i i}$ to zero, so the subplane scattering matrix $\sigma^{(i)}$ in the CSM equation Eq. 3.34 is replaced by the atomic scattering phase shifts. Similar simplifications can be made within the RSP formalism.

The KSA approach can be carried further by including only selected intersubplane scattering terms, for example in the benzene case discussed above, including only multiple scattering within benzene molecules, but not between the two inequivalent molecules. The simplest approximation is to consider multiple scattering only between neighboring atoms (those pairs of atoms forming chemical bonds). ${ }^{10}$ The more extreme approximations are useful for structural searches, but the results should be confirmed by more accurate calculations for final structure determination.

\subsubsection{Inter-layer approximations}

A useful approximation to inter-layer scattering is possible in the common case that the overlayer has lower translational symmetry than the substrate. If the overlayer unit cell is $n$ times the area of the substrate unit cell, then there will be $n$ times as many LEED beams in the plane-wave representation $\{g\}$. These can be divided into $n$ "beam sets" $\{\boldsymbol{g}\}_{i}$. The "substrate beams" are those beams that appear in the diffraction pattern in the absence of the overlayer and the remaining beams are called "overlayer beams" . Only the overlayer scattering matrix $M_{\mathrm{gg}}^{ \pm \ddagger}$ couples all beams in $\{\mathrm{g}\}$. The substrate reflection matrix $R_{\mathrm{gg}^{\prime}}$ can be block-diagonalized into $n$ blocks, so that each beam-set $\{\boldsymbol{g}\}_{i}$ scatters 
independently inside the substrate. In this description the overlayer can be an arbitrarily complex composite layer and $M$ is calculated including multiplescattering effects, either exactly using the RSP or CSM schemes, or with some of degree of approximation as discussed in the previous section. Likewise the substrate reflection matrix $R$ is calculated including multiple scattering effects.

Scattering between overlayer and substrate can be expanded as a perturbation series of non-zero angle scattering events. Since only overlayer scattering couples different beam sets, these beam sets can influence each other only through third- and higher-order scattering paths. Note that multiple scattering paths within the overlayer and the substrate are included to all orders. The beam set neglect (BSN) approximation ${ }^{11}$ includes first- and second-order overlayersubstrate scattering paths and neglects third- and higher-order overlayersubstrate scattering paths.

In this approximation the calculation of the intensities of any beam set involves calculation of the substrate reflection matrix $R_{\mathbf{g g}}(\mathbf{k})$ for at most two values of $\mathbf{k}$, at $\mathbf{k}_{\mathrm{o}}$ and also at $\mathbf{k}_{\mathrm{o}}+\mathbf{g}_{i}$ for a superlattice beam set, where $\mathbf{g}_{i}$ is an element of the superlattice beam set. The spherical-wave part of the calculation of the overlayer diffraction matrix $M_{\mathrm{Gg}}^{ \pm \neq}$is unchanged, but the time required to project the spherical-wave representation onto the plane-wave representation is reduced by $\sim n^{-1}$ and this is often the slowest part of the calculation if the RSP scheme can be used to calculate $M$. A comparison of I-V curves calculated for benzene adsorbed on $\operatorname{Rh}(111)$ with the BSN scheme and with an "exact" calculation agree quite well. 11 
Most of the terms neglected in the BSN scheme involve multiple backscattering and are quite weak. One third-order term is potentially more significant. This term includes non-zero angle forward-scattering in the overlayer before and after back-diffracting from the surface. Since the forward scattering cross-sections are significantly larger than back-scattering cross-sections, this term is probably larger than the other third- and higher-order terms and may be the limiting factor in the application of the BSN approximation.

\subsubsection{Beam set neglect and disorder}

The BSN scheme can speed up calculations somewhat for overlayers with large unit cells; however, this approximation provides and enormous simplification for overlayers without long range order. A modification of the BSN approximation is used in chapter 12 to calculate the diffracted intensities for an incommensurate overlayer. This scheme efficiently provides a calculation correct to second order of diffracted intensities for a problem that cannot be easily analyzed in the traditional LEED model, since an incommensurate overlayer would correspond to an infinitely large unit cell with an infinite number of beam sets.

The BSN approximation can also be used to calculate diffuse LEED intensities in the case of a disordered overlayer. Instead of using a XANES type cluster calculation as described in section 3.10 to calculate diffuse intensities, a planewave scattering matrix can be calculated for an adsorbate atom or molecule and this can be used in the BSN scheme. The diffracted intensity at an arbitrary value of momentum transfer $\mathbf{s}$ requires the calculation of the substrate reflection 
matrices $\boldsymbol{R}_{\mathbf{g g}}\left(\mathbf{k}_{\mathbf{o}}\right)$ and $\boldsymbol{R}_{\mathbf{g g}_{\mathbf{g}}}\left(\mathbf{k}_{\mathbf{o}}+\mathbf{s}\right)$. The difiuse iziensity calculation for an adsorbate at a given momentum $\mathbf{k}^{\prime}$ then requires the the same effort as a "normal" LEED calculation for a ( $2 \times 1)$ overlayer of the same adsorbate using the KSA approximation in the overlayer -- a substantial savings over a XANES calculation. Saldin et al. have compared the results of BSN and diffuse LEED cluster calculations for oxygen adsorbed on $\mathrm{Ni}(100) \cdot{ }^{16}$ In this case the BSN results compare favorably with the results of more complex and more exact calculations.

\subsection{Conclusion}

LEED structure determination is a powerful tool for investigating the solidvacuum interface. Using the theoretical techniques described in chapter 3 and the methods of structure determination described above, a wide range of surface geometries can now be investigated. Recent theoretical and experimental advances are extending the range of LEFD structure determination to systems with large unit cells, complex molecular overlayers, incommensurate and disordered overlayers. Some results illustrating these extensions are presented in part III, using the experimental tools described next in part II. 


\section{References}

1. M. Reed and B. Simon, Functional Analysis, Academic Press, New York and London, 1972.

2. L. J. Clarke, Surface Crystallography; An Introduction to Low Energy Electron Diffraction, Wiley-Interscience, Chichester, 1985.

3. J. B. Pendry, Low Energy Electron Diffraction, Academic Press, London, 1974.

4. R. J. Koestner, M. A. Van Hove, and G. A. Somorjai, Surface Science, vol. 107, p. 439, 1881.

5. Hu Zi-Pu, D. F. Ogletree, M. A. Van Hove, and G. A. Somorjai, Surface Science, to be published.

6. W. Moritz and D. Wolf, Surface Science, vol. 163, p. L655, 1985.

7. D. L. Adams, H. B. Nielsen, and M. A. Van Hove, Physical Review B, vol. 20, p. $4789,1979$.

8. J. R. Noonan and H. L. Davis, Physical Review B, vol. 29, p. $\{349,1984$.

8. M. A. Van Hove, R. J. Koestner, J. C. Frost, and G. A. Somorjai, Surface Science, vol. 129, p. 482, 1983.

10. M. A. Van Hove and G. A. Somorjai, Surface Science, vol. 114, p. 171, 1982.

11. M. A. Van Hove, R. F. Lin, and G. A. Somorjai, Physical Review Letters, vol. 51, p. 778, 1983. 
12. D. F. Ogletree, M. A. Van Hove, and G. A. Somorjai, Surface Science, in press.

13. M. A. Van Hove, F.. F. Lin, G. A. Blackman, and G. A. Somorjai, Acta Crystallograpirica, to be published.

14. M. A. Van Hove, R. F. Lin, and G. A. Somorjai, Journal of the American Chemical Society, vol. 108, p. 2532, 1986.

15. W. S. Yang, F. Jona, and P. M. Marcus, Physical Review B, vol. 27, p. 1394, 1983.

16. D. K. Saldin, J. B. Pendry, M. A. Van Hove, and G. A. Somorjai, Physical Review B, vol. 31, p. 1216, 1985. 


\section{Figure Captions for Chapter 4}

4.1 Data-subdivision illustrated for carbon monoxide chemisorbed on $\mathrm{Rh}(111)$ in the $\left(V_{3} \times V_{3}\right) R 30^{\circ}$ arrangement. Individual I-V curve $r_{5}$ values as a function of $d_{\mathrm{C}-\mathrm{O}}$ and $d_{\mathrm{Rh}-\mathrm{C}}$ for top and bridge adsorption sites. Note the agreement in the individual r-factors for the top site at $d_{\mathrm{Rh}-\mathrm{C}}=2.0 \AA$ and $d_{\mathrm{C}-\mathrm{O}} \approx 1.0 \AA$.

4.2 R-factors for carbon monoxide chemisorbed on $\mathrm{Pt}(111)$ in the $\mathrm{c}(4 \times 2)$ arrangement (see chapter 10). Contour plots of $r_{5}$ averaged over all beams at $\theta=0^{\circ}$ and $\theta=5^{\circ}$ calculated for $d_{\mathrm{C}-0}=1.15 \AA$. Note the agreement in the optimum values of the structural parameters for the different data subsets, also the relatively greater accuracy for $d_{\perp \mathrm{C}-\mathrm{Pt}}$ compared to $d_{\perp \mathrm{C}-\mathrm{C}}$. 


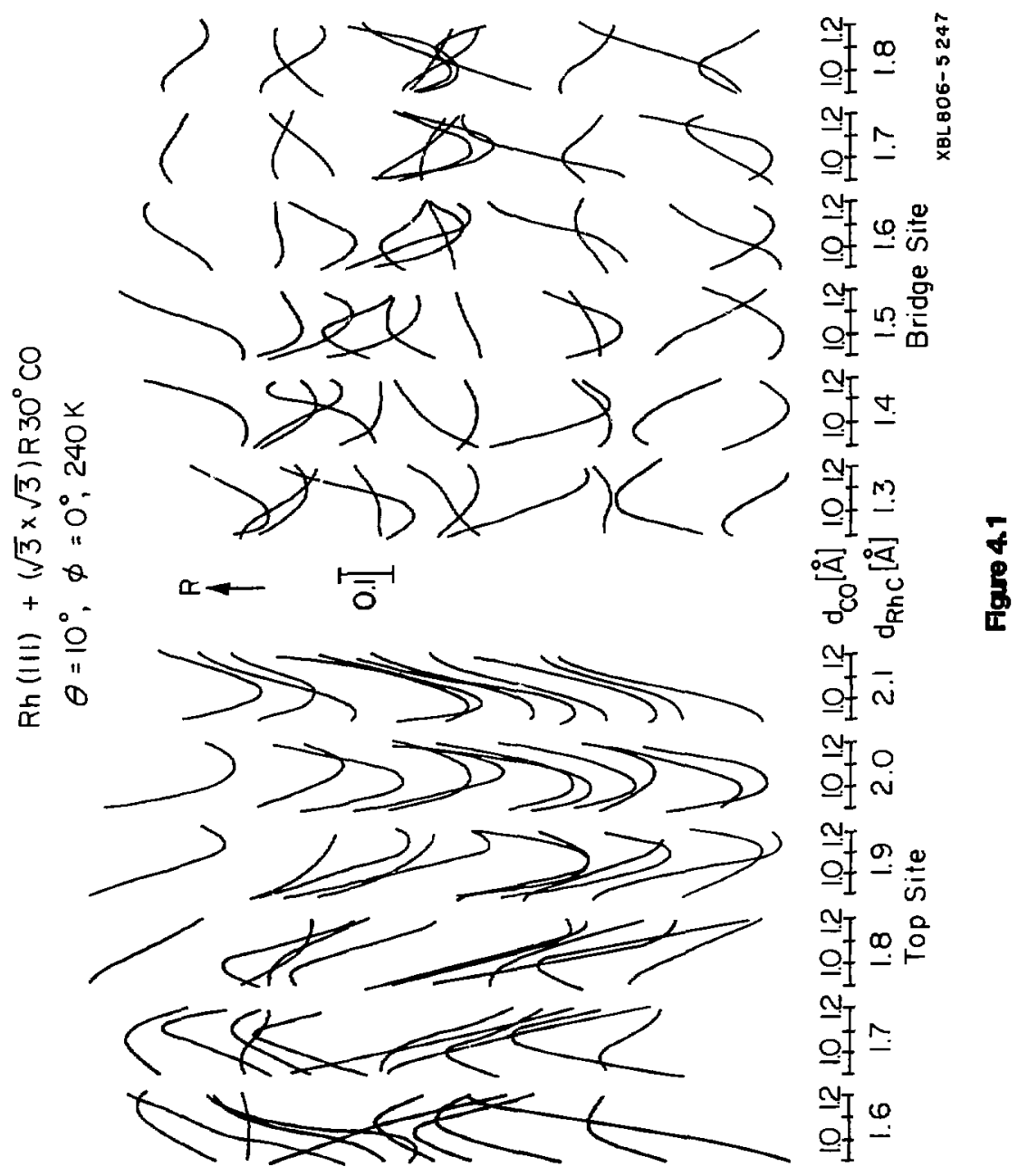




$$
\begin{gathered}
233 / 234 \\
\mathrm{Pt}(\mathrm{III})-\mathrm{C}(4 \times 2)-2 \mathrm{CO}
\end{gathered}
$$

$\mathrm{R}$-factor contour plots

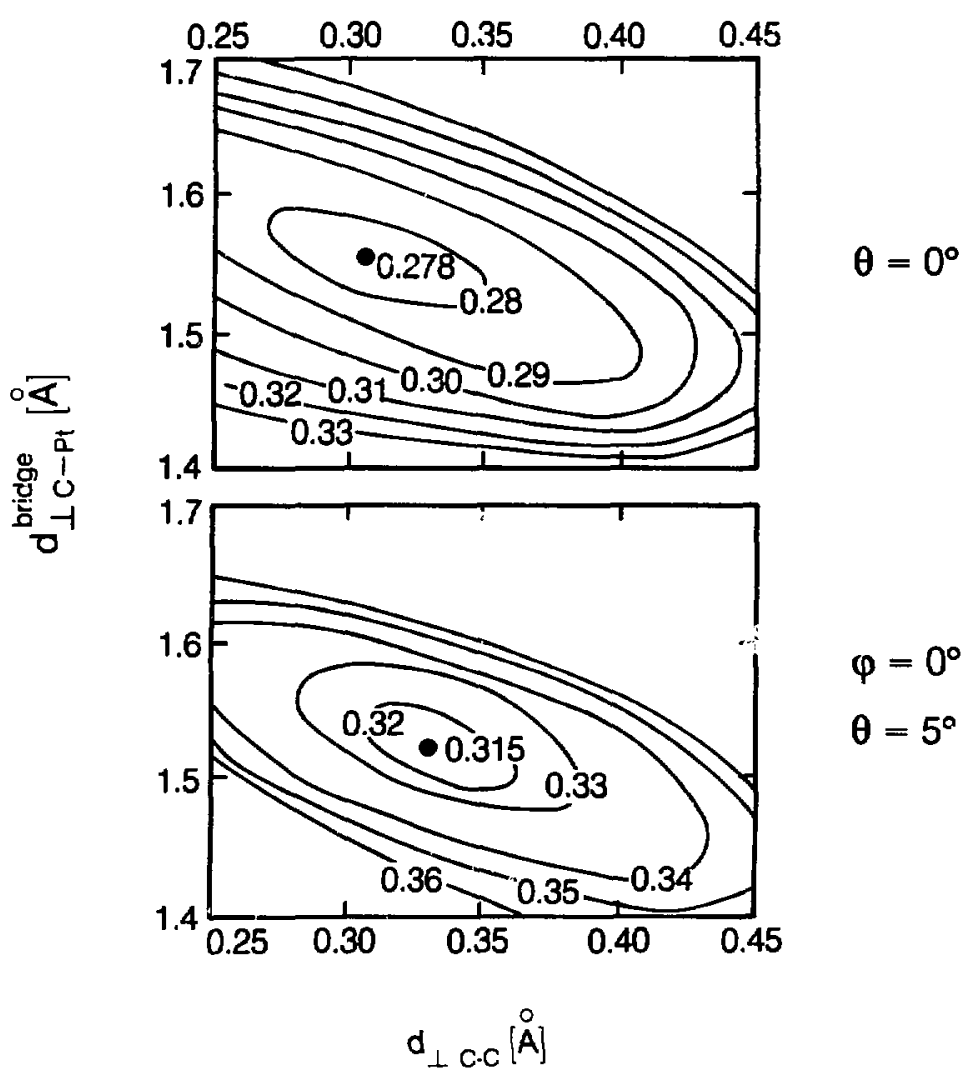

Fgre 4.2 
Part II

Experimental Developments in Low Energy Electron Diffraction 


\section{Chapter 5 \\ Video Techniques and LEED Intensity Measurement}

\subsection{Introduction}

Most LEED surface structure determination studies are now performed with a standard display LEED optics, consisting of a retarding-field energy analyzer and a post-accelerated phosphor-screen display. Rapid data collection is a major advantage for structure determination experiments. A large data-base is required fo: reliable surface structure determination calculations and surface contamination or electron beam damage can modify the surface during data collection. Fast data collection minimizes these problems.

As discussed above in chapter 2, piotographic recording of an entire LEED pattern, followed by photometric analysis, is a most efficient form of data collection. Video recording is superior to film photography because of its intrinsic linearity and the relative ease of analysis (see chapter 2).

Several video-camera systems for measuring LEED I-V curves and beam profiles were developed in the $70^{\prime}$ s. $^{1,2,3,4,5}$ These systems digitize one point from each horizontal video scan, then a computer builds up a LEED beam profile perpendicular to the video scan direction. Later, systems with faster electronics were developed that could collect data along video scans, ${ }^{2}$ or at arbitrary angles cutting across the video image. ${ }^{6}$ In these systems the operator selects a spot and computer programs track the given diffraction beam as the incident electron 
energy is varied. Each run generates one I-V curve and the process has to be repeated for each beam. Later systems with more sophisticated software could digitize profiles through several points in each image, ${ }^{5}$ although some systems can only digitize one LEED spot in a vertical column. Recording the LEED patterns on video tape for later analysis allows a high data collection rate. ${ }^{3,5}$

A vicieo camera with a full image digitizer was used for the first time for LEED and ultra-violet photo-electron spectroscopy (UPS) measurements in $1979 .^{7}$ This system had limited resolution and required a special computer interface and the data acquisition rate was slow compared to standard video systems.

During the late 70's and early 80's image processing developed rapidly with the introduction of computer based systems that digitize and operate on video images in real time with full video resolution..$^{8,9}$

A new LEED I-V data system based on a modern video processor has been developed. This system uses a video image digitizer to generate a two dimensional intensity-map of the entire LEED pattern at high resolution in real time. The major hardware components for this system are available commercially. Analysis software tracks all the beams in the pattern and calculates the location, width, peak and integrated intensities for each diffraction spot. The instrumental background is automatically subtracted and an algorithm corrects for the local diffuse electron scattering background. LEED image analysis and I-V curve generation is discussed in detail in chapter 7.

This system operates in real-time, so the rate of data acquisition is limited only by video scan rates. Because the entire LEED pattern is available for 
analysis, it is possible to distinguish with greate accuracy between elastically scattered (zero-phonon) and quasi-elastically scattered electrons from the shape of the intensity distribution in the neighborhood of diffraction beams. Complex diffraction patterns may also be analyzed with less chance of confusion between adjacent LEED spots.

\subsection{Video camera concepts}

A two-dimensional video image is built up from a series of horizontal scans across the image. A (black and white) video signal consists of timing pulses to align and synchronize the video scans, interspersed with a voltage signal which modulates the intensity of a scanning electron beam to produce a light and dark image on the TV. A video camera is built up of four basic components; lenses and other optical components which form an image, a transducer which converts the optical image into a current signal, an amplifier stage to produce the video signal from the transducer output and scanning circuits which control the transducer and add the synchronization pulses to the video output signal.

Video signals are digitized by identifying particular scan lines from the synchronization pulses, then locating points along the scan with a timing circuit and digitizing the analog voltage relative to the dark level over some interval to determine the image intensity for those particular pixels (picture-elements). The performance of the different video-camera components determines the accuracy and resolution of video-based LEED intensity measurements. 


\subsubsection{Optical components}

The optical performance of a typical video camera is quite good compared to the requirements of LEED measurements. The ultimate optical performance for a video camera can be characterized by a point-spread function. The full-width at half-maximum of the optical point spread function for a combined system of vidicon tube, camera lenses and phosphor screen has been determined to be $\sim$ $0.042^{\circ},{ }^{2}$ much less than the angular width of the beam from a typical LEED electron gun. A high-resolution vidicon camera can have a spatial resolution of 1200 lines across the height of the image. ${ }^{10}$ The U.S. video standard is 480 lines across the image, so if the entire LEED screen is imaged, one video line corresponds to $0.16^{\circ}$ for a $10 \mathrm{~cm}$ diameter LEED screen with a $7 \mathrm{~cm}$ radius of curvature. Although this image quality is more than adequate for LEED intensity measurements, it is significantly less than that of a good $35 \mathrm{~mm}$ film camera, so it is possible to make video lenses smaller, faster (smaller f-stop number) and cheaper than equivalent film camera lenses.

\subsubsection{Video transducer}

The video image transducer puts out an electrical current proportional to the illumination levels in the visible image. Vidicon tubes are normally used as image transducers in closed-circuit video cameras. A similar device, the video orthicon, is used for television studio cameras. The main advantage of a video orthicon is a faster response to changing light-levels. If a vidicon camera is swept across a room a characteristic streaking is seen in the image, because the signal takes 20 to 
$150 \mathrm{~ms}$ to decay after the external light source is removed. Although this is not good for broadcast television, it normally presents no problem for LEED applications, so the simpler and more compact vidicon camera is used.

The inside of the faceplate of a vidicon tube is coated with a photoconductive "target" layer on top of a transparent conductor at ground potential. An electron beam scans across the back of this target, charging it up to the beam voltage. The photoconductive target layer acts as a capacitor, with an opposite charge induced at each point on the front surface conductor to match the charge deposited on the back surface of the target. When light falls on the photoconductive layer electron-hole pairs are created, which locally short out the target capacitance. A charge-density image is formed on the back of the target equivalent to the light image falling on the input side of the target. When the electron beam next scans over this region of the target the charge is replaced and this induces an instantaneous current flow in the transparent conductive layer, which is the vidicon output signal. The electron beam is scanned over the target by magnetic deflection coils located outside the vidicon tube and the scanning pattern is synchronized with the scanning pattern used to reconstruct the video image at the monitor, digitizer or video tape recorder.

The vidicon dark current is due to the temperature-dependent concentration of charge carriers within the photoconductive layer which shorts out the target capacitance in the same way as a photo-current. The dark current will double with an $\sim 8 \mathrm{~K}$ increase in faceplate temperature. The response of the vidicon to light is linear until the charge on the target becomes depleted, at which point the 
vidicon saturates. As vidicons approach saturation they tend to "bloom", i.e. the apparent image diameter of a point-source of illumination increases. (This effect can partly compensate for accidental saturation in LEED intensity measurements when integrated intensity values are used.) The vidicon in effect integrates the detected light signal between scans. It is possible to increase the sensitivity (output-current/faceplate illumination) by reducing the scan rate below its normal value, although this will increase the dark current, as well as decreasing the spatial resolution because of charge diffusion across the target.

Vidicon tubes are available in 18 and $25 \mathrm{~mm}$ diameters with image heights of 6.6 and $9.6 \mathrm{~mm}$, respectively. The larger format has about twice the sensitivity and higher spatial resolution than the smaller format. High-sensitivity vidicon tubes will have dark-currents of $\sim 8$ to $10 \mathrm{nA}$ in an ambient temperature of $20^{\circ}$ $\mathrm{C}$ and the response to light will become non-linear at a target current of $\sim 700$ to $800 \mathrm{nA}$, corresponding to a faceplate illumination of $\sim 1$ lumen $/ \mathrm{m}^{2}$ of $2856 \mathrm{~K}$ tungsten light. The sensitivity to visible light is $\sim 20 \%$ of this value. The peak spectral response varies with target composition and the response between 4000 to $8000 \AA$ is at least $50 \%$ of the peak response, which is at $\sim 7500 \AA$ for the Newvi$\operatorname{con}^{11}$ and $\sim 5500 \AA$ for the Ultricon. ${ }^{12}$

In standard vidicon tubes the sensitivity may be varied by varying the target voltage. Many commercial vidicon cameras have "automatic gain control" circuits which vary the vidicon sensitivity by changing the target voltage to maintain a "good image" as the overall light level changes. To make accurate intensity measurements this feature must be disabled. This can be done by using 
high-sensitivity tubes like the Newvicon ${ }^{11}$ which have a constant target voltage, or by using instrumentation cameras where the target voltage is under external control. 10

A two-dimensional CCD (charge-coupled device) array may be used as a transducer in place of a vidicon tube. High-resolution two-dimensional CCD arrays are only now being developed. Ultimately, CCD cameras should be superior to vidicon-based cameras for LEED intensity measurements. A CCD array can have higher sensitivity and higher dynamic range.

\subsubsection{Video amplifier}

The video camera needs a high-bandwidth low-noise amplifier to avoid degrading the vidicon output signal. A $1 \mathrm{~V}$ peak-to-peak composite video signal has a $0.65 \mathrm{~V}$ range corresponding to the camera dark-to-light range, with the remaining $0.35 \mathrm{~V}$ used for synchronization pulses. A vidicon output current of $\sim$ 75 to $150 \mathrm{nA}$ corresponds to a full-scale video output signal. When the amplifier in a video camera is saturated a "comet-tail" effect is usually created, i.e. a bright spot in the video image will appear to have a triangular "tail" to the right, since the video scan direction is from left to right.

The amplifier bandwidth determines the camera spatial resolution along the scan lines. A single video scan lasts $64 \mu \mathrm{s}$ and about $1 / 5$ of this time is used for timing pulses. If a scan-line is digitized into 512 pixels this corresponds to a rate of $10^{7}$ points/s and an analog signal must have a bandwidth corresponding to twice the discrete sampling rate to avoid signal degradation. For high-resolution 
a vidicon camera should have a $20 \mathrm{MHz}$ bandwidth. A high-quality camera is required to achieve this bandwidth without excessive amplifier noise.

\subsubsection{Video scanning}

There are various video standards in use in the U.S. and Europe. The RS170 standard is the common standard for closed-circuit black and white video in the U.S. In the RS-170 format 30 video images are collected per second, each with 480 horizontal scans lasting $64 \mu$ s. A 2:1 interlace is used, i.e. the odd numbered scans are collected in the first $1 / 60 \mathrm{~s}$ and then the even numbered scans in the next $1 / 60 \mathrm{~s}$. This makes the video image appear more stable to the eye. This can also be treated as a lower-resolution video image at 60 images/s. After 240 image scans have been transmitted several synchronization and timing scans are transmitted. These signals align the overall image and distinguish between the even and odd interlace parts of the image. The European standard for video, based on a $50 \mathrm{~Hz}$ power-line frequency, has 25 interlaced images/s with 525 lines per image.

\subsection{Data Acquisition}

Most of the LEED I-V measurements discussed here have been performed on molecular chemisorption systems. Since these systems are often sensitive to electron beam damage or chemical contamination, I-V data should be collected quickly. Data acquisition is faster than analysis for this LEED system. An integrated LEED image may be acquired in less than one $s$ and data analysis 
takes $\sim 0.5 \mathrm{~s}$ per LEED spot at each energy (see chapter 7 ). If data analysis is done in an interactive mode the rate is even slower. A complex diffraction pattern may have 50 or more spots, so the diffraction patterns are recorded on video tape for later analysis. This minimizes the data collection time and also provides a permanent record of the LEED experiment.

The data acquisition system is shown schematically in figure 5.1(a). A camera with a fast lens and a high sensitivity vidicon tube images the entire phosphor screen of a conventional four-grid LEED optics. The camera is enclosed in a light-tight box attached to the view-port of the vacuum system. The video signal is recorded by a video cassette recorder (VCR) using standard VHS video cassettes and is simultaneously displayed on a video monitor.

Data are acquired with the LEED electron gun power supply under computer control. The Varian ${ }^{13}$ LEED power supply has been modified to allow for external beam voltage programming (figure 5.1(b)). Tìe beam voltage amplifier input is at approximately twice the beam: voltage in the Varian supply, so a $2.5 \mathrm{kV}$ opticai isolation stage is ised in the control circuit. A feedback control loop was added which measures the voltage between the electron gun filament center-tap and the crystal ground to compensate for non-linearity in the power supply. This also helped to increase the response time of the power supply. Using the external control circuit of figure $5.1(\mathrm{~b})$, the Varian supply has a $10 \%$ to $90 \%$ rise time of $290 \mathrm{~ms}$.

Character output from the computer controlling the video system is used to label the video tape record of an experiment. An RS-170 video cuiput from the 
computer terminal is mixed with the video output from the camera, which is synchronized to the terminal video output signal. This mixed signal is recorded. A diode circuit clamps the video terminal output so only high-intensity portions of the signal, such as bright letters, are mixed with the LEED image. This prevents noise from the video terminal or computer from degrading the quality of the LEED images. The video camera signal level was monitored to detect saturation of the camera amplifier, which occurs when the video signal level exceeds $1.0 \mathrm{~V}$. If the signal saturated a warning light came on until the system was reset. In practice this precaution was not required since a saturated LEED spot clearly shows a "comet tail".

The VCR runs continuously during an experiment. A short text describing the experiment is recorded on the video tape cassette before the LEED images, including information on surface preparation and angles of incidence. After the LEED images are collected an incident beam current calibration table, used later to normalize the I-V curves, is added to the tape.

The LEED patterns for an experiment are recorded in sequence. Information is superimposed on the recorded LEED image that shows beam voltage and the periods when the electron beam voltage is changing. After each change in beam voltage time is allowed for the LEED power supply to stabilize. A computer generated flag is superimposed on the video image while 1 to 256 video frames are recorded at each energy. When the video tape is analyzed these frames are averaged together. The number of frames to be averaged should be a power of 2 and depends on the signal-to-noise ratio of the LEED image. Data acquisition 
typically takes $1 / 2$ to 3 s per energy.

The recorded video image contains an instrumental background. A vidicon camera has a dark output level that may be 10 to $20 \%$ of the maximum linear signal output level. This is a combination of the vidicon dark current and the video camera amplifier offset voltage. In addition there may be stray light in the LEED image from the electron gun filament or from field emission flares on the phosphor screen. A zero beam-voltage instrumental background image is recorded before and after the LEED images for each experiment. This instrumental background image is subtracted from the recorded LEED images during data analysis. Any remaining experimental background is from scattered electrons.

\subsection{Video digitizer}

The digital video processor is the heart of the video LEED system. Real-time digital video processors now are available from several manufacturers. A system developed by Imaging Technology, Inc. is used here. ${ }^{14}$ There are three modules in this system: the analog processor, the frame buffer and the arithmetic logic unit. Each module is a circuit board that plugs directly into the backplane of the LSI11/23 computer 15 used for image analysis and I-V curve generation. The modules have two to ten directly addressable 16-bit control/data registers with addresses located in the $\mathrm{I} / \mathrm{O}$ page of the computer memory. All of the video processor functions are under program control.

The analog processor module converts a RS-170 video input signal to a stream of digital data (the analog processor is also compatible with other video 
standards, including European standards). Video pixels are digitized, processed and stored at a rate of $10 \mathrm{MHz}$ (figure 5.2). Data transfer over the special highspeed video data bus linking the image processor modules is synchronized with the video scan. Each of the $\mathbf{4 8 0}$ horizontal video scan lines that make up an image is broken into 512 pixels and each pixel is digitized with 8-bit resolution. The incoming data are passed through look-up tables which can be programmed to compensate for non-linearities in the input data. No changes are made in the input data for LEED experiments. The digital output of the analog processor goes onto a special high-speed video data bus that links the processor modules. The video system can operate without attention from the computer CPU. Likewise, the analog processor can simultaneously convert a stream of stored digital data from a memory into an analog black and white or color (RGB) video output signal. Output pixel values may be modified as they are transmitted to the video monitor to enhance the image contrast.

The frame buffer module can store a $512 \times 512$ video image with 8-bit resolution and a pair of modules may be coupled to give 16-bit resolution. The frame buffer memories can transfer images in real time on the video bus and the com-r CPU can independently read or write to the video frame buffer memory.

The arithmetic logic unit module performs operations on data streams from frame buffer memories or the analog processor. These operations include addition, subtraction, multiplication and logical comparisons. The logic module is a "pipeline" processor so it is able to handle operations on 16-bit video data at the realtime $10 \mathrm{MHz}$ video rate. 


\subsection{Video data processing}

During data analysis a video tape of the LEED experiment is played into the video processor. This image is digitized with 8-bit accuracy, or 256 "gray levels". Up to 256 consecutive 8-bit images at a given incident electron energy may be summed together into the 16-bit memory. When $N$ frames are summed the signal-to-noise ratio in the final image will be increased by $\sqrt{N}$ over a single image, however the pixel noise level cannot be less than \pm 1 in a digital system. Typically 16 frames are summed together, for a final image with 12 significant bits of data (two 8-bit frame buffer memories are coupled, so an image of up to 16 significant bits can be stored). A background image, consisting of the 8 most significant bits of the integrated image at zero beam voltage, previously stored in another frame buffer, is subtracted from the 8 most significant bits of the integrated diffraction pattern.

The output from the video processor is a two dimensional digital intensity map, $512 \times 480$ pixels, of the original LEED pattern. The image signal-to-noise ratio has been improved by integrating several video frames together and the instrumental background has been subtracted. The processed image (figure 5.3) is displayed on a video monitor, with computer enhanced contrast to aid in the visual interpretation of the diffraction pattern. The analysis programs use this digita! intensity map to calculate the integrated intensities of the different diffracted beams in the LEED pattern.

The data acquisition rate can be greatly increased, at the cost of reduced spatial resolution and a decreased signal to noise ratio for experiments where time 
resolution, speed, or beam-damage is a crucial factor. ${ }^{16}$ The maximum data acquisition rate at full spatial resolution is 30 images/s (with no image averaging). The background noise increases, reducing the dynamic range and the I-V curve signal to noise ratio, but this is only a serious problem for weak diffraction beans. Video data can be acquired as fast as 60 images/s if the video resolution is reduced to $512 \times 240$ pixels (no interlace). At this rate LEED I-V profiles can be acquired in a few seconds, provided the LEED power supply can change beam voltage this rapidly. Most commercial LEED supplies are not this fast, for example, the Varian supply ${ }^{13}$ has a rise time of $0.29 \mathrm{~s}$, tion slow to change beam voltage in $16 \mathrm{~ms}$.

\subsection{Instrumentation}

Two different video cameras have been used for LEED I-V measurements. The initial work with the video digitizer system was done with an inexpensive high-sensitivity vidicon camera sold for security applications, a Panasonic WV1550 camera equipped with an $18 \mathrm{~mm}$ Newvicon type $S 4075$ vidicon tube with a cadmium/zinc telluride target. This is a high sensitivity camera tube with a linear response to incident light $(\gamma=1)$ and the camera does not vary the vidicon target voltage for automatic gain control. The performance of this camera was good, with the only problems being the limited sensitivity and the absolute lack of technical documentation for commercial video products. Sensitivity was not a problem for LEED patterns from well-ordered metal surfaces. For less wellordered LEED patterns from chemisorbed molecules in large unit cells, where the 
average diffracted veam intensity was at least an order of magnitude less, sensitivity was a noticeable limitation. The camera output noise was $6 \mathrm{mV}$ peak-topeak.

The second camera is a scientific instrumentation camera manufactured by Dage-MTI ${ }^{10}$ equipped with a $25 \mathrm{~mm}$ RCA Ultricon II type $4532 / \mathrm{U}$ silicon target vidicon. This camera has a $52 \mathrm{~dB} 18 \mathrm{MHz}$ video amplifier and it was ordered with external manual controls for bandwidth, gain, black level and target voltage, which allows optimization of the camera sensitivity for weak diffaction patterns. The noise level was $4 \mathrm{mV}$ at $6 \mathrm{MHz}$ bandwidth and $6 \mathrm{mV}$ at $18 \mathrm{MHz}$. The $25 \mathrm{~mm}$ silicon target vidicon had a higher sensitivity and resolution and was a better spectral match to the P-11 phosphor used in the LEED optics. Both cameras were used with a $25 \mathrm{~mm} / / 0.85$ lens which images the entire LEED screen.

The LEED experiments were recorded on a Panasonic NV-8050 video recorder, also designed for security applications, using Fugi HG VHS video cassettes. This is an adequate choice, but there has been significant improvement in inexpensive VCR's over the last few years and better quality recorders are available. The bandwidth of the video recorder is less than for the camera and the synchronization: stability is just sufficient for the digitizer. In addition the video-recorder gain drifts as it warms up, so unless the recorder has reached thermal equilibrium there can be errors in intensities and background subtraction. The VCR output noise is $\sim 15 \mathrm{mV}$ peak-to-peak.

The practical dynamic range of our system for I-V curve measurement is somewhat over two orders of magnitude in integrated intensity. This dynamic 
range is usually enough to generate an I-V curve for a given beam. When there are large differences in intensity between different diffraction beams, for example between substrate and superlattice diffraction beams, the same data can be recorded at two different camera lens openings ( $f$-stops). In one run the strong I$V$ curves are measured, although the weak beams may be lost in the system noise. In the second run the camera lens opening is increased while the weaker I-V curves are measured and the strong beams inay saturate the video camera electronics at times. This two-pass method insreases the effective dynamic range of the system. The lens $f$-stop ranges from 0.85 to 32 , which changes the vidicon illumination by three orders of magnitude. If multiple-passes for data acquisition are tolerable, the effective dynamic range of $10^{5}$ is sulficient for most LEED measurements, although the ultimate sensitivity is still limited.

In any system using analog-to-digital conversion the sensitivity and dynamic range is limited by the conversion step. The digitizer resolves the video dark to light range into 256 steps and the background noise in a single frame digitized from the video recorder is $\sim 3$ to 4 steps. Integrating 16 fames is sufficient to reduce the noise to \pm 1 step, the limit for the system. The dynamic range for a single pixel is no more than 256 ; however, a typical leed spot has an area of $\sim 20$ to 60 pixels, so the range of integrated intensity values is significantly larger. In practice integrated intensities between $\sim 200$ and 50000 are reproducible (for a fixed !ens opening). An integrated intensity of $\sim 200$ corresponds to the effective noise level for video intensity measurement. 


\subsection{Performance}

The video LEED data system and analysis programs have been operating reliably since 1983. I-V curves were measured for the clean $\mathrm{Pt}(111)$ surface as a test of the equipment. There was excellent agreement with previously published I-V curves for this surface ${ }^{17,18,19}$ The first new system to be studied with the video LEED apparatus was the surface structure of the $(\sqrt{3} \times \sqrt{3})$ R30 $0^{\circ}$ reconstructed phase on the (111) face of a $\alpha$-copper-16 at. \% aluminum alloy crystal. ${ }^{20}$ This work was the first structure determination for an ordered alloy surface where there was no long range order in the bulk alloy (see chapter 13). The excellent agreement among I-V curves for symmetry related diffraction beams and the reproducibility of the $\mathrm{I}-\mathrm{V}$ measurements for the $\mathrm{Cu}-\mathrm{Al}$ alloy system are shown in figure 5.4 .

The video LEED system has been used to study other systems, including the structure of carbon monoxide on $\mathrm{Pt}(111)$ in the $\mathrm{c}(4 \times 2)$ phase ${ }^{21}$ (chapter 10), the structure of incommensurate graphitic overlayers of carbon on $\mathrm{Pt}(111)^{22}$ (chapter 12), the structure of benzene and carbon monoxide co-adsorbed on $\mathrm{Rh}(111)$ in the $\mathrm{c}(2 \sqrt{3} \times 4)$ rect pattern ${ }^{23}$ and in the $(3 \times 3)$ pattern. ${ }^{24}$

I-V curves were successfully calculated for the $(2 \sqrt{3} \times 4)$ rect LEED pattern of benzene and carbon monoxide co-adsorbed on the (111) surface of platinum ${ }^{25}$ (chapter 11), the most complex diffraction pattern analyzed to date with our video LEED system. I-V curves for 70 different beams were recorded between 20 and $150 \mathrm{eV}$. The video LEED system was able to resolve I-V curves for beams separated in reciprocal space by only $12 \%$ of the substrate unit cell size. 


\section{References}

1. P. Heilmann, E. Lang, K. Heinz, and K. Müller, Applied Physics, vol. 9, p. $247,1076$.

2. D. G. Welkie and M. G. Lagally, Applications of Surface Science, vol. 3, p. $272,1979$.

3. E. Lang, P. Heilmann, G. Hanke, K. Heinz, and K. Müller, Applied Physics, vol. 19, p. 287, 1979.

4. H. Leonhard, A. Gutmann, and K. Hayek, Journal of Physics E: Scientific Instruments, vol. 13, p. 298, 1980.

5. P. Heilmann, E. Lang, K. Heinz, and K. Müller, in Determination of Surface Structure by LEED, ed. P. M. Marcus and F. Jona, Plenum Press, New York, 1984.

6. B. Bölger and P. K. Larsen, Review of Scientific Instruments, vol. 57, p. $1363,1886$.

7. S. P. Weeks, J. E. Rowe, S. B. Christman, and E. E. Chaban, Review of Scientific Instruments, vol. 50, p. 1249, 1979.

8. Digital Design, p. 78, October 1982.

9. Electronic Design, p. 215, May 1982.

10. 68 Series $^{\odot}$ instrumentation camera, Dage-MTI Inc., Michigan City, Indiana 46360. 
11. Panasonic Newvicon type S4076.

12. RCA Ultricon II ${ }^{\oplus}$ type $4532 / \mathrm{U}$.

13. Varian model $881-2145$, Varian Corp., Palo Alto, CA.

14. IP-512 System, Imaging Technology, Inc., Wobern, MA.

15. LSI-11/23 Q-bus ${ }^{\circledast}$, Digital Equipment Corp., Maynard, MA.

16. K. Müller, E. Lang, L. Hammer, W. Grimm, P. Heilmann, and K. Heinz, in Determination of Surface Structure by LEED, ed. P. M. Marcus and F. Jona, Plenum Press, New York, 1984.

17. D. L. Adams, H. B. Nielsen, and M. A. Van Hove, Physical Review B, vol. 20, p. 4788,1979 .

18. P. C. Stair, T. J. Kaminska, L. L. Kesmodel, and G. A. Somorjai, Physical Review B, vol. 11, p. 623, 1975.

10. P. C. Stair, Ph.D. Thesis, Lawrence Berkeley Laboratory Report, LBL-6221, University of California at Berkeley, 1977.

20. R. J. Baird, D. F. Ogletree, M. A. Van Hove, and G. A. Somorjai, Surface Science, vol. 165, p. 345, 1986.

21. D. F. Ogletree, M. A. Van Hove, and G. A. Somorjai, Surface Science, vol. 173, p. 351. 1986.

22. Hu Zi-Pu, D. F. Ogletree, M. A. Van Hove, and G. A. Somorjai, Surface Science, to be published. 
23. M. A. Van Hove, R. F. Lin, and G. A. Somorjai, Journal of the American Chemical Society, vol. 108, p. 2532, 1986.

24. M. A. Van Hove, R. F. Lin, G. A. Blackman, and G. A. Somorjai, Acta Crystallographica, to be published.

25. D. F. Ogletree, M. A. Van Hove, and G. A. Somorjai, Surface Science, in press. 


\section{Figure Captions for Chapter 5}

5.1 a) Video LEED Data Acquisition System block diagram, showing video signal and synchronization connections.

b) Voltage-isolated LEED power supply control circuit.

5.2 Digital Video Processor block diagram, showing video signals, video data flow, computer data flow and computer control lines.

5.3 LEED images of the $c(4 \times 2)$ structure of carbon monoxide, chemisorbed on the (111) surface of platinum, recorded on video tape and displayed on the video monitor.

a) A single video frame.

b) The same image with contrast enhanced by the video processor. Both the LEED spots and the noise are more prominent.

c) The same image with 16 frames integrated together and the instrumental background subtracted. Note the reduced noise.

d) The same surface structure at a different energy. One LEED spot has been selected by the analysis program.

5.4 I-V curves produced by the video LEED system. These data are from the $(\sqrt{3} \times \sqrt{3}) \mathrm{R} 30^{\circ}$ reconstruction on the (111) surface of an $\alpha$-Cu-Al alloy single crystal, recorded at normal incidence at $150 \mathrm{~K}$ (see chapter 13). The data have been normalized for incident beam current, but are otherwise unprocessed. The upper part of the figure shows I-V curves for three symmetryrelated substrate beams and one of the same beams from a second experiment. The lower part of the figure shows three symmetry-related 
superlattice beams. 
258

\section{LEED DATA ACQUYSITION SYSTEM}

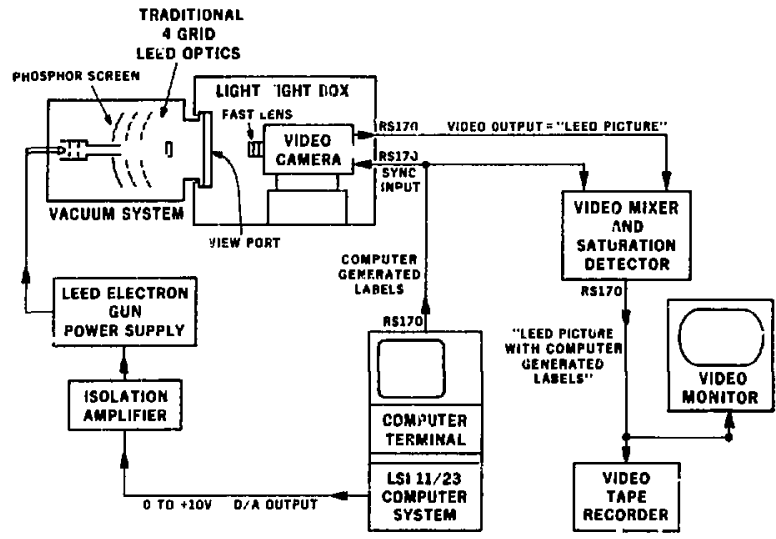

Video Data Acquisition

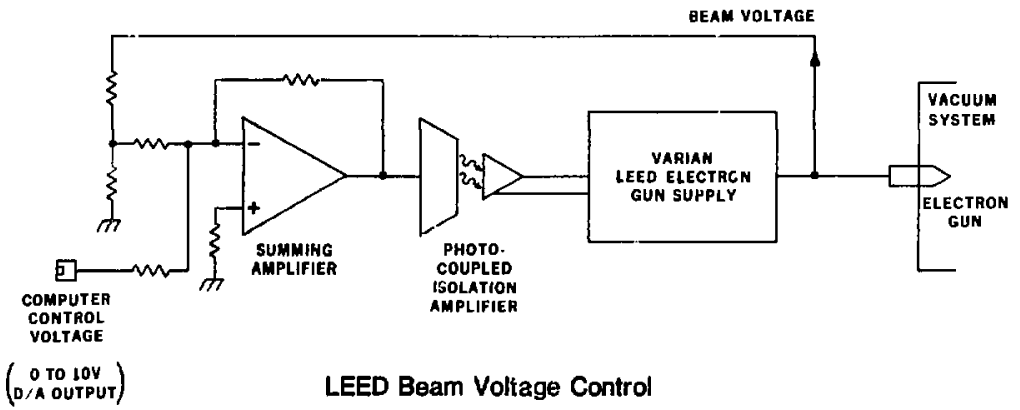




\section{Digital Video Processor Block Diagram}

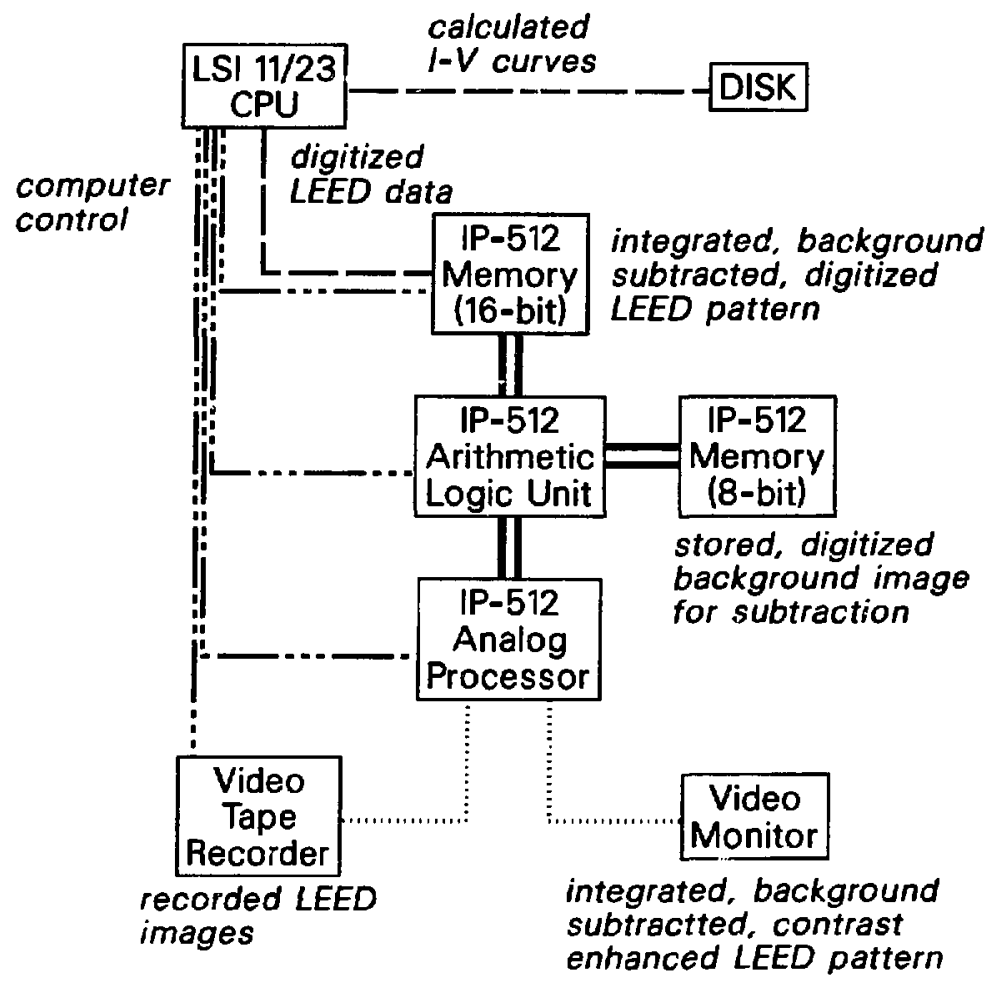

$10 \mathrm{MHz}$ video data bus

RS -170 video sinnal CPU data CPU control lines 


\section{LEED Patterns Displayed on Video Monitor}

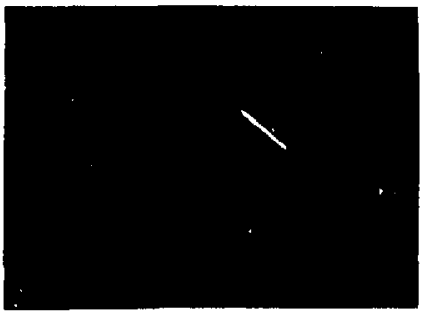

a) raw image from video tape recorder



c) integrated and background subtracted image

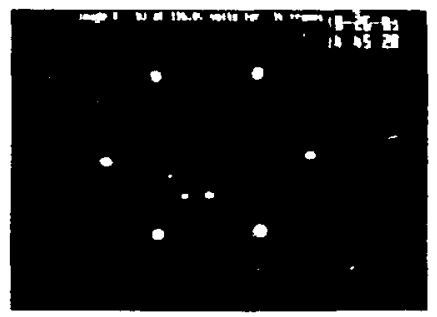

b) contrast enhanced image

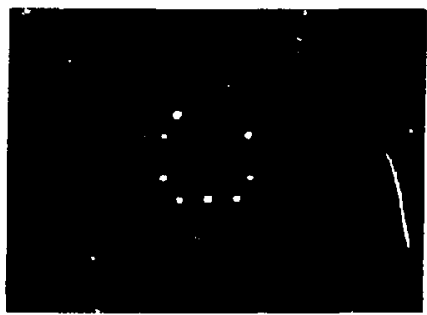

d) LEED spot selected for analysis XBE $365-3580$

Figre 5.3 


\section{$\alpha$-CuAl (111) I-V Curves}

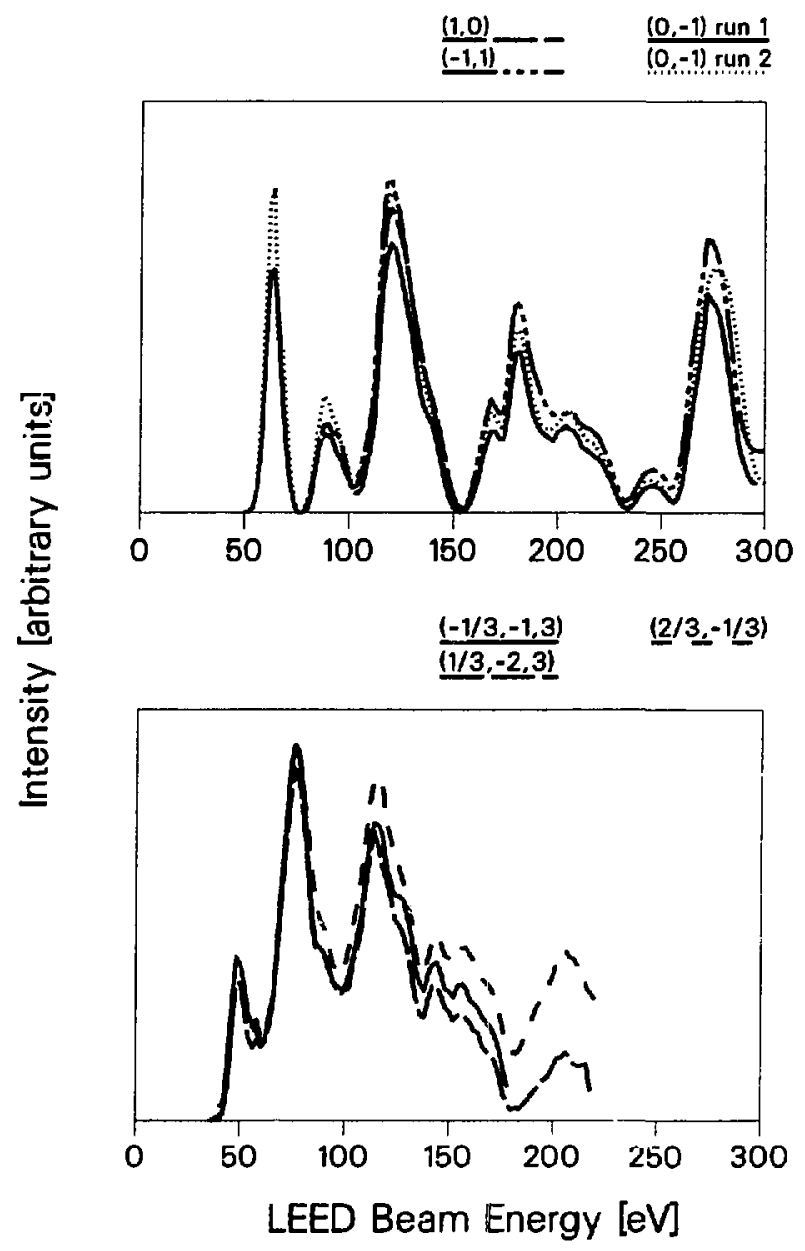

Fgre 5.4

XBL $865-1734$ 


\section{Chapter 6 \\ Electron Counting Techniques and \\ LEED Intensity Measurernents}

\subsection{LEED data acquisition}

The relative advantages of area (image) and point (Faraday cup) detection for LEED were discussed in chapter 2. Area detection is desirable for LEED structure studies since a large fraction of the diffraction pattern may be recorded at one time, which eliminates the need for spot tracking or analysis during data collection. Further, knowledge of the intensity distribution in the neighborhood of a diffraction beam allows the calculation of the integrated diffraction beam intensity with local corrections for the phonon and incoherent electron scattering background (see chapters 2 and 7 ).

Faraday cup detection, on the other hand, provides high spatial resolution and direct measurements of the diffracted electron current so that absoiute reflectivities $R_{\mathbf{g g}^{\prime}}\left(\mathbf{k}_{\mathbf{o}}\right)$ can be determined. When an electron multiplier is incorporated into a Faraday cup the diffracted electrons can be counted directly, with noise then determined by Poisson statistics. In the electron-counting mode the scnsitivity and dynamic range are limited only by the count-rate and the practical counting times.

It is now possible to construct position-sensitive electron counting detectors based on channel-plate electron multiplier arrays that combine the advantages of 
area detection and electron-counting. The construction of such a "digital" LEED detector is described below. An additional advantage of electron-counting is the reduction of the incident electron beam currents by a factor of $10^{3}$ to $10^{6}$ ard of the total electron flux incident during a LEED experiment by $\sim 10^{4}$ to $10^{5}$. The ability to collect a complete set of I-V curves with less than one electron incident per surface unit cell eliminates the problem of electron beam damage during LEED experiments for even the most delicate physisorbed or molecular overlayers.

\subsection{Comparison of video and digital LEED}

The video-digitizer system for LEED data acquisition described in the previous chapter has many advantages for routine experiments. The system is portable and easy to use, it is constructed from commercially available components and it can easily be adapted to existing LEED experimental systems; however, there are intrinsic limitations to the sensitivity and the dynamic range of a video camera based system. For many types of surfaces traditionally studied by LEED, including clean surfaces, surfaces with simple reconstructions and atomic adsorbates with small unit cells the video LEED system has quite adequate performance.

Data acquisition is difficult for surfaces with weak beam intensities. The: sensitivity of the video system was only marginal for the experiments involving chemisorbed benzene (chapter 11), since the overlayer did not order as well as for some simpler adsorbates, and there were forty times more LEED beams than for 
the clean metal because of the large unit cell with three rotationally inequivalent domains.

In practice the effective dynamic range of the video camera based system is somewhat over $10^{2}$, while $10^{3}$ or better is desirable for typical LEED experiments. For diffuse LEED exp sriments on systems without long-range order the backscattered electron intensities may be only $10^{-2}$ to $10^{-3}$ of typical LEED beam intensities and both the sensitivity and the dynamic range of a vidco system are inadequate.

The digital system described below can extend the range of LEED studies. The high sensitivity and dynamic range are suitable for studying surfaces with large unit cells or without long-range order. The very low incident beam current makes it possible to study sensitive systems, including molecular adsorbates, physisorption and molecular crystal surfaces. A completely new detector must be constructed for digital LEED measurements. ithis detector is more complex and more delicate than the traditional display LEED optics because of the use of channel plates. Also the total count rate of the position sensitive detector is limited.

At the current state of development it takes 10 to 100 times longer to record an image of a LEED diffraction pattern at a given signal to noise ratio with the digital LEED system than with the video system, provided the sensitivity and dynamic range of the video system is adequate. The digital system; however, uses far lower incident beam currents and can detect features in LEED patterns that cannot be recorded at all by the video system. 


\subsubsection{Improvements in video techniques}

Channel plates together with a phosphor screen can be used as an analog image amplifier to increase the sensitivity of display LEED systems. Such systems bave been used to study ordering in physisorbed overlayers through analysis of the LEED patterns., ${ }^{1,2}$ There are problems in using channel plates as analog amplifiers for intensity measurements since there is a wide random fluctuation in the channel plate gain when used as an analog amplificr. This is not a problem in pulse counting applications, but it does increase the random and systematic noise in analog applications.

It is more difficult to improve the dynamic range of video detectors. The current generation of video analog-to-digital converters have 8-bit resolution at a $10 \mathrm{MHz}$ sampling rate. A significant improvement in the dynamic range and sensitivity would require 14 to 16 bit analog-to-digital conversion with the video amplifier noise less than $\sim 50 \mu \mathrm{V}$ at $10 \mathrm{MHz}$ and this does not seem likely in the near future.

Two-dimensional charge-coupled device (CCD) arrays are becoming available. A CCD based video camera could offer several advantages over a conventional vidicon camera. The noise level in a CCD system is quite low, so a dynamic range of $10^{3}$ to $10^{4}$ is possible. ${ }^{3,4}$ The effective sensitivity can also be increased since it is much easier to vary integration times without signal degradaticn for CCD's than for conventional vidicons.

Data acquisition systems involving modified video or CCD cameras using non-standard image scanning with channel plates as analog amplifiers seem even 
more complex than pulse counting systems, without all of the advantages, so a position sensitive detector was chosen as a method of extending the limits of LEED detection rather than a hybrid imaging system.

\subsection{Channel plates}

Several different types of position-sensitive electron detectors are being developed for a wide range of applications. All of these detectors use channe] plates (channel electron mulïiplier arrays or microchannel plates) as electron amplifiers. A number of studies of channel plate performance have been published. $5,6,7,8,9$ Channel plates are composed of continuous dynode electron multipliers as small as $8 \mu \mathrm{m}$ in diameter. Arrays up to $75 \mathrm{~mm}$ in diameter are available, with lerger sizes possible on special order. ${ }^{10}$

Channel plates are sensitive to photons above $\sim 10 \mathrm{eV}$ and to charged particles. A single incident low-energy electron can trigger a charge-pulse with a quantum efficiency of over $50 \%$. The gain of a channel is limited by ion-feedback, caused by ions traveling back up the channels. This can be prevented by stacking two channel plates in a "chevron" array, where the channels in the two plates are not parallel. Electrons change dire ion to follow the channels more easily than ions, so high electron gain is possible while ion feedback is suppressed. ${ }^{5,6}$ A pair of channel plates in a chevron arrangement can have a gain up to $\sim 5 \cdot 10^{7}$.

When channel plates are operated in a saturated-gain pulse-counting mode there is a relatively wide output pulse height distribution, -50 to $150 \%$ of the mean pulse height. The gain fluctuations are even greater in the analog mode. 
Background or dark-count rates below 1 count $/ \mathrm{s}-\mathrm{cm}^{2}$ are commonly obtained for channel plates in a chevron array. The spatial resolution of channel plate detectors is ultimately limited by the channel size. The plates used for the LEED detector have $25 \mu \mathrm{m}$ diameter channels in a hexagonal array spaced $32 \mu \mathrm{m}$ apart. In a chevron stack one excited channel in the input plate triggers several channels in the output plate, which degrades the spatial resolution for analog imaging applications.

\subsubsection{Counting rates for channel plates}

The total counting rate of the chevron array is the main factor that limits the performance of the digital LEED detector. The quantrm efficiency, spatial resolution, gain and dark-count rate are all sufficient to excellent for the LEED application.

The channels in a channel plate are coated with a layer of semiconducting glass with a high secondary electron emission coefficient. When electrons strike this layer several secondaries are generated. Electron multiplication generates a pulse of electrons traveling down the channel. The gain saturates as the positively charged channel walls left behind the traveling pulse slow the electrons until the secondary electron emission coefficient drops to one.

Aster a pulse the charge in a channel is depleted and a certain time is required to recharge the channel. An estimate based on the $R C$ time constant of a single channel gives $\tau \approx 0.02 \mathrm{~s}^{5} \mathrm{~A}$ more sophisticated analysis, considering the effects of polarizing the adjacent channels gives a time constant of $\approx 5 R C .^{9}$ For a 
channel plate with $32 \mu \mathrm{m}$ channel spacing there are about 1100 channels $/ \mathrm{mm}^{2}$. The time-constant estimate would imply a maximum count-rate of $\sim 1$ $\mathrm{KHz} / \mathrm{mm}^{2}$. In high count-rate applications the actual limit on the count rate comes from the bias current of the channel plate. ${ }^{8}$ The gain starts to drop significantly when the electron output current exceeds $\sim 20 \%$ of the bias current. For standard $75 \mathrm{~mm}$ channel plates the bias current is rated at $12 \mu \mathrm{A},{ }^{10}$ so the maximum pulse output is $\sim 600 \mathrm{pA} / \mathrm{mm}^{2}$. The measured average outpu pulse size is $\sim 6.5 \mathrm{pC}$, for a gain of $\sim 4 \cdot 10^{7}$. This implies that the channel plate will start to saturate at $\sim 100$ counts $/ \mathrm{mm}^{2}$. Channel plates are now being fabricated with substantially higher bias currents ${ }^{10}$ which should allow pulse counting rates of $10^{3}$ to $5 \cdot 10^{4}$ counts $/ \mathrm{mm}^{2}$, depending on the gain.

\subsection{Position sensitive detectors}

Detectors using channel plates with position-sensitive anodes have been used for many applications, inc,uding space-borne EUV telescopes, ${ }^{11,12} \approx$-ray spectrometers, ${ }^{8}$ dispersive electron spectrometers ${ }^{13}$ and LEED. ${ }^{14,15}$ A number of different detection types of anodes have been used, including multi-anode schemes, coincidence detection and charge division, ${ }^{16,13}$ depending on the resolution and counting rates required. A charge division anode was chosen for the LEED detector. This approach requires only three of four amplifier channels, compared to dozens in coincidence and multi-anode schemes. 


\section{1.1. Resistive anode charge-division}

The first charge-division scheme used a resistive anode. ${ }^{17}$ Charge sensitive amplifiers are connected to each corner of a square thick-film resistor on a ceramic substrate which is placed on the output side of the channel plates. When this resistor is terminated with line-resistors along a circular are the charge collected at each corner is a linear function of position. ${ }^{18}$ Since the anode is linear the anode detects the centroid of the charge distribution, so charge spreading caused hy multiple channel excitation in the second channel plate of a chevron array does not degrade the spatial resolution provided the charge-cloud has radial symmetry, unlike in an optical imaging system. The 1 sistive anode was used by Stair ${ }^{14}$ in the first position sensitive LEED detector and similar detectors are now available commercially. ${ }^{19}$ Using a resistive anode count rates up to $5 \cdot 10^{3}$ with $100 \times 100$ resolution are possible for a detector using $75 \mathrm{~mm}$ channel plates. A resistive anode was also used in the first version of the digital detector described below (see figure 6.1)

The main disadvantage of the resistive anode is that detector performance is limited by the anode properties. Charge division anodes can only detect one charge pulse at a time. If a second pulse arrives before the first has dissipated the position calculation can have large errors. The speed of the resistive anode is determined by the RC time constant. For the $75 \mathrm{~mm}$ detector the parasitic capacitance was $\sim 100 \mathrm{pF}$ and the anode resistance was $\sim 10 \mathrm{~K} \Omega$ /square for a time constant of $\sim 1 \mu$ s. The resulting pulse pair resolution was $\sim 8 \mu$ s (i.e. the minimum time separating valid events). This is longer than the time constant 
because the amplifiers must return to the baseline between events for accurate position calculation.

Resistive anodes work quite well for $25 \mathrm{~mm}$ channel plates; however, for 75 $\mathrm{mm}$ plates the time constant is nine times longer due to the increased capacitance. The resistance cannot be reduced in proportion because the chargesensitive amplifiers become unstable if the inputs are connected by a low impedance path. The linearity of the resistive anode position determination is controlled by the uniformity of the resistive film and the accuracy of the resistive edge terminaticn. In practice the deviations from linearity are $\sim 5 \%$ across the anode. The resolution is limited by the electrical noise of the amplifiers and thermal noise in the resistor.

\subsubsection{Wedge-and-strip anode charge-division}

In order to increase the maximum count rate a new detector using a "wedge-and-strip" anode was constructed. ${ }^{20,11}$ This anode involves three metal conductors in an interlocking pattern with a spatial period $\sim 1 \mathrm{~mm}$. The anode is separated from the channel plate by several millimeters so the output charge cloud spreads out over several anode periods. The relative area of each conductor in the area of the charge "foot print" is a .ıear function of position.

The performance of the wedge-and-strip anode is determined by the system electronics and statistical noise. Wedge-and-strip anodes can be fabricated by photo-lithography to any desired degree of accuracy, so linearity is aot a problem. The $R C$ time constant of the anode and signal cables is $\sim 10^{-8} \mathrm{~s}$, so the count 
rate and resolution are limited by the channel-plate or the digitizing electronics. The wedge-and-strip anode is also better able to discriminate against puise pileup. If two charge pulses arrive at the anode in a time shorter than the processing time the resulting position calculations will be in error. It is much easier to distinguish coincidence events with the $\sim 50$ ns rise time of a metal wedge-and-strip anode than with the $\sim 1 \mu$ s rise time of a resistive anode.

\subsection{Detector construction}

The digital LEED detector is build around a chevron pair of $75 \mathrm{~mm}$ channel plates with a $6 \mathrm{~mm}$ hole in the center. The electron beam passes through this hole and is back-diffracted from the crystal (see figures 6.2 and 6.3).

Three hemispherical grids from a small (100 mm O.D.) Phi LEED optics ${ }^{21}$ are mounted in front of the channel plate. The first grid is grounded so the diffracted electrons travel in a field-free region. The second grid acts as a retarding field energy analyzer. It is biased at $\alpha V_{\text {beam }}-\Delta V$ where $\alpha$ is between 0.8 and 1.0 and $\Delta V$ is between $-10 \mathrm{eV}$ and $+10 \mathrm{eV}$. The energy analyzer parameters are controlled by the power supply (figure 6.4). The third grid is at the front channel plate potential, which floats on $V_{\text {beam }}$ and is biased 100 to $300 \mathrm{~V}$ positive, so electrons are incident on the channel plate at a constant energy independent of beam voltage. The channel plates are biased at $\sim 1000 \mathrm{~V}$ per plate to operate in the saturated-gain mode.

The wedge-and-strip anode is mounted $\sim 6 \mathrm{~mm}$ behind the output side of the channel plates to allow the output charge pulse room to spread. The anode is 
biased by +75 to $+600 \mathrm{~V}$ positive relative to the output side of the channel plates. The exact bias voltage has only a slight influence on the spatial resolution of the anode. A 100-mesh grid is stretched across the back of the channel plate holder to insure a uniform electric field between the channel plates and the anode. The initial design used a $15 \mathrm{~mm}$ anode to channel plate spacing with two field termination rings to allow sufficient time for the charge-clouds to expand before landing on the wedge-and-strip anode ${ }^{12}$ however, a $6 \mathrm{~mm}$ gap without field termination was found to perform just as well, even for a wedge-and-strip anode with a $1.5 \mathrm{~mm}$ anode period. There is a slight radial distortion of the LEED pattern since it is projected onto the flat channel plates and anode. This is easily corrected by the computer display software if necessary.

The electron gun is enclosed in a " $\mu$-metal" magnetic shield and a second shield encloses the entire detector. The electron gun beam tube at ground potential passes through the anode and channel plates at $\sim 2 \mathrm{KeV}$. This produces a strong electrostatic field which distorts the charge cloud diffusion between the channel plates and the anode, so an electrostatic shield is wrapped around the beam tube and biased to a voltage intermedjate between the anode and the output side of the channel plate. The exact bias voltage does not seem to effect the anode performance provided $V_{\text {output }} \leq V_{\text {bias }} \leq V_{\text {anode }}$. 


\subsubsection{Data system}

The digitizer electronics put out a 16-bit address ( 8 bits each for $\mathrm{X}$ and $\mathrm{Y}$ ) after each valid event. This corresponds to a detector spatial resolution of $256 \times 256$ pixels where each pixel is $\sim 1 / 3 \mathrm{~mm}$ square. Analog voltages proportional to the electron position are also produced to drive a live oscilloscope display which provides a real-time image of the LEED pattern independent of the computer system.

The digital addresses of detected electrons are stored in a "histograming memory". This is a memory with $256 \mathrm{~K}$ channels. Each time an electron is detected one count is put in the appropriate channel. Each channel can store up to $64 \mathrm{~K}$ counts and the memory runs at $2 \mathrm{MHz} .^{22}$ The histogram memory can collect data independently of the computer system under the control of a scaler/timer, so one LEED pattern can be collected while the previous image is stored or analyzed. The histogram memory is a CAMAC module and a 256 $256 \times 16$-bit LEED pattern can be transferred through the CAMAC controller to the computer $\mathrm{CPU}$ in $\sim 0.5 \mathrm{~s}$. The same computer system used for the digital LEED system is also used to analyze video LEED data (chapter 5) and so the digital video processor can be used to enhance and display the digitized LEED patterns from the digital LEED detector. 


\subsubsection{Digitizer electronics}

The position sensitive detector consists of the three-electrode wedge-and-strip anode with an amplifier circuit for each channel. This amplifier circuit includes a fast, charge-sensitive pre-amplifier followed by a shaping amplifier, a peak-detect and sample-and-hold circuit and a fast 12-bit analog-to-digital converter. Position calculating logic determines the position of electron event from the digitized outputs of the three charge-sensitive amplifier channels. The digitizer electronies can calculate positions at a rate of greater than $1 \mathrm{MHz}$ with a spatial resolution of 8 to 10 bits for a resolution of 256 to 1024 pixels across the anode diameter. The anode and all of the digitizer electronics were designed and constructed at LBL. (Circuit diagrams and construction specifications are available on request - see appendix I for details.)

\subsection{The wedge-and-strip anode}

Figure 6.5 shows a schematic drawing of the wedge-and-strip anode. This anode has three electrodes; the wedges (A), the strips (B) and the remaining area between the wedges and strips (C). If a charge cloud lands on the left side of the anode, on the narrow strips, the $x$ coordinate is small and if it lands on the right side, where the strips are wide, the $x$ coordinate is larger. Likewise, if the charge cloud lands near the botiom of the anode, near the peak of the wedges, the $y$ coordinate is small and if it lands near the top of the anode, at the base of the wedges, the $y$ coordinate is large. The positions are proportional to

$$
x=\frac{Q_{B}}{Q_{A}+Q_{B}+Q_{C}} \text { and } y=\frac{Q_{A}}{Q_{A}+Q_{B}+Q_{C}}
$$


The exact coordinates are scaled depending on the ratio of the maximum and minimum widths of the strips and wedges.

\subsubsection{Anode resolution}

Several studies have been made of the performance of wedge-and-strip anodes. ${ }^{11,23,12,24,25,26}$ The spatial resolution of the wedge-and-strip anode depends on the system noise. There are two main sources of noise for a wedgeand-strip anode, partition noise and amplifier noise. Partition noise arises because of statistical fuctuations in the division of electrons betwcen the discrete electrodes. This can be significant since only $10^{6}$ to $10^{7}$ are incident on the anode. Siegmund et al. ${ }^{23,26}$ bave shown that the spot-broadening due to partition noise for a wedge-and-strip anode is

$$
\frac{\Delta L}{L}=\frac{2.35[f(1-f)]^{1 / 2}}{f_{\max }-f_{\min }} \cdot \frac{1}{N}
$$

where $N$ is the number of electrons incident on the anode, $f N$ is the number of electrons collected on the strip (wedge) at the given $x(y)$ position and $f_{\max }$ and $f_{\text {min }}$ are the limits on $f$. The factor or 2.35 comes from the anode geometry. For an idealized anode $f_{\max }=0.5$ and $f_{\min }=0$; but this range is smaller for a real anode since there must be an insulating gap between electrodes, and there is also capacitive coupling between electrodes. The effect of partition noise is largest near the center of the anode.

The charge sensitive amplifier is the other noise source in the system. The anode is used with an integrating pre-amp with a slow decay time. A typical 
pre-amp wave form is shown in figure 6.4. The sharp steps in the pre-amp output voltage correspond to the integrated charge on the given electrode for an incident charge pulse. The shaping amplifier differentiates this waveform. The pre-amp noise, in terms of charge at the input, is

$$
N_{\text {noisc }}=\frac{1}{e} C_{i n} V_{\text {in }}
$$

where $C_{i n}$ is the input capacitance due to the anode, $V_{i n}$ is the arnplifier noise referred to the input and $\tau$ is the rise-time of the input charge pulse. The input noise for a typical FET pre-amp is $\sim 1 \mathrm{nV} / \mathrm{V}_{\mathrm{Hz}}$. The effect of amplifier noise on resolution in the $x$ direction is

$$
\frac{\Delta L}{L}=\frac{2.35\left[N_{A}^{2}(1-f)+f^{2}\left(N_{B}^{2}+N_{C}^{2}\right)\right]^{4 / 2}}{f_{\max }-f_{\min }} \cdot \frac{1}{\sqrt{N}}
$$

where $N_{A}$ is the amplifier noise for channel $A$ in units of electrons. A similar expression holds for spot-broadening in the $y$ direction with $N_{B}$ and $N_{A}$ exchanged. The electronic noise contribution is largest near the edges of the anode. The overall resolution is better for large $N$ and large $f_{\max }-f_{\min }$ and the amplifier noise contribution decreases with small $C_{i n}$. A good anode design should maxirize $f_{\max }-f_{\min }$ and minimize $C_{i n}$.

The amplifier noise is related to the band-width $\left(\frac{1}{\tau}\right)$ of the pre-amp. Channel plates produce very sharp output pulses, $\sim 1$ ns in the chevron configuration ${ }^{5}$ and the measured pre-amp rise time is less than 50 ns. The rise time of a charge sensitive amplifier is given by ${ }^{27}$ 


$$
\tau_{\text {ribe }}=Z_{\text {in }} C_{\text {in }}=\frac{1}{g_{m}} \frac{C_{o}}{C_{f}} C_{i n}
$$

where $Z_{i n}$ is the amplifier input impedance, $g_{m}$ is the input amplifier transconductance, $C_{f}$ is the feedback capacitance and $C_{0}$ is the output capacitance. The feedback capacitance $C_{f}$ is set by the pre-amp gain, which gives a $40 \mathrm{mV}$ output pulse for an input charge of $10^{7}$ electrons, or $1.8 \mathrm{pC}$ and $g_{m}$ is a property of the amplifier. The output capacitance $C_{0}$ can be increased to "roll off" the pre-amp frequency response and decrease the noise bandwidth; however, this causes a proportional increase in the pre-amp input impedance and this in turn increases the coupling between the $\mathrm{A}, \mathrm{B}$ and $\mathrm{C}$ electrodes, which degrades the anode performance.

\section{B.6.2. Anode coupling}

Capacitive coupling between the discrete electrodes on the wedge-and-strip anode distorts the image. If a certain charge is deposited onto one electrode some charge will be induced on the other electrodes. If $Q_{A}, Q_{B}$ and $Q_{C}$ are the incident charge distribution due to the anode geometry, the measured charge distribution will be

$$
\begin{aligned}
& Q_{a}=(1-\alpha-\gamma) Q_{A}+\gamma Q_{B}+\alpha Q_{C} \\
& Q_{b}=(1-\beta-\gamma) Q_{B}+\gamma Q_{A}+\beta Q_{C} \\
& Q_{c}=(1-\alpha-\beta) Q_{C}+\alpha Q_{A}+\beta Q_{B}
\end{aligned}
$$

where $\alpha, \beta$ and $\gamma$ are the coupling constants between electrodes A-C, B-C and AB, respectively. This coupling will be proportional to the inter-electrode 
capacitance, since the induced charge $\Delta Q=C \Delta V$. For an ideal pre-amplifier the input is a virtual ground and so $\Delta V$ would be zero. Since the real amplifier has finite gain the input impedance is non-zero from Eq. 6.5 and the input signals are coupled through the voltage change $\Delta V$.

Instead of the geometrical position $(x, y)$ being calw.inted from the geometrical charge distrijution $Q_{A}, Q_{B}$ and $Q_{C}$, a distorted position

$$
\begin{aligned}
& x^{\prime}=(1-2 \alpha-\gamma) x+(\gamma-\alpha) y+\alpha \\
& y^{\prime}=(1-2 \beta-\gamma) y+(\gamma-\beta) x+\beta
\end{aligned}
$$

is calculated from the measured charge distribution $Q_{a}, Q_{b}$ and $Q_{c}$. This coupling has two effects; first the overall size of the image is reduced, since the effective $f_{\max }-f_{\min }$ is reduced and second the image is distorted because of the coupling bitween $x$ and $y$. From the form of Eq. 6.7 this distortion corresponds to a compression of distances in the image along the $x=-y$ diagonal relative to distances along the $x=y$ diagonal.

For the wedge-and-strip anode $\alpha \approx \beta>\gamma$ since the $\mathrm{A}$ and $\mathrm{B}$ electrodes are separated by the $\mathrm{C}$ electrode. If an additional external capacitor connecting the wedge (A) and strip (B) electrodes is added to the anode to equalize the interelectrode capacitances $C_{A B}=C_{B C}=C_{A B}$ then the coupling constants will be equal and the anode image will be undistorted, with

$$
x^{\prime \prime}=\epsilon x+x_{0} \text { and } y^{\prime \prime}=\epsilon y+y_{0}
$$

where $\epsilon=1-3 \alpha$ is a scale factor and $(x, y)$ is the geometrical position 
The coupling constants were measured experimentally by connecting the anode to the pre-amplifier and driving one channel with a test pulse, then measuring the shaping amplifier output pulse heights. This gave $\alpha, \beta \approx=0.18$ and $\gamma \approx 0.12$ with the maximum pre-amp band-width (with no external roll-off capacitance $C_{0}$ for minimum $Z_{i n}$ ). When the inter-electrode capacitances were balanced the scale factor $\epsilon \approx 0.4$. This decrease in size has the effect of increasing the relative spot broadening due to partition noise and electrical noise. When the pre-amp rise time was rolled off to $\sim 1 \mu$ s the pre-amp input impedance was increased by a factor of about 20 and the image size scale factor $\epsilon$ dropped below 0.10 .

The measured wedge-and-strip anode capacitances were $C_{A B}=256 \mathrm{pF}$ and $C_{A C}=C_{B C}=441 \mathrm{pF}$ for the anode alone. When the detector was installed in the vacuum chamber and the pre-amp cables were connected $C_{A B}=545 \mathrm{pF}$ and $C_{A C}=C_{B C}=721 \mathrm{pF}$. After the inter-electrode capacitances were balanced $C_{A B}=C_{B C}=C_{C A}=1010 \mathrm{pF}$. This method of correcting the anode distortions increases the amplifier noise (Eq. 6.2) by $\sim 50 \%$ and decreases the image size by $\sim 1 / 3$ for a further reduction in resolution.

There is an alternative solution to the anode distortion problem. For the case when $\alpha=\beta \neq \gamma$, which is a good approximation for the wedge-and-strip geometry, a new position calculation algorithm

$$
x=\frac{Q_{b}-\rho Q_{c}}{Q_{a}+Q_{b}+Q_{c}}+x_{a} \text { and } y=\frac{Q_{a}-\rho Q_{c}}{Q_{a}+Q_{b}+Q_{c}}+y_{o}
$$

nuay be used in place of the original position algorithm Eq. 6.1, where 
$f=\frac{\gamma-\alpha}{1-3 \alpha}$ and $Q_{a}, Q_{b}$ and $Q_{c}$ are the experimentally measured charges. This algorithm gives the undistorted geometrical position directly in terms of the measured charges without the addition of external capacitors. With $f$ as defined, the scale factor $\epsilon=1-\alpha-2 \gamma$, so with the anode described above $\epsilon=0.6$, compared to $\epsilon=0.4$ for the compensated anode. With the algorithm of Eq. 6.9 the resolution is better by $\sim 50 \%$ and the input capacitance is snialler. The price for this improvement is more complex digitizer logic.

\subsubsection{Anode design}

For optimum resolution the anode should have low inter-electrode capacitance and $f_{\max }-f_{\min }$ should be large. The anode period was chosen to be 1.5 mm with an insulating gap of $44 \mu \mathrm{m}$ between electrodes. A large period reduces the inter-electrode capacitance since the total length of the electrode border is reduced. A larger period also reduces the fraction of the anode area occupied by insulating gaps. There are four gaps per period, so for a $1.5 \mathrm{~mm}$ period $\sim 88 \%$ of the area of the anode is occupied by the electrodes. The size of the gap was limited by fabrication considerations -. with a smaller gap shorts between the electrodes were a significant problem. The minimum and maximum strip and wedge widths were $50 \mu \mathrm{m}$ and $662 \mu \mathrm{m}$ so $f_{\min }=0.038$ and $f_{\max }=0.500$.

The active area of the anode was restricted to a circle $47 \mathrm{~mm}$ in radius (see figure 6.6(a)). This allows $10 \mathrm{~mm}$ outside the $37.5 \mathrm{~mm}$ active area of the channel plate for charge cloud spreading. For a square anode $f_{\max }$ must be less than 0.5 , 
otherwise the maximum width strip and the base of the adjacent wedge will intersect. The wedge-and-strip pattern is distorted at the center of the anode to allow the electron gun to pass through a $3 \mathrm{~mm}$ radius hole in the center (see figure 6.6(b)). The pattern is normal for $r>3.65 \mathrm{~mm}$. There is an inactive area of the channel plate near the center hole so the distorted region of the anode does not cause spurious counts.

\subsubsection{Anode fabrication}

The wedge-and-strip anode was produced by photo-lithography. A quartz glass substrate was chosen for its stability and low dielectric constant. The surface was abraded with 300 grit alumina for better adhesion, then $\sim 5 \mu \mathrm{m}$ of copper was evaporated after plasma cleaning the substrate.

A computer program (available on request, parameters are included in appendix iI) was used to draw a four times full scale mask of the wedge-and-strip anode using a Gerber photo-plotter and this mask was photographically reduced onto plastic. The masks were produced by an outside vendor from a computer tape. $^{28}$

The copper-plated substrate was coated with photo-resist and baked at $150^{\circ}$ $\mathrm{C}$ and then exposed for $30 \mathrm{~s}$ to UV light. A vacuum hold-down jig kept the mask in contact with the anode surface during the photoresist exposure. After exposure the anode was developed and the copper was etched in a ferric chloride solution ( $50 \mathrm{~g} \mathrm{FeCl}_{3}$ in $100 \mathrm{~cm}^{3}$ of distilled water) for $\sim 100 \mathrm{~s}$. The photo-resist was then removed with acetone. 
The finished anodes usually had a number of minor shorts between the conductors, probably because the anode fabrication was not carried out under cleanroom conditions. These shorts were removed by (tedious) selective etching or mechanical abrasion under a binocular microscope.

\subsection{Digitizer design}

The position calculation algorithm is implemented in high speed digital logic, rather than with the analog ratioing circuitry and successive-approximation digitization used in commercial resistive-anode based position sensitive detectors. ${ }^{19}$ The outputs of the three charge-sensitive integrating pre-amps go to differentiating shaping amplifiers which produce a smoothed pulse on a DC baseline with a height proportional to the integrated charge output of the pre-amp.

The three pre-amp outputs are summed by a fourth shaping amplifier (see figure 6.5). The output of this amplifier is used for threshold, overload and peak detection. The charge of the average output pulse is about $6.5 \mathrm{pC}$ and the threshold and overload levels are set to $\sim 1$ and $10 \mathrm{pC}$, respectively. Any events which fall outside these limits are not considered valid. A peak detection circuit on the sum output triggers the separate analog-to-digital converters on the $\mathrm{A}, \mathrm{B}$ and $\mathrm{C}$ channels. These are 12-bit ADC's (Burr-Brown $803 \mathrm{CM}$ ) with a conversion time of $\sim 750 \mathrm{~ns}$.

To maintain the potential resolution of the anode ( $\sim 500$ lines) the charge on each channel must be calculated with high accuracy. The average pulse height for a single channel is $\sim 1 \mathrm{~V}$. To determine positions with 8-bit accuracy the 
charges should be calculated with 9 to 10 bit accuracy, or $\pm 2 \mathrm{mV}$. This means the shaping amplifier offset and gain must be accurate on this level and the shaping amplifiers must return to the baseline within $\sim 2 \mathrm{mV}$ between pulses. Once the charges are digitized the position calculation circuitry maintains the initial accuracy.

\subsubsection{Pulse pile-up}

If a charge-pulse arrives on the wedge-and-strip anode before the shaping amplifiers have returned to the baseline from the previous pulse there can be significant position errors. When two bright features are observed at high count rates with a resistive anode a third "ghost" feature can sometimes be seen halfway between them because of coincidences or pulse pile-up. ${ }^{13}$

The digital detector has fast pulse pile-up rejection logic to prevent this problew. A fast summirg amplifier is also attached to the pre-amp outputs (see figure 6.5) along with the the four slower shaping amplifiers. When a pulse is detected above a certain thresnold (which can be set independently of the charge threshold described above) the logic is enabled. If a second pulse arrives before the analog-to-digital conversion is complete, both events are rejected. Once the analog-to-digital conversion is complete the first position is calculated. The digitizer logic will not accept a new input pulse until the shaping amplifier has had time to return to the baseline. If another pulse arrives before this point the delay time is reset. 
The pulse pile-up logic can resolve pulses separated by only $50 \mathrm{~ns}$, approximately the pre-amp rise time. This means that the detector resolution is independent of count rate. At high event rates the valid count-rate drops because of pulse pile-up and reductions in the channel plate gain. The shaping amplifier pulse length is now set at $4.5 \mu \mathrm{s}$, which corresponds to a theoretical maximum count rate of $\sim 220 \mathrm{KHz}$. For randorì events (Poisson statistics) the maximuin effective count rate will be about $100 \mathrm{KHz}$. This is not a limitation on the amplifier, since the shaping time could be reduced to $\sim 1 \mu$ at the cost of a larger noise band-width; however, LEED patterns usually include a number of bright diffraction beams and the channel plates will probably be locally saturated before the average count rate reaches $100 \mathrm{KHz}$. With the new, high bias-current channel plates higher total count-rates may be practical.

\subsubsection{Digital calculations}

Digital logic computes the diffracted electron positions using the algorithm of Eq. 6.9 (see figure 6.7). The 12-bit outputs of the analog-to-digital converters are summed to calculate $A+B+C$ and the inverse $\frac{k}{A+B+C}$ is calculated using a 16-bit look-up table with a 12-bit address. The product $f C$ is also calculated with a look-up table. Both look-up tables are EPROMS (electrically programmable read-only memories) so the scale factor $k$ and the anode coupling compensation constant $f$ can be easily changed. The values of $f$ and $k$ depend on the anode geometry through the inter-electrode capacitances and on the pre-amp input impedance. The $f$ value is first chosen to produce an undistorted image 
and then $k$ is chosen so the actual image of the detector fills the $256 \times 256$ pixel address space. Arbitrary digital offsets $x_{0}$ and $y_{0}$ may be subtracted from the computed $x$ and $y$ positions to center the digital image of the detector in the address space. The offsets are set by 12-bit dip switches. At this time there are 8 significant bits in the $x$ and $y$ outputs. The digitizer electronics are capable of up to 10 significant bit accuracy (1024 $\times 1024$ pixels) if the anode and the analog-todigital conversion are accurate enough to justify this precision.

\subsection{Electron gun}

The very low incident beams currents used for digital LEED experiments ( 0.1 to $100 \mathrm{pA}$ ) allow for a significant improvement in the LEED instrumental resolution. As disctssed in chapter 2, the main factor limiting the "coherence length" or angular resolution of mosc LEED instruments is the angular divergence of the electron gun as seen from the detector. For a display LEED optics the requirement of $\sim 1 \mu \mathrm{A}$ beam currents at $\sim 100 \mathrm{~V}$ makes it difficult to achieve a beam divergence less than $\sim 1^{\circ}$. A reduction in beam current by $>10^{4}$ should permit improved electron gun performance.

At this time the digital LEED system uses an electron gun of conventional design that has been used for LEED together with channel plate analog amplifiers by several other groups. ${ }^{29}$ This gun, shown schematically in figure $6.2,{ }^{30}$ consists of a triode electron gun with a beam-limiting aperture followed by an Einzel condenser lens. ${ }^{31}$ 
The digital LEED power supply controls the electron gun. The thoriated iridium filament is heated by a DC current of 4 to $5 \mathrm{~A}$. The next lens element is the Wehnelt, or control grid. This is biased negative relative to the filament by 12 to $18 \mathrm{~V}$ (for pA beam currents). The next electrode, the "first anode" is biased 300 to $400 \mathrm{~V}$ positive with respect to the filament independent of beam voltage. A beam-limiting aperture is attached to the first anode so that it falls at the cross-over (focus) of the triode electron source made up of the filament, Wehnelt and first anode. Apertures from $5 \mu \mathrm{m}$ to $625 \mu \mathrm{m}$ are available and a 37 $\mu \mathrm{m}$ aperture was used for the digital LEED gun. The alignment of the first three elements is critical for the gun performance and the guns are factory aligned under an optical microscope. ${ }^{30}$

The condenser lens images the beam cross-cver onto the sample. This is an Einzel $^{31}$ lens, so the first and final elements are at ground and the center, focusing element is adjusted for optimum performance. The gun is equipped with two sets of deflection plates. The deflection voltages are proportional to the beam voltage and they are adjusted so the beam passes through the $4 \mathrm{~mm} \mathrm{O.D.} \mathrm{beam} \mathrm{tube.}$

Depending on the beam voltage the electrons are decelerated (low voltages) or accelerated (high voltages) between the first anode and the condenser lens. The beam voltage and the conderser lens voltage are independently controlled by the computer system. For beam voltages over $\sim 75 \mathrm{~V}$ the gun is well focused when the condenser lens is at $\sim 70 \%$ of the beam voltage. For low voltages the focus must be adjusted as the beam voltage changes to maintain the optimum spot size. Operating the first stage of the electron gun at constant voltages 
irdependent of the beam voltage has two important advantages. First, the magnetic field effects are constant, so the gun performance is more uniform and secondly, the gun current is approximately independent of the beam voltage.

When the electron gun is directed into the LEED detector the spot size can be focused down to 1 to 2 pixels. The spot size produced by this type of gun has been measured at $\sim 300 \mu \mathrm{m} .^{32}$

\subsection{Performance}

The digital LEED detector and electronics have been tested by several methods. The anode and digitizer can be bench tested using a shielded probe attached to a pulse generator. A voltage pulse on the probe will capacitively couple charge onto the wedge-and-strip electrodes. As the probe is moved over the anode the digitized position changes. This kind of test can be used to set the gain, compensation and offset values $\left(k, f, x_{0}\right.$ and $\left.y_{0}\right)$. The detector was tested in vacuum by placing a mask was over the input side of the channel plates with a $5 \mathrm{~mm}$ grid of pin-holes and sweeping a defocused electron beam across the surface. This was used to check the linearity and resolution of the detector under operating conditions. Finally, the LEED electron gun was mounted opposite to the detector and the spot profile was measured. This tested the detector, power supply and electron gun.

The linearity of the detector was quite good once the digitizer parameters were calibrated and adjusted. The incident electron gun spot could be imaged over the entire detector, with no distortion at the outer edges of the channel 
plates. There is a dead region in the center 10 to $12 \mathrm{~mm}$ in diameter, which is reasonable since the anode is distorted over a $7.2 \mathrm{~mm}$ diameter. When a single spot is focused on the detector the effects of channel plate saturation are clearly visible for count rates greater than $300 \mathrm{~Hz}$ for a well focused spot. For distributed counts the detector works well at $\sim 30 \mathrm{KHz}$ with $\sim 80$ to $85 \%$ valid counts. Counts are rejected for pulse pile-up or too small or t,o large events.

\subsubsection{LEED statistics}

The performance of the digital LEED system is limited by the effective counting rate. The number of counts in a image required to resolve a given feature can be estimated from the counting statistics. For a random process like electron diffraction the statistical fluctuation or noise associated with an image feature is given by the square root of the total number of counts in the feature, so the signal-to-noise ratio is $\left(\frac{s}{n}\right)=\sqrt{N}$ where $N$ is the number of counts.

Let a certain image feature cover an area $\Delta A$ of the detector area and include a fraction $\alpha$ of the total counts collected by the detector. If the image has a uniform background (due to thermal and incoherent scattering) so the integrated background intensity is a fraction $\beta$ of the total counts, then the number of counts needed to measure the intensity of this feature at a given signal to noise ratio is

$$
N \geq \frac{\left(\frac{s}{n}\right)^{2}(\alpha+\beta \Delta A)}{\alpha^{2}}
$$


A typical LEED spot is $\sim 10^{-3}$ of the detector area. If this beam has $\sim 10^{-3}$ of the detected electron current, apprcximatcly the limit of detection for a video LEED system and $2 / 3$ of the detected electrons are part of the incoherently scattered background, then the intensity can be measured with a signal-to-noise ratio of 10 with $\sim 1.5 \cdot 10^{5}$ electrons in the image. At a count rate of $5 \cdot 10^{4} \mathrm{~Hz}$ this image can be acquired in $\sim 3 \mathrm{~s}$. If this feature were an order of magnitude weaker, or $10^{-4}$ of the total diffracted intensity, $160 \mathrm{~s}$ would be needed to measure the intensity with a signal-to-noise ratio of 10 . This last kind of measurement would be impossible with a video-type LEED system.

\subsubsection{Operating conditions}

Because of its extreme sensitivity the detector must be protected from other sources of excitation during data acquisition. Turning on an ion gauge, for example, will saturate the detector. There was a measurable background caused by electrons escaping from the ion pump when the LEED detector was operated at pressures $\sim 10^{-7}$ torr even though there was no line-of-sight path from the ion pump. Electrons had to travel around several corners and through two grounded grids to reach the detector. This electron background was proportional the system pressure, so it was not a problem under ultra-high vacuum conditions.

Light shining through the windows of the vacuum chamber could also trigger the channel plates. Indeed, even a $0.5 \mathrm{~mW}$ helium neon laser $(\sim 5000 \AA)$ could produce a bright spot visible on the oscilloseope display when pointed into the detector. 
The channel plates are very sensitive and surface damage or contamination can cause field-emission discharges which deposit a substantial amount of charge, $\geq 100 \mathrm{pC}$, onto the anode. This was enough to over-drive the pre-amplifier to the point that it took $\sim 50 \mathrm{~ms}$ to recover. This kind of channel plate damage significantly degrades the performance of the detector.

\subsubsection{Applications}

Only preliminary LEED measurements have been carried out with the digital LEED detector. An example of this is shown in figure 6.9, a digital LEED image of graphitic carbon on the $\mathrm{Pt}(111)$ surface at $60 \mathrm{eV}$ (see chapter 12). The specular beam, substrate beams and two orienterl domains of the incommensurate overlayer are visible. Preliminary digital LEED I-V curves for Pt(111) at near-normal incidence are shown in figure 6.10. These can be compared with the video LEED results shown in chapter 10 .

There are a number of interesting applications for the LEED system. First is the study of systems without long-range order by diffuse LEED calculations. This type of experiment requires the measurement of the back-scattered electron intensity over the sntire detector at two or three adjacent energies. The digital detector can make such measurements with a large dynamic range in just a few minutes and the detected intensities are immediately available in digital form.

This LEED system can also be applied to the many surface systems that interact strongly with incident electron beams. One such example would be the high and low coverage ordered phases of carbon monoxide on $\mathrm{Pt}(111)$ discussed in 
chapter 10. Another interesting case is the study of molecular crystals, for example, water ice has been grown epitaxially on a number of transition metal substrates. This would be an interesting structure to determine by LEED. Lastly, the surfaces of poor electrical conductors and insulators could be investigated with much less problems from charging using the very low beam currents of the digital LEED system. 


\section{References}

1. M. D. Chinn and S. C. Fain, Jr., Physical Review Lellers, vol. 39, p. 146, 1977.

2. R. D. Diehl and S. C. Fain, Jr., Surface Science, vol. 125, p. 116, 1983.

3. N. M. Allinson, Nuclear Instruments and Methods, vol. 201, p. 53, 1982.

4. D. H. Lumb, G. R. Hopkinson, and A. A. Wells, Nuclear Instruments and Methods, vol. 221, p. 150, 1984.

5. J. L. Wiza, Nuclear Instruments and Methods, vol. 162, p. 587, 1979.

6. J. G. Timothy, Review of Scientific Instruments, vol. 52, p. 1131, 1981.

7. G. W. Fraser, J. F. Pearson, G. C. Smith, M. Lewis, and M. A. Barstow, IEEE Transactions on Nuclear Science, vol. NS-30, p. 455, 1983.

8. G. W. Fraser, Nuclear Instruments arid Methods, vol. 221, p. 115, 1984.

8. E. Gatti, K. Oba, and P. Rehak, IEEE Transactions on Nuclear Science, vol. NS-30, p. 461, 1983.

10. Galileo Electro-optics Corp., Sturbridge, MA 01518.

11. C. Martin, P. Jelinsky, M. Lampton, R. F. Malina, and H. O. Anger, Review of Scientific Instruments, vol. 52, p. 1067, 1881.

12. O. H. W. Siegmund, R. F. Malina, K. Cobern, and D. Werthimer, IEEE Transactions on Nuclear Science, vol. NS-31, p. 776, 1984.

13. L. J. Richter and W. Ho, Review of Scientific Instruments, vol. 57, p. 1469, 1986. 
14. P. C. Stair, Review of Scientific Instruments, vol. 51, p. 132, 1980.

15. E. G. McRae, R. A. Malic, and D. A. Kapilow, Review of Scientific Instruments, vol. 56, p. 2077, 1985.

16. J. G. Timothy, IEEE Transactions on Nuclear Science, vol. NS-32, p. 427, 1985.

17. M. Lampton and F. Paresce, Review of Scientific Instruments, vol. 45, p. 1098, 1974.

18. M. Lampton and C. W. Carlson, Review of Scientific Instruments, vol. 50, p. $1093,1878$.

19. Surface Science Laboratories, Palo Alto, CA.

20. H. O. Anger, U.S. Patent \$, 209, 201, 1965.

21. Physical Electronies, Inc., LEED model 10-120, Eden Prarie, MN.

22. Bi Ra Systems, Inc., Histogram Memory 5904A, Albuquerque, NM 87101.

23. O. W. H. Siegmund, S. Clotheir, J. Thornton, J. Leman, R. Harper, I. M. Mason, and J. L. Culhane, IEEE Transactions on Nuclear Science, vol. NS30, p. 503, 1983.

24. J. R. Arlington-Smith, I. M. Mason, H. E. Schwartz, and J. L. Culhane, IEEE Transactions on Nuclear Science, vol. NS-32, p. 119, 1985.

25. H. E. Schwartz and J. S. Lapington, IEEE Transactions on Nuclear Science, vol. NS-32, p. 433, 1985. 
26. O. W. H. Siegmund, M. Lampton, J. Bixler, S. Bowyer, and R. F. Malina, to be published.

27. V. Radeka, IEEE Transactions on Nuclear Science, vol. 21, p. 51, 1974.

28. Phototech, Inc., Redwood City, CA 94063.

29. S. C. Fain, Seattle.

30. Cliftronics, Ine., Model CE406, Clifton, NJ 07013.

31. P. Grivet, Electron Optics, Pergammon Press, New York, 1965.

32. S. C. Fain, Jr., private communication. 


\section{Figure Captions for Chapter 6}

6.1 Sketch of the resistive anode version of the Digital LEED detector. The outline of the resistor is shown on the square anode substrate. The arcs on the four edges provide a linear termination of the anode.

6.2 Photograph of the digital LEED detector.

6.3 Schematic of the digital LEED system, showing the detector construction and data system. The digitizer puts out 8-bit $x$ and $y$ addresses after each detected electron. The addresses are stored in a histograming memory during data acquisition, which is later read out by the LSI $11 / 23$ computer for analysis.

6.4 Digital LEED power supply schematic. This supply controls the channel plates, anode bias and electron gun. The electron gun beam voltage and focusing are under computer control.

6.5 Schematic drawing of the wedge-and-strip anode and amplifier circuit for the digital LEED detector. Typical wave-forms for the pre-amp and shaping amplifier outputs are shown. The $4.5 \mu$ shaping amplifier time-constant was chosen based on noise and channel plate count-rate considerations. The electronics can operate as fast as $750 \mathrm{~ns} /$ event.

6.6 Wedge-and-strip anode pattern for $85 \mathrm{~mm}$ diameter anode with $1.5 \mathrm{~mm}$ period. The left and right sides of the " $\mathrm{C}$ " electrode are externally connected. The full size anode is shown in (a), and an enlarged detail of the 6 $\mathrm{mm}$ diameter center hole is shown in (b). 
6.7 A block diagram of the digital position calculation logic, using the algorithm of Eq. 6.9. The anode dependent functions are stored in EPROM look-up tables for easy adjustment.

6.8 A digitized LEED image. This is an inage of an incommensurate graphite overlayer cn $\mathrm{Pt}(111)$ at $60 \mathrm{eV}$ (see also figure 12.1). The intense specularly reflected beam, a platinum overlayer beam and short arcs of the graphite ring from two domains of different angular orientations can be seen.

6.9 I-V curves collected with the digital LEED detector at near normal incidence for $\mathrm{Pt}(111)$. These I-V curves may be compared with video LEED I-V curves for clean $\mathrm{Pt}(111)$ in chapter 10 . These $\mathrm{I}-\mathrm{V}$ curves represent, preliminary results - the crystal was only approximately at normal incidence and the vacuum was relatively poor so the surface was contaminated with (nongraphitic) disordered carbon. 


\section{DIGITAL LEED DETECTOR}

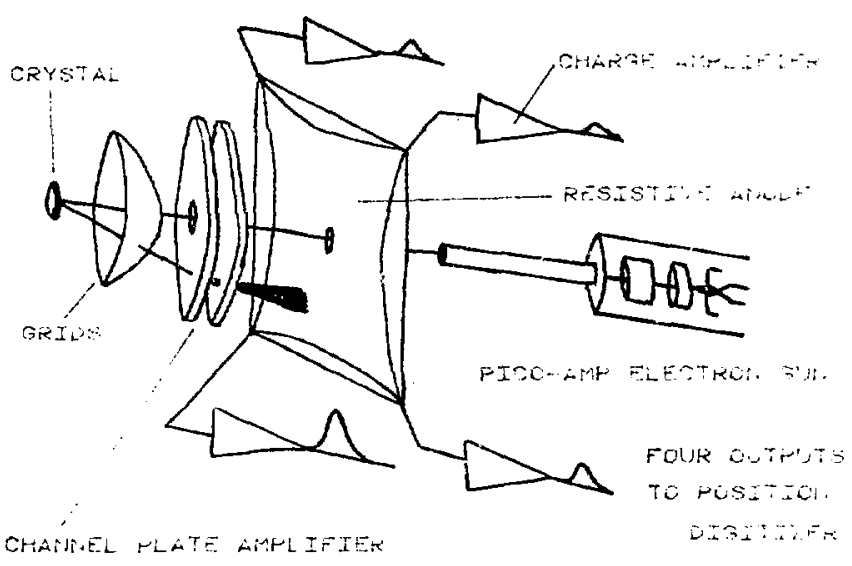

- WHEN a single difFracted Electron hits the chanNel PLATE, a PULSE of BeTweEN $10^{\circ}$ and $10^{\circ}$ ELECTRONS IS PRODUCED.

- the charge pulse. diffuses to the corners of the anode, WHERE AMPLIFIERS PRODUCE OUTPUT PULSES PROPORTIONAL TO THE AMOUNT OF CHARGE COLLECTED.

- the charge deposited on the anode by a oIfFracted ELECTRON MUST DISSIPATE BEFORE THE NEXT EVEITT IS DETECTED. THIS LIMITS THE MAXIMUM COUNT RATE TO $50 \mathrm{KHZ}$. 


\section{Digital LEED Detector Assembly}
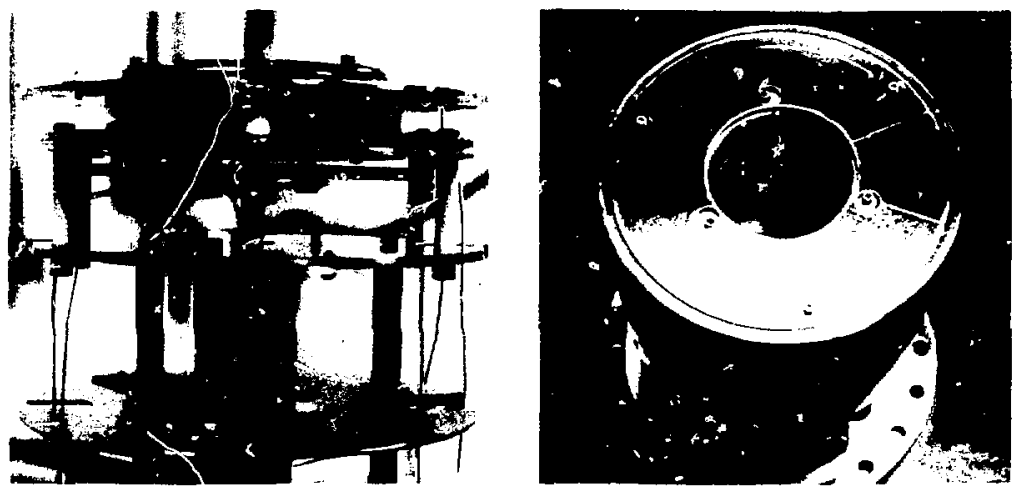

$X B: 3850-10025$

a) Detector assembly showing grid mount, channel plate supports, anode on quartz substrate, and electron gun shield. Note electrostatic shield around electron beam drift tube as it passes through the arode.

b) LEED detector before installatiun in the UHV system. showing hemispherical grids, the end of the electron peam drift tube, and the magnetic shield which encloses the detector assembly. 


\section{Digital LEED Detector}

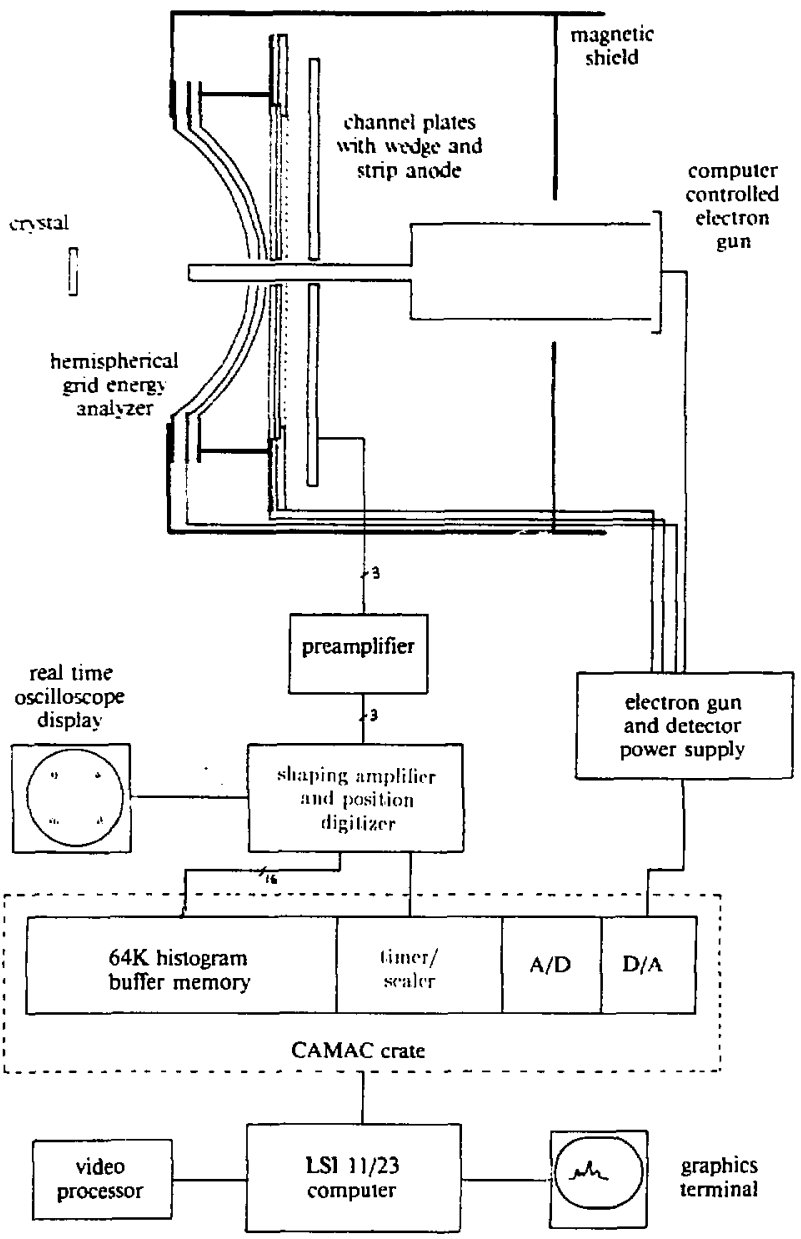

XBL $8512-5024$

Fgre 63 


\section{Digital LEED Detector Power Supply Voltages}

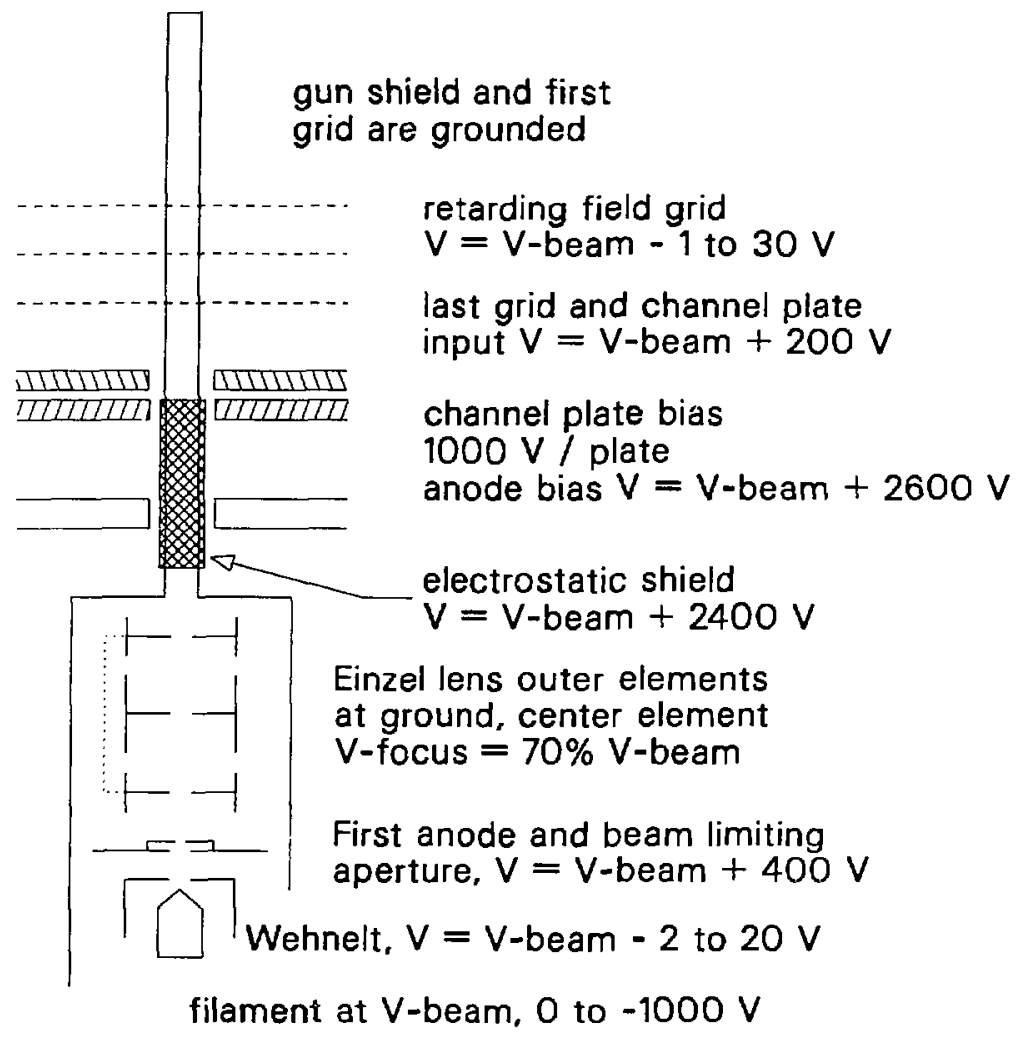

XBL $8612-4851$

Fgre 64 


\section{Position Sensitive Anode and Amplifier Circuit}

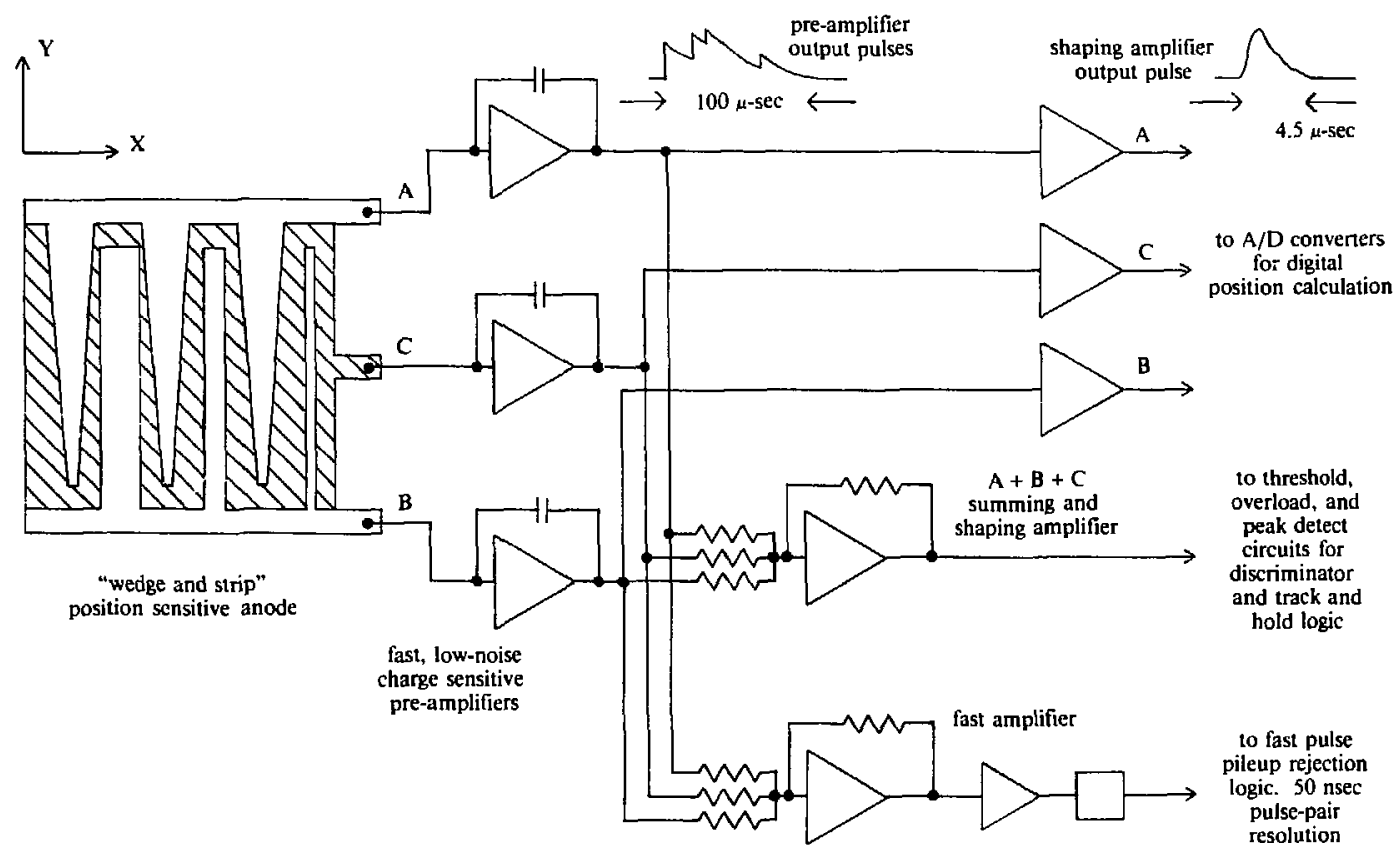


302

\section{Wedge and Strip Anode (actual size)}

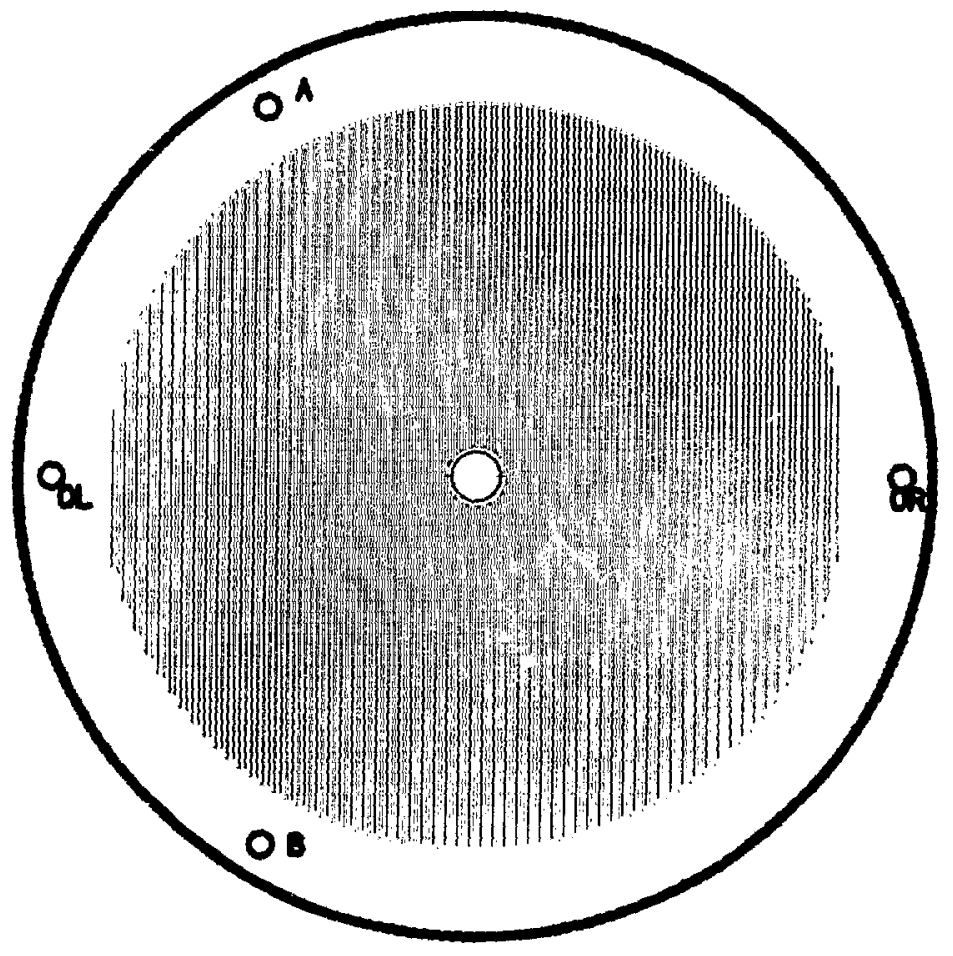

XBL $879-3865$

Figure 6.6(a) 


\section{Wedge and Strip Anode center detail}



Figure 6.8(b) 


\section{LEED Digital Position Calculation Circuit}






\section{Graphite on Pt(111) at $60 \mathrm{eV}$ by Digital LEED}



Figure 6.8 


\section{Pt (111) I-V Curves at $\theta=0$ by Digital LEED}

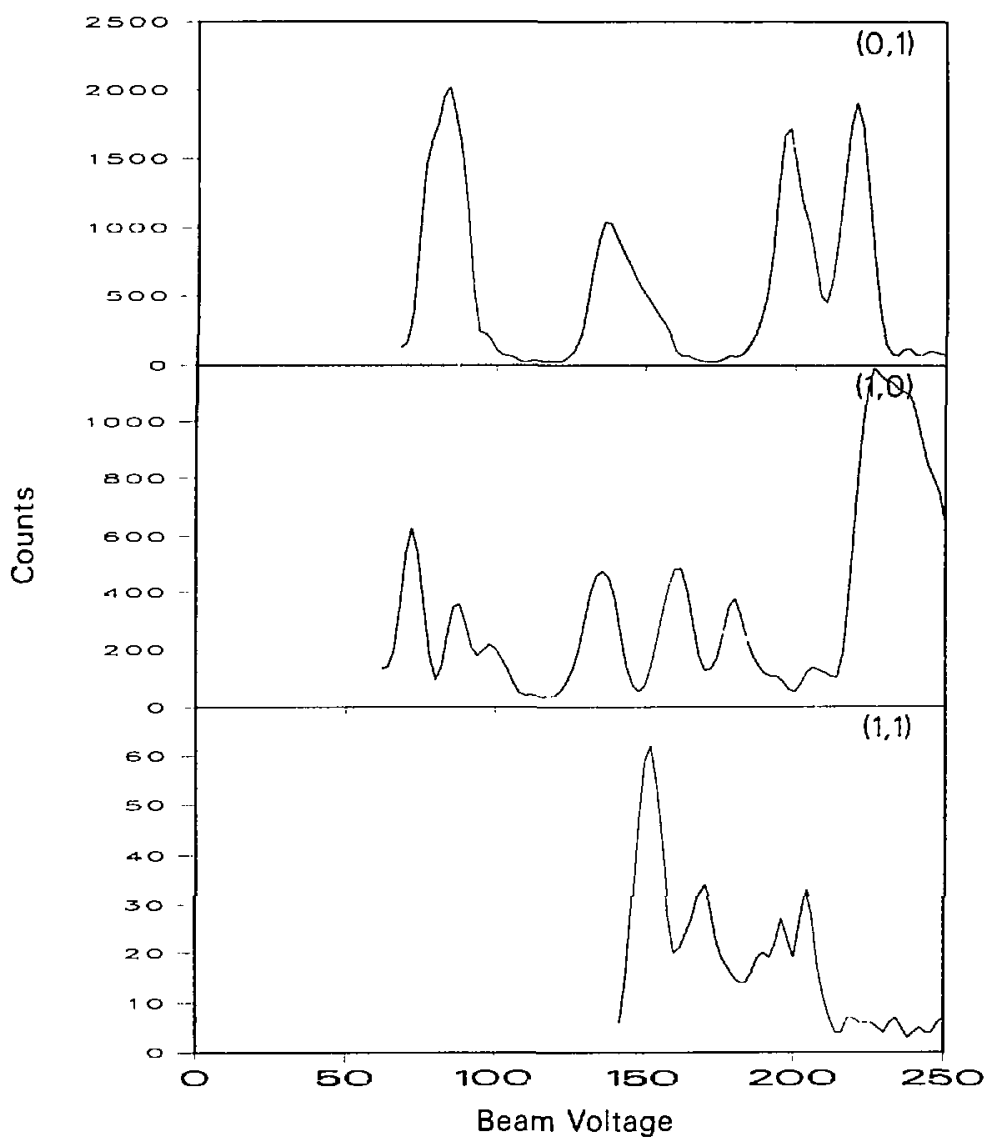

XBL $8612-4845$

Figure 6.9 


\section{Chapter 7 \\ I-V Curve Generation from \\ Digitized LEED Patterns}

\subsection{Introduction}

LEED data acquisition systems based on a display LEED optics with a realtime video digitizer and on a position-sensitive electron detector have been described in the two previous chapters. Both of these systems produce digitized images of the entire electron diffraction pattern. This chapter describes computer software that can generate a complete set of I-V curves from a sequence of digitized LEED patterns.

In the past the measurement of LEED I-V curves has been a difficult task, usually undertakien only as part of a structure determination experimeist. LEED intensity measurements can also be used for surface characterization for some systems and this would be practical if the effort required to obtain LEED data were similar to the effort involved in obtaining XPS or HREELS data. For example, comparison of the substrate LEED I-V curves for the (11i) surface of the $\alpha$-CuAl alloy and bulk $\mathrm{Cu}$ showed the similarity of the alloy surface structure to that of the bulk crystal (see chapter 13). LEED I-V curves could also be used as a measure of surface ordering by comparing I-V curves after different surface preparation procedures. Finally there are some systems where different surface phases produce the same LEED patterns, as in the case of sulfur on molybdenum (100), 
where a (2x2) LEED pattern is observed at sulfur coverages of both $1 / 4$ and $3 / 4 .^{1}$

The programs described here can generate LEED I-V curves in real time during experiments, or automatically from recorded data for simple LEED patterns. More complex LEED patterns can be analyzed interactively under computer control.

Both the video and digital LEED systems are controlled by menu-driven computer programs which can acquire and display LEED data using enhanced contrast and image manipulation, display I-V curves as the data are collected, store and retrieve images from disk storage, set up experimental parameters to acquire LEED I-V data sets and generate I-V curves from sequences of LEED images. Only the I-V curve generation routines are described in detail here. (The complete programs are available on request, see appendix I).

\subsection{LEED programs}

In this program the entire diffraction pattern is tracked instead of a single spot. This approach is much more reliable than single spot tracking. There is no danger of losing a spot at an intensity minimum since its position can be calculated from other, visible spots. Also the chance of accidentally switching from one spot to an adjacent spot in a dense LEED pattern is greatly reduced since the entire LEED pattern is available for reference. This approach of tracking the entire diffraction pattern was first used by Dr. Peter Stair ${ }^{2,3}$ to analyze digitized LEED photographs and this concept was later expanded in a set of computer programs by Dr. Jack Frost. ${ }^{4}$ This work was used a guide to develop the present 
interactive I-V curve generation program and the spot tracking part of the program in particular draws heavily on their work. The spot evaluation algorithms are mostly new.

The integrated intensity of a local LEED spot is corrected for the local background due to thermal and incoherent electron scattering and also for the effects of neighboring LEED spots. This is possible because the local intensity variation in the neighborhood of the spot is known. Both local background correction and the tracking of all the LEED spots at once are possible because of area detection of the LEED patterns. With a point detector like a Faraday cup or a spot photometer the total intensity accepted by the aperture of the detector is used for the spot intensity and spots are either tracked one at a time or manually by the observer.

\subsubsection{Program structure}

There are two basic steps in I-V curve generation, first the location of the LEED spot corresponding to a particular diffraction beam and second the evaluation of the integrated intensity of the LEED spot. A flowchart of the I-V curve generation program is shown in figure 7.1. The program is started by listing the reciprocal space coordinates of all the LEED beams in the pattern for which I-V curves are desired. The operator then positions a cursor over a few spots in the first (highest or lowest energy) LEED image and identifies the spots in terms of the reciprocal space coordinates. This gives enough information to determme the surface unit cell. There are also a number of adjustable program parameters to 
set (see Table 7.1), some that describe the data (number of images, starting and ending energy, etc.) and some that control the spot search and evaluation routines. These depend on the type of surface, i.e. the size of the unit cell, the typical spot width, etc.).

Once the initial conditions are set the I-V curves are generated as subroutine IVDATA loops over the sequential LEED images. The unit cell vectors are used to search locally for LEED spots. The program loops over each spot in the image, evaluating and testing the LEED spot and determining the integrated intensity.

\subsection{Spot tracking}

The LEED pattern is defined by an origin vector and two basis vectors $H$ and $\mathbf{K}$. The location of each spot in reciprocal space is given by coordinates $(h, k)$ where

$$
\mathbf{r}_{\text {spot }}=\mathbf{O}+h \mathbf{H}+k \mathbf{K}
$$

The reciprocal space coordinates $(h, k)$ of up to 75 spots are stored in the array $\operatorname{POSHK}(75,2)$ and the components of the three unit cell vectors are stored in $\mathrm{XY}(6)$.

When a spot is located by the searching routine, or when its initial position is given by the operator, this location is given as the memory address of the pixel with the maximum intensity in the spot. These values are stored in $\operatorname{IPOSXY}(75,2)$. Before the unit cell vectors can be calculated the pixel addresses must be mapped into an "image" space. Subroutine MAPMK translates the 
memory addresses IPOSXY into image space coordinates POSXY. The origin for the image space coordinate system is at the center of the image and the maximum dimension of the image is chosen to be 100.0 units.

This mapping is different for the digital and video LEED systems. Video images are rectangular, with a 5:4 horizontal to vertical "aspect ratio" and the digitized video image has 512 pixels horizontally and 480 vertically. The video LEED version of MAPKM simply scales the horizontal and vertical coordinates of the image. The digital LEED system does not have a curved screen like a display LEED optics. Instead the spherical image of the diffraction pattern is radially projected onto the flat position sensitive detector. This gives a radial distortion in the image and the digital LEED version of MAPKM transforms this distorted LEED patteru into image space coordinates POSXY.

Once the spot positions have been calculated in images space coordinates POSXY, subroutine RGRESS is called to calculate the unit cell vectors $\mathbf{O}, \mathbf{H}$ and K using a linear regression algorithm

$$
\begin{aligned}
\mathbf{H}_{x} & =\frac{\left(\sum_{i=1}^{n}\left(x_{i}-\bar{x}\right)\left(h_{i}-\bar{h}\right)\right)\left(\sum_{j=1}^{n}\left(k_{j}-\bar{k}\right)^{2}\right)}{\left(\sum_{i=1}^{n}\left(h_{i}-\bar{h}\right)^{2}\right]\left[\sum_{j=1}^{n}\left(k_{j}-\bar{k}\right)^{2}\right)-\left(\sum_{i=1}^{n}\left(h_{i}-\bar{h}\right)\left(k_{i}-\bar{k}\right)\right]} \\
& -\frac{\left(\sum_{i=1}^{n}\left(x_{i}-\bar{x}\right)\left(k_{i}-\bar{k}\right)\right]\left(\sum_{j=1}^{n}\left(h_{j}-\bar{h}\right)\left(k_{j}-\bar{k}\right)\right]}{\left(\sum_{i=1}^{n}\left(h_{i}-\bar{h}\right)^{2}\right)\left[\sum_{j=1}^{n}\left(k_{j}-\bar{k}\right)^{2}\right)-\left(\sum_{i=1}^{n}\left(h_{i}-\bar{h}\right)\left(k_{i}-\bar{k}\right)\right]}
\end{aligned}
$$

where $\bar{h}, \bar{k}$ and $\bar{x}$ denote averages. Similar expressions can be obtained for $\mathbf{H}_{y}$, 
$\mathbf{K}_{x}$ and $\mathbf{K}_{y}$ by substituting $y$ for $x$ or by interchanging $h$ and $k$. After the vectors $H$ and $K$ have been determined the unit cell origin is

$$
\mathbf{O}_{x}=\bar{x}-\bar{h} \mathbf{H}_{x}-\bar{k} \mathbf{K}_{x} \text { and } \mathbf{O}_{y}=\bar{y}-\check{h} \mathbf{H}_{y}-\bar{k} \mathbf{K}_{\mathbf{y}}
$$

Only spots with known positions, identified by IBFLG(75), are used in the unit cell vector calculation.

Subroutine HKCALC, which calls RGRESS, scales these results to the energy of the next LEED image

$$
\mathbf{H}^{\prime}=\left(\frac{E}{E+\Delta E}\right)^{1 / 2} \mathbf{H}, \quad \mathbf{K}^{\prime}=\left(\frac{E}{E+\Delta E}\right)^{1 / 2} \mathbf{K} \text { and } \mathbf{O}^{\prime}=\mathbf{O}
$$

The regression calculation is indeterminate if there are fewer tilan four noncolinear spots available for the calculation. In this case the previous unit cell vectors are extrapolated using Eq. 7.4. HKCALC also checks the percentage change in the unit cell vectors from the previous image and if this exceeds a preset limit HKCALC either asks for confirmation in the interactive mode or uses the previous unit cell vectors.

The new unit cell vectors $\mathbf{H}, \mathbf{K}$ and $\mathbf{O}$ are used to calculate new image space coordinates POSXY using Eq. 7.1 and these are transformed back into memory addresses in IPOSXY using subroutine MAPKM. These addresses are the starting points tu search for spots in the next image.

This approach to spot tracking works well. The limit on unit cell vector change is typically set at $2 \%$ and this is rarely exceeded. The program is able to find any spot that is visible by eye in an enhanced contrast image and the 
program does not confuse adjacent spots.

\subsection{Spot search}

Subroutine LOCMAX searches for spots around their predicted positions stored in IPOSXY. This routine reads out a $51 \times 51$ pixel region, centered on the predicted spot position, into an array in the program. (The RT-11 operating system used for the LSI 11/23 lab computer does not support virtual memory mapping, so the digitized image, which occupies $128 \mathrm{~K}$ bytes or $512 \mathrm{~K}$ bytes for the digital and video LEED detectors, respectively, cannot be stored in the program. The video image is left in the frame-buffer memory (see chapter 5) and the digital image is stored in high memory (see appendix II for details)).

LOCMAX makes a constrained search for a local maximum in the region around the predicted spot position. The data are usually smoothed before the spot search, using a "nine-point smoothing" algorithm

$$
\begin{aligned}
& I_{i j}{ }^{\prime}=\frac{1}{16}\left(8 I_{i j}+I_{i-1, j-1}+I_{i-1, j}+I_{i-1, j+1}\right. \\
& +I_{i, j-1}+I_{i, j+1}+I_{i+1, j-1}+I_{i+1, j}+I_{i+1, j+1}
\end{aligned}
$$

This smoothing algorithm is fast to implement and preserves the integrated intensities. The spot shapes are not affected much since LEED spots are typically five to fifteen pixels wide. This smoothing operation is usually appied twice, according to a preset parameter.

Smoothing the digitized image data makes it easier tc locate correctly weak spots which are near the noise level. The smoothing routine SMOOTH and the 
image data transfer routines RDFBD (for video LEED) and RDIGIT (for digital LEED) are written in DEC MACRO-11 assembly language for speed. All of the other programs are written in FORTRAN IV.

After smoothing LOCMAX searches for a local maximum within a range determined by the smaller of two preset limits; first, a given number of pixels and second, a fraction of the magnitude $|\mathbf{H}|$ (or $|\mathbf{K}|$, whichever is smaller). This second limit prevents LOCMAX from finding the wrong spot. For example, with a $7 \times 7$ superlattice FLM might be set to $\pm \frac{1}{14}$. For dense LEED patterns the search area shrinks at high energies. For a given pixel to be a local maximum it must be more intense than its four nearest neighbors. This condition prevents LOCMAX from considering a pixel on the edge of the search region to be the spot location if the slope at that point is non-zero. This is important when the shoulder of a strong spot overlaps the search area around a weak spot.

\subsection{Spot Evaluation}

Calculating the intensity of a spot is relatively easy for simple LEED patterns, such as from unreconstructed low Miller index surfaces. For more complex patterns like those discussed in part III (see figure 7.2) adjacent spots can easily influence the calculated intensity of a spot, especially when the neighboring spots are more intense. A detailed flowchart of the spot evaluation process is shown in figure 7.3. Subroutine DOBEAM calculates the spot background, widths and integrated intensity. 


\subsubsection{Local background}

The quantity that should be compared to theoretical LEED I-V calculations is the integrated spot intensity of the diffraction beam without contributions from incoherently (defect) and thermally (phonon) scattered electrons. The local background calculation is designed to correct for these factors. The instrumental background has already been subtracted from the digitized image in the video LEED system and the digital LEED instrumental background is completely insignificant. The background contribution due to multi-phonon scattering and scattering from disordered point-defects in the surface varies slowly with angle and energy, while the one-phonon scattering contribution forms a "halo" around each LEED spot.

Subroutine BKGRND calculates the background around the local maximum corresponding to the spot position. The background is calculated on an ellipse around the local maximum twice the size of the spot integration area, defined by INTXLM and INTYLM. An ellipse is used since the video pixels are rectangular. The background intensity is defined as the most frequently occurring intensity value along this ellipse. A histogram is made of the these intensities, with the interval width chosen to give reasonable statistics. This width is typically two counts for the digital LEED and 1/2048 of the maximum intensity of 4096 for the video $\mathrm{LEED}$.

This local-background algorithm was found to be much less sensitive to perturbations by adjacent spots than algorithms based on averaging, since an adjacent spot influences only a fraction of the perimeter of the local spot. If the 
shoulders or one-phonon halos of adjacent spots affect less than half of the spot background ellipse the calculated background is almost unaffected. The perfoi: mance of this background algorithm has been observed interactively in different LEED experiments. It gives stable and reasonable values for background intensity, with better performance than an averaging approach.

If the spot peak intensity is less than a preset noise level after the local background is subtracted, the spot is rejected and the spot evaluation fails.

\subsubsection{Spot width}

Spot widths are calculated in both horizontal and vertical directions. The width in pixels is determined by counting outward from the peak position until the intensity drops below a threshold value. This threshold is a fraction BGRAD of the difference between the peak and background intensities or the noise level, whichever is larger.

If the spot width is less than IWXo (or IWY0) the spot is rejected as an artifact. IWXO and IWYO correspond to the instrumental resolution. Any intensity feature narrower than this is not a diffaction beam but an artifact, such as a field.emission fiare on the phosphor screen of a display LEED optics.

\subsubsection{Spot intensity}

The integrated intensity is calculated by subroutine SPOINT by summing the pixel intensities, less background and noise, within an elliptical spot area with semi-axes INTXZM and INTYLM. The program gave the best results with a 
fixed integration area. When the measured spot widths were used to determine the integration limits the I-V curves were very noisy as a small change in the spot width could cause a large change in the integrated intensity.

A spot is rejected if any pixel in the integration area has an intensity greater than the peak intensity. This can happen if a weak spot is adjacent to a strong spot in a dense lattice. The local background algorithm cannot compensate for a background that changes significantly on the scale of the spot width.

Only the relatively intense spots are used in the unit cell vector calculation. Weak spots, where the spot location is more uncertain, are left out of this calculation. This is determined by calculating a "signal to noise" ratio, defined as the ratio of the spot sum to the number of pixels contributing to the sum times the background plus the noise level. This empirical figure of merit is compared to the parameter SNLIM to decide if a spot position is reliable.

\subsubsection{Interactive analysis}

The I-V curve generation program has a number of interactive features and the degree of interaction is controlled by a parameter INTER. At one extreme each decision made by the program is displayed and the operator is given the option to override decisions. At the other extreme I-V curve generation is completely automatic. The highly interactive modes can be used with new data to determine appropriate values of the analysis program parameters (Table 7.1). Even in the automatic modes the LEED images are displayed on a video monitor and a cursor marks the different LEED spots as the program locates them in the 
image. In the interactive mode some or all of the spot profiles may be displayed (figure 7.4). Horizontal and vertical cross sections through a LEED spot are shown, along with a "derivative" of the spot intensity in the two directions. The background, widths and noise level determined by the analysis program are shown.

\subsection{Performance}

The I-V curve generation programs described above have been used to generate I-V curves for a number of different surface systerns, including all of the I-V curves shown in chapters $6,10,11,12$ and 13 . Reasonable values of the analysis parameters can be found for a new type of LEED pattern after interactively analyzing a few LEED images. Once appropriate analysis parameters are chosen the program is able to make reasonable decisions for all spots that are visible to the eye in image-enhanced LEED patterns. When the program fails it rejects the LEED spot as too weak, to narrow, etc., rather than generating a spurious value.

For relatively good data, for example the $\mathrm{c}(4 \times 2)$ phase of carbon monoxide on $\mathrm{Pt}(111)$ (figure $7.2(\mathrm{a})$ and chapter 10 ) the progrim will run reliably in the non-interactive mode. For marginal data, such as the coinplex benzene structure (figure 7.2(b) and chapter 11) at higher energies, some additional information can be squeezed out of the data through highly interactive operation of the program. 


\section{References}

1. D. Kelly, private communication.

2. P. C. Stair, Ph.D. Thesis, Lawrence Berkeley Laboratory Report, LBL-6221, University of California at Berkeley, 1977.

3. P. C. Stair, T. J. Kaminska, L. L. Kesmodel, and G. A. Somorjai, Physical Review $B$, vol. 11, p. 623, 1975.

4. M. A. Van Hove, R. J. Koestner, J. C. Frost, and G. A. Somorjai, Surface Science, vol. 128, D. $482,1983$. 


\section{Figure Captions for Chapter 7}

7.1 Flow chart of data analysis program IVDATA.

7.2 Photos of LEED patterns for chemisorbed molecules with large unit cells.

7.3 Detailed flow chart of the LEED spot evaluation routines LOCMAX and DOBEAM.

7.4 After the analysis program locates and analyzes a LEED spot the results can be displayed interactively, as shown here in photographs of the computer terminal display. The upper plots show cross sections through the LEED spot along (left) and perpendicular (right) to the video scan direction. The lower plots show the "derivatives" (changes in intensity between adjacent pixels) of these cross sections. The solid horizontal line is the calculated local background for the spot and the solid vertical lines show the calculated spot width. The spot integrated intensity is the volume of the spot included between the dashed vertical lines, the preset integration area and above the dashed horizontal line, the (calculated) background plus a preset noise level. The spots in this figure were not smoothed. 


\section{LEED I-V Analysis Flowchart}

\begin{tabular}{|c|}
\hline Input \\
Analysis parameters \\
Reciprocal space coordinates \\
for each I-V curve needed \\
Position of some LEED spots \\
in the first image \\
\hline
\end{tabular}

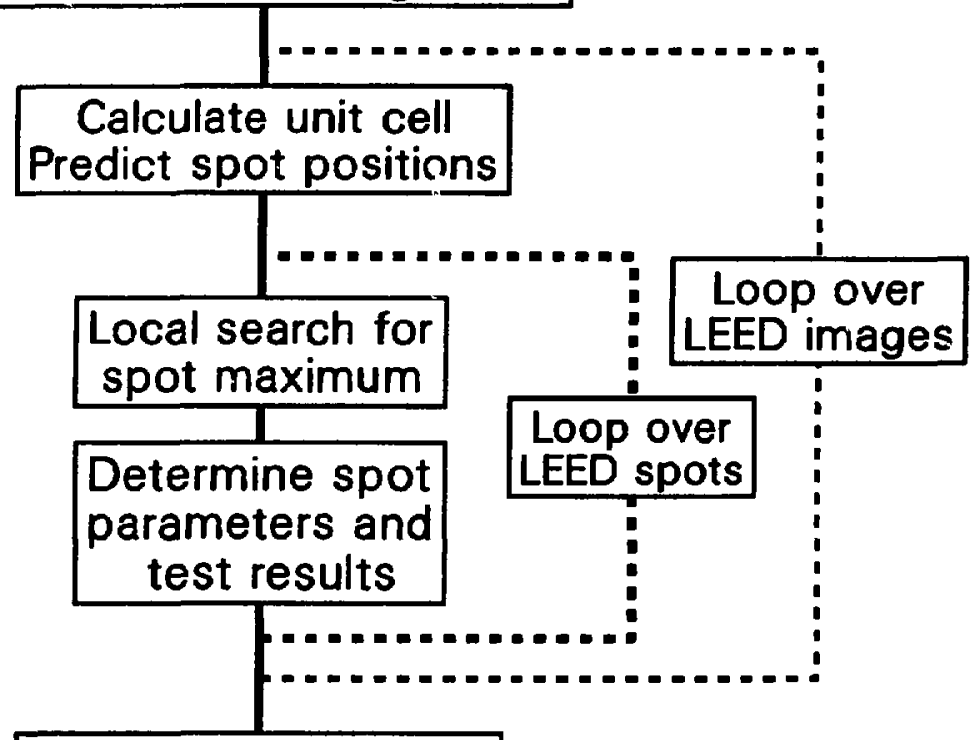

Display I-V curves

Figure 7.1

XBL $865-1736$ 
LEED Patterns of

Chemisorbed Molecules

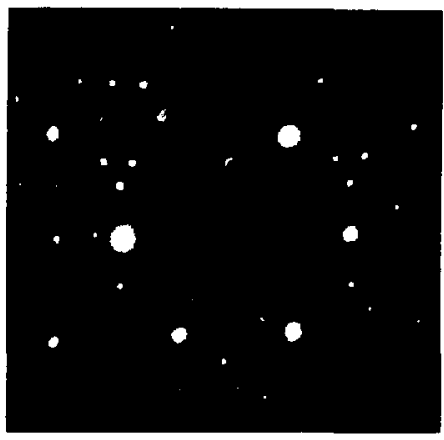

a) Pt(111)-c(4×2)-Carbon monoxide $130 \mathrm{eV}$ at normal incidence

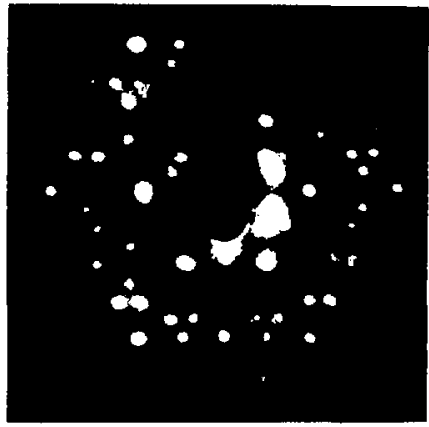

b) $\operatorname{Pt}(111)-(2 \sqrt{ } 3 \times 4)$ rect-Benzene

$54 \mathrm{eV}$ at near-normal incidence XBB $864-3604 A$

Figure 7.2 


\section{LEED Spot Analysis Flowchart}
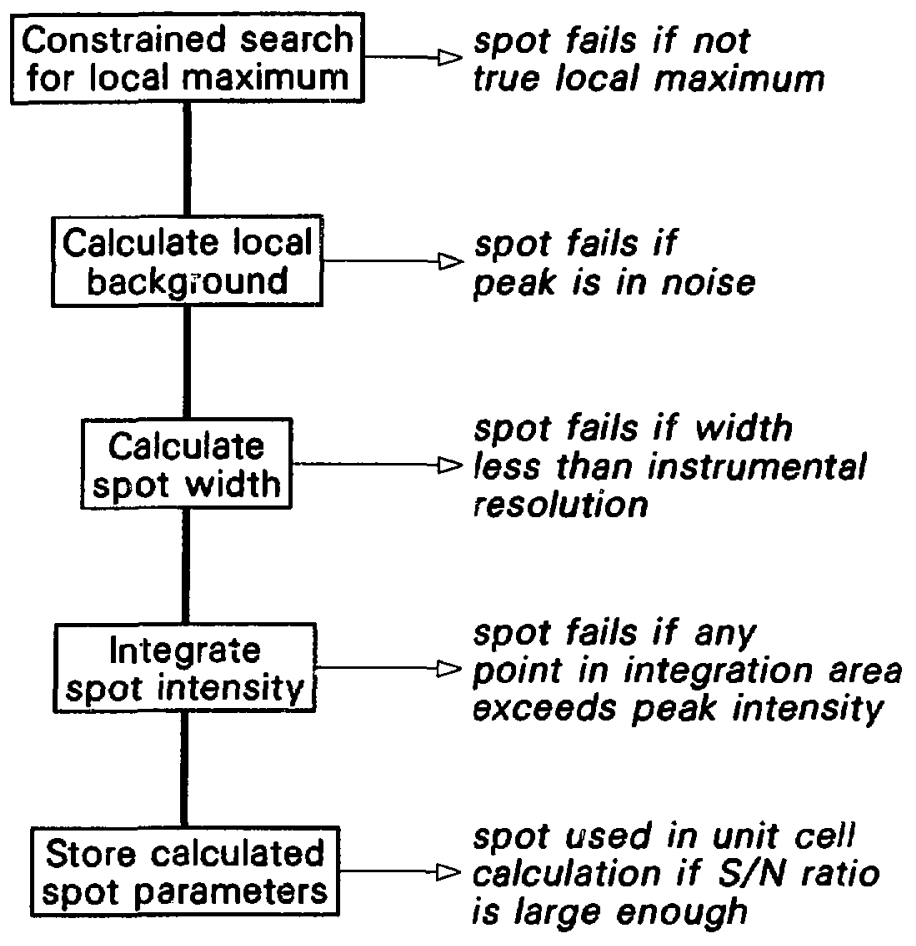

XBL $865-1819$

Figure 7.3 


\section{LEED Spot Analysis}

Interactive Display

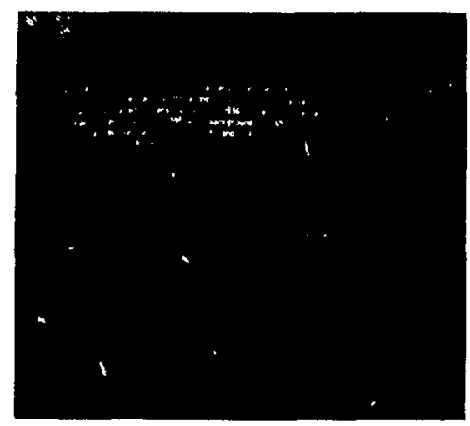

a) strong spot

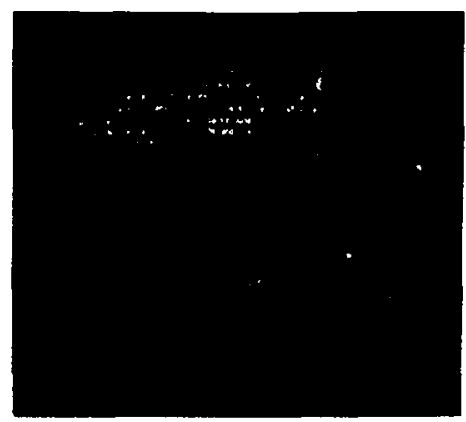

XBB 865-3579

b) weak spot

Figure 7.4 
Table 7.1 - LEED I-V analysis parameiers

\begin{tabular}{|c|c|c|}
\hline parameter & typical value & function \\
\hline BGRAD & 0.5 & $\begin{array}{l}\text { the spot width is measured at BGRAD } \\
\text { times (height - background). when } \\
\text { BGRAD is } 0.5 \text { the measured widths are } \\
\text { FWHM }\end{array}$ \\
\hline CLIM(3) & 0.02 & $\begin{array}{l}\text { maximum fractional change in the unit } \\
\text { cell vectors } \mathbf{O}, \mathbf{H} \text { and } \mathbf{K} \text { between images }\end{array}$ \\
\hline FLM & $0.3-0.06$ & $\begin{array}{l}\text { spot search range as a fraction of the unit } \\
\text { cell size }\end{array}$ \\
\hline INTXLIM & $6-8$ & $\begin{array}{l}\text { semi-axis of the spot integration ellipse in } \\
\text { the horizontal }(x) \text { direction }\end{array}$ \\
\hline INTYLIM & $6 \cdot 8$ & $\begin{array}{l}\text { semi-axis of the spot integration ellipse in } \\
\text { the vertical (y) direction }\end{array}$ \\
\hline ISEE & $6-10$ & spot search range in pixels \\
\hline ISM & 2 & number of times image is smoothed \\
\hline IWXo & $2-3$ & minimum acceptable horizontal spo width \\
\hline IWYO & $2-3$ & minimum acceptable vertical spot width \\
\hline NOISE & $2-10$ & $\begin{array}{l}\text { only pixels greatcr than NOISE above the } \\
\text { local background can contribute to the } \\
\text { integrated spot intensity }\end{array}$ \\
\hline SNLIM & 0.5 & $\begin{array}{l}\text { only spots with a "signal to noise ratio" } \\
\text { greater than SNLIM are used in the unit } \\
\text { cell calculation }\end{array}$ \\
\hline
\end{tabular}




\section{Chapter 8}

\section{An Improved LEED Manipulator}

\subsection{Introduction}

Modern surface studies often make use of a combination of different experimental techniques incorporated into the same vacuum system. When a sample is studied by one technique it may be out of alignment for other instruments.

Several methods have been used to mount multiple surface science instruments within one ultra-iligh vacuum chamber, including mounting instruments on large bellows so tinat they may be retracted when not in use and mounting instruments in different horizontal planes and using manipulators with large vertical travel. A simple solution is to mount the instruments in the same horizontal plane, with the focal points of different instruments all falling on a circle in this plane. I se rotation of an "L-shaped" sample holder around a vertical axis on the center-line of the chamber then moves the saniple from one instrument focus to the next. Pre-fabricated ultra-high vacuum systems in this "off-axis" configuration are commereially available, with a focal circles typically $10 \mathrm{~cm}$ and $12.5 \mathrm{~cm}$ in diameter. ${ }^{1}$

The advantages of this design are a compact vacuum chamber of simple construction with good pumping conductance and a sample manipulator that has short support arms, which provides good mechanical stability and simplifies electrital and thermal connections to the sample. This design was used for the 
vacuum chamber constructed for the electron-counting LEED detector, which was equipped with a conventional display LEED optics, a cylindrical mirror analyzer (CMA) for electron spectroscopy, a quadrupole mass spectrometer for thermal desorption spectroscopy, a directional gas doser and a collimated alkali atom source, in addition to the electron-counting LEED detector. All of these instruments are mounted in the same plane.

In LEED the diffracted electron intensity is a function of the angle between the incident electron beam and the sample normal (tilt angle $\theta$ ) and of the angular orientation of the sample (azimuthal angle $\phi$ ). The off-axis configuration complicates the sample-holder design.

A practical sample-holder for LEED needs to have two independent angular degrees of freedom and changing the angular position of the sample should not move the sample out of the instrument focus. Similar requirements also apply to several other common experimental techniques, including angle-resolved ultraviolet photo-emission spectroscopy (ARUPS), photoelectron diffraction, angleresolved photo-electron fine-structure spectroscopy (ARPEFS) and high-resolution electron energy-loss spectroscopy (HREELS).

\subsection{Design requirements}

The manipulator motions are defined in figure 8.1. Tine vertical sample holder rotation axis ( $\omega$-axis) is located along the center-line of the vacuum chamber. A $270^{\circ}$ rotation around this axis moves the sample from one instrument to the next. The rotation axis can also be translated in the $X, Y$ and $Z$ 
directions. These motions can compensate for alignment errors of the manipulator and the different insiruments.

The sample-tilt axis ( $\theta$-axis) should be perpendicular to the surface normal and lie along the front face of the sample, so there will be no coupling between rotational and translational motions. The sample can be brought to a normalincidence position even if the $\theta$-axis is not perpendicular to the sample normal because of small errors in sample alignment. Tilting the $\theta$-axis compensates for angular misalignment and also translates the sample. If the $\theta$-axis is horizontal, a small change $\Delta \omega$ can correct the error with a small trans'ation because of the short lever-arm, $\sim 5 \mathrm{~cm}$. If the $\theta$-axis is vertical, the waxis itself must be tilted to compensate, with a larger translation because of the longer lever-arm, $\sim 30$ cm. Therefore the $\theta$-axis should lie in the horizontal plane. For LEED intensity studies the range in $\theta$ should be at least from $0^{\circ}$ to $45^{\circ}$ and a $-45^{\circ}$ to $+45^{\circ}$ range is useful.

The range of motion required around the $\phi$-axis depends on the symmetry of the single-crystal sample surface. For a surface with 2-, 3-, 4-, or 6-fold rotational symmetry a $90^{\circ} \phi$-range allows any possible orientation if the tilt-angle $\theta$ can be either positive or negative. Most LEED intensity-voltage (I-V) curves are measured with the incident electron beam at normal incidence or in a surface mirrorplane -- this gives an internal check of the data, since symmetry related I-V curves should be identical; and the symmetry also reduces the cost of LEED structure calculations. A $90^{\circ} \phi$-range can reach the mirror-planes in a surface with 3-, 4-, or 6-fold rotational symmetry and can reach one mirror plane in a 
surface with $c 2 m m$ symmetry. The sample normal should be parallel to the $\phi-$ axis and a practical manipulator should have some method of fine-tuning the sample alignment after mounting.

The sample-holder must be non-magnetic and vacuum-compatible, with no insulators exposed to electrons to prevent charging problems. The sample should be electrically isolated to allow for sample current measurements, with provisions for heating, cooling and temperature measurement.

\subsection{Mechanical design}

Different types of sample-holder designs described in the literature meet some of the requirements described above. The problem of providing angular motions for an off-axis sample holder has produced a wide range of solutions. Angular motions have been driven by gears and cranks, ${ }^{2}$ by rack and pinion drives, ${ }^{3}$ around a virtual axis by support arms moving along epicycloidal tracks, ${ }^{4}$ by bevel gears, ${ }^{5,6}$ by cables and pulleys, ${ }^{7}$ by fixed levers ${ }^{8}$ and by gear, chain and pulley drives. 9

The sample-holder design described here uses a co-axial linear motion feedthrough on the main rotary feed-through to drive the $\theta$-motion and a cable in a flexible sheath to drive the $\phi$-motion. The rotary feed-through itself provides the w-motion. This design is simple to build and has fewer moving parts than most of the dosigns described above. Parts are 304 stainless steel unless otherwise noted. 
The sample holder is mounted on a commercial ultra-high vacuum manipulator that consists of a rotary feed-through with a co-axial linear motion feedthrough on an XYZ translation stage. ${ }^{10}$ This manipulator is constructed on a 150 mm conflat flange. Its rigid design minimizes the coupling between different translational and rotational motions. The feed-throughs required for the $\phi-$ motion drive, sample heating, cryogenic cooling and temperature measurement are mounted on the same flange.

Rotation around the $\phi$-axis is driven by a spring-loaded cable in a flexible sheatl. The cable is $.025^{\prime \prime}$ soft-temper 304 stainless steel wire and the cable sheath is a tightly coiled $0.075^{\prime \prime}$ diameter spring wound of $0.010^{\prime \prime}$ stainless steel wire. This cable can bend $360^{\circ}$ on a $3 \mathrm{~cm}$ radius, so it easily follows the XYZ, $\theta$ and $\omega$-motions of the manipulator.

A micrometer-drive linear motion feed-through controls th cable motion. The drive cable connects to a grooved wheel mounted on the end of the $\phi-$ rotation axle (figure 8.2 ). A beryllium-copper spring ${ }^{11}$ attached to the opposite side of ihe drive wheel loads the cable. When the linear motion feed-through pulls on the cable the sample azimuthal changes. This arrangement allows a motion of $\sim 120^{\circ}$ around the $\phi$-axis.

The sample is mounted on a copper block, which is attached to the $\phi$-axle with a gimbaled mount. It is important for the sample normal to be parallel to the $\phi$-axis. Two pairs of set-screws located at the back of the gimbal tilt the sample around orthogonal axes perpendicular to the $\phi$-axis. The sample is aligned by adjusting the gimbaled mount until a laser reflected off the front face of the 
sample is independent of the $\phi$-angle. The $\phi$-axle runs in a bearing and the front face of the sample is set at the $\theta$ rotation axis by adjusting the bearing housing.

During $\theta$-motion the whole sample mount assembly, figure 8.2 , pivots around the $\theta$-axis (see figure 8.3). This motion is controlled by a lever-arm driven by the coaxial linear motion feed-through, with a beryllium-copper spring to provide the return force. The manipulator is adjustable in $\theta$ from $+50^{\circ}$ (up) to $-30^{\circ}$ (down). A larger range of motion is possible using a lever with a larger mechanical advantage, but this reduces the accuracy of the $\theta$-motion.

There is a slightly non-linear relationship between the setting of the linear motion feed-through and the $\theta$-motion. This motion was calibrated externally during the set-up using laser reflection. Once in the vacuum system the normalincidence $\left(\theta=0^{\circ}\right)$ position is determined absolutely within $\sim 1 / 4^{\circ}$ by observing the symmetry of the LEED pattern and off-rormal angles are set from the external calibration or by observing LEED spot positions as a function of energy.

\subsection{Heating and cooling}

The sample is spot-welded between two tantalum wires, each connected io one half of a split copper disk (figure 8.4). A $0.005^{\prime \prime}$ chromel-alumel thermocouple spot-welded to one edge of the crystal measures sample temperature. A $30 \mathrm{~A}$ current flowing through the support wires heats the sample to $\sim 1300 \mathrm{~K}$ and the support wires provide enough thermal isolation so the copper disk is not overheated. Even with prolonged annealing at sample temperatures above 1300 $\mathrm{K}$ the disk temperature does not exceed $\sim 300 \mathrm{~K}$. 
Each side of the copper disk is cooled by a copper braid connected to an electrically insulated liquid nitrogen reservoir. These copper braids also carry the sample heater current. Quartz spacers electrically and thermally insulate the copper disk from the rest of the sample mount. All insulators are out of the line of sight to the crystal to prevent charging by electrons, which interferes with LEED measurements.

The copper braids are flexible enough to follow tise sample through the full range of $\omega, \theta$ - and $\phi$-motions. The thermal conductivity is limited by the $\sim 10$ $\mathrm{cm}$ long braids, so the minimum sample temperature is $\sim 130 \mathrm{~K}$. A similar sample holder using a copper braid connected to a single-stage recirculating helium gas refrigerator, is able to cool the sample to $\sim 30 \mathrm{~K} \cdot{ }^{12}$ No additional thermal isolation or thermal shielding is used.

The samrle reaches its minimum temperature $\sim 30$ minutes after the liquid nitrogen reservoirs are chilled, because of the large thermal mass of the split copper disk. Once the sample holder has reached thermal equilibrium, thə crystal can cool back to $200 \mathrm{~K}$ in $\sim 45 \mathrm{~s}$ and to $150 \mathrm{~K}$ in $\sim 90 \mathrm{~s}$ after flashing to $1000 \mathrm{~K}$.

The cold finger design (figure 8.4, left) uses a cryogenic and vacuum compatible liquid feed-through. ${ }^{13} \mathrm{~A}$ copper reservoir is brazed onto one end of the feedthrough and the other end is welded into a $33 \mathrm{~mm}$ conflat ${ }^{\circledR}$ flange. A $3 \mathrm{mr}$ teflon tube connected to a self-pressurized liquid nitrogen dewar and inserted through the stainless steel and alumina feed-through brings nitrogen into the reservoir. Boiled-off nitrogen and excess liquid pass back up the steel tube. A 25 liter dewar self-pressurized to $\sim 6$ psig will cool the two cold fingers for 8 to 10 
hours.

A copper wire connects a high-current feed-through to the cold finger and the copper braid carries the heater current to the sample. The heat-leak from the room-temperature high-current feed-through to the nitrogen reservoir is insignificant compared to the cooling capacity of the cold-finger.

\subsection{Performance}

The manipulator and sample-holder have been in use since 1985 on the electron-counting LEED detestor chamber. Initial trials determined the correct spring tension for reliable functioning of the $\theta$ - and $\phi$-motions. At first 304 stainless steel springs were 11sed, however the $200^{\circ} \mathrm{C}$ bake-out temperatures annealed the springs. Beryllium-copper springs are more satisfactory -- there is no residual unagnetism, the springs are stiffer and the working temperature range extends to $300^{\circ} \mathrm{C}$.

As in any spring-londed mechanism, there is a certain amount of backlash. The most reproducible angular settings are obtained by approaching from the direction of motion that works against the spring tension. With this precaution angles are reproducible to better than $0.1^{\circ}$. The $\phi$-angles have not been measured explicitly; however; the $\phi$-angles equivalent to mirror-planes are easily set and maintained with the $\sim 1 / 4^{\circ}$ accuracy required for LEED; and other angles may be set using the diffraction pattern at various voltages (see chapter 9).

A cable in a flexible sheath provides a simple and accurate method to control an additional manipulator motion. The cable sheath wound of spring-tempered 
304 stainless steel wire has a slight residual magnetisr?. A non-magnetic cable drive can be constructed using beryllium-copper in place of stainless steel. 


\section{References}

1. Varian, Inc., Palo Alto, CA; Perkin EImer, Inc., Mountain View, CA.

2. M. K. Debe, Review of Scientific Instruments, vol. 47, p. 39, 1976.

3. C. J. Russo and R. Kaplow, Journal of Vacuum Science and Technology, vol. 13, p. 487, 1976.

4. E. E. Chaban and H. D. Hagstrum, Review of Scientific Instruments, vol. 47, p. $828,1976$.

5. T. N. Tommet and S. L. Bernasek, Review of Scientific Instruments, vol. 48, p. $399,1977$.

6. W. A. Royer, Review of Scientific Instruments, vol. 51, p. 386, 1980.

7. J. Larscheid and J. Kirschner, Review of Scientific Instruments, vol. 49, p. 1486, 1978.

8. P. Morgan, T. Juul, and E. Larsen, Journal of Vacuum Science and Technology, vol. 16, p. 89, 1979.

9. C. A. Crider, Review of Scientific Instruments, vol. 52, p. 1156, 1981.

10. Vacuum Generators, Ltd., Model HPT-205/RD2S, West Sussex, England, U. K..

11. Instrument Specialties Co., Delaware Watergap, PA.

12. G. S. Blackman, private communication.

13. Insulator Seal Co., Model G01A0331-1, Hayward, CA. 


\section{Figure Captions for Chapter 8}

\subsection{Manipulator motion definitions.}

8.2 Side view of the sample mount, showing details of the cable drive and the gimbaled sample mount. This entire assembly pivots around the horizontal $\theta$-axis, located perpendicular to the $\phi$-axis at the front face of the sample.

8.3 Photograph of sample holder mounted on the manipulator in the vacuum chamber. The sample, facing to the right, is on line with the $\theta$-axis. The pivot point for the $\theta$-axis is visible on the end of the support arm. The lever controlling the $\theta$-motion comes down from above to attach to the sample mount assembly. The $\phi$-drive cable sheath is visible at the right side of the photograph and the drive cable connects to a grooved wheel on the end of the $\phi$-axle. The copper heating and cooling braids and the thermocouple leads connect to the crystal from below.

8.4 A front view of the sample mount (at left) shows the sample attached to the split copper disk. The sample is heated by a current flowing through the support wires. At right the cold finger is shown. Each copper braid attaches to an electrically isolated liquid nitrogen reservoir inside che vacuum system. The heater current is brought in to the nitrogen reservoir - the copper braids then carry the current to the crystal.

8.5 Photograph of manipulator translation stage and feed-throughs. The linear motion micrometer feed-through at the lower left side of the manipulator flange drives the $\phi$-motion. The small diameter flexible tubes at the right 
bring in liquid nitrogen for cooling and the larger diameter tubes are for exhaust gas. A pair of high-current feed-throughs carry the crystal heater current and the thermocouple feed-through is partly visible at the left rear of the manipulator flange. 


\section{Manipulator Motions}

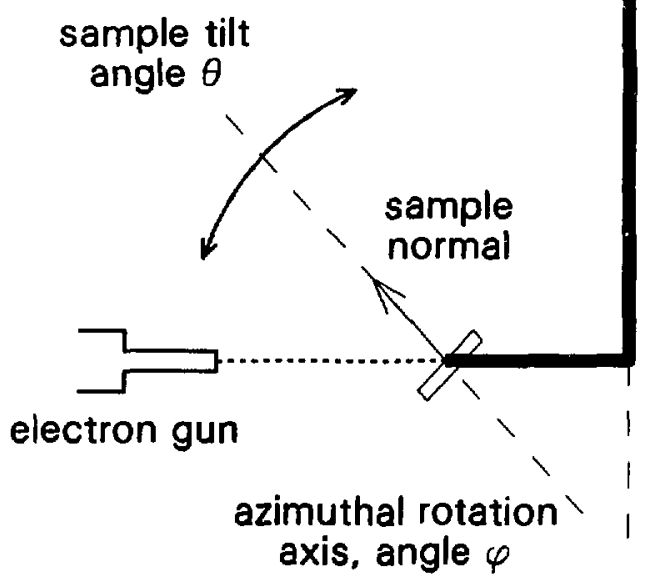

sample holder

rotation axis angle $\omega$

XBL $865-1923$

Figure 8.1 


\section{Sample Mount Side View}

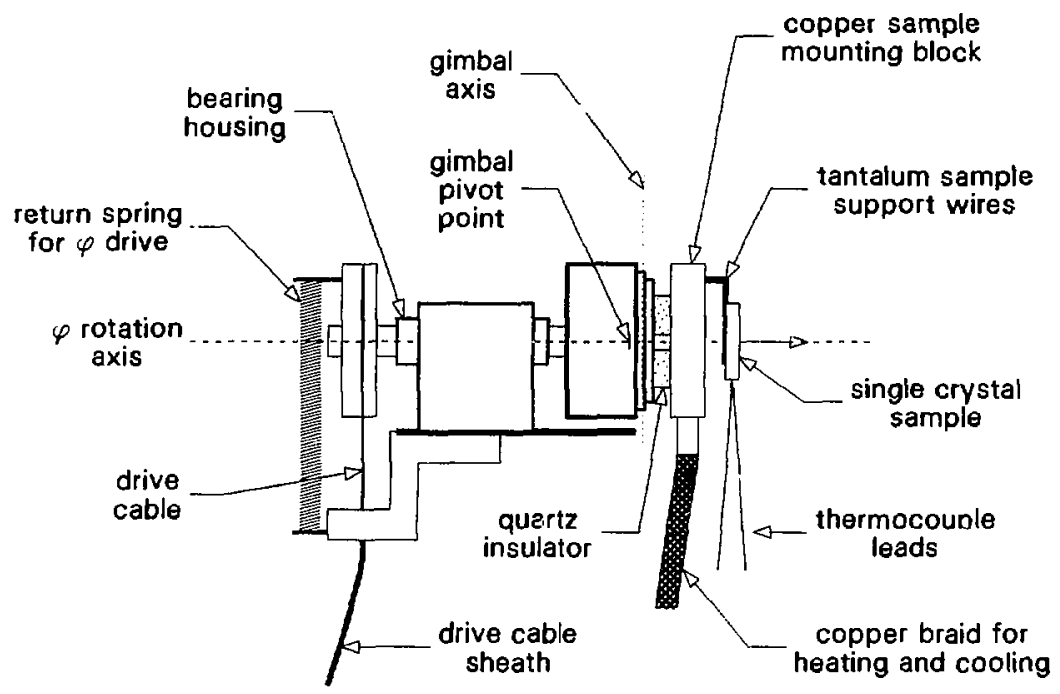

XBL 865-1922

Figure 8.2 



\section{Sample Heating and Cooling}

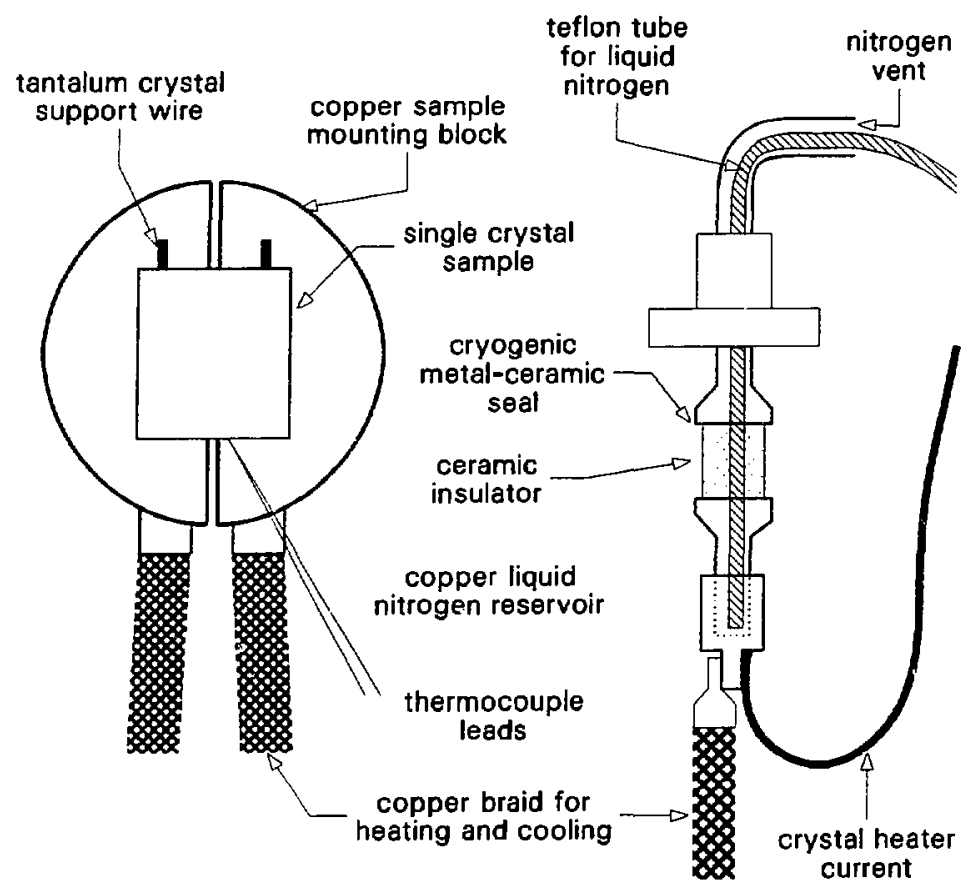

Sample Mount front view

Cold Finger side view

XBL 865-1921

Figure $\mathbf{8 . 4}$ 


\section{2}

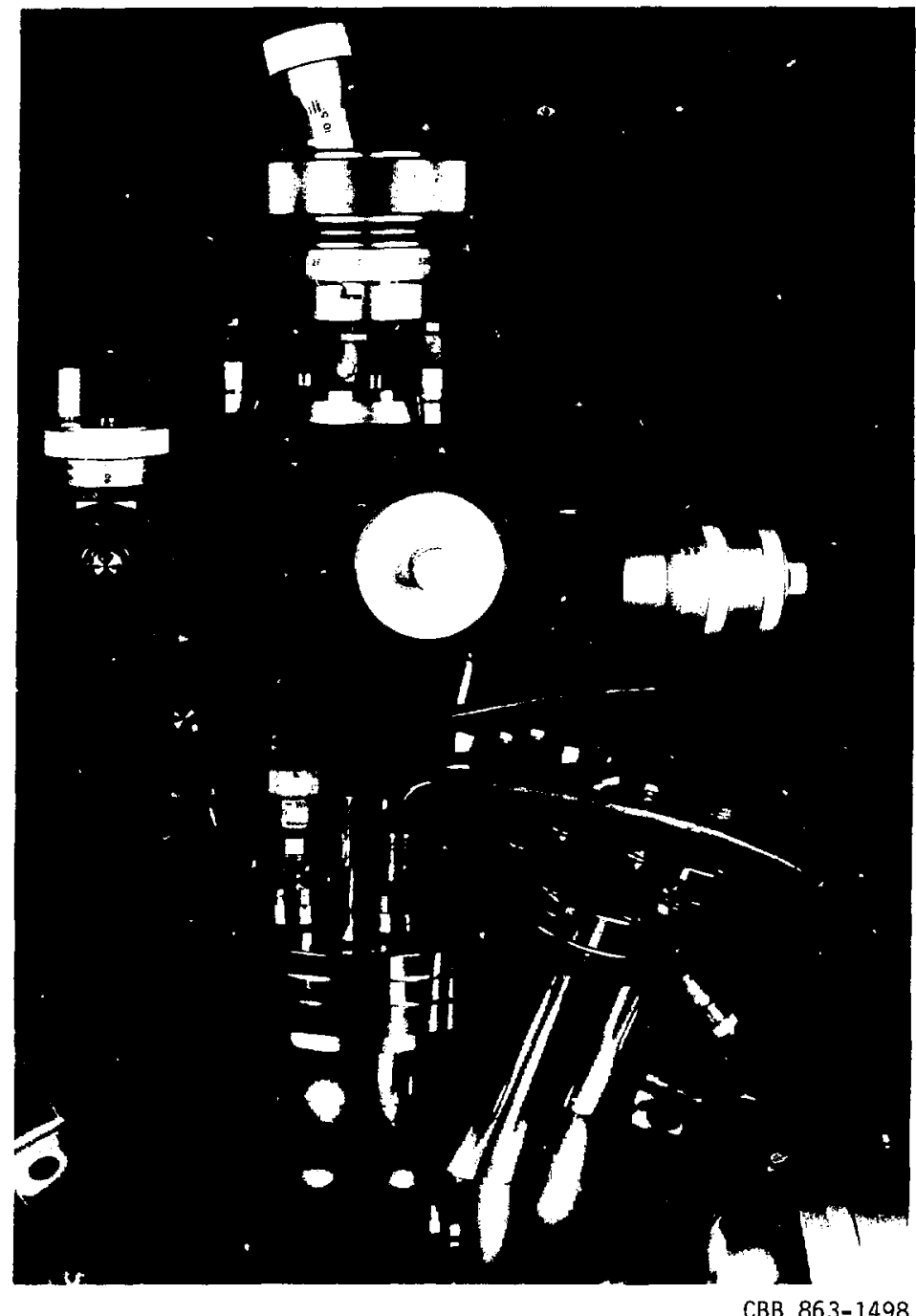

Figure 8.5 
Part III

Experimental Results for

Low Energy Flectron Diffraction

Structure Determination 


\section{Chapter 8}

\section{Experimental Techniques for LEED Structure Determination}

\subsection{Introduction}

Reliable surface structure determination experiments require well characterized surfaces prepared under known conditions. The single crystal surfaces must be free from contamination and chemisorbed overlayers must be formed from pure adsorbates under controlled conditions. This chapter describes the vacuum techniques, surface preparation and characterization methods and conditions for LEED data collection used for the structure determination experiments discussed in the following chapters.

\subsection{Vacuum techniques}

The LEED structural studies were conducted in a standard ion-pumped, bakeable, stainless steel ultra-high vacuum system. This systein was equipped with a Varian four-grid LEED optics with an "off-axis" LEED electron gus:" and an auxiliary glancing incidence electron gun used for ion-core excitation in Auger electron spectroscopy (AES). An EAI quadrupole mass spectrometer with an electron multiplier was used for residual gas analysis and thermal desorption spectroscopy (TDS) measurements and a $2 \mathrm{KV}$ sputter-ion gun was used for crystal cleaning. 
Pressure measurements were made with a standard ion gauge. No calibration was made beyond adjustment of the emission current. All of the pressures reported are nominal ion gauge readings, with no corrections for chamber geometry, conductance effects or changes in ionization cross-sections relative to the nominal $\mathrm{N}_{2}$ calibration of the ion gauge.

The primary vacuum pump was a Varian $240 \mathrm{l} / \mathrm{s}$ diode ion-pump. Secondary pumping was provided by a titanium sublimation pump in the ultra-high vacuum chamber and by a $60 \mathrm{l} / \mathrm{s}$ triode ion pump, a pair of zeolite sorption pumps and a mechanical vacuum pump attached to the gas manifold. The system was normally rough-pumped by the sorption pumps to a pressure of $\sim 10^{-2}$ torr, where the ion pumps could be started. The mechanical pump was equipped with an optically dense liquid nitrogen cold trap to prevent oil back-streaming, a possibie source of contamination. To minimize back-streaming the mechanical pump was only operated at pressures above 50 torr.

The sorption pumps were always baked-out into the mechanical pump and the mechanical pump was vented into vacuum lines in the laboratory fume hoods. This assured that toxje gases used in surface experiments, including carbon monoxide and benzene vapor, were not released into the laboratory. This is important as sorption pumps can trap and rclease hazardous quantities of these materials during typical surface preparation procedures.

Poor chamber design limited the effective pumping speed in the ultra-high vacuum system. The main vacuum pump was connected to the chamber through 4" tubing with two corners. Because of the low conductance ${ }^{2}$ the measured 
pumping speed for oxyzen in the vicinity of the crystal was only $60 l / \mathrm{s}$ at $10^{-8}$ torr, which corresponds to a nitrogen pumping speed of $35 \mathrm{l} / \mathrm{sec}$. The working base pressure of the system is determined by the balance between the pumping speed and the effusion or sut-gassing rate of the materials in the ultra-high vacuum chamber. Low pumping speed requires that the system be extremely clean to achieve a good base-pressure.

The vacuum system was baked-out using an oven that completely enclosed the vacuum chamber, which heats the system uniformly without cold spots. Thermal stresses are minimized and temperature control is reliable for an oven compared to heating schemes using locally applied heating elements. The system was baked-out at an oven air temperature of $185^{\circ} \mathrm{C}$. After opening the vacuum system to air, approximately three days were required to heat the system to bake-out temperature without stalling the ion-pump. This delay is caused mainly by the slow icn-pumping rate for water vapor. Baking for an additional day or two at $185^{\circ} \mathrm{C}$ produced a reasonable base pressure. The optimum base pressure obtained was $\sim 2 \cdot 10^{-10}$ torr. Some experiments required high pressure exposures $\left(\sim 10^{-6}\right.$ torr) of hydrocarbon vapors. During these experiments the base pressure increased to $\sim 2 \cdot 10^{-9}$ torr. The main residual gasses were hydrogen and carbon monoxide. 


\subsection{Surface preparation}

The current state of the art of surface structure determination is limited to relatively simple overlayers or reconstructions on defined single-crystal planes. The results of structure-sensitive experiments are normally compared to theoretical predictions made from surface models. If the experimental system is not well characterized, then surface models must include many parameters; and the results may be ambiguous or unreliable. In most $s^{t}$ ructure experiments an oriented single crystal substrate is prepared with a known surface plane, then this surface must be checked for order and cleanliness. Finally the desired surface phase must be formed through chemical and thermal processing.

\subsubsection{Crystal preparation}

Most of the structure determination experiments described here were conducted on the platinum (111) crystal face. (The preparation of the copper aluminum alloy surface is described in chapter 13.) The platinum crystals were previously grown from a melt of high-purity zone-refined platinum. The platinum crystal was then oriented by Laue X-ray back-diffraction and cut with a diamond saw. After cutting the crystals were polished using diamond pastes and standard metallographic techniques. Platinum is relatively soft and anneals well, so the crystals were not chemically etched.

The (111) crystal used for most experiments was a rectangle approximately 6 $\mathrm{mm}$ by $7 \mathrm{~mm}$ and $0.8 \mathrm{~mm}$ thick. The surface was cut and polished within $\sim 1 / 2^{\circ}$ of the [111] direction. 


\subsubsection{Sample cleaning in vacuum}

The sample can be contaminated both by bulk metallic impurities which segregate to the crystal surface, or by gas phase impurities adsorbed on the surface. The metallic impurities present in the platinum crystals included, in order of importance, sulfur, calcium, silicon, phosphorus and carbon. The main gas phase contarninants are carbon monoxide, hydrogen, various hydrocarbons and oxygen.

A clean $\operatorname{Pt}(111)$ surface was prepared with a combination of ion bombardment, annealing cycles and oxygen treatments. With prolonged heating at high temperatures $(\sim 1300 \mathrm{~K})$ sulfur and calcium segregate to the surface. Occasionally phosphorus or silicon were also detected. These metallic impurities were removed from the surface by ion-bombardment. Ion bombardment damages the surface, so the crystal had to be annealed before LEED measurements could be made.

\subsubsection{Ion bombardment}

During ion bombardment the main ion pump was valved off and the chamber was pumped with the auxiliary triode ion pump on the gas manifold, which had a much higher pumping speed for inert gases. A new layer of titanium was evaporated in the sublimation pump before sputtering, to increase the pumping speed for reactive gases. The crystal was sputtered in an argon partial pressure of $\sim 5 \cdot 10^{-5}$ torr at an ion energy of $500 \mathrm{eV}$, with the crystal heated to $\sim$ $1000 \mathrm{~K}$. The background pressure of the residual gasses in the chamber was 
$\sim 10^{-7}$ torr during sputtering. The combination of relatively low ion energy and a heated crystal minimized the surface damage to the crystal. When the surface was sputtered cold, longer annealing times were needed and bulk impurities would often segregate to the surface. When the crystal was heavily contaminated or the impurities were difficult to remove, as sometimes happened after an extended bake-out, sputtering in $\sim 10^{-6}$ torr of oxygen greatly increased the effective sputtering rate. As impurities segregated to the surface they would oxidize and become trapped at the surface, where they were quickly sputtered away.

The surface composition was monitored during sputtering by AES. When the crystal surface was clean, the argon ion beam was shut of and the chamber pumped down to ultra-high vacuum with the crystal still at $1000 \mathrm{~K}$. Annealing for 300 sat this temperature was sufficient to obtain good, low background LEED patterns.

The crystal used for the LEED experiments was relatively pure and repeated cycles of sputtering and annealing could deplete the bulk impurity concentrations in the near surface region. Then chemisorption experiments could often be conducted for several weeks before bulk impurities again segregated to the surface.

\subsubsection{Auger spectroscopy}

Auger data were obtained using the glancing incidence electron gun for excitation and the LEED optics retarding field energy analyzer (RFA) as the detector. AES was most commonly used to detect impurities. In this mode the electron beasn was incident at $\sim 60^{\circ}$ from the crystal normal at $2 \mathrm{KeV}$ and $\sim 75 \mu \mathrm{A}$. The 
RFA pass energy was modulated at $\sim 2.5 \mathrm{KHz}$ with a $10 \mathrm{~V}$ peak-to-peak modulation. The electron current was collected with the IEED screen biased to $+300 \mathrm{~V}$ by a battery with a $10 \mathrm{~K} \Omega$ resistor in series. The signal current was capacitively decoupled and detected with a lock-in amplifier operated in the second harmonic mode. An older tuned-channel lock-in (PAR JB-5) was used. This model actually performs better for Auger spectroscopy than a modern broad-band amplifier, since the tuned signal channel suppresses the signal harmonics. It was necessary to ground the final RFA grid between the LEED screen and the energy-resolving grids to reduce the capacitive coupling, otherwise the lock-in amplifier was overdriven at the fundamental modulation frequency.

Auger spectra recorded in this relatively low resolution, high beam current mode provided a quick check of the surface cleanliness. The high beam current would seriously damage chemisorbed overlayers, however, so diagnostic Auger spectra were recorded only after the LEED data were acquired. When adsorption uptake and coverage measurements were made much lower beam current densities were used. The intense Auger electron beam could affect surface reactions, for example, the rate of oxidation of surface carbon was significantly higher under the influence of the Auger beam and if the beam was left on during cleaning, the part oi the crystal illuminated by the electron beam would be clean well before the rest of the crystal surface.

It is possible to record high-quality high resolution Auger spectra with a LEED optics RFA, despite claims to the contrary. See for example figure 13.1, where copper and aluminum Auger doublets separated by only three eV were 
easily resolved using a $1 \mathrm{~V}$ peak-to-peak modulation and a $0.3 \mathrm{~s}$ time constant.

Surface cleanliness was primarily monitored by Auger spectroscopy. Since the platinum Auger transitions are relatively weak, most of the common contaminants are easily detected. Sulfur, for example, overlaps a platinum Auger line at $\sim 150 \mathrm{eV}$, but the sulfur Auger cross section is much larger than the platinum $150 \mathrm{eV}$ cross-section, so small sulfur concentrations are still detectable by comparing Auger peak height ratios. When calcium or silicon segregated to the surface during annealing they usually formed surface oxides. If an oxygen peak was seen in the platinum Auger spectrum it usually indicated the presence of silicon or calcium. These oxides could only be removed by sputtering.

An Auger spectrum from a contaminated Pt(111) surface is shown in figure 9.1. This spectrum was recorded after annealing the crystal, so most of the surface carbon, usually the main contaminant, had disolved into the bulk crystal. It should be noted that the commonly used Auger reference spectrum for platinum ${ }^{3}$ includes unlabeled impurity peaks due to silicon $(93 \mathrm{eV})$, argon $(217 \mathrm{eV})$ and carbon $(272 \mathrm{eV})$.

\subsubsection{Oxygen treatment}

Usually the last $30 \%$ or so of a monolayer of surface carbon could not be removed by sputtering. This was probably because residual gas-phase hydrocarbons would react with the hot crystal surface during annealing. This remaining carbon was removed by oxygen treatments. The crystal was heated to $\sim 1000 \mathrm{~K}$ in an atmosphere of $2 \cdot 10^{-7}$ torr of oxygen. Under these conditions a fraction of a 
monolayer of carbidic carbon (see chapter 12) could be oxidized and evaporated in $\sim 120 \mathrm{~s}$. The oxidation rate for graphitized carbon was at least an order of magnitude slower. Extended oxygen treatments, or reaction with oxygen at higher temperatures usually resulted in bulk impurity segregation to the surface, which could only be removed by ion sputtering.

After oxygen treatment the crystal was quickly flashed to $\sim 1300 \mathrm{~K}$ and cooled to room temperature. This desorbed any oxygen adsorbed on the platinum surface and dissolved remaining trace amounts of carbon on the surface into the bulk platinum crystal. This procedure produced a clean surface with no impurities detectable by AES or LEED observations.

LEED observations provided a final check on crystal cleanliness. The LEED background intensity, especially when the crystrl is cooled to $150 \mathrm{~K}$, is very sensitive to disorder. If the background did not appear sufficiently dark to the eye, it often indicated some residual contamination which could be resolved by more careful AES measurements. The surface oxides of silicon and calcium form ordered islands which produce complex and distinctive LEED patterns made up of very sharp spots. These patterns can be strikingly visible in LEED when the surface concentration of the oxides is barely above the Auger detection threshold.

\subsection{Crystal heating and cooling}

Crystal temperature control is important for surface structure studies. The single crystal surface needs to be heated to high temperatures for crystal cleaning and annealing. LEED I-V measurements are most sensitive when made at low 
temperatures and the ordering of chemisorption systems often depends on temperature. The crystal temperature was monitored by a 0.005 " chromel-alumel thermocouple spot-welded to the edge of the platinum crystal. An optical pyrometer was used to check the thermocouple performance.

Two different methods were used to heat the platinum crystal. In the earlier work a "button heater" was used. This is a molybdenum cylinder $\sim 9 \mathrm{~mm}$ in diameter containing a toroidally-wound tungsten wire heating coil potted in a ceramic compound. ${ }^{4}$ The maximum current of four $A$ could raise the heater temperature to $\sim 1250 \mathrm{~K}$. The main advantage of this heater is the toroidal winding, which contains the magnetic field produced by the heater current. LEED and electron spectroscopy measurements can be made undisturbed while the heater is operating. The main disadvantage is the limited current. At low temperatures the filament resistance is reduced and the total power output is restricted, so the crystal heats slowly. If a large-area sample is heated the thermally radiated power increases and so the temperature at the maximum heater current drops.

In later experiments resistive heating was used. The crystal was spot-welded to a pair of of tantalum foil strips and a current was passed through the supports to the crystal. Resistive heating at the spot-welds heats the crystal. With resistive heating the crystal can be heated as fast as desired, which is useful for thermal desorption and the temperature is limited only by the thermocouple or crystal melting points. Thermal stresses can break the spot welds to the crystal and then the vacuum system must be brought up to air for repair. Heater currents up to 50 A may be required, which have severe affects on LEED and other electron 
spectroscopies. This was tolerable since most LEED measurements were carried out at room temperature or at the minimum obtainable temperature.

The crystal was cooled by conduction from liquid nitrogen reservoirs through copper braids, as described in chapter 8 . These braids also carry the crystal heater current. The crystal holder is constructed so there is a relatively large thermal mass near the crystal. The crystal itself is relatively weakly coupled to this mass. The initial time needed to cool the sample holder is $\sim 30$ minutes. Once the crystal holder reaches thermal equilibrium, the crystal itself can be quickly flashed to a high temperature without much affect or the sample holder temperature. The small thermal mass of the crystal cools back to equilibrium teinperature of the sample holder very quickly. If the crystal is flashed to $\sim 550$ $\mathrm{K}$ any carbon monoxide or hydrogen adsorbed during the initial cooling will desorb intact from the platinum surface. The crystal will cool back to $\sim 200 \mathrm{~K}$ in ahout $50 \mathrm{~s}$ and to $\sim 150 \mathrm{~K}$ in about 3 minutes. Even when the crystal is flashed to $\sim 1300 \mathrm{~K}$ the cooling time is increased by less than a minute.

\subsubsection{Thermal desorption}

Thermal desorption spectroscopy (TDS) was used to characterize the surface adsorption sites and energies and to measure surface coverage. TDS measurements were made by manually setting the mass spectrometer to the desired mass and connecting the output to the $y$-axis of a chart recorder. The $x$-axis of the chart recorder was connected to the thermocouple (the chart recorder had high impedance inputs and built in amplifiers). The crystal was heated resistively 
using a constant current power supply and a heater current was chosen that gave a linear heating rate of 10 to $20 \mathrm{~K} / \mathrm{s}$ in the relevant temperature range.

The tantalum foil strips that support the crystal : $1 \mathrm{~d}$ carry the heater current heat very quickly during the TDS experiment $(>100 \mathrm{~K} / \mathrm{sec}$ ) so desorption from the foil produces a sharp spike at the start of the TDS spectrum which does not interfere with the platinum desorption data.

\subsection{Chemisorption techniques}

The chemisorption experiments involved gas-phase adsorbates. These were admitted to the vacuum system from a gas manifold using a standard leak valve. The leak valve was connected to a stainless-steel doser tube $1.5 \mathrm{~mm}$ in diameter. The end of the doser tube was located about $5 \mathrm{~cm}$ away from the crystal position so it did not block the LEED screen. When the crystal was moved away from the doser the tube was aimed at the mass spectrometer ionizer. Experiments with carbon monoxide adsorption showed that the effective gas exposure, measured by the nominal ion-gauge reading, was enhanced by a factor of five when the doser was used in place of a second leak valve which was not in the line of sight of the crystal.

Before each exposure the gas manifold was pumped down to a pressure below $10^{-7}$ torr and then flushed with the gas or vapor to be used in the adsorption experiment. The manifold was pumped out and this procedure was repeated for a second time before the actual exposure. The gases used for adsorption (carbon monoxide, propylene, butenes, etc.) were Matheson CP grade or better (99.8\%+ 
purity) and were used without further purification. The benzene exposures were made with spectroscopic grade benzene. The benzene samples were placed in a glass vessel attached to the vacuum line and frozen. The glass container was pumped out, closed off and the sample was thawed. This procedure was repeated several times to remove dissolved atmospheric gasses. Benzene has a vapor pressure of $\sim 100$ torr at room temperature, so benzene vapor could be admitted through the leak valve in the same way as the gases.

All of the gases used for chemisorption were checked for purity using the mass spectrometer. Before each adsorption the crystal was flashed above $500 \mathrm{~K}$ tr desorb any hydrogen or carbon monoxide on the crystal surface. Adsorption was done ai various crystal temperatures and a various pressures, depending on the specific experiment.

\subsection{LEED technique}

Reliable LEED structure determination requires accurate I-V data. LEFD I$V$ curves are a strong function of incidence angle. To be useful I-V curves must be measured at well-defined and controlled angles. This requires control of the electrostatic and magnetic fields, since they have an energy-dependent effect on electros trajectories. The ineasured LEED beam intensities must be corrected for variations in the incident beam current or in detector efficiency. 


\subsubsection{The LEED optics}

The LEED measurements were made using a standard Varian four-grid LEED optics. The relative orientations of the grids were adjusted to minimize the Moiré patterns in the visible images. The pass-energy of the RFA was adjusted for each set of $\mathrm{I}-\mathrm{V}$ curve data. At the maximum I-V curve energy the RFA pass-energy was increased until the LEED spots started to de-focus, then reduced slightly. Since the pass energy is a fixed amount less than $V_{\text {beam }}$ the defocusing effect is greater at higher energies.

The bias on the LEED screen ranged up to $7 \mathrm{KV}$. Sometimes bright flares of light were visible on the screen, caused by field-emission from dust or microscopic projections on the grid wires. The flares are more intense at high screen voltages. The video LEED system can subtract a certain degree of background intensity, but too much stray light degrades the signal quality. It was often possible to "burn-off" the source of such flares by disconnecting the electronics from the vacuum chamber and applying a Tesla coil (a high-voltage generator with negligible current) to the grid connections. (This trick will fix a wide variety of electron gun and electron optics problems.) The screen bias was set at the maximum value at which the screen flares were tolerable to maximize the brightness of the diffraction spots. 


\subsubsection{Voltage measu wement}

The experimentally measured quantity is the voltage difference between the Fermi levels of the filament and the crystal sample. This is the voltage measure used in all of the LEED I-V data reported here. The Varian power supply was modified to allow direct measurement of the center-tap voltage of the tungsten hair-pin filament in the LEED electron gun. To calculate the beam energy in vacuum, for example to determine diffaction angles, the experimental voltage must be corrected for the difference in work functions between the sample and the

filament, so $V_{v a c u u m}=V_{\text {filament-crystal }}+\phi_{\text {crystal }}-\phi_{\text {filament }}$. The work function is $\sim 4.5 \mathrm{~V}$ for tungsten and $\sim 6 \mathrm{~V}$ for platinum. Likewise, to determine the effect of electron lenses the measured voltage should be corrected by $\Delta V=\phi_{\text {lens }}-\phi_{\text {filament }}$. Usually these correations of a volt or two are not particularly important for LEED, especially since the inner potential is treated as an adjustable parameter in structure calculations.

\subsubsection{The LEED electron gun}

The LEED experiments were pe:formed using a siandard Varian "off-axis" electron gun. ${ }^{1}$ In this gun design electrostatic deflection plates bend the beam through a small angle $\left(\sim 20^{\circ}\right)$ so the filament is not in the line-of-sight of the final electron beam. This allows a high-temperature and relatively durable tungsten filament to be used, since most of the light emitted from the filament is trapped in the gun and does not reflect off the crystal and interfere with LEED measurements. 
The Varian power supply is designed so that one single supply provides the potentials for all the gun lenses through a voltage divider. This (rather economical) design means that the beam currelıt will be a strong function of the beam voltage. Electron emission from the filament can take place in two regimes. In space-charge limited operation the emission is limited by the charge density built up around the filament, but not by the specific emission of the flament. The emitted current will depend on the geometry and voltages in the region of the filament. At higher operating voltages the charge density around the filament is depleted and the beam current is limited by the specific emission of the filament, which depends on temperature. Figure 9.2 shows beam current plotted against voltage for various filament temperatures. The beam current rises monotonically and roughly linearly while the gun is space-charge limited, then the emission current reachs a plateau, which depends on the filament temperature.

During I-V experiments the filament temperature was set sufficiently high so that the gun operated in the space-charge limited mode throughout the I-V curve. The I-V curves were later normalized for constant incident beam current. This arrangement is advantageous experimentally. The beam current is insensitive to fluctuations in the filament heater voltage. The maximum parallel momentum transfer is proportional to $\sqrt{V_{\text {beam }}}$, so the number of visible beams is proportional to $V_{\text {beam }}$. Since the incident beam current also increases in proportion to $V_{\text {beam }}$, the incident electron current per difiracted beam is approximately constant; and less dynamic range is required for the LEED detector. 


\subsubsection{Magnetic and electrostatic flelds}

Magnetic fields deflect electron trajectories and as the electron energy changes the amount of deflection will change. In a standard display LEED optics the electrons travel in a nominally field-free region, so as beam energy changes, the angle of incidence will change in the presence of a magnetic field. If this change is $\sim 1^{\circ}$ over the energy range of an I-V curve the I-V curve can be significantly affected. The angular deflection due to the magnetic field is, for small angles

$$
\Delta \theta=\frac{\Delta \mathrm{p}_{\perp}}{\mathrm{p}}=\frac{F \Delta t}{\mathrm{p}}
$$

and $\Delta t=\frac{m l}{\mathrm{p}}$ where $l$ is the path-length and $F=\frac{e}{m c} \mathrm{p} B_{\perp}$, so

$$
\Delta \theta=\frac{e l}{c \mathrm{p}} B_{\perp}=\frac{e B_{\perp} l}{\sqrt{2 m c^{2} e V}}
$$

Here the angular deflection is measured in radians. Recognizing that the first factor in the denominator is twice the rest mass of the electron and multiplying by a factor of 299.8 to convert [esu gauss $\mathrm{cm}$ ] into [eV],

$$
\Delta \theta(\text { milli-radians }) \approx \frac{l(\mathrm{~cm}) B_{\perp}(\text { Gauss })}{\sqrt{V(\text { volts })}}
$$

For a display LEED oplics with a screen radius of $7 \mathrm{~cm}$ the angular defiection over the incident electron trajectory at $25 \mathrm{eV}$ due to the earth's magnetic field ( 0.5 gauss) will be $\sim 12^{\circ}$. To reduce the incident beam magnetic field deflection

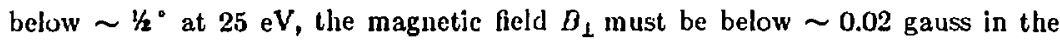
vicinity of the LEED optics. Magnetic fields also affect the opsration of electron 
guns. Near the filament the electrons have low energy and are easily deflected. If the deflection is large enough the beam can miss the lens apertures and be distorted or blocked. With no magnetic shielding or field correction typical electron guns stop working around 50 or $60 \mathrm{eV}$.

The ambient magnetic field can be neutralized by Helmhoitz coils or attenuated by magnetic shielding materials. Two pairs of Helmholtz coils one meter square were aligned perpendicular to the incident electron beam direction to neutralize the magnetic field. As the beam voltage changes the specular spot position will move because of magnetic field deflection. The Helmholtz coil currents were adjusted until the specular spot did not move significantly over the energy range of the I-V curve. A one degree deflection would cause motion of the specular beam spot on the order of $l \Delta \theta=1.2 \mathrm{~mm}$.

It is helpful to use magnetic shielding around the electron gun even if Helmholz coils are used for the LEED optics. Otherwise the coils must be adjusted to produce a good beam from the electron gun and at the same time prevent deflection of the incident beam once it leaves the gun. It is difficult to satisfy both conditions simultaneously, even with the help of deflection plates in the gun.

Electrostatic fields can cause severe distortions of the LEED patterns, but they are relatively easy to control. A charged insulator or ungrounded conductor in the vicinity of the LEED optics typically will produce a gross distortion of the LEED pattern bolow a given voltage. This problem can be prevented if there are no charged surfaces within $\sim 10 \mathrm{~cm}$ of the crystal. This means no insulation on 
thermocouple wires, heating/cooling leads or on the sample holder in the line-ofsight of the crystal. Also the shields of other instruments in the chamber should be grounded.

\subsection{Crystal orientation}

As discussed in chapter $\mathbf{8}$, it is important to be able to vary independently the polar angle $\theta$ and the azimuthal angle $\phi$ at which the LEED electron beam is incident on the crystal. LEED I-V curves are sensitive to changes in this angle of incidence on the order of $\sim 1 / 2$.

\subsubsection{Manipulator geometry}

The video LEED vacuum chamber was constructed with the "on-axis" geometry so the scattering geometry and manipulator construction are simpler than for the "off-axis" manipulator described in chapter 8 . The idealized manipulator geometry is shown in figure 9.3 . The incident LEED electron beam $\mathbf{k}_{\mathrm{o}}$ is in the horizontal plane and the polar $(\theta)$ rotation axis $\hat{\mathbf{r}}_{\text {, }}$ is vertical. A rotary feed-through is mounted on an XYZ translation stage to provide the $\theta$ motion. The azimuthal $(\phi)$ rotation axis $\hat{\mathbf{r}}_{\phi}$ is nerpendicular to $\hat{\mathbf{r}}_{\boldsymbol{g}}$ and a co-axial linear motion feed-through along the $\theta$ rotation axis controls the azimuthal angle $\phi$. The XYZ translation stage is adjusted so the $\hat{\mathbf{r}}_{\mathbf{q}}$ axis intersects the incident beam direction $\mathbf{k}_{\mathrm{o}}$ and so the crystal is at the focus of the LEED optics. When the crgstal is not at the focus the energy resolution of the retarding-field analyzer (RFA) is degraded. This is more important for Auger spectroscopy using the retarding- 
field energy analyzer than for LEED, since the RFA pass-energy is set at $80 \%$ to $90 \%$ of $V_{\text {beam }}$ so as not to de-focus the LEED beams. The focus position can be determined by setting the LEED gun voltage to $\sim 500 \mathrm{eV}$ and detecting the elasticly scattered electrons in the Auger mode at high $(<1 \mathrm{~V})$ resolution. The crystal is at the focus when the elastic peak is the sharpest.

When the single crystal is mounted on the manipulator sample hold?r, the front face of the crystal should lie along the polar axis $\hat{\mathbf{r}}_{\theta}$; and the unit vector $\hat{\mathbf{n}}$ normal to the crystal should be parallel to the azimuthal rotation axis $\hat{\mathbf{r}}_{\phi}$.

The sample holder has a gimbaled crystal mount controlled by set-screws so the crystal can be aligned after it is spot-welded to the tantalum supports and the crystal position along the $\hat{\boldsymbol{r}}_{\phi}$ axis can be adjusted so the crystal face can be placed along the $\hat{\mathbf{r}}_{0}$ :xis. After the crystal has been mounted and the axial position has been adjusted the gimbaled mount is set. A laser beam is reflected off the crystal surface while the azimuthal angle is varied. The gimbal is adjusted until the laser refiection angle is independent of the azimuthal angle.

\subsubsection{Crystal alignment}

It is difficult to align the crystal on the manipulator within the tolerance required for LEED data collection. The final calibration must be checked by observing the LEED patterns in vacuum. Even if the laser-alignment is perfect, there may still be some alignment error because the "optical" surface normal and the "electron diffraction" surface normal are not equivalent. For example, suppose a fec-metal single crystal is cut $1^{\circ}$ away from the (111) surface. This will 
produce a surface with (111) terraces $\sim 130 \AA$ wide separated by one-atom high steps. For low energy electrons $(\lambda \sim 1 \AA)$ the "electron diffraction" surface normal will be in the [111] direction. For visible light $(\lambda \sim 5000 \AA)$ the surface normal will appear perpendicular to the macroscopic surface $1^{\circ}$ away from the [111] direction.

The first step in the crystal alignment is to set the azimuthal angle. A simple metiod is to take a photograph of the LEED pattern using Folaroid film. A multiple exposure is made at different values of $\theta$. When the multiple images of the $(c, 0),(1,0)$ and $(\overline{1}, 0)$ form a single line the azimuthal angle corresponds to a mirror plane. (Polaroid photographs, including most of the LEED pattern illustrations, were made with Polaroid Type 57 film, ASA 3000, using a 4" format view camera, with exposures of 1 to $60 \mathrm{~s}$ and $f$-stops between 3.5 and 32.) Once the azimuthal angle is set correctly it does not need to be changed until a new cr:stal is mounted.

The next step is to check that the crystal normal $\hat{\mathbf{n}}$ is parallel to the incident LEED beam $\mathbf{k}_{\mathbf{0}}$. A simple check is made by measuring the perpendicular distance $d_{1}$ between the image of the LEED beam tube and the horizontal line made by the images of the $(0,0)$ LEED spot at different $\theta$ angles. If the alignment is perfect $d_{\perp}=0$, otherwise $d_{1}=2 l \Delta \theta$ where $\Delta \theta$ is the alignment error. For a $1^{\circ}$ error $d_{\perp}=$ $2.4 \mathrm{~mm}$, so this test is aceurate within $\pm 1 / 2^{\circ}$.

If the first test shows relatively close agreement a more sensitive test can be used. This is based on the visual observation of the beam symmetries. For the fec (111) surface the LEED pattern intensities should show three fold symmetry 
at normal incidence. If there is an alignment error $\Delta \theta$ then there is no nearnormal incidence angle where all three beams will have the same intensity. A very sensitive test can be made by finding an energy where the intensity of the beams in a particular symmetry group are a strong function oi the angle of incidence for near-normal incidence. For platinum $(111)$ the $(1,0),(0, \overline{1})$ and $(\overline{1}, 1)$ beams at $217 \mathrm{eV}$ satisfy this condition.

If the electron beam is not at normal incidence then the three beams will not have equal intensity, however different pairs of beams will have equal intensity at somewhat different angles. This can be used to calculate the alignment error $\Delta \theta$. A group of three symmetry related beams are pictured in reciprocal space in figure 9.4 with the origin at the specular beam " 0 ". At true normal incidence the vector $\mathbf{k}_{\mathrm{o}}$ ll lies along the dashed horizontal line labeled " $\Delta \theta=0$ ". When there is an alignment error, then the vector $\mathbf{k}_{\mathrm{o}}$ ll lies along the dashed horizontal line labeled " $\Delta \theta \neq 0$ ". When $\mathbf{k}_{\mathrm{o}}$ ll lies along the diagonal line labeled $M_{12}$, then spots " 1 " and " 2 " should have equal intensities; likewise when $\mathbf{k}_{\mathrm{oll}}$ lies along the diagonal line labeled $M_{23}$ then spots " 2 " and " 3 " should have equal inteasities. To make the test the angles $\theta_{12}$ and $\theta_{23}$ are determined where the the pairs of beams 1-2 and 2-3 have equal intensities. Using these angles the alignment error $\Delta \theta$ can be calculated. From the geometry shown in figure 9.4,

$$
k_{0} \| \tan \frac{\pi}{6}=k_{1-2 \|}-k_{2-3 \|}
$$

then

$$
\sin \Delta \theta=\frac{\sqrt{3}}{2} \sin \frac{\theta_{12}-\theta_{23}}{2}
$$


and the angles are small so that $\Delta \theta \approx \sqrt{3} / 2\left(\theta_{12}-\theta_{23}\right)$.

By observing the $(1,0)$ group of spots on $\mathrm{Pt}(111)$ near $217 \mathrm{eV}$ the angles $\theta_{12}$ and $\theta_{23}$ can be reproducibly determined $\pm 0.1^{\circ}$ using a vernier scale inscribed on the rotary feed-through housing, so the alignment error $\Delta \theta$ can be calculated accurately. If $\Delta \theta>1 / 2^{\circ}$ beams in the same symmetry group at normal incidence can have significantly different I-V curves. For $\Delta \theta<1 / 3^{\circ}$ th.e agreement between symmetry related I-V curves is quite good. This test does nut work very well for $\Delta \theta>2^{\circ}$ since the I-V curves for the three LEED beams start to diverge.

There are two ways to adjust $\Delta \theta$ without opening the vacuum chamber. The off-axis electron gun deflection angle can be varied by $\sim \pm 1 / 2^{\circ}$ without degrading the beam quality. With the deflection direction set in the vertical plane this allows some adjustment in the direction of $\mathbf{k}_{\mathbf{0}}$. The rotary motion feed-through that provides the $\theta$ motion was mounted on an "accu-port" flange ${ }^{5}$ that could tilt the B,r axis by $\pm 10^{\circ}$. This could be used to correct for $\Delta \theta$; however, this angular adjustment has a long lever arm $(\sim 30 \mathrm{cris})$ so a $1^{\circ}$ adjustment will translate the crystal by $0.51 \mathrm{~mm}$. The $\mathrm{XYZ}$ translation stage must be used to return the crystal to the focus of the LEED optics and this motion was limited to about $\pm 0.8 \mathrm{~cm}$.

If the error in alignment was too large to correct with the electron gun deflection plates or by tilting the $\hat{\mathbf{f}}$ axis, a window was opened in the vacuum system; and the sample holder gimbal was adjusted. The pitch of the gimbal setscrews was known and also the sign and magnitude of $\Delta \theta$, so a single adjustment 
could set $\Delta \theta=0.0 \pm 0.3^{\circ}$.

\subsubsection{Angle and spot labeling conventions}

The LEED substrate spots for the (111) surface are indexed in terms of the two basis vectors $(1,0)$ and $(0,1)$. These vectors are separated by $60^{\circ}$ and the angle going from $(1,0)$ to $(0,1)$ is counter-clockwise as seen in the LEED screen. There are two inequivalent beams that can be labeled $(1,0)$. The platinum LEED beams were indexed so that the I-V curve labels in this work agree with those in Adams et al. ${ }^{6}$ The overlayer beams are indexed in terms of these substrate beam basis vectors. The angle $\theta$ is defined such that the specularly reflected beam moves to the right as seen in the LEED screen when $\theta$ increases. The azimuthal angle $\phi$ is defined to be zero when the $(1,0)$ beam is parallel to $\mathbf{k}_{\mathrm{o}} \|$ and points to the right as seen in the LEED screen and $\phi$ increases in the counter-closkwise direction.

All of the experimental LEED data were recorded with $\mathbf{k}_{\mathrm{o}+}$ in a mirror plane, i.e. with $\phi=0^{\circ}$ or $180^{\circ}$. Normal incidence was defined using Eq. 9.5 so $\theta_{\text {zero }}=1 / 2\left(\theta_{12}+\theta_{23}\right)$. Off-normal polar angles were set relative to $\theta_{z e r o}$ using the scale inscribed on the rotary feed-through.

\subsection{LEED data reduction}

The r-factor calculations used in LEED structure determination are most reliable for a large data base. For complicated surface structures ther may be several geometrical parameters in the structural model that must be determined. 
A good data base would include I-V curves for five to ten non-degenerate diffraction beams recorded at known angles of incidence over the full energy range used for the structural calculations. LEED calculations are normally limited to energies above $\sim 20 \mathrm{eV}$, since the approximations used in LEED calculations break down below this energy. The complexity of LEED calculations expands rapidly with increasing energy, so I- $V$ curves are usually not calculated above 150 to $250 \mathrm{eV}$, depending on the size of the surface unit-cell and the complexity of the structure. The absolute angles of incidence must be known and maintained during I-V curve collection.

In the structure determination experiments LEED data sets were recorded at normal incidence and at least two off-normal angles. All of the off-normal data were recorded with the incident beam in a mirror plane of the surface. Two independent experiments were preformed for each data set, that is the crystal was cleaned by ion sputtering, re-annealed, re-oriented and a new overlayer formed between the experiments. The consistency of the independent data sets provided a check on crystal cleanliness, surface preparation and angular oritntation. Symmetry related beams were also checked for degeneracy. At normal incidence this is an absolute check on orientation, while at off-normal incidence it can only confirm that $\mathbf{k}_{\mathbf{o}}$ is in the surface mirror plane.

I-V curves were calculated for all beams visible in the digitized diffraction patterns using the methods of chapter 7 . The resulting I-V curves were not used in the final data set if the signal to noise ratio was too bad due to limited camera sensitivity, if too much of the I-V curve had been obscured as the diffraction 
beam passed behind obstacles such as the sample holder and its electrical connections, or if the beam first appeared on the LEED screen too close to the maximum beam voltage.

Diffraction patterns were recorded and the beam intensities calculated at two volt intervals. For electron-beam sensitive overlayers the data were collected as a function of jncreasing beam voltage to minimize the mean beam dose at each energy. The data consist of intensities $I_{1}, I_{2}, \cdots, I_{N}$ for each beam. The experimental intensities have been normalized to a $1 \mu \mathrm{A}$ incident beam current. The data were not corrected for the variation in the LEED screen illuminance or for the variation in optical transmission of the LEED grids with angle (see section 2.5.3.2)

The absolute intensity values used in the I-V curves are the integrated intensity of the LEED spots normalized to $1 \mu \mathrm{A}$ incident beam current when the entire LEED screen imaged by the video camera. Full video intensity is $2^{12}=4096$ counts per pixel and the image resolution is $512 \times 480$ pixels. All of the I-V data presented here were recorded with ihe Panasonic/Newvicon camera (see chapter 5) with an $f / 0.85$ lens. Intensity values scale as $(f-s t o p)^{-2}$ and this value is reported when $I-V$ data are discussed.

All of the acceptable I-V curves were included in the final data set. Symmetry related beams from the two independent runs were averaged together to improve the I-V curve signal to noise ratio. Some of the $r$-factors, in particular $r_{Z-\rho}$ and $r_{\text {Pendry }}$ involve derivatives of the beam intensities, so the three-point smoothing routine was applied to the experimental I-V curves before they were 
compared to the theoretical calculations. The smoothing algorithm is given by

$$
I_{n}^{\prime}=\frac{1}{4}\left(I_{n-1}+2 I_{n}+I_{n+1}\right)
$$

This algorithm does not change the integrated intensity of the I-V curve. Intensity peaks are typically 10 or more $\mathrm{eV}$ wide so the smoothing routine does not broaden the peaks significantly, especially since the theoretical calculations are usually made on a $5 \mathrm{eV}$ grid. 


\section{References}

1. Varian model 981-2145, Varian Corp., Palo Alto, CA.

2. J. F. O'Hanlon, A User's Guide to Vacuum Technology, Wiley-Interscience, New York, 1980.

3. L. E. Davis, N. C. McDonald, P. W. Palmberg, G. E. Ricah, and R. E. Weber, Handbook of Auger Electron Spectroscopy, Perkin-Elmer, Eden Prarie, MN, 1976.

4. Spectromat, Inc., Watsonville, $\mathbf{C A}$.

5. Huntington Vacuum Laboratories, Model VF-175-10, Mountain View, CA.

6. D. L. Adams, H. B. Nielsen, and M. A. Van Hove, Physical Review B, vol. 20, p. $4789,1979$. 


\section{Figure Caplions for Chapter 9}

9.1 Auger spectra for $\mathrm{Pt}(111)$ contaminated with carbon, silicon, sulfur, calcium, phosphorus and oxygen.

9.2 The typical beam current of the electron gun used for the video LEED experiments, plotted as a function of beam voltage at various filament temperatures.

9.3 The "on-axis" LEED manipulator geometry, showing the $\hat{\mathbf{r}}_{\theta}$ and the $\hat{\mathbf{r}}_{\phi}$ rotation axes and the incident beam direction $\mathbf{k}_{\mathbf{p}}$.

9.4 A schematic diagram of the LEED pattern shows the effect of an alignment error $\Delta \theta$. For $\Delta \theta \neq 0$ the vector $\mathbf{k}_{\mathbf{o}}$ il traces out the horizontal line labeled $\Delta \theta \neq 0$ as $\theta$ is varied. When the line $\Delta \theta$ crosses the lines $M_{12}$ or $M_{23}$ then $\mathbf{k}_{\mathrm{o} \|}(\theta)$ is in the mirror plane and the intensities of spots " 1 " and "2" or "2" and "3" are equal. 


\section{Pt Auger Spectrum \\ Contaminated Surface After Anneal}

Platinum peaks: 151, 168, 187, 201, 360, 392 and 466

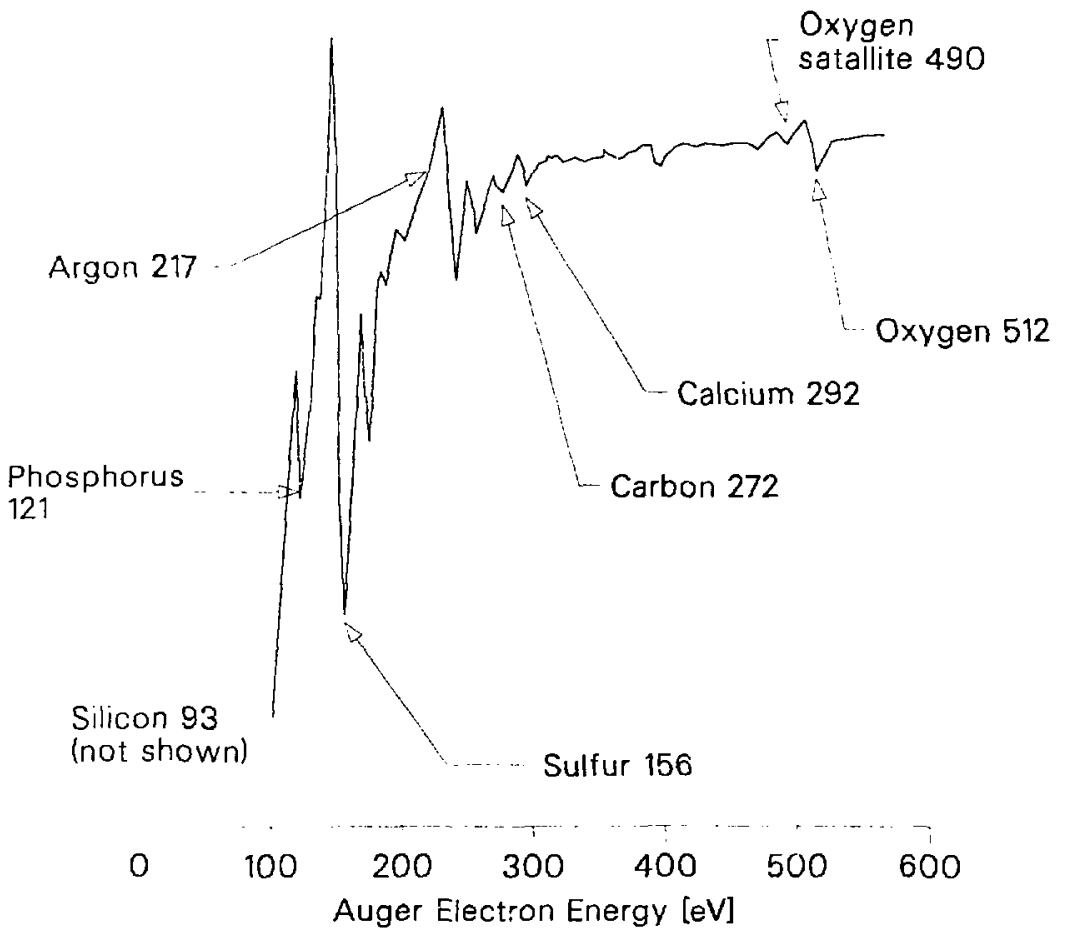

XBL $8612-4848$

Fgre 9.1 
374

\section{Video LEED Gun Current}

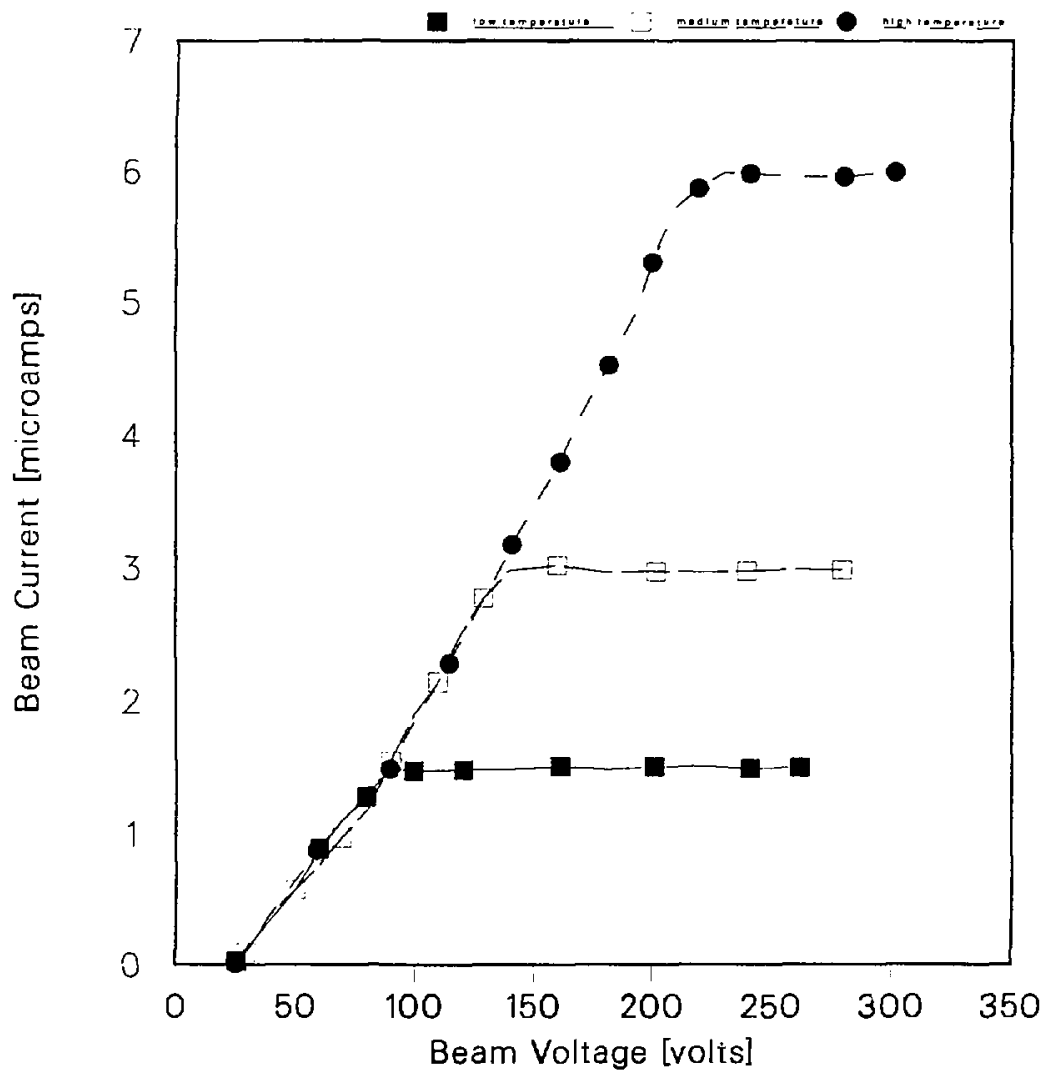

$X B L \quad 8612-4847$

Figure 9.2 


\section{On-Axis Manipulator}

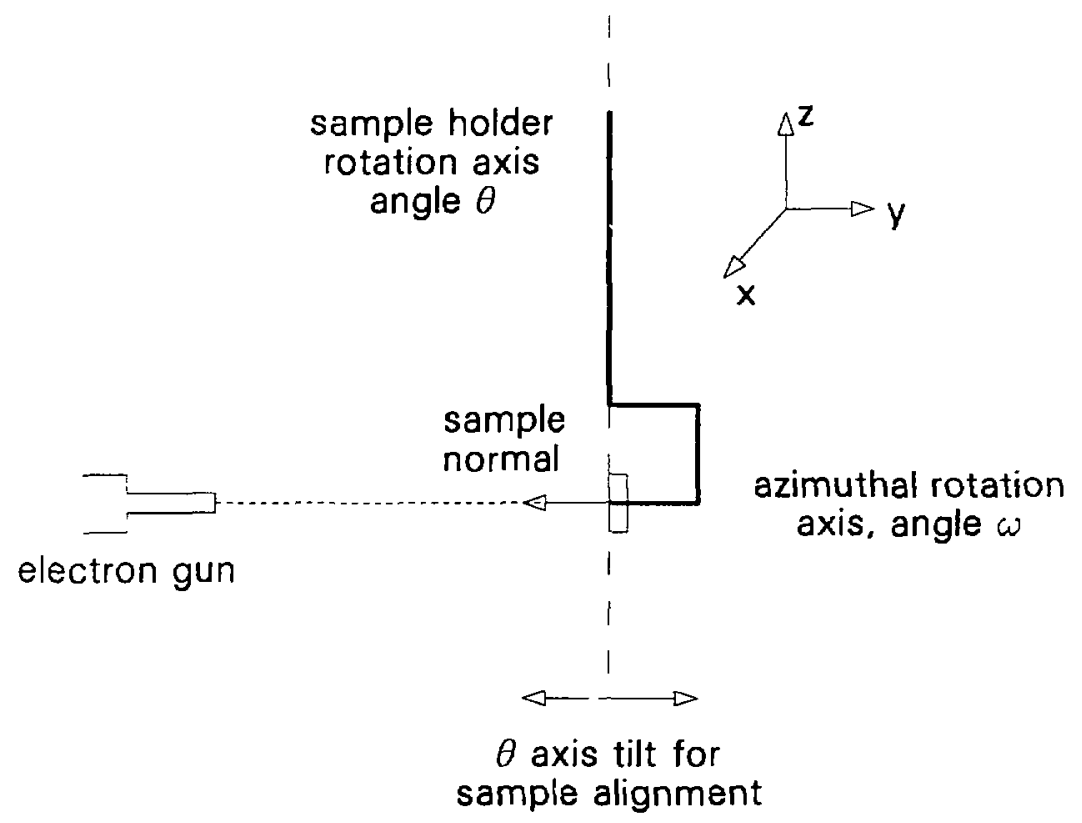

XBL $8612-4850$

Figure 9.3 
376

Normal Incidence Condition

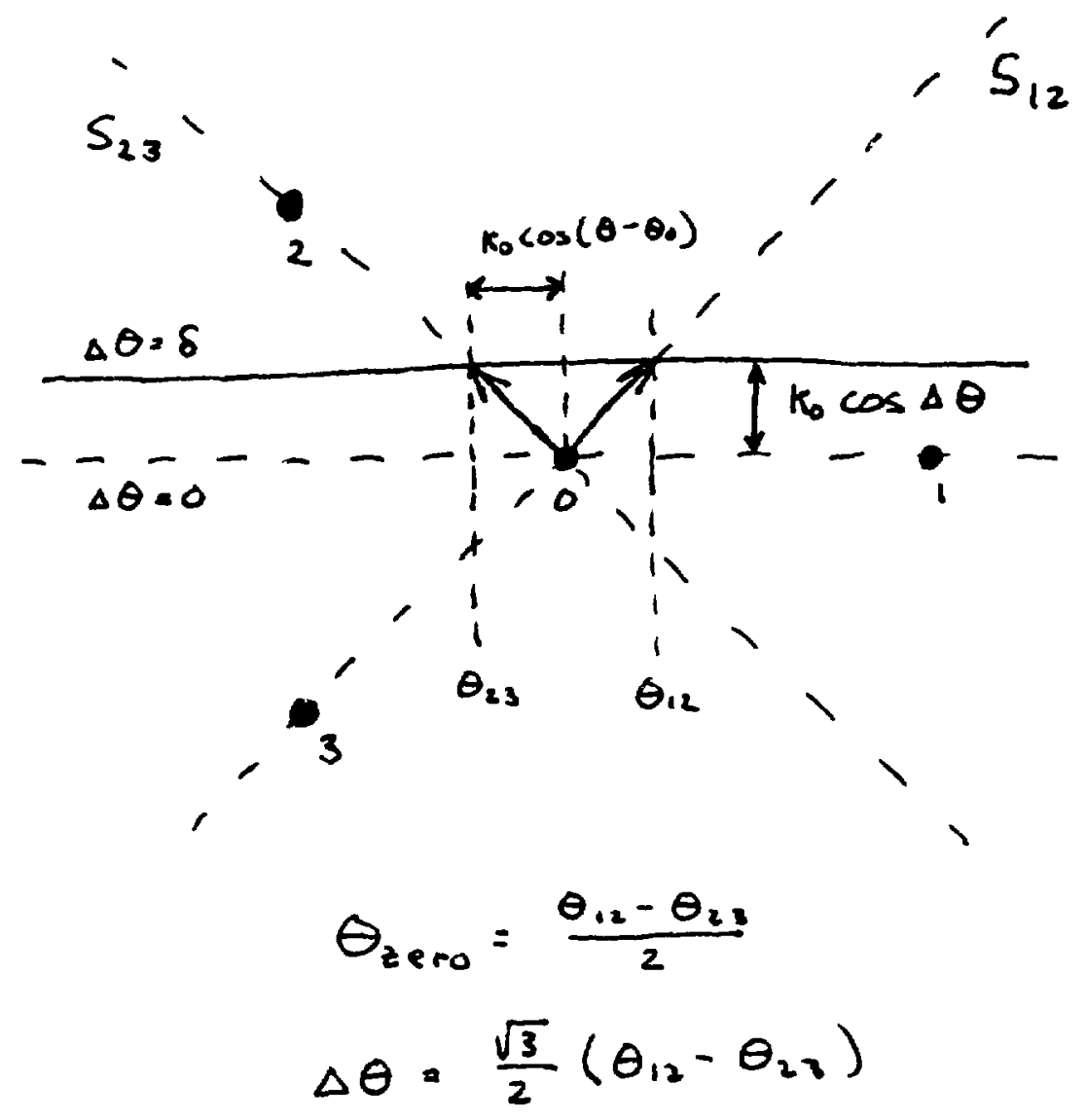

Figure 0.4 


\section{Chapter 10}

\section{The Structure of $c(4 \times 2)$ Carbon Monoxide on $\operatorname{Pt}(111)$}

\subsection{Introduction}

Carbon monoxide adsorption on transition metal surfaces has been studied extensively by many techniques (see table 1.9). This has become the prototype system for molecular chemisorption and more structural studies have been done for carbon monoxide than for all other molecular adsorbates comtined. Carbon monoxide adsorbed on platinum is of particular interest, sisce the platinumcarbon monoxide reaction is one of the main functions of platinum-based automotive exhaust-gas treatment catalysts.

The chemisorption of carbon monoxide on the $\mathrm{Pt}(111)$ surface has been the subject of many studies using a variety of techniques, including thermal desorption spectroscopy (TDS), $1,2,3$ work-function measurements, ${ }^{1,4}$ angle-resolved photoemission spectroscopy, ${ }^{5,6}$ infra-red reflectance adsorption spectroscopy (IRAS), ${ }^{4,7}$ electron energy loss spectroscopy (HREELS) $2,3,8,9,10$ and the analysis of LEED patterns. ${ }^{1,2}$

Tnese studies have shown that carbon monoxide inolecules adsorb intact on $\mathrm{Pt}(111)$ and the molecules desorb intact in TDS experiments in a single peak at $\sim$ $475 \mathrm{~K}$ for low coverages. As the coverage increases toward saturation the thermal desorption peak broadens and moves to lower temperatures. ${ }^{1,3}$ Carbon monoxide molecules bond to the surface carbon end down with the molecular axis 
perpendicular to the surface..$^{5,6}$

\subsection{The carbon monoxide-Pt(111) system}

Carbon monoxide chemisorbed on the platinum (111) surface has a conplex phase diagram resulting from the balance between adsorbate-adsorbate and adsorbate-substrate interactions. The carbon monoxide molecules are known to change adsorption site as a function of coverage and at least six different ordered LEED patterns have been observed as a function of surface temperature and coverage. In spite of intensive investigation, there are still unanswered questions about the carbon monoxide-Pt(111) system.

This is the first structural study of carbon monoxide on $\operatorname{Pt}(111)$. Only the structure of the $c(4 \times 2)$ phase was investigated, since beam damage problems and insufficient crystal cooling prevented LEED iñtensity measurements for the other phases. There are two competing models to explain the high-coverage phase diagram; uni-axial compression of the carbon monoxide unit cell ${ }^{\mathbf{1}}$ and changes in the density of domain-wall anti-phase boundaries as the mechanism of phase transitions. ${ }^{10,11,12}$ New structural results and other observations on the carbon monoxide-Pt(111) systemn help to answer these questions.

\subsubsection{Low coverage $(\theta \leq 0.35)$}

At low coverages $(\theta<0.35$, where $\theta$ is the ratio of adsorbed carbon monoxide molecules to first-layer platinum surface atoms) a diffuse, poorly ordered $(\sqrt{3} \times \sqrt{3}) \mathrm{R} 30^{\circ} \mathrm{LEED}$ pattern was observed at room temperature. On cooling to 
$\sim 130 \mathrm{~K}$, the minimum temperature obtainable with the LEED sample holder, there was very little change in the LEED pattern (see figure 10.1) and the spots do not get sharper.

Recent low temperature LEED, TDS and HREELS studies ${ }^{3,13}$ have shown that below $\sim 100 \mathrm{~K}$ two new, well ordered LEED patterns are observed, one at $\theta$ $=0.17$ and another af $\partial=0.33$, with an intermediate streaked LEED pattern observed at $\theta \approx 0.22$. Another study failed to observe these LEED patterns under similar conditions. ${ }^{7}$ Each of the diffuse spots in the " $(\sqrt{3} \times \sqrt{3})$ R30 phase" is resolved into a triangle of closely spaced spots in the two low-temperature ordered phases. The "intermediate" pattern visible at a reported coverage of 0.22 is shown in figure 10.2. HREELS data show that only one site is occupied in the $\theta$ $=0.17$ phase with a loss-peak at $2100 \mathrm{~cm}^{-1}$, presumably the top-site, while a seccnd site with a loss at $1850 \mathrm{~cm}^{-1}$ starts to be occupied at the carbon monoxide coverage increases. ${ }^{3}$

\subsubsection{The $c(4 \times 2)$ phase}

The $c(4 \times 2)$ LEE.) pattern can be seen on $\mathrm{Pt}(111)$ for carbon monoxide coverages $0.35 \leq \theta \leq 0.5$, implying island formation. ${ }^{3}$ A complete $c(4 \times 2)$ layer covers the surface at $\theta=0.5$. There are two inequivalent carbon monoxide molecules per unit cell, which are assumed to occupy different sites corresponding to the two HREELS loss peaks observed at $2100 \mathrm{~cm}^{-1}$ and $1850 \mathrm{~cm}^{-1}$ These losses have been assigned to the top and bridge sites, respectively $y^{2,3}$ (see figure 10.3). 
The superiattice spots in the $c(4 \times 2)$ LEED pattern become very dim for $\mathrm{T}>$ $270 \mathrm{~K}$ and they cannot be seen for $\mathrm{T}>300 \mathrm{~K}$. This appears to be due to a reversible $\mathrm{c}(4 \times 2) \underset{300 \mathrm{~K}}{\rightarrow}$ lattice gas order-disorder transition, since no appreciable amount of carbon monoxide desorption occurs below $\sim 350 \mathrm{~K}$ for $\theta=1 / 2$ and the $c(4 \times 2)$ LEED pattern reappears on cooling below $\sim 270 \mathrm{~K}$. LEED I-V curves were recorded for the $c(4 \times 2)$ phase and the structure was determined through LEED intensity calculations as described below.

\subsubsection{High coverage $(\theta \geq 0.5)$}

Three different LEED patterns have been observed for $\theta \geq 0.5$, along with streaked LEED patterns at intermediate coverages. In 1977 Ert] et al. ${ }^{1}$ reported ordered LEED patterns at $\theta=3 / 5$ (see figure 10.4) and at $\theta=2 / 3$ (see figure 10.5). These high coverage structures have unit cells that are best described with the rectangular unit cell notation introduced by Bibérian and Van Hove.11,12 in this notation the overlayer is described by a rectangular unit cell on a substrate witb three-fold rotational symmetry. The first number is the width of the unit cell in the [11 $\overline{2}]$ dirention (perpendicular to rows of atoms) and the second number is the length of the unit cell in the $[1 \overline{1} 0]$ direction (along a row). A "c" denotes a centered unit cell, as in the Wood notation. In this notation the $\mathrm{c}(4 \times 2)$ unit cell at $\theta=1 / 2$ is called $(\sqrt{3} \times 2)$-rect, the $\theta=3 / 5$ phase is $c(\sqrt{3} \times 5)$ rect and the $\theta=$ $2 / 3$ phase is $(\sqrt{3} \times 3)$-rect.

Ertl at al. determined the equilibrium surface coverage $\theta$ as a function of temperature and carbon monoxide background pressure for $\theta \geq 0.5$. They found 
that the carbon monoxide adsorption energy drops sharply above $\theta=0.5$, then continues to decrease with increasing coverage. As the adsorption energy drops, the electron stimulated desorption cross-section increases substantially. Although I-V curves could be recorded for the $(\sqrt{3} \times 2)$-rect phase with reasonable precautions, this was not possible for the higher coverage structures. Indeed, under the influence of a LEED incident beam current of $\sim 1 \mu \mathrm{A}$ the LEED pattern transformed continuously from $(\sqrt{3} \times 3)$-rect $\rightarrow c(\sqrt{3} \times 5)$-rect $\rightarrow(\sqrt{3} \times 2)$-rect in less than $60 \mathrm{~s}$. Useful I-V curve data from the high coverage carbon monoxide structures cannot be collected without a low-current, channel-plate amplified LEED system.

A third high-coverage carbon monoxide phase was reported more recently by Steininger et al.. ${ }^{3}$ They observed this LEED pattern after saturating the srystal with carbon monoxide at $100 \mathrm{~K}$ then annealing to $260 \mathrm{~K}$. According to the thermal equilibrium carbon monoxide coverage data of Ertl et al. ${ }^{1}$ this should result in a coverage $\theta<0.60$. Steininger et al. identified the observed LEED pattern, well-ordered with slightly elongated LEED spots, as an incommensurate quasihexagonal " $\sqrt{3 / 2} \times \sqrt{3 / 2}) \mathrm{R} 15$ " pattern at $\theta=0.58$. This pattern can also be described as a commensurate $c(\sqrt{3} \times 9)$-rect nhase with $\theta=5 / 9=0.55$ (see figure 10.6). If this $\mathrm{c}(\sqrt{3} \times 9)$-rect unit cell were described as a "incommensurate" coincidence lattice, the unit cell vector would be $\left(\frac{3}{2} \cdot \frac{97}{96}\right)^{\frac{1}{2}}$, very close to the reported $\sqrt{3 / 2}$ 


\subsection{Uni-axial compression and domain-walls}

Two distinct models have been proposed to explain the changes with coverage in the observed LEED patterns of carbon monoxide adsorbed on metal surfaces. Ertl et al. ${ }^{1}$ proposed the "vni-axial compression" model. In this model carbon monoxide molecules are adsorbed along the [1 $1 \overline{1} 0]$ rows of surface metal atoms. As $\theta$ increases, the carbon monoxide molecules maintain a constant separation as they are compressed along the [110] rows. The carbon monoxide overlayer is incommensurate in this model, although coincidence lattices will occur when $\theta$ is equal to a small rational number ratio. Bibérian and Van Hove ${ }^{11,12}$ have proposed another model, the "domain-wall" model. In this model increasing carbon monoxide coverage introduces anti-phase domain-walls into a commensurate overlayer, forming new ordered phases when the Homain-walls are periodic. Molecules are adsorbed only at high-symmetry adsorption sites. They have shown that this model is consistent with the available LEED and vibrationa] spectroscopy data for carbon monoxide adsorbed on (100) and (111) metal surfaces. 11,12

The uni-axial compression model and the domain-wall model have different implications for the understanding of the carbon monoxide- $\mathrm{Pt}(111)$ system. The uni-axial compression model requires that the interactions between adsorbed carbon monoxide molecules are strong compared to the variation in adsorption energy at different points on the surface. This model also raises questions about the correlation between the carbon-oxygen stretch frequencies and the adsorption sites determined by HREELS (see chapter 1 and figure 1.1). The domain-wall 
model, on the other hand, implies that the local carbon monoxide adsorption site geometry is important and the carbon monoxide-metal interaction is stronger than the inter-molecular interaction, while these inter-molecular interactions are important in controlling the sequence of ordered surface phases.

Careful analysis of the observed carbon monoxide-Pt(111) LEED patterns will be used to show that the "uni-axial compression" model is inconsistent with observations for carbon monoxide on $\mathrm{Pt}(111)$, while the "domain-wall" model helps to explain the LEED and vibrational spectroscopy data for this system.

\subsubsection{LEED pattern analysis}

The observed LEED pattern are explained in different ways using the two models. In the domain-wall model a series of commensurate overlayers produce the sharp LEED patterns. At intermediate coverages anti-phase domain-walls ar: formed. When these domains walls are ordered, sharp spots will be visible in the LEED patterns and when the domain-walls are randomly distributed, the overlayer spots will be streaked. Since the overlayer is commensurate, all of the LEED spots can be explained in the kinematic (single) scattering approximation.

The uni-axial compression model assumes an overlayer that is incommensurate in the $[1 \overline{10}]$ direction. The unit cell for this incommensurate layer can be described as $c\left(\sqrt{3} \times \frac{1}{\theta}\right)$-rect. The LEED spots in an incommens rate overlayer diffraction pattern can be classified into three groups: substrate, overlayer and combination or double-diffraction spots. If the overlayer unit cell is described by 
vectors $\{\mathbf{g}\}$ and the substrate unit cell by vectors $\{\mathbf{G}\}$, then these three sets of beams can be labeled by $\mathbf{g}, \mathbf{G}$ and $\mathbf{g}+\mathbf{G}$, respectively (see chapter 12). Only the spots $\mathbf{g}$ and $\mathbf{G}$ are present in the kinematic approximation, as the combination beams $\mathbf{g}+\mathbf{G}$ require scattering in the overlayer and the substratie.

When the overlayer and substrate unit cells are incommensurate, a large number of combination beams will contribute to the observed LEED pattern. When $\theta$ is a simple rational fraction like $1 / 2,3 / 5$ or $2 / 3$ the uni-axial compression model leads to a coincidence lattice. In this case different combination beams will coalesce, producing a relatively simpler diffraction pattern with fewer, brighter spots.

\subsubsection{Combination beam limits}

Many of the bright spots in the coincidence lattice patterns correspond to combination beams, yet as Bibérian and Van Hove have pointed out, ${ }^{11}$ these beams are usually significantly less intense than beams with single scattering contributions. For example, weak combination beams are visible in figure 12.1(a) of an incommensurate graphite overlayer on $\mathrm{Pt}(111)$. This photograph was taken near the maximum intensity of the overlayer beams and the combination beams are barely visible, approximately two orders of magnitude less intense than the overlayer beams. The density of carbon atoms in the graphite overlayer is comparable to the density of carbon and oxygen atoms combined for $0.5 \leq \theta \leq 0.6$.

There is an additional restriction on combination beam intensities. In principle there are an infinite number of combination beams that appear inside the 
substrate Brillouin zone for an incommensurate overlayer. In practice many of these $\mathbf{G}+\mathbf{g}$ combinations include individual vectors $\mathbf{g}$ and $\mathbf{G}$ of large absolute magnitude. If the magnitude of $\mathbf{g}$ or $\mathbf{G}$ is greater than the incident beam magnitude, the beam is evanescent and the intensity of the corresponding combination beam will be sharply attenuated. This condition occurs for some of the prominent beams in the observed diffraction patterns of carbon mcnoxide on $\operatorname{Pt}(111)$.

The maximum parallel momentum transfer $\Delta \mathbf{s}$ for normal incidence can be derived from Eq. 3.16. For a (111) surface, this is

$$
\alpha=\frac{\Delta \mathrm{s}}{\mathbf{G}_{01}}=\frac{a_{o} \sqrt{6 m\left(E-V_{o r}\right)}}{4 \pi h}=\left(\frac{E-V_{o r}}{26.1 e V}\right)^{\frac{1}{2}}
$$

where $a_{o}$ is the metal nearest-neighbor distance (2.77 $\AA$ for platinum) and momentum transfer $\alpha$ is measured in units of $\mathbf{G}_{01}$, a substrate unit cell vector.

Figure 10.7 is a schematic diagram of one domain of the $c(\sqrt{3} \times 5)$-rect LEED pattern at $38 \mathrm{eV}$ and normal incidence (the first published LEED photograph for this pattern ${ }^{1}$ ). At $38 \mathrm{eV}$ the maximum momentum transfer for a propagating plane wave is 1.21 in the vacuum and 1.38 in the metal (in units of $\mathbf{G}_{01}$ ), using the value of $12 \mathrm{eV}$ for $V_{0 r}$ determined in the LEED analysis for the $(\sqrt{3} \times 2)$-rect carbon monoxide phase. The $(2 / 5,2 / 5)$ beam in the LEED pattern fin terms of the substrate unit cell) is easily visible, but this beam is a combination beam. The two largest contributions to this beam are $(1,1)+(-3 / 5,-3 / 5)$ and $(0,-1)+$ $(7 / 5,2 / 5)$. In the first term the $(1,1)$ substrate beam is evanescent with magnitude $\sqrt{3}=1.73$ and in the second term the overlayer beam $(7 / 5,2 / 5)$ is evanescent, 
with magnitude 1.64. For these evanescent beams $k_{\perp}=i 1.04 \AA^{-1}$ and $i 0.881$ $\AA^{-1}$, respectively. Taking the substrate-overlayer spacing to be $1.55 \AA$, the distance between the metal and the closest overlayer atom, (the bridge-bonded carbon atom determined by LEED for the $(\sqrt{3} \times 2)$-rect phase), the intensities of these evanescent beams are attenuated by factors of 25.1 and 15.3. If the distance between the metal surface and the center of the carbon monoxide layer (2.1 $\AA)$ is used, these attenuation factors are 40 and 79.

A similar analysis could be applied to a number of other beams for various carbon monoxide-Pt(111) LEED patterns at different energies. The point is that some prominent beams in the observed LEED patterns are identified as combination beams in the uni-axial compression model and these beams are strongly attenuated. This attenuation factor is in addition to the overall weakness of second-order beams (as defined in chapter 12). The combination beams also have higher-order contributions from non-evanescent waves, such as in the example above, where $(1,0)+(0,1)+(-3 / 5,-3 / 5)$ also contributes to the observed $(2 / 5,2 / 5)$ beam. This contribution involves triple hack-scattering between the overlayer and the substrate, however, so it will be extremely weak.

\subsubsection{Interphase regions}

The uni-axial model and the domain-wall model also make different predictions for the LEED patterns at coverages intermediate between the observed coincidence lattices or ordered structures. Streaked or blurred spots in LEED patterns are usually associated with some degree of surface disorder. The uni-axial model 
does not predict disorder, so there should be no streaked spots; however, a number of different combination beams contribute each LEED spot for coincidence lattices. As the coverages changes, these terms no longer will coincide and this splitting could give the appearance of streaking. Figure 10.8 shows a schematic uni-axial LEED pattern for $\theta=0.53$. This pattern shows combination beam "satellites" around the substrate beams. Also, some of the split spots are far enough apart to be resolved as separate spots, for example, those near the $(1 / 3,1 / 3)$ and $(1 / 2,0)$ positions.

Some experimental diffraction patterns corresponding to coverages between $1 / 2$ and $3 / 5$ are shown in figure 10.9. There is no visible splitting or streaking associated with the specular spots and the streaks do not appear to be composed of discrete round spots.

The basic domain-wall model ${ }^{11,12}$ applied to carbon monoxide on $\operatorname{Pt}(111)$ is shown in figure 10.10. For coverages between $1 / ?$ and $3 / 5$ the surface is covered with unit celis of the $(\sqrt{3} \times 2)$-rect phase. Anti-phase domain-walls are introduced at right angles to the $[1 \overline{1} 0]$ rows of atoms. Fach new anti-phase domain-wall increases the average density of the overlayer. When a "domain-wall" has been inserted between each pair of $(\sqrt{3} \times 2)$-rect unit cells, a new ordered structure is formed, the $c(\sqrt{3} \times 5)$-rect phase at $\theta=3 / 5$. Different combinations of top and bridge sites are possible $e^{12}$ and the model chosen predicts an increase in the top to bridge site ratio for $\theta>1 / 2$ as observed in HREELS ${ }^{10}$ and IRAS. ${ }^{7}$

At intermediate coverages between $1 / 2$ and $3 / 5$ there is some disorder in the LEED pattern. There have been a number of investigations into the LEED 
patterns of partially disordered surfaces, most using a kinematic analysis. ${ }^{14}$ The, problem of anti-phase domains has been investigated both experimentally and through models based on kinematic diffraction theory. McKee et al. have analyzed the problem of anti-phase islands. ${ }^{15}$ They found that random anti-phase domains will split the superlattice spot into closely spaced spots, which will be seen as streaked spots when the effects of instrumental resolution are included. If the domains are widely separated islands, streaking is predicted for integral order spots. When the domains are in contact, only the superlattice spots are streaked.

\subsubsection{Streaks and domains}

Oxygen adsorbed on $\mathrm{Ni}(110)$ provides a good exampie of domain effects. When oxygen is adsorbed on $\mathrm{Ni}(110)$ at $570 \mathrm{~K}$ a $(2 \times 1)$ phase is formed with an oxygen coverage of half a monolayer. ${ }^{16}$ As the oxygen coverage increased to $2 / 3$, the $1 / 2$ order spots split continuously with increasing coverage until a $(3 \times 1)$ LEED pattern is formed. The authors showed that evenly-spaced domain-walls could produce this pattern. When the same experiment was performed at room temperature the $1 / 2$ order spots started to streak istead and the the streaks contracted to form the $1 / 3$ order spots as the oxygen coverage reached $2 / 3 .^{16}$ This was explained in terms of random domain-wall boundaries, which did not form a uniform distribution because of kinetic limitations on domain-wall motion at the lower temperature. Houston and Park ${ }^{17,18}$ carried out a more sophisticated analysis of domain-wall effects. They showed that a narrow distribution of domain-wall spacings will produce split spots, with increased splitting for closely 
spaced domain-walls and a broad distribution of domain-wall spacings will produce streaks.

This analysis can be applied to the carbon monoxide-Pt(111) system. The LEED patterns observed between $\theta=1 / 2$ and $3 / 5$ at $150 \mathrm{~K}$ (figure 10.9) show streaks. These start at spot positions for the $(\sqrt{3} \times 2)$-rect phase, grow outward toward the $c(\sqrt{3} \times 5)$-rect spot positions, then coalcsce in the new positions for $\theta=$ 3/5. The $c(\sqrt{3} \times 9)$-rect LEED pattern observed by Steininger ${ }^{3}$ was not seen at this temperature. The experiments of Steininger et al. were carried out at $100 \mathrm{~K}$. When the surface was saturated with carbon monoxide at this temperature a streaked pattern was observed. After annealing at $260 \mathrm{~K}$ and cooling back to 100 $K$ a well-ordered LEED pattern was observed, identified above as $c(\sqrt{3} \times 9)$-rect. This pattern can be explained in terms of ordered domain-walls after each pair of $(\sqrt{3} \times 2)$-rect unit cells (figure $10.10(d)$ ), i.e. strips of the $(\sqrt{3} \times 2)$-rect phase 4 atoms wide separated by anti-phase domain-walls.

The fact that the $\mathrm{c}(\sqrt{3} \times 9)$-rect phase is observed at $100 \mathrm{~K}$ but not $150 \mathrm{~K}$ gives an indication of the domain-wall repulsion energy. At $100 \mathrm{~K}$ the domain walls are ordered in the $c(\sqrt{3} \times 9)$-rect phase so that there is the maximum average separation between the domain walls. By $150 \mathrm{~K}$ the entrophy term becomes larger than the domain-wall repulsion energy and the domain walls disorder. If the domain-walls did not repel, then macroscopic island of $(\sqrt{3} \times 2)$-rect and $c(\sqrt{3} \times 5)$-rect would co-exist at intermediate coverages. This also favors the 2 2-2-2 domain-wall model for the $c(\sqrt{3} \times 9)$-rect phase over a 1-3-1-1-3-1 model, which would also give the correct coverage and surface unit cell. 


\subsection{High coverage discussion}

The domain-wall model can explain the LEED and HREELS observations on the carbon monoxide-Pt(111) system, while the predictions of the uni-axial compression model are not supported by observations. First, the uni-axial compression model and double-diffraction arguments cannot explain the observed LEED spot-patterns for carbon monoxide adsorbed on $\mathrm{Pt}(111)$ since some of the LEED spots are too strong to be combination beams involving evanescent waves. The domain-wall model is consistent with the observed patterns. Secord, the domain-wall model does a better job of predicting the LEED patterns at intermediate coverages. Third, the domain-wall model can explain the observation of the $c(\sqrt{3} \times 9)$-rect phase at $100 \mathrm{~K}$ but not at $150 \mathrm{~K}$. An incommensurate coincidence lattice at $\theta=5 / 9$ should not be more strongly affected by increasing temperature than the coincidence lattices at $\theta=3 / 5$ or $1 / 2$.

The domain-wall model is also consistent with vibrational spectroscopy data. There is no sign of inhomogeneous broadening in the HREELS ${ }^{3,10}$ or IRAS $^{7}$ speetra for $\theta<2 / 3$. High resolution IRAS spectra $\left(\sim 5 \mathrm{~cm}^{-1}\right)$ show no evidence of peak broadening and the coverage-dependent carbon-oxygen stretch frequency shifts are no more than $15 \mathrm{~cm}^{-1} .7$ This is not consistent with carbon monoxide molecules adsorbed in a range of intermediate sites between the symmetric top and bridge sites, whose carbon-oxygen stretch frequencies are separated by 250 $\mathrm{cm}^{-1}$.

Lastly, there is a conceptual problem with the uni-axial compression model. This type of model makes sense on a fcc (110) surface, were there is a clear 
directionality, but not on the (111) surface. If the adsorption site does not matter along the $[1 \overline{10}]$ direction, it is not clear why should it matter perpendicular to this direction. A uniform compressed hexagonal overlayer, such as is observed for Xe on $\mathrm{Ag}(111)^{19}$ or $\mathrm{K}$ on $\mathrm{Pt}(111)^{20}$ would seem more likely than uni-axial compression.

\subsection{Clean $\operatorname{Pt}(111)$ surface structure}

A number of studies have been made of the structure of the clean $\operatorname{Pt}(111)$ surface with LEED $^{21,22,23,24,25,26}$ and with ion channeling. ${ }^{27,28}$ The LEED results are somewhat unsatisfactory in that the agreement between the calculated and experimental I-V curves is worse than for other clean, unreconstructed metal surfaces (e.g. $\mathrm{Al}(110)^{29}$ and $\mathrm{Cu}(110)^{30}$ ). This poror agreement persists despite extensive variations of the non-structural and some structural parameters.

Before undertaking LEED structure calculations for carbon monoxide adsorbed on $\mathrm{Pt}(111)$, further calculations were made for the clean $\mathrm{Pt}(111)$ surface in an attempt to refine the LEED structural results. This lays the basis for the investigation of the surface structure of the $c(4 \times 2)$ phase of carbon monoxide adsorbed on $\mathrm{Pt}(111)$ and also to the studies of benzene adsorbed on $\mathrm{Pt}(111)$ (chapter 11) and incommensurate graphite adsorbed on $\mathrm{Pt}(111)$ (chapter 12). Theoretical calculations based on new, more elaborate surface models have been compared with new LEED data for the clean Pt(111) surface (see figure 10.11) in an attempt to improve the fit between theory and experiment. 


\subsubsection{New surface models}

A previous LEED study of $\mathrm{Pt}(111)$ varied the first inter-layer spacing along with a number of non-structural parameters, including the effect of relativistic corrections to the potential. ${ }^{24}$ In addition to Andersen's platinum potential, used in earlier studies, ${ }^{21,22,24}$ a new fully relativistic potential calculated by Wang ${ }^{31}$ was used for the clean $\mathrm{Pt}(111)$ scattering calculations.

lon scattering data indicate that the $\mathrm{Pt}(111)$ surface has a small expansion in the first inter-layer spacing of $0.03 \pm 0.01 \AA^{27}$ or $0.03 \pm 0.02 \AA .^{28}$ No changes in the registry parallel to the surface were observed within a similar sensitivity of \pm 0.02 $\AA$.

New LEED calculations were made for $\mathrm{Pt}(111)$ using the first three interlayer spacings $d_{1-2}, d_{2-3}$ and $d_{3-4}$ as independent geometrical parameters. The first two inter-layer spacings were varied by $\pm 0.10 \AA$ around the bulk value of $2.265 \AA$, while $d_{3-4}$ was contracted by 0.0 or $0.05 \AA$ relative to the bulk value. Clean iridium, gold and platinum surfaces all tend to reconstruct. ${ }^{32}$ The reconstructions of $\operatorname{Ir}(100), \operatorname{Pt}(100), A u(100)$ and $A u(111)$ involve average top-layer bond-length reductions of $1 \%, 2 \%, 4 \%$ and $4 \%$, respectively. Since contractions parallel to the surface are important for other $5 d$ metal surfaces, a few cases were investigated for $\mathrm{Pt}(111)$. 


\subsubsection{Structural results for $\operatorname{Pt(111)}$}

Using the Wang potential and assuming identical vibrational amplitudes for all layers at $\theta_{D}=302 \mathrm{~K}$, the optimum values of the geometrical parameters are $\Delta d_{1-2}=0.0 \pm 0.025 \AA, \Delta d_{2-3}=-0.025 \pm 0.025 \AA$ and $\Delta d_{3-4}=0.0 \pm 0.05 \AA$ (negative values indicate contraction). From the normal incidence data the optimal value for the clean surface inner potential was found to be $V_{o r}=14.0 \pm 0.5 \mathrm{eV}$. The minimum $r$-factor values were $r_{5}=0.24, r_{Z-J}=0.29$ and $r_{P e n d r y}=0.50$. The introduction of layer dependent atomic vibrational amplitudes had 2 negligible effect on the minimum $r$-factor values.

Next changes in the top-layer registry were investigated. First a $2 \%$ reduction in the size of the translational unit cell was considered. Since no superlattice due to reconstruction is seen in the $\mathrm{Pt}(111)$ LEED pattern, all layers were contracted equally. This contraction increased the value of $r_{5}$ from 0.24 to 0.25 . A change in the translational surface geometry would probably bive shown up in ion scattering and channeling measurements in any case. Finally, a rigid shift in the top laver registry of $0.2 \AA$ was considered. This breaks the 3-fold rotational symmetry of the fcc (111) surface, but the superposition of the LEED patterns from the three non-equivalent domains would restore apparent rotational symmetry to the observed LEED pattern. This registry shift increased the minimum r-factor values even more than a uniform lattice contraction.

The results of these additional calculations still indicate that the equilibrium $\mathrm{Pt}(111)$ surface resembles the terminated bulk structure within $\pm 0.025 \AA$. Nonbulk lattice terminations such as hep stacking are ruled out by the ion-scattering 
studies, which would have easily picked up such a gross rearrangement of the surface structure. The only improvement in the fit was a minor change in $r_{5}$ from 0.247 to 0.240 when the Wang putential was substituted for the Anderson potential. The relatively large absolute value of the clean $\mathrm{Pt}(111)$ r-factor minima may be due to local relaxations or aperiodic distortions or to unknown and unusual features of the inelastic clamping, the atomic vibration amplitudes or the atomic scattering potential for platinum.

\subsection{Carkon Monoxide structure determination}

The $c(4 \times 2)$ phase of ordered carbon monoxide on $\mathrm{Pt}(111)$ was formed by cooling the clean Pt(111) crysta! and sample holder to thermal equilibrium at $140 \mathrm{~K}$, then flashing the crystal to $550 \mathrm{~K}$ to desorb residual carbon monoxide and hydrogen. The crystal temperature returned to $\sim 150 \mathrm{~K}$ in approximately $100 \mathrm{~s}$. As the temperature reached $\sim 270 \mathrm{~K}$ the crystal was exposed to a nominal pressure of $5 \cdot 10^{-8}$ iorr of carbon monoxide for $20 \mathrm{~s}$. The crystal surface was facing a stainless steel doser tube $0.15 \mathrm{~cm}$ in diameter located $10 \mathrm{~cm}$ away from the crystal surface. With this arrangement a 0.1 Langmuir exposure, based on the uncorrected ion gauge reading, corresponds to a 1 Langmuir exposure on the work of Ertl, et al. ${ }^{1}$ Because of the sharp drop in the adsorption energy and sticking coefficient for carbon monoxide on $\mathrm{Pt}(111)$ at a coverage of $1 / 2$ monolaver $^{1}$ the exact carbon monoxide exposure was not critical and this procedure gave a reproducible and well-ordered LEED pattern for the $c(4 \times 2)$ phase of carbon monoxide adsorbed on $\operatorname{Pt}(111)$. 


\subsubsection{Electron beam damage}

The LEED electron beam interacts strongly with adsorbed carbon monoxide. The main effect is electron stimulated desurption of the carbon monoxide molecules, which disorders the carbon monoxide surface structure and induces phase transitions on the surface. The cross-section for electron stimulated decomposition of carion monoxide is approximately an order of magnitude smaller at LEED energies, but this also contributes to the electron beam damage. The desorption and decomposition effects depend linearly on beam current, but seem approximately independent of beam voltage in the LEED energy range.

During I-V data collection the exposure of the carbon monoxide covered surface to the LEED electron beam was limited to $45 \mu \mathrm{A}-\mathrm{s}$ (LEED beam diameter $1 \mathrm{~mm}$ ). This limit was empirically determined to cause no significant beaminduced changes in the $c(4 \times 2)$ diffraction pattern or intensities. A full set of $I-V$ curves was recorded in $100 \mathrm{~s}$ using the video LEED data system (chapter 5 ). The running total of the electron beam exposure was monitored and data acquisition was stopped when the exposure limit was reached. The crystal was then flashed above $550 \mathrm{~K}$, desorbing the carbon monoxide molecules and a new exposure was made, forming a new $\mathrm{c}(4 \times 2)$ carbon monoxide phase, before data acquisition was resumed. 


\subsubsection{LEED data}

All of the carbon monoxide I-V curve data were collected at a crystal temperature of $\sim 150 \mathrm{~K}$ to optimize the contrast in the LEED pattern. After each set of I-V data were collected the crystal was checked by Auger spectroscopy for contamination, with particular attention given to carbon deposised on the surface by electron-beam induced decomposition.

$\mathrm{I}-\mathrm{V}$ data for the $\mathrm{c}(4 \times 2)$ phase of carbon monoxide adsorbed on $\mathrm{Pt}(111)$ were collected at normal incidence and with the electron beam rotated $5^{\circ}$ and $15^{\circ}$ toward the $[1 \overline{10}]$ direction, preserving the mirror symmetry in the LEED pattern. Two independent experiments were performed at each angle of incidence and the final I-V curves are an average of symmetrically related I-V curves from both data sets. The experimental energy range was 20 to $200 \mathrm{eV}$. The normal incidence data set has I-V curves for 9 symmetrically independent beams with 30 significant peaks over a cumulative $1000 \mathrm{eV}$ energy range. The $5^{\circ}$ and $15^{\circ}$ data sets each have 14 symmetrically independent beams with more than 35 peaks over a cumulative energy range greater than $1300 \mathrm{eV}$ at each angle.

\subsubsection{Theoretical methods}

Standard theoretical methods (see chapter 3) were used to calculate the model I-V curves for the clean $\mathrm{Pt}(111)$ surface and for the $\mathrm{c}(4 \times 2)$ phase of carbon monoxide adsorbed on $\mathrm{Pt}(111)$. The carbon monoxide overlayer was treated with the CSM (spherical wave) method using the RSP perturbation scheme. This layer contains two inequivalent carbon monoxide molecules in the unit cell, for a total 
of four non-coplanar atomic sublayers, two for oxygen and two for carbon. The RFS perturbation scheme was used to stack the substrate metal layers and the combined overlayer. This same calculational approach was successfully used to determine the structure of the $(2 \times 2)$ phase of carbon monoxide adsorbed on $\operatorname{Rh}(111),{ }^{33}$ which has three inequivalent carbon monoxide molecules per unit cell adsorbed in near-top and bridge sites. This is an "exact" treatment of the LEED multiple scattering within the context of the basic assumptions of LEED scattering theory and multiple scattering effects are included to all orders.

Two sets of phase shifts were used for the clean platinum experiments, phase shifts derived from a potential calculated by Anderson which were used in previous investigations of the clean platinum surface ${ }^{24}$ and spin-averaged phase-shifts derived from a new relativistic potential calculated by Wang. ${ }^{31}$ The new relativistic potential gave slightly better results for the clean platinum calculations, so these phase-shifts were used for the carbon monoxide calculations. The carbon monoxide scattering phase-shifts used in this study are the same that were used in several previous studies of carbon monoxide adsorbed on other metal surfaces. ${ }^{34,35,36,33,37}$ Phase shifts up to $l_{\max }=7$ were used for the clean platinum calculations and up to $l_{\max }=5$ for the carbon monoxide overlayer case.

An inelastic damping potential proportional to $E^{1 / 3}$ was used with $V_{o i}=3.8$ $\mathrm{eV}$ at an electron energy of $90 \mathrm{eV}$. The Debye temperature was initially chosen to be $302 \mathrm{~K}$ for the clean $\mathrm{Pt}(111)$ surface, the optimum value found by Bibérian et al. $^{38}$ and $255 \mathrm{~K}$ for carbon monoxide covered platinum, closer to the bulk Debye temperature of about $234 \mathrm{~K}$. The carbon and oxygen atoms were initially given 
the mean square arnplitude of the underlying metal atoms, multiplied by two to account for surface enhancement in the vibrational amplitude. Theoretical calculations were compared to the normal-incidence experimental data over the 20 to $200 \mathrm{eV}$ data range using the $r_{5}$ r-factor. Once the best structural model was chosen, additional calculations were made for the off-normal data sets.

\subsection{Carbon monoxide surface models}

All of the structural moàels considered for the $c(4 \times 2)$ phase of carbon monoxide adsorbed on $\mathrm{Pt}(111)$ assumed intact carbon monoxide molecules adsorbed with the molecular axis normal to the surface and the carbon atom closest to the surface, as indicated by ARPES measurements. ${ }^{5,6}$ There are two carbon monoxide molecules per unit cell in the $\mathrm{c}(4 \times 2)$ phase, as indicated by thermal desorption measurements. ${ }^{1}$ HREELS measurements suggest two nonequivalent carbon monoxide molecules in the unit cell adsorbed in high-symmetry sites, ${ }^{3}$ which were identified as top and bricige sites by the assignment of the HREELS losses. All of the structural models considered assume that the carbon monoxide molecules are adsorbed in high-symmetry sites -- the top, bridge and the two types of three-fold hollow sites on the fcc (111) surface. The platinum substrate was assumed to have the platinum terminated bulk geometry, since no evidence of significant relaxation was found in the clean platinum structural study.

Six different structural models were considered for the unit cell of the $c(4 \times 2)$ phase of carbon monoxide adsorbed on $\mathrm{Pt}(\mathbf{1 1 1})$ : 
A One carbon monoxide molecule in a top site and one in a bridge site (see figure 10.12).

B One carbon monoxide molecule in a top site and one in an fec-type hollow site.

C One carbon monoxide molecule in a top site and one in an hep-type hollow site.

D One carbon monoxide molecule in a bridge site and one in an fcc-type hollow site.

E One carbon monoxide molecule in a bridge site and one in an hep-type hollow site.

F Only one molecule in a top site. The other molecule is assumed to be disordered, possibly switching rapidly between the bridge site and the adjacent fec and hep hollow sites, as suggested by Hayden and Bradshaw. ${ }^{7}$

\subsection{Structural search}

The main structural parameters were the perpendicular carbon-metal distances for the two inequivalent carbon monoxide molecules in the unit cell. These distances were varied independently. The carbon-oxygen bond length was assumed to be the same for the two inequivalent molecules. This distance was fixed at $1.15 \AA$ for models $B$ through $F$. Once model $A$ was shown to be the best model in the preliminary calculations, additional calculations were made for a carbon-oxygen bond length of 1.10 and $1.20 \AA$. 
Theoretical calculations were made for all six models using the normal incidence data, for a total of $\mathbf{1 4 6}$ different geometries. The top-and-bridge site model (model A) clearly was the best of the six considered. Calculations for model A were also made at $\theta=5^{\circ}$ and $15^{\circ}$ for 20 geometries. Figure 10.13 shows I-V curves for the $c(4 \times 2)$ phase and figure 10.14 shows the $r_{5}$ contour plot for the $\theta=$ $0^{\circ}$ and $\theta=5^{\circ}$ data sets. Well-defined minima are seen at both energies at approximately the same parameter values.

For the $\theta=15^{\circ}$ data set the Zanazzi-Jona $r$-factor $r_{Z J}$ had erratic values close to the assumed minimum. The other $r$-factors in the $r_{5}$ average $\left(r_{\text {Pendry }}, r_{1}\right.$, $r_{2}$ and $r_{\text {slope }}$ ) were all well behaved and confirmed the optimum result found with the $\theta=0^{\circ}$ and $5^{\circ}$ data. Only the $\theta=0^{\circ}$ and $5^{\circ}$ data were used to calculate $r_{5}$ for the determination of the optimum parameters for model $\mathrm{A}$.

The optimum muffin tin zero was found to be $V_{o r}=12 \pm 1 \mathrm{eV}$ and the optimum carbon-oxygen bond length was $1.15 \pm 0.10 \AA$. The perpendicular carbon-metal spacings for the top and bridge sites were $1.85 \pm 0.10$ and $1.55 \pm 0.10$ $\AA$, respectively, corresponding to platinum-carbon bond-lengths of $1.85 \pm 0.10 \AA$ and $2.08 \pm 0.07 \AA$. For these parameters $r_{5}=0.29$, while $r_{Z J}=0.50$ and $r_{P e n d r y}=$ 0.61. In comparison, the $r$-factor values for the $(2 \times 2)$ phase of carbon monoxide adsorbed on the $\operatorname{Rh}(111)$ surface, which has three non-equivalent carbon monoxide molecules per unit cell, were $r_{5}=0.19, r_{Z-J}=0.25$ and $r_{P e n d r y}=0.47$. This difference is probably due primarily to the r-factors for the clean metal surfaces, which are much smaller for $\mathrm{Rh}(111)$ than for $\mathrm{Pt}(111)$. The clean $\mathrm{Pt}(111) \mathrm{r}$-factors are $r_{5}=0.24, r_{Z-J}=0.29$ and $r_{P e n d r y}=0.50$. 


\subsection{Discussion of structural results}

The top and bridge site model (model $A)$ for the $c(4 \times 2)$ phase resulting from the LEED calculations is consistent with the HREELS assignments for this structure $^{3}$ The bond-length results indirectly support the conclusion from photoemission studies that the carbon-oxygen bond axis is perpendicular to the surface. If the molecule were tilted, then the $1.15 \AA$ vertical spacing between the carbon and oxygen sublayers found by LEED would predict an unreasonably long carbonoxygen bond-length.

Hayden and Bradshaw ${ }^{7}$ resolved the $1850 \mathrm{~cm}^{-1}$ band observed by HREELS into two components in their high-resolution IRAS study. They assigned the two components to bridge and hollow sites, with some hollow site occupation at $\theta=$ $1 / 2$ and $T>100 \mathrm{~K}$. The results of the LEED structure analysis show no evidence of hollow site occupation. The best fit was obtained for top and bridge site occupation. The LEED results indicate that the majority of the carbon monoxide molecules are in top or bridge sites at $150 \mathrm{~K}$. This LEED study would not have detected a small fraction $(\sim 15 \%)$ of carbon monoxide molecules randomly adsorbed in hollow sites.

\subsection{Conclusions}

LEED structure determination has confirmed top and bridge site adsorption for the $c(4 \times 2)$ phase of carbon monoxide on $\mathrm{Pt}(111)$. The carbon-metal perpendicular spacings are $1.85 \pm 0.10 \AA$ and $1.55 \pm 0.10 \AA$, respectively and the carbonoxygen bond length was found to be $1.15 \pm 0.05 \AA$. 
The domain-wall model has been shown to explain the LEED and HREELS observations for the high-coverage phases of carbun monoxide adsorbed on $\mathrm{Pt}(111)$, while the uni-axial compression model is inconsistent with LEED observations. Based on the LEED observations and the domain-wall model, a rough phase diagram for the carbon monoxide-Pt(111) system can be drawn (see figure 10.15). An ordered domain-wall phase, $\mathrm{c}(\sqrt{3} \times 9)-r e c t$ at $\theta=5 / 9$, has been proposed, which disorders between $100 \mathrm{~K}$ and $150 \mathrm{~K}$. The structures of the other ordered phases of carbon monoxide on $\mathrm{Pt}(111)$ are good candidates for LEED structure determination for a low beam-eurrent, low-temperature LEED system. 


\section{References}

1. G. Ertl, M. Neumann, and K. M. Streit, Surface Science, vol. 64, p. 393, 1977.

2. H. Hopster and H. Ibach, Surface Science, vol. 77, p. 108, 1978.

3. H. Steininger, S. Lehwald, and H. Ibach, Surface Science, vol. 123, p. 645, 1982.

4. K. Horn and J. Pritchard, Journal de Physique, vol. 38-C4, p. 164, 1977.

5. P. Hoffmann, S. R. Bare, N. V. Richardson, and D. A. King, Solid State Communications, vol. 42, p. 645, 1982.

6. M. Trenary, S. L. Tang, R. J. Simonson, and F. R. McFeely, Surface Science, vol. 124, p. 555, 1983.

7. B. E. Hayden and A. M. Bradshaw, Surface Science, vol. 125, p. 787, 1883.

8. H. Froitzheim, H. Hopster, H. Ibach, and S. Lehwald, Applied Physics, vol. 13, p. $147,1977$.

0. A. M. Baró and H. lbach, Journal of Chemical Physics, vol. 71, p. 4812, 1979.

10. N. R. Avery, Journal of Chemical Physics, vol. 74, p. 4202, 1981.

11. J. P. Bibérian and M. A. Van Hove, Surface Science, vol. 118, p. 443, 1982.

12. J. P. Bibérian and M. A. Van Hove, Surface Science, vol. 138, p. 361, 1984.

13. B. Poelsma, R. L. Palmer, and G. Comsa, Surface Science, vol. 123, p. 152, 1982. 
14. C. S. McKee, M. W. Roberts, and M. L. Willians, Advances in Colloid and Interface Science, vol. 8, p. 29, 1977.

15. C. S. McKee, D. L. Perry, and M. W. Roberts, Surface Science, vol. 39, p. 1761973.

16. L. H. Germer, J. W. May, and R. J. Szostak, Surface Science, vol. 7, p. 430, 1967.

17. J. E. Houston and R. L. Park, Surface Science, vol. 21, p. 209, 1970.

18. R. L. Park, J. E. Houston, and D. G. Schreiner, Review of Scientific Instruments, vol. 42, p. 60, 1971.

19. P. I. Cohen, J. Unguris, and M. B. Webb, Surface Science, vol. 58, p. 429, 1976.

20. E. L. Garfunkel and G. A. Somorjai, Surface Science, vol. i15, p. 441, 1982.

21. L. L. Kesmodel and G. A. Somorjai, Physical Review B, vol. 11, p. 630, 1975.

22. L. L. Kesmodel, P. C. Stair, and G. A. Somorjai, Surface Science, vol. 64, p. $64,1977$.

23. R. Feder, Surface Science, vol. 68, p. 229, 1977.

24. D. L. Adams, H. B. Nielsen, and M. A. Van Hove, Physical Review B, vol. 20, p. $4789,1979$.

25. P. Bauer, R. Feder, and N. Müller, Surface Science, vol. 99, p. L395, 1980.

26. R. Feder, H. Pleyer, P. Bauer, and N. Müller, Surface Science, vol. 109, p. $419,1981$. 
27. J. A. Davies, D. P. Jackson, N. Matsunami, P. R. Norton, and J. N. Andersen, Surface Science, vol. 78, p. 274, 1978.

28. J. F. van der Veen, R. G. Smeenk, R. M. Tromp, and F. W. Saris, Surface Science, vol. 79, p. 219, 1979.

29. J. R. Noonan and H. L. Davis, Physical Review B, vol. 29, p. 4349, 1984.

30. D. L. Adams, H. B. Nielsen, and J. N. Andersen, Surface Science, vol. 128, p. $294,1983$.

31. S.-W. Wang, unpublished.

32. M. A. Van Hove, R. J. Koestner, P. C. Stair, J. P. Bibérian, L. L. Kesmodel, I. Bartos, and G. A. Somorjai, Surface Science, vol. 103, p. 218, 1981.

33. M. A. Van Hove, R. J. Koestiner, J. C. Frost, and G. A. Somorjai, Surface Science, vol. 129, p. 482, 1983.

34. R. J. Behm, K. Christmann, G. Ertl, M. A. Van Hove, P. A. Thiel, and W. H. Weinberg, Surface Science, vol. 88, p. L59, 1979.

35. R. J. Behm, K. Christmann, G. Ertl, and M. A. Van Hove, Journal of Chemical Physics, vol. 73, p. 2984, 1980.

36. R. J. Koestner, M. A. Van Hove, and G. A. Somorjai, Surface Science, vol. 107, p. $439,1981$.

37. M. A. Van Hove, R. F. Lin, and G. A. Somorjai, Sournal of the American Chemical Society, vol. 108, p. 2532, 1986.

38. J. P. Bibérian and M. A. Van Hove, in Vibrations at Surfaces, ed. R. Caudano, J.-M. Gillis and A. A. Lucas, p. 91, Plenum, New York, 1982. 


\section{Figure Captions for Chapter 10}

10.1 LEED patterns of the " $(\sqrt{3} \times \sqrt{3})$ R30" phase of carbon monoxide on $\mathrm{Pt}(11 \mathrm{i})$ at $150 \mathrm{~K}$. This structure was formed by a nominal 0.1 Langmuir carbon monoxide exposure at $300 \mathrm{~K}$ before cooling to $150 \mathrm{~K}$. The upper pattern was recorded at $72 \mathrm{eV}$ and the lower at $135 \mathrm{eV}$.

10.2 LEED pattern of the "intermediate" low coverage phase at $35 \mathrm{eV}$ and near-normal incidence at $130 \mathrm{~K}$.

10.3 LEED patterns of the $c(4 \times 2)$ (or $(\sqrt{3} \times 2)$-rect) phase formed when $1 / 2$ monolayer of carbon monoxide is adsorbed on the $\mathrm{Pt}(111)$ surface. The carbon monoxide adsorption took place at $270 \mathrm{~K}$, then the surface was cooled to $150 \mathrm{~K}$, before the LEED intensity data were recorded. Three rotated domains of the $c(4 \times 2)$ unit cell are superimposed in the LEED patterns. Normally these domains have approximately equal intensity, giving rise to apparent three-fold rotational symmetry in the LEED patterns, as seen in (a) and (b). A schematic diagram of the LEED pattern indicating the domain structure is shown in (c). Occasionally, as in (d), one domain is predominant.

10.4 The LEED pattern of the $c(\sqrt{3} \times 5)$-rect phase of carbon monoxide on $\mathrm{Pt}(111)$ (three domains) at $88 \mathrm{eV}$. This structure was formed after exposure of the $\mathrm{Pt}(111)$ crystal to $10^{-7}$ torr of carbon monoxide for 20 minutes (120 Langmuir) at $237 \mathrm{~K}$. 
10.5 The LEED pattern of the $(\sqrt{3} \times 3)$-rect phase of carbon monoxide on $\mathrm{Pt}(111)$ (three domains) at $111 \mathrm{eV}$. This structure was observed at $145 \mathrm{~K}$ with a carbon monoxide background pressure of $8 \cdot 10^{-8}$ torr.

10.6 LEED patterns and real-space unit celis schematics for the $(\sqrt{3} \times 2)$-rect, $c(\sqrt{3} \times g)-r e c t, c(\sqrt{3} \times 5)-r e c t$ and $(\sqrt{3} \times 3)-r e c t$ phases of carbon monoxide on $\operatorname{Pt}(111)$ at $\theta=1 / 2,5 / 9,3 / 5$ and $2 / 3$, respectively. The LEED patterns are shown for three superimposed, rotationally equivalent domains.

10.7 Schematic diagram of the diffraction pattern for one domain of the $c(\sqrt{3} \times 5)$-rect phase at $38 \mathrm{eV}$. The substrate beams are indicated by large filled circles, the overlayer beams (in the uni-axial compression model) by small filled circles and the combination beams by open circles. The outer circle gives the maximum parallel momentum iransfer $\Delta \mathbf{s}$ allowed for a non-evanescent beam inside the crystal and the smaller circle shows the maximum $\Delta \mathbf{s}$ for a beam in vacuum, assuming normal incidence. (The limits are different because of the inner potential $V_{o r}$.) Only evanescent beams contribute to the observed spots, represented by open circles, which are note connected by dashed lines.

10.8 Schematic diagram of the LEED pattern predicted by the uni-axial model for $\theta=0.53$. The filled circles show the overlayer beams, the large filled circles show substrate beams and the open circles show combination beams involving substrate vectors. 
10.9 LEED patterns of carbon monoxide on $\mathrm{Pt}(111)$ at $150 \mathrm{~K}$ and various beam eneigies, for $1 / 2<\theta<3 / 5$. Parallel streaks arc from the same rotational domain. The streaks connect the spot positions of the $(\sqrt{3} \times 2)$-rect and the $c(\sqrt{3} \times 5)$-rect LEED patterns. There is no observed streaking associated with the substrate spots.

10.10 The domain-wall model in real space.

(a) The $(\sqrt{3} \times 2)$-rect phase and one "( $\sqrt{3} \times 2)$-rect unit".

(b) A single domain-wall.

(c) The $\mathrm{c}(\sqrt{3} \times 5)$-rect phase, with the maximum number of domain-walls, forming a new unit cell.

(d) An ordered domain-wall model for $\theta=5 / 9$. This gives the I.EED pattern for the $c(\sqrt{3} \times 9)$-rect phase.

10.1) Clean platinum (111) I-V curves at normal incidence, recorded at $300 \mathrm{~K}$.

10.12 The optimum structure of adsorbed carbon monoxide in the $\mathrm{c}(4 \times 2)$ (or $(\sqrt{3} \times 2)-r e c t)$ phase, as determined by LEED structure calculations (model A). The broken circles in the lower right corner of the figure indicate the carbon monoxide inter-molecular spacing if the bridge site molecule should move to either hollow site, as in models $\mathrm{A}, \mathrm{C}$ or $\mathrm{F}$.

10.13 I- $\mathrm{V}$ curves recorded at $\theta=0^{\circ}, 5^{\circ}$ and $15^{\circ}$ for the $c(4 \times 2)$ phase of carbon monoxide on $\mathrm{Pt}(111)$. The data were recorded at $150 \mathrm{~K}$.

10.14 Contour plots of $r_{5}$ as a function of the metal-carbon perpendicular distance for the two inequivalent carbon monoxide molecules $\mathrm{fc}^{\mathrm{r}}$ the geometry 
of model A. The carbon-oxygen bond length was fixed at $1.15 \AA$ for this plot.

10.15 A possible phase diagram for carbon monoxide adsorbed on $\mathrm{Pt}(111)$. 


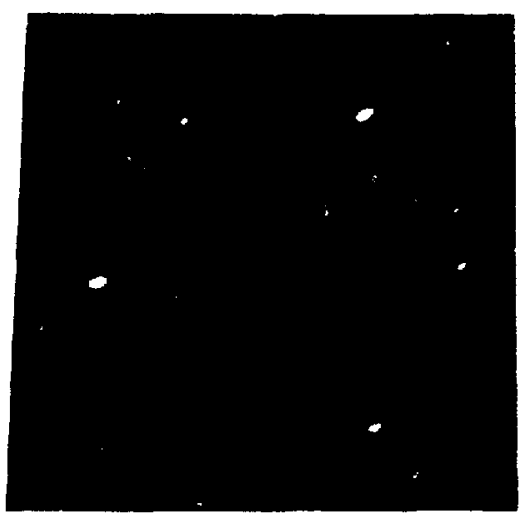

\section{$72 \mathrm{eV}$}

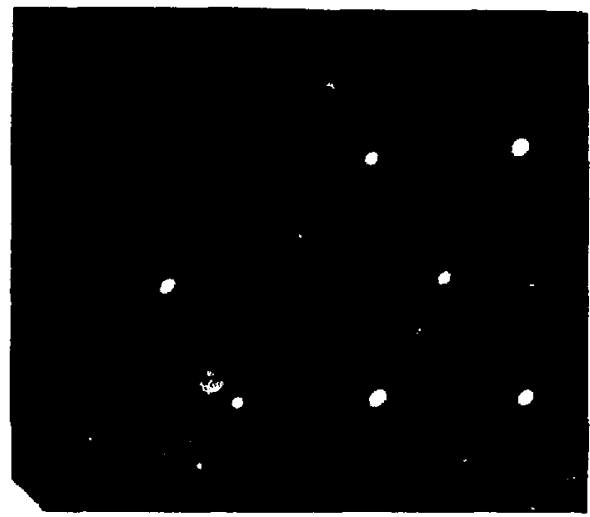

$135 \mathrm{eV}$

XBB $829-8610$

$(\sqrt{3} \times \sqrt{3}) R_{30}{ }^{\circ}$ on $\operatorname{Pt}(111)$

0.1 Langmuir at $150 \mathrm{~K}$

Figare 10.1 




XBB $860-8991$

\section{Low Coverage CO on $\mathrm{Pt}(111)$ $135 \mathrm{~K}$ at $35 \mathrm{eV}$}

Figure 10.2 


\section{Pt (111) - c $(4 \times 2)-2 \mathrm{CO}$ LEED Patterns $T=150 \mathrm{~K}$}

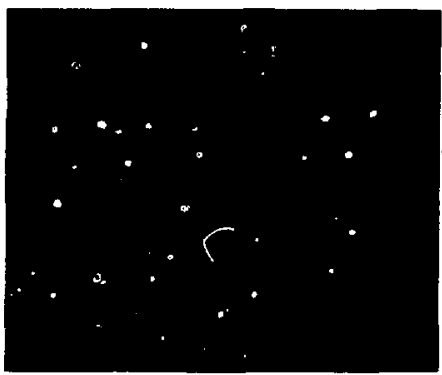

a) $38 \mathrm{eV}$

-

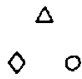

$\diamond \Delta \circ \Delta \Delta \Delta 0$

00

$\circ 0$

$0 \Delta 0$



$\circ 0$

- $\Delta$

c) LEED Pattern Schematic

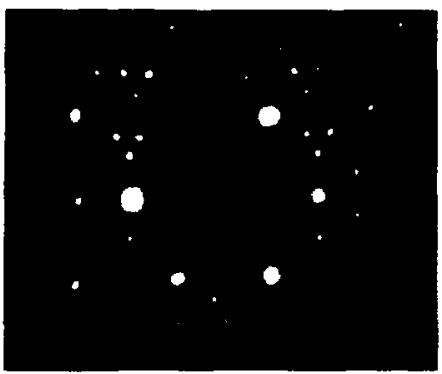

b) $130 \mathrm{eV}$

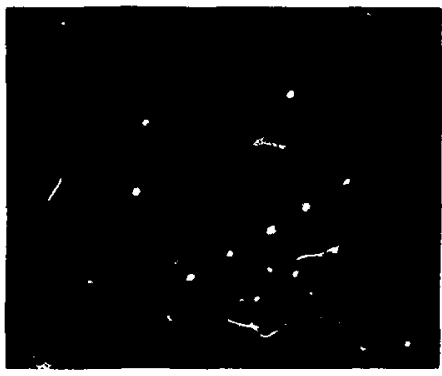

d) $110 \mathrm{eV}$

One domain predominant

- substrate spots

- 1st domain

$\triangle$ 2nd domain

$\diamond$ 3rd domain 

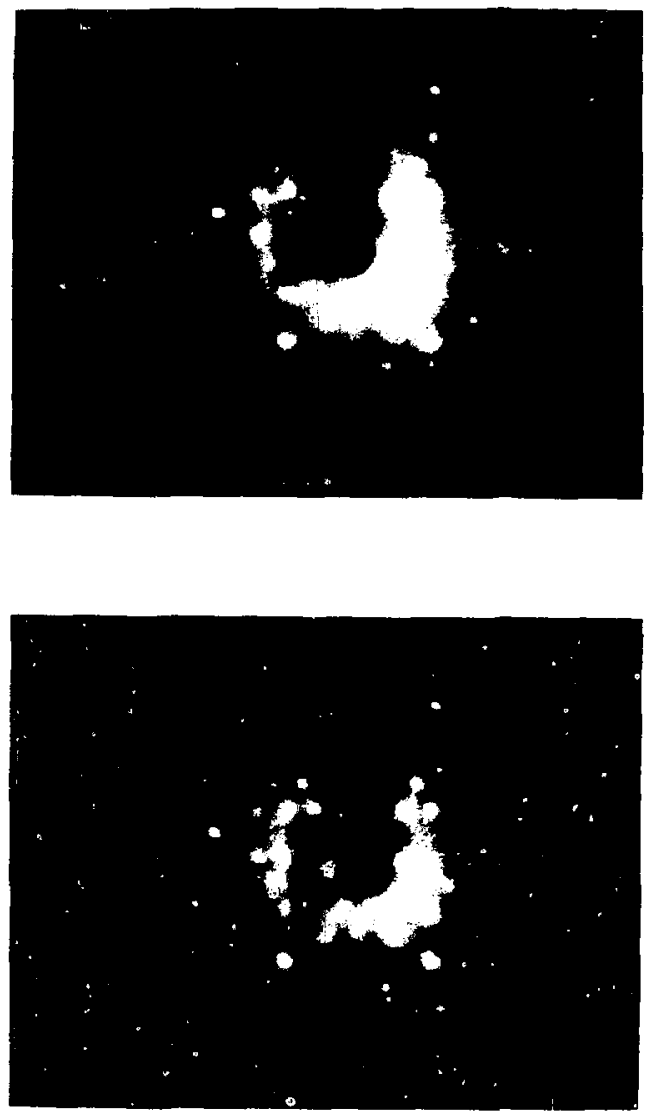

XBB 829-8613

\section{$\mathrm{CO} c(\sqrt{3} \times 5)$ rect on $\operatorname{Pt}(111)$ $10^{-7}$ torr at $237 \mathrm{~K}, 88 \mathrm{eV}$}

FIgure 10.4 


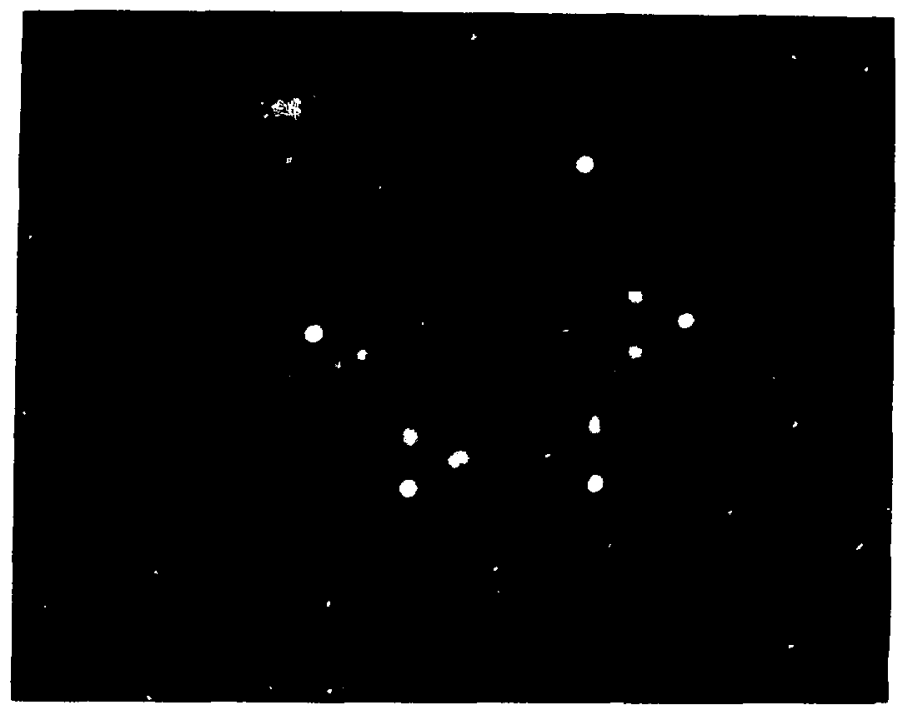

XBB $829-8617 B$

\section{CO $(\sqrt{3} \times 3)$ rect on $\operatorname{Pt}(111)$ $8 \cdot 10^{-8}$ torr at $145 \mathrm{~K}, 111 \mathrm{eV}$}

Figure 10.5 
Carbon Monoxide on $\operatorname{Pt}(111)$

LEED Patterns for $\theta \geq 1 / 2$

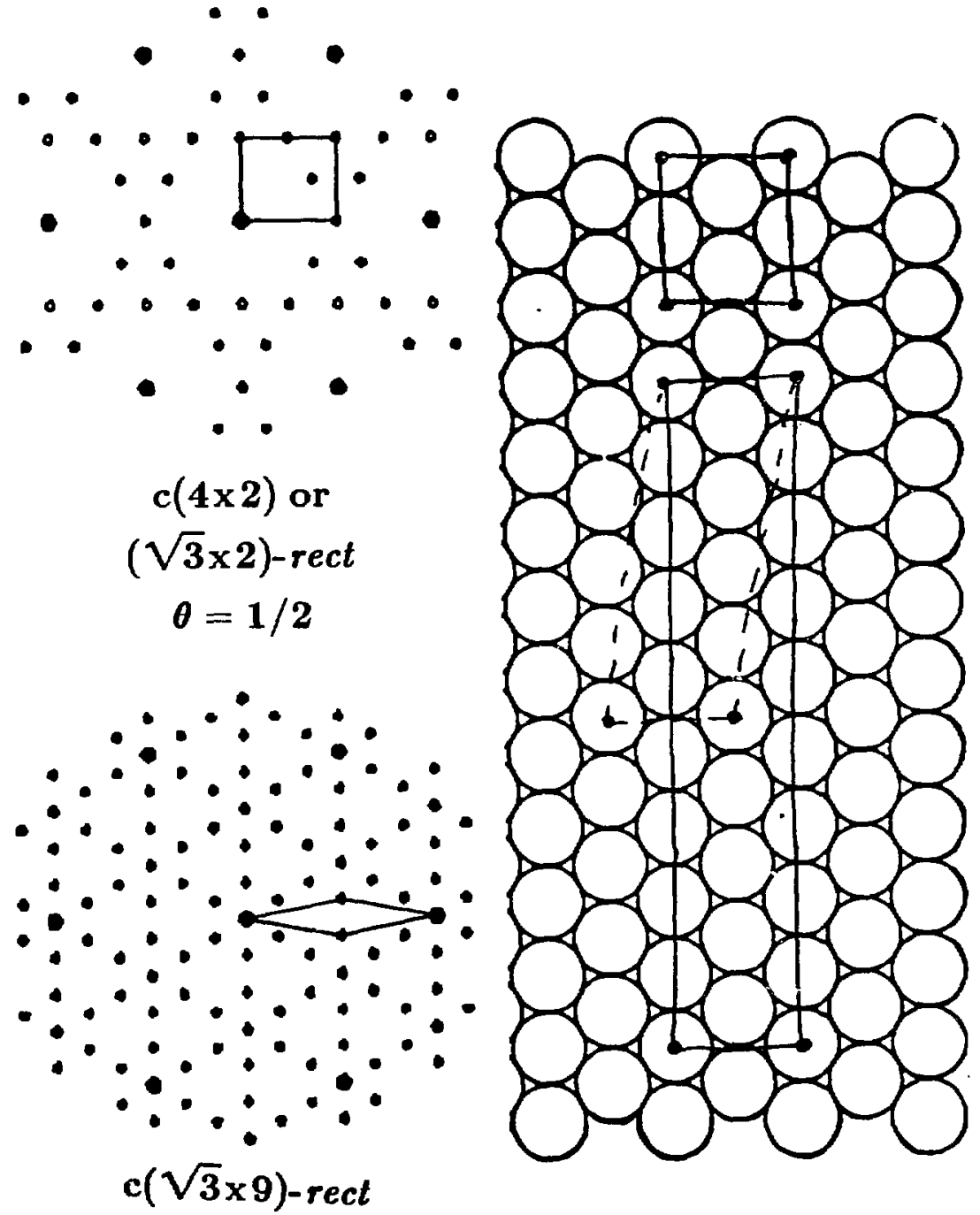

$\theta=5 / 9 \quad$ Figure $10.6 a$ 
Carbon Monoxide on $\mathrm{Pt}(\mathbf{1 1 1})$

LEED Patterns for $\theta \geq 1 / 2$

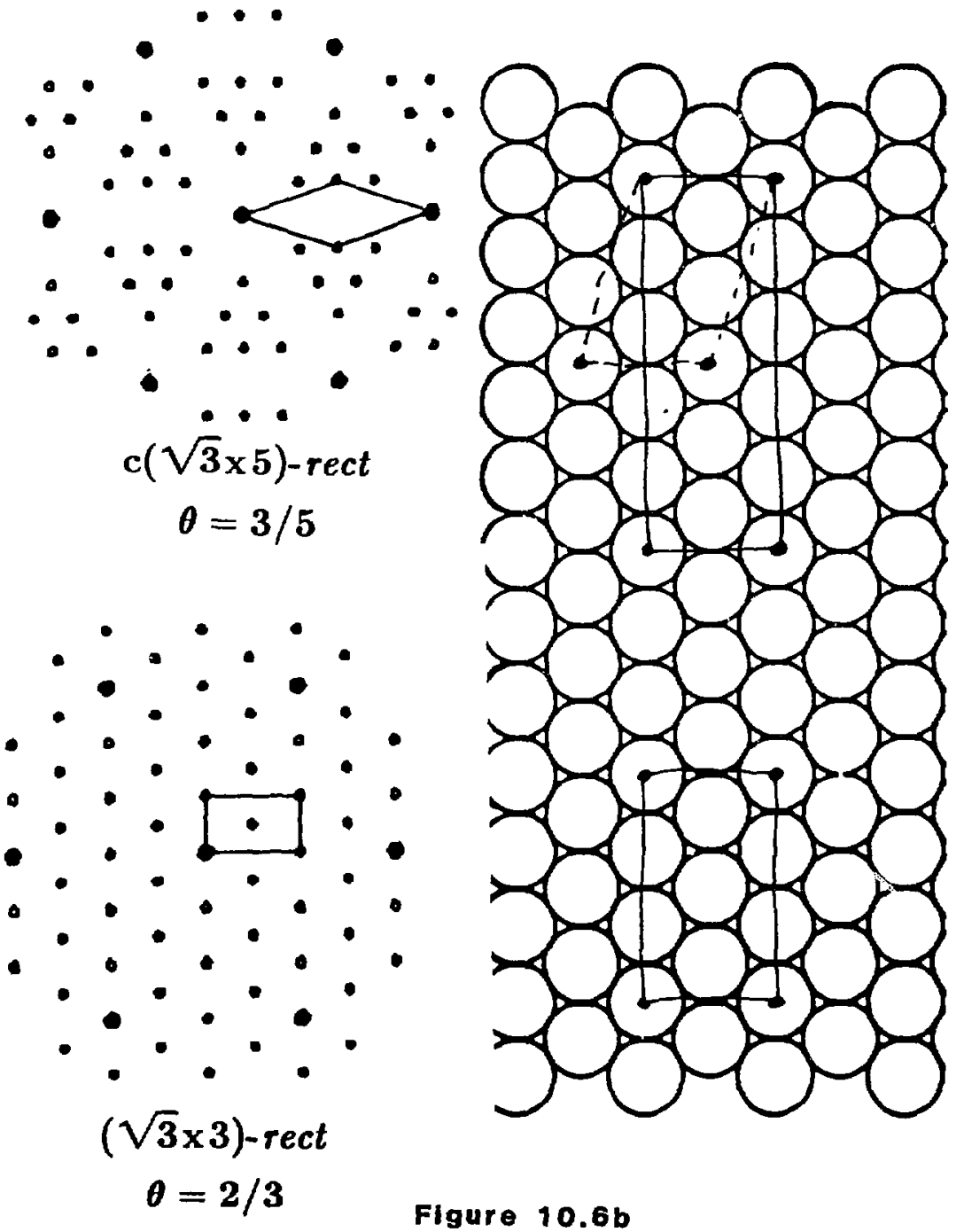


417

LEED Pattern for CO on Pt(111) in the Uni-axial Compression Model for $\theta=2 / 3$ at $38 \mathrm{eV}$ Only evanescent beams contribute to the observed spots, represented by open circles, which are not, connected by dashed lines.

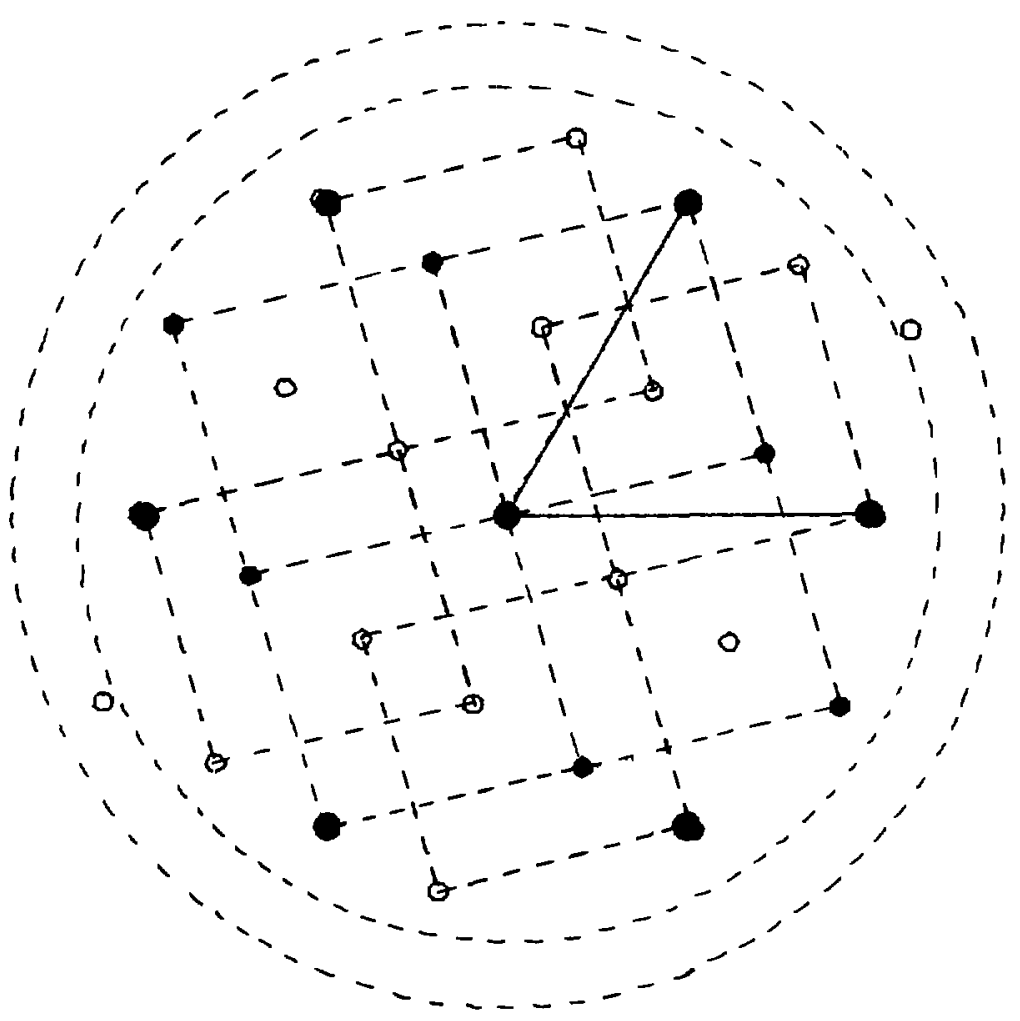

XmL $8612-4843$ 




Figure 10.8 


\section{$23 \mathrm{eV}$}

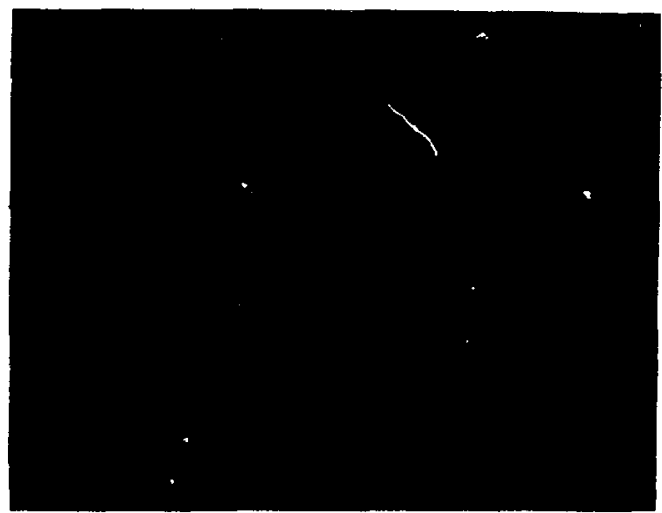

$38 \mathrm{eV}$

XBB 829-8609

$\mathrm{CO}$ on $\mathrm{Pt}(111)$

$(\sqrt{3} \times 2)$ rect $\rightarrow c(\sqrt{3} \times 5)$ rect 0.5 Langmuir at $150 \mathrm{~K}$

Flgure 10.9a 


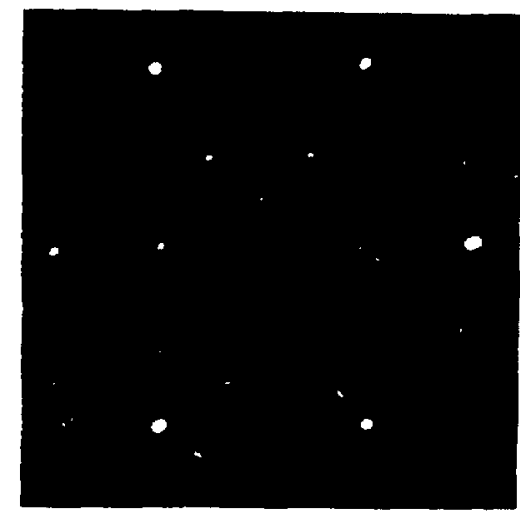

\section{$66 \mathrm{eV}$ \\ $0.5 \mathrm{~L}$}

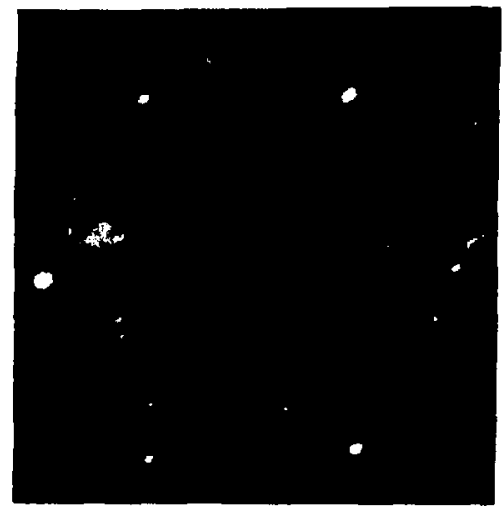

$59 \mathrm{eV}$ $5.0 \mathrm{~L}$

XBB $829-8612$

CO on Pt(111)

$(\sqrt{3} \times 2)$ rect $\rightarrow c(\sqrt{3} \times 5)$ rect

Figure $10.8 b$ 


\section{Carbon Monoxide on $\operatorname{Pt}(\mathbf{1 1 1})$ Domain Wall Model}

a sirigle

$(\sqrt{3} \times 2)$ unit

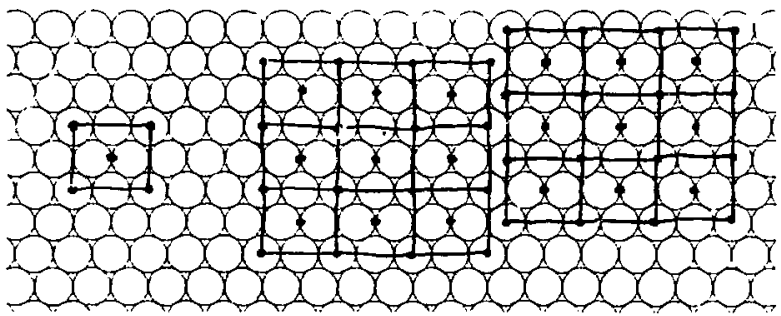

$c(\sqrt{3} \times 5)$ phase

domain wall model $(\sqrt{3} \times 2)$ phase with one domain wall

$c(\sqrt{3} \times 9)$

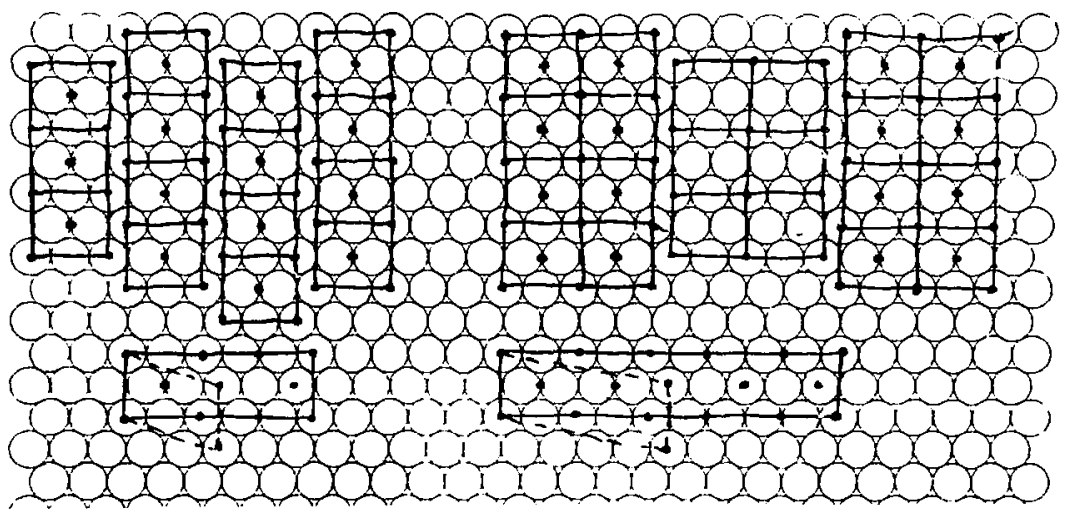


422

\section{Pt (111) I-V Curves \\ Normal Incidence at $300 \mathrm{~K}$}

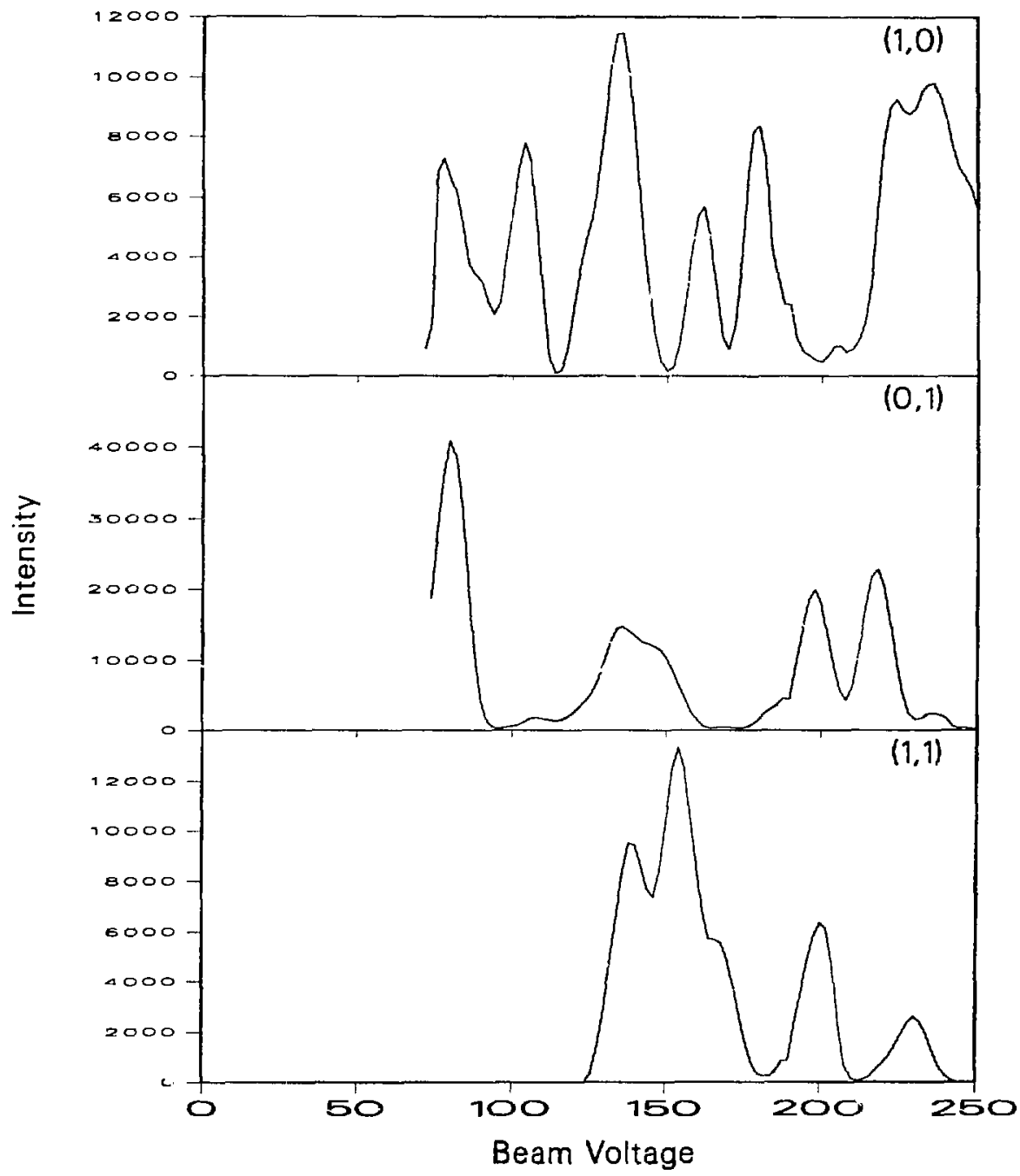

FIgure $10.11 \mathrm{a}$ 
423

Pt(111) I-V Curves

Normal Incidence at $300 \mathrm{~K}$

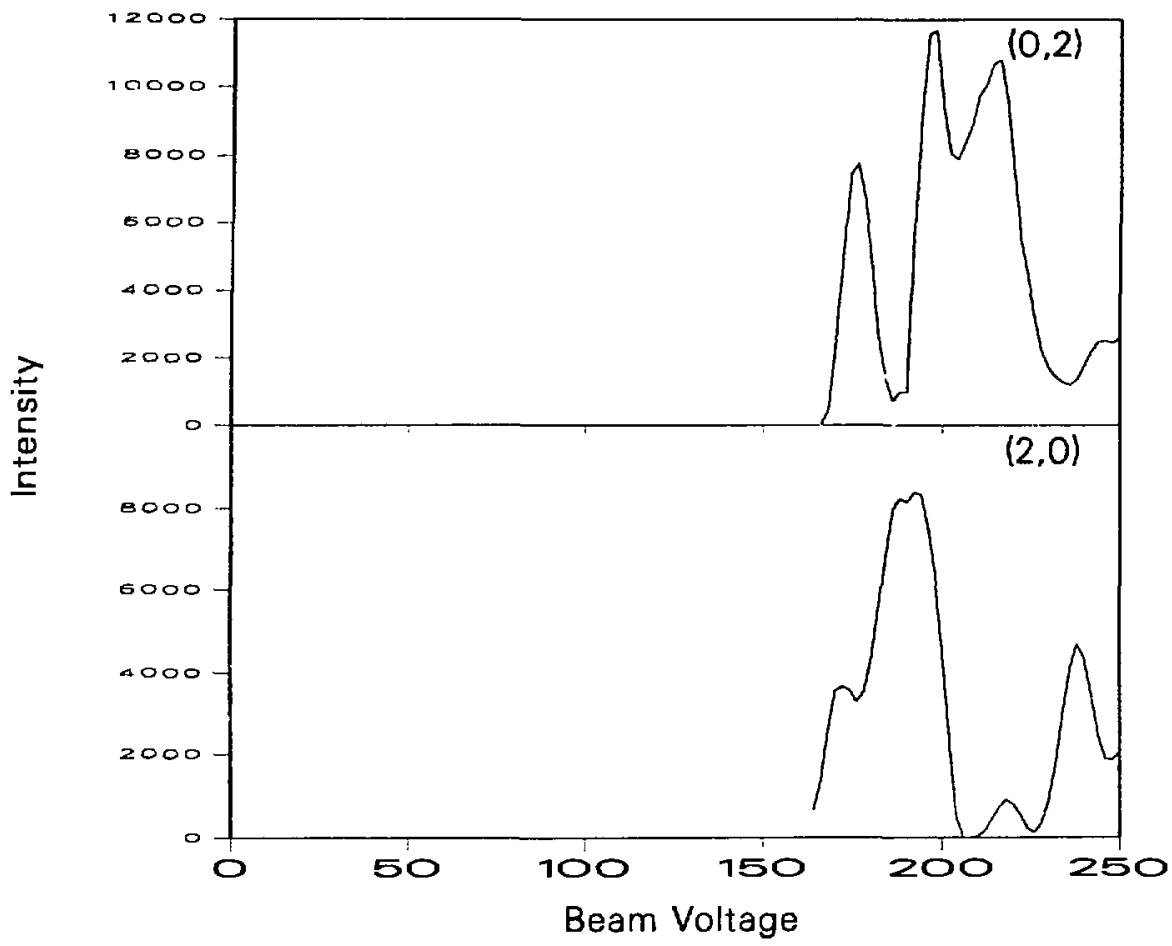

Figure 10.11b 


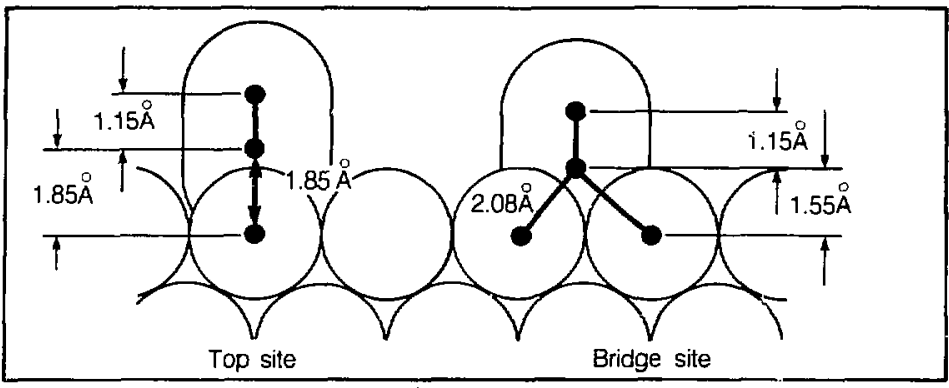

Side view

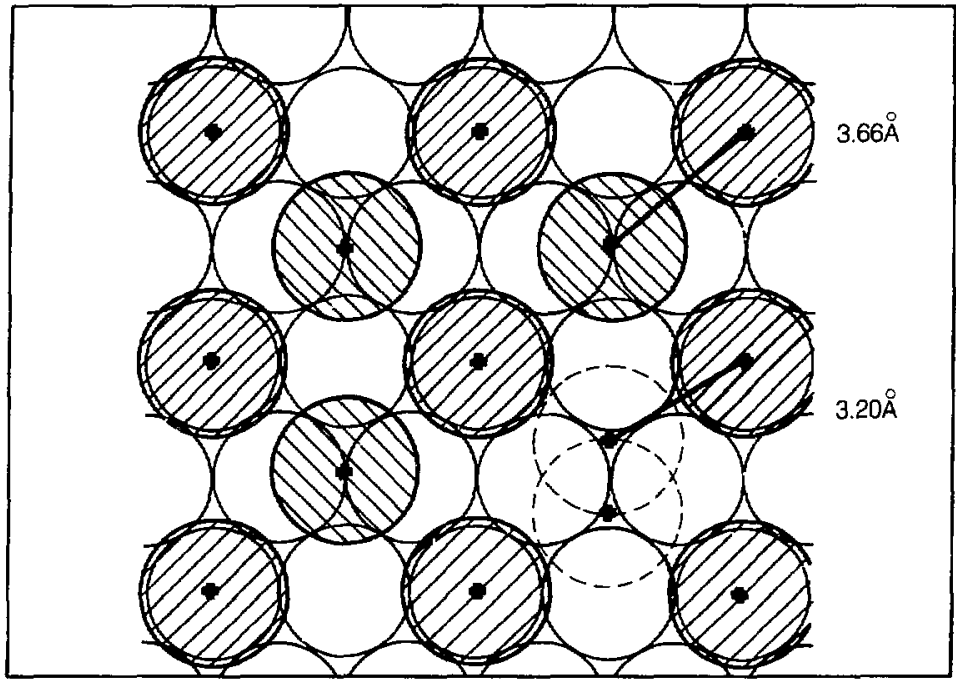

Top view

Pt (111) - c $(4 \times 2)-2 \mathrm{CO}$

LEED Structure at $\mathrm{T}=150 \mathrm{~K}$

XBL $857-3135$

Figure 10.12 


\section{5 \\ $\mathrm{CO}$ c( $4 \times 2)$ on $\mathrm{Pt}(111)$ \\ Normal Incidence at $150 \mathrm{~K}$}

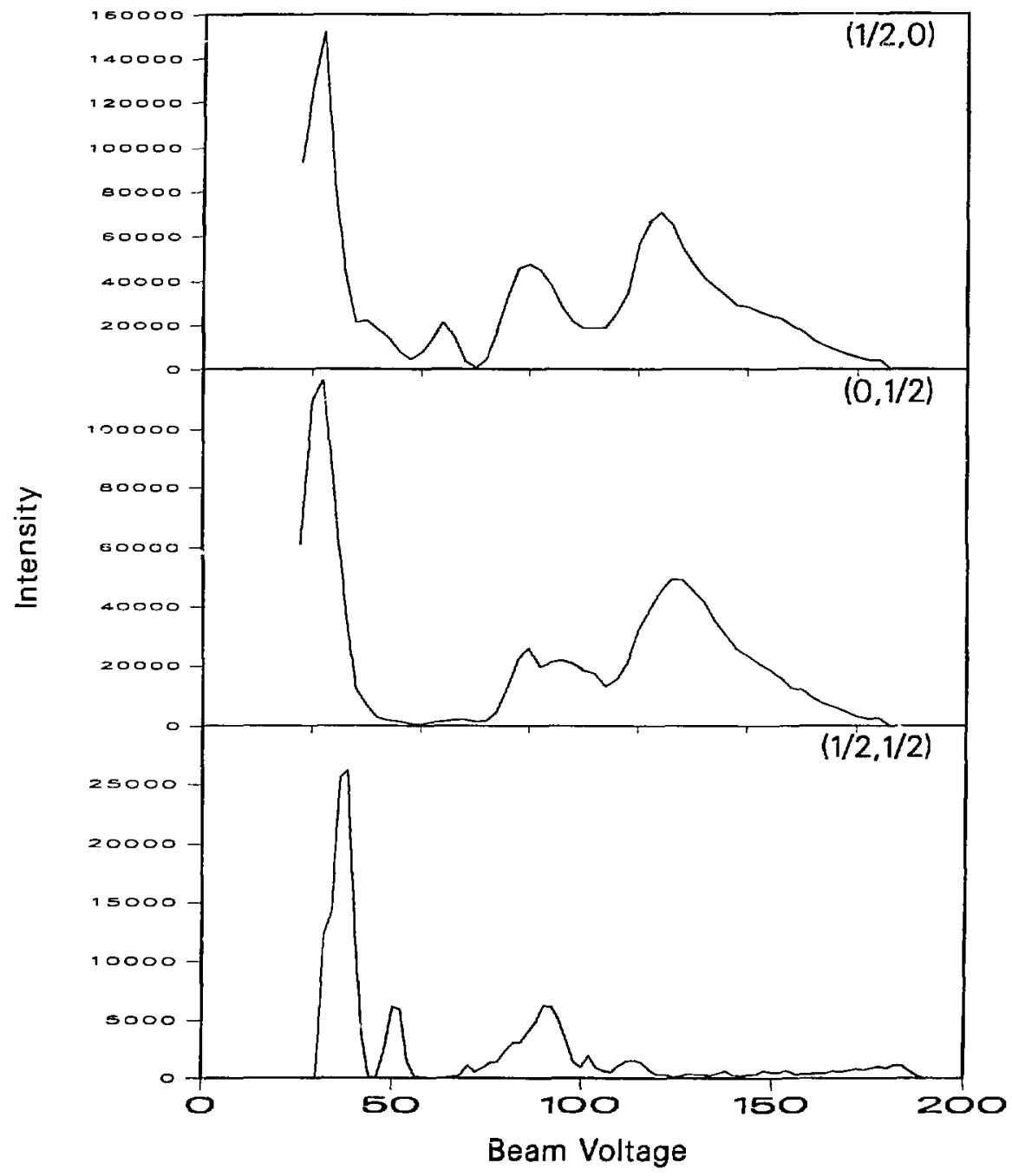

FIgure 10.13a 


\section{$\mathrm{CO}(4 \times 2)$ on $\mathrm{Pt}(111)$ Normal Incidence at $150 \mathrm{~K}$}

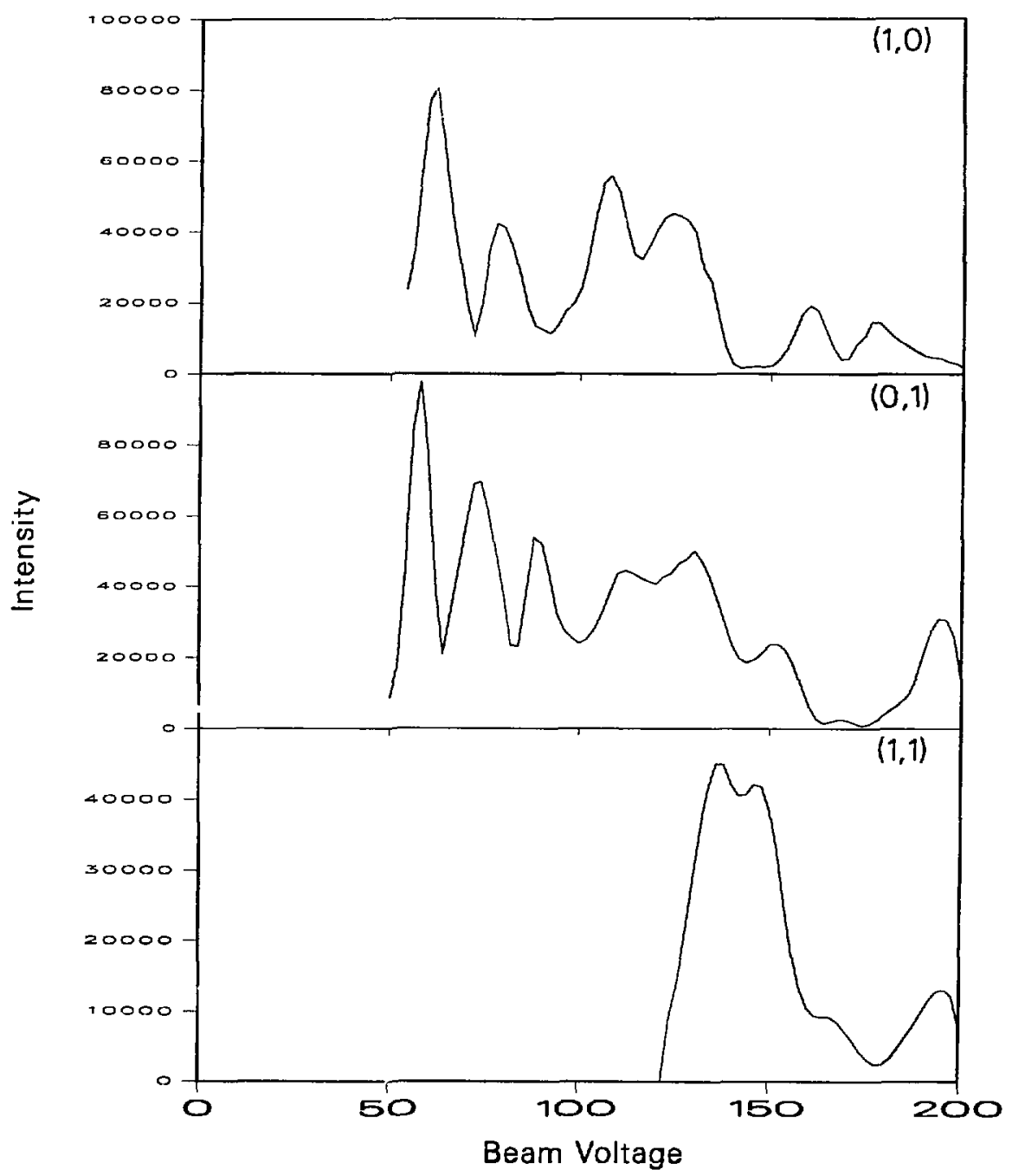

Figure 10.13b 


\section{7 \\ $\mathrm{CO} c(4 \times 2)$ on $\mathrm{Pt}(111)$ Normal Incidence at $150 \mathrm{~K}$}

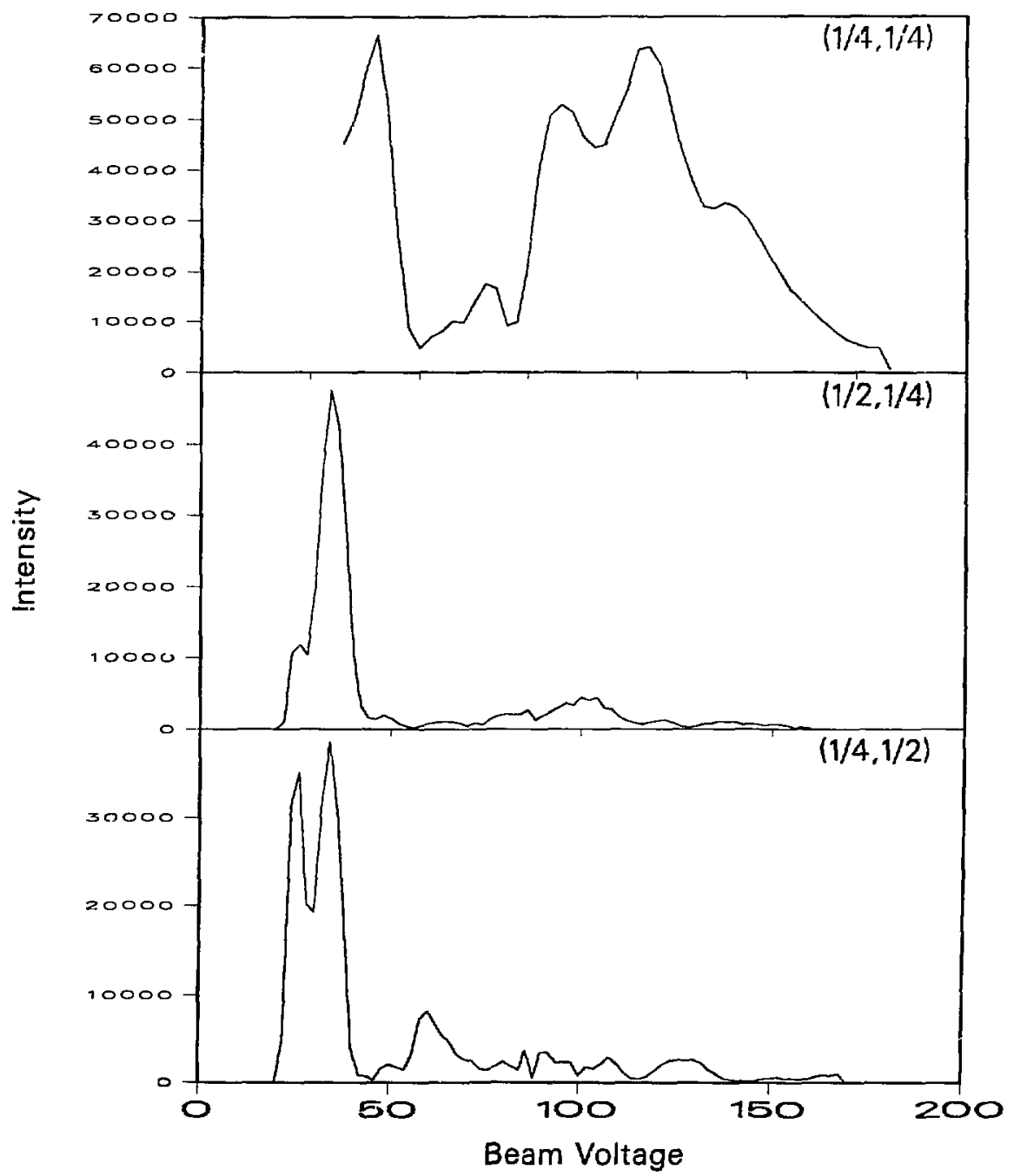

Figure $10.13 \mathrm{c}$ 


\section{8 \\ $\mathrm{CO} \mathrm{c}(4 \times 2)$ on $\mathrm{Pt}(111)$ \\ Normal Incidence at $150 \mathrm{~K}$}



Figure 10.13d 


\section{9 \\ $\mathrm{CO} \mathrm{c}(4 \times 2)$ on $\mathrm{Pt}(111)$ Normal Incidence at $150 \mathrm{~K}$}



Beam Voltage

Figure 10.13e 
430

$\mathrm{CO}$ c(4x2) on $\mathrm{Pt}(111)$
$\theta=5$ at $150 \mathrm{~K}$

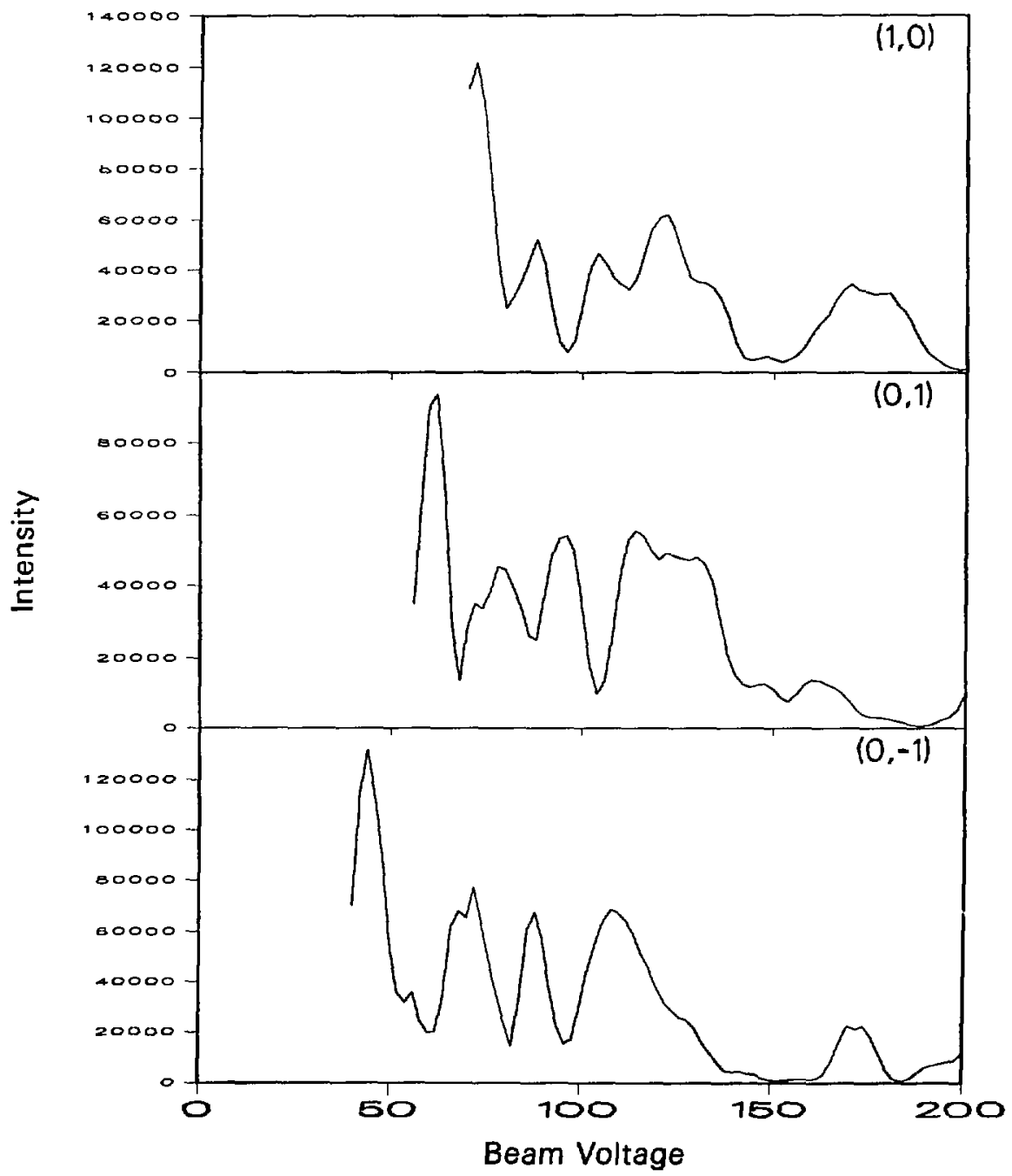

Figure 10.131 


\section{1 \\ $\mathrm{CO} \mathrm{c}(4 \times 2)$ on $\mathrm{Pt}(111)$ \\ $\theta=5$ at $150 \mathrm{~K}$}

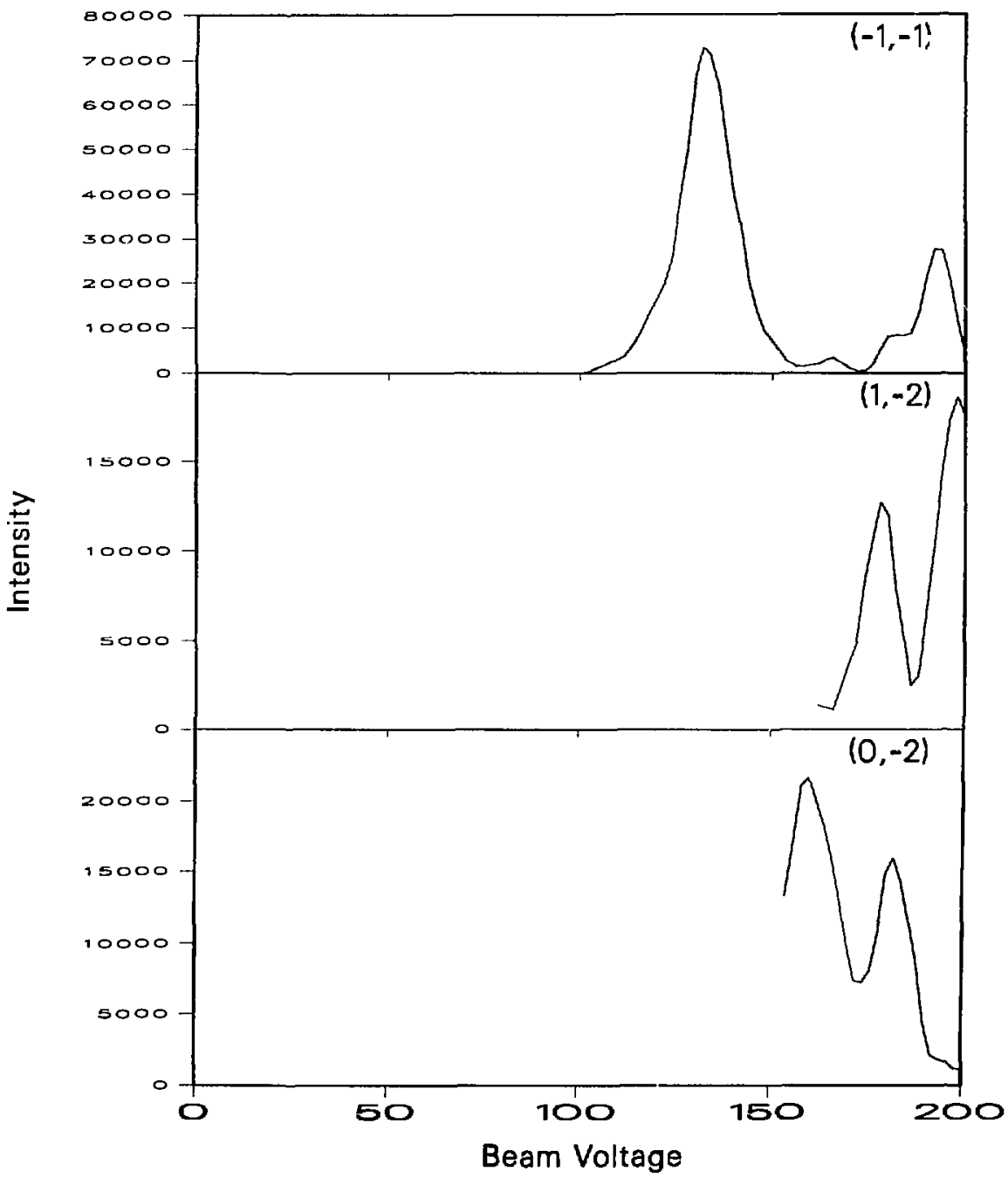

Figure 10.139 


\section{2 \\ $\mathrm{CO} \mathrm{c}(4 \mathrm{x} 2)$ on $\mathrm{Pt}(111)$ \\ $\theta=5$ at $150 \mathrm{~K}$}

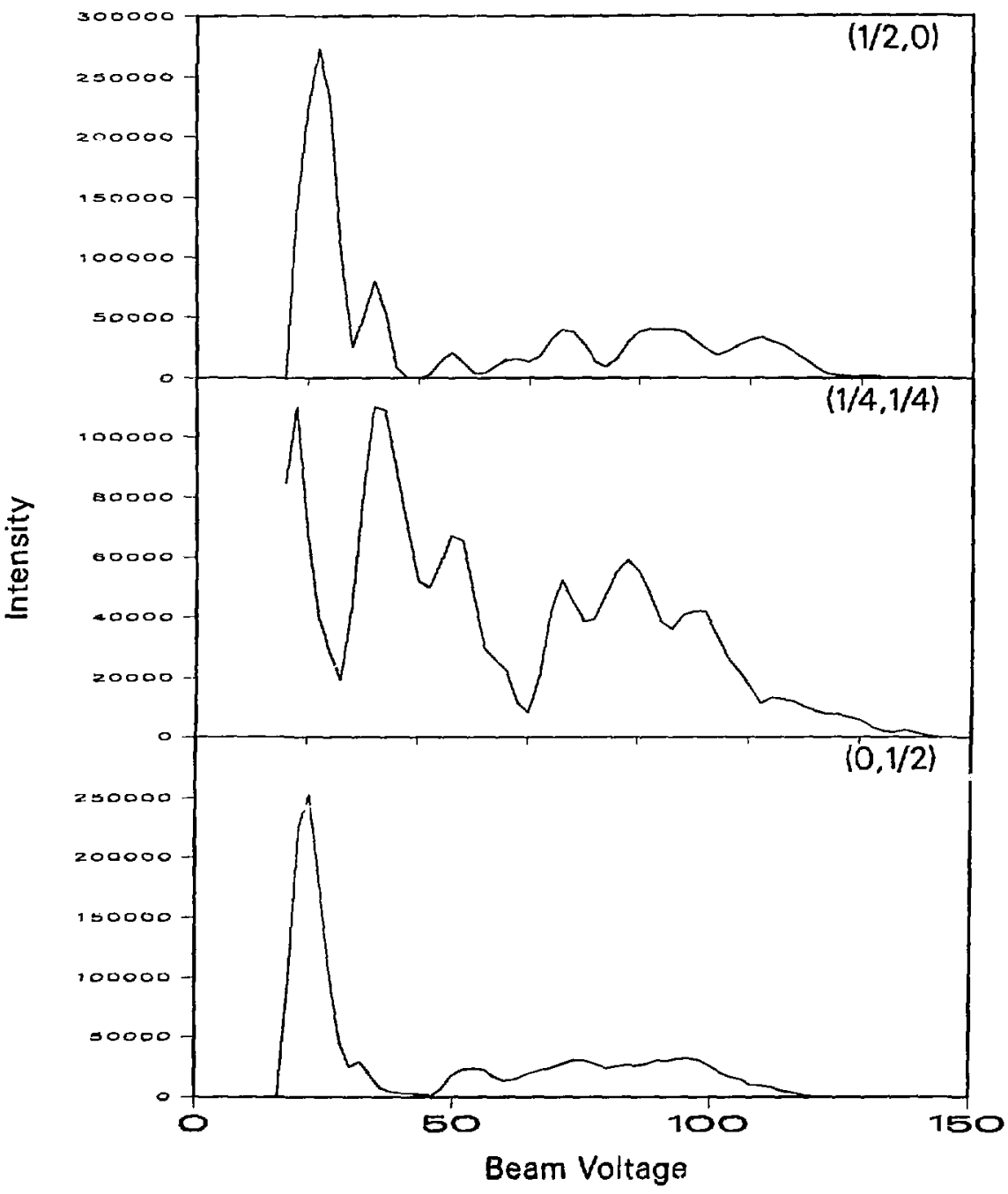

Figure 10.13h 


\section{3 \\ $\mathrm{CO} c(4 \times 2)$ on $\mathrm{Pt}(111)$ \\ $\theta=5$ at $150 \mathrm{~K}$}

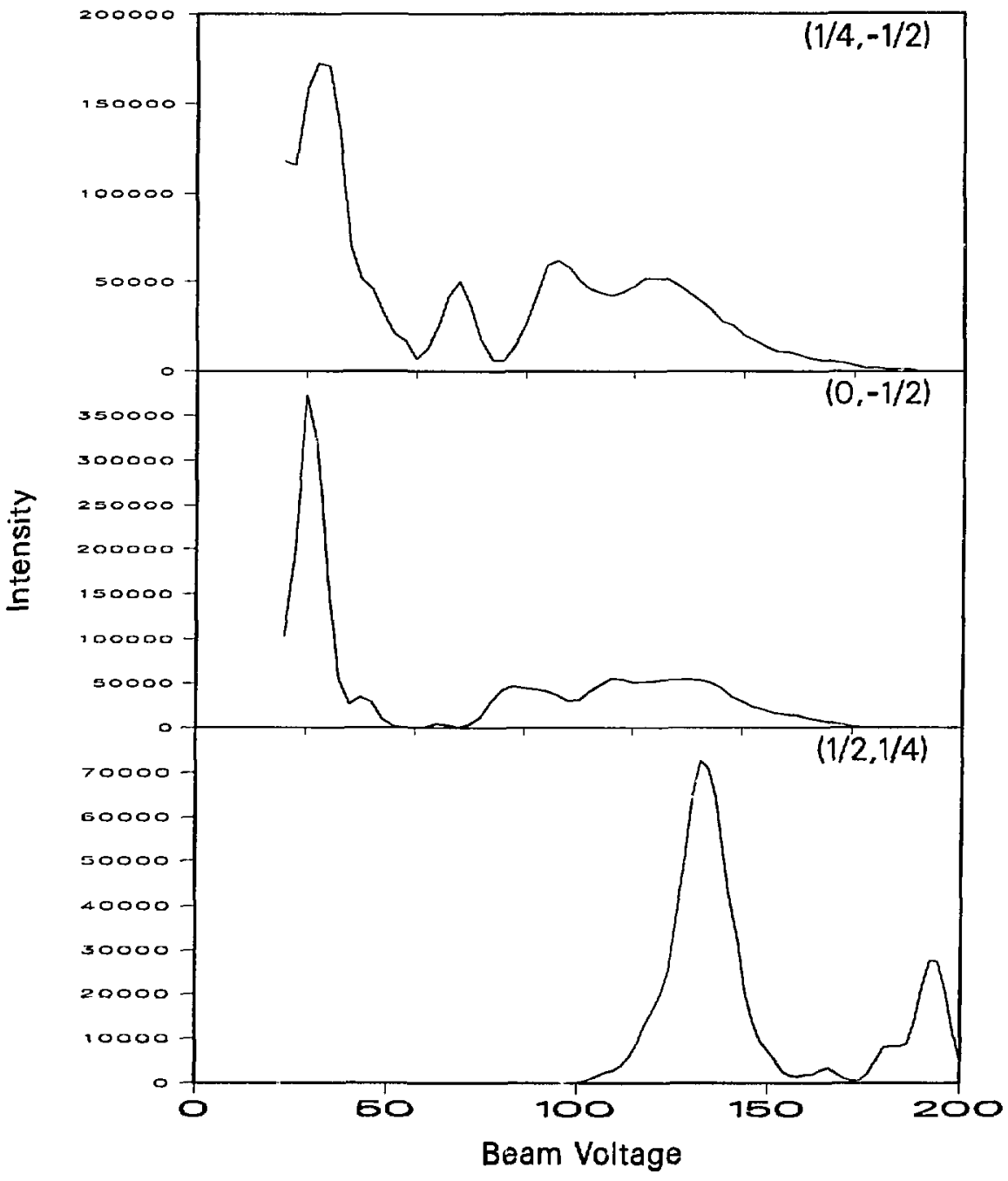

Figure 10.131 


\section{4 \\ $\mathrm{CO} c(4 \times 2)$ on Pt(111) \\ $\theta=5$ at $150 \mathrm{~K}$}



Figure 10.13 J 


\section{5 \\ $\mathrm{CO} \mathrm{c}(4 \mathrm{x} 2)$ on $\mathrm{Pt}(111)$ \\ $\theta=5$ at $150 \mathrm{~K}$}

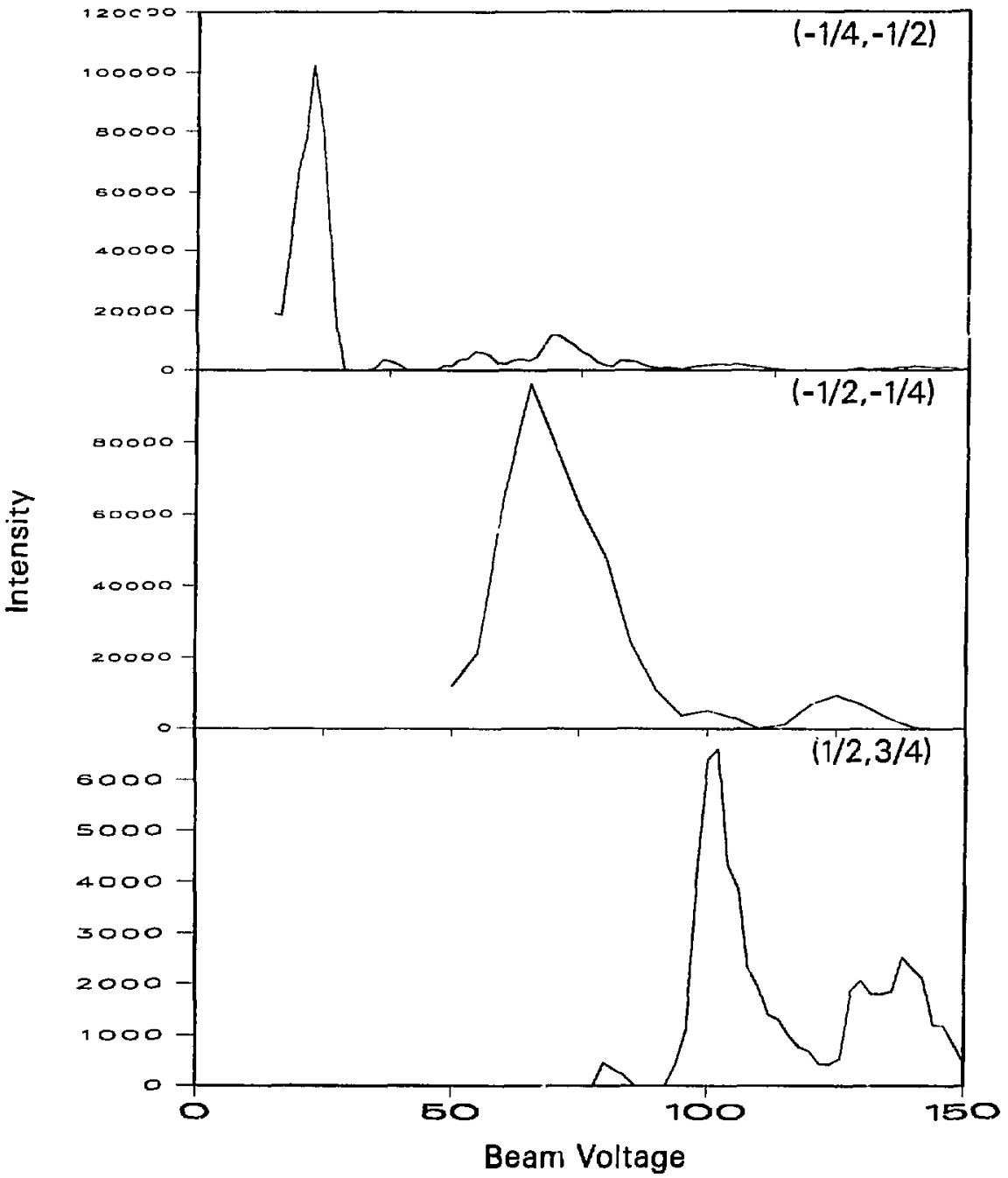

Figure 10.13k 


\section{6 \\ $\mathrm{CO} c(4 \times 2)$ on $\mathrm{Pt}(111)$ \\ $\theta=5$ at $150 \mathrm{~K}$}

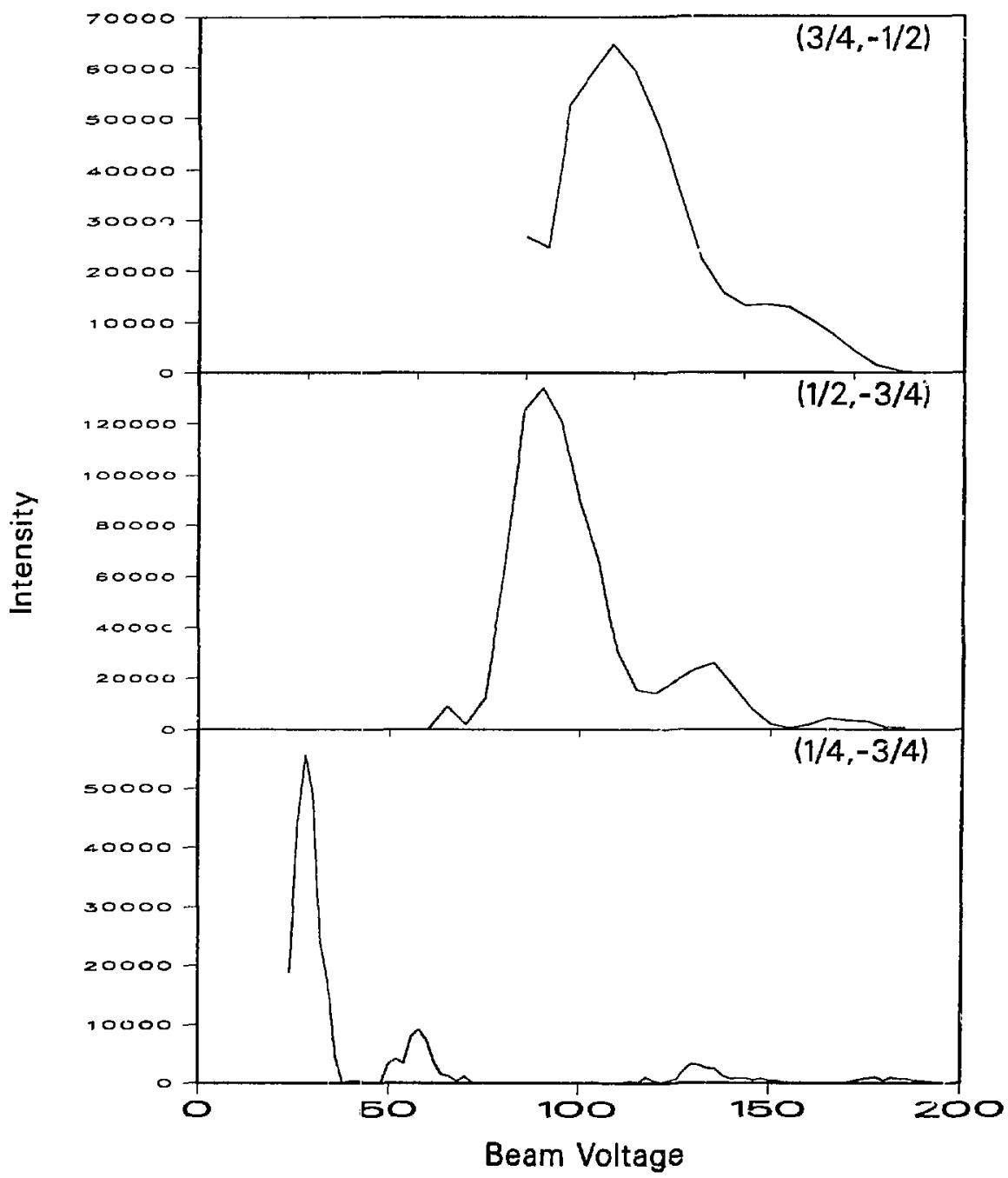

Figure $10.13 i$ 


\section{7 \\ $\mathrm{CO} c(4 \times 2)$ on $\mathrm{Pt}(111)$ \\ $\theta=5$ at $150 \mathrm{~K}$}

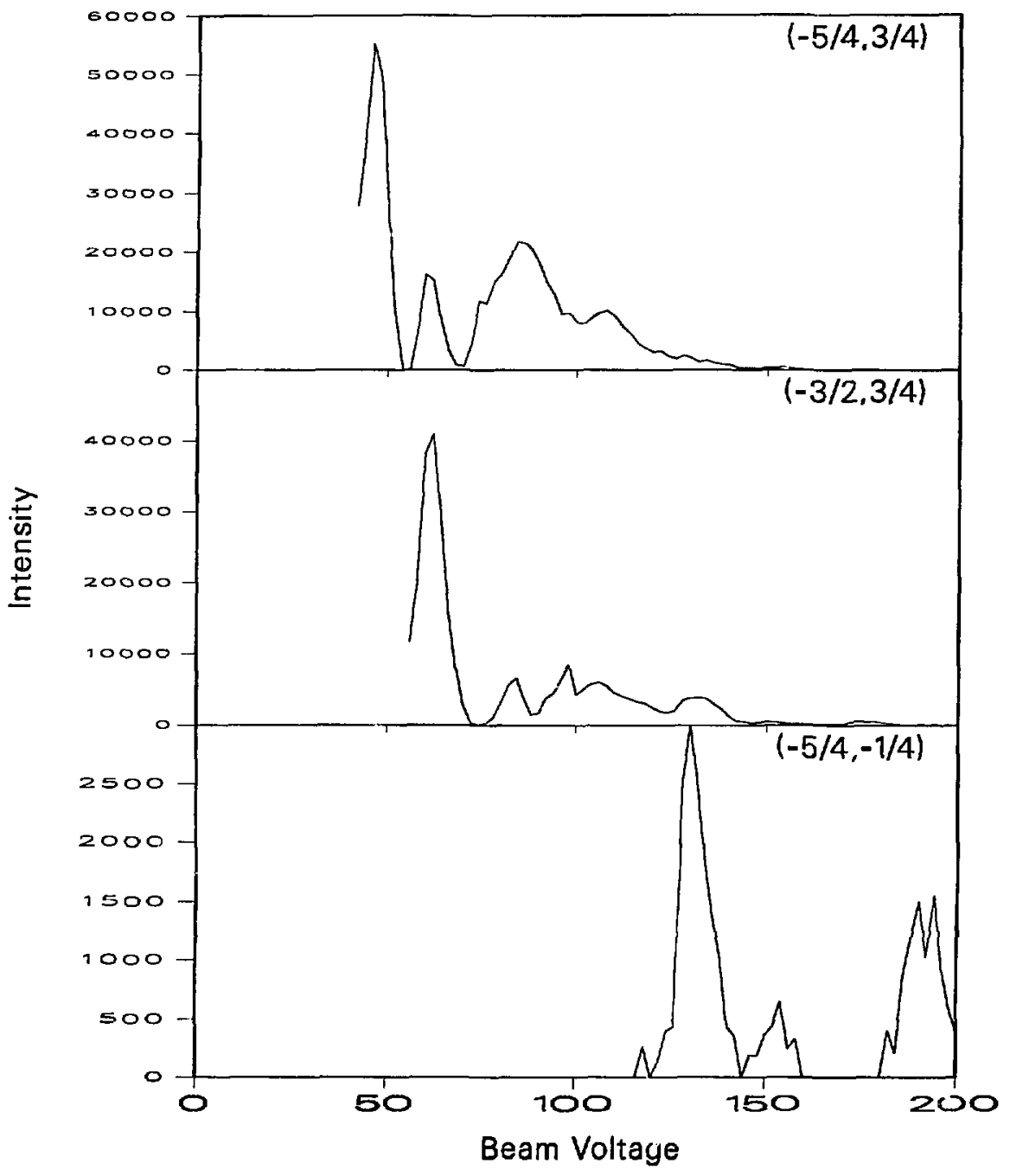

Figure $10.13 \mathrm{~m}$ 
438

\section{$\mathrm{CO}$ c(4x2) on $\mathrm{Pt}(111)$ \\ $\theta=15$ at $150 \mathrm{~K}$}

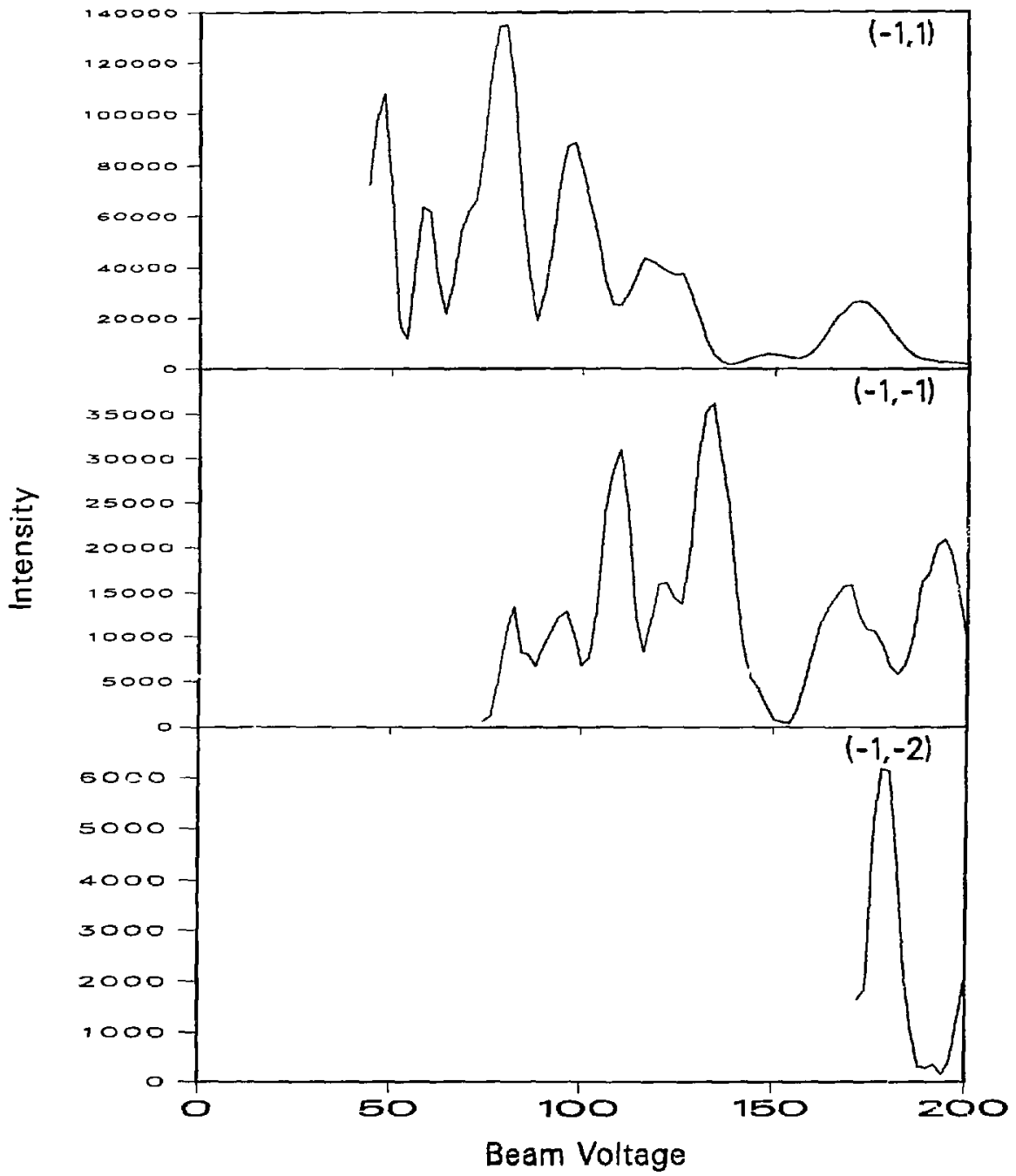

Figure 10.13n 


\section{9 \\ $\mathrm{CO} c(4 \times 2)$ on $\mathrm{Pt}(111)$ \\ $\theta=15$ at $150 \mathrm{~K}$}

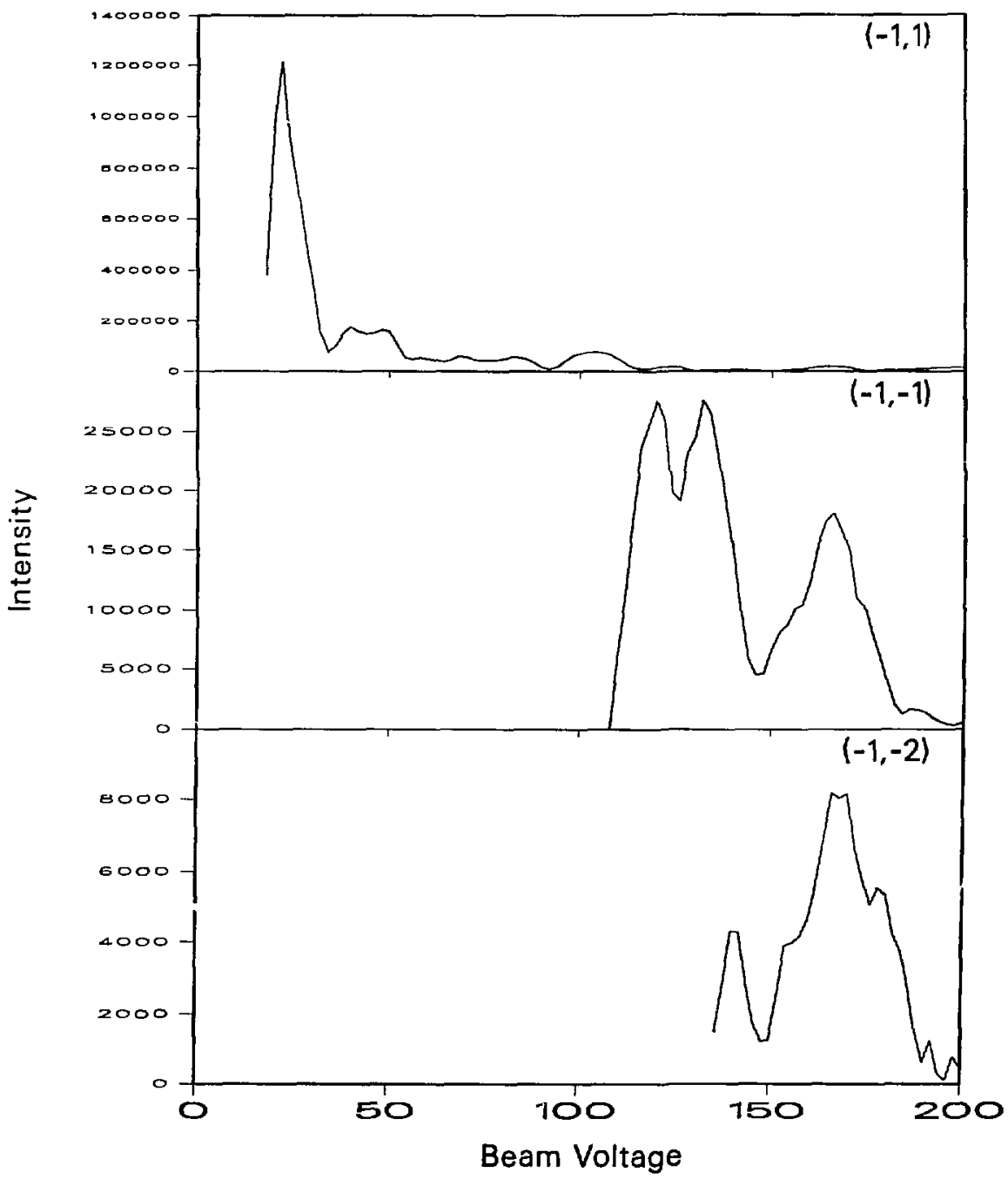

Figure 10.130 


\section{0 \\ $\mathrm{CO} c(4 \times 2)$ on $\mathrm{Pt}(111)$ \\ $\theta=15$ at $150 \mathrm{~K}$}



Figure 10.13p 
441

\section{$\mathrm{CO}$ c $(4 \times 2)$ on $\mathrm{Pt}(111)$ \\ $\theta=15$ at $150 \mathrm{~K}$}

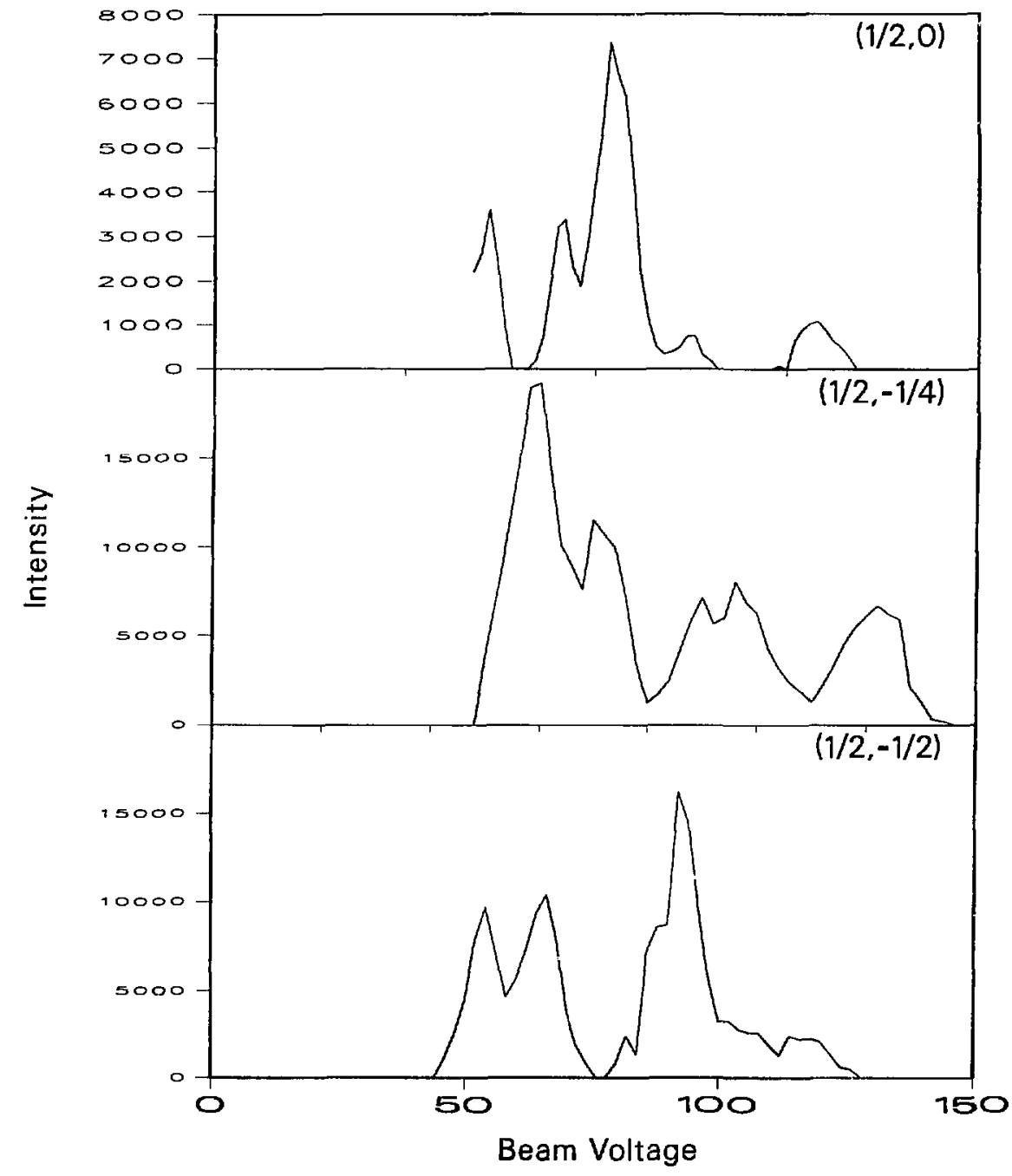

Figure 10.13q 


\section{2 \\ $\mathrm{CO} \mathrm{c}(4 \mathrm{x} 2)$ on $\mathrm{Pt}(111)$ \\ $\theta=15$ at $150 \mathrm{~K}$}

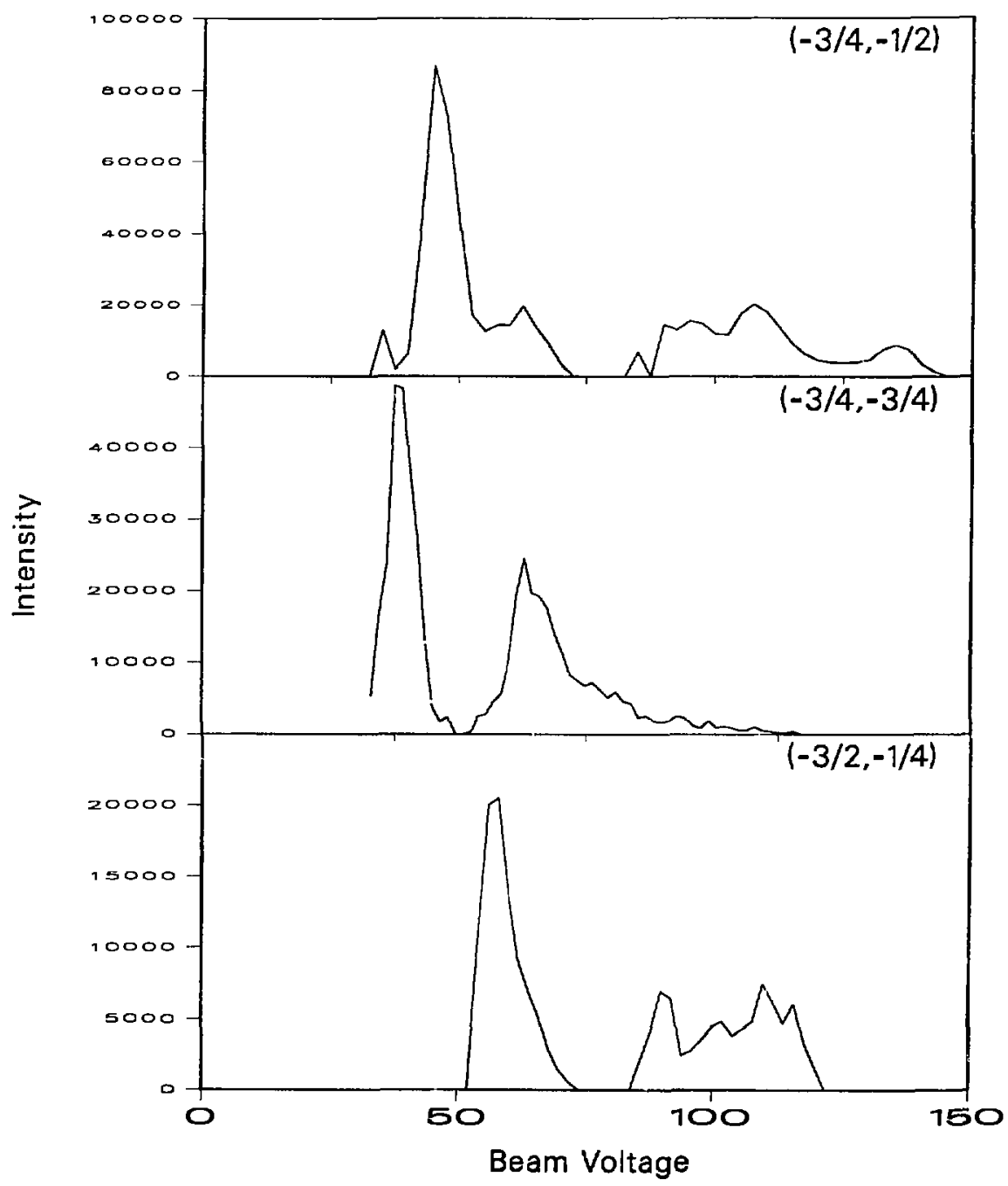

Figure $10.13 r$ 
443

$\mathrm{CO}$ c( $(4 \times 2)$ on $\mathrm{Pt}(111)$
$\theta=15$ at $150 \mathrm{~K}$

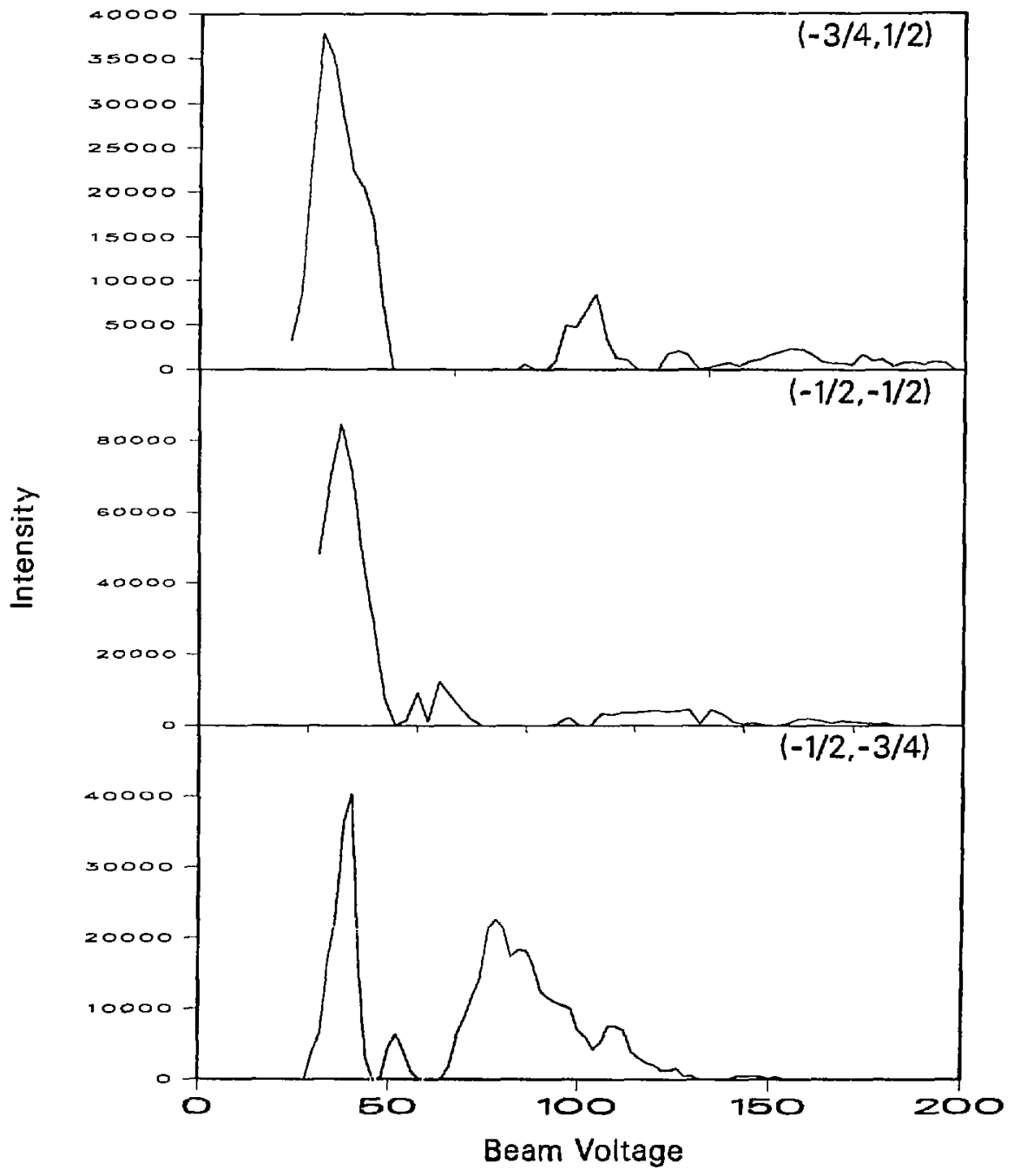

Figure 10.13s 
444

Pt (III) $-\mathrm{c}(4 \times 2)-2 \mathrm{CO}$

R-factor contour plots

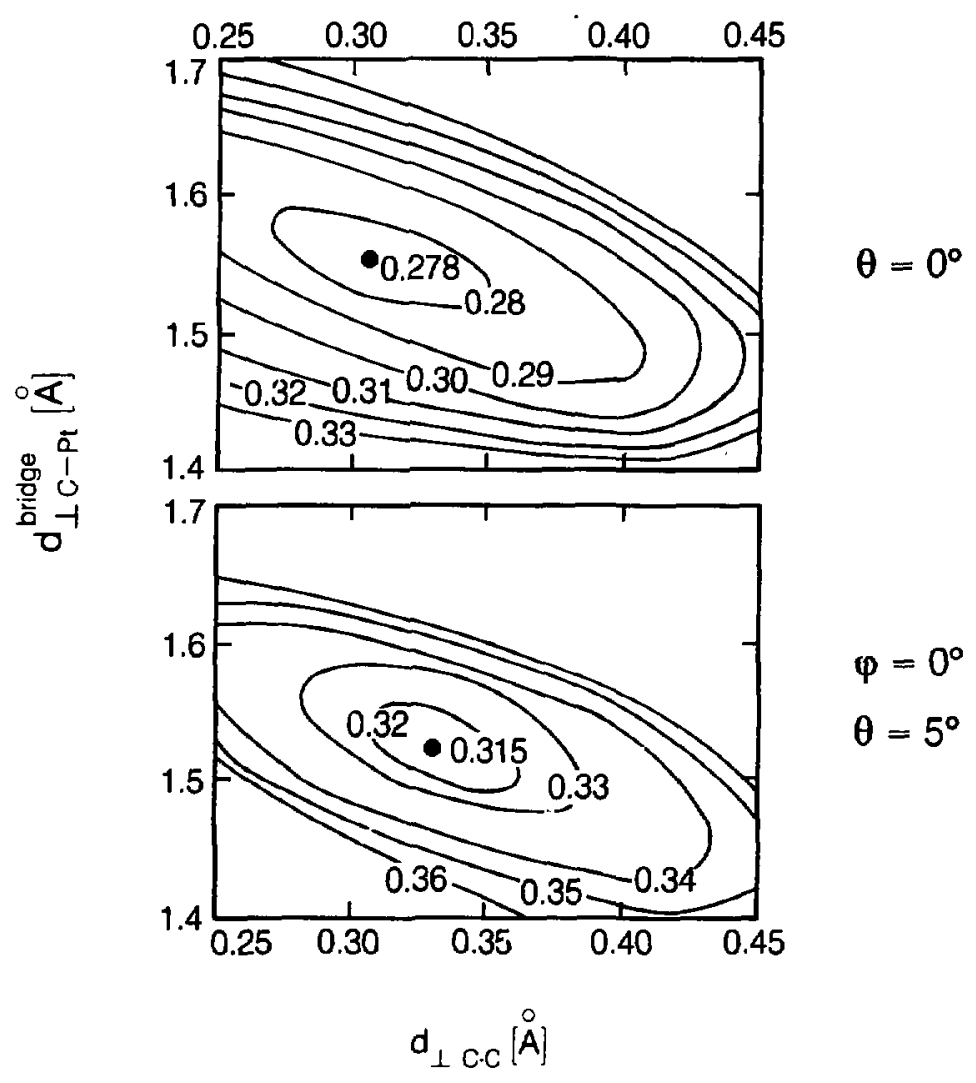

FIgure 10.14

XBL 855-10512 


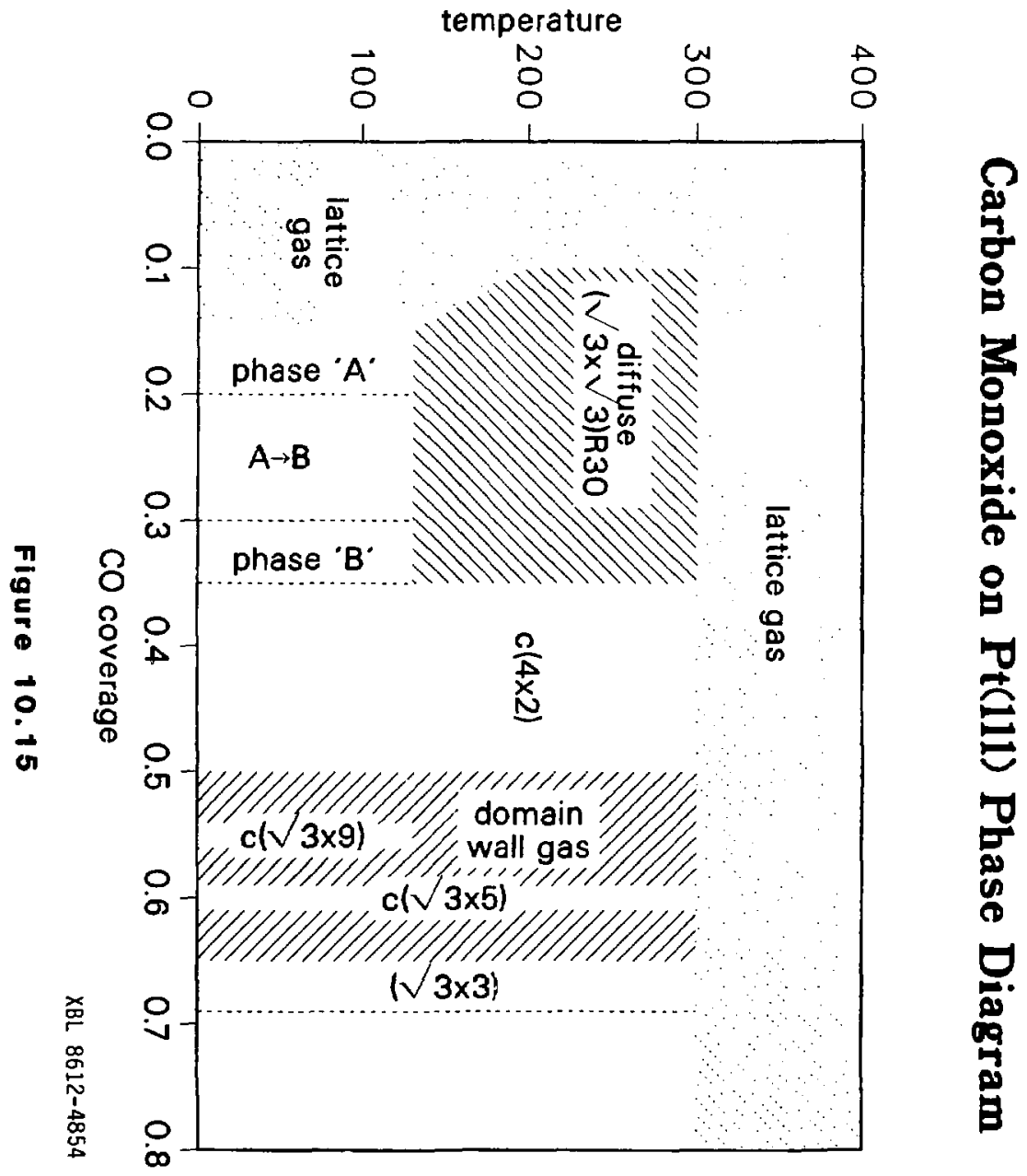




\section{Chapter 11 \\ Benzene Ordering on Pt(111)}

\subsection{Introduction}

The structure of benzene adsorbed on platinum and rhodium surfaces has been under intensive investigation. Both of these metals are key active ingredients in important catalytic processes, ${ }^{1}$ and an understanding of the structure of hydrocarbon molecules adsorbed on the surfaces of these metals is a necessary step toward understanding the mechanism of catalytic processes on a molecular level.

Detailed structural results have been obtained for only a few hydrocarbon molecules adsorbed on metal surfaces. The structure of ethylidyne ( $\equiv \mathrm{C}-\mathrm{CH}_{3}$ ) chemisorbed on $\mathrm{Pt}(111)^{2}$ and on $\mathrm{Rh}(111)^{3}$ after ethylene $\left(\mathrm{CH}_{2}=\mathrm{CH}_{2}\right)$ exposure, as determined through LEED structural studies, has become the structural prototype for understanding the adsorption of straight-chain (alkyene) hydrocarbon molecules on transition metal surfaces. The structure of chemisorbed benzene is being investigated to develop a similar understanding of the structure of aromatic hydrocarbon nolecules adsorbed on transition metal surfaces.

One important catalytic step in petroleum refining is dehydrocyclization (ring-forming) as in the formation of benzene from n-hexane or n-heptane, which increases the octane number of the gasoline product. Platinum is an excellent dehydrocyclization catalyst, while on rhodium the hydrocarbon molecules 
fragment instead (hydrogenolysis). A comparison of the structural results for monolayers of benzene adsorbed on $\mathrm{Pt}(111)$ and $\mathrm{Rh}(111)$ may shed some light on the reasons for the different chemistry of the two metal surfaces.

\subsection{Benzene on platinum (111)}

Benzene chemisorption on platinum surfaces has been investigated with a number of different surface sensitive techniques, including work-function measurements, ${ }^{4}$ LEED, ${ }^{4,5}$ electron energy loss spectroscopy (HREELS), ${ }^{6,7}$ thermal desorption spectroscopy (TDS), ${ }^{8,9}$ ultra-violet pl:stuemission spectroscopy (UPS), ${ }^{10}$ and photoemission fine-structure (NEXAFS). ${ }^{11,12}$ The initial LEED studies fcund two ordered structures on $\mathrm{Pt}(111)$ formed by benzene exposure. Later studies using other techniques showed that benzene adsorbs intact on the Pt(111) surface with the benzene ring parallel to the surface, and that the structure of benzene is not greatly changed from the gas phase.

\subsubsection{LEED observations}

Two ordered LEED patterns formed when $\operatorname{Pt}(111)$ was exposed to benzene vapor were first observed by Gland ${ }^{4}$ in a LEED and work-function survey of hydrocarbon adsorption on $\mathrm{Pt}(111)$ and $\mathrm{Pt}(100)$. These LEED patterns are most easily described using the rectangular unit-cell notation of Bibérian and Van Hove ${ }^{13}$ discussed in chapter 10. In this notation the benzene structures are $(2 \sqrt{3} \times 4)$ rect and $(2 \sqrt{3} \times 5)$ rect. These large rectangular unit cells have sixteen and twenty times the area of the $P t(111)$ unit cell, respectively. A rectangular 
unit cell has three possible orientations on the $\mathrm{Pt}(111)$ surface, and so three rotational domains are normally seen superimposed in the observed LEED patterns.

These structures were observed only after long exposure to benzene vapor. A $\mathrm{Pt}(111)$ crystal was exposed to $\sim 10^{-6}$ tors of benzene vapor, the work-function changed by $\Delta \phi=-1.8 \mathrm{eV}$, and a diffuse LEED pattern was observed. With continued exposure to benzene flux $\Delta \phi$ decreased to $-1.4 \mathrm{eV}$ in 5 to 20 minutes and the $(2 \sqrt{3} \times 4)$ rect LEED pattern was observed. With continued benzene exposure $\Delta \phi \rightarrow-1.0 \mathrm{eV}$ and the $(2 \sqrt{3} \times 5)$ rect LEED pattern was observed. The transition to the second benzene LEED pattern took 20 to 100 minutes depending on the benzene pressure. This transition also occurred in vacuum in about 10 hours. The work-function change approached a limit as $\Delta \phi \rightarrow-0.7 \mathrm{eV}$. Benzene was the only hydrocarbon molecule in Gland's 4 survey to show substantial work-function changes after the initial saturation exposure of the surface. ${ }^{4}$

Gland and Somorjai proposed that benzene was initially adsorbed in a $\pi$ bonded configuration with the benzene ring roughly parallel to the surface. With increasing exposure the molecule would change to a $\sigma$-bonded configuration with the carbon-ring tilted or perpendicular to the surface. The change in orientation could explain the decreasing work-function with increasing coverage. ${ }^{4}$

Stair ${ }^{5}$ performed a more detailed LEED study of benzene adsorption on $\mathrm{Pt}(111)$ and obtained similar results. The $(2 \sqrt{3} \times 4)$ rect structure formed after a $700 \mathrm{~s}$ exposure to $10^{-7}$ torr of benzene vapor at room temperature. After an additional 50 minutes at this pressure the $(2 \sqrt{3} \times 4)$ rect $\rightarrow(2 \sqrt{3} \times 5)$ rect transition was complete. This transition was observed to take place in vacuum in $~$ 
200 minutes at room temperature or in $\sim 50$ minutes at $325 \mathrm{~K}^{5}$

Stair also measured the carbon to platinum atom ratio from Auger peak heights, using observations of ethylene, acetylene and propylene adsorption on $\operatorname{Pt}(111)$ as a calibration. He reported carbon coverages of $2.46 \pm 0.40$ and $3.06 \pm 0.20$ monolayers for the $(2 \sqrt{3} \times 4)$ rect and $(2 \sqrt{3} \times 5)$ rect structures, respectively. ${ }^{5}$ This was interpreted as additional support for a model involving tilted benzene molecules, since the most densely packed plane of solid benzene would correspond to a coverage of 1.46 , and benzene packed on $\mathrm{Pt}(111)$ in a layer with the carbon ring perpendicular to the surface would only give a coverage of 2.07 , neglecting attenuation effects. Stair also attempted to record LEED I-V curves for the $(2 \sqrt{3} \times 4)$ rect and $(2 \sqrt{3} \times 5)$ rect phases of benzene on Pt(111).

\subsubsection{Glide symmetry}

Certain LEED spots in the benzene LEED patterns are systematically missing from the recorded LEED data. Koestner ${ }^{14}$ showed that this was due to the presence of glide-line symmetries in the $[11 \overline{2}]$ direction in both the $(2 \sqrt{3} \times 4)$ rect and $(2 \sqrt{3} \times 5)$ rect LEED patterns. This means that a translation along the glide line by half the unit cell size combined with a reflection across a plane perpendicular to the surface along the glide line is a symmetry operation. This glide symmetry causes the first, third, fifth, etc. spots along the $(1,0)$ and $(\overline{1}, 0)$ directions in the LEED pattern to vanish whenever $\mathbf{k}_{\mathrm{o}}$ il is along the [11, $]$ direction. At normal incidence this condition is satisfied simultaneously for all three rotational domains. 
The existence of a glide symmetry greatly restricts the possible surface models. Because of the glide sy nmetry the unit cell must contain an even number of adsorbed molecules, and the adsorption sites must be arranged to preserve the glide symmetry.

\subsubsection{HREELS results}

Benzene adsorption has been investigated by HREELS on severa! transition metal surfaces, including platinum, ${ }^{6,7}$ nickel, ${ }^{6,15}$ rhodium ${ }^{16}$ and palladium. ${ }^{17}$ The HREEL spectra for these different metals are similar. All are dominated by a strong loss betweer 720 and $830 \mathrm{~cm}^{-1}$ assigned to the $\gamma_{\mathrm{CH}}$ or carbon-hydiogen bond out-of-plane bending mode. All of these spectra have been interpreted in terms of intact benzene molecules adsorbed parallel to the surface. These assignments were made using the dipole selection rules for HREELS scattering.

In their HREELS study of benzene on $\operatorname{Pt}(111)$ Lehwald et al. ${ }^{6}$ argued that the benzene molecule must be adsorbed in a high-symmetry site since the number of observed vibrational loss modes was small and was consistent with benzene adsorbed in sites of $C_{3 v}(\sigma)$ symmetry. Only the three-fold sites -- top, fcc-hollow and hcp-hollow -- with certain orientations of the benzene molecule -- have this symmetry. The $\gamma_{\mathrm{CH}}$ mode for platinum is spiit into two peaks, one at $830 \mathrm{~cm}^{-1}$ and one at $020 \mathrm{~cm}^{-1}$. The ratio of these loss-peaks changes with coverage. Lehwald et al. proposed that this splitting is due to benzene molecules adsorbed in two inequivalent sites of $C_{3 v}(\sigma)$ symmetry. Lehwald et al. did not observe the ordered LEED patterns reported by Gland and Somorja1. 
In a more recent HREELS study of benzene adsorbed on palladium Waddill and Kesmodel ${ }^{17}$ obtained a HREEL spectra for $\operatorname{Pd}(111)$ that was consistent with sites of $C_{3 v}(\sigma)$ synmetry; however, they also obtained very sinilar spectra for benzene adsorbed on $\mathrm{Pd}(\mathbf{1 0 0})$, and there are no sites of three-fold symmetry on the (100) surface. The authors have proposed an altarnative interpretation of the observed spectra assuming a benzene adsorption site with $C_{8}(\sigma)$ symmetry corresponding to bridge sites. This would be consistent with both the (100) and (111) surface data. They also show that the apparent splitting of the $\gamma_{\mathrm{CH}}$ losspeak and the two-peak TDS spectrum seen for benzene on palladium can be explained in terms of one adsorption site rather than two inequivalent adsorption sites of the same symmetry.

As Mate ${ }^{7}$ has remarked, HREELS may be relatively insensitive to the benzene adsorption site. Although adsorption in a site of reduced symmetry should split the highly degenerate vibrational modes of gas-phase benzene, it is possible that this splitting is too small to be resolved in HREELS and so HREELS alone cannot reliably determine the adsorption site of benzene on transition metal surfaces. Although the adsorption site is uncertain, the HREELS data slearly indicate that benzene is adsorbed intact and parallel to the metal surface.

\subsubsection{Thermal desorption results}

Tsai and Muettertjes ${ }^{8}$ conducted as series of TDS experiments using benzene and labeled benzene on $\mathrm{Pt}(111)$ and a stepped $\mathrm{Pt}(111)$ surface. Desorption as intact benzene molecules and decomposition on the surface are competing 
processes. Benzene desorption peaks were observed at 370 to $400 \mathrm{~K}$ and 470 to $490 \mathrm{~K}$ after heating at $25 \mathrm{~K} / \mathrm{s}$. A sharp hydrogen desorption peak was observed at $540 \mathrm{~K}$ along with a broad peak at $650 \mathrm{~K}$ and, for saturation coverage, a broad peak at $440 \mathrm{~K}$.

When benzene and deuterated benzene $\left(\mathrm{C}_{6} \mathrm{D}_{6}\right)$ were adsorbed together on $\operatorname{Pt}(111)$ only $\mathrm{C}_{6} \mathrm{H}_{6}$ and $\mathrm{C}_{6} \mathrm{D}_{6}$ were deicated in the TDS experiment. Likewise when deuterated benzene and hydrogen were adsorbed together, only $\mathrm{H}_{2}$ and not $\mathrm{HD}$ was desorbed below the benzene decomposition temperature. (Hydrogen is desnrbed from $\mathrm{Pt}(111)$ at $\sim 320 \mathrm{~K}$ with or without benzene adsorbed on the surface.) In a third experiment the $\mathrm{Pt}(111)$ surface was saturated with deuterated benzene and then exposed to unlabeled benzene at $10^{-8}$ torr for $180 \mathrm{~s}(1.8 \mathrm{~L})$. The TDS results showed mostly unlabeled benzene and not deuterated benzene desorbing from the $\mathrm{Pt}(111)$ crystal. ${ }^{8}$ These results indicated that intact benzene adsorbs reversibly on $\mathrm{Pt}(\mathbf{1 1 1})$. There is no evidence for hydrogen exchange between benzene molecules or between benzene and atomic hydrogen on the surface. Further, benzene molecules can be displaced from the surface.

Similar experiments showed that the initial fraction $(<10 \%$ of the saturation coverage) of adsorbed benzene cannot be displaced and does not desorb intact during TDS experiments. This may be due to adsorption at defect sites. When benzene was adsorbed on a stepped $\mathrm{Pt}(111)$ surface the irreversibly adsorbed fraction was increased and the fraction of benzene desorbed as intact molecules decreased. ${ }^{8}$ 
The thermal desorption results clearly show that most benzene molecules chemisorbed on $\mathrm{Pt}(111)$ are intact. The failure to detect $\mathrm{H}-\mathrm{D}$ exchange in the TDS data when $\mathrm{C}_{6} \mathrm{H}_{6}$ and $\mathrm{C}_{6} \mathrm{D}_{6}$ were co-adsorbed together on $\mathrm{Pt}(111)$ rules out a benzene bonding mechanism where one or more carbon-hydrogen bonds are broken to form carbon-metal $\sigma$-bonds. These results give additional support to the hypothesis that intact benzene is $\pi$-bonded parallel to the $\mathrm{Pt}(111)$ surface, as indicated by the HREELS and photoemission results.

\subsubsection{Photoemission results}

Ultra-violet photoemission studies of benzene on $\mathrm{Pt}(\mathbf{1 1 1})^{10}$ are consistent with intact benzene $\pi$-bonded parallel to the $\mathrm{Pt}(111)$ surface. This study also confirms the large $(>1 \mathrm{eV})$ decrease in the work-function of the $\mathrm{Pt}(111)$ surface for benzene adsorption. ${ }^{10}$

Near-edge $\mathrm{x}$-ray adsorption fine structure (NEXAFS) measurements have been carried out for benzene adsorbed on $\mathrm{Pt}(111)$. In these experiments the $\sigma$ and $\pi$ shape-resonances of the benzene valence orbizals are detected. By changing the polarization and angle of incidence of the photon beam relative to the surface the orientation of the benzene molecule can be determined. The NEXAFS data ${ }^{11,12}$ show that benzene is adsorbed with the carbon ring parallel " the surface and further, using an empirical correlation between the $\sigma$ shape-resonance energy and the carbon-carbon bond length, that this bond length is the same as that for gasphase benzene within the accuracy of the NEXAFS data $(1.40 \pm 0.02 \AA)$. 


\subsection{Benzene chemisor, tion experiments}

Additional benzene chemisorption experiments were performed on $\mathrm{Pt}(111)$ to obtain LEED I-V data for struciure determination experiments. Stair ${ }^{5}$ had made I-V measurements for both the $(2 \sqrt{3} \times 4)$ rect and $(2 \sqrt{3} \times 5)$ rect phases of benzene adsorbed on $\operatorname{Pt}(111)$ at several angles of incidence, however the signal-to-noise ratio for these I-V curves was very low. The I-V structure was superimposed on a monotonically decreasing signal, and the difference between adjacent I-V maxima and minima was less than $10 \%$ of the average integrated (background) intensity. Also there were many breaks in the I-V curves where the LEED spots were lost in the background intensity. Overall, the LEED data-base was not sufficient for reliable structure determinaticn.

\subsubsection{Reproducibility}

It was not possible to reproduce the ordered $(2 \sqrt{3} \times 4)$ rect and $(2 \sqrt{3} \times 5)$ rect LEED patterns reported by Gland ${ }^{4}$ and $\mathrm{Stair}^{5}$ for benzene on $\operatorname{Pt}(111)$. Benzene exposures under a variety of different dosing pressures, surface temperatures and exposures produced only a disordered LEED pattern with no resolved superlattice spots, only a diffuse hexagonal ring in the LEED pattern with a radius (in reciprocal space) of $\sim 1 / 3$ of the $\mathrm{Pt}(111)$ unit cell (see figure 11.1). 


\subsubsection{Benzene uptake}

A series of Auger measurements were made to determine the coverage of benzene on the $\operatorname{Pt}(111)$ surface as a function of the nominal benzene exposure. The crystal was exposed to benzene vapor at $10^{-8}$ torr for $20 \mathrm{~s}$ intervals $(0.2$ Langmuir increments) and the ratio of the Auger peak heights for the carbon 272 $\mathrm{eV}$ and platinum $237 \mathrm{eV}$ peaks was measured. After a total exposure of $2 \mathrm{~L}$ the exposure increments were increased to first $0.5 \mathrm{~L}$ and then to $5 \mathrm{~L}$. The results are shown in figure 11.2. The Auger peak ratio saturates at $\mathrm{C} / \mathrm{Pt} \approx 2.2$ after a benzene exposure of $\sim 2 \mathrm{~L}$. The figure shows the results for exposures up to $15 \mathrm{~L}$, however this experiment was continued up to a total exposure of $400 \mathrm{~L}$ with no further changes in the carbon coverage on $\mathrm{Pt}(111)$ as measured by AES.

The carbon coverage for the benzene saturated $\mathrm{Pt}(111)$ surface is significantly smaller than the values of $2.46 \pm 0.4$ and $3.06 \pm 0.2 \mathrm{C} / \mathrm{Pt}$ reported by Stair ${ }^{5}$ for the $(2 \sqrt{3} \times 4)$ rect and $(2 \sqrt{3} \times 5)$ rect structures of benzene on $\operatorname{Pt}(111)$. The results presented here imply a significantly lower carbon coverage, $\sim 1 \mathrm{C} / \mathrm{Pt}$, which is consistent with results reported by $\mathrm{Tsai}^{8}$ and Davis et al. ${ }^{18}$ for benzene coverage on $\mathrm{Pt}(111)$. Determination of the absolute carbon coverage by Auger is difficult since many factors affect the result, including incidence angle, modulation voltage, excitation energy and the effect of the incident electron beam on the surface. In another experiment the crystal was exposed to a single $240 \mathrm{~L}$ dose of benzene at $270 \mathrm{~K}$ and then the Auger $\mathrm{C}-272 / \mathrm{Pt}-237$ ratio was measured at fifteen different points on the crystal. The average ratio was 1.02 with a standard deviation of 0.13 , and the range was 0.80 to 1.24 . Such Auger coverage measurements 
can be used as a guide and to determine trends, but they should not be treated as quantitative measures of the absolute carbon coverage.

Auger measurements were also made after benzene exposure to check for any surface contamination that might affect the benzene ordering, but only carbon and platinum peaks were found in the Auger spectrum.

\subsubsection{Benzene thermal desorption}

A series of thermal desorption experiments was performed after benzene exposure to look for possible coadsorbed molecules. Carbon monoxide was always dctected in the TDS experiments after benzene exposure (see figure 11.3). The desorption temperature for carbon monoxide coadsorbed with benzene is 40 to 50 $\mathrm{K}$ lower than for similar amounts of carbon monoxide alone on $\mathrm{Pt}(111)$.

No oxygen was seen the Auger spectra, however detection of co-adsorbed carbon monoxide by AES would be difficult because of the strong interaction between the incident electron beam and adsorbed carbon monoxide molecules (see chapter 10). Since Auger spectra were typically recorded with increasing energy, by the time the spectrometer reached the $500 \mathrm{eV}$ oxygen transition the beam would have had time to desorb or decompose many of the carbon monoxide molecules within the beam area.

TDS measurements of carbon monoxide adsorbed on $\mathrm{Pt}(111)$ were made to calibrate the area under the TDS curves. The spectra show pressure as a function as crystal temperature. The area under the curve would only be proportional to the amount desorbed for a linear heating rate. Tests showed that when the 
crystal was heated with a constant current the heating rate was linear in the temperature range of interest.

The $\mathrm{Pt}(111)$ surface was saturated with carbon monoxide at room temperature and then cooled to show the ordered $c(4 \times 2)$ LEED pattern, which corresponds to 0.5 monolayers of carbon monoxide for a complete layer. This experiment was repeated several times to get a consistent value. TDS area for the diffuse " $(\sqrt{3} \times \sqrt{3}) \mathrm{R} 30$ " phase of carbon monoxide was about $2 / 3$ of that for the $c(4 \times 2)$ phase.

When this calibration was applied to the benzene TDS data the amount of coadsorbed carbon monoxide ranged from 0.10 to 0.18 monolayers (see figure 11.3). When a clean $\mathrm{Pt}(111)$ surface was held at $150 \mathrm{~K}$ for the time required for a Denzene exposurc less than $\sim 0.02$ of a monolayer of carbon monoxide was adsorbed, so the background carbon monoxide concentration was not sufficient to account for the amount of coadsorbed carbon monoxide. The clean $\mathrm{Pt}(111)$ crystal was then exposed to argon gas at $5 \cdot 10^{-7}$ torr for $60 \mathrm{~s}$. After this $30 \mathrm{~L}$ exposure TDS showed 0.16 monolayers of adsorbed carbon monoxide (argon does not adsorb on $\mathrm{Pt}(111)$ at $\geq 15 \cup \mathrm{K})$. The partial pressure of carbon monoxide apparently increases during benzene or argon exposure, possibly because the gasses are contaminated with carbon monoxide, or because carbon monoxide is released from the ion pump when the overall chamber pressure increases.

Several attempts were made to minimize the amount of carbon monoxide coadsorbed during benzene exposures. The best results were obtained by flashing the erystal to $\sim 550 \mathrm{~K}$ and then starting the benzene exposure at $5 \cdot 10^{-7}$ torr as 
soon as the crystal temperature dropped below $400 \mathrm{~K}$, below the minimum temperature of benzene decomposition on $\mathrm{Pt}(111)$ as determined by $\mathrm{H}_{2}$ TDS measurements. ${ }^{8}$ This procedure reduced the amount of carbon monoxide coadsorbed to $~$ 0.05 monolayers, but this was not sufficient to produce an ordered LEED pattern.

\subsubsection{Ordering with large exposures}

Ordered ben:ene LEED patterns were finally formed with very large benzene exposures. The clear. $\mathrm{Pt}(111)$ crystal was exposed to $10^{-6}$ torr of benzene for 10 minutes, an exposure of $600 \mathrm{~L}$, and the $(2 \sqrt{3} \times 4)$ rect $\mathrm{LEED}$ pattern was visible (see figure i1.4). As the exposure was increased to $1500 \mathrm{~L}$ the $(2 \sqrt{3} \times 4)$ rect LEED pattern transforıned to the $(2 \sqrt{3} \times 5)$ rect LEED pattern (see figure 11.5).

\subsection{Benzene -- carbon monoxide coadsorption}

The mechanisin of benzene ordering was explained when HREELS and LEED measurements were made in the same vacuum chamber at the same time.? The correlated techniques showed that benzene orders on $\mathrm{Pt}(111)$ only when coadsorbed with carbon monoxide. HREELS is very sensitive to carbon monoxide because of its large dynamic d. Jle moment. Only the disordered (figure 11.1) LEED pattern was seen when HREELS showed the surface was carbon monoxide free, and carbon monoxide was always present when the ordered LEED patterns were observed.

The $(2 \sqrt{3} \times 4)$ rect and $(2 \sqrt{3} \times 5)$ rec $t$ LEED patterns could be formed either by first exposing the clean $\operatorname{Pt}(111)$ surface to a measured amount of carbon 
monoxide and then saturating the surface with benzene, or by saturating the clean $\mathrm{Pt}(111)$ surface with benzene and then exposing the surface to carbon monoxide. ${ }^{7}$ If the surface was first saturated with benzene a disordered LEED pattern was observed. An exposure to $0.5 \mathrm{~L}$ of carbon monoxide produced the $(2 \sqrt{3} \times 4)$ rect LEED pattern, and an exposure of $1.0 \mathrm{~L}$ produced the $(2 \sqrt{3} \times 5)$ rect pattern. For exposures of carbon monoxide $>5$ L the LEED pattern was again disordered, and exposures $>20 \mathrm{~L}$ displaced almost all of the benzene from the surface.

HREEL spectra recorded for disordered, $(2 \sqrt{3} \times 4)$ rect and $(2 \sqrt{3} \times 5)$ rect benzene on $\operatorname{Pt}(111)$ at $77 \mathrm{~K}$ are shown in figure 11.6. There is no significant difference in the benzene loss peaks in the three different spectra. Some peaks shift by up to $15 \mathrm{~cm}^{-1}$, and the relative intensity of the benzene losses to the carbon monox: de losses decreases as $(2 \sqrt{3} \times 4)$ rect $\rightarrow(2 \sqrt{3} \times 5)$ rect.

Three loss peaks due to carbon monoxide are seen in the HREEL spectra. When similar spectra were recorded at $300 \mathrm{~K}$ the two peaks between $1700 \mathrm{~cm}^{-1}$ and $1800 \mathrm{~cm}^{-1}$ appeared as one broad peak and the peak at $\sim 2030 \mathrm{~cm}^{-1}$ was not observed. Since the LJEDD pattern did not change from $300 \mathrm{~K}$ to $77 \mathrm{~K}$, except for a reduction in background, the $2300 \mathrm{~cm}^{-1}$ peak is probably due to carbon monoxide adsorbed outside of the ordered carbon monoxide-benzene overlayer. 


\subsubsection{Co-adsorption and ordering}

A similar series of ordered benzene and carbon monoxide coadsorption systems has been found on the $\operatorname{Rh}(111)$ surface. ${ }^{7}$ Benzene alone on the $\mathrm{Rh}(111)$ surface forms a poorly ordered $(2 \sqrt{3} \times 3)$ rect LEED pattern with a glide-line symmetry which has two benzene molecules per unit cell. As increasing amounts of carbon monoxide are coadsorbed first a $c(2 \sqrt{3} \times 4)$ rect phase with one benzene and one carbon monoxide molecule per unit cell is formed, and then a $(3 \times 3)$ phase with one benzene and two carbon monoxide molecules per unit cell. ${ }^{7}$ On $\operatorname{Pt}(111)$ both ordered phases have two benzene molecules jer unit cell. The glide symmetry requires an even number of adsorbed molecules, and it is not possible to fit four nat-lying benzene molecules into the unit cell. From TDS measurements Mate $^{7}$ estimates that the $(2 \sqrt{3} \times 4)$ rect and $(2 \sqrt{3} \times 5)$ rect unit cells contain four and six carbon monoxide molecules, respectively, or 0.25 and 0.30 monolayers of carbon monoxide.

An additional benzene LEED pattern has been observed, which is probably also due to carbon monoxide coadsorption although it was not observed by Mate, a $(2 \sqrt{3} \times 2 \sqrt{3}) R 30^{\circ}$ or $\mathrm{c}(\sqrt{3} \times 6)$ rect pattern (see figure 11.7$)$. From the size, the unit cell probably contains one benzene molecule and at least four carbon monoxide molecules. When this pattern was observed the role of carbon monoxide coadsorption was not yet, understood, and it was not possible to reproduce the LEED pattern.

Since the observation of coadsorbate-induced ordering for benzene and carbon monoxide on $\mathrm{Pt}(111)$ and $\mathrm{Rh}(111)$, similar ordering has been observed for 
several other atoms or molecules coadsorbed with carbon monoxide or with nitric oxide (NO), including potassium, ethylidyne (from ethylene adsorption) propylidyne (from propylene adsorption, $\mathrm{CH}_{2}=\mathrm{CH}-\mathrm{CH}_{3}$ ) and butylidyne (from cis-, trans- or 1-butene adsorption, $\mathrm{CH}_{3}-\mathrm{CH}=\mathrm{CH}-\mathrm{CH}_{3}$ and $\mathrm{CH}_{2}=\mathrm{CH}-\mathrm{CH}_{2}-\mathrm{CH}_{3}$ ) on $\operatorname{Rh}(111)$ or $\operatorname{Pt}(111)$ surfaces. ${ }^{19}$

\subsection{LEED I-V data}

I-V data were collected for the $(2 \sqrt{3} \times 4)$ rect and $(2 \sqrt{3} \times 5)$ rect LEED patterns at normal incidence and at $\theta=15^{\circ}$. These data were recorded using the video data system at a camera lens opening of $\int / 0.85$ at $300 \mathrm{~K}$ and also at $150 \mathrm{~K}$. It is difficult to generate $\mathrm{I}-\mathrm{V}$ curves from the benzene data. The average LEED beam intensities are weak since the total back-diffracted intensity is distributed over so many beams. Also, this overlayer of adsorbed molecules is less wellordered than some other complex LEED patterns (e.g. Si(111)-(7x7)) so the spots are relatively broad, and disordered molecules contribute to the diffuse quasielastic background in addition to thermal scattering effr :ts.

For the $(2 \sqrt{3} \times 4)$ rect structure the unit cell is sixteen times the size of the substrate cell. Furthermore there are three rotationally inequivalent domains, so the number of beams is multiplied by forty-eight. All three domains contribute to the half-order beams (i.e. $(1 / 2,0),(1,1 / 2)$, etc.), so there are a total of thirtyeight distinct beams in the substrate Brillouin zone instead of only one for the clean surface. The beams in the observed LEED pattern fall on an $8 \times 8$ lattice, so the closest beams (from different domains) are separated by $1 / 8$ of the substrate 
reciprocal lattice vector. The situation is even worse for the $(2 \sqrt{3} \times 5)$ rect LEED pattern. Here there are sixty beams in che substrate Brillouin zone, and none of the beams from the different domains coincide. These beams fall on a $20 \times 20$ lattice.

It was not possible to get useful I-V curves from the room temperature data, or from the $(2 \sqrt{3} \times 5)$ rect structure at either temperature. The best results resembled the earlier attempts of Stair, ${ }^{5}$ i.e. weak J-V peaks superimposec on a large background due to diffuse quasi-elastic scattering, with a very bad overall signalto-noise ratio.

It was possible to resolve useful I-V curves fiom the $(2 \sqrt{3} \times 4)$ rect LEED patterns recorded at $150 \mathrm{~K}$. This was possible because of the improved image signal-to-noise ratio of the LEED video data system (chapter 5) due to video image integration and hardware background subtraction, and because of the improved algorithms for local quasi-elastic background subtraction (chapter 7). For the most closely spaced LEED beams in the $(2 \sqrt{\therefore} \times 4)$ rect pattern, separated by only $0.052 \AA^{-1}$, the overlap of the single-phonon scattering "halo" of the stronger beam with the weaker beam can make a significant contribution to the measured intensity (see chapter 2). The median-hackground approach was much better at correcting for this overlap than the local-average approach used by Stair. 20 


\subsubsection{Data acquisition}

Only the normal incidence data were used in the LEED structure calculations for the $(2 \sqrt{3} \times 4)$ rect structure. The large number of diffraction beams gives a large data set using only one angle of incidence. Also the LEED calculations are complex even at normal uncidence. At off-normal incidence calculations must be made for each of the three rotated domains, and the advantage of symmetry is reduced.

The LEED data for benzene on $\mathrm{Pt}(111)$ were collected before the role of carbon monoxide coadsorption was understood. To form the $(2 \sqrt{3} \times 4)$ rect LEED pattern, the crystal was cleaned and then flashed to $550 \mathrm{~K}$. As soon as the crystal had cooled to $330 \mathrm{~K}$ the crystal was exposed to benzene vapor at $5 \cdot 10^{-7}$ torr for four minutes, then the benzene pressure was increased to $1 \cdot 10^{-6}$ torr for the next eight minutes, for a total exposure of $600 \mathrm{~L}$. After the benzene leak valve was closed forty minutes was required for the background pressure to drop to $2.2 \cdot 10^{-9}$ torr. (The erystal cooling could not be operated during these large exposures since multi-layers of benzene would condense on all low-temperature surfaces.) Another thirty minutes elapsed while the crystal cooled to $150 \mathrm{~K}$ where the video data were recorded.

LEED I-V curves were generated for the $(2 \sqrt{3} \times 4)$ rect pattern between 10 and $150 \mathrm{eV}$. I-V curves were initially generated for a total of seventy-five beams. Some of these I-V eurves had to be discarded because the signal-to-noise ratic as too low or because there were too many gaps where the intensity dropped below the detection threshold. After the remaining symmetry-equivalent beams from 
two independent data sets were averaged together, there were a total of three independent substrate beams and ten superlattice beams for a cumulative energy range of $978 \mathrm{eV}$ (see figure 11.8). The I-V curves obtained include: $(1.0),(0,1)$, and $(1,1)$ for the substrate beams, and $(-3 / 8,1 / 4),(3 / 8,-1 / 4),(-1 / 2,1 / 2),(1 / 2,-$ $1 / 2),(1,-1 / 2),(3 / 8,3 / 4),(-1 / 8,-3 / 4),(1 / 4,-1),(-5 / 8,-1 / 4)$ and $(-1,-1 / 4)$ for the superlattice beams.

TDS experiments were performed for the ordered benzene overlayers after the LEED data were recorded. The $\mathrm{H}_{2}$ TDS data were very similar to that reported by $\mathrm{Tsai}^{8}{ }^{8}$ but the benzene ( $78 \mathrm{AMU}$ ) TDS results were different for disordered, $(2 \sqrt{3} \times 4)$ rect and $(2 \sqrt{3} \times 5)$ rect benzene. These spectra are shown in figure 11.9. The relative amount of intact benzene in the $390 \mathrm{~K}$ peak increases from disorder $\rightarrow(2 \sqrt{3} \times 4)$ rect $\rightarrow(2 \sqrt{3} \times 5)$ rect, and the peaks are better resolved, while the total amount of benzene desorbed decreases. It is not clear if different sites contribute to the lower temperature peak, or if some collective adsorbateadsorbate interaction accounts for the changes.

\subsection{Structural models}

The benzene-carbon monoxide coadsorption structures on $\operatorname{Pt}(111)$ and $\operatorname{Rh}(111)$ are some of the most complex structures ever analyzed by LEED structure calculations. The $(2 \sqrt{3} \times 4)$ rect unit cell on $\operatorname{Pt}(111)$ covers sixteen metal atums and includes twelve carbon atoms in the benzene molecules and (probably) four more carbon atoms and four oxygen atoms in the carbon monoxide molecules. To make this calculation possible a number of approximations were 
made in the LEED calculations to sereen possible structures, and then increasingly accurate calculational schemes were used as the structure search converged on a solution.

\subsubsection{Model constraints}

A number of assumptions were made about the structure of the $(2 \sqrt{3} \times 4)$ rect phase on benzene and carbon monoxide on $\mathrm{Pt}(111)$ to restrict the range of the structural starch. First the $\mathrm{Pt}(111)$ substrate was assumed to have the terminated bulk geometry, since this has been found in a range of previous investigations of the $\mathrm{Pt}(111)$ surface with and without adsorbates (see chapter 10). Second the benzene molecules were assumed to be intact and to lie with the carbon ring parallel to the surface, consistent with the HREELS, TDS, UPS and NEXAFE results discussed above. Third, the glide symmetry inferred from the systematically missing spots in the $(2 \sqrt{3} \times 4)$ rect LEED pattern was assumed. This means that each pair of molecules in the unit cell have adsorption sites related by the glide symmetry. Finally, both the benzene and the carbon monoxide molecules were assumed to be adsorbed in high-symmetry sites, i.e. top, bridge or hollow sites.

Plausible structures were selected by considering the Van der Waals radii of the adsorbed molecules. Adsorption geometries with a substantial overlap of the Van der Waals radii of adjacent molecules are unlikely. Based on the size of the unit cell and the benzene molecules only models with two benzenes per cell were considered. Any model with four flat-lying benzene molecules would involve a 
second benzene layer, and this was ruled out by the HREELS results of Lehwald et al. ${ }^{6}$ which showed a substantial change in the HREEL spectrum with multileyer benzene adsorption. LEED calculations were carried out for some model geometries where the Van der Waals radii of adjacent molecules did overlap.

\subsubsection{Internal degrees of freedom}

Even with the constraints discussed above there are still many possible model adsorption geometries. The benzene molecules may adsorb on top, fec-hollow, hep-hollow or three different bridge sites. The distance $d_{\perp M-C}$ between the toplayer metal atoms and the carbon ring is a structural parameter to be determined. In addition the molecule has internal structure.

The internal structure of the adsorbed benzene molecules was assumed to have the same symmetry as the adsorption site, i.e. molecules adsorbed in top or hollow sites were assumed to have $C_{3 v}\left(\sigma_{d}\right)$ symmetry, and molecules in bridge sites to have $C_{2 v}$ symmetry (or $C_{s}$ symmetry when the stacking of the deeper metal layers in considered). The benzene molecule can rotate around an axis perpendicular to the surface through the center of the molecule - this is defined to be the azimuthal angle $\phi$. There are only three values of $\phi$ consistent with the local site symmetry: $\phi=0^{\circ}$, where a line connecting adjacent sites (i.e. parallel to the $[1 \overline{1} 0]$ direction) intersects two carbon atoms of the benzene ring (figure $11.10(\mathrm{~b})), \phi=30^{\circ}$, where the same line bisects a carbon-carbon bond in the benzene ring (figure $11.10(a)$ ), and a random ("spinning") orientation. 
Different distortions of the benzene carbon ring relative to gas-phase benzene are possible depending on the adsorption site. An overall expansion or contraction of the ring is possible in any site. In a 3-fold site the Kekulé distortion (figure 11.10(e)) is possible for the $\phi=0^{\circ}$ orientation, with alternate long and short carbon-carbon bonds. At this angle no out-of-plane distortion is possible. In three-fold sites with $\phi=30^{\circ}$ an out of plane buckling, where alternate carbon atoms move up and down is possible, as in the cyclohexane $\left(\mathrm{C}_{6} \mathrm{H}_{12}\right)$ case where the carbon atoms adopt tetrahedral $s p^{3}$-bond angles instead of planar $s p^{2}$-bond angles.

In a two-fold bridge site with $\phi=30^{\circ}$ the allowed in-plane distortion is a change in the length of the two carbon-carbon bonds parallel to the bridge axis relative to the other four carbon-carbon bonds. The only out-of-plane distortion consistent with $C_{2 v}$ symmetry is the "boat" shape were the carbon atoms along the bridge axis move up or down. (The "chair" shape is consistent with $C_{8}$ symmetry, where the two end carbon atoms move in opposite directions.) At the $\phi=$ $0^{\circ}$ orientation the same kind of distortions are allowed, with the benzene molecule rotated by $90^{\circ}$, except that the "chair" shape out-of-plane distortion is not allowed.

So far the carbon monoxide molecules have not been considered. The adsorption site and number (two or four) of these molecules add additional possible models, along with the two additional geometrical parameters, the metalcarbon $\left(d_{\perp M-C}\right)$ and carbon-oxygen $\left(d_{\perp C-O}\right)$ bond lengths. The carbon monoxide molecules are assumed to bond carbon-atom down and perpendicular to the metal 
surface.

\subsection{LEED Theory}

The vast range of passible structures for the $(2 \sqrt{3} \times 4)$ rect phase on benzene and carbon monoxide coadsorbed on $\mathrm{Pt}(111)$ was analyzed with a range of theoretical methods. Calculationally efficient schemes were used to survey the range of possible structures, and then the more promising structures were treated with more exact aric more complex calculations.

The overlayer was treated in the CSM (spherical-wave) scheme, with the combined effects of the benzene and carbon monoxide molecules described by a single overlayer scattering matrix. Multiple scattering within the metal substrate was treated exactly: the individual metal layer diffraction matrices were calculated by matrix inversion and combined using RFS. The beam-set neglect (BSN) approximation $^{21}$ was used to combine overlayer and substrate scattering in the RFS scheme, so certain high-order multiple-scattering events between the overlayer and substrate were neglected.

The non-structural parameters used in the benzene calculations were the same as in the carbon monoxide calculations described in chapter 10. The effect of hydrogen was neglected, as has been done in other hydrocarbon structure calculations. 2,3 Phase shifts up to $l_{\max }=4$ were used, and the $r_{5}$ average was used for the $\mathbf{r}$-factor calculations. 


\subsubsection{Overlayer calculations}

Various approximation schemes for overlayer calculations involving hypothetical benzene-like molecules have been analyzed by Van Hove. ${ }^{22}$ Because of the large unit celi, and because the hydrogen atoms serve to keep adjacent molecules well separated, approximations to intra-layer multiple scattering are appropriate for the benzene-carbon monoxide overlayer. The final calculations were believed to be accurate on the order of $\pm 0.10 \AA$, depending on which struetural parameter is considered.

The most extreme approximation for overlayer scattering is the kinematic approximation, where intra-layer scattering is neglected completely. This rough approximation was used to survey rapidly possible geometries. Within the framework of the renormalized scattering perturbation (RSP) the kinematic sublayer addition (KSLA) approximation is useful. In this approximation intra-layer scattering between equivalent atoms in different unit-cells is neglected, while scattering between inequivalent atoms is included to all orders. This approximation can be modified so that only some multiple scattering events are included, sucb as multiple scattering by nearest neighbors, or only within molecules. Better accuracy was obtained when the complete RSP approximation was applied with all intra-layer scattering included, and for the most accurate calculations the individual molecules were treated with matrix inversion, and then the molecular scattering matrices were combined. 


\subsubsection{Glide symmetry theory}

Some modifications to the theory are needed to treat the overlayer glide symmetry. Normally LEED calculations include only symmetrical combinations of wave-functions at symmetrical angles of incidence. For glide symmetries either symmetrical or anti-symmetrical combinations of ware-functions are used, depending on which diffraction beam is being considered. These modifications to the formalism are discussed in Van Hove et al. ${ }^{23}$

\subsection{Structural search}

The LEED structural search for the $(2 \sqrt{3} \times 4)$ rect phase on $\mathrm{Pt}(111)$ drew on results previously obtained for the $c(2 \sqrt{3} \times 4)$ rect phase or benzene and carbon monoxide coadsorbed on $\mathrm{Rh}(111),{ }^{21,24}$ and on a parallel LEED structure determination analysis of the (3x3) structure of benzene and carbon monoxide coadsorbed on $\mathrm{Rh}(111) \cdot{ }^{25}$ Some possible models were explored in detail in the rhodium calculations and were found to be unfavorable, and so they were not treated in detail in the platinum calculations.

The range of structures considered at the different levels of approxination are summarized in Table 11.1. First calculations for benzene only (without carbon monoxide) tested the different adsorption sites and azimuthal orientations. Bridge site adsorption at $\phi=30^{\circ}$ was preferred, the structure that had the best fit based on Van der Waals considerations. Next in the kinematic approximation carbon monoxide was added in different sites, with benzene in bridge sites, while the metal-carion bond-lengths were varied. Out-of-plane "boat" distortions were 
considered in this approximation. Finally, detailed calculations were made to dei,iermine the benzene ring distortions for benzene and carbon monoxide in bridge sites with four carbon monoxide molecules per unit cell. Altogether, 750 different structures were tested for the $(2 \sqrt{3} \times 4)$ rect structure on $\operatorname{Pt}(111)$.

A similar range of structures was tested for the (3x3) structure on $\mathrm{Rh}(111)$, except that for this structure coadsorbed carbon atoms, and coadsorbed $\mathrm{C}_{2}$ perpendicular to the surface (like the ethylidyne skeleton) were tested in addition to coadsorbed carbon monoxide. The "spinning" benzene orientation was tested on $\mathrm{Rh}(111)$, along with a more extensive range of out-of-plane distortions. These more exotic structures did not show any signs of improving the agreement between theory and experiment. More than 1600 different configurations were tested for the $\operatorname{Rh}(111)-(3 \times 3)$ phase.

\subsubsection{R-factor results}

Benzene adsorbed in bridge sites with $\phi=30^{\circ}$ was clearly favored in the $\mathrm{r}$ factor calculations. The two types of bridge sites (differing in the registry of deeper metal layers) had $r_{5}$ minima of 0.316 and 0.327 in the approximation used, and this difference is insufficient to distinguish between them. The carbon ring was found to be $2.10 \pm 0.10 \AA$ above the metal surface, so all of the metal-carbon bonds are $2.25 \AA$ within $\pm 0.10 \AA$.

Out-of-plane distortions of the benzene molecules substantially worsened the r-factor fit, from $\sim 0.28$ to $\sim 0.38$ for up or down distortions. Similar results were found for the $\mathrm{Rh}(111)$ structures. There was a definite in-plane distortion, 
with the radii (relative to the center of the molecule) $1.79 \pm 0.15$ and $1.72 \pm 0.15 \AA$ for the two end and four side carbons, respectively, and the azimuthal distortion angle $\beta=1.5 \pm 2.5^{\circ}$ (see figure 11.10(d)). This leads to carbon-carbon bondlengths of $1.65 \pm 0.15 \AA$ for the two shorter carbon-carbon bonds (parallel to the bridge) and $1.76 \pm 0.15$ for the four longer bonds. The shorter bonds lie over single metal atoms, while the longer bonds bridge over pairs of metal atoms.

There is less certainty about the location of the carbon monoxide molecules. The best r-factors for two bridge or hollow site carbon monoxide molecules were 0.315 and 0.321 , while the best r-factor for four bridge-site carbon monoxide molecules was 0.316. This is not a large enough difference to distinguish on the basis of the r-factors, however all of the carbon monoxide geometries had the same optimum metal-carbon spacing of $1.45 \pm 0.10 \AA$, which gives more reasonable bond lengths for bridge site adsorption. Bridge sites for the carbon monoxide molecules also give the best fit in terms of the Van der Waals radii. Based on the thermal desorption results of Mate and in figure 11.3 four carbon monoxide molecules per cell was chosen as the optimum geometry.

The final structure is shown in figure 11.12. This structure had r-factors of $r_{5}=0.28, r_{Z-J}=0.42$ and $r_{P e n d r y}=0.54$. This can be compared to the optimum results for the $c(2 \sqrt{3} \times 4)$ rect phase on $\operatorname{Rh}(111),{ }^{24}$ with $r$-factors of $r_{5}=0.31, r_{Z-J}$ $=0.40$ and $r p_{e n d r y}=0.56$, and $r$-factors of $r_{5}=0.21, r_{Z-J}=0.24$ and $r_{P e n d r y}=$ 0.41 for the (3x3) phase on $\operatorname{Rh}(111) .25$ The LEED structure for $\operatorname{Rh}(111)$. $c(2 \sqrt{3} \times 4)$ rect is shown in figure 11.13 , and for $\operatorname{Rh}(111)(3 \times 3)$ in figure 11.14 . LEED calculations have not been periormed for the $\operatorname{Rh}(111)-(2 \sqrt{3} \times 3)$ rect 
benzene only structure, but a model suggested by Mate ${ }^{7}$ based on the observed glide symmetry is shown in figure 11.15. Table 11.2 compares the benzene structure for the different ordered structures on $\mathrm{Pt}(111)$ and $\mathrm{Rh}(111)$ with cluster and gas-phase values, and with the carbon-carbon bonds in other molecules adsorbed on metal surfaces.

\subsection{Discussion}

Benzene has been shown to order on $\mathrm{Pt}(111)$ only when coadsorbed with carbon monoxide. This fact explains the difficulty in reproducing the ordered benzene structures. The apparent discrepancy in the conditions for ordering and for transformation from the $(2 \sqrt{3} \times 4)$ rect phase to the $(2 \sqrt{3} \times 5)$ rect phase are now explained -- ordering was controlled by the amount of carbon monoxide contamination present in the vacuin chamber. Carbon monoxide coadsorption can also explain the work function changes observed by Gland. The initial decrease in the work function was caused by benzene adsorption, and then as carbon monoxide coadsorbed the work function increased toward the original clean $\mathrm{Pt}(111)$ value. An analogous effect was observed by Kudo et al. ${ }^{10}$ in a UPS study of carbon monoxide and potassium coadsorbed with benzene. When carbon monoxide is adsorbud on clean Pt(111) they found the work function decreased by $\sim 0.3 \mathrm{eV}$. When 0.3 monolayers of potassium was adsorbed the work function decreased substantially, by more than $3 \mathrm{eV}$, and when carbon monoxide was coadsorbed with the potassium the work function now increased by close to $1 \mathrm{eV}$. 
The structure of the $(2 \sqrt{3} \times 4)$ rect phase of carbon monoxide and benzene adsorbed on $\mathrm{Pt}(111)$ has been determined. Benzene is found to be adsorbed on bridge sites with an expansion of the carbon ring relative to the gas phase. There is a possible discrepancy between the LEED results and the HREELS data. The HREEL spectra shows peaks at 1700 and $1800 \mathrm{~cm}^{-1}$, but the LEED structure puts both carbon monoxide molecules on bridge sites. There is not room in the unit cell to fit four carbon monoxide molecules unless they are on bridge sites, and the glide symmetry requires paired molecules for each type of site. One bridge-site carbon monoxide is in contact with a single benzene molecule, and the other carbon monoxide is in contact with three benzenes. This may be sufficient to cause a $100 \mathrm{~cm}^{-1}$ shift, since there is already a significant shift from the 1850 $\mathrm{cm}^{-1}$ frequency for bridge site carbon monoxide on $\operatorname{Pt}(111)$ without benzene, or there may be some other explanation. Only one carbon monoxide lass peak is seen for the ordered carbon monoxide-benzene structures on $\operatorname{Rh}(111)$, but the models also predict that all of the carbon monoxide molecules are in equivalent sites.

The third carbon monoxide loss peak at $2050 \mathrm{~cm}^{-1}$ is probably not related to the ordered benzene structure, since this loss only appeared when the $\mathrm{Pt}(111)$ surface was cooled to $70 \mathrm{~K}$. It is probably due to adsorption of residual carbon monoxide as the crystal cooled.

Some trends are apparent for benzene adsorption on $\mathrm{Pt}(111)$ and $\mathrm{Rh}(111)$. First, that benzene alone adsorbs on bridge sites. This is based on the observation of glide plane symmetries for the two ordired phases on $\mathrm{Pt}(111)$ and for the 
carbon monoxide free phase on $\mathrm{Rh}(111)$. Secondly, the benzene ring tends to expand on adsorption, so the mean carbon-carbon bond-length is $1.58 \pm 0.15$, $1.51 \pm 0.15$ and $1.72 \pm 0.15 \AA$ for the $\operatorname{Rh}(111)-c(2 \sqrt{3} \times 4)$ rect, $\operatorname{Rh}(111)-(3 \times 3)$ and $\operatorname{Pt}(111)-(2 \sqrt{3} \times 4)$ rect structures, respectively. On $\operatorname{Rh}(111)$ carbon monoxide coadsorption shifts the benzene molecules to hollow sites. On all three surfaces the molecule shows in-plane distortions of the local site symmetry, while there is no evidence for out-of-plane distorîions. Similar distortions have been found for some benzene organometallic complexes, ${ }^{24}$ and some Extended Hückel calculations also indicate benzene ring expansion an a possible Kekulé distortion for cluster models. ${ }^{26}$ The strong asymmetric distortion of the benzene ring found for the $\operatorname{Rh}(111)-c(2 \sqrt{3} \times 4)$ rect structure may be a hint toward the different catalytic behavior of $\mathrm{Rh}(111)$ and $\mathrm{Pt}(111)$, since benzene is more likely to decompose on the former under catalytic conditions.

The benzene carbon monoxide metal system is quite complicated, and this work certainly does not represent the "last word" on the structure of aromatic molecules on metal surfaces. Hopefully future studies will be able to build on these initial results and establish a stronger link between catalytic processes and monolayer surface structures. 


\section{References}

1. G. A. Somorjai, Chemistry in Two Dimensions: Surfaces, Cornell Univereity Press, Ithaca, New York, 1981.

2. L. L. Kesmodel, L. H. Dubois, and G. A. Somorjai, Journal of Chemica! Pliysics, vol. 70, p. $2180,1979$.

3. R. J. Koestner, M. A. Van Hove, and G. A. Somorjai, Surface Science, vol. 121, p. 321, 1982.

4. J. L. Gland and G. A. Somorjai, Surface Science, vol. 38, p. 157, 1973.

5. P. C. Stair and G. A. Somorjai, Journal of Chemical Physics, vol. 6ri, p. $4361,1977$.

6. S. Lehwald, H. Ibach, and J, E. Demuth, Surface Science, vol. 78, p. 577, 1878.

7. C. M. Mate and G. A. Somorjai, Surface Science, vol. 160, p. 542, 1985.

8. M. C. Tsai and E. L. Muetterties, Journal of the American Chemical Society, vol. 104, p. 2534, 1982.

9. E. L. Garfunkel, M. H. Farias, and G. A. Somorjai, Journal of the Arierican Chemical Society, vol. 107, p. 349, 1985.

10. M. Kudo, E. L. Garfunkel, and G. A. Somorjai, Journal of Physical Chemistry, vol. 89, p. 3207, 1985.

11. J. A. Horsley, J. Stöhr, A. P. Hitehcock, D. C. Newbury, A. L. Johnson, and F. Sette, Jotırnal of Chemical Physics, vol. 83, p. 6090, 1985. 
12. J. A. Horsley, J. Stöhr, and R. J. Koestner, Journal of Chemical Physics, vol. 83, p. 3146, 1985.

13. J. P. Bibérian and M. A. Van Hove, Surface Science, vol. 118, p. 443, 1982.

14. R. F. Lin, R. J. Koestner, M. A. Van Hove, and G. A. Somorjai, Surface Science, vol. 134, p. 161, 1983.

15. J. C. Bertolini and J. Rousseau, Surface Science, vol. 89, p. 467, 1979.

16. B. E. Koel, J. E. Crowell, C. M. Mate, and G. A. Somorjai, Journal of Physical Chemistry, vol. 88, p. 1988, 1984.

17. G. D. Waddill and L. L. Kesmodel, Physical Review B, vol. 31, p. 4940, 1985.

18. S. M. Davis, B. E. Gordon, M. Press, and G. A. Somorjai, Journal of Vacuum Science and Technology, vol. 19, p. 231, 1981.

19. G. S. Blackman, C. T. Kao, and G. A. Somorjai, to be published.

20. P. C. Stair, Ph.D. Thesis, Lawrence Berkeley Laboratory Report, LBL-6221, University of California at Berkeley, 1977.

21. M. A. Van Hove, R. F. Lin, and G. A. Somorjai, Physical Review Letters, vol. 51, p. 778, 1983.

22. M. A. Van Hove and G. A. Somorjai, Surface Science, vol. 114, p. 171, 1982.

23. M. A. Van Hove, W. H. Weirberg, and C.-M. Chan, Low Energy Electron Diffaction: Experiment, Theory and Structural Determination., Springer Verlag, Heidelberg, 1986. 
24. M. A. Van Hove, R. F. Lin, and G. A. Somorjai, Journal of the American Chemical Society, vol. 108, p. 2532, 1986.

25. M. A. Van Hove, R. F. Lin, G. A. Blackman, and G. A. Somorjai, Acta Crystallographica, to be published.

26. C. Minot, E. L. Garfunkel, A. Gavezzotti, and M. Simonetta, to be published. 


\section{Figure Captions for Chapter 11}

11.1 LEED pattern of disordered benzene on $\mathrm{Pt}(111)$. The surface was saturated with benzene vapor at $300 \mathrm{~K}$.

11.2 Carbon coverage on the $\mathrm{Pt}(111)$ surface as a function of benzene exposure at room temperature. An exposure of $\sim 2 \mathrm{~L}$ corresponds to saturation coverage of the crystal as measured by AES. No further changes were seen up to $400 \mathrm{~L}$ exposures. The nominal exposure is the product of the uncorrected ion-gauge reading and the exposure time.

11.3 Thermal Desorption spectra recorced after benzene adsorption for 28 AMU (carbon monoxide). For comparison TDS data from the $c(4 \times 2)$ and the diffuse " $(\sqrt{3} \times \sqrt{5}) R 30^{\circ}$ " phases of carbon monoxide on $\mathrm{Pt}(111)$ are stown.

11.4 The $(2 \sqrt{3} \times 4)$ rect LEED pattern produced by benzew: adsorption on $\operatorname{Pt}(111)$.

(a) A photograph of the LEED pattern taken near normal incidence at approximately $60 \mathrm{eV}$.

(b) A schematic diagram of the LEED pattern. The circles, squares and triangles indicate the superlattice beams for the three rotated domains. The open circles indicate spots that are absent because of glide symmetries. (c) The benzene unit cell in real space shown relative to the $\operatorname{Pt}(1110$ surface. The area of the unit cell is sixteen times that of the clean $\operatorname{Pt(111)}$ unit cell. 
11.5 The $(2 \sqrt{3} \times 5)$ reci LEED pattern at approximately $60 \mathrm{eV}$ and near normal incidence.

11.6 HREEL spectra for benzene and for the ordered phases of coadsorbed carbon monoxide and benzene on $\mathrm{Pt}(111)$ recorded at $77 \mathrm{~K}$. At $300 \mathrm{~K}$ the two carbon monoxide loss peaks between 1700 and $1800 \mathrm{~cm}^{-1}$ appeared as one broad peak, and the carbon monoxide loss peak at $\sim 2030 \mathrm{~cm}^{-1}$ was not observed.

11.7 Photograph of the benzene $(2 \sqrt{3} \times 2 \sqrt{3})$ R30 ${ }^{\circ}$ LEED pattern on $\operatorname{Pt}(111)$ at $64 \mathrm{eV}$ and $\theta=19^{\circ}$ taken at $300 \mathrm{~K}$.

11.8 LEED I-V curves for the $(2 \sqrt{3} \times 4)$ rect phase recorded at normal incidence at $150 \mathrm{~K}$. Symmetry equivalent beams have been averaged together.

11.9 Benzeae (78 AMU) TDS results from $\mathrm{Pt(111)}$ for a disordered saturation coverage of benzene, the $(2 \sqrt{3} \times 4)$ rect phase and the $(2 \sqrt{3} \times 5)$ rect phase of coadsorbed carbon monoxide and benzene.

11.10 Possible surface models for the $(2 \sqrt{3} \times 4)$ rect phase on $\mathrm{Pt}(111)$.

(a) and (b) show two possible azimuthal orientations of the benzene molecules. The Van der Waal radii of two benzene and four carbon monoxide molecules are shown in the unit cell. The dashed vertical lines indicated the glide planes.

(c) Possible benzene adsorption sites consistent with the glide symmetry. Shown are the top site $(\mathrm{aABC})$, hcp hollow site (bABC), fec hollow site (cABC) and bridge site (d'ABC + d"ABC). The symbols $d$, d' and d" 
indicate different bridge site orientations. The sites d' and d" differ in the relative position of the second and deeper metal layers, similar to the difference between fec and hcp hollows.

(d) In-plane distortion consistent with local $C_{2 v}$ symmetry. The two carbon-carbon bonds parallel to the bridge bond can have a different length from the other bonds.

(e) In-plane (Kekulé) distortion consistent with local $C_{3 v}\left(\sigma_{d}\right)$ symmetry for $\phi=0^{\circ}$.

11.11 Typical r-factor plots for different pairs of geometrical parameters for the $(2 \sqrt{3} \times 4)$ reet phase on $\operatorname{Pt}(111)$.

11.12 The best structural model from the results of LEED structure determination calcr]ations for the $(2 \sqrt{3} \times 4)$ rect phase on benzene and carbon monoxide coadsorbed on $\mathrm{Pt}(111)$.

11.13 LEED structural result for the $\mathrm{Rh}(111)-\mathrm{c}(2 \sqrt{3} \times 4)$ rect LEED structure with one benzene and one carbon monoxide per unit cell.

11.14 LEED structural result for the $\mathrm{Rh}(111)$ - (3x3) LEED structure with one benzene and two carbon monoxide per unit cell.

11.15 A possible surface model for the $\operatorname{Rh}(111)-(2 \sqrt{3} \times 3)$ rect structure with two benzene molecules per unit cell. 


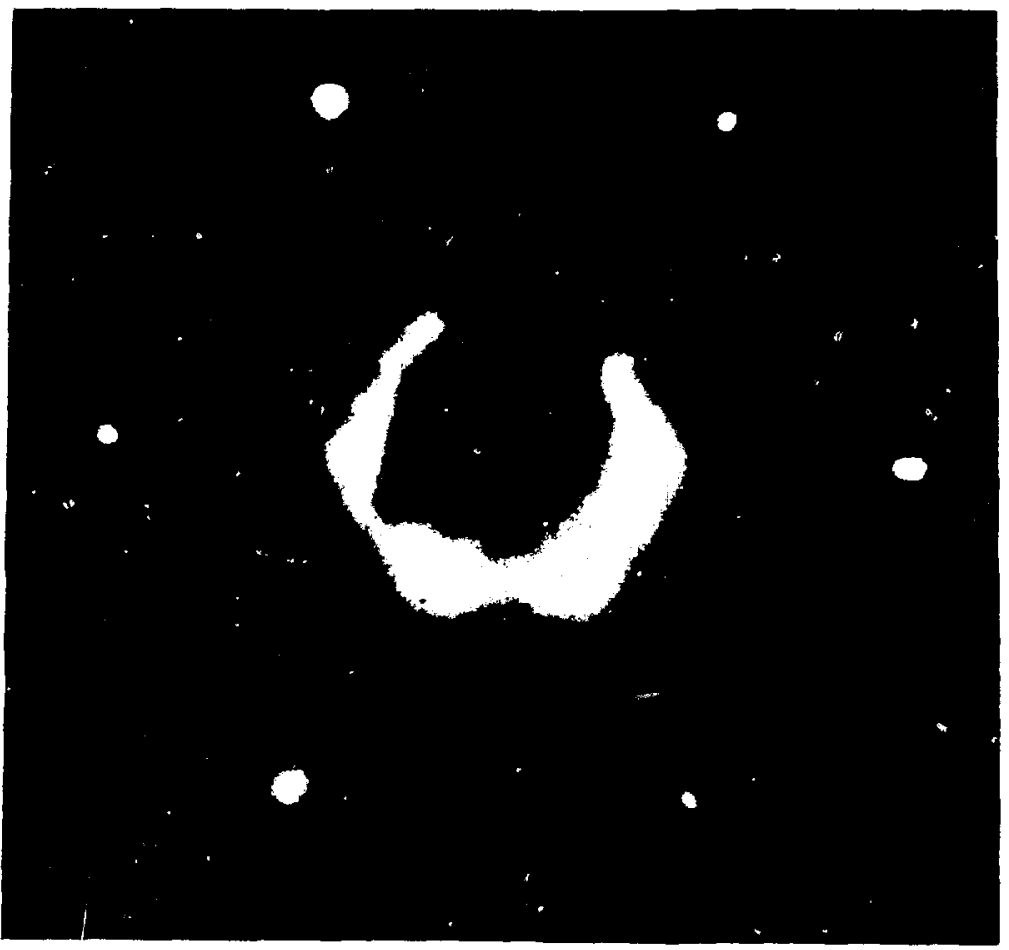

XBB $860-8990$

Disordered Benzene on $\operatorname{Pt}(111)$ $60 \mathrm{eV}$

Find 11.1 
483

\section{Benzene Uptake on Pt(111)}

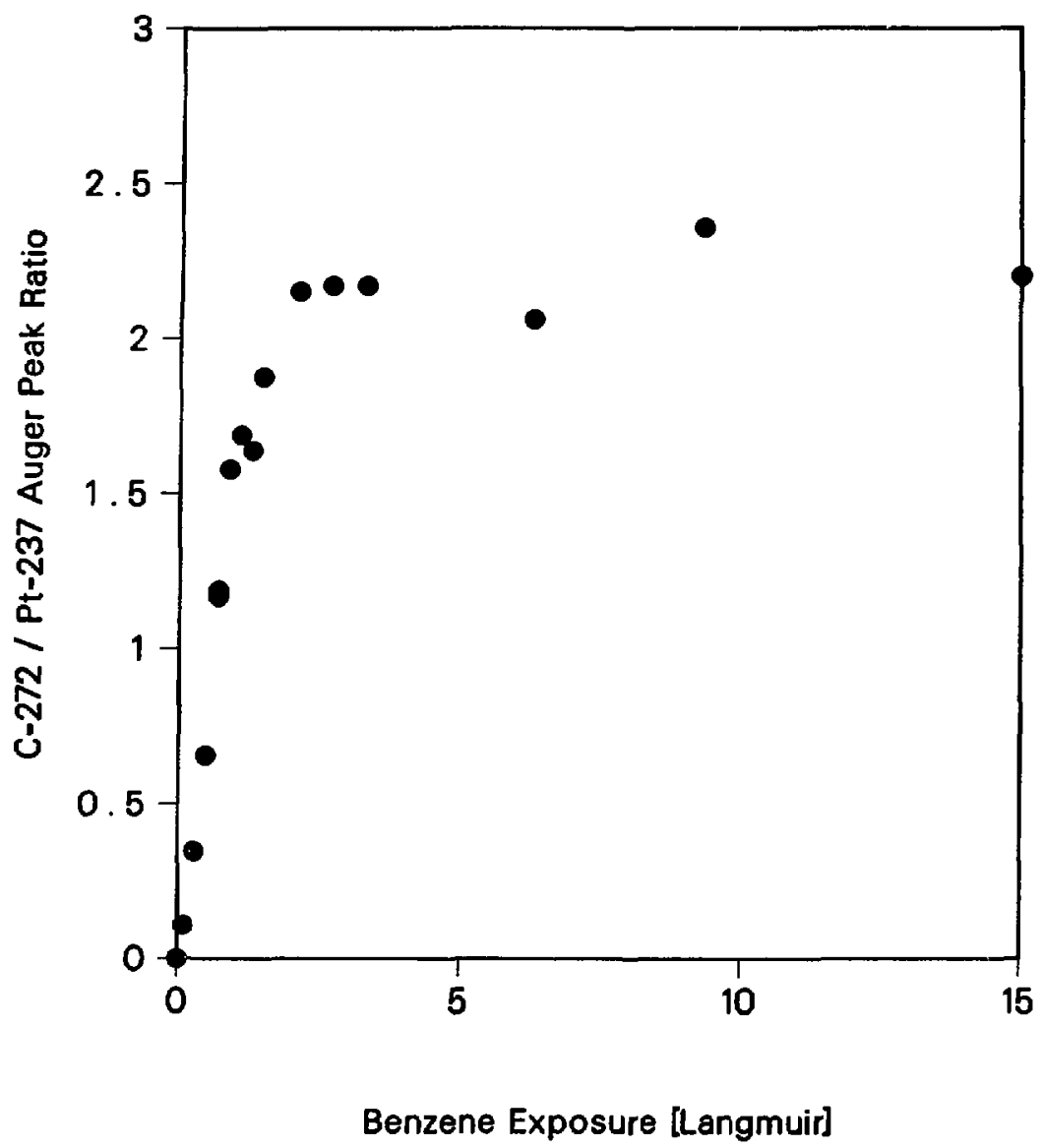

XBL $8611-4247$

Fgre 11.2 


\section{CO Thermal Desorption Data}



Figure 11.3 


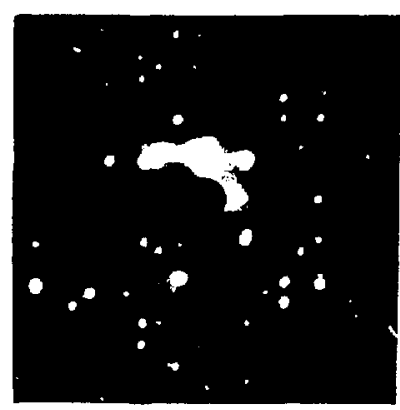

(a)

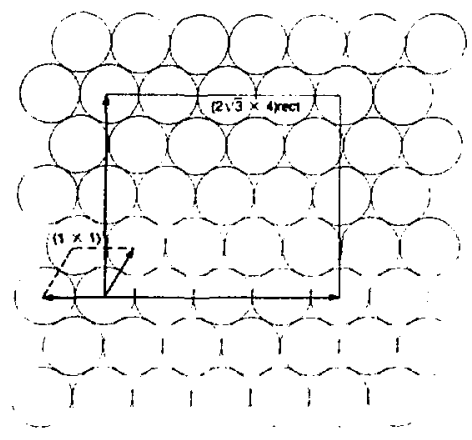

(c)

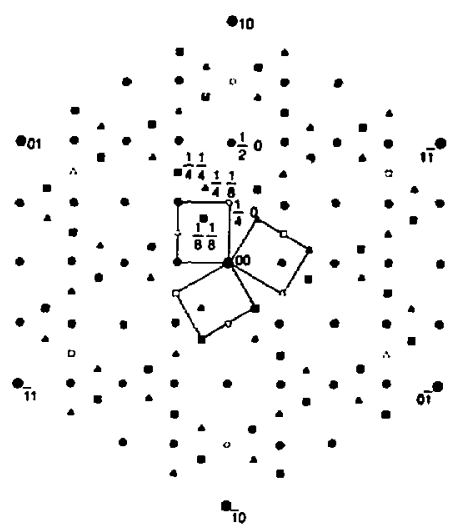

(b)

XBB 864-3004B 


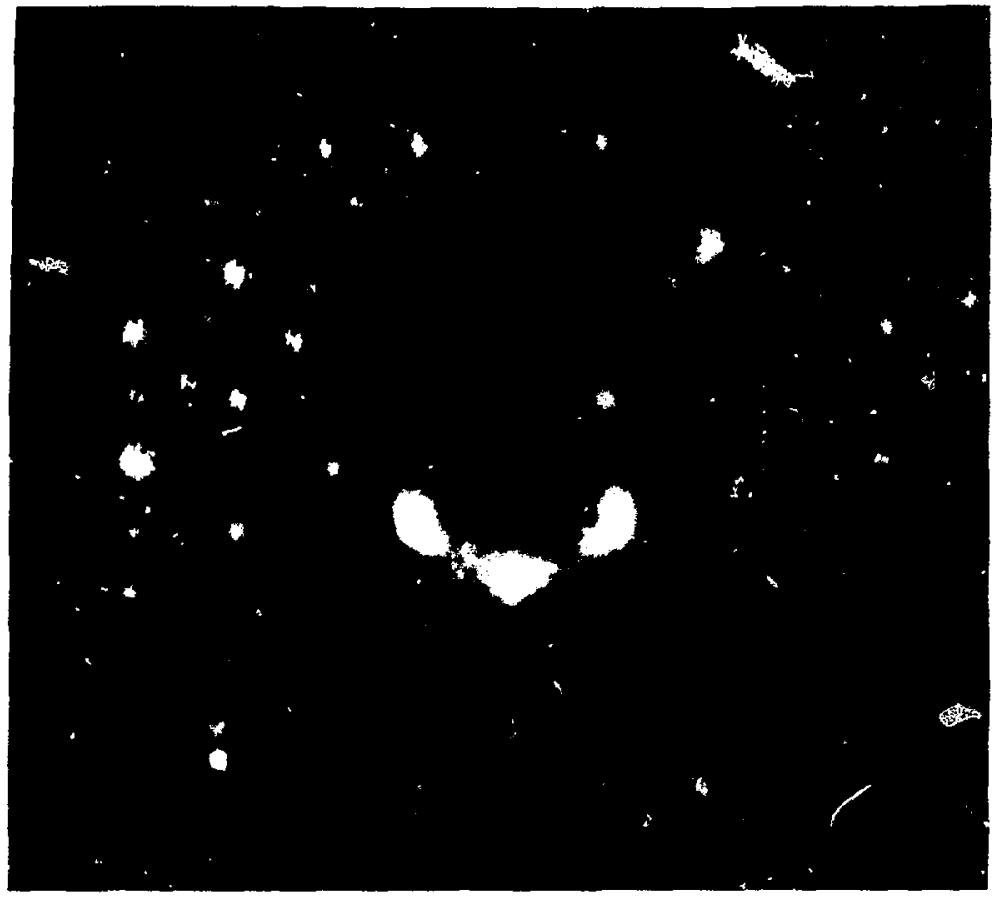

ХВВ $860-8989$

\section{$(2 \sqrt{3} \times 5)$ rect}

Benzene and Carbon Monoxide Coadsorbed on Pt(111)

Fore 11.5 




Fene 11.8 


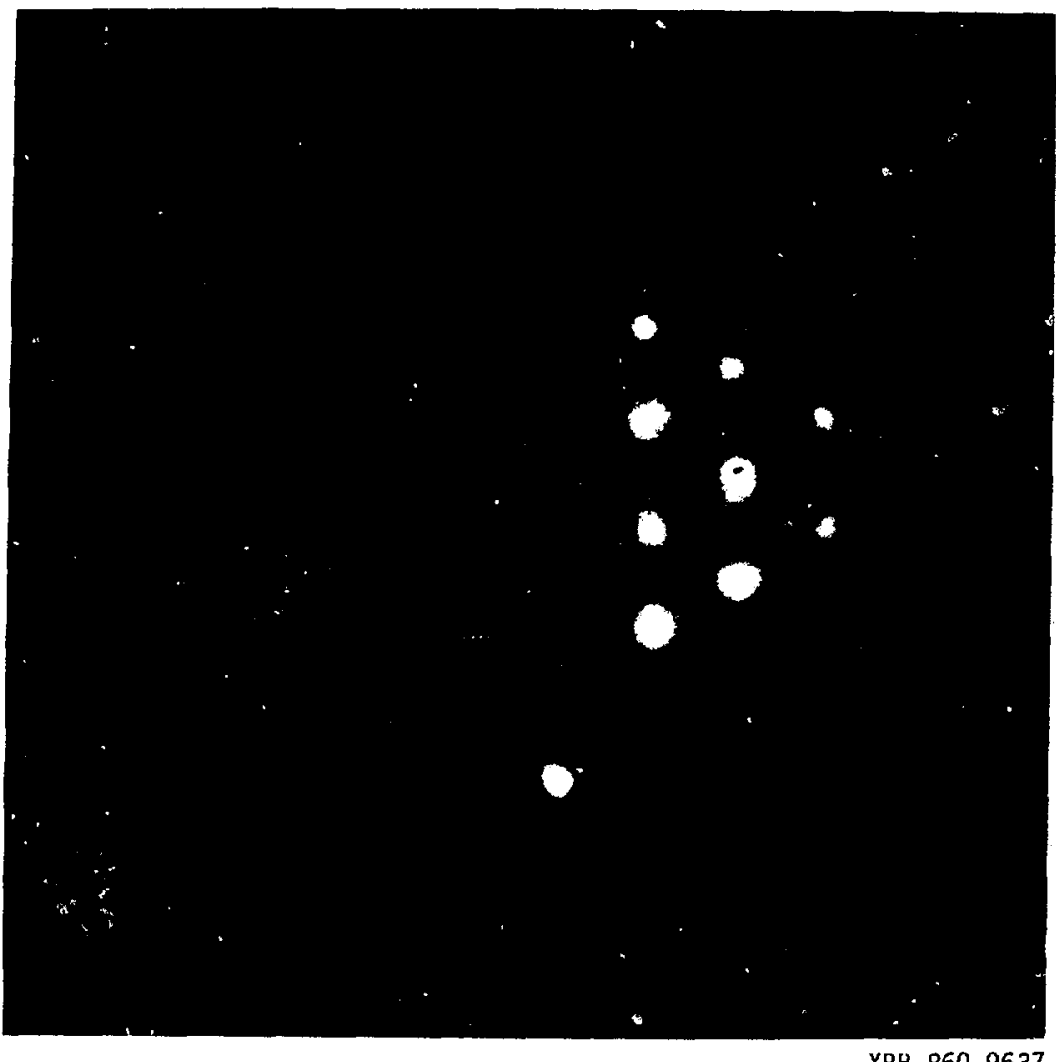

XBB $860-9637$

$(2 \sqrt{3} \times 2 \sqrt{3}) R 30^{\circ}$

Benzene and Carbon Monoxide Coadsorbed on Pt(111) 
489

Coadsorbed $(2 \sqrt{ } 3 \times 4)-$ rect Benzene and $\mathrm{CO}$ on $\mathrm{Pt}(111)$ at $150 \mathrm{~K}$

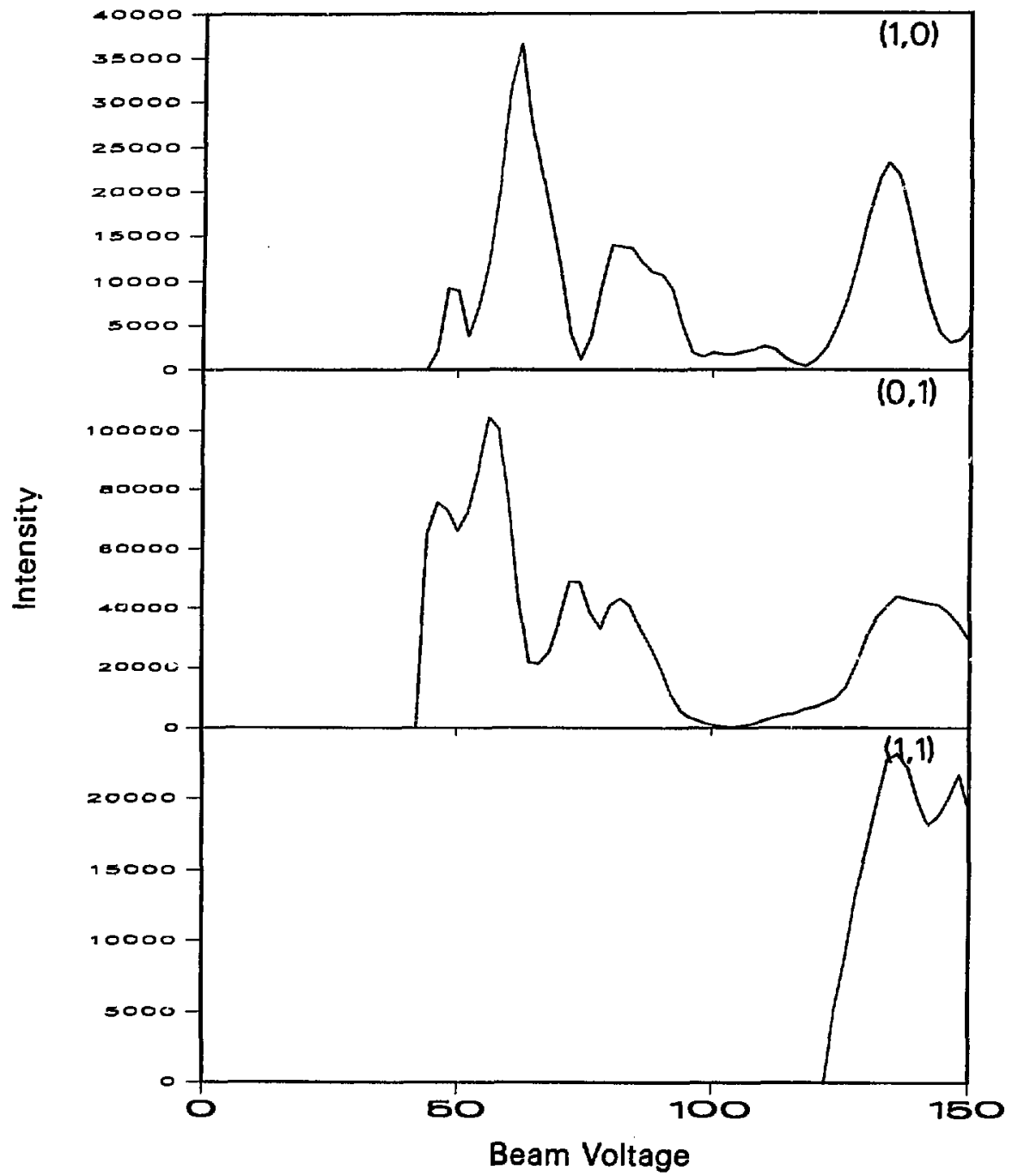

Fare 118f ic 


\section{Coadsorbed $(2 \sqrt{ } 3 \times 4)$-rect Benzene and $\mathrm{CO}$ on $\mathrm{Pt}(111)$ at $150 \mathrm{~K}$}

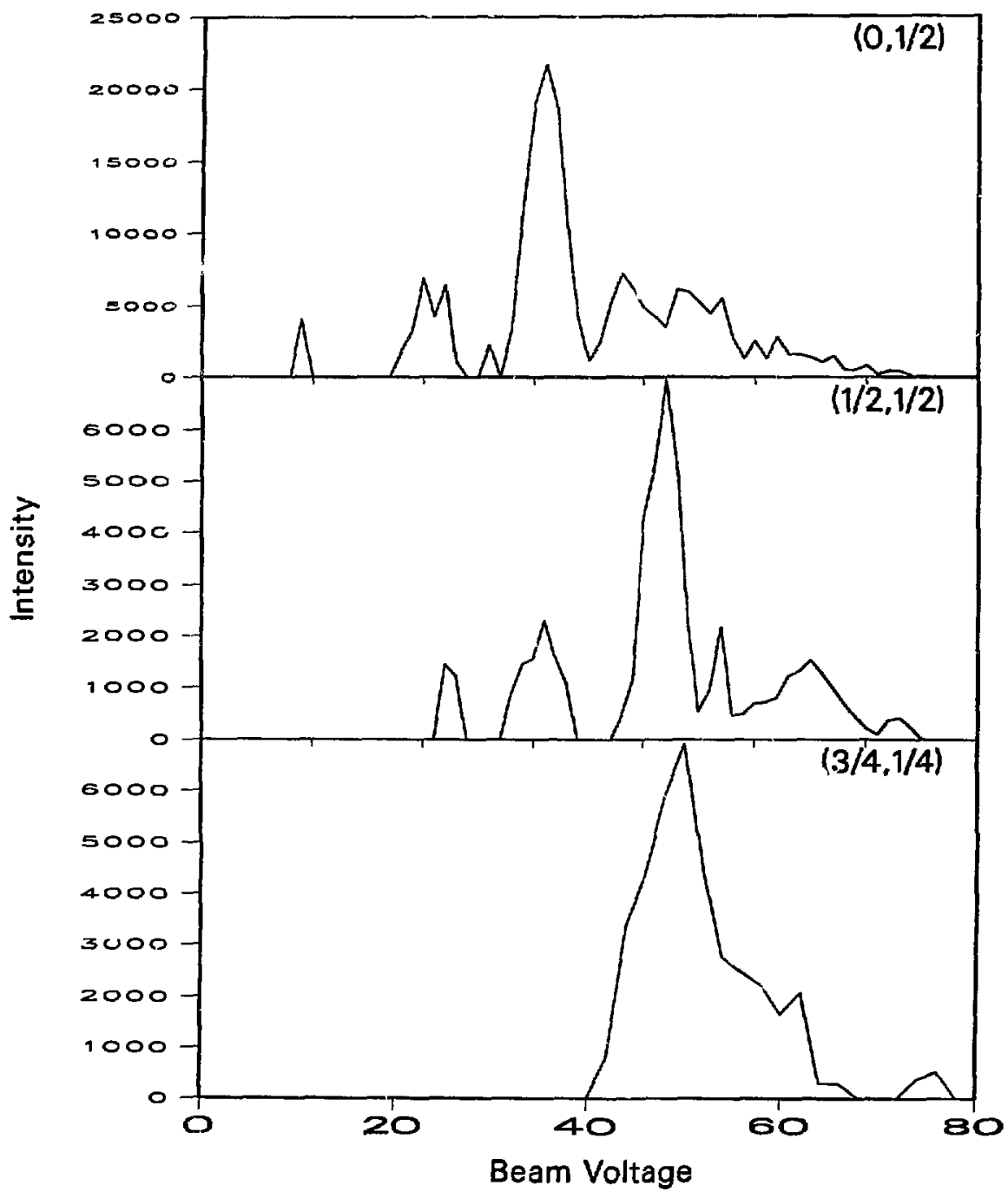




\section{Coadsorbed $(2 \sqrt{ } 3 \times 4)$-rect Benzene and $C O$ on $\mathrm{Pt}(111)$ at $150 \mathrm{~K}$}

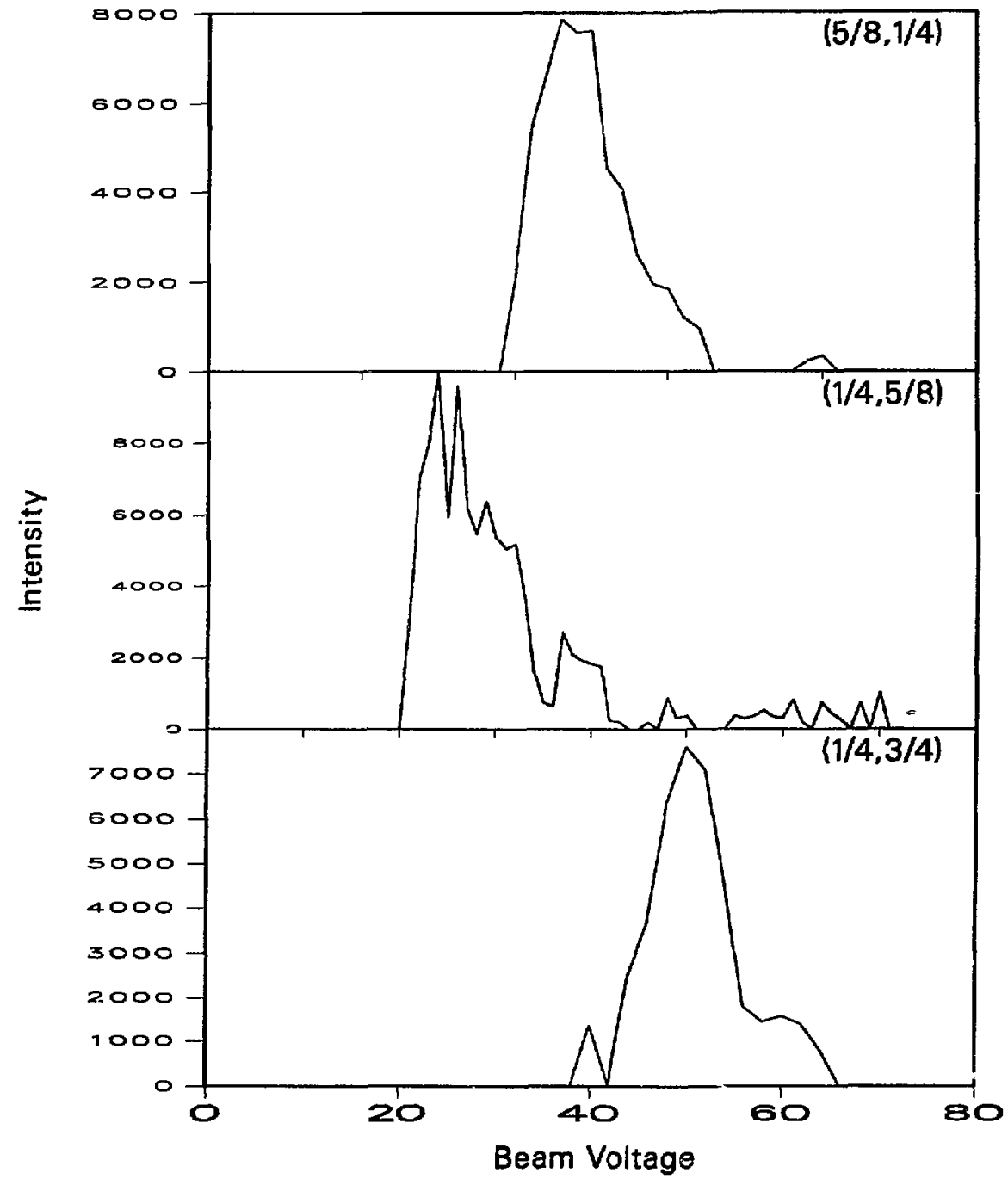

Fgme 11.6') 


\section{Coadsorbed $(2 \sqrt{ } 3 \times 4)-$ rect Benzene and $\mathrm{CO}$ on Pt(111) at $150 \mathrm{~K}$}

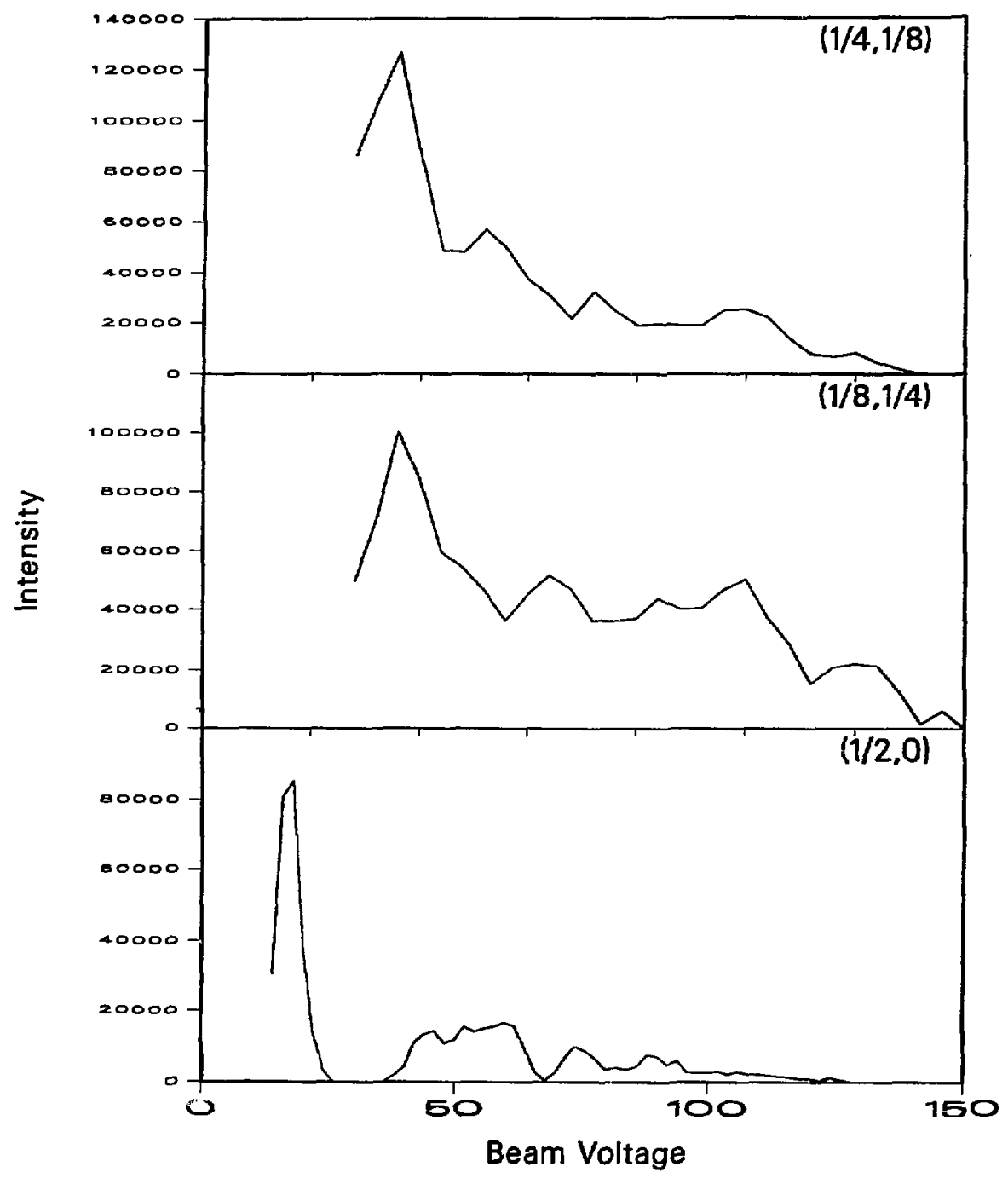

Fine 11.8d 


\section{. \\ Coadsorbed $(2 \sqrt{ } 3 \times 4)$-rect Benzene and $\mathrm{CO}$ on $\mathrm{Pt}(111)$ at $150 \mathrm{~K}$}

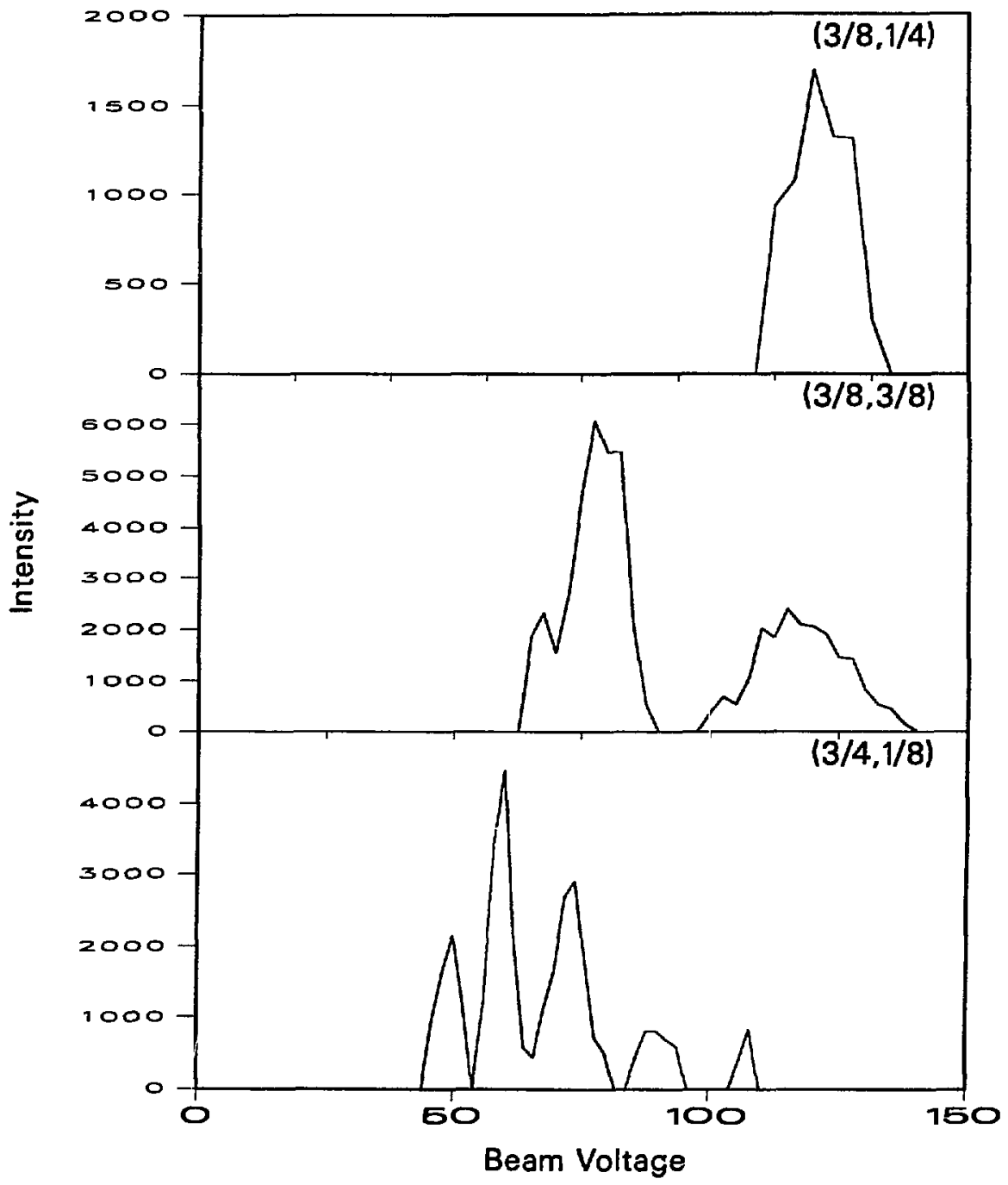

Fin 118(s) 


\section{Benzene Thermal Desorption Data}

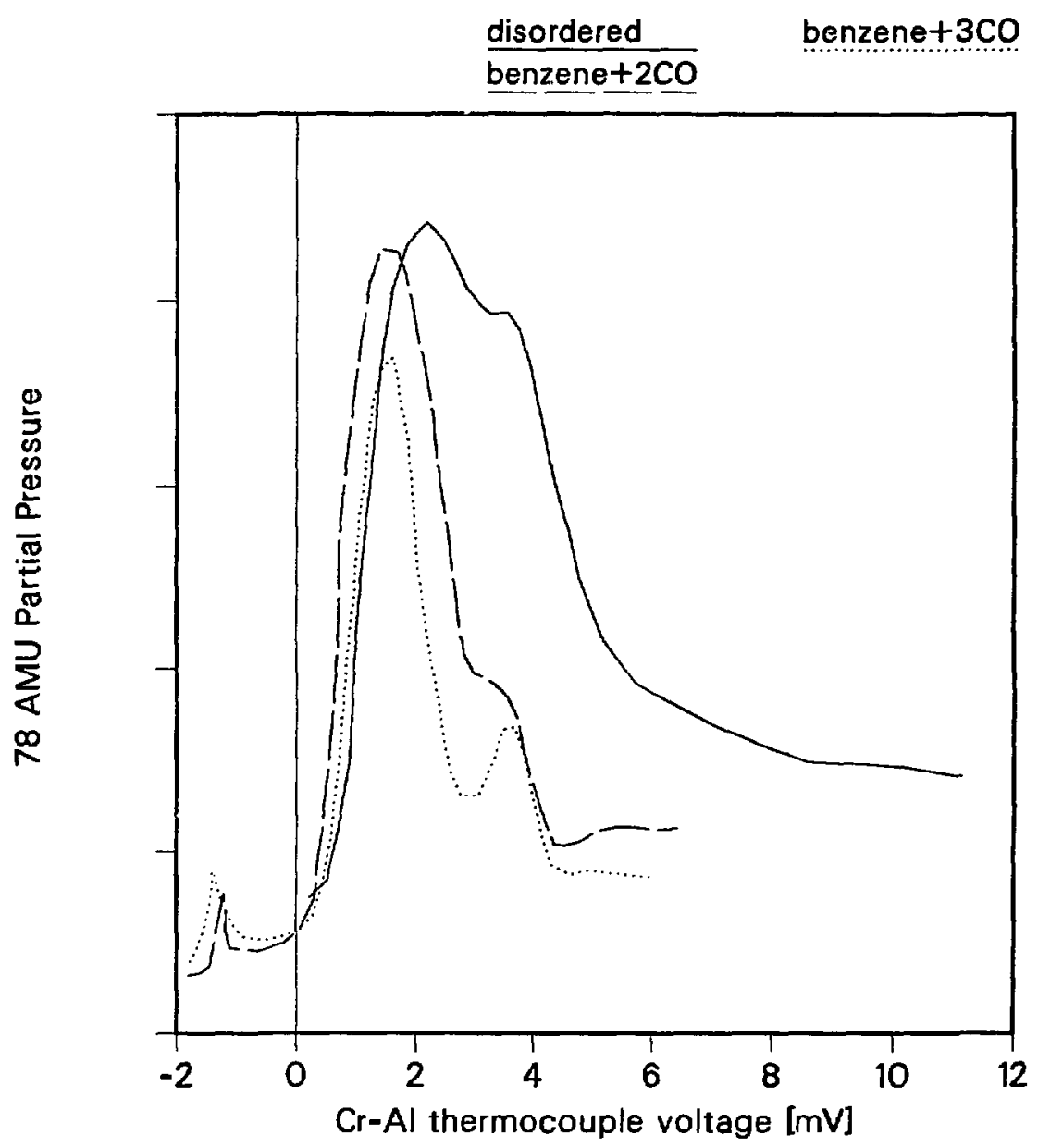

Figure 11.9 

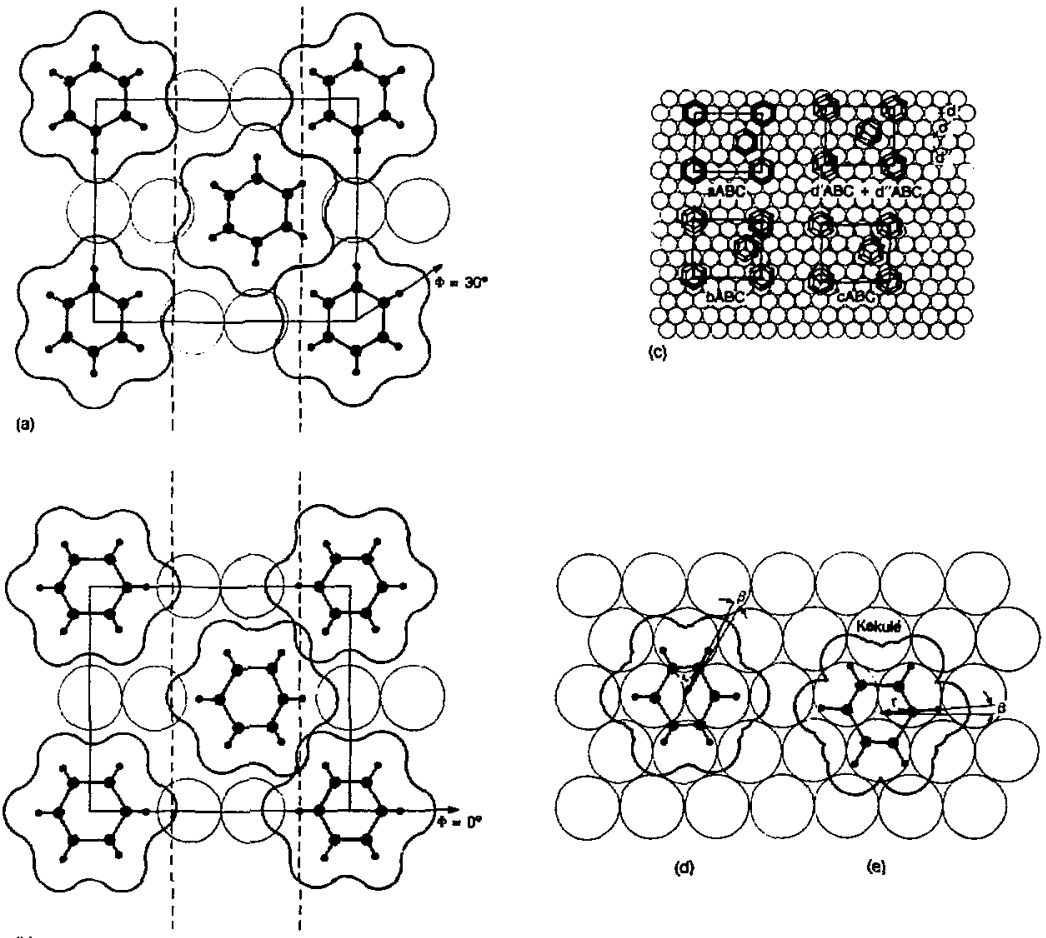

(d)

(e)

XBL $867-2544$

Fgro 11.10 


\section{8}

$$
\begin{gathered}
\mathrm{Pt}(111)-(2 \sqrt{3} \times 4) \text { rect }-2 \mathrm{C}_{6} \mathrm{H}_{6}+4 \mathrm{CO} \\
\text { R-factor contour plots }
\end{gathered}
$$
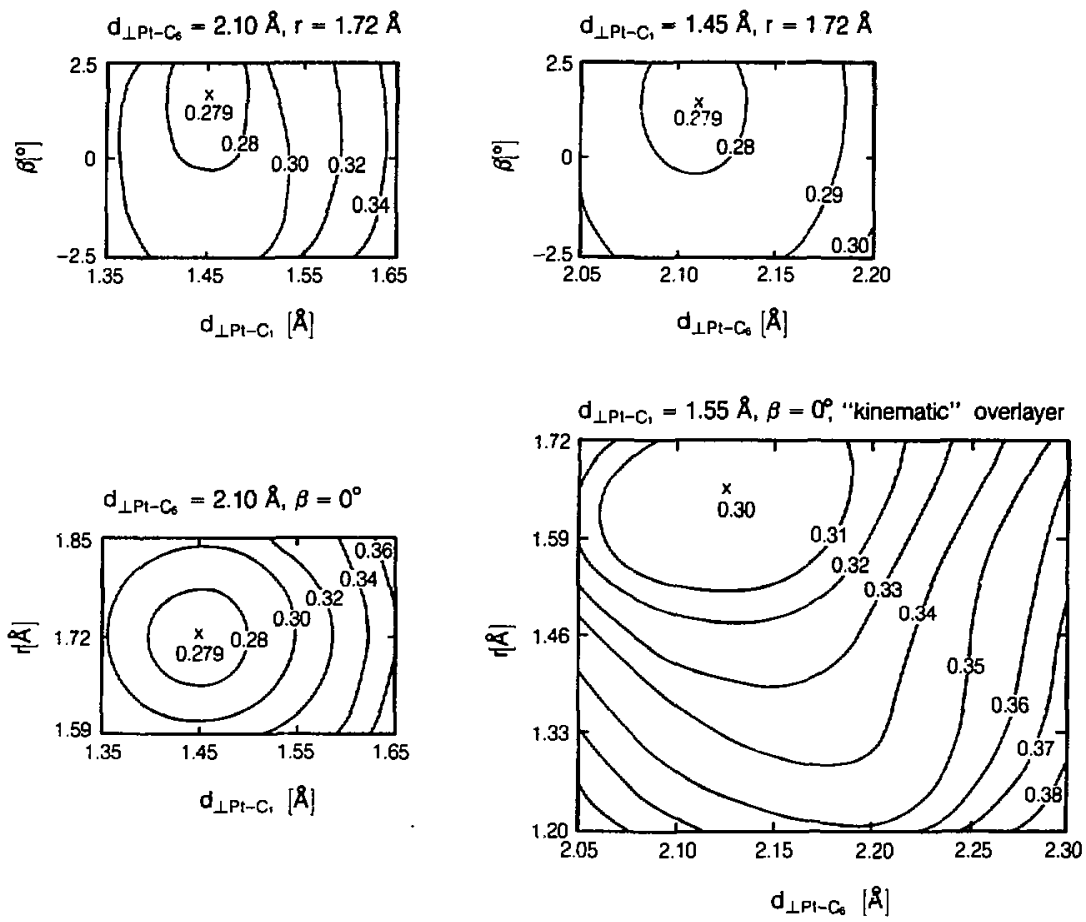

Fgre 11.11 

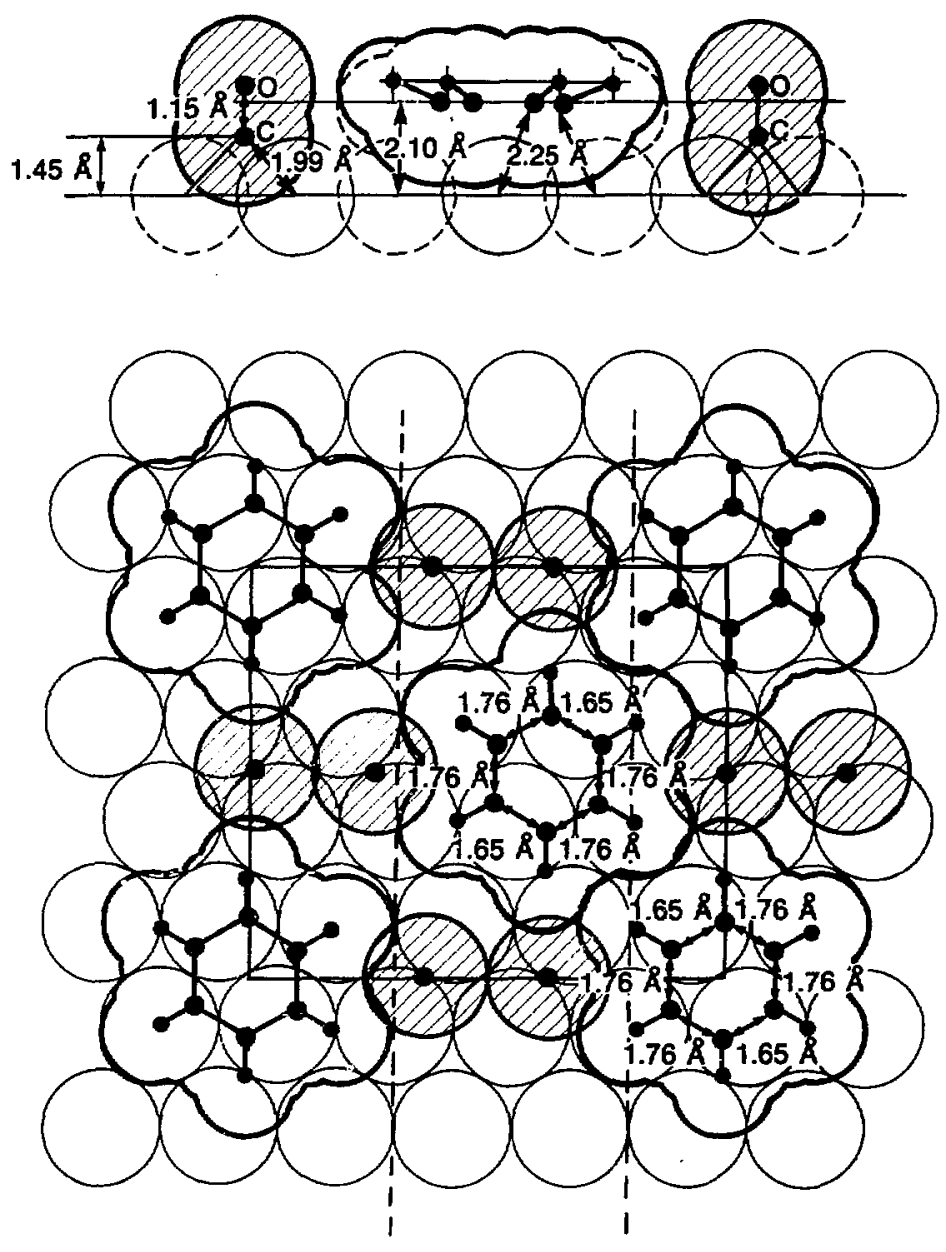

$\mathrm{Pt}(111)-(2 \sqrt{3} \times 4)$ rect $-2 \mathrm{C}_{6} \mathrm{H}_{6}+4 \mathrm{CO}$ 

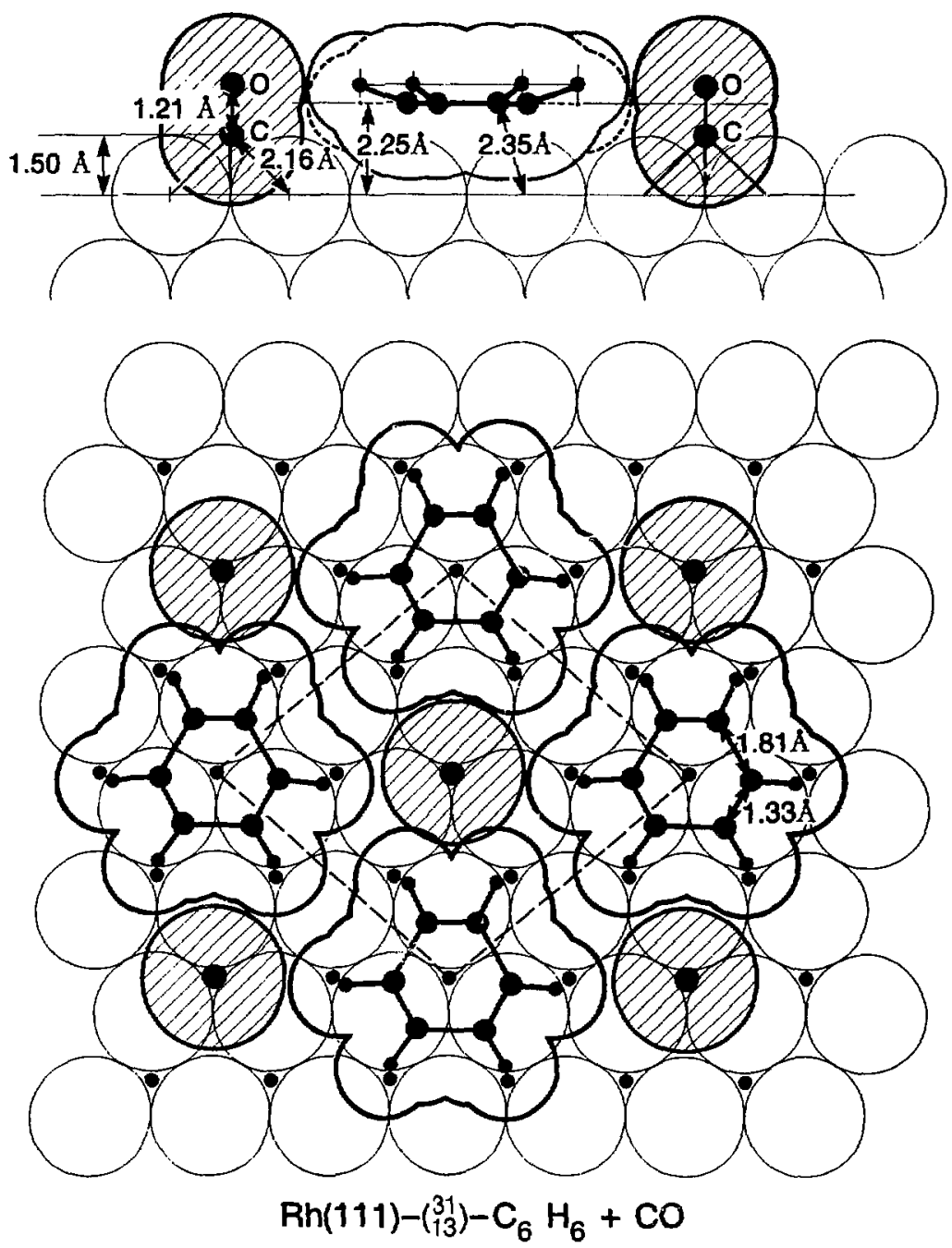

XBL 851-9917 A

Pgre 11.13 

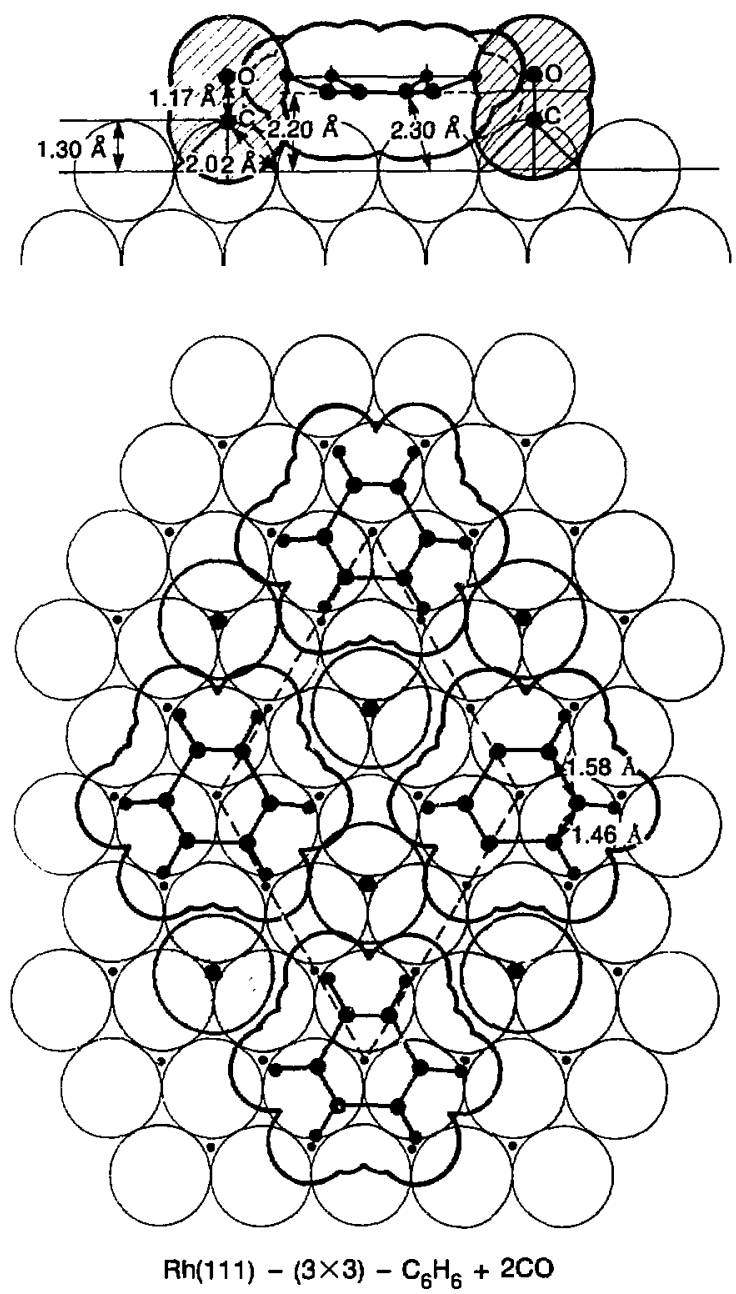

Fgre 11.14 
500

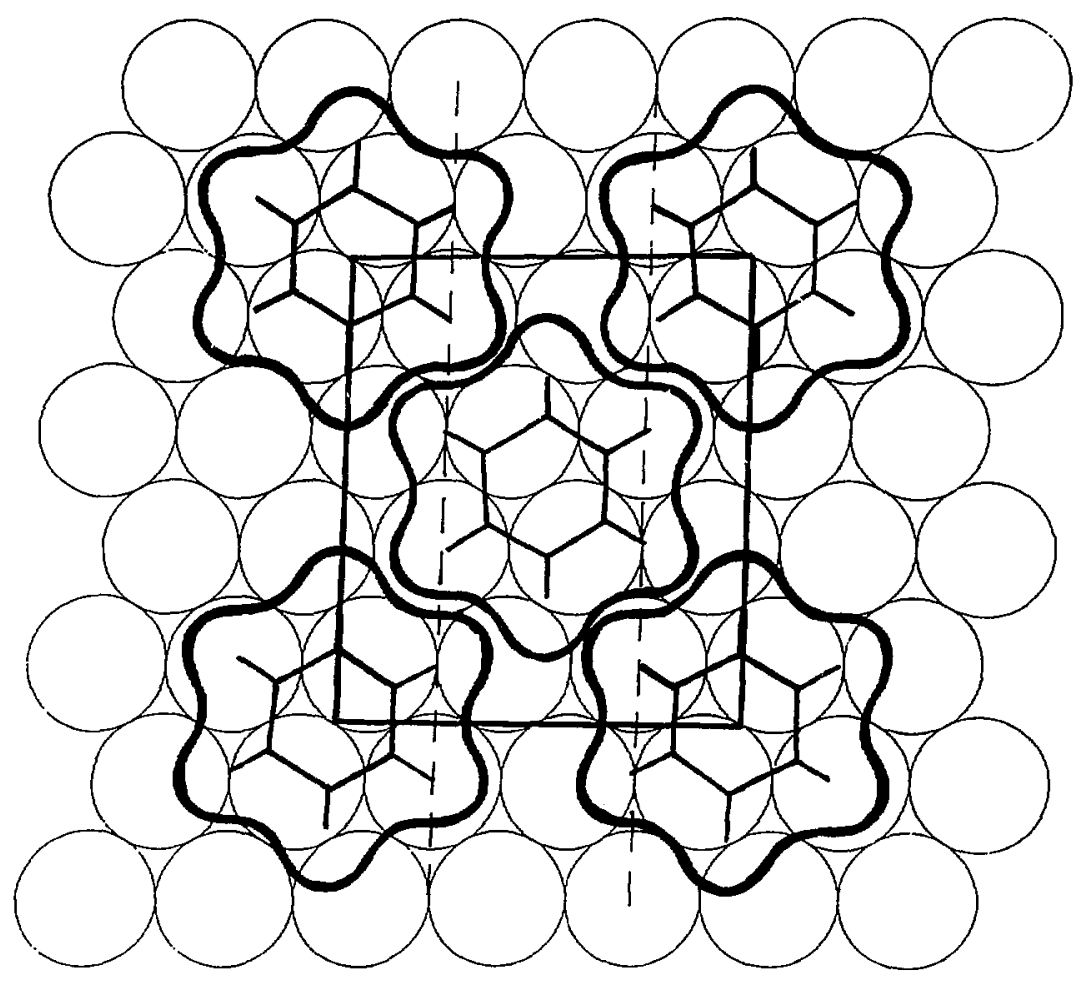

$\operatorname{Rh}(111)+(2 \sqrt{3} \times 3)$ rect $\mathrm{C}_{6} \mathrm{H}_{6}$ 
Table 11.1 - Benzene structures examined by LEED

\begin{tabular}{|c|c|c|c|c|c|c|c|c|}
\hline \multicolumn{6}{|c|}{ Benzene } & \multicolumn{2}{|c|}{$\mathbf{C O}$} & \multirow{3}{*}{ Method } \\
\hline \multicolumn{6}{|c|}{ carbon ring distortions ${ }^{e}$} & \multirow[b]{2}{*}{ site } & \multirow[b]{2}{*}{$d_{\perp \mathbb{N}-c^{c}}$} & \\
\hline site $^{a}$ & $\phi^{b}$ & $d_{L M-C} c^{c}$ & buekling ${ }^{d}$ & $\mathbf{r}(\boldsymbol{A})$ & $\beta$ & & & \\
\hline top & 0,30 & $1.2(.1) 2.1$ & & 1.397 & & & & BSN + partial RSP \\
\hline hep-hollow & $\mathbf{0 , 3 0}$ & $1.5(.1) 2.4$ & & 1.397 & & & & BSN + partial RSP \\
\hline fec-hollow & 0,30 & $1.5(.1) 2.4$ & & 1.397 & & & & BSN + partial RSP \\
\hline briäge & 0,30 & $1.5(.1) 2.4$ & & 1.397 & & & & BSN + partial RSP \\
\hline bridge & 0,30 & $1.5(.1) 2.4$ & & 1.44 & $\pm 4^{f}$ & & & BSN + partial RSP \\
\hline bridge & 0,30 & $1.5(.1) 2.4$ & & 1.44 & \pm 4 & & & BSN + partial RSP \\
\hline bridgeg & 0,30 & $1.8(.1) 2.4$ & & 1.397 & & $\begin{array}{l}\text { bridge } \\
\text { and hollow }\end{array}$ & $\begin{array}{c}1.3(.1) 1.6 \\
1.27(.1) 1.57\end{array}$ & kinematic \\
\hline bridge $^{g}$ & 30 & $1.9(.1) 2.4$ & +0.36 & 1.397 & & 2 bridge ${ }^{g}$ & $1.3(.1) 1.6$ & kinematic \\
\hline bridge $e^{g}$ & 30 & $1.54(.1) 2.04$ & -0.36 & 1.397 & & 2 bridge & $1.3(.1) 1.6$ & kinematic \\
\hline
\end{tabular}




\begin{tabular}{|c|c|c|c|c|c|c|c|c|}
\hline \multicolumn{6}{|c|}{ Benzene } & \multicolumn{2}{|c|}{$\mathrm{CO}$} & \multirow[t]{3}{*}{ Method } \\
\hline & & & \multicolumn{3}{|c|}{ carbon ring distortions ${ }^{e}$} & & & \\
\hline site $^{a}$ & $\phi^{6}$ & $d_{1 M-C} c^{c}$ & buckling ${ }^{d}$ & $\mathbf{r}(\boldsymbol{A})$ & $\beta$ & site & $d_{\perp M-C} c^{c}$ & \\
\hline bridge & 0 & $1.4(.1) 1.9$ & +0.36 & 1.397 & & 2 bridge & $1.3(.1) 1.6$ & kinematic \\
\hline bridge & 30 & $1.9(.1) 2.4$ & & 1.397 & & bridge & $1.3(.1) 1.6$ & kinematic \\
\hline bridge & 30 & $1.9(.1) 2.4$ & & 1.397 & & hep-hollow & $1.3(.1) 1.6$ & kinematic \\
\hline bridge & 30 & $2.05(.05) 2.3$ & & $1.2(.13) 1.85$ & & 2 bridge & 1.55 & kinematic \\
\hline bridge & 30 & $2.05(.05) 2.3$ & & 1.59 & $-2.5(5) 7.5$ & 2 bridge & 1.55 & BSN+KSLA+MINV \\
\hline bridge & 30 & $2.05(.05) 2.3$ & & $1.59(.13) 1.85$ & & 2 bridge & $1.35(.1) 1.65$ & BSN+KSLA+MINV \\
\hline bridge & 30 & $2.05(.05) 2.3$ & & 1.72 & $-2.5,1.5,2.5$ & 2 bridge & $1.35(.1) 1.65$ & $\mathrm{BSN}+\mathrm{KSLA}+\mathrm{MINV}$ \\
\hline bridge & 30 & $2.05(.05) 2.3$ & \pm 0.35 & 1.72 & -1.5 & 2 bridge & $1.35(.1) 1.65$ & BSN+KSLA+MINV \\
\hline bridge & 30 & $2.05(.05) 2.3$ & & $1.59 / 1.72$ & 1.5 & 2 bridge & $1.35(.1) 1.65$ & $\mathrm{BSN}+\mathrm{KSLA}+\mathrm{MINV}$ \\
\hline bridge & 30 & $2.05(.05) 2.3$ & & $1.85 / 1.72$ & 1.5 & 2 bridge & $1.35(.1) 1.65$ & BSN+KSLA+MINV \\
\hline bridge & 30 & $2.05(.05) 2.3$ & & $1.72 / 1.85$ & 1.5 & 2 bridge & $1.35(.1) 1.65$ & BSN+KSLA+MINV \\
\hline
\end{tabular}




\begin{tabular}{|c|c|c|c|c|c|c|c|c|}
\hline \multicolumn{6}{|c|}{ Benzene } & \multicolumn{2}{|c|}{ CO } & \multirow[t]{3}{*}{ Method } \\
\hline & & & carb & ring dist & $\mathbf{n s}^{e}$ & \multirow[b]{2}{*}{ site } & \multirow[b]{2}{*}{$d_{L M-c}{ }^{c}$} & \\
\hline site $^{a}$ & $\phi^{6}$ & $d_{\perp M-c^{c}}$ & buckling $^{d}$ & $r(\boldsymbol{A})$ & $\beta$ & & & \\
\hline bridge & 30 & $2.05(.05) 2.3$ & & $1.72 / 1.59$ & 1.5 & 2 bridge & $1.35(.1) 1.65$ & BSN+KSLA+MINV \\
\hline bridge & 30 & $2.05(.05), 2.3$ & & $1.79 / 1.72$ & $-1,0,4$ & 2 bridge & $1.35(.1) 1.65$ & $\mathrm{BSN}+\mathrm{KSLA}+\mathrm{MINV}$ \\
\hline bridge & 30 & $2.05(.05) 2.3$ & & $1.79 / 1.72$ & 1.5 & 2 bridge & $1.35(.1) 1.65$ & BSN+KSLA+MINV \\
\hline
\end{tabular}

For all of the structures tested the carbon monoxide $\mathcal{C}-\mathrm{O}$ bond length was assumed to be $1.15 \AA$ with the bond axis perpendicular to the surface. The glide symmetry was also assumed, so for each benzene or carbon monoxide molecule listed, a second molecule is positioned in the unit cell. If the CO site is given as "2 bridge" it implies two benzenes and four CO's in the unit cell. 


\section{Notes}

a The carbon ring is centered over this site.

$b$ The azimuthal orientation of the benzene ring, as defined in figure 11.10.

c The first and last numbers give the range of 1 layer spacings in $\AA$, and the number in parenthesis is the increrrental step size. For the first entry 1.2(.1)2.1 means that LEED calculations were made for metal-carbon ring layer spacings of $1.2,1.3,1.4, \ldots 2.1 \AA$.

d Out of plane buckling at a bridge site is restricted to the cyclohexane "boat" shape, with two opposite carbon atoms tilted up or down relative to the plane defined by the other four carbons.

$e \quad$ In plane distortions are characterized by $r$ and $\beta$ as defined in figure 11.10 for Kekule and $C_{2 v}$ sites. The notation $r_{1} / r_{2}$ indicates different radii for the two "end" carbons $\left(r_{1}\right)$ and the four "side" carbons $\left(r_{2}\right)$ at bridge sites.

$f$ A Kekule distortion was tested at the bridge site for comparison with the $\mathrm{Rh}(111)$ results, although this is not consistent with the local site symmetry.

g Both possible registries for the deeper metal layers were tested. 
Table 11.2 - Benzene Adso-otion Geometries

\begin{tabular}{|c|c|c|c|c|c|}
\hline system & $d \delta-C$ & $d \vec{c}-c$ & $d_{\perp M-C}$ & $d_{M-C}$ & site \\
\hline $\operatorname{Rh}(111)-c(2 \sqrt{3} \times 4)-r e c t-\mathrm{C}_{6} \mathrm{H}_{6}+\mathrm{CO}^{1}$ & $1.33 \pm 0.15$ & $1.81 \pm 0.15$ & $2.25 \pm 0.05$ & $2.35 \pm 0.05$ & hollow \\
\hline $\mathrm{Rh}(111)-(3 \times 3)-\mathrm{C}_{6} \mathrm{H}_{8}+2 \mathrm{CO}^{2}$ & $1.46 \pm 0.15$ & $1.58 \pm 0.15$ & $2.20 \pm 0.05$ & $2.30 \pm 0.05$ & hollow \\
\hline $\operatorname{Rh}(111)-(3 \times 3)-\mathrm{C}_{6} \mathrm{H}_{6}+2 \mathrm{CO}$ (theory) $^{3}$ & 1.50 & 1.64 & 2.1 & 2.15 & hollow \\
\hline $\mathrm{Pt}(111)-(2 \sqrt{3} \times 4)-r e c t-\mathrm{C}_{6} \mathrm{H}_{6}+4 \mathrm{CO}^{4}$ & $1.65 \pm 0.15$ & $1.76 \pm 0.15$ & $2.10 \pm 0.10$ & $2.25 \pm 0.10$ & bridge \\
\hline $\mathrm{Pt}(111)-\mathrm{C}_{2} \mathrm{H}_{2}{ }^{5}$ & $1.40 \pm 0.02$ & & & & disordered \\
\hline $\mathrm{C}_{6} \mathrm{H}_{6}$ on metal clusters ${ }^{6}$ & 1.39 & 1.48 & & 2.27-2.32 & hollow \\
\hline $\mathrm{C}_{6} \mathrm{H}_{6}$ mulecule & 1.397 & & & & gas phase \\
\hline $\operatorname{Pt}(111)-(2 \times 2)-\mathrm{C}_{2} \mathrm{H}_{3}$ (ethylidyne) ${ }^{7}$ & $1.50 \pm 0.1$ & & $1.20 \pm 0.1$ & $2.00 \pm 0.07$ & hollow \\
\hline $\mathrm{Rh}(111)-(2 \times 2)-\mathrm{C}_{2} \mathrm{H}_{3}$ (ethylidyne $)^{8}$ & $1.45 \pm 0.1$ & & $1.31 \pm 0.1$ & $2.03 \pm 0.07$ & hollew \\
\hline $\mathrm{Pt}(111)-\mathrm{C}_{2} \mathrm{H}_{3}$ (ethylidyne) ${ }^{9}$ & $1.49 \pm 0.02$ & & & & \\
\hline $\operatorname{Pt}(111)-\mathrm{C}_{2} \mathrm{H}_{3}$ (ethylidyne) ${ }^{10}$ & $1.47 \pm 0.03$ & & & & \\
\hline
\end{tabular}




\begin{tabular}{|c|c|c|c|c|c|}
\hline system & $d \widehat{c}-G$ & $d \vec{C}-C$ & $d_{\perp M-C}$ & $d_{M-C}$ & site \\
\hline $\mathrm{C}_{2} \mathrm{H}_{6}$ molecule & 1.54 & & & & \\
\hline $\mathrm{Pt}(111)-\mathrm{C}_{2} \mathrm{H}_{4}{ }^{11}$ & $1.48 \pm 0.03$ & & & & \\
\hline $\mathrm{C}_{2} \mathrm{H}_{4}$ molecule & 1.33 & & & & \\
\hline $\mathrm{Pt}(111)-\mathrm{C}_{2} \mathrm{H}_{2}{ }^{11}$ & $1.45 \pm 0.03$ & & & & \\
\hline $\mathrm{C}_{2} \mathrm{H}_{2}$ molecule & 1.20 & & & & \\
\hline
\end{tabular}

All distances measured in Angstroms.

\section{References}

1. M. A. Van Hove, R. F. Lin, and G. A. Somorjai, Journal of the American Chemical Society, vol. 108, p. $2532,1986$.

2. M. A. Van Hove, R. F. Lin, G. A. Blackman, and G. A. Somorjai, Acta Crystallographica, to be published.

3. C. Minot, E. L. Garfunkel, A. Gavezzotti, and M. Simonetta, to be published. 
4. D. F. Ogletree, M. A. Van Hove, and G. A. Somorjai, Surface Science, in press.

5. J. A. Horsley, J. Stöhr, A. P. Hitchcock, D. C. Newbury, A. L. Johnson, and F. Sette, Journal of Chemical Physics, vol. 83, p. 6099, 1985 .

6. M. P. Gomez-Sal, B. F. G. Johnson, T. Lewis, P. R. Raithby, and A. H. Wright, Journal of the Chemical Society; Chemical Communications, p. 1682, 1985.

7. L. L. Kesmodel, L. H. Dubois, and G. A. Somorjai, Journal of Chemical Physics, vol. 70, p. 2180, 1979.

8. R. J. Koestner, M. A. Van Hove, and G. A. Somorjai, Surface Science, vol. 121, p. 321, 1982.

9. P.-K. Wang, C. P. Slichter, and J. J. Sinfelt, Journal of Fhysical Chemistry, vol. 89, p. 3606, 1985.

10. J. A. Horsley, J. Stöhr, and R. J. Koestner, Journal of Chemical Physics, vol. 83, p. 3146, 1985.

11. J. Stöhr, F. Sette, and A. L. Johnson, Physical Review Lellers, vol. 53, p. 1684, 1984. 


\section{Chapter 12}

\section{Incommensurate Graphite on $\mathrm{Pt}(111)$}

\subsection{Introduetion}

Ordered monolayers of adsorbates on solid surfaces often form incommensurate structures, in the sense that an ordered overlayer exists with a lattice that is not simply related to the substrate lattice which supports it. The two lattices may be mis-aligned in angle, and they may have lattice constants that form no simple ratio. In an incommensurate system the adsorbate atoms or molecules occupy many different positions, or sites, relative to the substrate atoms. This is the physically important aspect of incommensurate alignment, since 2 mathematical definition of "incommensurability" is somewhat ambiguous for adsorbates with ordered domains of finite extent, and some coincidence lattice is bound exist on a sufficiently large scale due to slight relaxations in substrate or adsorbate geometry.

The incommensurate "graphitic" carbon overlayer on the platinum (111) surface has been analyzed by LEED. The graphite-metal spacing was found to be

$3.70 \pm 0.05 \AA$, larger than expected. This large lattice spacing has been explained by the presence of additional "intercalated" carbon atoms adsorbed in-between the graphite overlayer and the platinum substrate. 


\subsection{Incommensurate overlayers}

Incommensurate overlayers are common for rare gas atoms and small molecules adsorbed on the graphite (0001) basal plane, $1,2,3,4,5,6$ and on metal surfaces. $1,7,8,9,10,11,12,13,14,15,16,17$ Incommensurate alkali metal overlayers have also been observed on the graphite basal plane. ${ }^{18,19}$ Many of these incommensurate overlayers involve a close-packed overlayer where the overlayer lattice is determined by the adsorbate density, hence it is often out of registry with the substrate lattice. There are also examples of incommensurate metal overlayers on metal substrates, $2,20,21$ including some metal reconstructions. ${ }^{22}$

There is another class of incommensurate systems where the overlayer has a well-defined geometry, which is not much influenced by the substrate geometry. Such systems include graphitized carbon layers on various metals $23,24,25,26,27$ and compound layers (i.e. oxides) grown on metal surfaces. ${ }^{28,29,30,31,32,33,34,35,36}$

In incommensurate overlayer systems, the interaction energy between the overlayer atoms or molecules is generally strong compared to the variation in the substrate-overlayer interaction energy across the surface. Foz example, the corrugation of the Van der Waals potential on the basal plane of graphite is quite small, hence the large number of observations of incommensurate physisorbed overlayers reported. ${ }^{2}$ 


\subsection{Incommensurate overlayer analysis}

Incommensurate overlayers show up in diffraction experiments, including LEED and $x$-ray diffraction. The major features of the diffraction patterns can be explained as the superposition of two independent diffraction patterns, one from the substrate alone and one from the overlayer alone. Additional weak beams may be present in LEED due to multiple scattering between the substrate and the overlayer. There could also be some degree of buckling induced in the overlayer by the substrate, or vis versa, which would show up in the diffraction pattern.

The overlayer part of the diffraction pattern may be sufficient to identify the structure of the overlayer. In the case of carbon on $\mathrm{Pt}(111)$ a ring-like diffraction pattern is sometimes observed, corresponding to randomly oriented incommensurate domains of "graphitic" carbon. Sharp LEED spots from the individual domains are superimposed, forming rings of well-defined radius centered around the specularly reflected beam. The radius of these rings corresponds to the graphite basal plane lattice spacing within $\pm 0.5 \%$, the accuracy of the LEED measurement. If the overlayer scattering is sufficiently strong similar ring-like patterns centered on other substrate beams would also be visible in the diffraction pattern.

To get additional information on the surface structure the intensities of the diffracted beams must be investigated. In this way the overlayer-substrate spacing and the internal structure of the overlayer (if applicable) may be determined. Diffraction techniques such as LEED are the only effective way to determine the overlayer-substrate spacing. Fine-structure techniques can be used to investigate t. internal structure of the overlayer, but not the overlayer-substrate geometry, 
since the signal from many different local adsorbate-substrate geometries will be superimposed. Similar problems limit the usefulness of diffuse LEED and photoemission methods.

\subsection{Carbonaceous layers}

Carbon is adsorbed on transition metal surfaces in three basic forms: as part of a chemisorbed molecule, as "carbidic" carbon, and as "graphitic" carbon. Significant changes in the chemical environment and bonding of a surface carbon atom will change the fine-structure of the carbon $K V V$ Auger band. This has been used as a fingerprint for different types of surface carbon. Carbon adsorbed in the "carbidic" form has a line-shape similar to that of bulk metal-carbides, implying that the carbon chemical environment is dominated by carbon-metal bonds, and carbon adsorbed in the "graphitic" form has a line-shape similer to bulk graphite, implying that the carbon chemical environment is dominated by carbon-carbon bonds. ${ }^{37,38}$ The Auger line-shape has also been used to distinguish between carbon in intact chemisorbed molecules and residual surface carbon due to molecular decomposition; an example is the observation of intact and decomposed carbon monoxide on transition metal surfaces. ${ }^{39}$

Carbonaceous layers on metal surfaces are known to play an important role in catalytic reactions and other surface processes, such as lubrication. ${ }^{40}$ Platinum is widely used in pure form and in combination with other materials for hydrocarLon catalysis. Experiment has shown that under catalytic conditions there is a significant amount of carbon chemisorbed on the metal surface. ${ }^{41}$ Neverless, very 
little is known about the structure and bonding of these layers, which can include chemisurbed carbon overlayers, graphite overlayers, and metal-carbide compound phases.

The only structural studies of carbon adsorbed on metals have been done for nickel: by LEED for carbon on nickel (100) with two carbon atoms adsorbed in a (2x2) unit cell, $42,43,44,45$ and by SEELFS for commensurate graphite on nickel (111), ${ }^{46}$ and for incommensurate graphite on nickel (110). ${ }^{38}$ The commensurate (2x2) overlayer of carbon on nickel (100) is an example of carbidic carbon. There is a strong metal-carbon interaction resulting in the lateral reconstruction of the first metal layer along with a $22 \%$ expansion in the first inter-layer spacing. Carbon atoms are adsorbed in four-fold hollow sites only $0.10 \pm 0.10 \AA$ above the surface. ${ }^{43}$ There is a close fit between the graphite lattice and the (111) face of nickel. A graphite lattice expansion of $1.3 \%$ is sufficient for the $1.42 \AA$ carboncarbon spacing to fit the $1.44 \AA$ separation between adjacent hollow sites on Ni(111). The overlayer-substrate spacing is $2.80 \pm 0.08 \AA .46$ Only an initial investigation of the graphite- $\mathrm{Ni}\left(11^{\prime}\right)$ surface has been made. Here the graphite lattice is approximately commensurate along the $[1 \overline{1} 0]$ direction, but incommensurate along the [001] direction. The authors of the SEELFS (electron fine-structure technique, see table 1.1) study propose a model with carbon atoms located in quasithree-fold hollow sites and above the second layer nickel atoms with a "hardsphere" carbon-nickel spacing of $1.95 \AA .38$ 


\subsection{Graphite overlayers on platinum}

Incommensurate, ring-like electron diffraction patterns, attributed to graphitic carbon overlayers, have been observed on the $(100),{ }^{47}(110)$ and $(111)^{48}$ surfaces of platinum. The observed ring radii match the lattice constant of the (0001) basal plane of bulk graphite, ${ }^{49}$ and the carbon $K V V$ Auger line-shape indicates that graphitic carbon is present on the surface. ${ }^{39}$ The characteristic rings seen in the LEED pattern correspond to incommensurate layer of graphite in randomly oriented domains. Several different preferred orientations of the graphite overlayer domains have been observed on the different faces of platinum. ${ }^{25}$

A graphitic carbon layer can be produced on a platinum crystal surface under ultra-high vacuum conditions either by exposing a hot platinum crystal to carbon-containing molecules, or by adsorbing carbon-containing molecules on the platinum surface at or below room-temperature and then annealing the crystal to decompose some of the adsorbed molecules. Graphite layers have been produced on $\mathrm{Pt}(111)$ by the decomposition of ethylene, cyclohexane, n-heptane, benzene, toluene, ${ }^{23}$ propylene and butene. The graphite morphology does not seem to depend on the original source of the carbon atoms. Heating the $\operatorname{Pt}(111)$ surface after hydrocarbon chemisorption results in selective dehydrogenation (decomposition) of the adsorbed molecules between 400 and $800 \mathrm{~K} .^{40}$ After annealing at a temperature sufficient for the complete dehydrogenation of the adsorbed molecules, the LEED pattern will show characteristic graphite rings. If the $\operatorname{Pt}(111)$ surface is heated above $\sim 1150 \mathrm{~K}$ the surface carbon starts to dissolve into the bulk platinum; after sustained heating above $1150 \mathrm{~K}$ the graphite 
diffraction pattern is no longer visible, and Auger measurements show only a small fraction of a monolayer of residual earbon on the surface.

\subsubsection{Preferred graphite orientations}

Certain preferred angular orientations have been observed for domains of the graphite lattice relative to the substrate lattice on $\mathrm{Pt}(111){ }^{25}$ Some typical diffraction patterns are shown in Figure 12.1. The rings in the graphite diffraction pattern have a well-defined radius; the radial width of the diffraction beams is approximately the same as the width of the substrate diffaction spots, indicating long range order in the overlayer on at least the scale of the instrument response function, $\sim 100 \AA$. When a hydrocarbon covered crystal is annealed at successively higher temperatures, the graphite diffraction rings at first appear with relatively isotropic intensity. As the annealing temperature increases, the rings break up into bright segments, often with a dim continuous ring joining the segments, indicating some orientations are preferred.

At least five distinct preferred orientations of graphite overlayer domains on $\mathrm{Pt}(111)$ have been observed. Usually the length of the bright arc on the graphite diffraction ring is several times its radial width. The orientation of the graphite ring will be specified by the angle between the platinum (10) reciprocal lattice vector and a line connecting the specular beam to a spot or the center of a bright arc on the first graphite ring (with radius $\sim 14 \%$ greater than the length of the platinum (10) vector). Approximate coincidence between the graphite and platinum lattices have been suggested as the possible reason for the preferrential 
orientation of the graphite domains on $\mathrm{Pt}(111)$. Lang ${ }^{25}$ has classified several coincidence lattices between graphite and platinum according to the degree of surface strain.

The most frequently observed orientation is shown in figure 12.1(a), with the graphite arcs at $\pm 19.1^{\circ}$, corresponding to a $(\sqrt{7} \times \sqrt{7})$ coincidence lattice with a $0.8 \%$ strain. The second most common orientation is shown in figure 12.1(b) and (d), with arcs at $\pm 23.4^{\circ}$, corresponding to a $(\sqrt{19} \times \sqrt{19})$ coincidence lattice with $1.8 \%$ strain. The orientation shown in figure 12.1(c) was only observed in one series of experiments. This orientation of $\pm 16.1^{\circ}$ corresponds to a $(\sqrt{39} \times \sqrt{39})$ coincidence lattice with $1.7 \%$ strain. When one rotation angle is seen as in figure 12.1(a), the domains rotated by $\pm \theta$ always had equal apparent intensities. Graphite domains have also been observed at orientations of $0^{\circ}$ and, on one occasion, $30^{\circ}$. These domains were never observed alone, but always in combination with one or more of the other rotated domains described above, as for the $0^{\circ}$ orientation seen with the $\pm 23.4^{\circ}$ orientation in figure $12.1(\mathrm{a})$ and (d). The $0^{*}$ orientation corresponds to a $(\theta \times 9)$ coincidence lattice with $1.3 \%$ strain.

\subsubsection{Overlayer growth}

Graphite overlayers apparently grow by island formation on $\mathrm{Pt}(111)$. A clean $\mathrm{Pt}(111)$ surface at $1100 \mathrm{~K}$ was initially exposed to a small amount $(1 \mathrm{~L})$ of propylene $\left(\mathrm{C}_{3} \mathrm{H}_{5}\right)$ vapor at $5 \cdot 10^{-8}$ torr. When the crystal was cooled, segmented graphite rings were already visible in the LEED pattern similar to those in figure 12.1(a), with the same sharpness as the substrate diffraction spots. As the crystal 
was exposed to increasing amounts of propylene vapor in increments of $1 \mathrm{~L}$, the only change in the diffraction pattern was an increase in the brightness of the overlayer diffraction features. The ratio of the carbon $272 \mathrm{eV}$ Auger peak to the platinum $237 \mathrm{eV}$ peak (measured with a retarding field energy analyzer in the second derivative mode at $10 \mathrm{~V}$ peak-to-peak modulation) increased approximately linearly with propylene exposure.

After a total exposure of $11 \mathrm{~L}$ of propylene, weak diffraction features due to multiple scattering between the platinum substrate and the graphite overlayer became visible (see Figure 12.1(a)). The $\mathrm{C} / \mathrm{Pt}$ Auger peak height ratio was 3.4. The multiple scattering features of the pattern were at least an order of magnitude weaker than the single scattering features due to the overlayer. Exposure to $13 \mathrm{~L}$ of propylene irsireased the $\mathrm{C} / \mathrm{Pt}$ Auger ratio to $\sim 3.7$; no further change in the $\mathrm{C} / \mathrm{Pt}$ Auger ratio or the LEED pattern was observed with increased hydrocarbon exposure.

In a second experiment the $\mathrm{Pt}(111)$ crystal was held at $1100 \mathrm{~K}$, and the Auger peak height ratio was monitored between incremental doses of propylene vapor. The results of this experiment are shown in figure 12.2. Once again the the $\mathrm{C} / \mathrm{Pt}$ Auger ratio reaches a maximum at $\sim 3.7$. After the final propylene exposure the crystal was cooled and the LEED pattern resembled that of figure 12.1(c). This apparent saturation of the graphite coverage in ultra-high vacuum conditions is consistent with results obtained from carbon monoxide decomposition. ${ }^{50}$ The carbon coverage corresponding to a $\mathrm{C} / \mathrm{Pt}$ Auger peak height ratio of $\sim 3.7$ is associated with a single complete sheet of graphite on the $\operatorname{Pt}(111)$ 
surface.

Graphite rings have been observed on the $\mathrm{Pt}(111)$ surface with a C/Pt Auger ratio significantly higher than 3.7 after the crystal was exposed to atmosphere and then baked out with the vacuum system. During bake-out the crystal is exposed to background gases at up to $\sim 5 \cdot 10^{-7}$ torr at $500 \mathrm{~K}$ for several days. Diffraction spots from the platinum substrate were almost invisible, and the graphite rings were continuous, with no sign of the angular variation in intensity typical of graphite layers formed under ultra-high vacuum conditions. In this case there were probably multiple layers of graphite or pyrographite on the $\mathrm{Pt}(111)$ substyate.

\subsection{LEED beam types}

The LEED beams from a surface with an incommensurate overlayer may be classified into four distinct groups: the specularly reflected beam $\mathbf{O}$, the set of substrate beams $\{\mathbf{G}\}$, the set of overlayer beams $\{\boldsymbol{\varepsilon}\}$ and the combination beams $\{g+G\}$ (see figure 12.3). The specular beam $O$ is important, since it is the only beam that contains information on both overlayer and substrate in the kinematic limit. The substrate beams $\{\mathbf{G}\}$ are defined by the translational symmetry of the substrate alone, and the overlayer beams $\{g\}$ are defined by the translationa! symmetry of the overl syer. In the kinematic limit these two sets of beams are independent of each other. The set of combination beams $\{\boldsymbol{g}+\boldsymbol{G}\}$ contain all beams not in the first three groups. The combination beams arise from multiple scattering, so they are absent in the kinematic limit. 
Together the four groups of beams are used in tha plane-wave representation of the LEED wave-function. In principle there are an infinite number of these beams, since some combination beam $\mathbf{g}+\mathbf{G}$ can reach any point in the substrate Brillouin zone for an incommensurate lattice. In practice the number of beams required in the plane-wave representation is limited by the arguments of section 3.6 and Eq. 3.17. Auy beam with momentum $|\mathbf{k}|>\left|\mathbf{k}_{\mathrm{o}}\right|$ is an evanescent beam and decays between layers, and beams that are attenuated by more than a factor of $t$ are excluded from the plane-wave representation. Only those combination beams for which $|\mathbf{g}|,|\mathbf{G}|$ and $|\mathbf{g}+\mathbf{G}|$ are all less than or near $\left|\mathbf{k}_{\mathbf{o}}\right|$ are included in a LEED intensity calculation.

This profusion of plane-waves can be organized with the concept of beam sets $^{15,51}$ introduced in section 4.9. Reflection from the substrate can only couple beams differing in $\mathbf{k}_{\|}$by $\{\mathbf{G}\}$. The calculation of the scattering in the substrate involves the beam set $\mathbf{O}+\{\mathbf{G}\}$ plus the beam sets $\mathbf{g}+\{\mathbf{G}\}$ for each $\mathbf{g}$ in $\{\mathbf{B}\}$. This description is complicated for the incommensurate case since the substrate heam sets are not all coupled by the overlayer - the overlayer scattering in turn can be calculated for beam sets $\mathbf{O}+\{\mathbf{g}\}$ and $\mathbf{G}+\{\mathbf{g}\}$ for each $\mathbf{G}$ in $\{\mathbf{G}\}$. This is a very complex calculation since the overlayer diffaction matrix and the substrate reflection matrix will have to be calculated at many difierent angles of incidence. For such calculations to be practical approximations must be introduced, especially for the case of graphite on $\operatorname{Pt}(111)$, where the diffraction patterns from overlayer domains of different orientations are superimposed in the experimental data. Figure 12.4 classifies the scattering paths which contribute to the different 
types of LEED beams in orders of overlayer-substrate scattering. 52 This classification serves as a guide to the information contained in different beams, and to the types of approximations that may be used.

\subsubsection{Structural information}

The specular beam $O$ will be strongly affected by both substrate and overlayer structure, since first-order substrate and overlayer terms interfere. In particular this beam should be sensitive to the substrate-overlayer spacing. The substrate beams $\{\mathbf{G}\}$ will be only weakly affected by the overlayer, which can only affeci the substrate beam intensities through third- and higher-order scattering paths. Comparison of the substrate beam intensities with and without an overlayer can give a sense of the importance of multiple scattering. There will be some changes caused by the overlayer in addition to overlayer ion-core scattering, because of the attenuation of the incident beam and the back-diffracted beams by the overlayer. The overlayer will change the surface potential barrier, and may change the surface vibrations. If any intensity peaks are influenced by surface resonances ${ }^{53}$ then these peaks will be strongly affected by the changed surface potential barrier. A few peak intensities could also be changed by resonant specular scattering between the substrate and the overlayer, as in an optical FabryPerot interferometer.

The relative independence of the substrate beam intensities on the overlayer structure means that the substrate structure can be determined in the presence of an incommensurate overlayer with a simple calculation. In particular the changes 
in inter-layer relaxations with adsorption could be investigated. An incommensurate overlayer would be unlikely to cause periodic changes in the substrate.

The overlayer beams $\{g\}$ are somewhat more sensitive to the effect of the substrate since second-order substrate terms contribut to these beams. The combination beams, like the specular beam, will be strongly sensitive to both substrate and overlayer geometry since both types of scattering must contribute to these beams in the sาme order.

\subsection{LEED data}

LEED I-V curves were collected for both clean and graphite covered $\mathrm{Pt}(111)$ between 20 and $400 \mathrm{eV}$. Data were recorded at $\theta=10^{\circ}, 15^{\circ}$ and $20^{\circ}$, all at room temperature with the azimuthal angle $\phi=180^{\circ}$. These angles are defined using the beam labeling convention of Adams, et al..$^{54}$ for platinum (111). I-V curves were recorded using the video data system (see chapter 5) with the lens aperture at $f / 2.0$ to prevent camera saturation.

The graphite overlayer was prepared by exposing the $\mathrm{Pt}(111)$ crystal at 300 $\mathrm{K}$ to benzene vapor at $5 \cdot 10^{-7}$ torr (uncorrected ion gauge reading) for $60 \mathrm{~s}$. This is sufficient to saturate the surface with benzene vapor (see chapter 11). The crystal was then annealed at $1100 \mathrm{~K}$ for $120 \mathrm{~s}$. To increase the intensity of the graphite diffraction features the crystal was exposed a second time to benzene vapor at rom temperature, and more benzene molecules were adsorbed on the exposed parts of the platinum surface, which was then annealed again. The LEED pattern indicated a superposition of graphite domain orientations, as shown in figure 
12.1(d).

I-V curves were calculated for the specular beams and three symmetry independent non-specular beams at each angle of incidence, and the I-V curves for one pair of beams related by mirror symmetry were compared at each angle of incidence to confirm the mirror symmetry at $\phi=180^{\circ}$. It was not possible to calculate I-V curves for the overlayer beams since these are much weaker than the substrate beams over most of the energy range, and the available intensity is further divided among several different rotationally oriented domains. (The diffraction patterns of figure $\mathbf{1 2 . 1}$ were recorded where the overlayer diffraction features had maximum intensity.)

The clean and graphite overlayer specular beams are compared in figure 12.5. There are substantial changes in the specular beam I-V curves due to the overlayer, and therefore structural information is contained in the specular beam data. The substrate beams, however, are changed only slightly by the graphite overlayer. Figure 12.6 compares substrate beams at the different angles of incidence. In general the peak positions agree well, and the graphite curves are attenuated relative to the clean platinum curves. There are some changes in relative intensity of adjacent peaks.

There are two cases where substrate beam I-V curve peaks have significantly greater intensity than the corresponding clean platinum peaks: at $\sim 330 \mathrm{eV}$ in the $\theta=10^{\circ}(0, \overline{1}) \mathrm{l}-\mathrm{V}$ curve, and at $\sim 370 \mathrm{eV}$ in the $\theta=20^{\circ}(2, \overline{1}) \mathrm{I}-\mathrm{V}$ curve. The reason for this is unclear. The overall good agreement makes a significant structural change unlikely. The intensity variation may be due to non-structural effects of 
the graphite overlayer - either a change in the surface potential barrier, or a reduction in the platinum surface vibrations.

\subsection{Theoretical analysis}

The goal of the LEED intensity analysis is to determire the overlayersubstrate spacing. The similarity of the substrate I-V curves with and without the graphite overlayer suggests that the substrate structure is not changed from the terminated bulk geometry of the clean platinum (111) surface (see chapter 10). In the following analysis the graphite overlayer is assumed to be a single (0001) plane of graphite, which is consistent with the overlayer LEED pattern. The substrate is assumed to have the terminated bulk geometry. An idealized incommensurate overlayer is assumed, i.e. no domain effects, coincidence lattice, or overlayer buckling. For an incommensurate layer the concept of registry between the overlayer and the substrate is undefined, so the overlayer-substrate spacing is the only unknown structural parameter.

Three different theoretical approaches have been used to analyze overlayersubstrate spacings for incommensurate systems. Cohen, et al. ${ }^{13}$ first used a variation of a constant momentum-transfer averaging quasi-kinematical scheme $\mathrm{e}^{55}$ to analyze an incommensurate xenon overlayer on silver (111). They found an overlayer-substrate spacing of $3.50 \pm 0.10 \AA$. This method was later used by Shaw, et $a_{0}{ }^{3}$ to analyze incommensurate argon and krypton overlayers on graphite (0001). 
Later Stoner et al..$^{15}$ did a relatively complete multiple-scattering calculation for xenon on silver (111) at normal incidence. The combination beams $\{g+G\}$ were left out of the plane-wave expansion of the wave-function, which is a reasonable approximation because of their low intensity, but the calculation was otherwise complete. Up to 29 substrate beams and 58 overlayer beams were used in the plane-wave expansion at the maximum energy. For each beam in the planewave representation a calculation at a new angle of either the overlayer scattering matrix or the substrate reflection matrix is required, so this calculation could take up to 84 times longer than a clean substrate calculation. The results of this analysis gave an overlayer-substrate spacing of $3.55 \pm 0.05 \AA$, consistent with the original quasi-kinematical analysis. Further, this study showed good convergence using only first order terms for the specular beam $\mathbf{O}$ and substrate beams $\mathbf{G}$, and first and second order terms for overlayer beams $\mathrm{g}$.

A new theoretical approach to incommensurate overlayers has been developed by Hu et al. ${ }^{52}$ to analyze graphite overlayers on platinum, based on the beam-set neglect (BSN) scheme. ${ }^{51}$ This method allows the calcult tion of incommensurate beam intensities to second order in overiayer-substrate scattering with great efficiency compared to the full plane-wave calculations used by Stoner et a1. 15

The LEED intensity data for graphite on platinum (111) has been analyzed using both the quasi-kinematic approach and the BSN approach. The plane-wave approach of Stoner et al. is not practical for graphite, since the already formidable calculation would have to be performed for many different graphite domain 
orientations and then averaged together. The quasi-kinematic analysis gave ambiguous results, so the more sophisticated BSN calculation was performed. Once the multiple-scattering BSN results were determined, the significance of the quasi-kinematic results became clear.

\subsection{BSN approximation for incommensurate overlayers}

The BSN approach greatly reduces the effort needed to caiculate any beam intensity to second order. When all third order terms are included the time required for the BSN calculations is s'ose to that required for a full plane-wave calculation correct to all orders. Figure 12.4 illustrates the various orders of overlayer-substrate scattering that contribute to different types of beams. The basic idea of the BSN method is that scattering from the overlayer or substrate couples certain specific subsets of the diffacted ?eams, and the scattering matrices for each subset can be calculated independently.

Overlayer scattoring is described by a scattering matrix $M_{s+8, g+\sigma^{\prime}}^{ \pm \pm}(\mathrm{s})$ where s can be reguarded as the "incident beam" momentum. This matrix describes the effect of overlayer scattering on the beam set $+\{\boldsymbol{B}\}$. Likewise substrate scattering is described by a reflection matrix $R_{0+G, a+G}(s)$, which describes substrate scattering for the beam set $\mathbf{s}+\{\mathbf{G}\}$. These matrices are calculated for any given angle of incidence by standard techniques (see section 3.8).

Incommensurate calculations are complex because a new substrate reflection matrix $R_{\mathbf{g}+\mathbf{G}, \mathbf{g}+\mathbf{G}}(\mathbf{O}+\mathbf{g})$ must be calculated for every overlayer beam $\mathbf{g}$, and a new overlayer scattering matrix $M_{\mathrm{G}^{\prime}+{ }_{\mathrm{z}}, \mathrm{G}+\mathrm{g}^{\prime}}(\mathrm{O}+\mathrm{G})$ must be calculated for every 
substrate beam G. However, in the BSN approximation any beam intensity may be calculated to second order using elements from only two to four scattering matrices. A general third-order term requires calculation of all scattering matrices; however, a few specific higher-order terms, in particular multiple specular reflections between overlayer and substrate before or after another event, can easily be included to all orders using standard layer stacking techniques and a few additional matrix elements.

\subsubsection{Specular beam intensity}

The specular beam intensity may be calculated to second order using only the matrix elements, $M_{\mathrm{O}}^{+-}(O) . M_{\mathrm{O}}^{++}(O), M_{\mathrm{OO}}^{-}(O)$ and $R_{\mathrm{OO}}(O)$. Multiple specular reflections are easily included with the addition of $M_{\mathrm{OO}}^{-}(O)$.

\subsubsection{Substrate beam intensities}

Any one substrate beam intensity can be calculated using the matrix elements $R_{\mathrm{GO}}(\mathbf{O}), M_{\mathrm{O}}^{++}(\mathbf{O})$ and $M_{\mathrm{O}}^{-}(\mathrm{G})$. With the addition of $M_{\mathrm{OO}}^{+}(\mathbf{G})$, $M_{\mathrm{OO}}^{-+}(O), R_{\mathrm{OO}}(O)$ and $R_{\mathrm{GG}}(\mathrm{O})$ multiple specular reflection can be included. For a different substrate beam $\mathbf{G}^{\prime}$ the new elements $M_{\mathbf{0 O O}}^{-}\left(\mathbf{G}^{\prime}\right)$ and $M_{\bar{O}}^{-+}\left(\mathbf{G}^{\prime}\right)$ must be calculated. 


\subsubsection{Overlayer beam intensities}

Any overlayer beam intensity can be calculated using the matrix elements $M_{\mathrm{g}}^{+-}(\mathbf{O}), M_{\mathrm{g}}^{-}(\mathbf{O}), M_{\mathrm{gz}}^{-}(\mathrm{O}), R_{\mathrm{OO}}(\mathbf{O})$ and $R_{\mathrm{OO}}(\mathrm{g})$. With the addition of $M_{\bar{O}}^{-}(\mathbf{O})$ and $M_{\mathrm{gg}}^{-+}(\mathbf{O})$ multiple specular reflection can be included. For a different overlayer beam $\mathbf{g}^{\prime}$ the new element $R_{\mathrm{OO}}\left(\mathbf{g}^{\prime}\right)$ must be calculated.

\subsubsection{Combination beam intensities}

In a similar way a combination beam intensity can be calculated to second order with only a few more terms: $M_{\mathrm{OO}}^{++}(\mathrm{O}), M_{\mathrm{gO}}^{++}(\mathrm{O}), M_{\mathrm{gO}}^{-}(\mathrm{G})$ and $M_{\mathrm{gg}}^{--}(\mathrm{G})$ for the overlayer and $R_{\mathbf{G O}}(\mathbf{O})$ and $\boldsymbol{R}_{\mathbf{G O}}(\mathbf{g})$ for the substrate. Once again multiple specular reflection is included with $M_{\mathrm{O}}^{-+}(\mathrm{O}), M_{\mathrm{GE}}^{-+}(\mathrm{O}), M_{\mathrm{OO}}^{-}(\mathrm{G})$ and $M_{\mathrm{gE}}^{-+}(\mathrm{G})$ for the overlayer and $R_{\mathrm{OO}}(\mathbf{O}), \boldsymbol{R}_{\mathrm{GG}}(\mathbf{O}), \boldsymbol{R}_{\mathrm{OO}}(\mathbf{g})$ and $\boldsymbol{R}_{\mathrm{GG}}(\mathrm{g})$ for the substrate.

\subsubsection{BSN application}

Using the BSN scheme any incommensurate overlayer beam intensity may be calculated using only one or two beam sets, so the total effort for a given beam is comparable to a calculation for a simple overlayer. Because the overlayer is incommensurate, the complexity increases linearly with the number of beams included in the calculation. In the case of graphite on platinum (111) only the specular beam intensity was calculated, since the substrate beam I-V curves contain little new information; and the overlayer and combination beams were too weak to obtain a useful range of I-V data. 


\subsection{Graphite-platinum calculations}

The specular beam I-V curves were calculated between 20 and $235 \mathrm{eV}$ at all three angles using the spin averaged phase shifts of figure $3.1^{56}$ and a Debye temperature of $255 \mathrm{~K}$ for platinum. The carbon atoms were given twice the vibrational amplitude of the platinum atoms. ${ }^{52}$ Only one graphite domain orientation was used in calculating the specular reflection and transmission matrix elements, the orientation where the (10) reciprocal lattice vectors were parallel for the graphite overlayer and the platinum substrate. In principle the specular beam reflection and transmission matrix elements can yary with the azimuthal orientation of the overlayer; however, it has been observed that this variation is small for small polar angles, and Clarke ${ }^{57}$ reports that this variation is appreciable only for $\theta>40^{\circ}$. Therefore it is a reasonable assumption to calculate the overlayer matrix elements for only one domain. The resulting theoretical curves were compared to the experimental data using the five r-factor average $r_{5}$ (see section 4.4), and the results are shown in figure 12.7. Multiple r-factor minima appear as a function of overlayer-substrate spacing. The data subdivision method is used to discriminate between the true minimum and the false minima (see section 4.5). When different energy ranges are used to calculate $r_{5}$ the false minima shift, and the optimum overlayer-substrate spacing is seen to be $3.70 \pm 0.05 \AA$ at $r_{5}=0.15$.

An overlayer-substrate spacing of $3.70 \AA$ is much larger than expected. Graphite on $\mathrm{Ni}(111)$ has a spacing or $2.80 \AA, 46$ and xenon physisorbed on $\mathrm{Ag}(111)$ has a $3.55 \AA$ spacing, approximately the sum of the $\mathbf{A g}$ and $\mathrm{Xe}$ atomic radii. Substituting the metallic radius of $\mathrm{Pt}$ for $\mathrm{Ag}$, and the Van der Waals radius of $\mathrm{Xe}$ (2.18 
A) with that of carbon (1.67 $\AA$ ) gives a spacing of $2.95 \AA$ for "physisorbed" carbon.

To explain this difference a second structural model was considered -- carbon atoms intercalated between the graphite layer and the substrate layer. Auger measurements of this system showed only carbon and platinum present, so other intercalates were ruled out. A second graphite layer is unlikely, since the graphite interlayer spacing is $3.35 \AA$, and $0.37 \AA$ would be too small a spacing for the first graphite layer. Also graphite multi-layers are not consistent with the observation of graphite island formation and then saturation in the carbon coverage. $A$ full double layer of graphite should give a $\mathrm{C} / \mathrm{Pt}$ Auger peak-height ratio larger than 3.7 and should attenuate the substrate LEED beam intensities substantially.

I-V calculations were carried out for two similar models, a single graphite overlayer with intercalated carbon in three-fold fec and hep hollow sites, respectively. The two structural parameters $d_{\text {graphite-carbon }}$ and $d_{\text {carbon-metal }}$ were varied independently. Figvire 12.8 shows an $r_{5}$ contour plot for intercalated carbon in bep hollows. The r-factor calculations failed to distinguish between the fce and hep hollow sites. In both cases the graphite platinum spacing was found to be $3.70 \pm 0.05 \AA$, the same as in the graphite only model. Also, this distance is determined with greater precision than the carbon-platinum spacing, which is $1.25 \pm 0.10 \AA$. 


\subsubsection{Disckssion of results}

This result for the carbon-platinum spacing is consistent with previous results. The structure of ethylidyne on $\mathrm{Pt}(111)$ has been determined, ${ }^{58}$ and there the terminal carbon atom is in a hollow site with a carbon-platinum spacing of $1.30 \pm 0.10 \AA$. Similar results were found for ethylidyne on $\operatorname{Rh}(111) .58,60$ When carbon-monoxide is co-adsorbed with benzene ${ }^{61}$ or ethylidyne $e^{60}$ on $\operatorname{Rh}(111)$ the carbon atom is also in a three-fold hollow site, and the carbon-metal spacings are $1.30 \pm 0.10 \AA$ and $1.45 \pm 0.10 \AA$, respectively.

These results are plausible for a graphite-intercalated carbon model. The calculated metal-carbon distance plus half of the covalent platinum radius plus half the bulk graphite inter-layer spacing is $1.25+0.77+1.67=3.69 \AA$, surprisingly close to the LEED result of $3.70 \pm 0.05 \AA$.

The absolute value of the $r_{5}$ minimum does not distinguish among the three models: the graphite only, graphite plus hep carbon, and graphite plus fec carbon $r_{5}$ values are $0.150,0.154$ are 0.154 , an insignificant difference. The models involving intercalated carbon, however, make better sense physically. The actual distribution of the intercalated carbon is unknown. There may be a continuous $1 \times 1$ layer below the graphite, patches of $1 \times 1$ carbon, or scattered intercalated carbon atoms that act as "tent poles" to support the graphite layer. Since the substrate beams for clean and graphite covered platinum match relatively well, a continuous $1 \times 1$ carbon layer is unlikely -- this would cause a greater perturbation to the substrate I-V curves. The intercalated carbon surface model is shown in figure 12.9, and a comparison of a typical experimental I-V curve with the near- 
optimum theoretical I-V curves for the different model geometries is shown in figure $1 * 10$.

\subsection{Quasi-kinemntical analysis}

The scattering from a surface with an incommensurate overlayer can be analyzed kinematically after Cohen et al. ${ }^{13}$ For the specular beam the amplitude diffracted from a clean substrate is

$$
A_{s}(\mathbf{S})=N_{s} f_{s} e^{i \delta_{d}} \sum_{n=0}^{\infty}\left(\alpha_{s} e^{-i S c}\right)^{n}=\frac{N_{s} f_{s} e^{i \delta_{b}}}{1-\alpha_{s} e^{-i S_{e}}}
$$

where $\mathbf{S}$ is the momentum transfer, which is $2 \mathbf{k}_{\mathrm{o}}$ fl for the specular beam. Here $c$ is the inter-layer separation, $N_{o}$ the atomic density, $f_{s} e^{i \phi_{0}}$ is the substrate atom scattering factor and $\alpha_{\mathrm{a}}$ is the attenuation of a substrate layer. The diffracted intensity $A_{s}^{*} A_{s}$ is then

$$
I_{s}(\mathrm{~S})=\frac{N_{a}^{2} f_{a}^{2}}{1+\alpha_{a}^{2}-2 \alpha_{a} \cos S c}
$$

Adding an adsorbate with density $N_{e}$, atomic scattering factor $f_{a} e^{i \delta_{c}}$ and attenuation $\alpha_{a}$ a distance $d$ above the substrate surface gives

$$
A(S)=N_{c} \int_{c} e^{i \delta_{c}} e^{i S d}+\frac{\alpha_{a} N_{b} f_{e} e^{i \delta_{t}}}{1-\alpha_{d} e^{-i S c}}
$$

The diffracted intensity for the overlayer system is then 


$$
\begin{gathered}
I=N_{a}^{2} \int_{a}^{2}+\alpha_{a}^{2} I_{\text {clean }}+2 \alpha_{a} \frac{N_{a} f_{a}}{n_{b} f_{b}} I_{\text {clean }} \\
\times\left(\cos \left(S d+\delta_{a}-\delta_{a}\right)-\alpha_{b} \cos \left(S(d-c)+\delta_{a}-\delta_{b}\right)\right)
\end{gathered}
$$

This expression can be simplified experimentally by dividing the specular beam intensity with the adsorbate layer by the specular beam intensity of the clean surface $I_{\text {clean }}$ to give

$$
\begin{gathered}
\frac{I}{I_{\text {clean }}}=\left[\alpha_{a}^{2}+\beta^{2}\left(1+\alpha_{a}^{2}\right)\right]-2 \alpha_{a} \beta^{2} \cos S c \\
+2 \alpha_{a} \beta\left(\cos \left(S d+\delta_{a}-\delta_{a}\right)-\alpha_{a} \cos \left(S(d-c)+\delta_{a}-\delta_{a}\right)\right)
\end{gathered}
$$

where $\beta=\frac{N_{a} f_{a}}{N_{b} f_{a}}$.

If the scattering phase shifts $\delta_{\mathrm{a}}$ and $\delta_{\mathrm{a}}$ change slowly compared to $S d$, then the intensity ratio should be dominated by oscillations at $S d$ and $S c$ in the kinematic limit. A Fourier transform of the ratio of specular beam intensities should yield the distances $d, c$ and $|d-c|$. To improve the kinematic approximation, specular beam I-V curves taken at different angles of incidence are averaged together at constant momentum transfer $\Delta \mathbf{k}_{\|}$. This should "average out" the multiple scattering effects in some sense, and approach the kinematic limit. ${ }^{55}$

For the case of xenon on silver analyzed by Cohen $^{13}$ and for argon or krypton on graphite analyzed by Shaw et al. ${ }^{3}$ the $c-d$ term is insignificant since $c \sim d$. The $S c$ term is relatively small since the overlayer density is small compared to the substrate density, and so the $S d$ term dominates the Fourier 
transform.

\subsubsection{Application to graphite-platinum}

The I-V curves for clean and graphite covered platinum for the three angles of incidence were averaged together at constant momentum transfer. The momentum transfer $\mathbf{S}$ for the specular beam is

$$
\mathbf{S}=2 \mathbf{k}_{\mathrm{o} \|}=\frac{2}{h} \cos \theta\left[2 m e\left(V-V_{o}\right)\right]^{1 / 2}
$$

where $\theta$ is the angle of incidence and $V_{0}$ is the inner potential. A value of $18 \mathrm{~V}$ ir 23 used for platinum, ${ }^{54}$ both clean and graphite covered. Figure 12.11 shows the clean platinum I-V curves as a function of momentum transfer. The Bragg peaks are indicated. There is clearly a significant amount of structure in addition to the 3ragg peaks in these curves. In figure $\mathbf{1 2 . 1 2}$ the momentum transfer averages of the clean and graphite covered curves are shown together.

\subsubsection{Fourier transform analysis}

If the quasi-kinematic analysis of Coinen et al..$^{13}$ is applicable to the case of graphite on platinum, a system with stronger multiple-scattexing effects than xenon on silver, then Eq. 12.5 can be used to determine the overlayer-substrate spacing. The specular beam intensity ratio for the graphite covered and clean platinum surfaces is shown in figure 12.13. The Fourier transiorm of this curve should give the overlayer-substrate spacing. 
The experimental ratio of specular beam intensities was measured from 6 to $20 \AA^{-1}$. If a Fourier transform is made of this data segment alone, the finite range of data will cause oscillations in the final transform. Barton ${ }^{62}$ describes various techniques used to treat ARPEFS data, where interatomic distances can be extracted from experimental data by a similar Fourier analysis. Following this treatment, a value of 1.0 is subtracted from the intensity ratio, since this ratio approaches 1.0 at large $k_{\|}$, and then the ratio data are multiplied by a Gaussian function centered on the data range with a width $5 / 8$ of the data range. The optical Fourier transform $F(d)$ is then given by

$$
F(d)=\left|\int_{S_{\min }}^{S_{\max }}\left(\frac{I_{\text {graphite }}}{I_{\text {clean }}}-1.0\right) e^{i S d} e^{(S-\bar{S})^{2} /\left(\frac{5}{16} \Delta S\right)^{2}} d S\right|
$$

The Fourier transform $F(d)$ for $d$ from 0.0 to $10.0 \AA$ is shown in figure 12.14.

When the quasi-kinematic analysis was first performed this Fourier transform data seemed inconclusive, since there was no clearly dominant peak, as there was in the xenon on silver case, ${ }^{13}$ and none of the peaks were near the expected value of the overlayer-substrate interlayer spacing. However, with the LEED result oi a $3.70 \AA$ overlayer-substrate spacing the Fourier transform of figure 12.14 can be reinterpreted. Eq. 12.5 predicts peaks at $d$ and $|d-c|$, where $d$ is the overlayer-substrate spacing and $c$ is the substrate inter-layer spacing. The graphite lattice is denser than the inert gas overlayers studied on silver and graphite substrate, so from Eq. 12.5 the substrate inter-layer spacing should be more prominent in the Fourier transform. In figure 12.1 1 there are clear peaks at $c, d, 2 c, 3 c$ and $4 c$. The peak at $\sim 1.1 \AA$ is somewhat below the predicted 
value of $1.44 \AA$ for the $|c-d|$ peak, and the peak at $3.70 \AA$ for $d$ agrees exactly with the calculated LEED/BSN result. The strong peak at $5.2 \AA$ in the experimental Fourier transform is not predicted by Eq. 12.5. The results of the quasikinematic analysis are consistent with the results of the LEED beam-set neglect calculations for overlayer-substrate spacing.

\subsection{Conclusion}

The application of the beam set neglect (BSN) approximation to incommensurate systems provides a quick and efficient method to calculate the overlayersubstrate spacing. This method has been applied to incommensurate graphite overlayers on platinum (111), the first non-inert gas incommensurate overlayer to be investigated by LEED intensity structure determination calculations. The LEED analysis gives an overlayer-substrate distance of $3.70 \pm 0.05 \AA$, along with the first observation of an incommensurate overlayer supported by intercalated atoms. The intercalated carbon atoms are adsorbed in three-fold hollow sites $1.25 \pm 0.10 \&$ above the platinum surface. This result is consistent with the carbon-metal spacings observed in other systems with carbon bound in three-fold hollow sites on transition metal surfaces. The results of the LEED/BSN analysis are shown to be consistent with a quasi-kinematical analysis of the specular beam intensities.

This study has not described all the structural details of graphite on platinum (111). The exact distribution and density of the intercalated carbon atoms is not known, and the LEED results do not distinguish between the fec and the 
hcp hollow sites. The intercalated atoms may occupy either of both sites. Also, the preferential angular orientation of graphite domains on the platinum (111) surface is not known. However, these results should describe the major structural features of the graphite-platinum (111) system. 


\section{References}

1. Ordering in Two Dimensions, p. 17, Elsevier, New York, 1980.

2. G. L. Price and J. A. Venables, Physics Letters, vol. 47A, p. 9, 1974.

3. C. G. Shaw, S. C. Fain, Jr., M. D. Chinn, and M. F. Toney, Surface Science, vol. 97, p. 128, 1980.

4. M. D. Chinn and S. C. Fain, Jr., Physical Review Letters, vol. 39, p. 146, 1977.

5. S. C. Fain, Jr., M. D. Chinn, and R. D. Diehl, Physical Review B, vol. 21, p. 4170, 1980.

6. R. D. Diehl and S. C. Fain, Jr', Strface Science, vol. 125, p. 116, 1983.

7. J. Perdereau and G. E. Rhead, Surface Science, vol. 24, p. 555, 1971.

8. P. W. Palmberg, Surface Science, vol. 25, p. 104, 1971.

9. M. A. Chesters, M. Hussain, and J. Pritchard, Surface Science, vol. 35, $\rho$. 161, 1973.

10. M. A. Chesters, M. Hussain, and J. Pritchard, Surface Science, vol. 54, p. $687,1976$.

11. A. Ignatiev, T. N. Rhodin, and S. Y. Tong, Surface Science, vol. 42, p. 37, 1974 .

12. M. F. Bridge, R. A. Marbrow, and R. M. Lambert, Surface Science, vol. 57, p. $415,1976$. 
13. P. I. Cohen, J. Unguris, and M. B. Webb, Surface Science, vol. 58, p. 429, 1876.

14. L. W. Bruch, P. I. Cohen, and M. B. Webb, Surface Science, vol. 59, p. 1, 1976.

15. N. Stoner, M. A. Van Hove, S. Y. Tong, and M. B. Webb, Physical Review Letters, vol. 40, p. $243,1978$.

16. L. L. Atanososka, J. C. Buchholtz, and G. A. Somorjai, Surface Science, vol. 72, p. $189,1978$.

17. J. Unguris, L. W. Bruch, E. R. Moog, and M. B. Webb, Surface Science, vol. 109 , p. 522, 1881.

18. Z. P. Hu and A. Ignatiev, Physical Reriew B, vol. 30, p. 4856, 1984.

19. Z. P. Hu, N. J. Wu, and A. Ignatiev, to be published.

20. W. S. Yang and F. Jona, Surface Science, vol. 109, p. L505, 1981.

21. E. L. Garfunkel and G. A. Somorjai, Surface Science, vol. 115, p. 441, 1982.

22. M. A. Van Hove, R. J. Koestner, P. C. Stair, I. P. Bibérian, L. L. Kesmodel, I. Bartos, and G. A. Somorjai, Surface Science, vol. 103, p. 218, 1981.

23. K. Baron, D. W. Blakeley, and G. A. Somorjai, Surface Science, vol. 41, p. $45,1974$.

24. J. L. Gland and G. A. Somorjai, Surface Science, vol. 41, p. 387, 1974.

25. B. Lang, Surface Science, vol. 53, p. 317, 1975. 
26. J. G. McCarty and R. J. Madix, Journal of Catalysis, vol. 38, p. 402, 1975.

27. D. G. Castner, B. A. Sexton, and G. A. Somorjai, Surface Science, vol. 71, p. $519,1078$.

28. F. P. Netzer and M. Prutton, Surface Science, vol. 52, p. 505, 1972.

28. S. Ekelund and C. Leygraf, Surface Science, vol. 40, p. 179, 1973.

30. C. Leygraf and S. Ekelund, Surface Science, vol. 40, p. 609, 1973.

31. W. N. Unertl and J. M. Blakeley, Surface Science, vol. 69, p. 23, 1973.

32. P. H. Holloway and J. B. Husdson, Surface Science, vol. 43, p. 141, 1874.

33. N. R. Avery, Surface Science, vol. 41, p. 533, 1974.

34. A. Glachant, J. P. Coulomb, and J. P. Bibérian, Surface Science, vol. 59, p. $619,1876$.

35. P. Légaré, G. Marie, B. Carriere, and J. P. Deville, Surface Science, vol. 68, p. $348,1877$.

36. R. Bastasz, C. A. Colmenares, R. L. Smith, and G. A. Somorjai, Surface Science, vol. 67, p. 45, 1977.

37. T. W. Haas, J. T. Grant, and G. T. Dooley, III, Journal of Applied Physics, vol. 43, p. 1853, 1972.

38. L. Papagno and L. S. Caputi, Physical Review B, vol. 29, p. 1483, 1984.

39. M. P. Hooker and G. T. Grant, Surfice Science, vol. 62, p. 21, 1977.

40. G. A. Somorjai, Chemistry in Two Dimensions: Surfaces, Cornell University Press, Ithaca, New York, 1981. 
41. S. M. Davis and G. A. Somoriai, Sournal of Catalysib, vol. 65, p. 78, 1980.

42. M. A. Van Hove and S. Y. Tong, Surface Science, vol. 52, p. 673, 1975.

43. J. H. Onuferko, D. P. Woodruff, and B. W. Holland, Surface Science, vol. 87, p. $357,1979$.

44. K. H. Rieder and H. Wilsch, Surface Science, vol. 131, p. 245, 1983.

45. T. S. Rahman and H. Ibach, Physical Review Letters, vol. 54, p. 1933, 1985.

46. R. Rosei, M. de Crescenzi, F. Sette, C. Quarvesima, A. Savoia, and P. Perfetti, Physical Review B, vol. 28, p. 1161, 1983.

47. A. E. Morgan and G. A. Somorjai, Surface Science, vol. 12, p. 405, 1968.

48. H. B. Lyon and G. A. Somorjai, Journal of Chemical Physics, vol. 46, p. $2530,1967$.

49. J. W. May, Surface Science, vol. 17, p. 267, 1969.

50. J. P. Bibérian and G. A. Somorjai, Applications of Surface Science, vol. 2, p. $352,1979$.

51. M. A. Var Hove, R. F. Lin, and G. A. Somorjai, Physical Review Letters, vol. 51, p. 778, 1983.

52. Hu Zi-Pu, D. F. Ogletree, M. A. Van Hove, and G. A. Somorjai, Surface Science, to be published.

53. J. B. Pendry, Low Energy Electron Difraction, Academic Press, London, 1974. 
54. D. L. Adams, H. B. Nielsen, and M. A. Van Hove, Physical Review B, vol. 20 , p. $4789,1979$.

55. T. C. Ngoc, M. G. Lagally, and M. B. Webb, Surface Science, vol. 35, p. 117, 1973.

56. S.-W. Wang, unpublished.

57. L. J. Clarke, Surface Crystallography; An Introduction to Low Energy Electron Diffaction, Wiley-Interscience, Chichester, 1985.

58. L. L. Kesmodel, L. H. Dubois, and G. A. Somorjgi, Journal of Chemical Physies, vol. 70, p. $2180,1879$.

59. R. J. Koestner, M. A. Van Hove, and G. A. Somorjai, Surface Science, vol. 121, p. 321, 1982.

60. R. J. Koestner, M. A. Van Hove, and G. A. Somorjai, to be published.

61. M. A. Van Hove, R. F. Lin, G. A. Blackman, and G. A. Somorjai, Acta Crystallographica, to be published.

62. J. J. Barton, to be published. 


\section{Figure Captions for Chapter 12}

12.1 Typical LEED patterns for graphite layers on $\mathrm{Pt}(111)$. Graphite domains exhibit a number of different angular orientations with respect to the platinum substrate. The observed orientation has no obvious relation to the conditions under which the graphitic layer was formed.

(a) The bright ring segments indicate two preferred domain orientations for graphite on $\mathrm{Pt}(111)$. This is the orientation that was observed most often. Weak streaks near the bright center (specular) beam are $\mathbf{g}+\mathbf{G}$ combination beams. These beams show the relative weakness of secondand higher-order scattering. The $\mathrm{Pt}(111)$ crystal was exposed to $12 \mathrm{~L}$ of propylene at $1100 \mathrm{~K}(\mathrm{C} / \mathrm{Pt}$ Auger ratio is 3.3).

(b) Several graphite ring features are shown. Three domain orientations dominate this image. The $\operatorname{Pt}(111)$ crystal was exposed to $\theta \mathrm{L}$ of propylene at $1100 \mathrm{~K}(\mathrm{C} / \mathrm{Pt}$ Auger ratio is 3.5).

(c) Two different domain orientations on $\mathrm{Pt}(111)$. The crystal was saturated with propylene at room temperature and then annealed to 1150 K.

(d) Several graphite domains on $\mathrm{Pt}(111)$. Some $\mathbf{g}+\mathbf{G}$ multiple scattering features are visible near the substrate beams. The $\mathrm{Pt}(111)$ crystal was exposed to $25 \mathrm{~L}$ of benzene vapor at room temperature, annealed at 1100 $\mathrm{K}$, exposed to an additional $25 \mathrm{~L}$ of benzene at room temperature, then annealed a second time at $1100 \mathrm{~K}$ for $180 \mathrm{~s}$. This picture was taken after recording the LEED I-V data analyzed in this chapter (C/Pt Auger ratio is 
1.4).

12.2 Auger uptake curve for graphite as a function of exposure to propylene vapor. The crystal was held at $1100 \mathrm{~K}$ throughout the experiment. The exposure was increased in increments with Auger measurements after each additional dose.

12.3 (a) The basic beam types for an incommensurate overlayer.

(b) The corresponding reciprocal lattice vectors in momentum space for one overlayer domain. A single combination beam $\mathbf{g}+\mathbf{G}$ is shown.

12.4 The scattering paths which contribute to the different types of LEED beams are listed in orders of overlayer-substrate scattering as in section 4.9. The rows are different types of beams, and the terms in the columns are $1^{\text {st }}$ through $4^{\text {th }}$ order in overlayer-substrate scattering. The beam set neglect (BSN) approximation for incommensurate overlayers includes the first two orders (boxed).

12.5 Specular beams for the clean and graphite covered $\mathrm{Pt}(111)$ surface compared for $\theta=10^{\circ}, 15^{\circ}$ and $20^{\circ}$, all at $\phi=180^{\circ}$. Note the significant changes in the I-V curves due to the graphite overlayer. These I-V curves have not been normalized to constant beam current so the high energy structure is visible. The beam current is approximately linearly proportional to voltage, reaching $\sim 6 \mu \mathrm{A}$ at $400 \mathrm{eV}$.

12.6 Non-specuiar beams for the clean and graphite covered Pt(111) surface compared for $\theta=10^{\circ}, 15^{\circ}$ and $20^{\circ}$, all at $\phi=180^{\circ}$. Note the general 
similarity of the beams except an for overall attenuation for the graphite covered surface. These curves have not been current normalized.

12.7 The value of $r_{5}$ as a function of overlayer-substrate spacing at $\theta=15^{\circ} \mathrm{cal}-$ culated over different energy ranges. The top three curves are displaced upward. The minima around $3.7 \AA$ is most stable when the data range used to calculate $r_{5}$ is changed.

12.8 Specular beam $r_{5}$ contour plot at $\theta=15^{\circ}$ for a graphite overlayer and intercalated carbon in hcp-hollow sites.

12.9 An incommensurate graphite overlayer with intercalated carbon atoms in hollow sites. Optimum layer spacings are shown.

12.10 The experimental specular beam I-V curve at $\theta=15^{\circ}$ is compared to near-optimum theoretical I-V curves for the three different model geometries.

12.11 Specular beam intensities (current normalized) at $\theta=10^{\circ}, 15^{\circ}$ and $20^{\circ}$ plotted as a function of momentum transfer on a logarithmic scale. The upper curves are displaced upward by a factor of 10 . The Bragg peaks are indicated.

12.12 Momentum transfer average for clean and graphite covered platinum plotted on a logarithmic scale. The upper curve is displaced upward by a factor of 10. The Bragg peaks are indicated.

12.13 The ratio of the constant momentum transfer average for the specular beams of graphite covered and clean platinum, as a function of momentum 
transfer.

12.14 The optical Fon rier transform of the intensity ratio from figure 12.13 plotted as a function of distance. The numbered arrows mark multiples of the bulk platinum (111) inter-layer spacing (2.26 $\AA$ ). The arrow labeled "d" is the graphite-platinum layer spacing of $3.70 \AA$, and the arrow labeled "d c" is at $3.70 \AA-2.26 \AA=1.44 \AA$. The peaks labeled by arrows are predicted by Eq. 12.5 . 


\section{LEED Patterns of Graphite on Pt(111)}

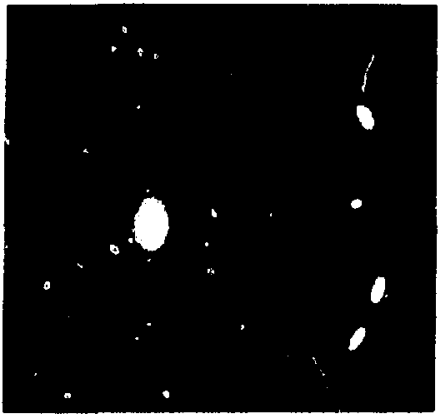

a) $46 \mathrm{eV}$ at $-10^{\circ}$

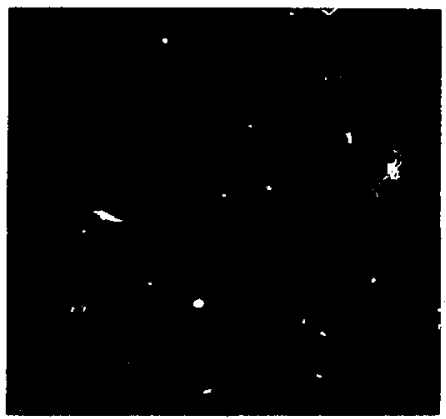

b) $64 \mathrm{eV}$ at $35^{\circ}$



c) $55 \mathrm{eV}$ at $0^{\circ}$



d) $45 \mathrm{eV}$ at $11^{\circ}$

XBB 864-3003 
548

Graphite Uptake Curve

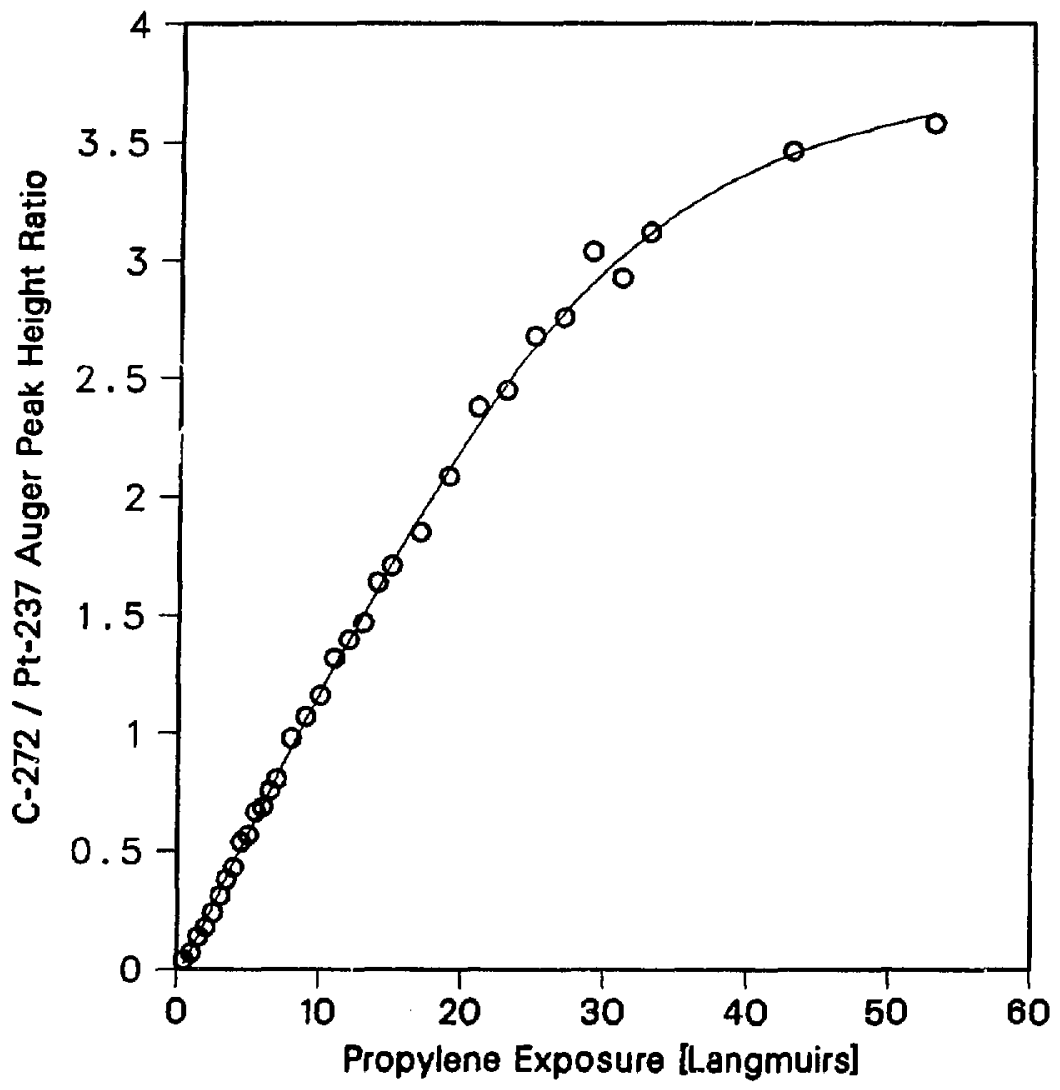

XBL $8611-4242$

Floure 12.2 


\section{Incommensurate beams and matterns}

a) Beam types

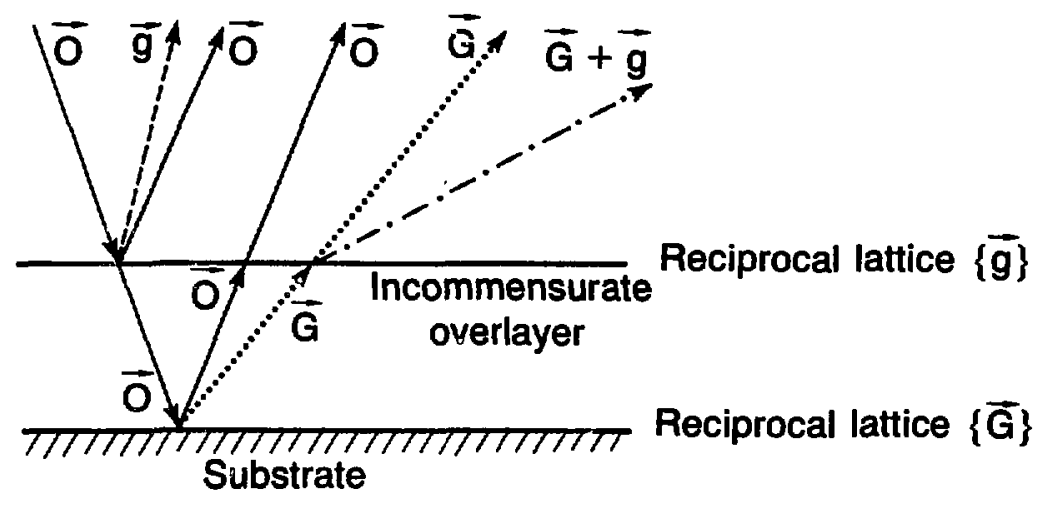

b) Reciprocal lattices

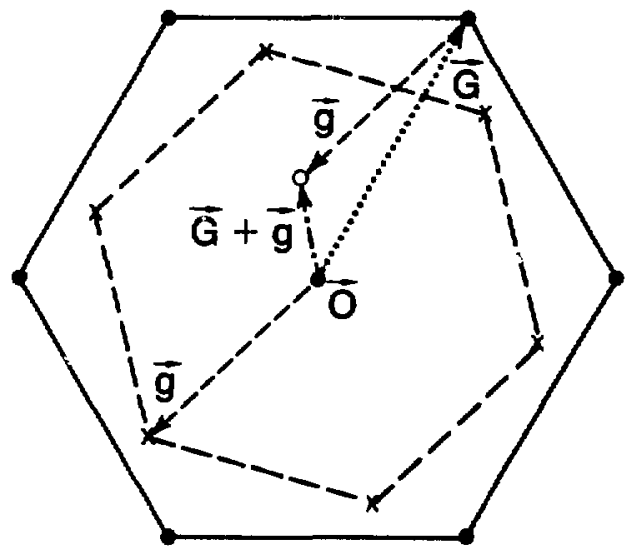


Incommensurate overlayers:

beam type vs. order of scattering

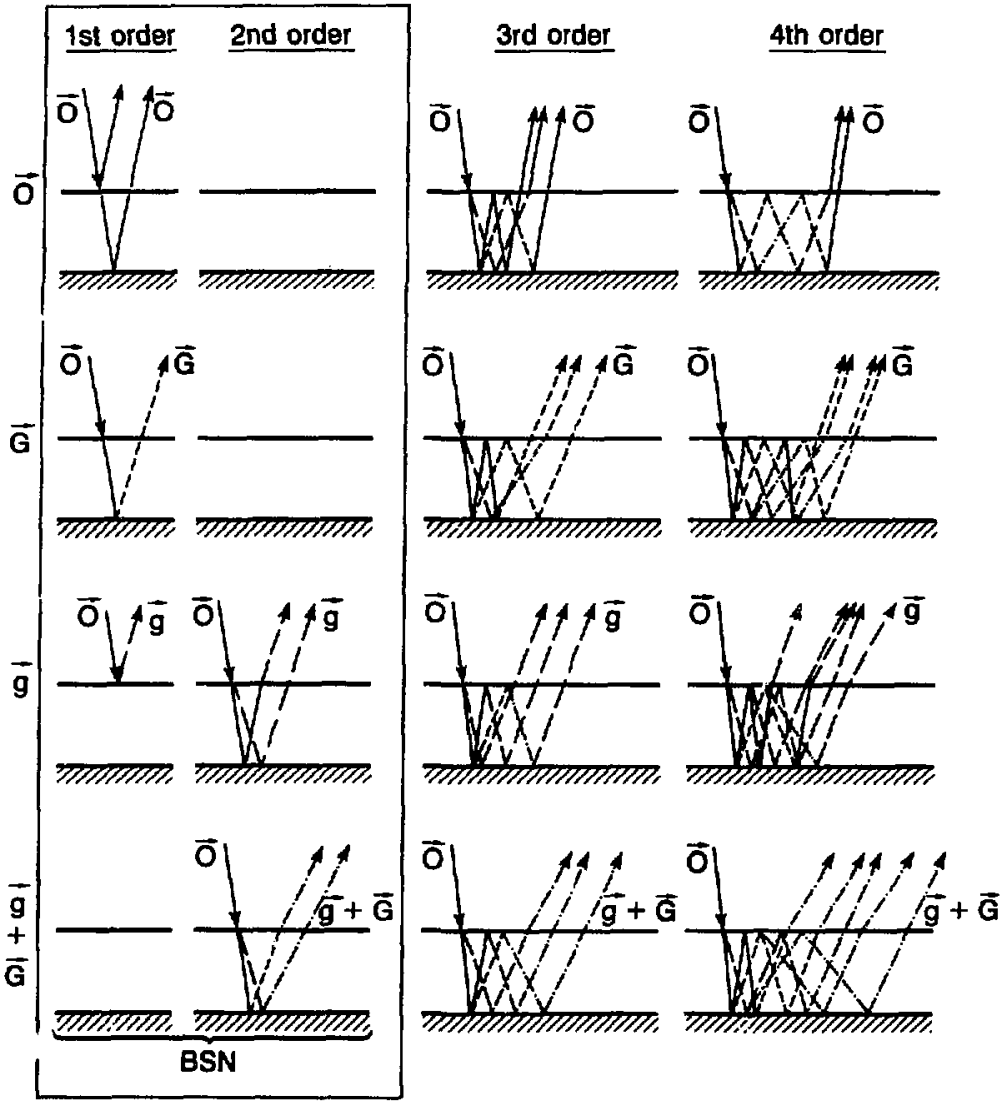


549

\section{Specular Beam Data at $\theta=10$}

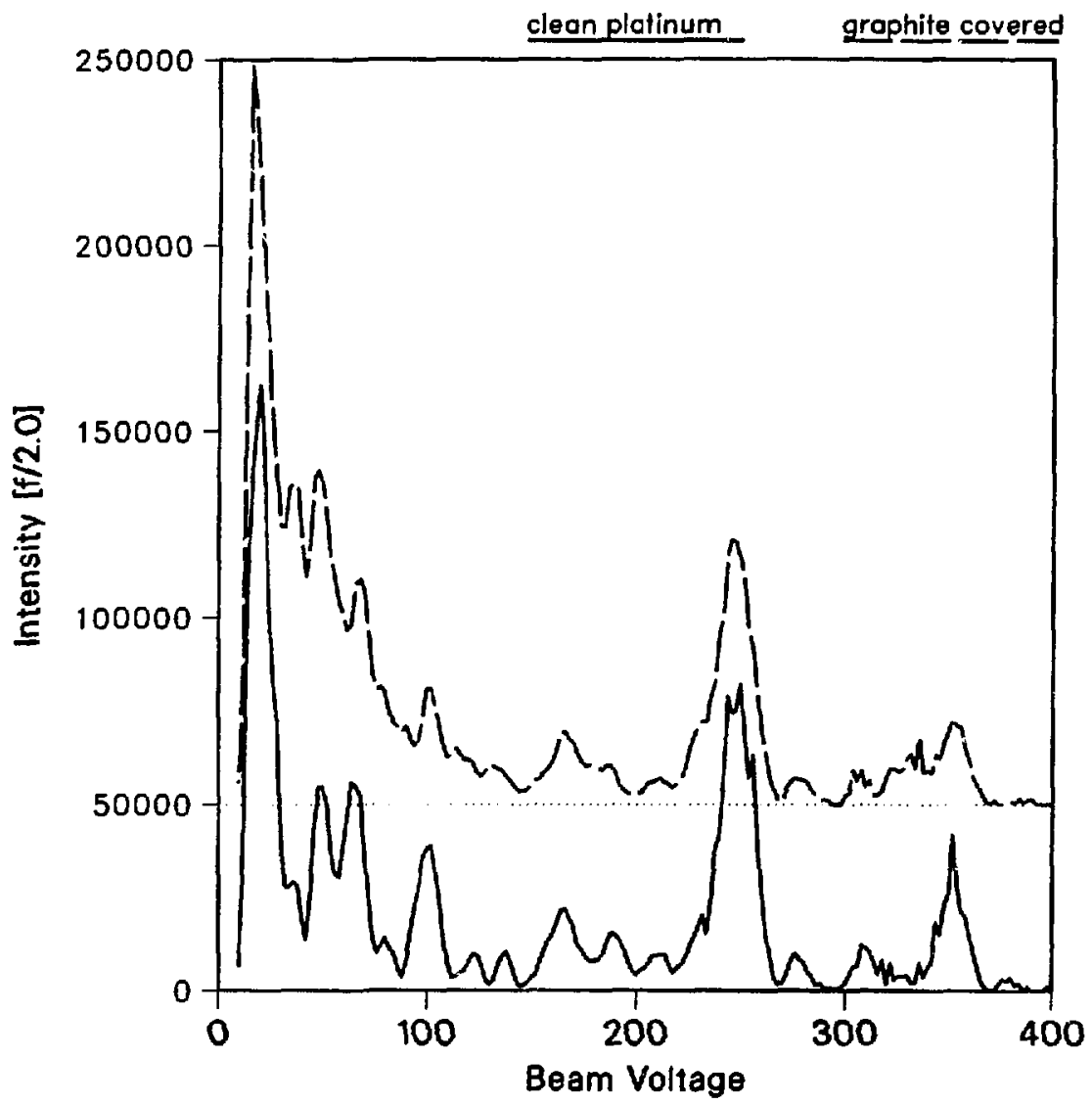

XBL $8611-4250$

Figure 12.5(a) 
Specular Beam Data at $\theta=15$

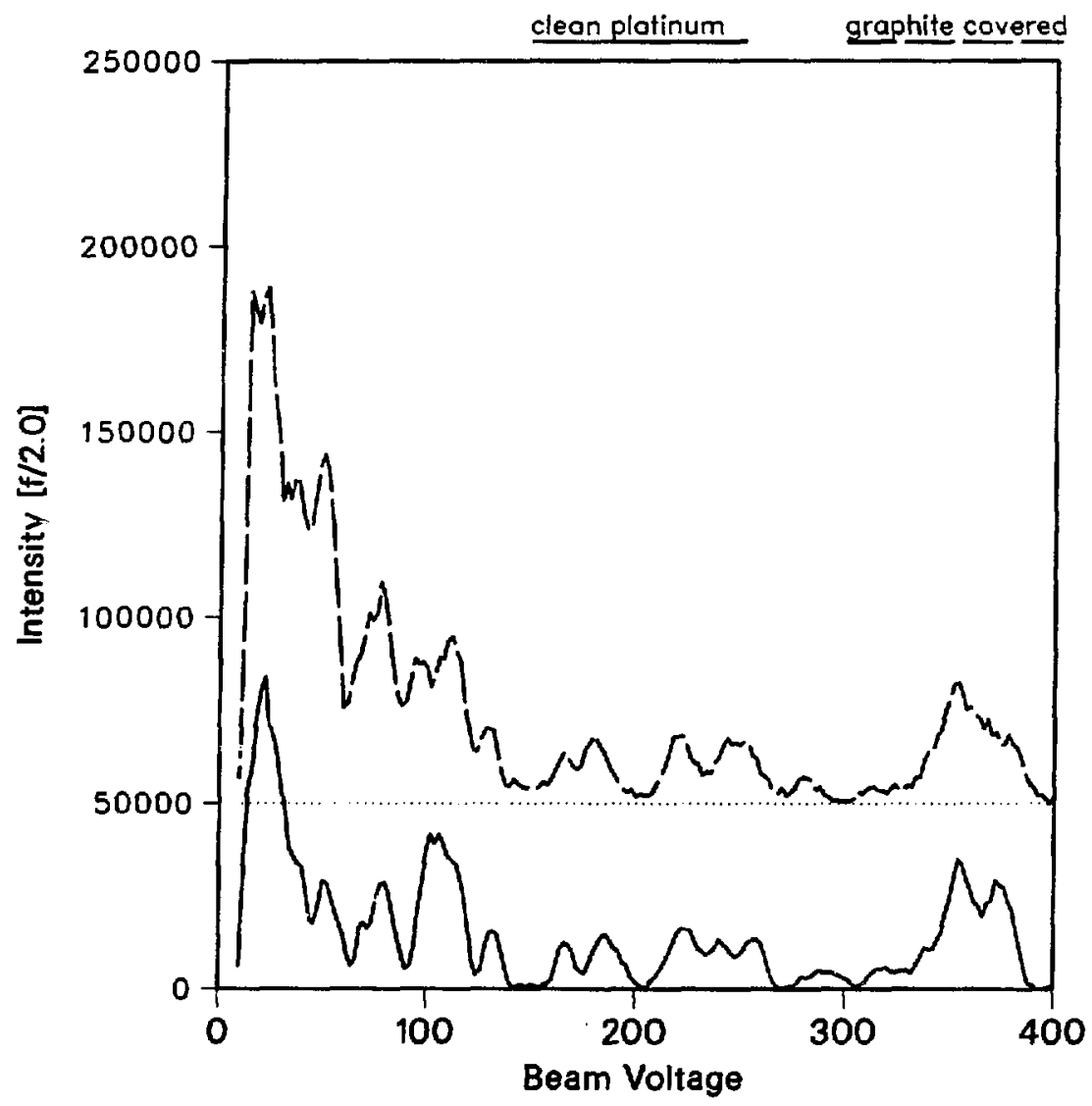

XBL 8611-4249

FIgure 12.5(b) 


\section{Specular Beam Data at $\theta=20$}

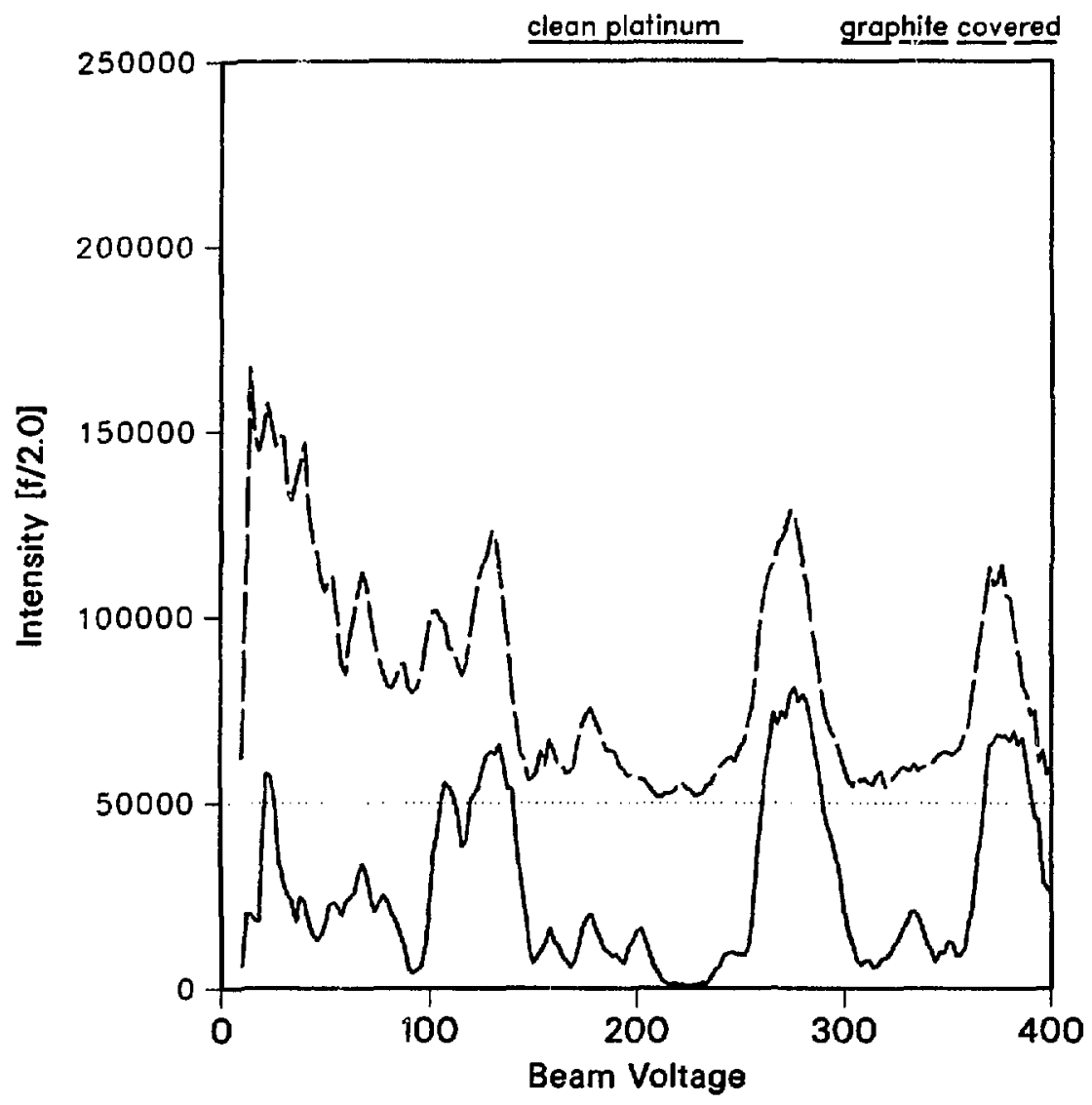

XBL 8611-4251

Figure 12.5(c) 


\section{Nonspecular Beams at $\theta=10$}

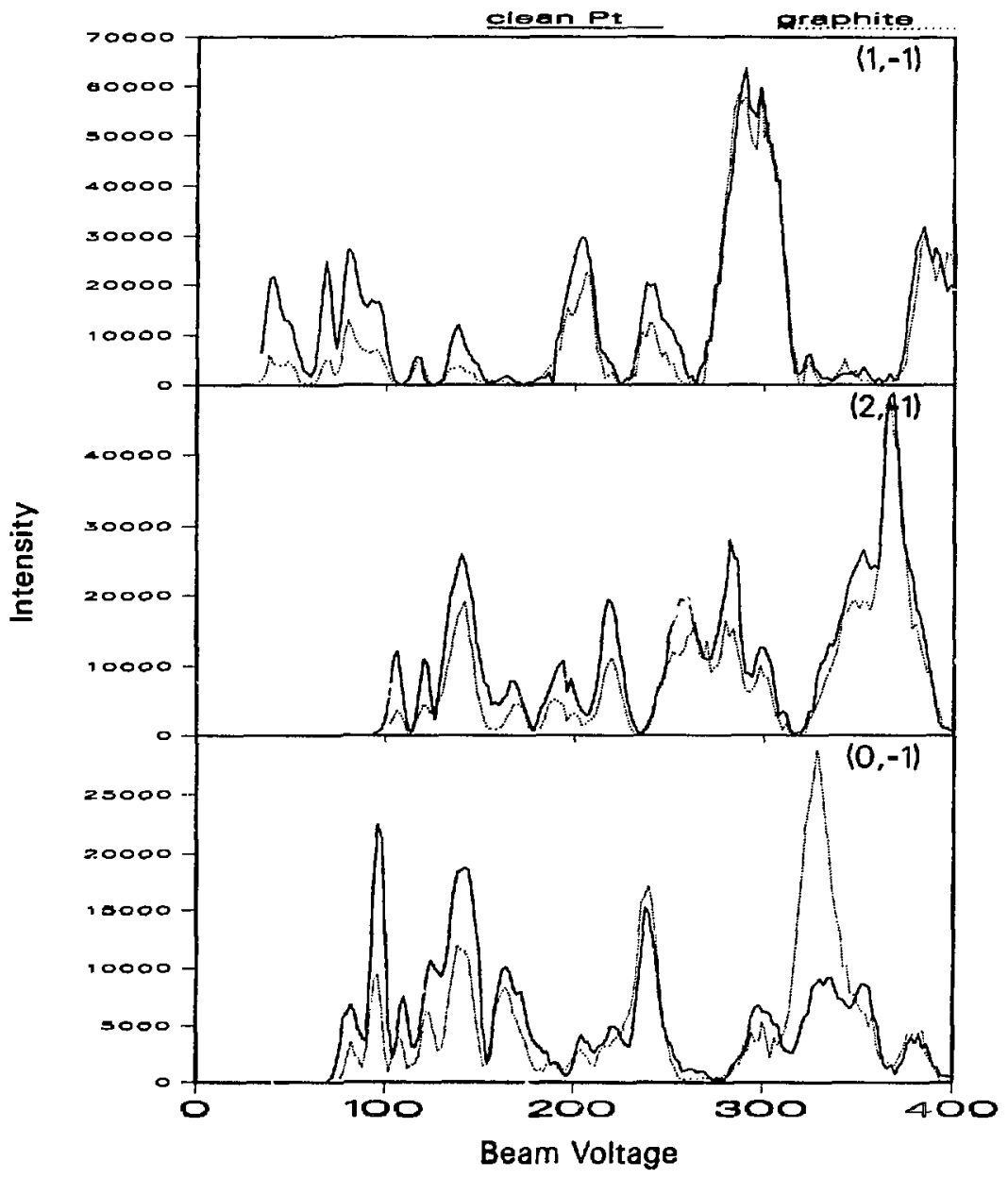

XBL 8612-4857

Flgure 12.6(a) 
553

\section{Nonspecylar Beams at $\theta=15$}

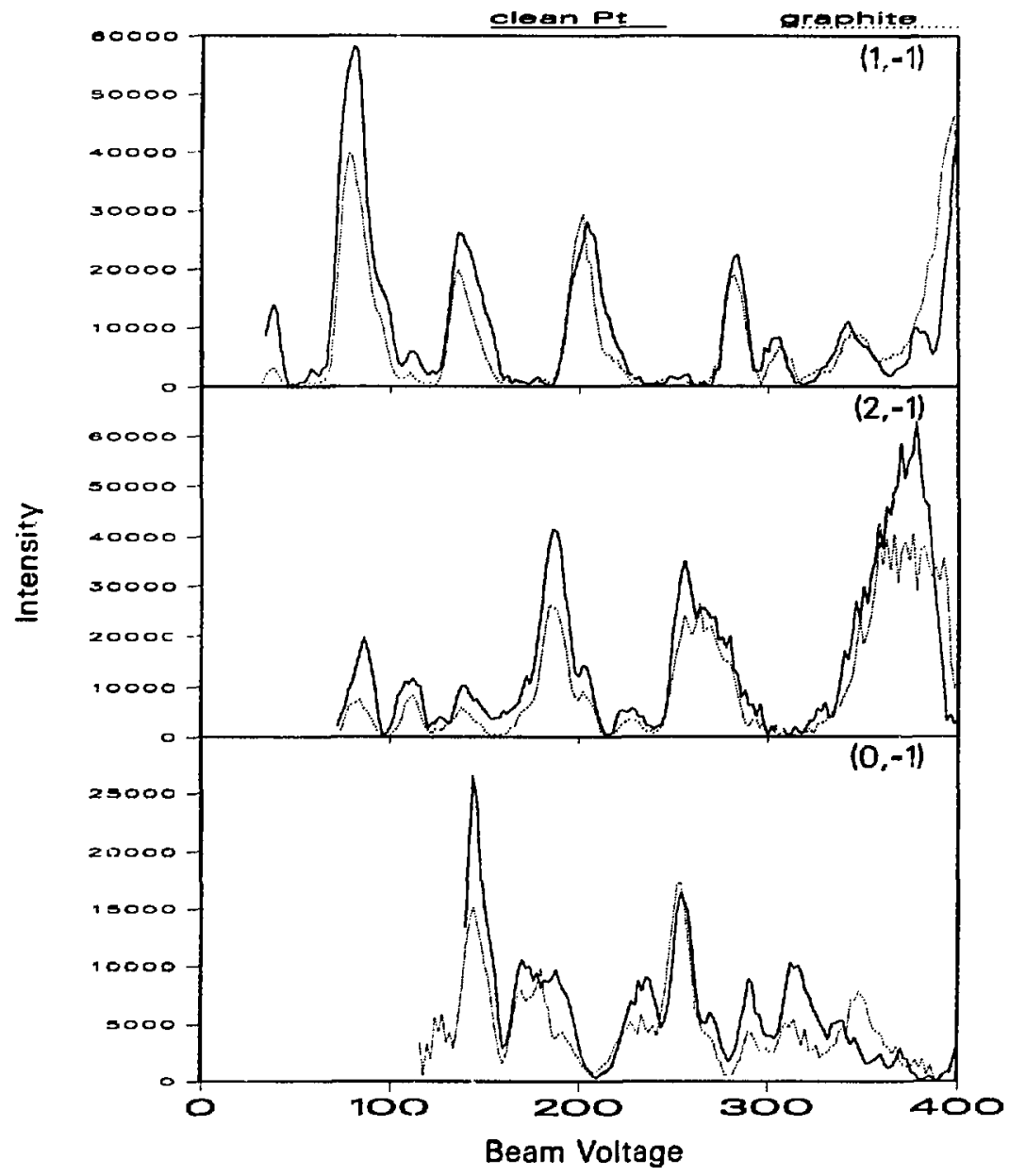

XBL $8612-4856$

Figure 12.6(b) 


\section{Nonspecular Beams at $\theta=20$}

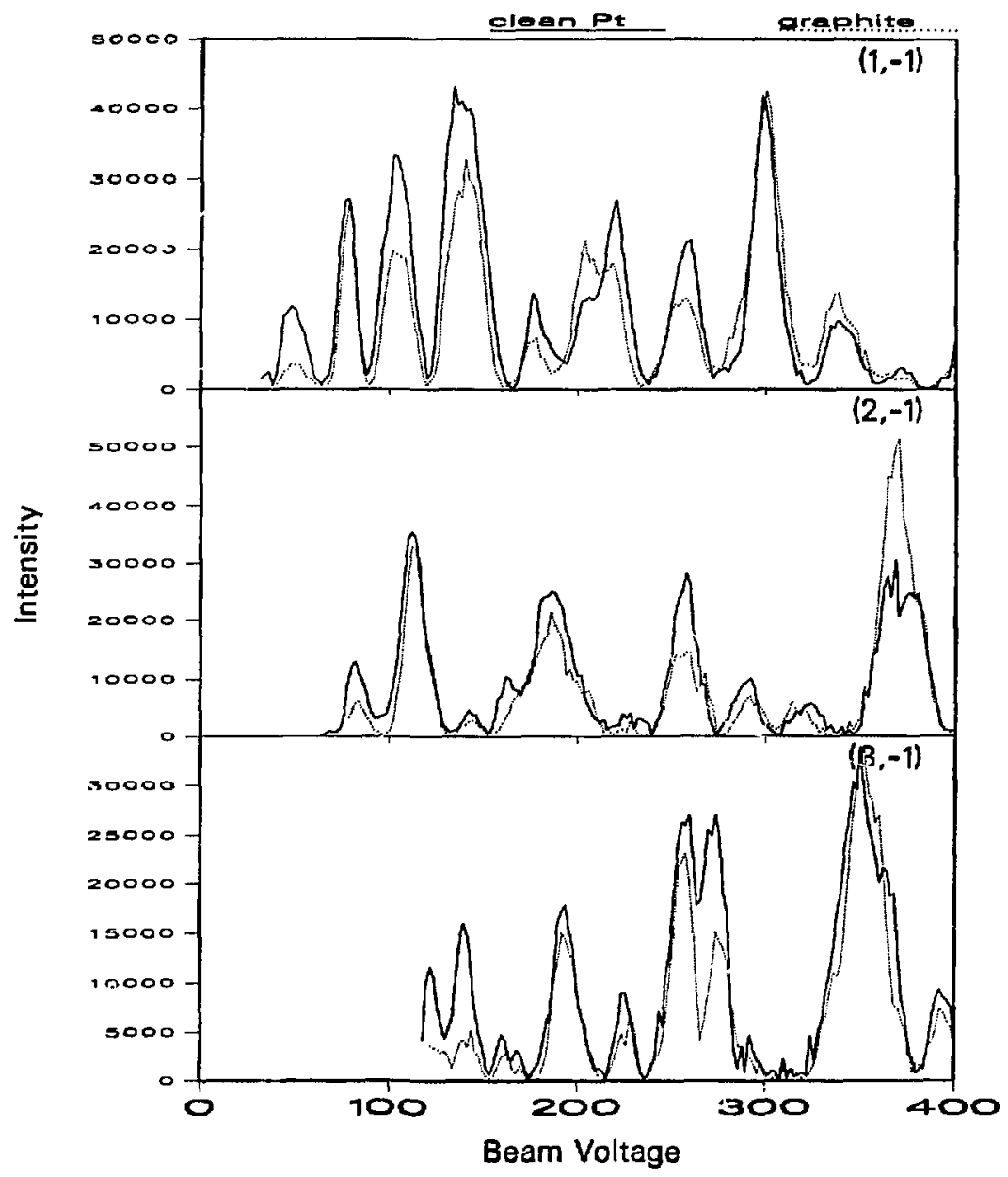

XBL $8612-4855$

Figure 12.8(c) 
Graphite/Pt (111)

$$
\begin{gathered}
\theta=15^{\circ}, \varphi=150^{\circ}, T=300 \mathrm{~K} \\
(00) \text { beam }
\end{gathered}
$$



XBL B512.12789

Figure 12.7 
Graphite/C/Pt (111) R-factor plot

(00) beam $\theta=15^{\circ}, \varphi=150^{\circ}$



XBL 8512-12791

Figure 12.8 

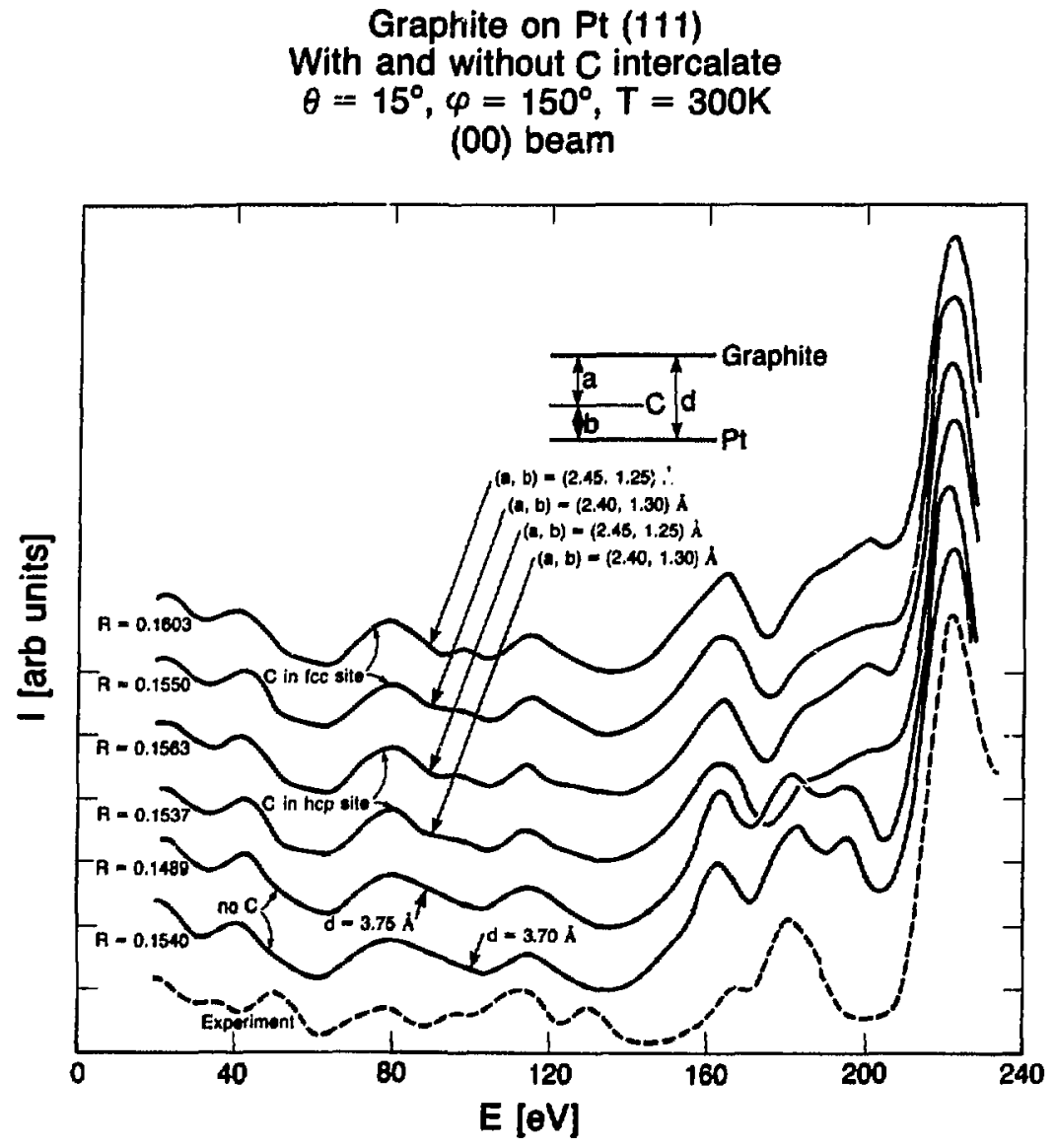

X8L 8512.12780

Figur 12.0 


\section{Incommensurate graphite on Pt (111) with $\mathrm{C}$ intercalate}

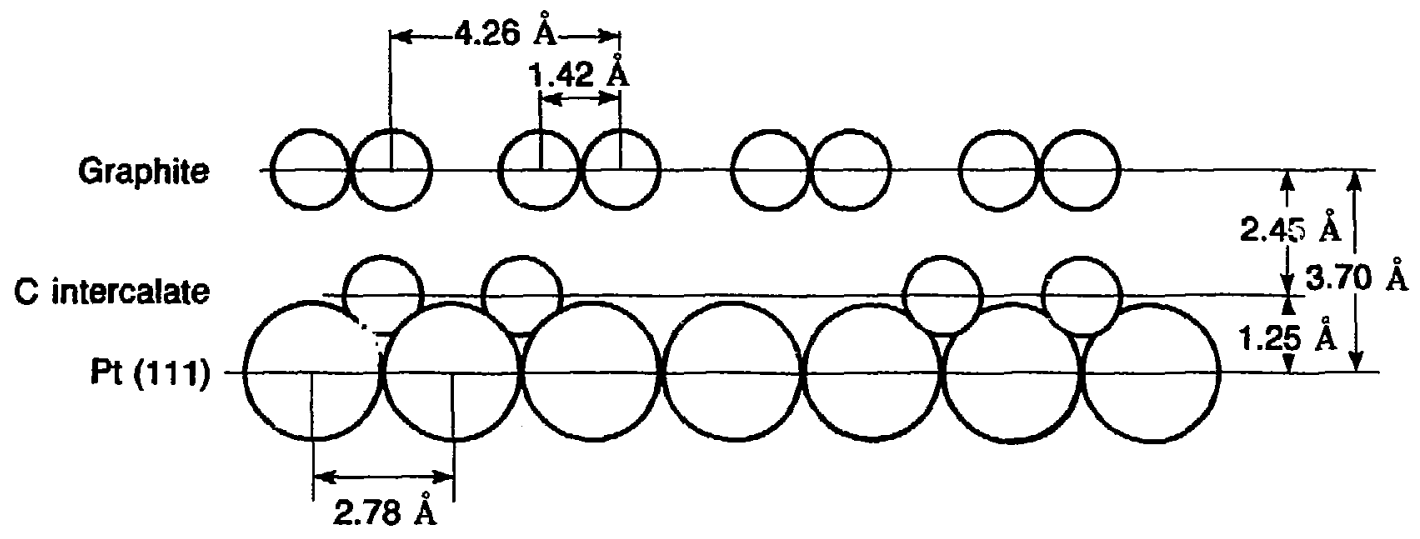

Figure 12.10 


\section{Clean Platinum Specular Beam Intensity}
$\theta=10$
$\theta=15$
$\underline{\theta=20}$



XBL 8611-4241

Figure 12.11 


\section{Specular Beam Momentum Transfer Average}

clean piatinum -

graphite covered

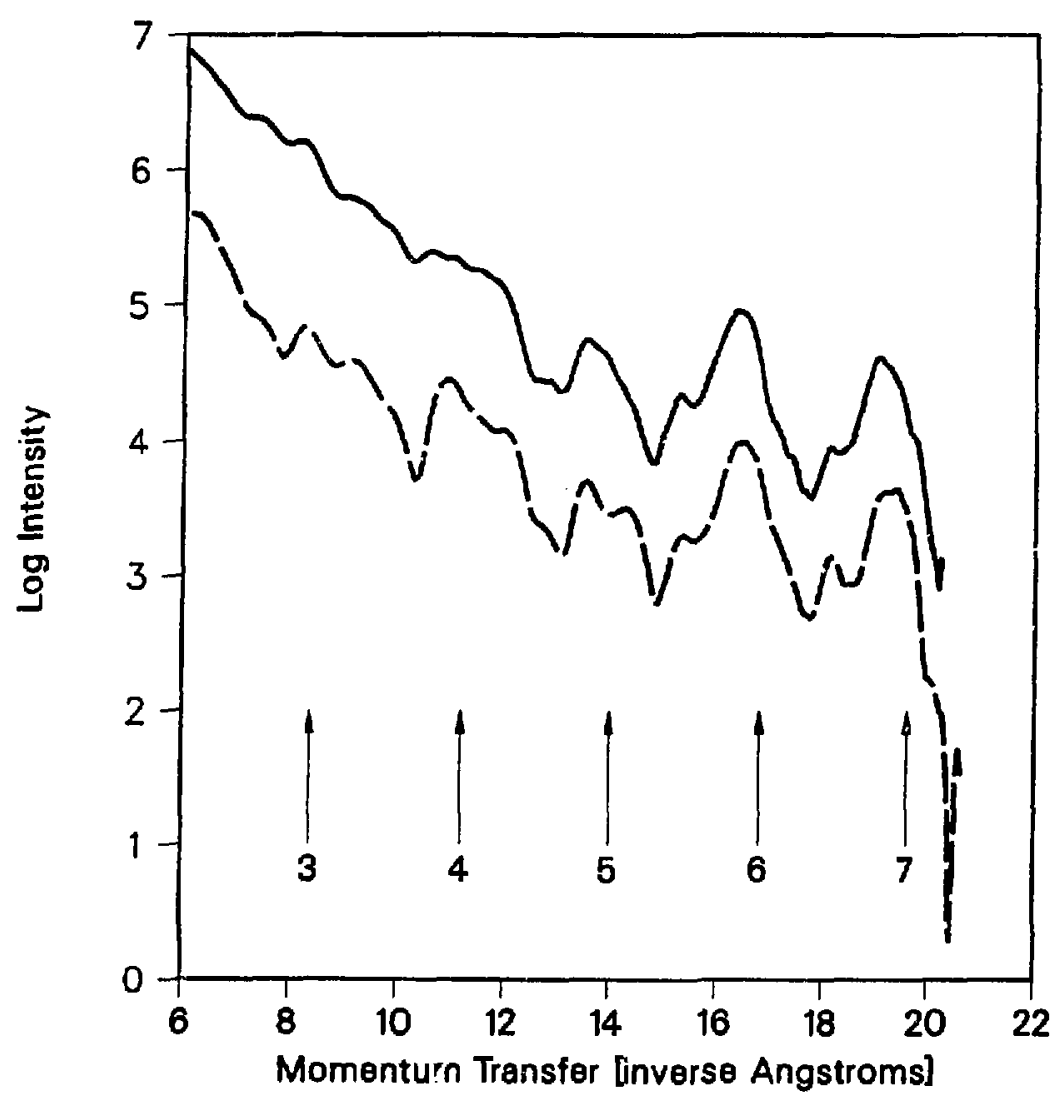

XBL $8611-4240$

Figure 12,12 


\section{Specular Beam Averaged Intensity Ratio}



XBL 8611-4239

Figure 12.13 
562

\section{Optical Fourier Transform of Intensity Ratio}

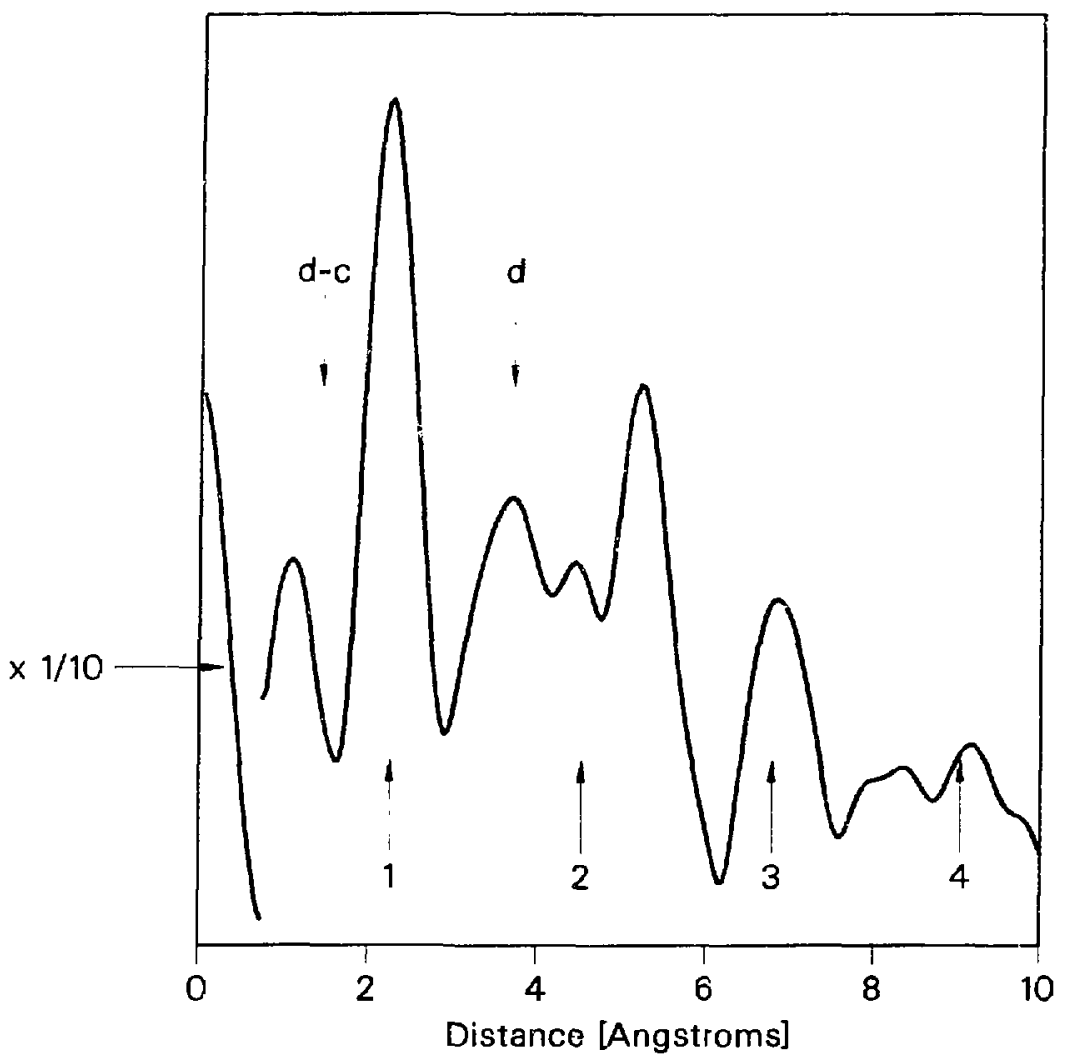

XBL $8611-4248$

Figure 1214 


\section{Chapter 13 \\ The Structure of the $(\sqrt{3} \times \sqrt{3}) R_{30}$ reconstruction of $\alpha$-CuAl (111)}

\subsection{Introduction}

The structure of alioy surfaces has only recently begun to be investigated. Several groups have undertaken studies of alloy surface structures: on NiAl (110);, ${ }^{1,2}$ on $\mathrm{Cu}_{3} \mathrm{Au}(100)$ and (111) and on $\mathrm{Ni}_{3} \mathrm{Al}(100),(110)$ and (111);,4 and on $\mathrm{Pt}_{3} \mathrm{Ti}(100){ }^{5}$ All of these stoichiometric alloys have an ordered bulk structure, and the surface order reflects the bulk structure. The case of the copper aluminum alloy is different. The $\alpha$ phase is a random substitutional alloy of aluminum in the copper lattice. A $(\sqrt{3} \times \sqrt{3}) R 30^{\circ}$ LEED pattern has been observed on the (111) surface of $\alpha-\mathrm{CuAl}^{6,7,8}$ for aluminum concentrations $\geq 9$ atomic $\%$. This surface superlattice does not reflect long-range order present in the bulk alloy. $\alpha-\mathrm{CuAl}$ is the first case where the structure of a reconstructed alloy surface has been investigated. ${ }^{9}$

\subsection{The $\alpha$-CuAl alloy system}

In bulk $\alpha$-CuAl the aluminum atoms occupy random substitutional sites without long range order. The $\alpha$ phase of the copper aluminum alloy exists between 0.0 and 19.4 atomic $\%$ aluminum atoms in the copper crystal lattice. ${ }^{10}$ Over this range the lattice parameter of the copper fec crystal changes by no more than $1 \%$ 
A number of investigations have been made of the short-range order in $\alpha$ CuAl using diffuse $x$-ray scattering measurements. ${ }^{11,12}$ These studies show a significant degree of short-range order. In particular, there are almost no aluminum nearest neighbor pairs, and a distorted tetrahedral arrangement of four next-nearest neighbor aluminum atoms is common. ${ }^{11}$ Aluminum atoms were found to occupy next-nearest neighbor sites much more often than would be predicted by a random distribution of aluminum atoms, and arrangements of aluminum atoms in (111) planes within the bulk crystal were common. The arrangement of three next-nearest neighbor aluminum atoms forming a triangle about a copper atom in a (111) plane was found to occur seven times more often than predicted for a random substitutional alloy in a $\alpha$-CuAl crystal with 9.6 atomic $\%$ aluminum. ${ }^{12}$

\subsection{Surface characterization}

The (100) and (111) surfaces of various $\alpha$-CuAl alloy crystals have been characterized by Baird et al. ${ }^{13,14}$ The surface composition of the (100) and (111) surfaces was measured with Auger electron spectroscopy (AES) and x-ray photoemission spectroscopy (XPS) for two $\alpha$-CuAl crystals with different aluminum concentrations, and no evidence was found for surface segregation. ${ }^{13}$ This is consistent with theoretical calculations for this system. ${ }^{15}$ 


\begin{tabular}{|c|c|c|c|}
\hline \multicolumn{4}{|c|}{ Surface Aluminum Concentration } \\
\hline $\begin{array}{c}\text { bulk } \\
\text { composition }\end{array}$ & (100) & (111) & method \\
\hline $5.7 \%$ & $\begin{array}{l}5.6 \% \\
6.2 \%\end{array}$ & $\begin{array}{l}5.9 \% \\
6.4 \%\end{array}$ & $\begin{array}{l}\text { AES } \\
\text { XPS }\end{array}$ \\
\hline $12.5 \%$ & $\begin{array}{l}12.4 \% \\
11.9 \%\end{array}$ & $\begin{array}{l}14.0 \% \\
12.0 \%\end{array}$ & $\begin{array}{l}\text { AES } \\
\text { XPS }\end{array}$ \\
\hline
\end{tabular}

There is some evidence for an increased concentration of aluminum at the surface, even though there is no evidence for net surface segregation. The low energy $(\sim \omega 0 \mathrm{eV})$ copper $M V V$ and aluminum $L V V$ Auger lines suggest that the aluminum concentration on the (111) surface is approximately twice that of the (100) surface. This cannot be calibrated since the aluminum $L V V$ transition splits into two lines when alloyed with copper. One explanation for these observations is that aluminum is redistributed within the topmost layers without net segregation. The low energy Auger lines are near the minimum value of the electron mean-free path, so they are most sensitive to the top layer, while electrons from the higher energy XPS lines $(\sim 120 \mathrm{eV})$ and the copper $L M M$ Auger line $(918 \mathrm{eV})$ or aluminum $K_{L} L L$ Auger line $(1392 \mathrm{eV})$ have a longer mean-free path and penetrate more deeply into the surface. 
The $\alpha$-CuAl (111) surface reconstruction is probably due to an order-disorder phase transition. The surface symmetry as seen in the LEED pattern transforms reversibly from $(\sqrt{3} \times \sqrt{3}) \mathrm{R} 30^{\circ}$ to $(1 \times 1)$ at $570 \mathrm{~K}$. Measurements of the LEED beam intensity as a function of temperature for a superlattice beam show a clear inflection point at $570 \mathrm{~K}$ for a 12.5 atomic $\% \alpha$-CuAl crystal, while the substrate beams show snly a smooth drcp in intensity with increasing temperature. ${ }^{16}$ There is no sign of hysteresis in these intensity measurements. (The intensity measurements were made with a spot photometer at a fixed angular aperture, with no corrections for temperature-dependent changes in the spot widths.) Careful measurements oi whe low energy copper and aluminum Auger doublets (55 to $70 \mathrm{eV}$, figure 13.1 ) above (690 K) and below (480 K) the phase transition temperature are almost identical, ${ }^{16}$ which suggests that a temperature-dependent change in the aluminum surface concentration is not the duiving force for the $(\sqrt{3} \times \sqrt{3}) \mathrm{R} 30^{\circ}$ $\rightarrow(1 \times 1)$ phase transition, but that this is a true order-disorder phase transition.

When the (111) surface of the 16 atomic $\% \alpha$-CuAl crystal was argon ion sputtered at room temperature there was a significant decrease in the intensity of the low energy aluminum $L V V$ Auger transition. After annealing the surface aluminum concentration increases toward the equilibrium value, as monitored by the $L V V$ Augcr reak. Once this equilibrium is reached, thermal treatments cause no further change in the surface aluminum concentration. This is further evidence for an increased concentration of aluminum in the topmost layer of the surface. The copper-aluminum system is a compound-forming system with a heat of formation for $\alpha-\mathrm{CuAl}$ of $-2 \mathrm{Kcal} / \mathrm{mol}^{17}$ This suggests that aluminum is more 
likely to be in substitutional sites within the first copper layer, rather than in adsorption sites outside of the first copper layer.

\subsection{Surface models}

Five surface model geometries were considered for the $(\sqrt{3} \times \sqrt{3}) \mathrm{R} 30^{\circ}$ reconstruction of the $\alpha$-CuAl (111) surface, two involving an aluminum overlayer, and the rest based on substitutional incorporation of aluminum into copper lattice sites. These models are:

A One third of a monolayer of aluminum adsorbed in three-fold fec hollow sites on bulk copper (111), with $d_{\perp \mathrm{Cu}-\mathrm{Al}}$ varied from $1.787 \AA$ to $2.237 \AA$ in steps of $0.05 \AA$.

B This is the same as model $\mathrm{A}$, with aluminum atoms in three-fold hep hollow sites instead of fcc hollow sites.

C One third of a monolayer of aluminum atoms substituted in ton-layer copper sites in a bulk copper crystal. The top layer was allowed to buckle, with the aluminum atoms located $0.0, \pm 0.10$ and $\pm 0.20 \AA$ above the plane of the copper atoms. The first inter-layer spacing was also varied independently between 1.887 and $2.287 \AA$ in $0.10 \AA$ steps.

D The same as model C, but with the top layer registry changed to hcp termination (layer stacking ABACBAC...) rather than fce termination (layer stacking $A B C A B C \ldots)$. 
E The same as model $\mathrm{C}$, but with aluminum atoms also substituted in one third of the sites in the $3^{\text {rd }}$, $5^{\text {th }}$, etc. layers. The layer registries for the deeper layers were chosen to preserve fec stacking and the point group symmetries (3-fold rotation axis and mirror planes) of the pure $\mathrm{Cu}(111)$ crystai surface.

\subsection{LEED structure determination}

The 16 atomic \% aluminum $\alpha$-CuAl crystal used in the LEED experiments was grown from the melt, cut and polished to a (111) orientation by Baird and Eberhardu. ${ }^{13}$ This crystal was a disk approximately $7 \mathrm{~mm}$ in diameter and $3 \mathrm{~mm}$ thick. The crystal was supported by two 0.012 " tungsten wires which passed through holes spark machined through tlie crystal parallel to the surface. The sample was heated by passing a current through the tungsten support wires, and cooled by conduction from liquid nitrogen reservoirs. The temperature was measured by a thermocouple inserted in a third hole spark machined part way through the crystal, and checked with an optical pyrometer. The crystal could be cooled to $\sim 130 \mathrm{~K}$ or heated to the melting point.

The crystal was cleaned by $\mathrm{Ar}^{+}$ion bombardment at $500 \mathrm{eV}$ in $5 \cdot 10^{-5}$ torr of argon at room temperature. Special care was taken to avoid oxidation of the surface aluminum atoms. The vacuum system base-pressure was $\sim 5 \cdot 10^{-10}$ torr for these experiments, and the aluminum $L V V$ Auger lines where checked after LEED data collection (see figure 13.1). The fine-structure of these lines is sensitive to the chemical environment, and changes significantly if the aluminum is 
oxidized. ${ }^{16}$ After ion bombardment the crystal exhibited a diffuse $(1 \times 1)$ LEED pattern. The crystal was heated to $670 \mathrm{~K}$, well above the $570 \mathrm{~K}$ phase transition temperature, then the crystal temperature was reduced in $50 \mathrm{~K}$ stages, annealing the crystal for $300 \mathrm{~s}$ at each stage down to $420 \mathrm{~K}$, to insure a reproducible and well ordered $(\sqrt{3} \times \sqrt{3})$ R30 $^{\circ}$ phase. After annealing a sharp $(\sqrt{3} \times \sqrt{3})$ R30 ${ }^{\circ}$ LEED pattern was observed.

An attempt was made to quench the crystal from above the phase transition temperature to freeze in the metastable $(1 \times 1)$ structure. After cooling at the maximum obtainable rate of $-10 \mathrm{~K} / \mathrm{s}$ a sharp and well-ordered $(\sqrt{3} \times \sqrt{3}) \mathrm{R} 30^{\circ}$ superlattice LEED pattern was still observed.

\subsubsection{LEED data}

LEED I-V curves were recorded for the $(\sqrt{3} \times \sqrt{3}) R 30^{\circ}$ superlattice between 20 and $300 \mathrm{eV}$ with the crystal at $\sim 150 \mathrm{~K}$. Data sets were collected at normal incidence and with the incident beam rotated by $10^{\circ}$ and $15^{\circ}$ toward the [1 $\left.\overline{10}\right]$ direction. Two independent sets of LEED intensity data were collected at each angle of incidence. The crystal was sputter-cleaned, re-annealed and re-oriented between the data sets. There was good agreement between the symmetry related beams, and between equivalent beams in the independent data sets (see figure 5.4).

I-V curves were generated for 24 beams at normal incidence. The symmetry related beams were averaged together, leaving seven independent beams with a total of 16 major peaks in a total energy range of $600 \mathrm{eV}$. The normal incidence 
I-V curves are: $(1,0),(0,1),(\overline{2}, 0),(2,0),(\overline{2}, 1), 1 / 3,1 / 3)$ and $(\overline{4 / 3}, 2 / 3)$. I-V curves were generated for 23 beams at $\theta=15^{\circ}$, and the 15 independent beams after symmetry averaging included 34 major peaks in a total energy range of $1200 \mathrm{eV}$. The $\theta=15^{\circ} \mathrm{I}-\mathrm{V}$ curves are: $(0,0),(\overline{1}, 1),(0,1),(\overline{1}, \overline{1}),(0, \overline{2}),(\overline{2}, \overline{1}),(\overline{1 / 3}, \overline{1 / 3})$, $(1 / 3, \overline{2 / 3}),(\overline{2 / 3}, \overline{2 / 3}),(\overline{5 / 3}, \overline{2 / 3}),(\overline{2 / 3}, \overline{5 / 3}),(\overline{7 / 3}, \overline{1 / 3}),(\overline{4 / 3}, \overline{4} / 3),(\overline{4 / 3}, \overline{1 / 3})$ and $(\overline{1 / 3}, \overline{4 / 3})$. The $\alpha$-CuAl I-V curves are shown in figure 13.2 .

\subsubsection{Theoretical methods}

LEED structure calculations were performed between 20 and $200 \mathrm{eV}$ using standard techniques (see chapter 3 ). The copper and aluminum icn-core scattering potentials were assumed to be the equal to those for bulk copper and bulk aluminum. The phase shifts were derived from the atomic potentials of Burdick $^{18}$ for copper and Snow ${ }^{19}$ for aluminum. The layer scattering matrices for the mixed copper and aluminum co-planar or near co-planar (buckled) layers were calculated with CSM matrix inversion. (The RSP perturbation scheme was tried, but this expansion failed to converge.) The atomic layers were stacked using the RFS scheme.

The non-structural parameters used included Debye temperatures of $335 \mathrm{~K}$ for copper and $514 \mathrm{~K}$ for aluminum, an initial inner potential $V_{o r}=10 \mathrm{eV}$, later adjusted to $V_{o r}=8 \mathrm{eV}$, and a damping potential $V_{0 i}=3.8 \mathrm{eV}$ at $90 \mathrm{eV}$ and proportional to $E^{1 / 3}$. 


\subsubsection{Structure search}

Theoretical calculations and $\mathbf{r}$-factor comparisons were made using the normal incidence and $15^{\circ}$ data sets. These provided a sufficient data base, so the $10^{\circ}$ data were not included in the structure determination calculations. The prelinninary structural search used only the normal incidence data. Once the best model was chosen additional calculations were made at $15^{\circ}$ to confirm the model choice and to optimize the model geometry.

The initial calculations at normal incidence clearly rule out models $A, B$ and D. The best $r_{5}$ values for these models were $0.338,0.324$ and 0.358 , respectively, compared to $r_{5}$ values of 0.206 and $\mathbf{0 . 2 1 3}$ for fcc-terminated substitutional models $\mathrm{C}$ and E. In figure $\mathbf{1 3 . 3}$ the experimental normal incidence I-V curves are compared with theoretical I-V curves the best overlayer geometry (model A) and the substitutional geometry (model C) with no buckling and the bulk copper interlayer spacing.

Additional calculations were erformed for models $\mathrm{C}$ and $\mathrm{E}$ using the $\theta=$ $15^{\circ}$ data. The $r_{5}$ minima was found at approximately the same parameter values as for normal incidence, although the absolute value of $r_{5}$ was a bit larger. Contour plots of $r_{5}$ as a function of inter-layer spacing and buckling (see figure 13.4) show well-defined minima in equivalent positions ior the two data subsets. This agreement shows that model $\mathrm{C}$ is a reasonable description of the surface structure of the $(\sqrt{3} \times \sqrt{3}) \mathrm{R} 30^{\circ}$ reconstruction of $\alpha-\mathrm{CuAl}(111)$. Extrapolating to the $r_{5}$ contour plot minima using both data sets, the optimum parameter values are an expansion of the first inter-layer spacing by $0.05 \pm 0.05 \AA$ and an inward buckling 
of the aluminum atoms by $0.025 \pm 0.05 \AA$. These values are not significantly different from the terminated bulk structure of copper with substitutional aluminum. The optimum r-factor values, calculated for both normal incidence and $\theta==$ $15^{\circ}$ data sets, are $r_{5}=0.218, r_{Z J}=0.300$ nd $r_{P e n d r y}=0.514$. When third layer aluminum is included (model $\mathrm{E}$ ) there is still a reasonable fit, but the minimum value of $r_{5}$ (normal incidence data only) increases from 0.10 to 0.24 . Clearly model $\mathrm{C}$ is a better description of the real surface geometry than model $\mathbf{E}$.

\subsection{Discussion of results}

None of the surface models used above have aluminum-aluminum nearest neighbor pairs, consistent with the diffuse $x$-ray scattering results. ${ }^{12}$ This means that the overlayer adsorption site models ( $\mathrm{A}$ and $\mathrm{B}$ ) must have a pure copper first layer, and the substitutional models (C-E) must have a pure copper second layer. An excess of aluminum in the top layer and a depletion in the second layer is consistent with by recent $A$ uger measurements. ${ }^{20}$

Ferrante $^{6}$ initially proposed an overlayer model for the $(\sqrt{3} \times \sqrt{3}) R_{30}{ }^{\circ}$ superlattice on $\alpha-\mathrm{CuAl}$; however, this was just suzgested as one model consistent with the LEED pattern in the Auger measurements, and no evidence was presented that would favor an overlayer model over a substitutional inodel.

Berning and Coleman ${ }^{21}$ have also investigated the structure of the $\alpha$-CuAl (111) surface by LEED. Their experimental results for substrate beams at normal incidence agree weli with the data of figure 13.2. They propose a model involving alıminum in interstitial sites and the bulk lattice, and forming an aluminum 
overlayer on the copper surface with aluminum atoms in top sites (not hollow sites). This model is based on a qualitative interpretation of the appearance of the substrate beam I-V curves, which is not a viable approach to structure determination. Their model assumptions are inconsistent with the normal behavior of metals -- all known structures for non-alkali metals on metals have metal adsorbates on the most highly coordinated sites (see chapter 1), and interstitial aluminum atoms in a close-packed copper crystal are inconsistent with $\mathrm{x}$-ray results and the lattice stress involved.

The optimum $r_{5}$ value for model $E$, with ordered aluminum in the third layer, was significantly worse than the fit for model $\mathrm{C}$, with aluminum in the top layer only. A model with alternating mixed copper-aluminum and pure copper layers would be consistent with the bulk composition of 16 atomic $\%$ aluminum, and is similar to the structure found for the (100) faces of $\mathbf{A}_{3} \mathrm{~B}$ alloys, $3,4,5$ where the first layer is an $\mathrm{AB}$ layer, followed by alternating pure $\mathrm{A}$ and $\mathrm{AB}$ layers.

The substrate beam I-V curves for $\alpha-\mathrm{CuAl}$ are close tu the experimental I-V curves for the pure copper (111) surface ${ }^{22,23,24}$ (see figure 13.6). Indeed, the differences between the CuAl(111) substrate beams and the different sets of experimental $\mathrm{Cu}(111)$ beams are no larger than the differences between the different $\mathrm{Cv}^{111}$ experiments. This supports a model where only the top layer aluminum atoms have long-range order. If many of the substrate aluminum atoms were in ordered sites, there would probably be a stronger affect on the LEED I-V curves. 


\subsection{Conclusion}

The structure of the $(\sqrt{3} \times \sqrt{3})$ reconstruction on the $\alpha$-CuAl (111) surface is described by the terminated bulk structure of $\mathrm{Cu}(111)$ with aluminum atoms substituted in $1 / 3$ of the top layer sites. There is no significant buckling of the mixed copper-aluminum layer and no large change in the inter-layer spacing. There is no evidence for ordered aluminum atoms in the deeper layers of the crystal. This description is consistent with the thermodynamic properties and the short-range order in the bulk alloy, and the observed lack of net aluminum segregation to the surface. 


\section{References}

1. H. L. Davis and J. R. Noonan, Journal of Vacuum Science and Technology A, vol. 3, p. 1507, 1985.

2. H. L. Davis and J. R. Noonan, Physical Review Letters, vol. 54, p. 566, 1985.

3. D. Sondericker, F. Jona, V. L. Moruzzi, and P. M. Marcus, in Proceedings of ICSOS-1, The Structure of Surfaces, ed. M. A. Van Hove and S. Y. Tong, vol. 2, Springer Series in Surface Sciense, Springer Verlag, Berlin, 1985.

4. D. Sondericker, F. Jona, V. L. Moruzzi, and P. M. Marcus, Solid State Communications, vol. 53, p. 175, 1985.

5. U. Bardi, M. Tossini, E. Zanazzi, G. Rovida, M. Maglietta, P. N. Ross, and M. A. Van Hove, poster presentation at ICSOS-1, Berkeley, CA, Auzust 1984.

6. J. Ferrante, Acla Metallurgica, vol. 19, p. 743, 1971.

7. J. Ferrante, Scripta Metallurgica, vol. 5, p. 1971, 1129.

8. H. P. Bonzel and H. B. Aaron, Scripta Metallurgica, vol. 5, p. 1971, 1057.

9. R. J. Baird, D. F. Ogletree, M. A. Van Hove, and G. A. Somorjai, Surface Science, vol. 165, p. 345, 1986.

10. M. Hanson, Constitution of Binary Alloys, 2ed edition, McGraw-Hill, New York, 1958.

11. B. Borie and C. J. Sparks, Acta Crystallographica, vol. 17, p. 827, 1964. 
12. J. E. Epperson, P. Fürnrohr, and C. Ortiz, Acta Crystallographica A, vol. 34, p. $667,1978$.

13. J. R. Baird and W. Eberhardt, Journal of Vacuum Science and Technology, vol. 18, p. 538,1981 .

14. R. J. Baird, Bulletin of the American Physical Society, vol. 29, p. 268, 1984 and to be published.

15. P. Wynblatt and R. C. Ku, Surface Science, vol. 65, p. 511, 1977.

16. R. J. Baird, private communication.

17. R. Hultgren, et. al., Selected Values of Thermodynamic Properties of Binary Alloys, American Society for Metals, Metals Park, OH, 1973.

18. G. A. Burdick, Physical Review, vol. 129, p. 138, 1963.

18. E. C. Snow, Physical Review, vol. 172, p. 708, 1868.

20. R. J. Baird and T. J. Potter, Journal of Vacuum Science and Technology A, vol. 3, p. 137i, 1985.

21. G. L. P. Berning and W. J. Coleman, Proceedings of the 9th International Vacuum Congress and 5th International Conference on Surface Science, p. 36, extended abstracts, Madrid, 1983 and to be published.

22. P. R. Watson, F. R. Shepherd, D. C. Frost, and K. A. R. Mitchell, Surface science, vol. 72, p. 562, 1978.

23. S. P. Tear, K. Röll, and M. Prutton, Journal of Physics C, vol. 14, p. 3297, 1981. 
24. S. A. Lindgren, L. Walldén, J. Rundgren, and P. Westrin, Physical Review $B$, vol. 29, p. 576, 1984 . 


\section{Figure Captions for Chapter 13}

13.1 Auger spectrum of 16 atomic $\% \alpha$-CuAl (111) after cleaning and annealing. This spectrum was recorded with the retarding-field energy analyzer of the LEED optics at $1 \mathrm{~V}$ peak-to-peak modulation amplitude.

13.2 LEED I-V curves for 16 atomic \% aluminum $\propto-\mathrm{CuAl}(111)$ recorded at 150 $\mathrm{K}$ for $\theta=0^{\circ}$ and $15^{\circ}$.

13.3 Comparison between theoretical calculations and experimental I-V curves at normal incidence for the near-optimal geometries of model A (a monolayer Al overlayer on bulk a bulk $\mathrm{Cu}$ (111) crystal) and model $\mathrm{C}$ ( of a monolayer of $\mathrm{Al}$ substituted into the top layer of a $\mathrm{Cu}(111)$ crystal).

13.4 Contour plots of $r_{5}$ as a function of the structural parameters of model C. Contour plots at both normal incidence and $\theta=15^{\circ}, \phi=0^{\circ}$ are shown.

13.5 Definition of the structural parameters of model C, the best model for the $\alpha-C u A l$ (111) surface: Outward expansion, relative to the bulk copper inter-layer spacing, of the topmost (substitutional copper-aluminum) layer from the second (pure copper) layer; and outward displacement of the aluminum atoms relative to the plane of the top-layer copper atoms.

13.6 Substrate beam I-V curves for $\alpha$-CuAl (111) compared to experimental I-V curves for pure copper (111). 


\section{CuAl(111) Auger Spectrum}

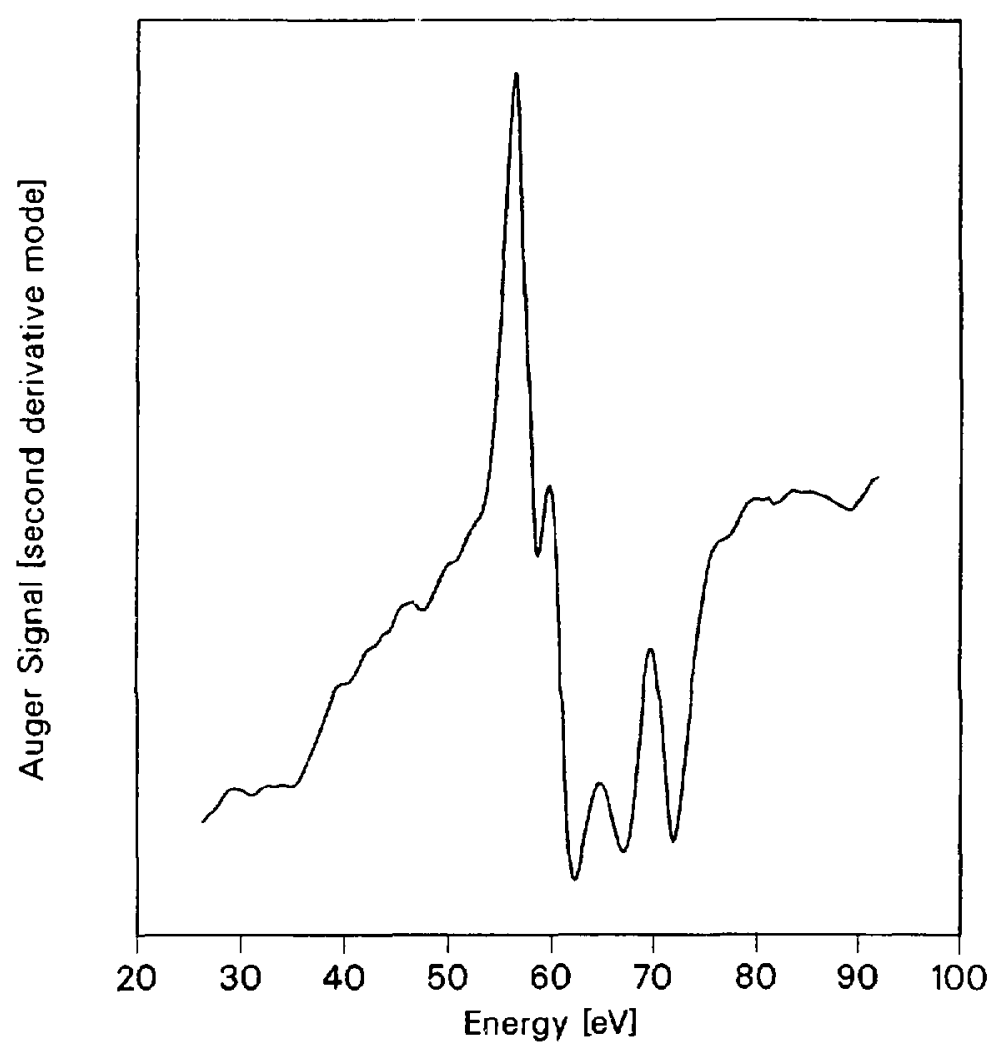

XBL 8611-4342

Figre 13.1 


\section{0 \\ CuA1 (111) I-V Curves
Normal Incidence at $150 \mathrm{~K}$}

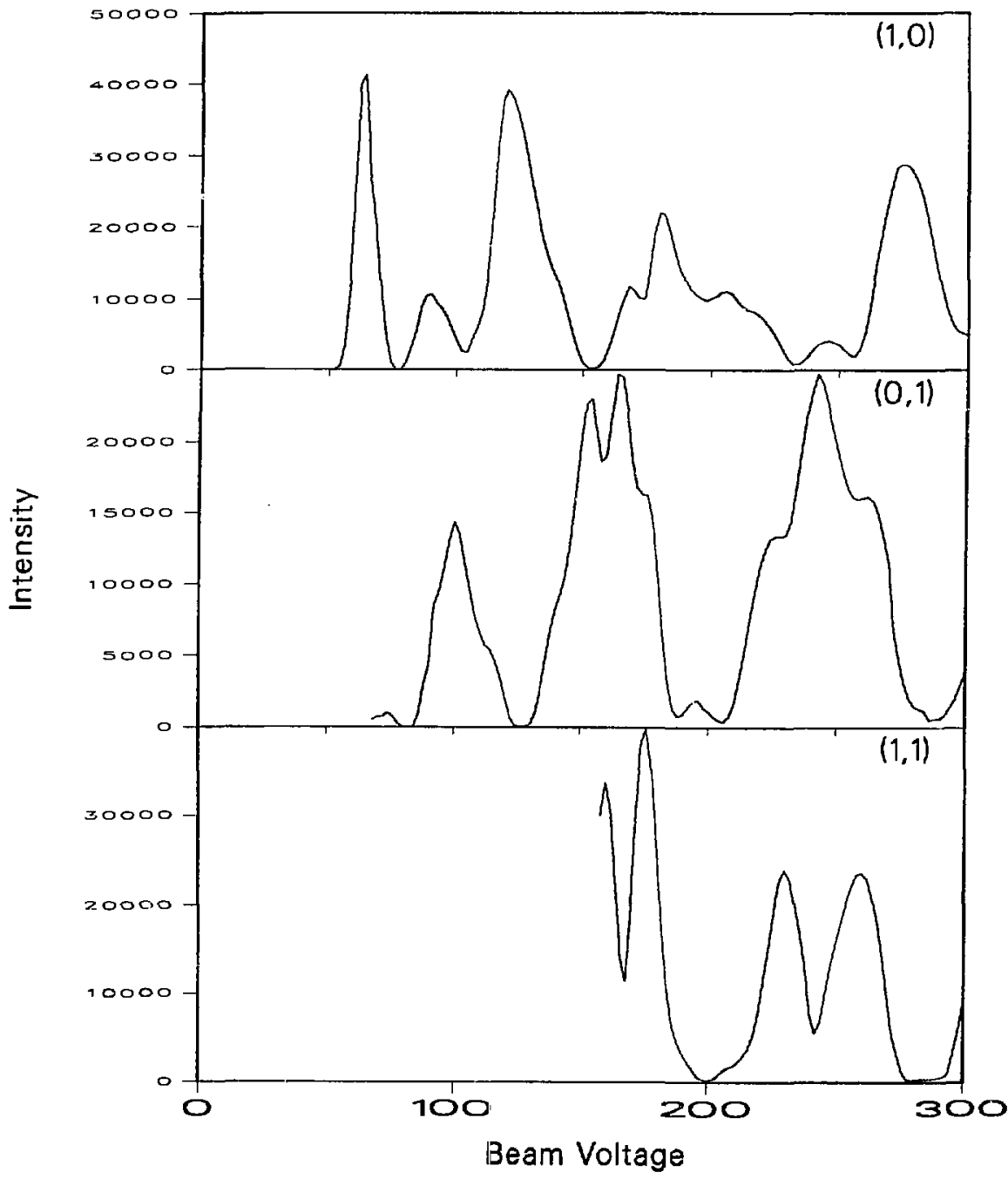

Figro 132(a) 
581

\section{CuA1 (111) I-V Curves \\ Normal Incidence at $150 \mathrm{~K}$}

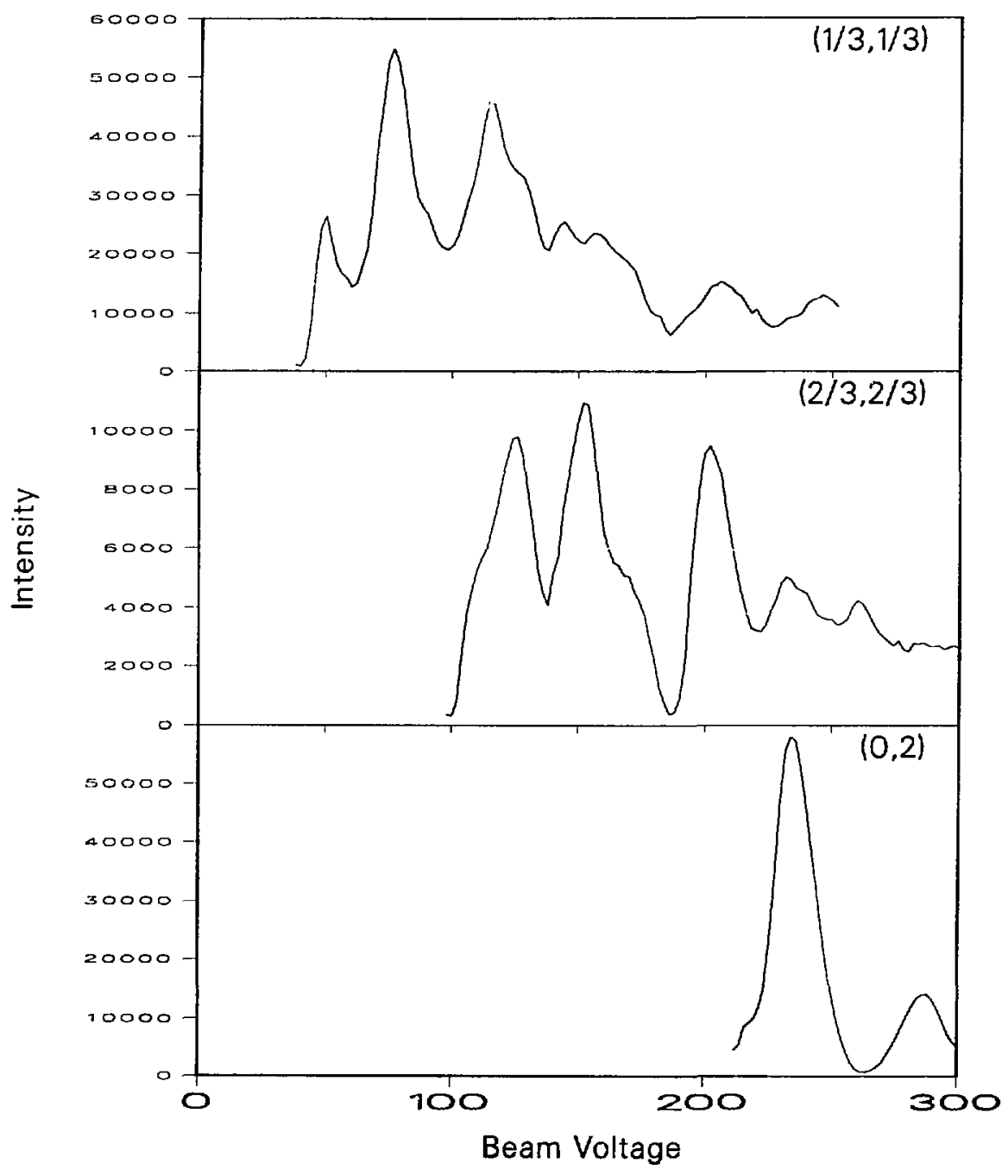

Flgre 13.2(b) 


\section{CuAl (111) I-V Curves $\theta=15$ at $150 \mathrm{~K}$}

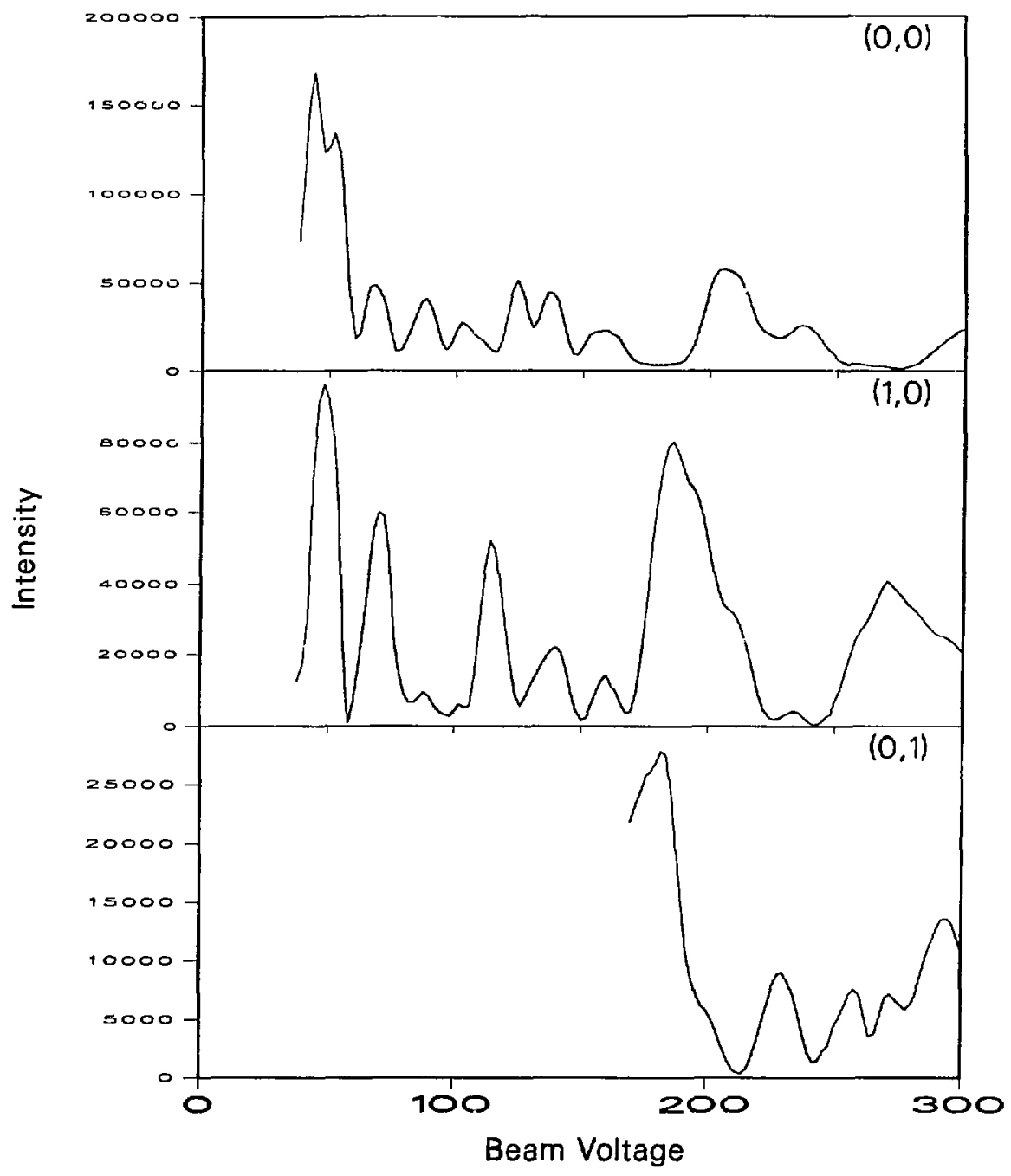

Fgre 13.2(c) 
583

\section{CuAl (111) I-V Curves \\ $\theta=15$ at $150 \mathrm{~K}$}

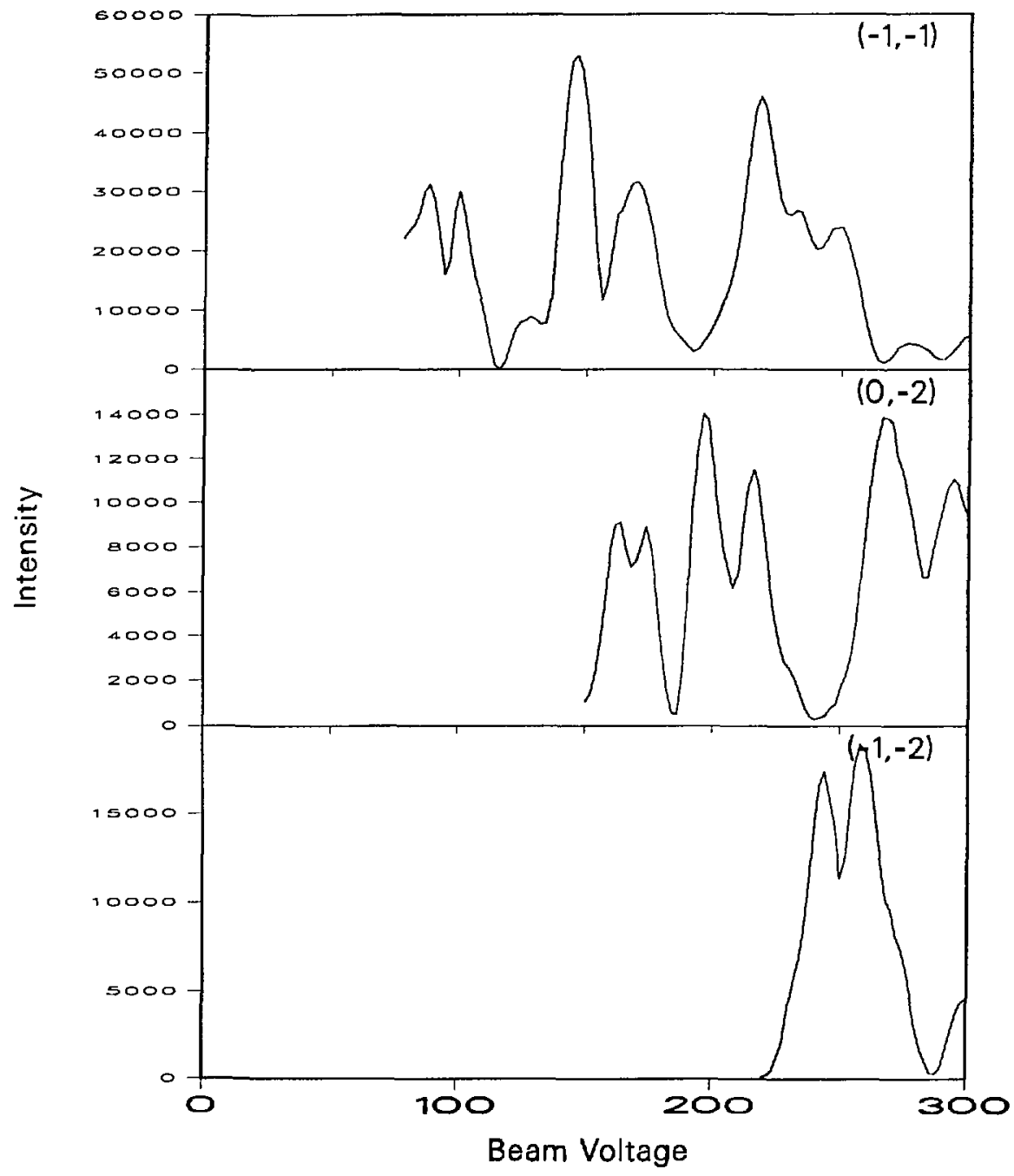

Fgre 13.2(d) 
584

CuAl (111) I-V Curves $\theta=15$ at $150 \mathrm{~K}$



Fgure 132(e) 
585

\section{CuA1 (111) I-V Curves \\ $\theta=15$ at $150 \mathrm{~K}$}

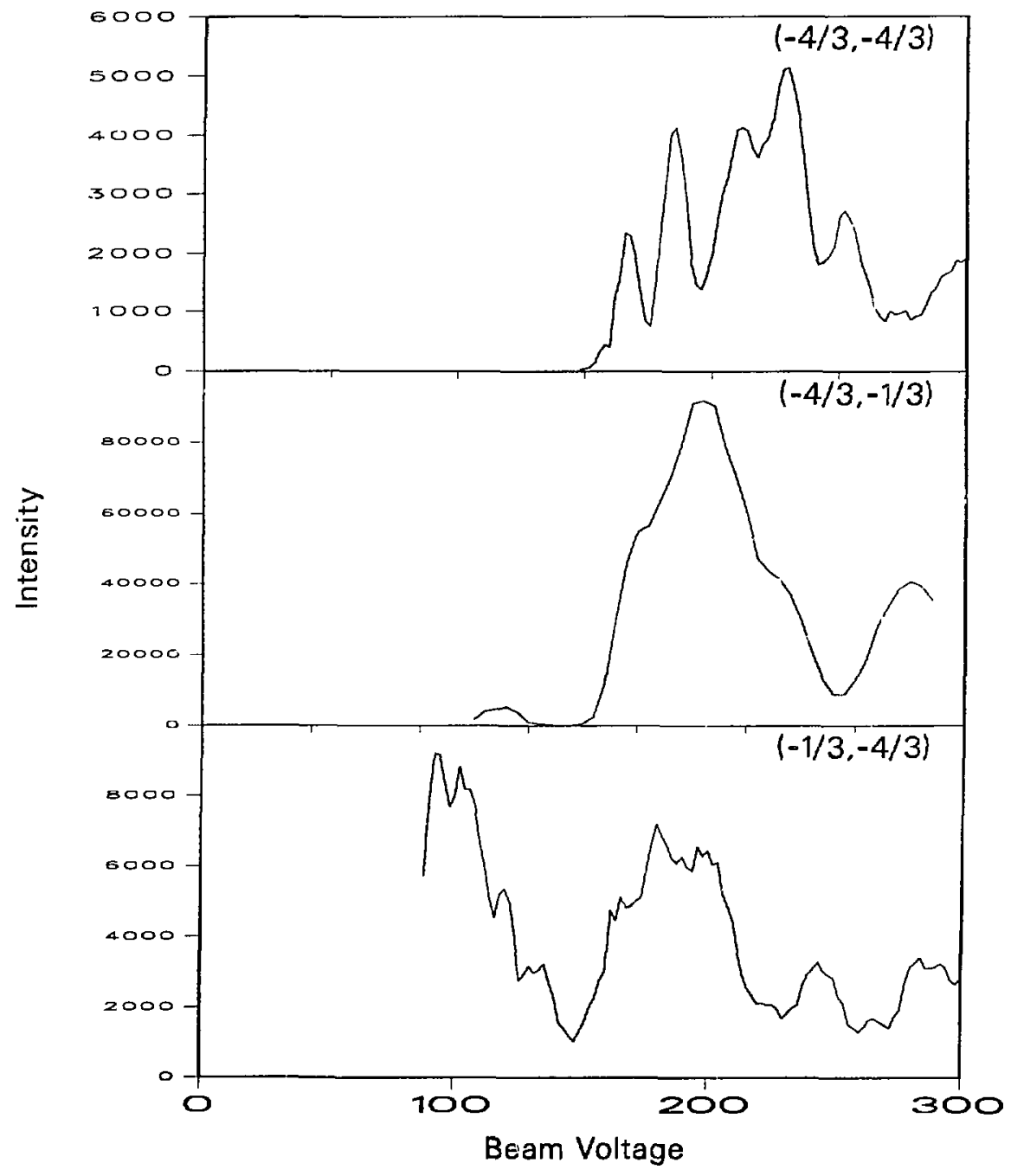

Agre 13.2(0) 
583

\section{CuA1 (111) I-V Curves \\ $\theta=15$ at $150 \mathrm{~K}$}

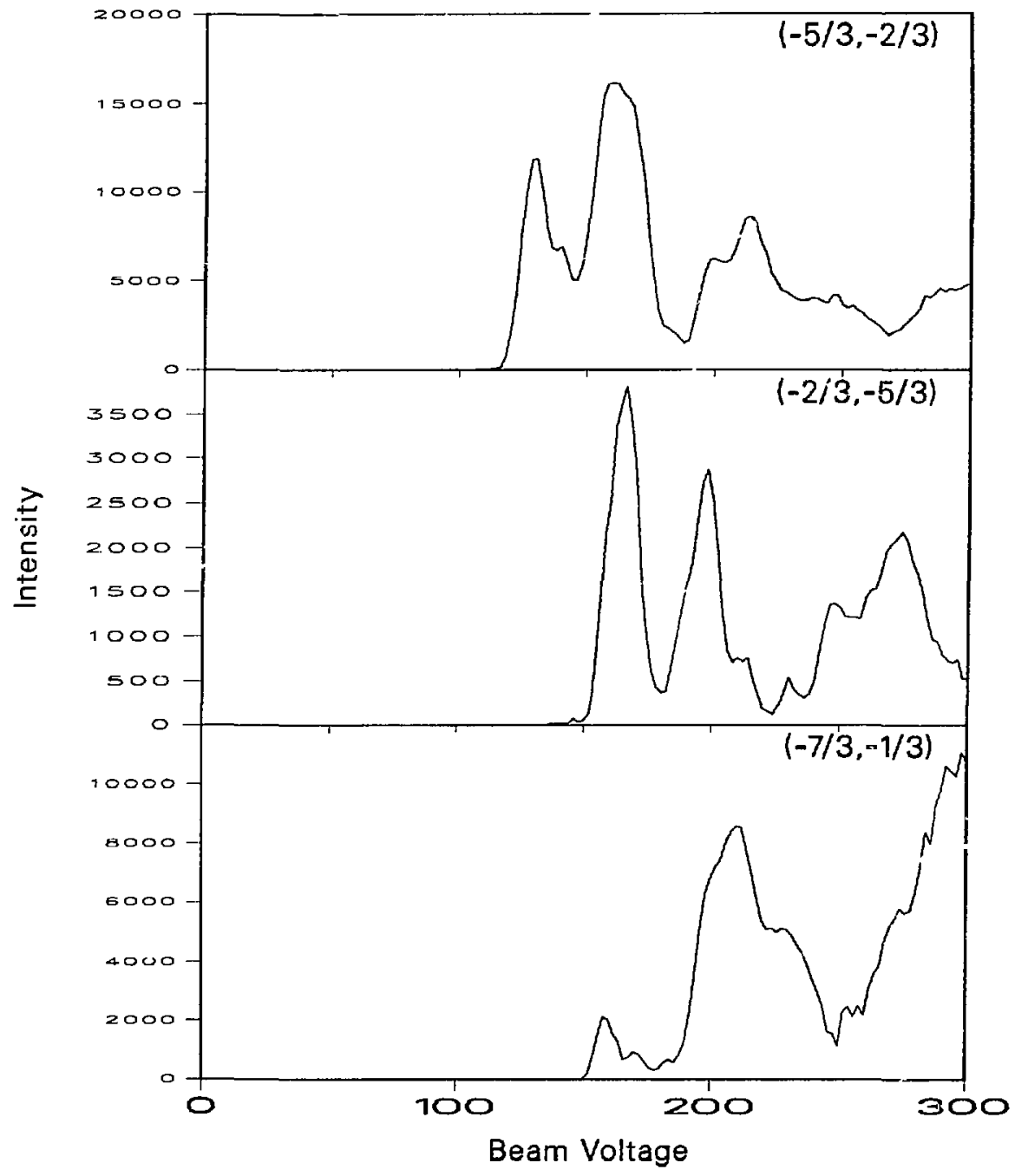

Fgre 13.2(g) 
587

\section{CuAl (111) I-V Curves \\ $\theta=15$ at $150 \mathrm{~K}$}

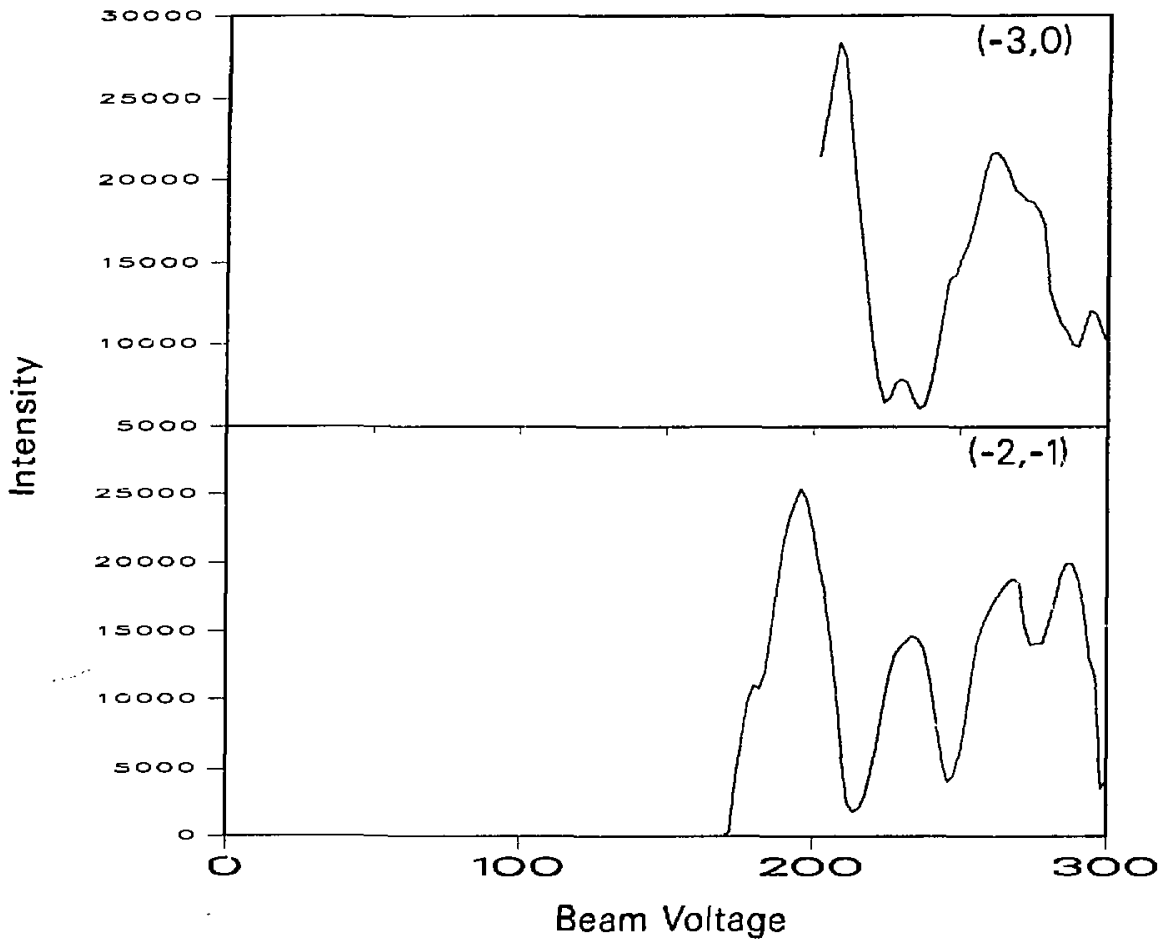

Figre 13.2(h) 
$a-\mathrm{Cu} A \mathrm{Al}$ (III) $-(\sqrt{3} \times \sqrt{3}) \mathrm{R} 30^{\circ}$ Structure

Comparison of Theory and Experiment



$(0,1)$ beam

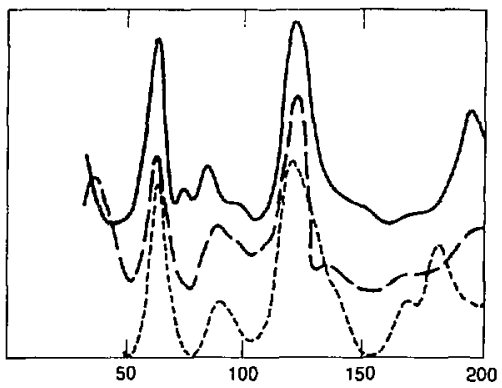

$(1,0)$ beam

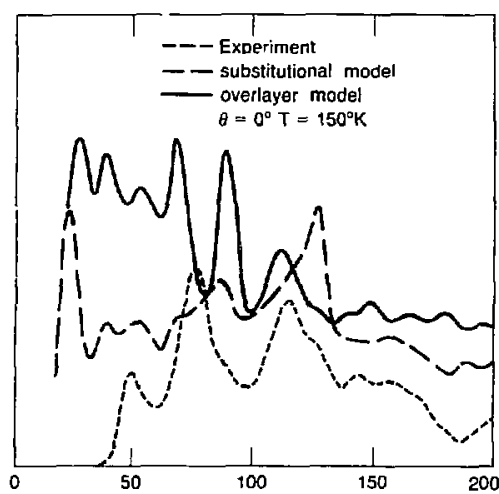

$(1 / 3,1 / 3)$ beam



$(2 / 3,2 / 3)$ beam 
$\alpha-\mathrm{Cu} \mathrm{Al}$ (III) $-(\sqrt{3} \times \sqrt{3}) \mathrm{R} 30^{\circ}$ Structure R - factor contour plots

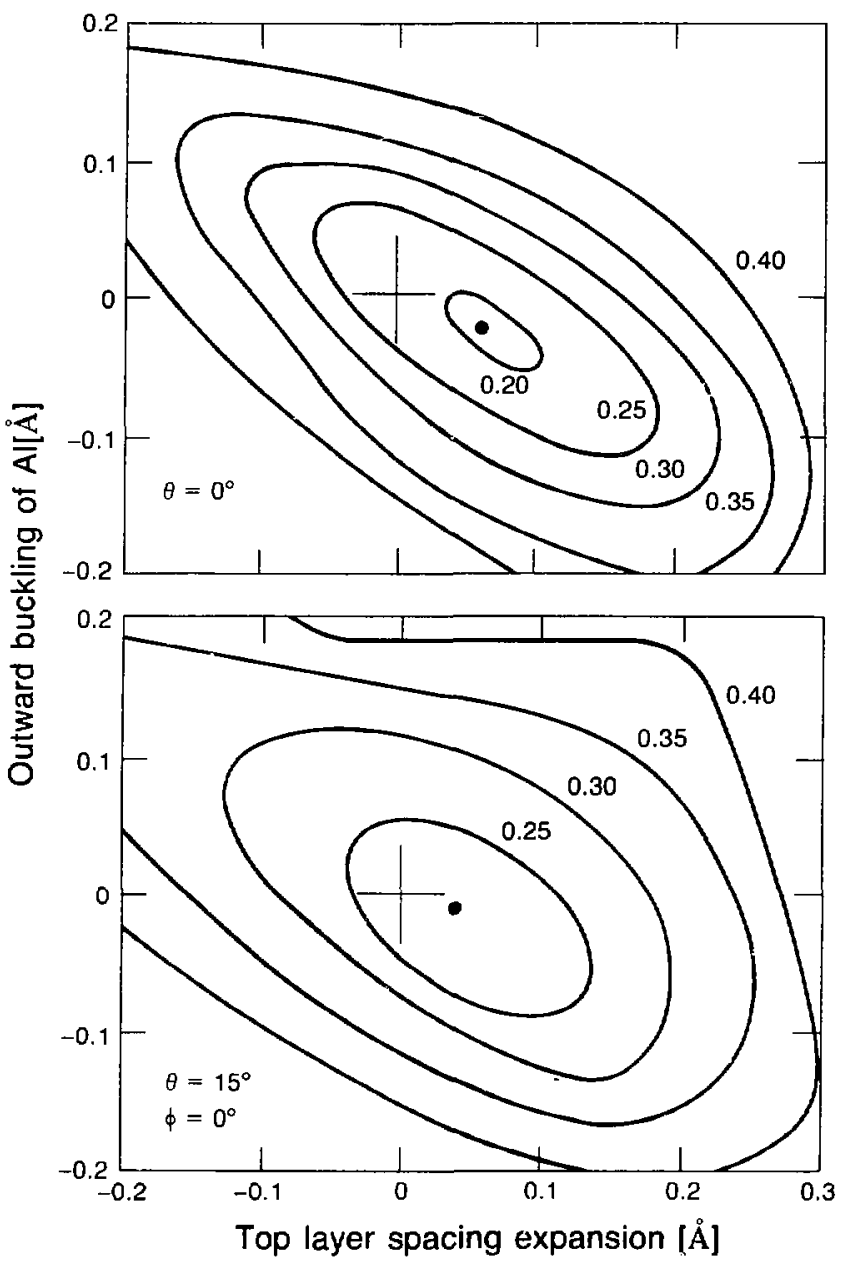

XBL 853-8027

Figure 13.4 
$\alpha-\mathrm{Cu}$ Al (III) $-(\sqrt{3} \times \sqrt{3}) \mathrm{R} 30^{\circ}$

Substitutional AI Model Parameters

Al Atom Shaded

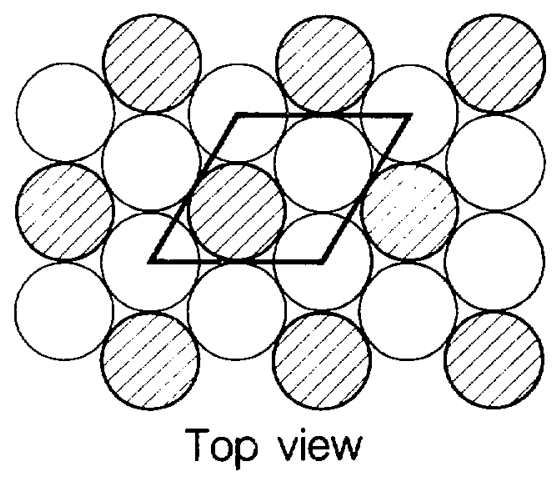

Outward

displacement

of $\mathrm{Al}$

Outward

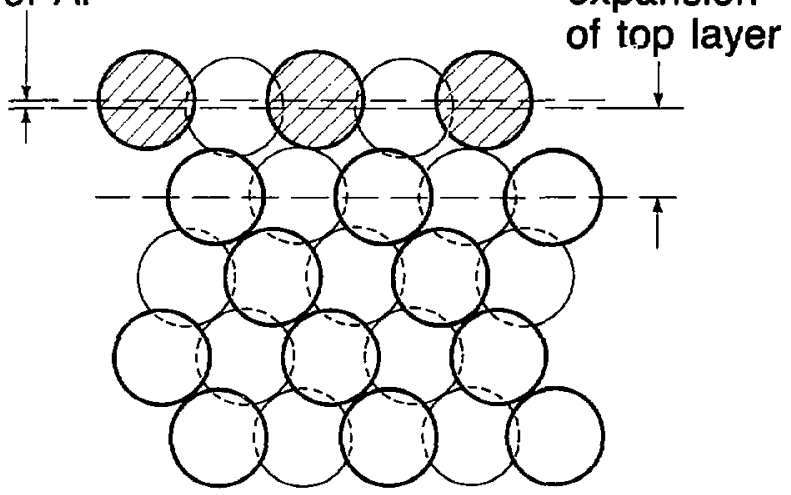

Side view

XBL 855-9971

Figre 13.5 
$591 / 592$

\section{Comparison of $\mathrm{Cu}(111)$ and CuAl(111) I-V Curves}



$(1,0)$ Beam at normal incidence

XBL. 8611-4341

Fgre 136 


\section{Conclusion}

Low energy electron diffraction is the most general and has been the most productive probe of the structure of surfaces in vacuum. A substantial majority of the known surface structures have been determined by LEED, and LEED investigations continue to provide a majority of the new structural results.

Two of the studies described above, the copper alloy study and the carbon monoxide study, fit within the frame work of "conventional" LEED structure determinations. In these studies exact electron scattering calculations, within the muffin-tin approximation, are made for surface phases with long-range order. The computational effort involved puts a practical limit on the complexity of surface structures that can be investigated. LEED scattering calculations will become prohibitive for structures involving more than four or five geometrical parameters within the surface model, and for structures with much more than six or eight inequivalent atoms within the unit cell.

Even within these "conventional limits" there remain many interesting classes of surface structures that have barely been investigated. The study of the reconstructed $\alpha-\operatorname{CuAl}(111)$ surface is the first structural study of a reccnstructed alloy surface, and one of the first half dozen investigations of any kind of alloy surface. The study of carbon monoxide in the $c(4 \times 2)$ phase on $\mathrm{Pt}(111)$ was only the second study of a system where molecules are adsorbed at more than on site on the surface, and again, one of only a few structural studies of surfaces where 
there is more than one atom or molecule in the unit cell.

\section{Extending the range}

The range of LEED structure determination is being qualitatively extended by new developments in both theoretical methods and experimental techniques. Theoretical developments are, on one hand, overcoming the requirement of longrange order for LEED calculations, and on the other hand, substantially increasing the complexity of structures that can be investigated by LEED.

Diffuse LEED methods can be applied to atomic or molecular adsorbates with lattice-gas discrder. From diffuse intensity calculations the local geometry of the adsorption site can still be determined. The beam-set neglect (BSN) approximation, by neglecting certain weak, high-order scattering paths, can handle surfaces with much larger unit cells than conventional calculations. Indeed, as the study of the graphite overlayer shows, the BSN approximation can handle calculations for incommensurate overlayers, equivalent to an infinite surface unit cell. Extensions of the BSN method may soon be applied to stepped surfaces.

The benzene-carbon monoxide coadsorption phases on $\mathrm{Pt}(111)$ and $\mathrm{Rh}(111)$ are much more complex than the kinds of structures traditionally investigated by LEED. This analysis is possible because approximations have been developed that allow hundreu's and even thousands of possible structures to by surveyed with rough but efficient calculations, which can be improved as the structural solution is refined. 


\section{Experimental methods}

The theoretical aGivances which are opening up complex and disordered systems to LEED investigation offer new experimental challenges. Structure determination is now possible provided a sufficient range of electron scattering intensity data can be acquired from a well-characterized surface system. Characterization is more difficult without long-range order, since diffraction is not available to filter-out deviations from the equilibrium geometry. Also it is more difficult to measure the intensity of elastically scattered electrons without long-range order, since the signal is no longer concentrated into intense beams.

The electron counting techniques for LEED intensity measurements are a natural complement to diffuse LEED studies. The inherent sensitivity of electron counting can detect the much weaker diffuse signals without damage from the incident electron beam, and the position-sensitive detector can simultaneously measure the scattered intensity at many different angles.

For more conventional structure problems, the computer-interfaced video nsinera together with interactive $\mathrm{I}-\mathrm{V}$ cur'se generation programs can now produce LEED I-V data with little more effort than collecting an XPS or HREEL spectra.

Now that the range of structure determination is opening up, new structural knowledge should help to advance the general level of surface science, of the understanding of the physical, chemical and materials properties of surfaces and interfaces. 


\section{Appendix I}

\section{I-V Data}

LEED beam I-V data are available in digital form on request. Requests should be directed to:

D. F. Ogletree or

M. A. Van Hove

Materials and Molecular Research Division

Lawrence Berkeley Laboratory

Berkeley, CA 94720

Note: All of the I-V plots in chapters 10 to 13 show raw I-V data. The intensities were normalized to 1 ; A incident beam current (except, as noted, for some of the graphite I-V curves), and symmetry-equivalent beams have been averaged together. The data have not been smoothed or otherwise processed.

I-V curves have been generated for the systems systems listed on the following table. This table also shows the temperatures, energy ranges and angles of incidence at which the data were collected. There is some additional data on video tape that was not reduced because it was not needed for the theoretical structure analysis. This includes clean $\mathrm{Pt}(111)$ data collected at room temperaiure for a number of different angles of incidence, $\alpha$-CuAl data collected at $150 \mathrm{~K}$ for $\theta=5^{\circ}$ and $(2 \sqrt{3} \times 5)$-rect data collected at $150 \mathrm{~K}$ at off $\theta=5^{\circ}$ and $15^{\circ}$.

Video data was recorded for the $(2 \sqrt{3} \times 5)$-rect system at normal and offnormal angles of incidence at both $150 \mathrm{~K}$ and $300 \mathrm{~K}$. The contrast is poor for this data and I-V curve generation would be difficult or impossible. 


\section{Electronics}

Complete circuit diagrams are available for the position sensitive electron detection system. The reference to the electron gun, channel plate and detector power supply plans is $13 \mathrm{X} 412-\mathrm{P} 1$, and the reference to the pre-amplifier, shaping amplifier and position calculation logic plans is 13X409-P1. These plans may be obtained from the Lawrence Berkeley Laboratory. Requests should be directed to:

D. F. Ogletree or

J. E. Katz

Department of Instrument Science and Engineering

Engineering and Technical Services Division

Lawrence Berkeley Laboratory

Berkeley, CA 94720 
LEED I-V Curve Data

\begin{tabular}{|c|c|c|c|c|c|}
\hline system & energy & $\theta, \phi$ angles & temperature & $f$-stop & video tape \\
\hline clean $\operatorname{Pt}(111)$ & $\begin{array}{l}40-250 \\
40-250 \\
10-400 \\
10-400 \\
10-400\end{array}$ & $\begin{array}{c}0,0 \\
4,0 \\
10,180 \\
15,180 \\
20,180\end{array}$ & $\begin{array}{l}300 \\
300 \\
300 \\
300 \\
300\end{array}$ & $\begin{array}{l}2.0 \\
2.0 \\
2.0 \\
2.0 \\
2.0\end{array}$ & $\begin{array}{l}\text { 1A0001, } 1 \mathrm{~A} 0886 \\
\text { 1A0952 } \\
1 \mathrm{D} 0360 \\
1 \mathrm{D} 0750 \\
\text { 1D0860 }\end{array}$ \\
\hline graphite on $\operatorname{Pt}(111)$ & $\begin{array}{l}10-400 \\
10-400 \\
10-400\end{array}$ & $\begin{array}{l}10,180 \\
15,180 \\
20,180\end{array}$ & $\begin{array}{l}300 \\
300 \\
300\end{array}$ & $\begin{array}{l}2.0 \\
2.0 \\
2.0\end{array}$ & $\begin{array}{l}\text { 1D1060 } \\
\text { 1D1248 } \\
\text { 1D1154 }\end{array}$ \\
\hline$c(4 \times 2)$ phase of $\mathrm{CO}$ on $\mathrm{Pt}(111)$ & $\begin{array}{l}20-200 \\
20-200 \\
20-200\end{array}$ & $\begin{array}{c}0,0 \\
5,0 \\
15,0\end{array}$ & $\begin{array}{l}150 \\
150 \\
150\end{array}$ & $\begin{array}{l}0.85 \\
0.85 \\
0.85\end{array}$ & $\begin{array}{l}110224 \\
110662 \\
110345,110405\end{array}$ \\
\hline$(2 \sqrt{3} \times 4)$-rect phase of $\mathrm{CO}$ and $\mathrm{C}_{6} \mathrm{H}_{6}$ on $\mathrm{Pt}(111)$ & $10-150$ & 0,0 & 140 & 0.85 & $1 \mathrm{H} 0566$ \\
\hline$(\sqrt{3} \times \sqrt{3}) \mathrm{R} 30^{\circ}$ phase of $\alpha-\mathrm{CuAl}(111)$ & $\begin{array}{l}40-300 \\
40-300\end{array}$ & $\begin{array}{c}0,0 \\
0,15\end{array}$ & $\begin{array}{l}150 \\
150\end{array}$ & $\begin{array}{l}2.8 \\
2.8\end{array}$ & $\begin{array}{l}\text { 1E0312,1F0189 } \\
\text { 1E0597, 1F0622 }\end{array}$ \\
\hline
\end{tabular}

Energies are measured in volts, angles in degrees and temperature in Kelvins. 


\section{Appendix II}

\section{Data Analysis Programs}

The source code for the the wedge-and-strip anode drawing program described in chapter 6 and the data reduction programs described in chapter 7 are available on magnetic media. Requests should be directed to:

D. F. Ogletree or

M. A. Van Hove

Materials and Molecular Research Division

Lawrence Berkeley Laboratory

Berkeley, CA 04720

\section{Wedge-and-strip Anode Program}

The FORTRAN program ANODE produces a control file for a Gerber plotter and several files that display the plotting commands in an easy to read format. All of the parameters to design the anode are listed in the first section of ANODE. This program could easily be adapted to other types of vector-based plotting devices. The Gerber plotter can draw vectors or arcs. 


\section{PROGRAM ANODE}

C Unit 10 is Gerber plotter output file

C Unit 11 is a short file appended to 20 , to provide labels

C Unit 15 is output file for GERVAX, using Tek Plotters

C Unit 20 is a user-friendly Gerber-like output file

$\mathrm{C}^{*}$

C

SET PARAMETERS, all dimensions in $\mathrm{mm}$

C PEN is insulation gap, open space fraction is $4.0^{\star}$ PEN/PERIOD

C PENX outlines the anode O.D.

C PENY labels connections

C STRP1, STRPN are minimum and maximum strip widths

C the aspect ratio is STRPN/STRP1

C R1 is the inner hole radius, now $3 \mathrm{~mm}$

C R5 is the effective radius of the anode

C R7 is the bolt circle radius, $2.125 "$

C R8 is the anode substrate radius, now 2.25 "

C PAD is the size of the pad at the end or each strip or wedge

C DELTA is the pattern offset, $-1.0<$ DELTA $<1.0$

C COND is the width of the conduction path around the ccenter hole

C SLOP is the additional allowance for misalignment of the hole

C IDSAVE $=0$ draws anode with hole, $=1$ without hole

C XFACTOR is the scale factor for plot (XFACTOR* real size)

$\mathrm{PEN}=0.044$

PERIOD $=1.500$

STRP 1 $=0.050$

$\mathrm{STRPN}=\mathbf{0 . 6 6 2}$

$\mathrm{RI}=\mathbf{3 . 0}$

$\mathrm{R} 5=47.0$

$\mathrm{R} 7=2.125 / 0.03937$

$\mathrm{R} 8=2.250 / 0.03937$

PENX $=1.0$

$\mathrm{PENY}=0.013$

$\mathrm{PAD}=0.5$

COND $=0.150$

DELTA $=0.35$

SLOP $=0.500$

IDSAVE $=0$ 


\section{I-V curve generation programs}

The I-V curve generation programs are linked into larger menu-driven programs which run the video or digital LEED experiments. Much of the dispiay and hard-copy output routines and the video and memory control routines are hardware dependent, and so they are not included here. A copy of thie complete code is available on request, and the command file to link and run the digital LEED program is included. This gives brief functional descriptions of the different program modules that are not included, so the structure of the program can be understood.

Many of the more important variables are grouped into common blocks. The functions of these are:

/BEAM/ Adjustable parameters which control the evaluation of a single LEED spot. These may change from data set to data set.

/FLXED/ These are not changed.

/RESULTS/ The results for the evaluation of a single spot at one energy, such as width, height, intensity, etc.

/ACQIRE/ Parameters that describe the data set, i.e. number of images, energy range, counting time, etc.

/SEARCH/ Adjustable parameters that control the spot search routines. /SPOTS/ Arrays that store spot addresses in image, reciprocal and memory space, along with flags for spot status. 
/PROFL/ Controls interactive functions.

\subsection{Programming environment}

These programs were written for an LSI 11/23 computer running RT-11 version 5.0B. The hardware included a CAMAC crate with a $512 \mathrm{~K}$ histogramming memory, timers, scalers, $D / A$ and $A / D$ converters, a digital video processor from Imaging Technology (see chapter 5) with $1736 \mathrm{~K}$ of video image memory, and 768 K RAM.

One major failure of the RT-11 cperation system is that it does not support virtual memory mapping in a manner compatible with heavy I/O usage. Also the DEC virtual array routines are (violently) incompatible with block-structured access to high memory. For this reason the RAM above $60 \mathrm{~K}$ was treated as a block-addressable device (a "virtual disk"), and a macro routine VDATA was used in place of Fortran virtual arrays. This routine constructs 2-dimensional arrays of arbitrary size and precision in high memory and accesses them through fast block transfer routines.

The limitation of the available memory to $60 \mathrm{~K}$ required a heavily overlaid program for the LEED data acquisition and analysis. This controls the way parts of the progranis are structured. 


\subsection{Fortran code for I-V curve generation}

The command file to link the LEED programs and the source code for the following FORTRAN modules is included: IVDATA, HKCALC, MAPMK, MAPKM, BEAMS, DOBEAMS, LOCMAX, BKGRND, XYSIZE and SPOINT. The macro code for the smooting routine SMOOTH and the virtual array substitute VDATA follows. 
! Command file XD.COM, to compile, link and run

! digital leed data acquistion and display program.

!FORTRAN XD:DLEED/LIST/WARN !/ONDEBUG

l root program

!FORTRAN XD:LEEDBD/LIST/WARN

! block data module, common initialization

!FORTRAN XD:INTRTN/LIST/WARN

I interrupt service for CAMAC timer, HM data acquisition

!MACRO XD:BIRAMS

I rontines to drive CAMAC crate modules

MACRO GR:VDATA

! sets up and uses VM array memory

!FORTRAN XD:IVDATA/LIST/WARN

! runs I-V experiment, loops over energies

FIRST REGION OVERLAYS

IFORTRAN XD:EGUN/LIST/WARN I/ONDEBUG

! controls leed supply beam voltage via CAMAC D/A module

!FORTRAN XD:STARTC/LIST/WARN!/ONDEBUG

! starts CAMAC timer, histogram memory to start counting

IFOR'IRAN XD:HISTRD/LIST/WARN !/ONDEBUG

! transfers HM data to VM array and FB after counting is done

!FORTRAN XD:CAMSUB/LIST/WARN !/ONDEBUG

! CAMAC operating routines, using BIRAMS.MAC drivers

!

IFORTRAN XD:DATPLT/LIST/WARN!/ONDEBUG

! displays HM data on video terminal

1

!FORTRAN VID:VIDEO/LIST/WARN !/ONDEBUG

$! \quad$ video processor control program

!FORTRAN VID:VIDSUB/LIST/WARN!/ONDEBUG

! high level video processor subroutines

IFORTRAN VID:VTR/LIST/WARN !/ONDEBUG

! controls video tape recorder through DRV-11C parallel $1 / O$ card

IFORTRAN XD:DISPLA/LIST/WARN !/ONDEBUG

! rescales video display for more esthetic picture, restores original

IFORTRAN XD:LDISK/LIST/WARN !/ONDEBUG

! stores DLEED image on disk DL1; , retrieves stored image

!FORTRAN XD;IVTNIT/LIST/WARN !/ONDEBUG

! sets up parameters to run I-V experiment, stores on disk

!FORTRAN GR:RTFLE/LIST/WARN

! utility routine to open, handle disk files

1

!FORTRAN XD:BEAMS/LIST/WARN

! locates and analyzes all diffraction beams at a given energy

IFORTRAN XD:BTEST/LIST/WARN

! analyze a single beam under operator control

IFORTRAN XD:DOBEAM/LIST/WARN !/ONDEBUG

! evaluates a single beam: position, width, height, sum, background !FORTRAN XD:LOCMAX/LIST/WARN !/ONDEBUG

! finds a beam in FB memory, transfers region to array in program

IFORTRAN XD:SPSHOW/LIST/WARN

! marks spots on video display aster run

IFORTRAN XD:XYSPOT/LIST/WARN 


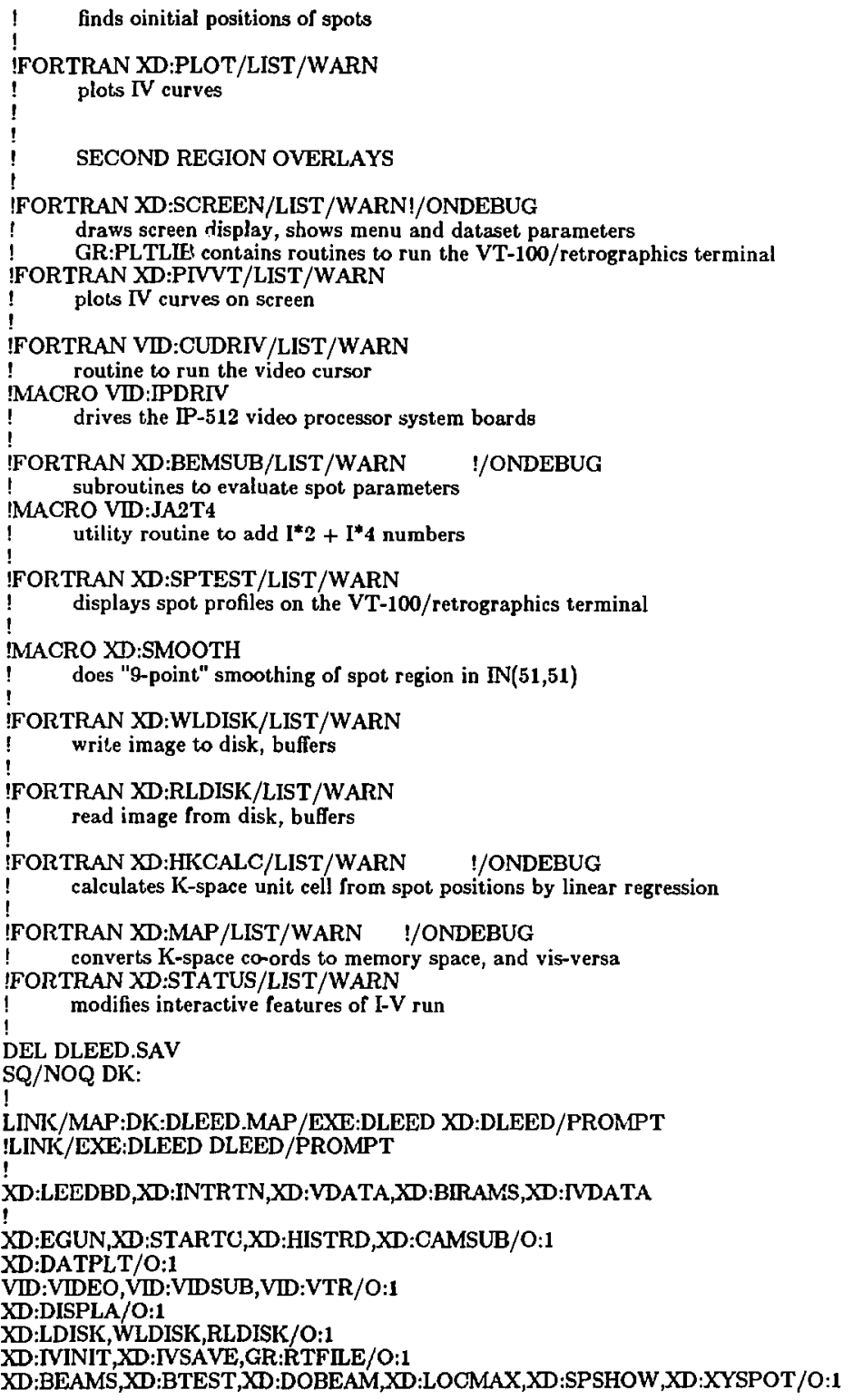


XD:PLOT/O:1

$!$

XD:SCREEN,XD:PIVVT,GR:PLTLIB,SY:SYSLIB/O:2

$\mathrm{XD}: \mathrm{PLFLE} / \mathrm{O}: 2$

$\mathrm{XD}: \mathrm{HKCALC} / \mathrm{O}: 2$

XD:MAP,XD:STATUS/O:2

VID:CUDRIV, VID:IPDRIV $/ 0: 2$

XD:BEMSUB,VID:JA2T $4 / O: 2$

XD:SPTEST $/ 0: 2$

$\mathrm{XD}$ :SMOOTH/O:2

/1

COPY/PREDELETE DLEED.SAV VM:

RUN VM:DLEED 
C

C

C

C COUNT AT INITIAL ENERGY

C

C

C

C

C

C

30

C

C

C

22

C

C

C

40 1

COMMON /PROFL/ IPROFL(75)

LOGICAL * 1 WORD, IVMODE

DATA JSW /"44/

CALL EGUN( EC, COND )

CALL STARTC( CTIME, o )

\section{LOOP OVER ENERGIES}

DO 10 IFFRAME = KSTART, KIMAGE

$\mathrm{KK}=$ KFRAME

WAT FOR DATA

IF ( FI .EQ. KSTART ) GO TO 20

TTOTAL = STOTAL

TVALID = SVALID

CALL SCREEN ( 0 )

TTOTAL $=\mathbf{0 . 0}$

TVALID $=0.0$

TYPE 1000

CET DATA

EC = ESTART + EINC*FLOAT(KK)

CALL EGUN( EC, COND )

CALL HISTRD $(0,0,0)$

STOTAL $=$ TTOTAL

SVALID = TVALID

DO $40 \mathrm{~J}=1$, NSPOT

SUBROUTINE IVDAT:

COMMON /CAMAC/ IHM, ITMER, IDA, LAD, INSET

COMMON /DPARAM/ TTIME, TTOTAL, TVALID, EC

COMMON /ACQIRE/ESTART, EINC, CTRE, ISM, KIMAGE, KSTART

COMMON /SEARCH/ FLM, ISEE, INTER, CLM(3), XY(6)

COMMON /BEAM/ IWX0, IWYO, INTXIM, INTYLM, NOISE, BGRAD, SNLIM

COMMON /FLXE/ NX, NY, DXMAX, IYMAX, IVMODE

COMMON /SPOTS/ POSHIK(75,2), POSXY $(75,2), \operatorname{IPOSXY}(75,2)$, IBFLG(75), IBSYM(75), NSPOT

$\mathrm{EC}=\mathrm{ESTART}+\mathrm{EINC} *$ FLOAT(KSTART - 1)

IF( INTER .EQ. 0) CALL IPOKE(JSW,IPEEK(JSW).OR."10000)

IF( INSET .EQ. 1 ) GO TO 22 !check for data ready flag

TYPE 1020, IFLTT, NSPOT, ITEST, MORET

IF( INSET .NE. 1 ) GO TO 20 !wail for data ready lag

!set next LEED beam energy

CALL STARTC( CTME, o ) Istart collecting next energy data

CALL VARRAY ( 2, KK, 1, XY, 6, 1) !STORE UNIT CELL

CALL VARRAY (2, KK, 7, EC, 1, 1) !AND ENERGY

CALL VARRAY $(1,76, \mathrm{KK}$, EC, 1, 1$)$ !AND ENERGY

\section{CALCULATE SPOT POSITIONS FROM UNIT CELL VECTORS}

$\operatorname{POSXY}(J, 1)=X Y(1)+\operatorname{POSHK}(\mathrm{J}, 1)^{*} \mathrm{XY}(3)+\operatorname{POSHK}(\mathrm{J}, 2)^{*} \mathrm{XY}(5)$

$\operatorname{POSXY}(J, 2)=X Y(2)+\operatorname{POSHK}(J, 1)^{*} X Y(4)+\operatorname{POSHK}(J, 2)^{*} X Y(6)$

CALL MAPIGM

!convert from K-space to memory address space 


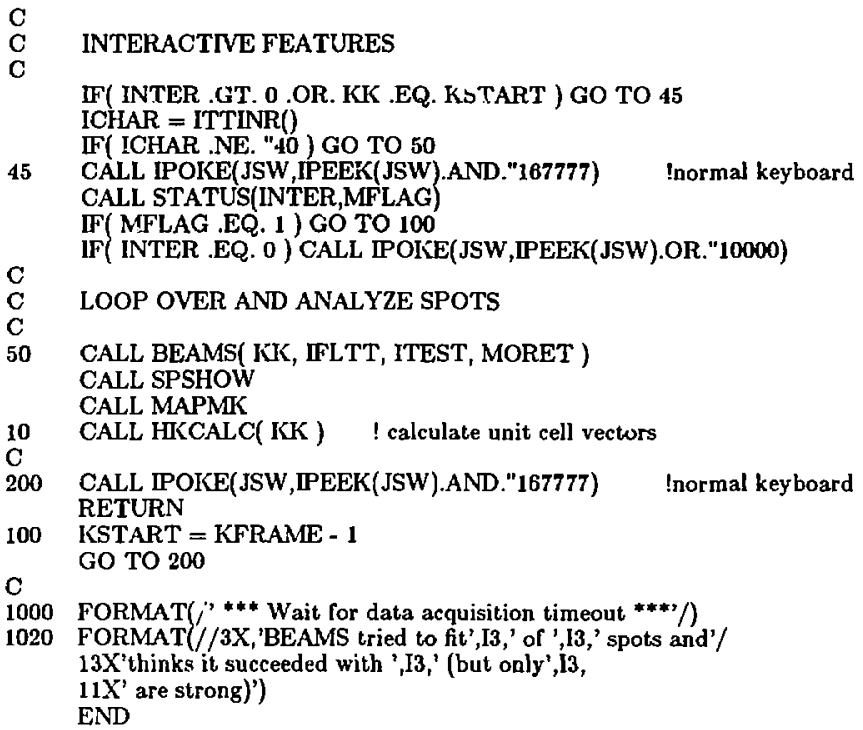


C
C
C
C
C
C

C

C

10

C

C

C

C

20

C

C

C

30

C

D

D11 FORMAT('AT \#',I3,' XY WAS ',6F8.2/

D 1 ' XY IS ',6F8.2)

D PAUSE' HKCALC RESULT'

RETURN

C

C

C

200

210

220

C

1000 FORMAT(' delta $\mathrm{O}=$ ',F6.3,' delta $\mathrm{H}=$ ',F6.3,

1' delta IK = ',F6.3/

1' Override ICLM $(\mathrm{Y} / \mathrm{N})$ ? '\$)

2000

The 2-D unit cell is calculated from experimantal spot positions

Frank Ogletree $1 / 15 / 86$

DIMIENSION DX(3), XYOO(6)

\section{LOGICAL * 1 WORD}

DO $10 \mathrm{~J}=1,6$

CALL RGRESS( XY, KFAIl ) !linear regression

IF( KFAIl .LT. 0 ) GO TO 200

$\mathrm{DNOM}=\mathrm{XY0O}(3)^{* * 2} 2+\mathrm{XY} 00(4)^{* * 2}$

$\mathrm{DO} 20 \mathrm{~J}=1,3$

(DNOM .EQ. 0.0) GO TO 20

IF ( INTER .LT. 1 .OiR. IGFAlL .EQ. 0 ) GO TO 30

WRITE $(5,1000)$ DX

$\operatorname{READ}(5,2000)$ WORD

EXTRAPOLATE CELL TO NEXT ENERGY

FAC $=$ SQRT $(($ ESTART + EINC * FLOAT $($ KK-1) $) /$

DO $40 \mathrm{~J}=3,6$

ESTART + EINC * FLOAT(KK)) )

$\mathrm{XY}(\mathrm{J})=\mathrm{XY}(\mathrm{J}) * \mathrm{FAC}$

IF RGRESS FAILS, EXTRAPOLATE OLD UNIT CELL

KFAIL $=-10$

DO $220 \mathrm{JJ}=1,6$

$\mathrm{XY}(\mathrm{JJ})=\mathrm{XYOO}(\mathrm{JJ})$

GO TO 30

FORMAT(70A1)

\section{SUBROUTINE HKCALC( KK )}

in POSXY, and this result is compared with the predicted unit cell.

COMMON /ACQIRE/ ESTART, EINC, CTIME, ISM, KIMAGE, KSTART

ON /SEARCH/ FLM, ISEE, INTER, CLIM(3), XY(6)

COMMON /SPOTS/ POSHK(75,2), POSXY $(75,2), \operatorname{IPOSXY}(75,2)$,

$=\operatorname{SQRT}\left(\left(\mathrm{XY}\left(2^{*} \mathrm{~J}\right)^{* *} 2+\mathrm{XY}\left(2^{*} \mathrm{~J}-1\right)^{* *} 2\right) / \mathrm{DNOM}\right)-1.0$

IF ( ABS(DX(J)) .GT. CLIM(J) AND. CLIM(J) .GT. 0.0 ) KFAIL=10

loverride limit on change? 
C END

$\mathrm{C}$

SUBROUTINE RGRESS( XY, KFAIL)

C Calculates unit cell vector components from spot positions.

C Frank Ogletree, last changed 1/14/86 COMMON /SPOTS/ POSHK(75, 2), POSXY(75,2), $\operatorname{POSXX}(75,2)$, 1 IBFLG(75), IBSYM(75), NSPOT

$\mathrm{C}$ DIMENSION XY(6)

$\mathrm{KFAIL}=0$

ITEST $=0$

$\mathrm{S} 1=0.0$

$\mathrm{S} 2=0 . \mathrm{G}$

DO $10 \mathrm{~J}=1$, NSPOT

IF( IBFLG(J) .NE. 1 ) GO TO 10

ITEST $=$ ITEST +1

$\mathrm{S} 1=\mathrm{S} 1+\mathrm{ABS}(\operatorname{POSH} \mathrm{K}(\mathrm{J}, 1))$

10 CONTINUE

$\mathrm{S} 2=\mathrm{S} 2+\mathrm{ABS}(\operatorname{POSHK}(\mathrm{J}, 2))$

C IF( ITEST .LT. 4 .OR. S1 .LT. O.1 .OR. S2 .LT. 0.1) GO TO 120

C CALCULATE AVERAGES

C

$\mathrm{AVEH}=\mathbf{0 . 0}$

AVEK $=0.0$

$\mathrm{AVEX}=\mathbf{0 . 0}$

$\mathrm{AVEY}=0.0$

TOT $=0.0$

$\mathrm{C}$

DO $30 \mathrm{~J}=1$, NSPOT

IF( IBFLG(J) .NE. 1 ) GO TO 30

TOT $=$ TOT +1.0

AVEH $=$ AVEH + POSHK $(\mathrm{J}, 1)$

AVEK $=$ AVEK + POSHK $(\mathbf{J}, 2)$

AVEX $=$ AVEX + POSXY $(J, 1)$

$30 \quad$ CONTINUE

C

AVEH $=$ AVEH $/$ TOT

AVEK $=$ AVEK $/$ TOT

AVEX $=$ AVEX / TOT

AVEY $=$ AVEY $/$ TOT

C CALCULATE BILINEAR TERMS

C

PRODHH $=0.0$

PRODKK $=0.0$

PRODHK $=0.0$

PRODIXX $=0.0$

PRODHY $=0.0$

PRODKX $=0.0$

C

$\mathrm{PRODKY}=\mathbf{0 . 0}$

DO $40 \mathrm{I}=1$, NSPOT 




\section{SUBROUTINE MAPIOM}

$\mathrm{C}$

C

Digital LEED version. This subroutine converts cartesian K-SPACE coordinates into integer memory coordinates. Conversion depends on the physical structlice of detector and buffer memory. The detector diameter is arbitrarily normalized to $100.0 \mathrm{~K}$-space units. Address space is $256 \times 256$ pixels. The circular detector is circumscribed by the square address space, with the center at $\mathrm{K}$-space coords $(0,0)$. The radial distortion due to the spherical projection of $\mathrm{K}$ into the delector plane is corrected. $R(k$-space $)=R($ observed $) * \cos ($ co-lattitude $)$

$$
\text { Frank Ogletree } \quad 1 / 14 / 86
$$

COMMON /SPOTS/ POSHK(75,2), POSXY(75,2), IPOSXY $(75,2)$,

C 1 IBFLG(75), IBSYM(75), NSPOT

DIST $=2.0 * 50.0 * * 2 \quad$ la guess, for theta $\max { }^{*} 45$ degrees SCALE $=128.0 / 50.0 / \operatorname{SQRT}(2.0)$

DO $10 \mathrm{~J}=1$, NSPOT

$Y=1,0-\left(\operatorname{POSXY}(\mathrm{J}, 1)^{* *} 2+\operatorname{POSXI}(\mathrm{J}, 2)^{* * 2} 2\right) / \mathrm{DIST}$

IF ( Y,GT, 0.0$)$ GO TO 5 


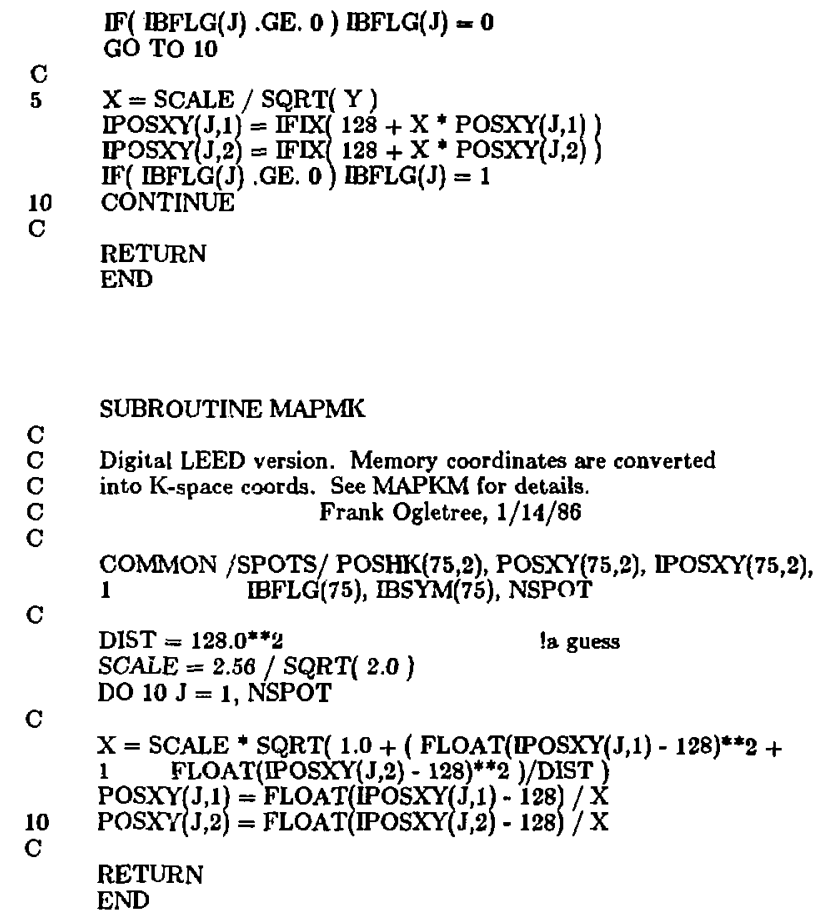


C

SUBROUTINE BEAMS( JJ, IFLTT, ITEST, MORET )

C

COMMON /SEARCH/ FLM, ISEE, INTER, CLM(3), XY(6)

COMMON /SPOTS / POSHK $(75,2), \operatorname{POSXY}(75,2), \operatorname{IPOSXY}(75,2)$, 1 IBFLG(75), IBSYM(75), NSPOT

COMMON /RESULT/IP, IQ, IHIGH, IBACK, IWX, IWY, IFAIL, 1 ITOT, SUM, IPC, IQC, IDX, IDY

COMMON /FIXED/ NX, NY, IXMAX, IYMAX

C

COMMON /PROFL/ IPROFL(75)

C LOOP OVER THE SPOTS

C

ITEST $=0 \quad$ !number of spots in bounds with IFAIL .GE. 0

IFLTT $=0$ !number of spots in bounds

C

MORET $=0$ !number of spots with good $S / N$ ratio

C

DO $10 \mathrm{~K}=1$, NSPOT

$\mathrm{KI}=\mathrm{K}$

IHIGH $=0$ !clear /RESULT/

IBACK $=0$

IWX $=0$

$\mathrm{IWY}=0$

ITOT $=0$

$\mathrm{IPC}=\mathbf{0}$

$\mathrm{IQC}=\mathbf{0}$

IDX $=0$

$\mathrm{IDY}=0$

$\mathrm{SN}=0.0$

C

$\mathrm{SUM}=0.0$

C

OUT OF BOUNDS ?

IFAIl $=-100$

IF( IPOSXY(K,1) .LT. 1 .OR. IPOSXY(K,1) .GT. NX .OR.

1 IPOSXY(K,2).LT. 1 .OR. IPOSXY $(K, 2)$,GT. NY .OR.

1 IBFLG(K) .EQ. -1 ) GO TO 10

C

IF( IBFLG(K) .GT. 0) IBFLG(K) =0

C ANALYZE SPOT

C

IFAIL $=0$

IFLTT $=$ IFLTT +1

TYPE 1040, K, POSHK(K,1), POSHK(K,2), IBSYM(K)

1040 FORMAT(3X'Locate spot number ',I3,' at (',F6.3,','F6.3,'),',

C

11X,'symmetry group ', (3)

$\mathrm{IP}=\operatorname{IPOSXY}(\mathrm{K}, 1)$

$\mathrm{IQ}=\mathrm{IPOSXY}(\mathrm{K}, \mathbf{2})$

CALL DOBEAM(IK,IBFLG(KK),PROFL(KK),POSHK(KK,1),POSHK(KK,2))

$\operatorname{POOSXY}(K, 1)=\mathbb{P}$

IPOSXY $(K, 2)=1 Q$

C

CALL VARRAY ( $1, \mathrm{KK}, \mathrm{JJ}$, SUM, 1, 1 )!WRITE SUM

10

IF ( IBFLG(K) .EQ. 1 ) MORET = MORET + 1

IF (IBFLG(K).GT, 0 ) ITEST $=$ ITEST +1

C

CONTINUE

RETURN 
END

C

C

C

C

C FIND LOCAL MAX NEAR CALCULATED SPOT POSITION

22

C

$20 \quad$ IFLM $=$ ISEE I** fix later - flm bere

CALI, LOCMAX( IN, IFLM)

IF( IFAIL .LE. - 100) GO TO 8 !spot too close to edge ?

C

C PUT CURSOR UP

C

IPDEL $=150$

INULL - IPLEL

CALL ROT(INULL)

INULL = INULL .OR. IPLXEL

CALL BOXON( 0, INULL )

IF( IVMODE .EQ. 'D' ) CALL TVBOX $\left(0,2^{*}\right.$ TE, $2^{*} I Q$, INULL )

IF (IVMODE .NE. 'D' CALL TVBOX( 0, IP, IQ, INULL)

100 CONTINUE

C

C

IF( IFAIL .LT. 0) GO TO 8 (or no true local maximum ? CALL BKGRND(IN) idetermine background IF (IHIGH - IBACK .LE. NOISE) IFALl $=-20$ !peak in noise? IF (IFAll .LT. 0 ) GO TO 8

CALL XYSIZE( IN) !determine spot width

CALL SPOINT(IN, IBFLG) !integrate spot

C IF( IFAIL .LT. 0) GO TO 8

C IS THE SPOT STRONG ? 


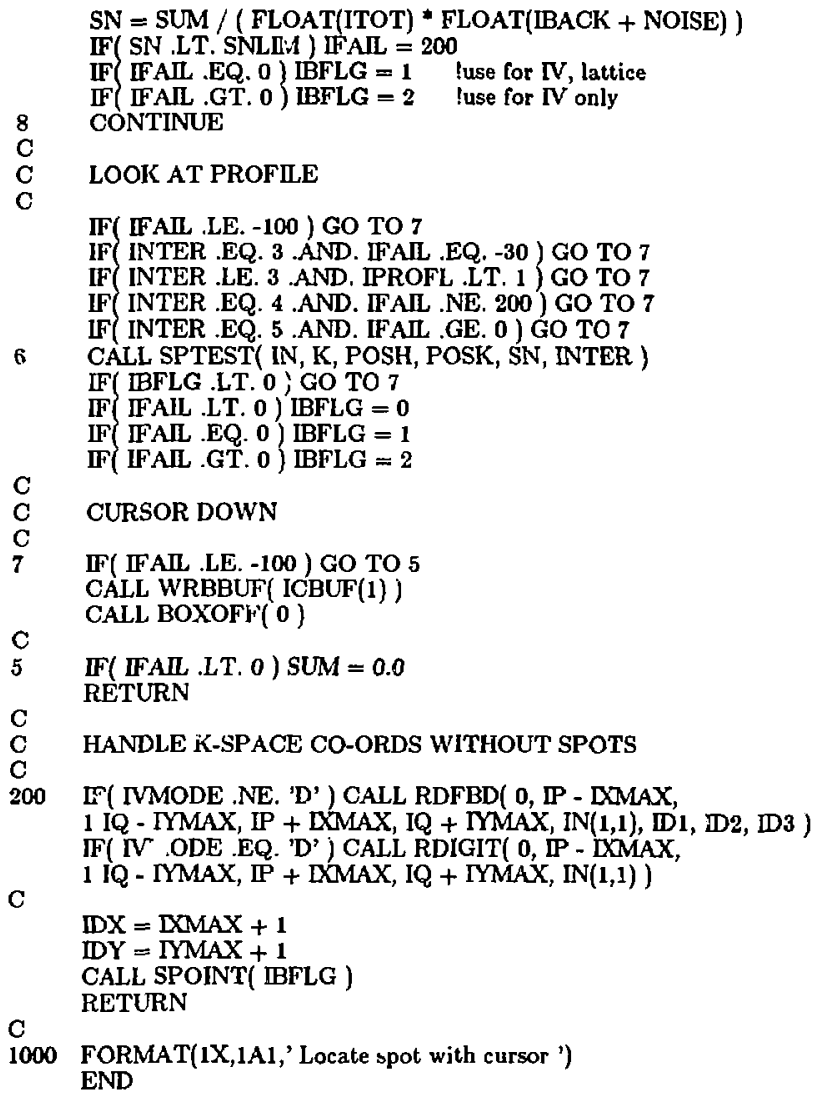

\section{SUBROUTINE LOCMAX( IN, IFLM)}

C
C
C
C

S?arch for local max in ISEE $x$ ISEE array of memory locations centered on extrapolated spot position

COMMON /ACQIRE/ ESTART, EINC, CTIME, ISM, KIMAGE, KSTART COMMON /SEAROH/ FLM, ISEE, INTER, CLIM(3), XY(6) COMMON /BEAM/IWX0, IWYO, INTXLM, INTYLM, NOISE, BGRAD, SNLIM COMMON /FDED/ NX, NY, DXMAX, IMMAX, IVMODE COMMON /RESULT/ IP, IQ, IHIGH, IBACK, IWX, IWY, IFAIL, C 1 ITOT, SUM, IPC, IQC, IDX, IDY

DMENSION IN $(\tilde{3} 1,51)$ 
C

C

C

C

D

D

C

20

10

C

C

C

C

C

C

100

200

C

C

$30 \quad \mathbb{P}=\mathbb{I P}+\mathbb{I D X}-\mathrm{IXMAX}-1$

$\mathrm{IQ}=\mathrm{IQ}+\mathrm{DY}-\mathrm{IYMAX}-1$

$\mathrm{C}$

C

C

IF( ISM .EQ. 0) GO TO 10

DO $20 \mathrm{I}=1$, ISM

CALL SMOOTH ( IN $(1,1))$

CONTINUE

ISX $=$ IFLM

ISY $=$ IFLM

DII = IXMAX + $1-$ ISX

$\mathrm{DX} 2=\mathrm{IXMAX}+1+\mathrm{ISX}$

IY1 $=$ IYMAX $+1-$ ISY

$I Y 2=I Y M A X+1+I S Y$

IIIIGH $=0$

DO $200 \mathrm{~J}=\mathrm{TY} 1$, TY2

DO $100 \mathrm{I}=\mathrm{DX} 1, \mathrm{LX} 2$

1 GO TO 100

$\mathrm{IHIGH}=\mathbf{I N}(\mathbf{I}, \mathbf{J})$

IDX $=$ I

IDY $=\mathbf{J}$

CONTINUE

CONTINUE

IDX $=$ IXMAX +1

$\mathrm{IDY}=$ IYMAX + 1

$\mathrm{IHIGH}=\mathrm{IN}(\mathrm{IDX}, \mathrm{DP})$

IF AIL $=-10$

RETURN

\section{LOGICAL"1 IVMODE}

GET AREA AROUND PREDICTED SPOT POSITION FROM MEMORY AND SMOOTH QUIT IF TOC CLOSE TO EDGE OF DETECTOR

IF ( DOMAX .GE. IP .OR. NX - DXMAX .LE. IP) IFAIL $=-110$

IF MMAX .GE. IQ .OR. NY - IYMAX .LE. IQ ) IFAIl $=-120$

IF ( FAIL .LT. 0) RETURN

IF( IVMODE .NE. 'D') CALL RDFBD( 0, IP - IXMAX,

1 IQ - TYMAX, IP + DXMAX, IQ + IYMAX, IN(1,1), ID1, ID2, ID3 )

IF( IVMODE .EQ. 'D') CALL RDIGIT( 0, IP - LXMAX,

$1 \mathrm{IQ}-\operatorname{TMMAX}, \mathrm{IP}+\mathrm{DMMX}, \mathrm{IQ}+\operatorname{TMMAX}, \mathrm{IN}(1,1))$

IF( IVMODE .EQ. 'V') PAUSE 'MODT = ' '

IF' IVMODE .EQ. 'D' ' PAUSE 'MODE $=$ ' '

DEFINE LOCAL SEARCH AREA

IF (ISX, GT. ISEE ) ISX = ISEE

IF (ISY .GT. ISEE ) ISY = ISEE

FIND LOCAL MAXIMUM IN LOCAL SEARCH AREA

IF( IN(I,J) .LE. IHIGH) GO TO 100

IF ( IN (I,J).LT. IN(I-1,J) .OR. IN(I,J) .LT. IN(I+1,J) .OR.

1 IN(I,J).LT. IN(I,J-1) .OR. IN(I,J) .LT. IN(I,J+1))

IF( IHIGH .GT, 0) GO TO 30

if no true local max default

! to predicted spot position

ROOM TO INTEGRATE SPOT ?, IF NOT,

GET AREA AROUND SPOT FRÓM MEMORY AND SMOOTH 
C

C

$\mathrm{C}$

C

50

C

$40 \quad \begin{aligned} & \text { IDX } \\ & =\text { DXMAX }+1 \\ & \text { IDY }=\text { IYMAX }+1\end{aligned}$

C

RETURN

END DO $50 \mathrm{I}=1$, ISM

IF( IDX .GT. INTXIM .AND. IDX+INTXIM .LT. 2*IXMAX .AND.

1 IDY .GT. INTYLM .AND. IDY+INTYLM .LT. 2*IYMAX ) RETURN

FF (XXMAX .GE. IP .OR. NX - DXMAX .LE. IP) IFAIL $=-110$

IF( IMMAX .GE. IQ .OR. NY - IYMAX .LE. IQ ) IFAIl $=-120$

IF ( IFAIL .LT. 0 ) RETURN

IF( IVMODE .NE. 'D') CALL RDFBD( 0, IP - IXMAX, $1 \mathrm{IQ}$ - TYMAX, IP + IXMAX, IQ + TYMAX, IN(1,1), ID1, ID2, ID3 ) IF ( IVMODE .EQ. 'D') CALL RDIGIT( 0, IP - IXMAX, 1 IQ - TYMAX, IP + DXMAX, IQ + IYMAX, IN $(1,1))$

IF( ISM .EQ. 0 ) GO TO 40

CALL SMOOTH ( IN $(1,1))$

C

SUBROUTINE BKGRND( IN )

C Median value background routine

C F. Ogletree $1 / 12 / 84$

C

COMMON /RESULT/ IP, IQ, IHIGH, IBACK, IWX, IWY, FFAIL, 1 ITOT, SUM, IPC, IQC, IDX, IDY

COMMON /BEAM/ IWX0, IWYO, INTXIM, INTYLM, NOISE, BGRAD, SNLIM

C COMMON /FDED/ NX, NY, DMMAX, IYMAX, IVMODE

DMMENSION IN $(51,51)$

INTEGER ICOUNT(256)

C

LOGICAL*1 IVMODE

C DETERMINE AREA LMITS

C

XXHI $=$ IDX $+2^{*}$ INTXLM

LXLO $=$ IDX $-2 *$ INTXLM

IYHI $=$ IDY $+2 *$ INTYLM

IYLO $=$ IDY $-2^{*}$ INTYLM

IF ( IXHI .GT. ${ }^{*}$ DXMAX) $\mathrm{DXHI}=2 *$ IXMAX

IF IYHI .GT. $2^{*}$ TYMAX $\{$ IYHI $=2 *$ IYMAX

IF (LXO ,LT. 2) LXLO = 2

C

IF( IYLO .LT. 2 ) IYLO = 2

C CALCULATE BACKGROUND - median value in AREA array IN

C

IDENOM $=2$ !only the $12 \mathrm{MS}$ bits from the $\mathrm{FB}$ are moved to $\mathrm{IN}$

DO $10 \mathrm{I}=1,256 \quad$ ! $\mathrm{max}$ bgrad is 512 of 4096

10 CONTINUE

C

DO $20 \mathrm{DX}=\mathrm{DX} \mathrm{C}, \mathrm{XXHI}$ 




SUBROUTINE XYSIZE( IN )

C Estimate spot size by searching radially from spot center

C IWX0, TWY0 is instrumental resolution half widtb

C IWX, IWY calculated half width

$\mathrm{C}$

COMMON /RESULT/ IP, IQ, IHIGH, IBACK, WX, IWY, IFAIL,

1 ITOT, SUM, IPC, IQC, IDX, IDY

COMMON /BEAM/ IWXO, IWYO, INTXIM, INTYLM, NOISE, BGRAD, SNLIM

$\mathrm{C}$

COMMON /FIXED/ NX, NY, IXMAX, IYMAX, IVMODE

C

DIMENSION IN $(51,51)$

LOGICAL"1 IVMODE

ITHRSH $=\operatorname{IFIX}($ FLOAT $($ IHIGH - BACK $) *$ BGRAD $)$

C

IF( ITHRSH .LT. NOISE) ITHRSH = NOISE

C CALCULATE 'SPOT WIDTH IN X

C

$\operatorname{WXX} 1=0$

ILOX $=$ IDX - 1

I2 = IN(IDX,IDY) - IBACK

200 II = IN ( I.OX, IDY ) - IBACK

IF( I1 .LE. ITHRSH .OR. I1 .GE. 12 + NOISE ) GO TO 210

$12=11$

$\Gamma W X 1=\Gamma W X 1+1$

ILOX $=$ LOX -1

$\mathrm{C}$

IF( II.OX.GT. 1 ) GO TO 200

$210 \quad \mathrm{WWX} 2=0$

IHDX $=\mathrm{IDX}+1$ 




SUBROUTINE SPOINT( IN, ITYPE)

C

COMMON /BEAM/ IVX0, IWY0, INTXLM, INTYLM, NOISE, BGRAD, SNLIM COMMON /RESULT/ IP, IQ, IHIGH, IBACK, IWX, IWY, IFAIL, 1 ITOT, SUM, IPC, IQC, IDX, IDY

C DIMENSION IN $(51,51)$

C INTEGER * 4 JSUM

CALL JICVT(0,JSUM) !spot integral ITOT $=0$ ?count points that contribute to sum

C

C INTEGRATE OVER AN ELLIPSE AROUND IP, IQ

C OF SEMI-AXIS INTXIM, INTYLM

C USING POINTS ABOVE BACKGROUND + NOISE

C

RA2 = FLOAT( INTXIM ** 2$)$

RAB $=$ RA2 / FLOAT( INTYLM * 2) 
C

NTEST $=$ IHIGH - BAACK

NSUB = BBACK + NOISE

IF (ITYPE .EQ. -2) NSUB $=0$

IF (ITYPE .EQ. -2 NTEST =0

DO $100 \mathrm{~J}=-$ INTYLM, INTYLM

$\operatorname{LXX}=\operatorname{IFLX}\left(\operatorname{SQRT}\left(\mathrm{RA} 2-\operatorname{RAB} * \operatorname{FLOAT}\left(\mathrm{J}^{* *} 2\right)+0.01\right)+0.5\right)$

DO $200 \mathrm{~K}=\mathrm{IDX}-\mathrm{IXX}, \mathrm{IDX}+\mathrm{DXX}$

$N=\mathrm{IN}(\mathbf{K}, \mathrm{J}+\mathrm{IDY})-\mathrm{NSUB}$

IF ( N .GT. NTEST) IFAIL $=-30$

IF (N .LT. 0) GO TO 200

GALL JA2T4( JSUM, N) !I*4 summation

200

ITOT $=$ ITOT +1

CONTINUE

100 CONTIVUE

C

SUM = AJFLT(JSUM)

C

IF ( SUM .LE. 0.0) IFAIL $=\mathbf{- 8 0}$

RETURN

END 


\section{Assembler code}

\section{;CALL SMOOTH( IN $(1,1))$}

;9 point smoothing routine for array $\mathrm{IN}(51,51)$, produces ;smoothed values inside a $\mathbf{4 9} \times \mathbf{4 9}$ block in IN.

;

SMOOTH:: ;iN(i+1,j)

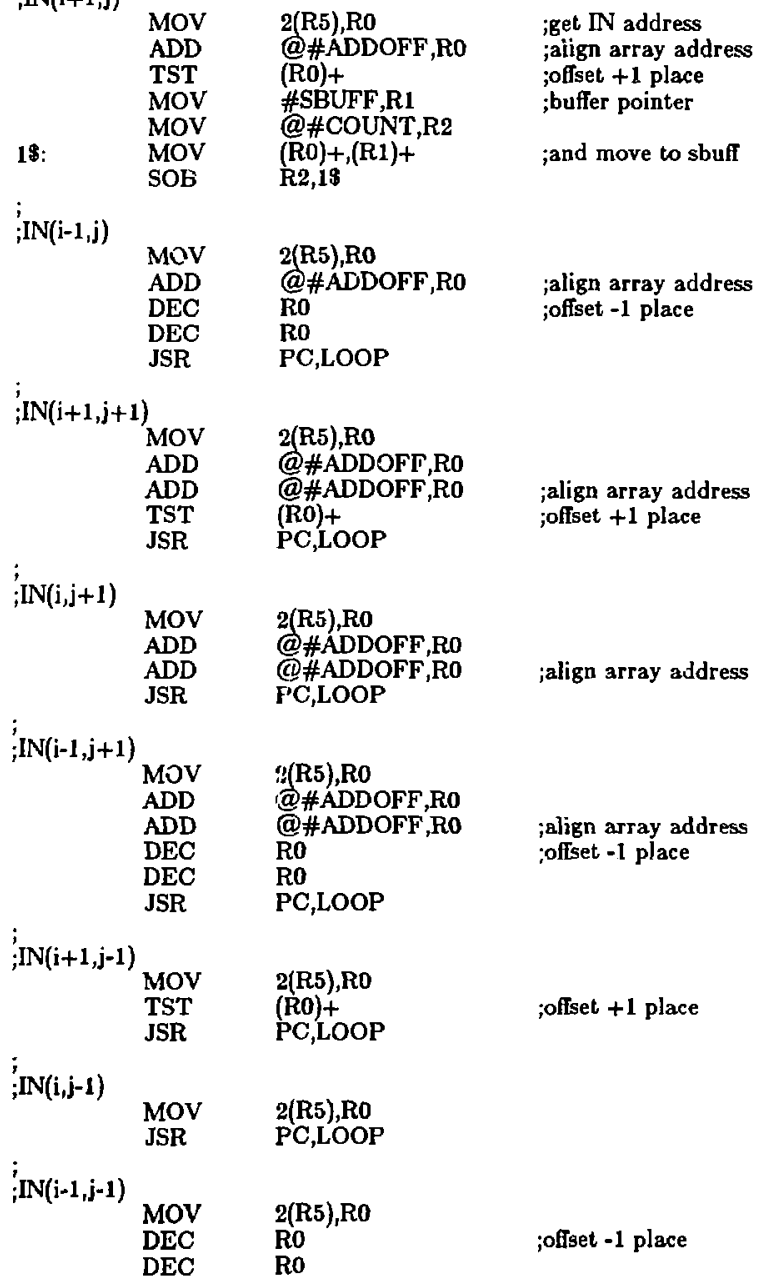




\begin{tabular}{|c|c|c|c|}
\hline & JSR & PC,LOOP & \\
\hline ' & $\begin{array}{l}\text { MOV } \\
\text { ADD } \\
\text { MOV } \\
\text { MOV }\end{array}$ & $\begin{array}{l}\text { 2(R5),R0 } \\
\text { @\#ADDOFF,R0 } \\
\text { \#SBIJF,R1 } \\
\text { @\#COUNT,R2 }\end{array}$ & $\begin{array}{l}\text {;calculate smoothed matrix element } \\
\text {;align array address }\end{array}$ \\
\hline 58: & $\begin{array}{l}\text { MOV } \\
\text { ASH } \\
\text { ADD } \\
\text { ASR } \\
\text { MOV } \\
\text { SOB }\end{array}$ & $\begin{array}{l}\text { (R1),R3 } \\
\# 75, \mathrm{R3} \\
(\mathrm{R} 0)+, \mathrm{R3} \\
\mathrm{R3} \\
\mathrm{R3},(\mathrm{R} 1)+ \\
\mathrm{R2}, \mathbf{5 3}\end{array}$ & $\begin{array}{l}\text {;get sum of adjacent matrix elements } \\
\text {; then divide by } 8 \text {. for weighted average } \\
\text {;add central element } \\
\text {;divide by } 2 \\
\text {;and store result }\end{array}$ \\
\hline ; & $\begin{array}{l}\text { MOV } \\
\text { ADD } \\
\text { MOV } \\
\text { MOV }\end{array}$ & $\begin{array}{l}\text { 2(R5),R0 } \\
\text { @\#ADDOFF,Ro } \\
\text { \#SBUFF,R1 } \\
\text { @\#ROW,R4 }\end{array}$ & $\begin{array}{l}\text {;skip first row } \\
\text {;move sbuff back to IN } \\
\text {;move next } 49 \text { rows }\end{array}$ \\
\hline 48: & $\begin{array}{l}\text { TST } \\
\text { TST } \\
\text { MOV }\end{array}$ & $\begin{array}{l}\mathrm{R} 0)+ \\
\mathrm{R} 1)_{+}\end{array}$ & ;skip first place \\
\hline 38: & $\begin{array}{l}\text { MOV } \\
\text { SOB } \\
\text { TST } \\
\text { TST }\end{array}$ & $\begin{array}{l}(\mathrm{Q} 1)+(\mathrm{RO})+ \\
\mathrm{R2}, 3 \mathbf{3} \\
(\mathrm{R} 0)+ \\
(\mathrm{R} 1)+\end{array}$ & $\begin{array}{l}\text {;move next } 49 \text { places } \\
\text {;skip last place }\end{array}$ \\
\hline ; & SOB & $\mathbf{R 4}, \mathbf{4 8}$ & ;loop over rows \\
\hline ', & RTS & $\mathrm{PC}$ & \\
\hline LOOP: & $\begin{array}{l}\text { MOV } \\
\text { MOV }\end{array}$ & $\begin{array}{l}\text { \#SBUFF,R1 } \\
\text { @\#COUNT,R2 }\end{array}$ & \\
\hline 28: & $\begin{array}{l}\text { ADD } \\
\text { SOB } \\
\text { RTS }\end{array}$ & $\begin{array}{l}(\mathrm{R} 0)+,(\mathrm{R} 1)+ \\
\mathrm{R} 2,2 \$ \\
\mathrm{PC}\end{array}$ & \\
\hline $\begin{array}{l}\text { COUNT: } \\
\text { ADDOFF: } \\
\text { ROW: } \\
\text { SBUFF: }\end{array}$ & $\begin{array}{l}\text { WORD } \\
\text {.WORD } \\
\text { WORD } \\
\text {.BLKW }\end{array}$ & $\begin{array}{l}2499 . \\
102 . \\
49 . \\
2499 .\end{array}$ & \\
\hline
\end{tabular}









\begin{tabular}{|c|c|c|c|}
\hline & $\begin{array}{l}\text { MOV } \\
\text { BIC } \\
\text { ADD }\end{array}$ & $\begin{array}{l}@ 2(\mathrm{Rs}), \mathrm{R} 1 \\
\# 177774, \mathrm{R} 1 \\
\# 12 ., \mathrm{R1}\end{array}$ & $\begin{array}{l}\text { ID NUMBER } \\
\text {; RANGE O TO } 3 \\
\text {; USE CHANNELS } 12 . \text { TO } 15 .\end{array}$ \\
\hline & $\begin{array}{l}\text { CMP } \\
\text { BEQ }\end{array}$ & $\begin{array}{l}\#-1, @ 4(R 5) \\
13\end{array}$ & ;PURGE? \\
\hline 1\$: & $\begin{array}{l}\text { CLOSE } \\
\text { RETURN } \\
\text {.PURGE } \\
\text { RETURN }\end{array}$ & $\begin{array}{l}\text { R1 } \\
\text { R1 }\end{array}$ & \\
\hline GETBLK:: & $\begin{array}{l}\text { CLRB } \\
\text { BR }\end{array}$ & $\begin{array}{l}\text { FLAG } \\
\text { START }\end{array}$ & ; CALI, GETBLK(D,BUFFER,NSTART,NBLOCK) \\
\hline PUTBLK:: & MOVB & $\# 1$, FLAG & ; CALL PUTBLK(ID,BUFFER,NSTART,NBLOCK) \\
\hline START: & $\begin{array}{l}\text { MOV } \\
\text { BIC } \\
\text { ADD }\end{array}$ & $\begin{array}{l}@ 2(\mathrm{R} 5), \mathrm{R} 1 \\
\# 177774, \mathrm{R} 1 \\
\# 12 . \mathrm{R} 1\end{array}$ & $\begin{array}{l}\text {; ID NUMBER } \\
\text {; RANGE 0 TO } 3 \\
\text {; USE CHANNELS 12. TO } 15 .\end{array}$ \\
\hline & MOV & A(R5),R2 & ;BUFFER ADDRESS \\
\hline & $\begin{array}{l}\text { MOV } \\
\text { SWAB } \\
\text { CLRB }\end{array}$ & $\begin{array}{l}@_{10}(\mathrm{R} 5), \mathrm{R3} \\
\mathrm{R3} \\
\mathrm{R3}\end{array}$ & $\begin{array}{l}\text { ¡BLOCKS TO DO } \\
\text {;WORDS TO DO }\end{array}$ \\
\hline & MOV & $@ 6(\mathrm{R} 5), \mathrm{R4}$ & ;STARTING BLOCK \\
\hline & $\begin{array}{l}\text { TSTB } \\
\text { BNE }\end{array}$ & $\begin{array}{l}\text { FLAG } \\
13\end{array}$ & \\
\hline 1\$: & $\begin{array}{l}\text {.READW } \\
\text { BCS } \\
\text { RETURN } \\
\text {.WRITW } \\
\text { BCS } \\
\text { RETURN }\end{array}$ & $\begin{array}{l}\text { \#AREA,R1,R2,R3,R4 } \\
\text { HRDERR } \\
\text { \#AREA,R1,R2,R3,R4 } \\
\text { HRDERR }\end{array}$ & \\
\hline HRDERR: & $\begin{array}{l}\text { MOVB } \\
\text { ADD } \\
\text { MOVB } \\
\text {.PRINT } \\
\text {.EXIT }\end{array}$ & $\begin{array}{l}\text { @\#ERRBYT,R1 } \\
\text { \#60,R1 } \\
\text { R1,ERR1 } \\
\text { \#ERR }\end{array}$ & $\begin{array}{l}\text {;WHICH ERROR } \\
\text {;ASCII DIGIT } \\
\text {;ERROR MESSAGE }\end{array}$ \\
\hline VSETUP:: & $\begin{array}{l}\text { MOV } \\
\text { BIC }\end{array}$ & $\begin{array}{l}\text {;CALL VSETUP( ID, I } \\
\text { @2(R5),R1 } \\
\# 177774, \mathrm{R} 1\end{array}$ & $\begin{array}{l}\text { IIZE, JSIZE, ITYPE ) } \\
\text {;GET ID \# } \\
\text {;MASK ID \# }\end{array}$ \\
\hline & $\begin{array}{l}\text { MOV } \\
\text { MOV } \\
\text { DEC } \\
\text { ASH }\end{array}$ & $\begin{array}{l}@ 6(\mathrm{R} 5), \mathrm{R2} \\
@ 10(\mathrm{R} 5), \mathrm{R} 3 \\
\mathrm{R3} \\
\mathrm{R3}, \mathrm{R2}\end{array}$ & $\begin{array}{l}\text {;NUMBER ELEMENTS FOR 2ED DIMENSION } \\
\text {;WORDS/ELEMENT } \\
\text {;NUMBER WORDS FOR 2ED DRMENSION }\end{array}$ \\
\hline & $\begin{array}{l}\text { MOV } \\
\text { ASH } \\
\text { ADD } \\
\text { MOV }\end{array}$ & $\begin{array}{l}\text { R1,R4 } \\
\# 2, R 4 \\
\# \text { JSIZE,R4 } \\
\text { R2,(R4) }\end{array}$ & $\begin{array}{l}\text {;FILE \# } \\
\text {;WORDS OFFSET } \\
\text {;GET ADDRESS } \\
\text {;STORE 2ED DIMENSION }\end{array}$ \\
\hline
\end{tabular}




\begin{tabular}{|c|c|c|c|}
\hline  & $\begin{array}{l}\text { MOV } \\
\text { MOV }\end{array}$ & $\begin{array}{l}\text { R3,2(R4) } \\
(\mathrm{RA}), @ 6(\mathrm{R} 5)\end{array}$ & $\begin{array}{l}\text {;AND WORDS/ELEMENT } \\
\text {;RETURN JSIZE IN WORDS }\end{array}$ \\
\hline & $\begin{array}{l}\text { MOV } \\
\text { MUL } \\
\text { BCS }\end{array}$ & $\begin{array}{l}@ 4(\mathrm{R5}), \mathrm{R2} \\
(\mathrm{R4}), \mathbf{R 2} \\
\text { DIMERR }\end{array}$ & $\begin{array}{l}\text {;GET 1ST DRMENSION } \\
\text {;WORDS IN ARRAY } \\
\text {; }>\text { 64K, ERROR }\end{array}$ \\
\hline & $\begin{array}{l}\text { MOVB } \\
\text { CLRB } \\
\text { SWAB } \\
\text { TST } \\
\text { BEQ }\end{array}$ & $\begin{array}{l}\text { R3,R2 } \\
\text { R3 } \\
\text { R3 } \\
\text { R2 } \\
\text { 18 }\end{array}$ & $\begin{array}{l}\text {;LOW BYTE WORDS } \\
\text {;HIGH BYTE BLOCKS } \\
\text {;\# BLOCKS NEEDED } \\
\text {;LEFT OVER WORDS? }\end{array}$ \\
\hline ;!\$: & $\begin{array}{l}\text { INC } \\
\text { MOV }\end{array}$ & $\begin{array}{l}\text { R3 } \\
\text { R3,@4(R5) }\end{array}$ & $\begin{array}{l}\text {;ONE MORE BLOCK } \\
\text { :RETURN \# BLOCKS }\end{array}$ \\
\hline 18: & $\begin{array}{l}\text { CALL } \\
\text { RETURN }\end{array}$ & IN1 & ;OPEN FILE \\
\hline DIMERR: & $\begin{array}{l}\text { MOVB } \\
. \text { PRINT } \\
\text { EXTT }\end{array}$ & $\begin{array}{l}\mathrm{R} 1, \mathrm{DMM} 1 \\
\text { \#DIM }\end{array}$ & \\
\hline VARRAY:: & MOVB & $\begin{array}{l}\text {;CALL VARRAY( ID, I, } \\
\text { @14(R5),FLAG }\end{array}$ & $\begin{array}{l}\text { J, IVALUE, N, IFLAG ) } \\
; 0 \text { FOR READ, } 1 \text { FOR WRITE }\end{array}$ \\
\hline & $\begin{array}{l}\text { MOV } \\
\text { BIC } \\
\text { MOV } \\
\text { ADD }\end{array}$ & $\begin{array}{l}@ 2(\mathrm{R5}), \mathrm{R4} \\
\# 177774, \mathrm{R4} \\
\mathrm{R4}, \mathrm{CHAN} \\
\# 12 . \text { CHAN }\end{array}$ & $\begin{array}{l}\text {;GET ID \# } \\
\text {;MASK ID \# } \\
\text {;SAVE IT } \\
\text {;CHANNEL \# }\end{array}$ \\
\hline & $\begin{array}{l}\text { ASH } \\
\text { ADD } \\
\text { MOV }\end{array}$ & $\begin{array}{l}\# 2, R 4 \\
\text { \#JSIZE,R4 } \\
(\mathrm{R4})+, \mathrm{R2}\end{array}$ & $\begin{array}{l}\text {;WORDS OFFSET } \\
\text {;GET ADDRESS } \\
\text {;GET JSIZE }\end{array}$ \\
\hline r & $\begin{array}{l}\text { MOV } \\
\text { DEC } \\
\text { MUL } \\
\text { BCS }\end{array}$ & $\begin{array}{l}@ 4(\mathrm{R5}), \mathrm{RO} \\
\text { R0 } \\
\text { R0,R2 } \\
\text { DIMERR }\end{array}$ & $\begin{array}{l}\text {;GET I } \\
\text {;START FROM } 0 \\
\text {;> 64K, ERROR }\end{array}$ \\
\hline & $\begin{array}{l}\text { MOV } \\
\text { DEC } \\
\text { ASH } \\
\text { ADD }\end{array}$ & $\begin{array}{l}@ 6(\mathrm{R} 5), \mathrm{R2} \\
\mathrm{R2} \\
\text { (R4),R2 } \\
\mathrm{R2}, \mathrm{R} 3\end{array}$ & $\begin{array}{l}\text {;GET J } \\
\text {;START FROM O } \\
\text {;WORDS/ELEMENT } \\
\text {;OFFSET OF VALUE }\end{array}$ \\
\hline & $\begin{array}{l}\text { MOV } \\
\text { CLRB } \\
\text { SUB } \\
\text { SWAB } \\
\text { MOV }\end{array}$ & $\begin{array}{l}\text { R3,R2 } \\
\text { R2 } \\
\text { R2,R3 } \\
\text { R2 } \\
\text { R2,BLIK }\end{array}$ & $\begin{array}{l}\text {;OFFSET IN BLOCK } \\
\text {;STARTING BLOCK }\end{array}$ \\
\hline ; & $\begin{array}{l}\text { MOV } \\
\text { ASH } \\
\text { MOV }\end{array}$ & $\begin{array}{l}@ 12(\mathrm{R} 5), \mathrm{R} 1 \\
(\mathrm{R} 4), \mathrm{R} 1 \\
\text { 10(R5),R4 }\end{array}$ & $\begin{array}{l}\text {;NUMBER ELEMENTS } \\
\text {;WORD COUNT } \\
\text {;/OO POINTER }\end{array}$ \\
\hline , & $\begin{array}{l}\text { MOV } \\
\text { ADD } \\
\text { CMP }\end{array}$ & $\begin{array}{l}\text { R3,WORDS } \\
\text { R1,WORDS } \\
\text { WORDS,\#256. }\end{array}$ & $\begin{array}{l}\text {;WORDS TO READ FROM DISK } \\
\text {;MORE THLAN ONE BLOCK? }\end{array}$ \\
\hline & $\mathrm{BHI}$ & MORE & \\
\hline
\end{tabular}




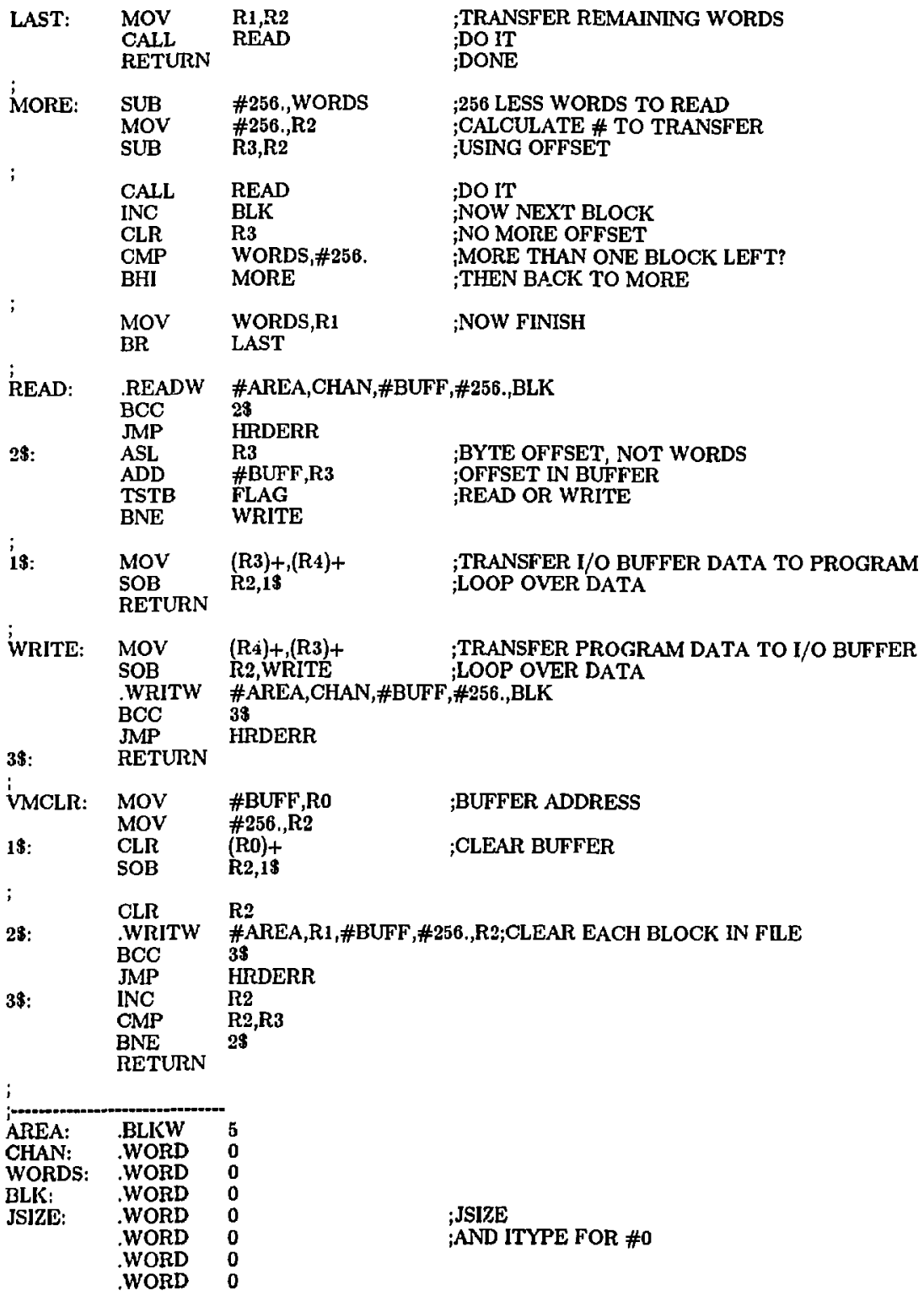




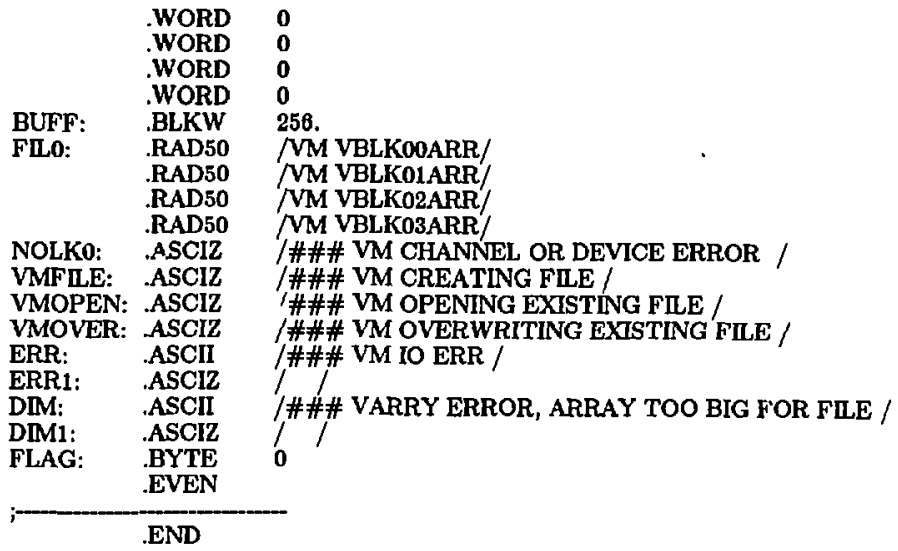

